\title{
Zur gekoppelten numerischen Modellierung von unterirdischem Hochwasser
}

\author{
Von der Fakultät für Bauingenieurwesen \\ der Rheinisch-Westfälischen Technischen Hochschule Aachen \\ zur Erlangung des akademischen Grades \\ eines Doktors der Ingenieurwissenschaften \\ genehmigte Dissertation
}

vorgelegt von

Bernhard Peter Josef Becker

Berichter: Universitätsprofessor Dr.-Ing. Jürgen Köngeter

Professeur dr ir Michel Pirotton

Universitätsprofessor Dr.-Ing. Holger Schüttrumpf

Tag der mündlichen Prüfung: 11. Juni 2010

Diese Dissertation ist auf den Internetseiten der Hochschulbibliothek online verfügbar. 




\section{Kurzfassung}

Unterirdisches Hochwasser ist ein Grundwasseranstieg, der durch Hochwasser in einem Fluss ausgelöst wird. Während im Fluss die Hochwasserwelle relativ schnell wieder abklingt, dauert der Rückgang des unterirdischen Hochwassers in der Regel länger an. Wesentliche nachteilige Folgen des unterirdischen Hochwassers sind Schäden an Gebäuden und an Infrastrukturbauwerken durch erhöhte Auftriebskräfte und in das Gebäude eindringendes Wasser.

An der Oberfläche kann die Wasserbewegung mit Hilfe der Navier-Stokes-Gleichungen oder Vereinfachungen dieser Gleichungen beschrieben werden. Für die Modellierung des Wassers auf einer Überflutungsfläche wird das Strömungsprogramm Ilmoflood vorgestellt. Im Untergrund gelten die Richards-Gleichung und die Grundwasserströmungsgleichung. Diese beiden Gleichungen sind im Programm Feflow umgesetzt.

Zwischen dem Wasser an der Oberfläche und dem Grundwasser wirken als Wechselwirkungsprozesse die Uferspeicherung, die Infiltration von einer überfluteten Fläche und der Qualmwasseraustritt. Wegen dieser Wechselwirkungsprozesse ist zur Prognose der Ausbreitung unterirdischen Hochwassers eine gekoppelte Modellierung erforderlich. Die Umsetzung einer solchen Modellkopplung für die beiden Programme Feflow und Ilmoflood mit Hilfe des OpenMI-Standards bildet einen Schwerpunkt der Arbeit. Die besondere Schwierigkeit besteht darin, das kommerzielle Programm Feflow mit einer OpenMI-Schnittstelle auszurüsten, ohne den Programmcode zu verändern. Dies gelingt mit Hilfe von Methoden der Netzwerkprogrammierung, die über die Feflow-Programmierschnittstelle implementiert werden. Damit ist es nun möglich, bereits bestehende Grundwassermodelle, die zum Beispiel für die Begleitung der Wasserversorgung entwickelt wurden, im Rahmen des Hochwasserschutzes einzusetzen.

Vor diesem Hintergrund werden verfügbare Programmfunktionen auf ihre Eignung zur Modellierung der Wechselwirkungsprozesse hin untersucht. Dazu werden für den Prozess der Uferspeicherung Felddaten eines in der Fachliteratur dokumentierten Uferspeicherungsereignisses analysiert und darauf aufbauend numerische Modelluntersuchungen durchgeführt. Für die Infiltration aus einer überfluteten Fläche werden analytische Infiltrationsformeln und die numerische Lösung der Richards-Gleichung ausgewertet. Für Qualmwasser stehen modelltechnische Aspekte im Vordergrund der Untersuchung. Wesentliche Ergebnisse sind, dass die Uferspeicherung gut mit den bestehenden Leakage-Funktionen in einem Grundwassermodell erfasst werden kann. Für die Infiltration von Wasser aus einer Überflutungsfläche sind Leakage-Ansätze eine Vereinfachung, aber mit Einschränkungen der Modellaussage für die vorliegende Fragestellung verwendbar.

Mehrere Anwendungsbeispiele dienen dazu, das Prozessverständnis des unterirdischen Hochwassers zu vertiefen und die Funktionsweise der Modellkopplung zu erläutern. 


\section{Résumé}

Une crue souterraine est par définition une hausse du niveau de la nappe phréatique induite par une élévation du niveau d'un cours d'eau. Alors que l'onde de crue s'estompe relativement rapidement dans le fleuve ou la rivière, la crue souterraine persiste généralement plus longtemps. Les conséquences indésirables qui peuvent accompagner une crue souterraine sont l'endommagement des bâtiments et d'autres ouvrages structurels causé par la force de poussée verticale exercée par la nappe ainsi que la pénétration d'eau au sein des habitations.

Le mouvement des eaux de surface est décrit mathématiquement par les équations de Navier-Stokes ou par des simplifications de celles-ci. Le programme Ilmoflood est présenté pour modéliser le mouvement de l'eau dans la zone d'inondation. Les équations de Richards et des écoulements souterrains sont appropriées pour décrire les écoulements souterrain. Ces équations sont implémenté au programme Feflow.

Plusieurs processus d'interactions lient les eaux de surface aux eaux souterraines telles que la capacité de stockage des rives, l'infiltration de l'eau dans les zones inondées et le resurgence des eaux venant de la nappe. Compte tenu de ces interactions, une modélisation couplée est nécessaire pour prévoir correctement la propagation de la crue souterraine. Cette thèse met ainsi un accent particulier sur le couplage des deux programmes Feflow et Ilmoflood à l'aide du standard OpenMI. Dans ce but, implémenter ce standard dans le code commercial Feflow sans en modifier le code source fut la principale difficulté. Celle-ci fut surmontée grâce aux méthodes de programmation en réseau qui sont implémentées via l'interface de programmation de Feflow. Il est dès lors possible d'utiliser pour la protection contre l'inondation des modèles d'eau souterraines déjà existants et développés pour d'autres applications telles que l'approvisionnement en eau.

Dans ce contexte, nous étudions ici les méthodes les plus adéquates pour modéliser les processus d'interactions à l'aide des fonctions disponibles dans les différents programmes. Un évènement de stockage de rive documenté dans la littérature est analysé et comparé avec les résultats d'une étude numérique. Le processus d'infiltration des eaux est analysé sur base de modèle d'infiltration analytique et de la solution numérique de l'équation de Richards. Pour le resurgence des eaux, des aspects de simulation numérique sont étudiés. Les résultats essentiels sont que le stockage de rive peut-être modélisé via l'imposition de conditions aux limites de type «leakage» qui sont déjà disponibles dans le programme Feflow. En ce qui concerne l'infiltration verticale dans une zone d'inondation, l'approche par conditions aux limites «leakage» représentent une simplification du phénomène réel. Eu égard à l'objectif de cette recherche, elles restent néanmoins adéquate sous certaines restrictions.

Quelques exemples d'application servent à approfondir la compréhension des processus d'une crue souterraine et à décrire le mode de fonctionnement du couplage des modèles. 


\section{Abstract}

A subsurface flood is defined as a groundwater head rise induced by high water in a river. While in the river the flood wave decays comparatively fast, the duration of the subsurface event is usually longer. Substantial unfavourable consequences of the underground flood is damage to buildings and infrastructure caused by increased uplift forces and the penetration of water into buildings.

At the surface, the water movement can be mathematically described by the Navier-Stokes equations or simplifications of these equations. As a programme to model the movement of water on an inundated area, Ilmoflood is presented. For the subsurface, the Richards equation and the groundwater flow equation are appropriate. The Feflow groundwater simulation programme solves these two equations numerically.

Between the water at the land surface and groundwater, bank storage, infiltration of water from an inundated area and return flow act as interchange processes. Thus, in order to predict the subsurface flood propagation, it is necessary to couple a surface flow model with a groundwater model. One focus of this dissertation work is the model coupling of Feflow and Ilmoflood with the OpenMI standard. The main challenge was to implement this standard into the commercial programme Feflow without changing the source code. This has been achieved with the help of network programming methods implemented into the Feflow programming interface. It is now possible, to use groundwater models already existing, which for example have been developed for water supply purpose, for the planning of flood protection measures.

Against this background, it is investigated how the interchange processes can be modelled best with the available programme functions. For the bank storage process, field data from the literature for one subsurface flood event is analysed and in addition, numerical model investigations are carried out. The infiltration process is analysed with the help of analytical infiltration models and the numerical solution of the Richards equation. For the return flow, modelling aspects are discussed. Key findings are, that the bank storage can be modelled well with the existing leakage boundary condition in a groundwater model. For the vertical infiltration from an inundation area, leakage approaches are a simplified representation of the process, but considering these limitations, they are appropriate for the objective of this investigation.

Several application cases are presented to give a better understanding of the subsurface flood processes and to explain, how the model coupling works. 


\section{Inhaltsverzeichnis}

\begin{tabular}{|ll}
\hline Abbildungsverzeichnis & XI
\end{tabular}

\begin{tabular}{lll}
\hline Tabellenverzeichnis & XVI
\end{tabular}

\begin{tabular}{lll}
\hline Symbolverzeichnis & XVII
\end{tabular}

$\begin{array}{lll}\mathbf{1} \text { Einleitung } & \mathbf{1}\end{array}$

1.1 Hochwasser und unterirdisches Hochwasser . . . . . . . . . . . . . . . . 1

1.2 Problemstellung . . . . . . . . . . . . . . . . . . . . . 6

$1.3 \quad$ Zielsetzung und Vorgehensweise $\ldots \ldots \ldots \ldots \ldots$

\begin{tabular}{|lll}
\hline 2 & Die Bewegung des Wassers im Untergrund & 14
\end{tabular}

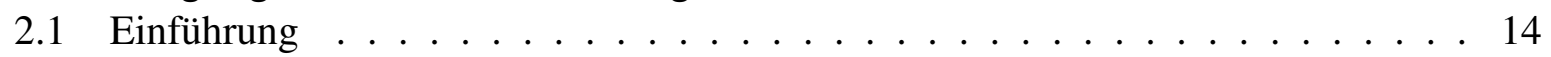

2.2 Die Bewegung des Wassers in der gesättigten Zone . . . . . . . . . . . . . . 16

2.3 Die Bewegung des Wassers in der ungesättigten Zone . . . . . . . . . . . . . . . 20

2.4 Modellierung der Bewegung des Wassers im Untergrund mit Feflow . . . . . . . 23

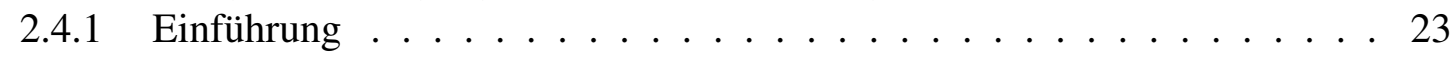

2.4 .2 Mathematische Grundlagen . . . . . . . . . . . . . . . . . 25

2.4 .3 Diskretisierung, Anfangs- und Randbedingungen . . . . . . . . . . . 26

2.5 Zusammenfassung des Kapitels . . . . . . . . . . . . . . . . . . . . . . . . 29

$\begin{array}{|lll|}3 & \text { Die Bewegung des Wassers an der Oberfläche } & 31\end{array}$

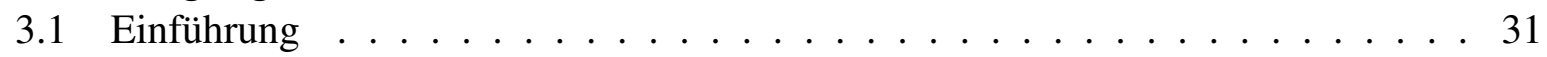

3.2 Die Navier-Stokes-Gleichungen $\ldots \ldots \ldots \ldots$. . . . . . . . . . . . . . 32

$3.3 \quad$ Zweidimensional-tiefenintegrierte Strömungsgleichungen . . . . . . . . . . . . 33

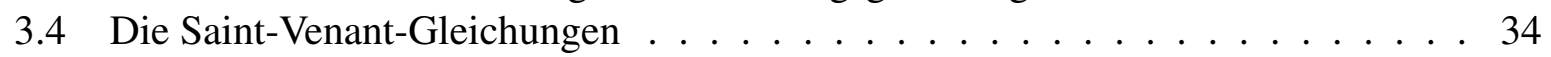

3.5 Das Modell der kinematischen Welle . . . . . . . . . . . . . . . . . . . 35

3.6 Das Modell der Diffusionswelle $\ldots \ldots \ldots \ldots$. . . . . . . . . . . . 35

3.7 Modellierung der Wasserbewegung an der Landoberfläche mit Ilmoflood . . . . . 35

$3.7 .1 \quad$ Allgemeine Beschreibung des Programms . . . . . . . . . . . . 35

3.7.2 Grundlagen des zweidimensionalen Überflutungsflächenmoduls . . . . . 36

3.8 Zusammenfassung des Kapitels . . . . . . . . . . . . . . . . . 38

4 Kopplung von Strömungsmodellen 39

4.1 Einführung . . . . . . . . . . . . . . . . . . . . . . . . . . . . 39

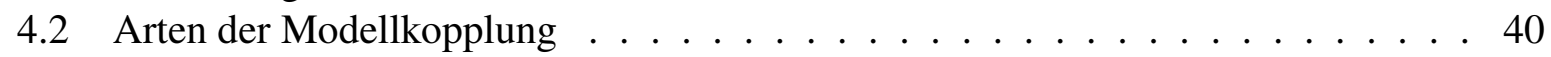

4.2 .1 Vollständig implizite Kopplung . . . . . . . . . . . . . . . . 40 
4.2 .2 Iterative Kopplung $\ldots \ldots$. . . . . . . . . . . . . . . . 40

4.2 .3 Externe Kopplung . . . . . . . . . . . . . . . . . . . . . . . . . . . . . . . . . . . . . . . 43

4.3 Auswahl einer Kopplungsart $\ldots \ldots \ldots$. . . . . . . . . . . . . . . . . . 43

4.4 OpenMI $\ldots \ldots \ldots \ldots \ldots \ldots \ldots \ldots \ldots$

$4.4 .1 \quad$ Allgemeines zum OpenMl-Standard . . . . . . . . . . . . . . . . . . 44

4.4 .2 Datenaustausch über OpenMI . . . . . . . . . . . . . . . . . 45

4.5 Zusammenfassung des Kapitels . . . . . . . . . . . . . . . . . . . . . . . . 48

5 Uferspeicherung 49

5.1 Einführung $\ldots \ldots \ldots \ldots \ldots \ldots \ldots$

5.2 Leakage-Ansätze . . . . . . . . . . . . . . . . . . . . . . 50

5.3 Zur Bestimmung des Leakage-Flusses $\ldots \ldots \ldots$. . . . . . . . . . . . . 55

5.4 Beispiel: Rheinhochwasser von 1983 im Neuwieder Becken . . . . . . . . . . . 58

5.4 .1 Zur Geologie des Neuwieder Beckens . . . . . . . . . . . . . . . 58

5.4 .2 Das Hochwasserereignis von $1983 \ldots \ldots$. . . . . . . . . . . . . . . . . . . . . . . . . . 58

$5.4 .3 \quad$ Leakage-Beziehung . . . . . . . . . . . . . . . . . . . . . 59

5.4 .4 Numerische Modellrechnungen . . . . . . . . . . . . . . . . 65

5.4.4.1 Einführung und Vorgehensweise . . . . . . . . . . . . . 65

5.4 .4 .2 Auswertung der Modellrechnungen . . . . . . . . . . . . 67

5.4 .5 Schlussfolgerungen und Diskussion . . . . . . . . . . . . . . 74

5.5 Zusammenfassung des Kapitels . . . . . . . . . . . . . . . . . . . . . 79

6 Infiltration aus einer Überflutungsfläche 81

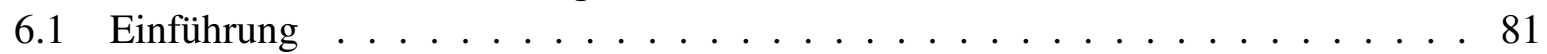

6.2 Mathematische Modelle für die Infiltration unter Einstau $\ldots \ldots$. . . . . . . . . 82

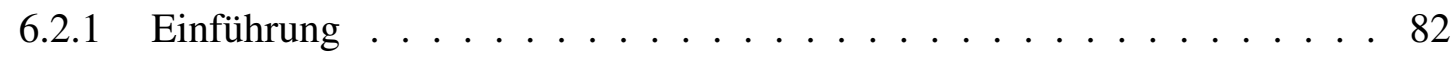

6.2 .2 Die Richards-Gleichung . . . . . . . . . . . . . . . . . . . . . . 83

$6.2 .3 \quad$ Das Infiltrationsmodell von Green und Ampt . . . . . . . . . . . . . . . 84

$6.2 .4 \quad$ Die Infiltrationsformeln von Haverkamp . . . . . . . . . . . . . . . 85

6.2 .5 Zur Berechnung der Sorptivität _ . . . . . . . . . . . . . . . 86

6.3 Auswertung des Haverkamp-Modells zur Frage des Fortschritts einer Sätti-

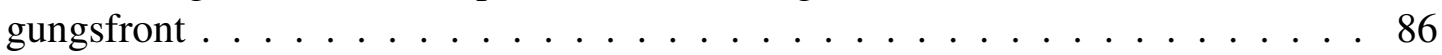

6.4 Möglichkeiten zur Abbildung der Infiltration unter Einstau in numerischen Mo-

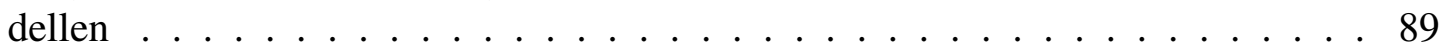

$6.4 .1 \quad$ Einführung . . . . . . . . . . . . . . . . . . . . . 89

6.4.2 Ungesättigte Modellierung des Infiltrationsvorgangs mit Lösung der Richards-Gleichung .......................... 91

$6.4 .3 \quad$ Vereinfachung durch gesättigte Modellierung der Infiltration . . . . . . . 96

6.4 .4 Schlussfolgerungen und Diskussion . . . . . . . . . . . . . . . 100

6.5 Zusammenfassung des Kapitels . . . . . . . . . . . . . . . . . . . . 102

7 Qualmwasseranstieg 103

7.1 Einführung . . . . . . . . . . . . . . . . . . 103 


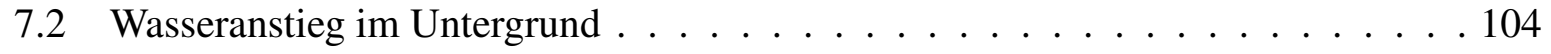

7.3 Modellierung des Wasseraustritts an der Landoberfläche . . . . . . . . . . . . 106

7.4 Zusammenfassung des Kapitels . . . . . . . . . . . . . . . . . . . . . . 107

8 Anwendungsbeispiele der Modellkopplung $\quad 108$

$8.1 \quad$ Einführung . . . . . . . . . . . . . . . . . . . . . . 108

8.2 Uferspeicherung und Infiltration $\ldots \ldots \ldots$. . . . . . . . . . . . . . . . . . . . . . . . . . . . . . . . . . . . . . .

$8.2 .1 \quad$ Einführung . . . . . . . . . . . . . . . . . . . . 108

$8.2 .2 \quad$ Rein gesättigte Modellierung der Grundwasserströmung . . . . . . . . . 111

8.2.2.1 Grundwassermodell und Modellkopplung . . . . . . . . . . . 111

$8.2 .2 .2 \quad$ Berechnungsergebnisse . . . . . . . . . . . . . . 112

8.2.2.3 Modelltechnische Aspekte der Kopplung . . . . . . . . . . . . 114

8.2 .3 Ungesättigte Grundwasserströmung . . . . . . . . . . . . . . . . . . . . 118

8.2.3.1 Grundwassermodell und Modellkopplung . . . . . . . . . . . 118

$8.2 .3 .2 \quad$ Berechnungsergebnisse . . . . . . . . . . . . . . . 119

8.2 .4 Schlussfolgerungen und Diskussion . . . . . . . . . . . . . . . . . . 124

8.3 Qualmwasser und Infiltration . . . . . . . . . . . . . . . . . . . . 125

8.3 .1 Einführung . . . . . . . . . . . . . . . . . . 125

8.3 .2 Modellaufbau . . . . . . . . . . . . . . . . . . 126

$8.3 .3 \quad$ Berechnungsergebnisse . . . . . . . . . . . . . . . . . . . 128

$8.3 .4 \quad$ Schlussfolgerungen und Diskussion . . . . . . . . . . . . . . . 133

8.4 Fallbeispiel $\ldots \ldots \ldots \ldots \ldots \ldots$

$8.4 .1 \quad$ Einführung . . . . . . . . . . . . . . . . . . . . . 137

$8.4 .2 \quad$ Modellaufbau und Modellkopplung . . . . . . . . . . . . . . . . . 138

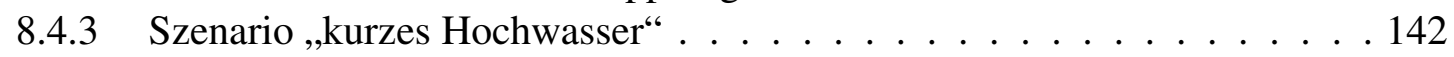

8.4.3.1 Rand- und Anfangsbedingungen . . . . . . . . . . . . . . 142

8.4.3.2 Ergebnisse . . . . . . . . . . . . . . . . . . 144

8.4 .4 Szenario ,langes Hochwasser" ${ }^{\circ}$. . . . . . . . . . . . . . . . 150

$8.4 .5 \quad$ Szenario ,langes Hochwasser und hoher Anfangswasserstand“" . . . . . . 152

8.4 .6 Schlussfolgerungen und Diskussion . . . . . . . . . . . . . . . 156

8.5 Zusammenfassung des Kapitels . . . . . . . . . . . . . . . . . . 158

9 Zusammenfassung der wesentlichen Ergebnisse, Bewertung und Ausblick $\quad 160$

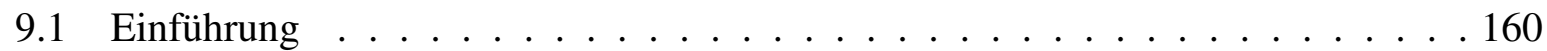

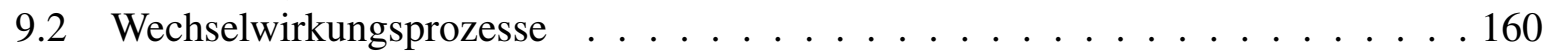

9.3 Modellkopplung . . . . . . . . . . . . . . . . . . . . . 162

9.4 Einsatz gekoppelter Modelle zum Schutz gegen unterirdisches Hochwasser . . . 167

9.5 Zusammenhängende Beschreibung von Hochwasserereignissen . . . . . . . . . . 169

$9.6 \quad$ Andere Einsatzmöglichkeiten für OpenMl-Modellkopplungen . . . . . . . . . . . 169

\begin{tabular}{ll}
\hline Literaturverzeichnis & 171
\end{tabular}

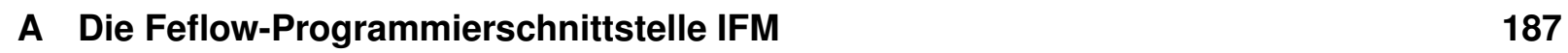


B Ausrüstung bestehender Programme mit einer OpenMI-Schnittstelle 190

C Aufbau eines OpenMI-Systems aus den Programmen Ilmoflood und Feflow 192

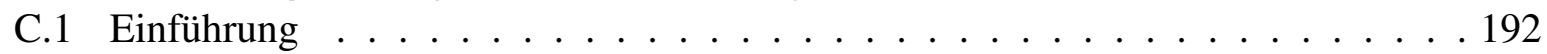

C.2 Migration von Ilmoflood . . . . . . . . . . . . . . . . . . . . . . . . . . . 192

C.3 Erweiterung von Feflow mit einer OpenMI-Schnittstelle . . . . . . . . . . . . . 193

C.3.1 Problemstellung und Lösungsansatz . . . . . . . . . . . . . . . . . . . 193

C.3.2 Umsetzung . . . . . . . . . . . . . . . . . . . . . . . . . . . 194

C.3.3 Austauschobjekte . . . . . . . . . . . . . . . . . . . . . . . . . 197

C.4 Verifikationsbeispiel ,Deichquerschnitt ${ }^{\circ}$. . . . . . . . . . . . . . . 198

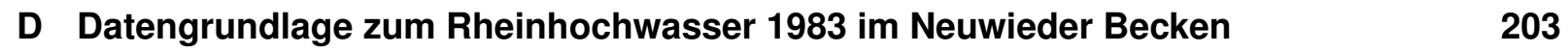

\begin{tabular}{lll}
\hline E Statistische Kennzahlen & 204
\end{tabular}

E.1 Empirischer Korrelationskoeffizient ～. . . . . . . . . . . . . . . . . . 204

E.2 Nash-Sutcliffe-Effizienz . . . . . . . . . . . . . . . . . 205

\begin{tabular}{ll}
\hline Danksagung & 206
\end{tabular} 


\section{Abbildungsverzeichnis}

1.1 Ganglinie der Grundwassermessstelle Hauptbahnhof Dresden und Ganglinie des Dresdner Elbpegels . . . . . . . . . . . . . . . . . . . 1

1.2 Systemskizze eines flussnahen Grundwasserleiters und Prozesse der Wasserbewegung beim unterirdischen Hochwasser $\ldots \ldots \ldots . \ldots 2$

1.3 Wirkung des Auftriebs auf ein Gebäude . . . . . . . . . . . . . . . . . . . . . . 4

1.4 Sofortmaßnahme gegen die Einwirkung des unterirdischen Hochwassers im St.-

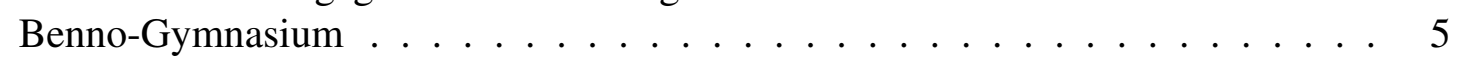

1.5 Ausschnitt aus einer Hochwassergefahrenkarte der Stadt Köln _ . . . . . . . . . . 7

1.6 Schematische Darstellung eines gekoppelten Systems aus Überflutungsflächenund Grundwassermodell, für das der Fluss die wesentlichen Randbedingungen vorgibt ............................. 9

1.7 Abdeckung des Stadtgebietes von Köln mit Grundwassermodellen des örtlichen

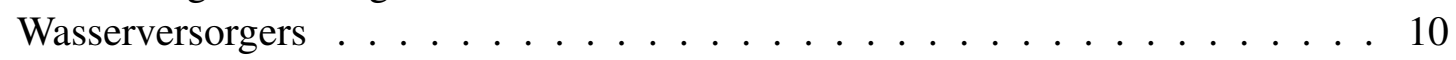

\begin{tabular}{lll}
1.8 & (Programm-)Komponenten des gekoppelten Systems zur Modellierung des un- \\
\hline & terirdischen Hochwassers $\ldots \ldots \ldots \ldots \ldots \ldots \ldots \ldots \ldots \ldots \ldots \ldots \ldots \ldots$
\end{tabular}

2.1 Einordnung der Bewegung des Wassers im Untergrund in die Prozesse der Wasserbewegung beim unterirdischen Hochwasser . . . . . . . . . . . . . . . 14

2.2 Definition der Standrohrspiegelhöhe für gespannte und ungespannte Verhältnisse 16

2.3 Abstands- und Filtergeschwindigkeit in porösen Medien . . . . . . . . . . . . . 17

2.4 Potenziale einer Bodensäule im Gleichgewicht, bei Versickerung und bei kapil-

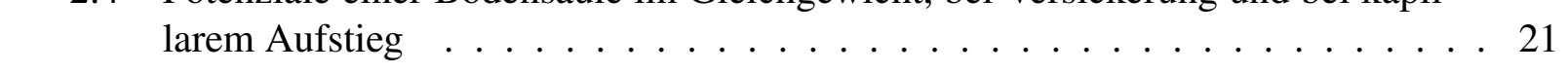

2.5 Abhängigkeit der hydraulischen Durchlässigkeit $k$ und des Wassergehalts $\theta$ vom Matrixpotential $\psi \ldots \ldots \ldots \ldots 22$

2.6 Einordnung von Feflow in das gekoppelte System zur Modellierung des unterirdischen Hochwassers . . . . . . . . . . . . . . . . . 24

$2.7 \quad$ Anwendungsbeispiele für h-Randbedingungen . . . . . . . . . . . . . . . 27

$2.8 \quad$ Anwendungsbeispiele für Randbedingungen zweiter Art . . . . . . . . . . . . . . 28

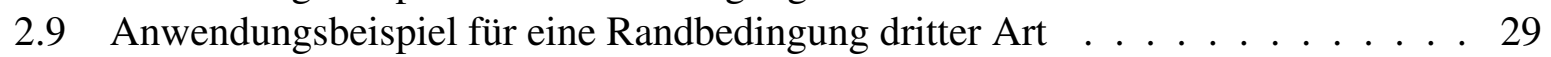

2.10 Anwendungsbeispiel für eine Randbedingung vierter Art . . . . . . . . . . . . 29

3.1 Einordnung der Bewegung des Wassers an der Landoberfläche in die Prozesse der Wasserbewegung beim unterirdischen Hochwasser . . . . . . . . . . . . 31

3.2 Einordnung von Ilmoflood in das gekoppelte System zur Modellierung des unterirdischen Hochwassers . . . . . . . . . . . . . . 36

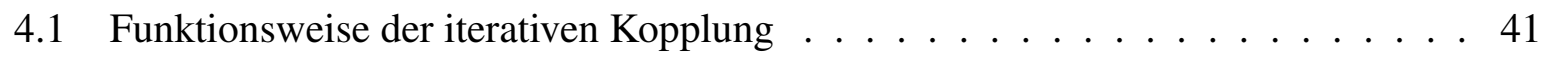


$4.2 \quad$ Funktionsweise der externen Kopplung . . . . . . . . . . . . . . . . . . . . . . 42

4.3 Einordnung von OpenMl in das gekoppelte System zur Modellierung des unterirdischen Hochwassers . . . . . . . . . . . . . . . . . . 45

4.4 Frage-Antwort-Mechanismus und Verbindungen zwischen OpenMI-Komponenten 46

5.1 Einordnung der Uferspeicherung in die Prozesse der Wasserbewegung beim un-

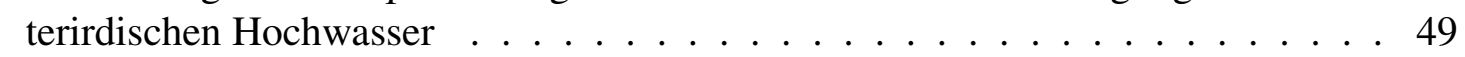

5.2 Querschnitt eines idealisierten Fließgewässers mit Druckhöhendifferenz $\Delta h$ sowie die Austauschrate $q$ für zwei verschiedene Fließzustände . . . . . . . . . . . 51

5.3 Leakage-Fluss $Q$ in Abhängigkeit von der Wasserstandsdifferenz $\Delta h$ für verschiedene Leakage-Ansätze . . . . . . . . . . . . . . . . . . . . . . 52

5.4 Prinzipskizze zur Methode der Volumenbetrachtung . . . . . . . . . . . . . . 57

5.5 Übersichtskarte des Neuwieder Beckens mit dem Grundwassermessprofil bei Urmitz . . . . . . . . . . . . . . . . . . . 59

5.6 Profilschnitt durch die Rheinniederung bei Urmitz . . . . . . . . . . . . . . . 60

5.7 Ganglinien des Rheins und dreier Grundwassermesstellen, Leakage-Fluss und

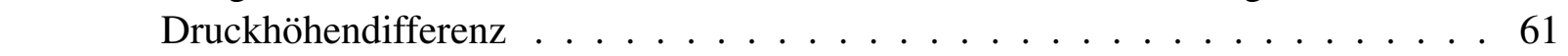

5.8 Druckhöhendifferenz über Leakage-Fluss für die Grundwassermessstelle U01 . . 62

5.9 Druckhöhendifferenz $\Delta h$ über Leakage-Fluss $Q$ für die Grundwassermessstellen U01, U03 und U05 . . . . . . . . . . . . . . . . . . . . . . . 63

5.10 Charakteristischer Korndurchmesser $d_{\mathrm{k}}$ für das von Schubert [2002) beschriebene Sedimentprofil des Rheins und überschläglich berechnete Reynolds-Zahlen . . 64

5.11 3D-Grundwassermodell des Messprofils Urmitz mit dem Fluss als h-Randbedingung und Kalibrierungsergebnisse . . . . . . . . . . . . . . . . 66

5.12 3D-Grundwassermodell des Messprofils Urmitz mit dem Fluss als LeakageRandbedingung und Kalibrierungsergebnisse . . . . . . . . . . . . . 68

5.13 Berechnete und konstruierte Grundwasseroberflächen des manuell kalibrierten

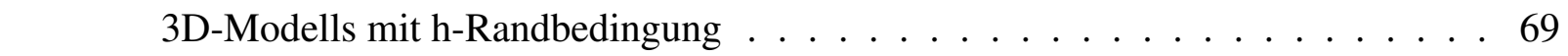

$5.14 Q$ - $\Delta h$-Diagramme aus den automatisch und manuell kalibrierten Modellvarianten mit Leakage- und h-Randbedingung für den Rhein. . . . . . . . . . . . . . 72

5.15 Benetzter Umfang und Leakage-Fluss bei unterschiedlichen Wasserständen und schichtweise unterschiedlichen Durchlässigkeitseigenschaften, aber gleicher

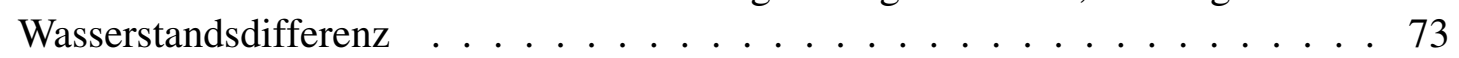

$5.16 Q$ - $\Delta h$-Diagramme aus der automatisch kalibrierten 2D-Modellvariante mit

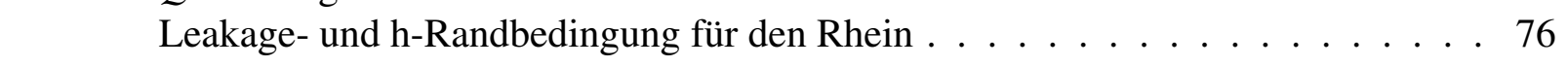

5.17 Messstellen im Stadtgebiet von Köln, die vom örtlichen Wasserversorger betrieben werden, und der Turnus der Messwerterfassung . . . . . . . . . . . . . 78

6.1 Einordnung der Infiltration aus einer überfluteten Fläche in die Prozesse der Wasserbewegung beim unterirdischen Hochwasser . . . . . . . . . 81

6.2 Sättigungssprofil einer Bodenprobe nach Infiltration aus eingestauter Bodenoberfläche ...................... . . . . . . . . 
6.3 Fortschritt einer Sättigungsfront für einen Sandboden bei zwei verschiedenen Anfangsbedingungen und unterschiedlichen Einstauhöhen $\ldots$. . . . . . . . . 87

6.4 Fortschritt einer Sättigungsfront für einen Tonboden bei verschiedenen Anfangs-

bedingungen und unterschiedlichen Einstauhöhen . . . . . . . . . . . 88

6.5 Verlauf der Infiltrationsrate über die Infiltrationszeit für einen Sandboden bei

\begin{tabular}{|l|l|l|}
\hline verschiedenen Anfangsbedingungen und jeweils unterschiedlichen Einstauhöhen 90 & 90
\end{tabular}

6.6 Berechnete Sättigungsprofile eines Sandbodens für unterschiedliche Anfangsbe-

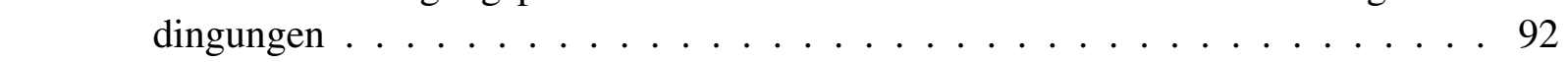

6.7 Infiltrationsraten ungesättigter Strömungsberechnungen mit Feflow für die Bo-

densäule mit homogenen Anfangsbedingungen und mit Kapillarsaum, Entwick-

lung des Grundwasserstands für das Beispiel mit Kapillarsaum sowie Infiltrationsraten des Haverkamp-Modells $\ldots \ldots . \ldots . \ldots 93$

6.8 Sättigungsprofile für eine Infiltration unter Einstau in eine Sand-Bodensäule mit

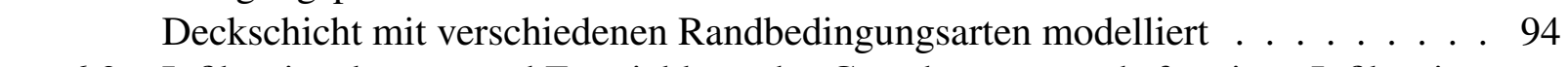

6.9 Infiltrationskurven und Entwicklung des Grundwasserstands für einen Infiltrationsvorgang in einem Sandboden mit Deckschicht auf zwei verschiedene Arten

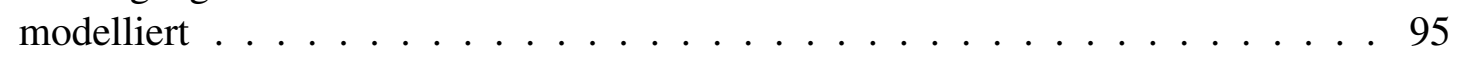

6.10 Angesetzter h-Randbedingungswert und mit Feflow als gesättigte Strömung berechnete Zuflussmenge über die Simulationszeit . . . . . . . . . . . . 97

\begin{aligned} & 6.11 Angesetzte Q-Randbedingungsmenge und berechneter Wasserstand über die Si- \\ & \hline mulationszeit $\ldots \ldots \ldots \ldots \ldots \ldots\end{aligned}$

6.12 Mit der Leakage-Randbedingung berechnete Zuflussmenge und berechneter

W Wasserstand . . . . . . . . . . . . . . . . . . 99

$7.1 \quad$ Einordnung der Bildung von Qualmwasser in die Prozesse der Wasserbewegung beim unterirdischen Hochwasser . . . . . . . . . . . . . . . . . . 103

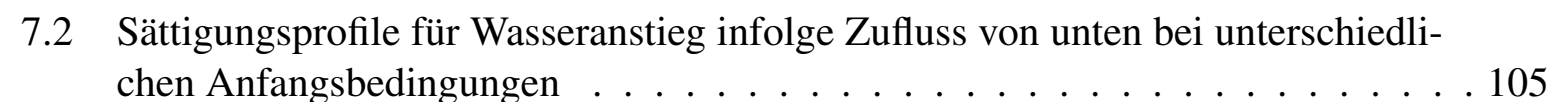

7.3 Grundwasseranstieg für verschiedene Anfangszustände . . . . . . . . . . . . 106

8.1 Die Wechselwirkungsprozesse Uferspeicherung und Infiltration im Zusammenhang mit dem unterirdischem Hochwasser . . . . . . . . . . . . 109

8.2 Modellkomponenten des gekoppelten Testmodells für die Prozesse Uferspeiche-

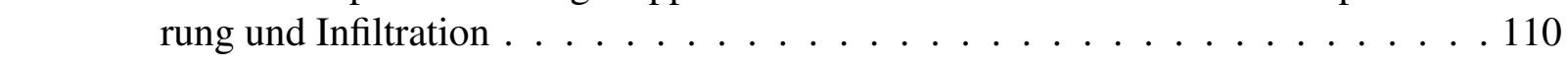

8.3 Kopplung der Ilmoflood-Zellen mit den Feflow-Knoten . . . . . . . . . . . . . . 111

$8.4 \quad$ Wasserstand im Fluss und im Untergrund für verschiedene Zeitpunkte . . . . . . 113

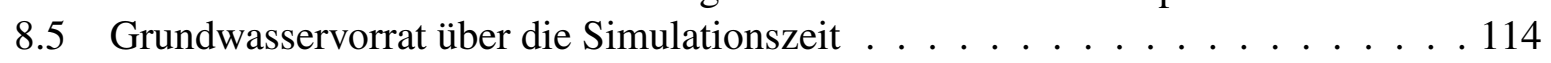

$8.6 \quad$ Frage-Antwort-Mechanismus für die bidirektionale Kopplung . . . . . . . . . . 115

8.7 Verlauf des Oberflächenwasserstands und des Grundwasserstands, der Wasserstandsdifferenz und der Austauschmenge sowie Abweichungen der Austauschmengen für ein Zellen-Knoten-Paar . . . . . . . . . . . . . . . 116

8.8 Ausschnitt aus dem Finite-Elemente-Netz des Richards-Modells und Bereiche der Kopplung . . . . . . . . . . . . . . . . . . 118 
8.9 Ergebnisse der gekoppelten Berechnung mit dem Richards-Grundwassermodell für Tag 2,6 . . . . . . . . . . . . . . . . . . . . . . . . . . . 119

8.10 Detail des Uferbereichs mit Druckpotenzial und Wasserstand im Fluss für zwei Simulationszeitpunkte. . . . . . . . . . . . . . . . . . 120

8.11 Ergebnisse der gekoppelten Berechnung mit dem Richards-Grundwassermodell für zwei Simulationszeitpunkte . . . . . . . . . . . . . . . . . . . . . . 121

8.12 Ergebnisse der gekoppelten Berechnung mit dem Richards-Grundwassermodell

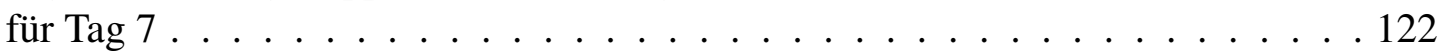

8.13 Grundwasservorratsänderung über die Simulationszeit des Richards-Modells und des rein gesättigten Modells . . . . . . . . . . . . . . . . . . . . 123

8.14 Die Wechselwirkungsprozesse Qualmwasser und Infiltration im Zusammenhang mit dem unterirdischem Hochwasser . . . . . . . . . . . . . . . . . . 125

8.15 Berechnungsnetze des Ilmoflood- und des Feflow-Modells . . . . . . . . . . . . 126

8.16 Grundwasserstand und Oberflächenwasserstand zur Simulationszeit 0,15 d . . . . 127

8.17 Grundwasserstand und Oberflächenwasserstand für die Simulationszeitpunkte 0,20 d, 0,32 d und 0,40 d (unterschiedliche Skaleneinteilung auf der Ordinate) . . 129

8.18 Grundwasserstand und Oberflächenwasserstand für die Simulationszeitpunkte 0,55 d und 2,0 d (unterschiedliche Skaleneinteilung auf der Ordinate) . . . . . . . 131

8.19 Grundwasserstand, Randbedingungswert und korrespondierender Durchfluss über einen Teil der Simulationszeit . . . . . . . . . . . . . . . . . . . . . 132

8.20 Zufluss der Zellen 2 und 3 und Wasserstand der Zelle 2 . . . . . . . . . . . . . 133

8.21 Ausschnitt aus dem FE-Netz des 3D-Vergleichsmodells mit Isolinien und Stromfaden im Vertikalschnitt . . . . . . . . . . . . . . . . . . . . . . . 134

8.22 Grundwasserstand, Randbedingungswert und korrespondierender Durchfluss für drei Knoten und einen Teil der Simulationszeit . . . . . . . . . . . . . . 136

8.23 Modellkopplung für das Anwendungsbeispiel . . . . . . . . . . . . . . . . . 137

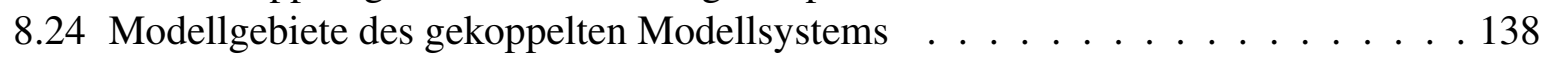

8.25 Detailausschnitt der Netze an der Nordostecke des llmoflood-Modellgebiets . . . 140

8.26 Einteilung der Kopplungsbereiche . . . . . . . . . . . . . . . . . 141

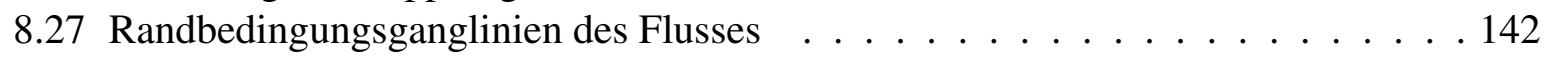

8.28 Wasserausbreitung auf der Überflutungsfläche und Isolinien des Grundwasserstands für den Simulationszeitpunkt 3d . . . . . . . . . . . . . . . . . 143

8.29 Wasserausbreitung auf der Überflutungsfläche und Isolinien des Grundwasserstands für den Simulationszeitpunkt 3,75d . . . . . . . . . . . . . . . . . . . . 144

8.30 Wasserausbreitung auf der Überflutungsfläche und Isolinien des Grundwasserstands für den Simulationszeitpunkt $8 \mathrm{~d}$. . . . . . . . . . . . . . . . . . 145

8.31 Wasserausbreitung auf der Überflutungsfläche und Isolinien des Grundwasserstands für den Simulationszeitpunkt 9,75d . . . . . . . . . . . . . . . . . . . 146

$8.32 \mathrm{Zu}-$ und Abflüsse des Grundwassermodells getrennt nach Kopplungsbereichen und Wasserstand im Fluss und auf der Überflutungsfläche für zwei Beobachtungspunkte aus gekoppelter und ungekoppelter Berechnung . . . . . . . . . . . 147

8.33 Verlauf des Grundwasserstands über die Zeit an verschiedenen Beobachtungspunkten sowie die Pegelganglinie des Flusses . . . . . . . . . . . . . . . . 149 
$8.34 \mathrm{Zu}-$ und Abflüsse des Grundwassermodells nach Kopplungsbereichen und Wasserstand im Fluss und auf der Überflutungsfläche für zwei Beobachtungspunkte $\quad$. 150

8.35 Vergleich der Grundwasserstände an den Beobachtungspunkten 2s, 3s und 4 für die Szenarien „langes“ und „kurzes Hochwasserereignis“ und die Ganglinie des Flusses für das lange Hochwasserereignis $\ldots \ldots \ldots . \ldots 151$

8.36 Qualmwasseraustritt nach acht Tagen Simulationszeit . . . . . . . . . . . . . . 152

8.37 Anfangsbedingungen des Szenarios „,langes Hochwasser und hoher Anfangs-

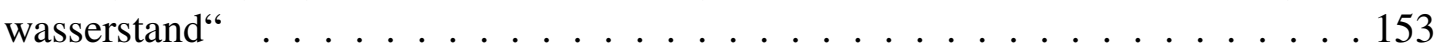

8.38 Verlauf des Grundwasserstands über die Zeit an verschiedenen Beobachtungspunkten und die Pegelganglinie des Flusses . . . . . . . . . . . . . . . . 154

8.39 Grundwasservorratsänderung über die Simulationszeit für die drei Szenarien des

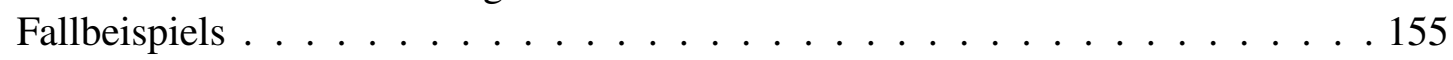

8.40 Flurabstände aus dem Szenario „langes Hochwasser mit hohem Anfangswasserstand" für zwei verschiedene Simulationszeitpunkte . . . . . . . . . . . . . . . 157

A.1 Möglichkeiten des Programmeingriffs in die Feflow-Grundwasserströmungsbe-

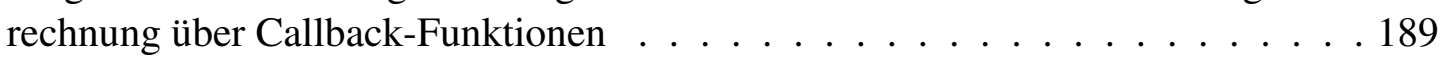

C.1 Funktionsprinzip der externen Steuerung des Programms Feflow 195

C.2 Berechnungsnetz des Deichquerschnitts und des Vorlands, Randbedingungen an der Landseite des Deichs, OpenMl-Kopplungsbereiche und die Lage zweier Zellen-Knoten-Paare . . . . . . . . . . . . . . . . . . . . . . . . 199

C.3 Bildschirmfoto des OpenMI-Editors mit dem OpenMI-System des Deichquer-

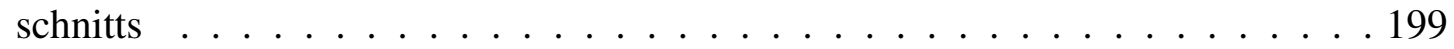

C.4 Ablauf des Frage-Antwort-Mechanismus und der Interpolation für die unidirektionale Verbindung . . . . . . . . . . . . . . . . . 200

C.5 Sickerlinie des Deichquerschnitts und Wassertand im Vorland für verschiedene

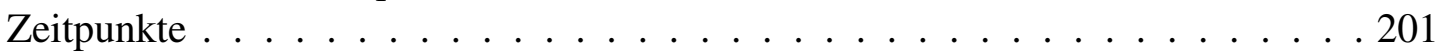

C.6 Ergebnis des Ilmoflood-Modells und angesetzter Randbedingungswert im Feflow-Modell für zwei verschiedene Zellen-Knoten-Verbindungen . . . . . . . . . 202

E.1 Beispiele für Korrelationen zweier Datenreihen $\ldots \ldots \ldots$. . . . . . . . . . . . . 204 


\section{Tabellenverzeichnis}

$2.1 \quad$ Knotenbasierte Randbedingungsarten des Programms Feflow . . . . . . . . . . . 26

4.1 Zusammenstellung der Vor- und Nachteile der verschiedenen Arten der Modellkopplung auf Basis der zitierten Literatur $\ldots . . . . . . .43$

5.1 Zur Berechnung der Leakage-Flüsse in Abbildung $5.3 \mid$ verwendete Werte . . . . . 53

5.2 Entfernung der Grundwassermessstellen des Messprofils Urmitz zum Ufer . . . . 59

5.3 Kennzahlen zur Kalibrierungsgüte der manuell kalibrierten Modelle . . . . . . . 68

$5.4 \quad$ Kennzahlen zur Kalibrierungsgüte der automatisch kalibrierten Modelle $\ldots . .70$

5.5 Summe der Kennzahlen zur Kalibrierungsgüte . . . . . . . . . . . . . . 70

$5.6 \quad$ Kennzahlen zur Kalibrierungsgüte der automatisch kalibrierten 2D-horizontalen

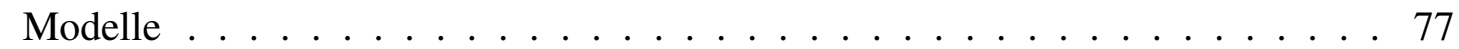

5.7 Summe der Kennzahlen zur Kalibrierungsgüte 2D-Modell $\ldots . \ldots$

6.1 Eigenschaften der Beispielböden und Anfangsbedingungen . . . . . . . . . . . . 89

$8.1 \quad$ Eigenschaften der Grundwassermodellvarianten $\ldots \ldots \ldots$. . . . . . . . . 111

8.2 Übersicht der Austauschobjekte zur Verbindung zwischen Ilmoflood-Zellen und Feflow-Knoten . . . . . . . . . . . . . . . . . . . . 112

8.3 Übersicht der Austauschobjekte zur Verbindung zwischen IImoflood-Zellen und Feflow-Knoten . . . . . . . . . . . . . . . . . . . . 128

$8.4 \quad$ Übersicht der Austauschobjekte zur Verbindung zwischen Ilmoflood-Zellen und Feflow-Knoten des 3D-Modells . . . . . . . . . . . . . . . . . . . 135

8.5 Eigenschaften und Parameter des Ilmoflood-Überflutungsflächenmodells . . . . . 139

8.6 Eigenschaften und Parameter des Feflow-Grundwassermodells . . . . . . . . . . 139

8.7 Horizontale Entfernung der Beobachtungspunkte vom Fluss und vom Längsdamm 140

8.8 Übersicht der Austauschobjekte $\ldots \ldots \ldots$. . . . . . . . . . . . . . . . . . . . . . .

9.1 Verfahren zur Erstellung von Hochwassergefahrenkarten und Gefahrenkarten des unterirdischen Hochwassers . . . . . . . . . . . . . . . 165

A.1 Beispiele für IFM-Funktionen . . . . . . . . . . . . . . . . . . . . 187

C.1 Austauschobjekte für das Überflutungsflächenmodul von Ilmoflood . . . . . . . . 193

C.2 Austauschobjekte für Feflow . . . . . . . . . . . . . . . . . . . 198

D.1 Messwerte für Wasserstände des Rheins und Grundwassermessstellen, Austauschflüsse und Wasserstandsdifferenzen . . . . . . . . . . . . . . . . . . 203 


\section{Symbolverzeichnis}

\section{Lateinische Zeichen}

Zeichen Beschreibung

Einheit

A Fläche $\quad \mathrm{m}^{2}$

$b \quad$ Breite $\mathrm{m}$

c kinematische Wellengeschwindigkeit $\mathrm{m} / \mathrm{s}$

$c_{1} \quad$ Leakage-Koeffizient $\quad 1 / \mathrm{s}$

C $\quad$ spezifische Feuchtigkeitskapazität (specific moisture capacity) 1/m

$C_{i} \quad$ Konstante, Parameter

$C_{\mathrm{K}} \quad$ Kapillaritätskoeffizient $\mathrm{m}$

$d \quad$ Dicke einer Sedimentschicht m

$d_{\mathrm{k}} \quad$ charakteristischer Korndurchmesser $\mathrm{m}$

$d_{n} \quad$ Kornduchrchmesser bei $n \%$ der Sieblinie $\quad \mathrm{m}$

ex Index für Exfiltration

$f_{i} \quad$ Komponente eines Kraftvektors $\mathrm{m} / \mathrm{s}^{2}$

g Gravitationskonstante $\mathrm{m} / \mathrm{s}^{2}$

h Wasserstand m m

H Wassertiefe m m

$h_{\mathrm{F}} \quad$ Flusswasserstand $\mathrm{m}$

$h_{\mathrm{G}} \quad$ Grundwasserdruckhöhe $\mathrm{m}$

$h_{\mathrm{p}} \quad$ Überstauhöhe (ponding depth) $\mathrm{m}$

$i \quad$ Laufindex

i Index für ,initial“ (Anfangsbedingung)

I kumulative Infiltration

$\mathrm{m}$

$I_{\mathrm{E}} \quad$ Energieliniengefälle

in Index für Infiltration

$i_{\mathrm{R}} \quad$ Regenintensität $\mathrm{m} / \mathrm{s}$

$j \quad$ Laufindex

$k \quad$ Beiwert der hydraulischen Durchlässigkeit $\mathrm{m} / \mathrm{s}$

$k_{0} \quad$ hydraulische Durchlässigkeit bei Sättigung (matching point, siehe $\mathrm{m} / \mathrm{s}$ Schaap \& van Genuchten 2006)

$k^{\prime} \quad$ relative hydraulische Durchlässigkeit

$k_{\mathrm{S}} \quad$ Beiwert der hydraulischen Durchlässigkeit einer Sedimentschicht im $\mathrm{m} / \mathrm{s}$

Flussbett

$k_{\mathrm{St}} \quad$ Strickler-Beiwert $\quad \mathrm{m}^{\frac{1}{3}}$ 


\begin{tabular}{|c|c|c|}
\hline$l$ & Länge & $\mathrm{m}$ \\
\hline$L_{\mathrm{G}}$ & Tortuositätsparameter im Mualem-van-Genuchten-Modell & \\
\hline$l_{\mathrm{U}}$ & benetzter Umfang & $\mathrm{m}$ \\
\hline$M$ & Mächtigkeit & $\mathrm{m}$ \\
\hline$m_{\mathrm{G}}$ & Van-Genuchten-Parameter & \\
\hline$n$ & natürliche Zahl & \\
\hline$n_{\mathrm{G}}$ & Van-Genuchten-Parameter & \\
\hline$n_{\mathrm{M}}$ & Manning-Beiwert & $1 / \mathrm{m}^{\frac{1}{3}}$ \\
\hline$n_{\mathrm{P}}$ & Porosität & \\
\hline$n_{\text {Pn }}$ & nutzbares Porenvolumen & \\
\hline$N S E$ & Nash-Sutcliffe-Effizienz & \\
\hline$p$ & Druck & $\mathrm{N} / \mathrm{m}^{2}$ \\
\hline$Q$ & Durchfluss & $\mathrm{m}^{3} / \mathrm{s}$ \\
\hline$q$ & Durchflussrate, Austauschrate, Infiltrationsrate & $\mathrm{m}^{3} / \mathrm{m}^{2} \mathrm{~s}, \mathrm{~m} / \mathrm{s}$ \\
\hline$Q_{\mathrm{QS}}$ & Quelle/Senke & $\mathrm{m}^{3} / \mathrm{s}$ \\
\hline$q_{\mathrm{QS}}$ & Quelle/Senke & $\mathrm{m}^{2} / \mathrm{s}$ \\
\hline $\mathrm{r}$ & Index für „,residual“ & $\mathrm{m}$ \\
\hline$r_{\mathrm{h}}$ & hydraulischer Radius & $\mathrm{m}$ \\
\hline$R$ & Korrelationskoeffizient & \\
\hline$R^{2}$ & Bestimmtheitsmaß & \\
\hline $\mathrm{RB}$ & Randbedingung & \\
\hline$R e$ & Reynolds-Zahl & \\
\hline$s$ & Sickerlänge & $\mathrm{m}$ \\
\hline$S$ & eindimensionale Raumkoordinate & $\mathrm{m}$ \\
\hline $\mathrm{s}$ & Index für ,gesättigt“‘ & \\
\hline$S$ & Sättigungsgrad & \\
\hline$S_{\mathrm{s}}$ & Speicherkoeffizient & \\
\hline$S_{\mathrm{sp}}$ & spezifischer Speicherkoeffizient & $1 / \mathrm{m}$ \\
\hline$S_{\mathrm{P}}$ & Sorptivität & $\mathrm{m} / \sqrt{\mathrm{s}}$ \\
\hline$t$ & Zeit & $\mathrm{s}$ \\
\hline$T$ & Transmissivität & $\mathrm{m}^{2} / \mathrm{s}$ \\
\hline$U$ & Ungleichförmigkeitszahl & \\
\hline$v$ & Geschwindigkeit & $\mathrm{m} / \mathrm{s}$ \\
\hline$v_{\mathrm{a}}$ & Abstandsgeschwindigkeit & $\mathrm{m} / \mathrm{s}$ \\
\hline$v_{\mathrm{f}}$ & Filtergeschwindigkeit oder Darcy-Geschwindigkeit & $\mathrm{m} / \mathrm{s}$ \\
\hline$V$ & Volumen & $\mathrm{m}^{3}$ \\
\hline$V_{\mathrm{W}}$ & Volumen des Wassers & $\mathrm{m}^{3}$ \\
\hline$V_{\mathrm{G}}$ & Volumen des Gesteins & $\mathrm{m}^{3}$ \\
\hline$x$ & Raumkoordinate in einem kartesischen System & $\mathrm{m}$ \\
\hline$x_{i}$ & Raumkoordinate in einem kartesischen System & $\mathrm{m}$ \\
\hline$y$ & Raumkoordinate in einem kartesischen System & $\mathrm{m}$ \\
\hline & Raumkoordinate in einem kartesischen System & $\mathrm{m}$ \\
\hline
\end{tabular}


$z_{\mathrm{B}} \quad$ Lage der Gewässersohle (B: bottom)

$\mathrm{m}$

$z_{\mathrm{s}} \quad$ Lage der Sättigungsfront bei einem Infiltrationsvorgang

$\mathrm{m}$

\section{Griechische Zeichen}

Zeichen Beschreibung

Einheit

$\alpha \quad$ Parameter

$\alpha_{\mathrm{G}} \quad$ Van-Genuchten-Parameter

$1 / \mathrm{cm}$

$\beta \quad$ Parameter

$\Delta \quad$ Differenz

$\theta \quad$ Wassergehalt

$\theta_{\mathrm{S}} \quad$ Wassergehalt bei Sättigung

$\theta_{\mathrm{i}} \quad$ Anfangswassergehalt

$v \quad$ kinematische Viskosität (Zähigkeit)

$\mathrm{m}^{2} / \mathrm{s}$

$\rho \quad$ Dichte

$\mathrm{kg} / \mathrm{m}^{3}$

$\rho_{\mathrm{W}} \quad$ Dichte des Wassers

$\mathrm{kg} / \mathrm{m}^{3}$

$\tau^{\mathrm{B}} \quad$ Sohlschubspannung

$\mathrm{N} / \mathrm{m}^{2}$

$\tau^{\mathrm{D}} \quad$ Impulsdispersion

$\mathrm{N} / \mathrm{m}^{2}$

$\tau^{\mathrm{W}} \quad$ Windschubspannung

$\phi \quad$ Potential bezogen auf die Masseeinheit des Fluids

$\mathrm{N} / \mathrm{m}^{2}$

$\phi_{\mathrm{m}} \quad$ Matrixpotential bezogen auf die Masseeinheit des Fluids

$\mathrm{m}^{2} / \mathrm{s}^{2}$

$\Phi \quad$ geographische Breite

$\psi \quad$ Druck- oder Matrixpotenzial

$\mathrm{m}^{2} / \mathrm{s}^{2}$

$\psi_{\mathrm{a}} \quad$ Druckpotenzial bei Lufteintritt (air entry pressure)

$\mathrm{m}$

$\psi_{\mathrm{m}}$

Matrixpotenzial

$\mathrm{m}$

$\psi_{\mathrm{p}}$

Druckpotenzial

$\mathrm{m}$

$\omega$

Winkelgeschwindigkeit

$\mathrm{m}$

$\frac{\mathrm{rad}}{s}$ 



\section{Einleitung}

\subsection{Hochwasser und unterirdisches Hochwasser}

Hochwasser wird der Zustand bei Gewässern genannt, bei dem der Wasserstand deutlich über dem normalen Pegelstand liegt. Hochwässer sind periodisch wiederkehrende natürliche Ereignisse, die erst durch die Besiedlung der Täler entlang der Flüsse und Ströme ihre besondere Brisanz bekommen. So entstanden in den vergangenen Jahren vermehrt große Schäden durch Hochwasser (siehe z. B. Münchener Rück 2003). Das Thema Hochwasser rückte damit wieder verstärkt in das öffentliche Interesse.

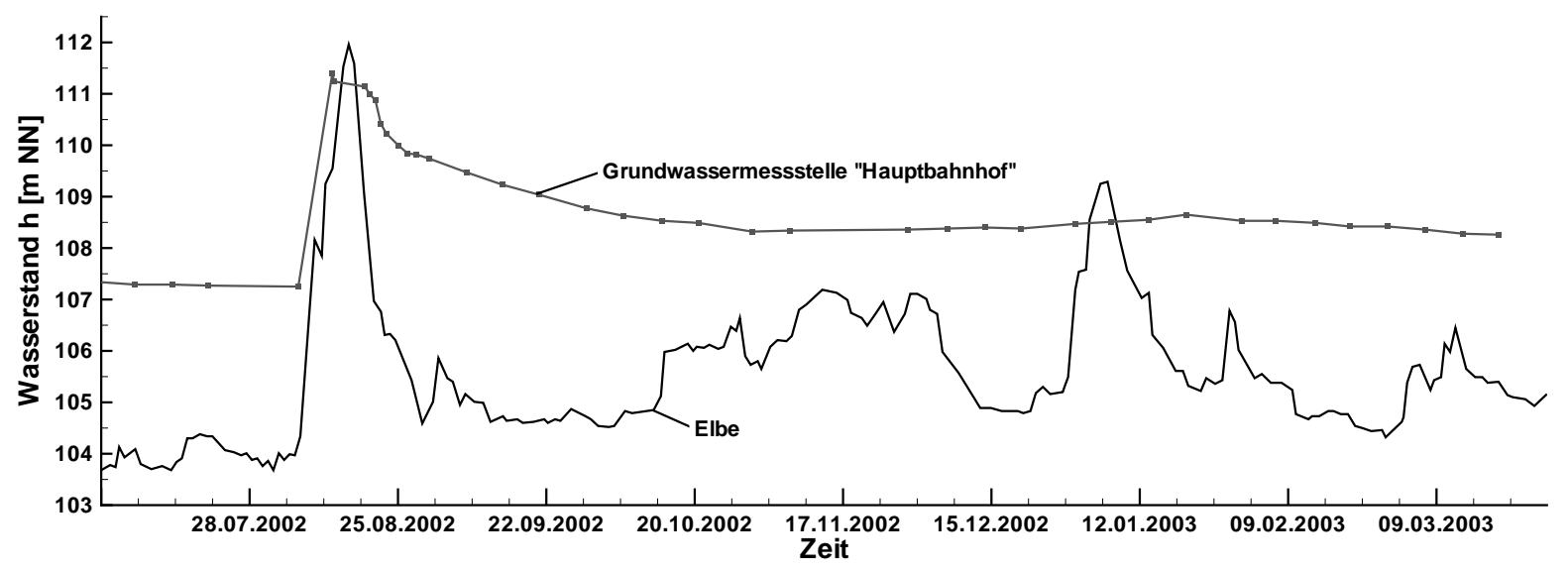

Abbildung 1.1: Ganglinie der Grundwassermessstelle Hauptbahnhof Dresden mit etwa $1900 \mathrm{~m}$ Entfernung zur Elbe und Ganglinie des Dresdner Elbpegels (nach Sächsisches Landesamt für Umwelt und Geologie 2003)

Eines der verheerendsten jüngeren Hochwasserereignisse in Deutschland war das AugustHochwasser im Jahr 2002. Zahlreiche Städte und Kommunen im Elbe-Einzugsgebiet waren davon betroffen. Eine Besonderheit dieses Ereignisses war der starke Grundwasseranstieg, unter dem die Bürger der Stadt Dresden zu leiden hatten: innerhalb kürzester Zeit kam es zu einem Anstieg des Grundwasserspiegels um bis zu sechs Meter auf ein zuvor noch nicht gemessenes Niveau. Abbildung 1.1 verdeutlicht das Problem durch den Vergleich der Ganglinie einer Grundwassermessstelle und der Ganglinie des Dresdner Elbpegels. Noch bevor in der Elbe der maximale Wasserstand erreicht wurde, stieg der Grundwasserstand an einer Grundwassermessstelle in der Nähe des Dresdner Hauptbahnhofs wegen der Überflutungen im Dresdner Stadtzentrum um mehr als drei Meter an. Das Abklingen des unterirdischen Hochwassers in diesem Bereich dauerte mehrere Wochen, wegen anhaltend hoher Niederschläge verblieb der 


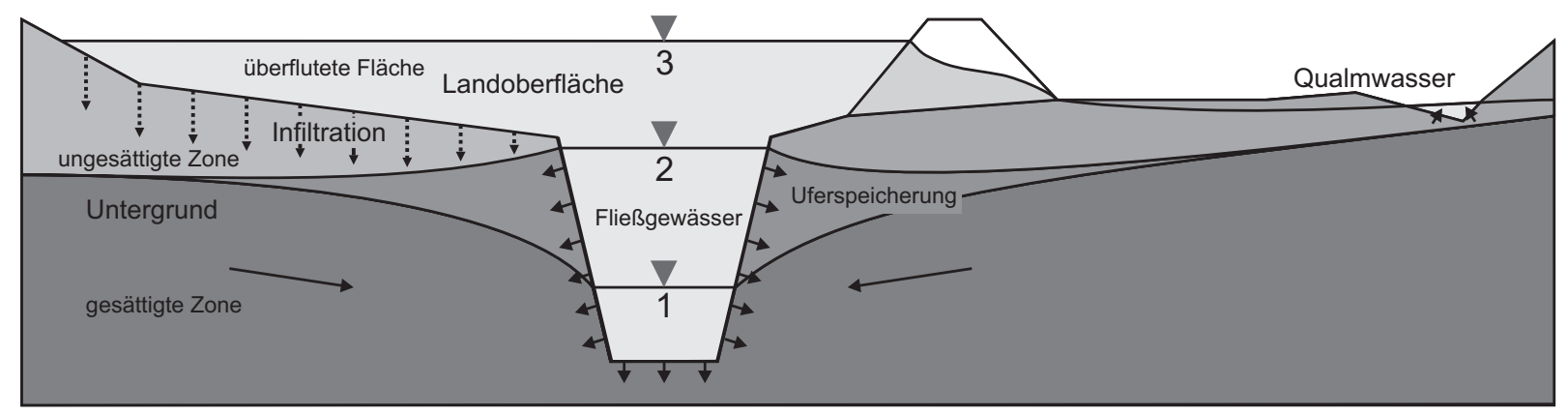

Abbildung 1.2: Systemskizze eines flussnahen Grundwasserleiters und Prozesse der Wasserbewegung beim unterirdischen Hochwasser

Grundwasserstand zudem lange deutlich über dem Mittelwasserbereich (Sächsisches Landesamt für Umwelt und Geologie 2003).

Ein Grundwasseranstieg, der durch Hochwasser eines Flusses ausgelöst wird, wird „unterirdisches Hochwasser“ genannt. Auch der Begriff „Grund-Hochwasser“ wird verwendet, Sommer \& Ullrich (2004) sprechen vom ,unsichtbaren Hochwasser“.

Für das August-Hochwasser 2002 wurden keine gesonderten Erhebungen für Schäden aus dem Grundwasser durchgeführt (Sommer \& Ullrich 2004). Huber et al. (2003) geben jedoch für Landesliegenschaften in Sachsen eine Quote von $16 \%$ der gesamten Schadenshöhe für Einwirkungen aus dem Grundwasser an. Kreibich \& Thieken (2008) befragen 1697 Haushalte im Einzugsgebiet der Donau und der Elbe nach Schäden aus Überflutungen. Aus den Umfrageergebnissen wurden 264 Haushalte identifiziert, bei denen ausschließlich Schäden aus dem Grundwasser auftraten. Als mittlerer Gebäudeschaden werden $14456 €$, als mittlerer Einrichtungsschaden (mobiler Schaden) $3769 €$ angegeben.

Die Ausbreitung des unterirdischen Hochwassers wird durch drei Wechselwirkungsprozesse des Grundwassers mit dem Oberflächenwasser bestimmt (siehe z. B. Winter et al. 1998):

- Uferspeicherung

- Infiltration von einer Überflutungsfläche

- Qualmwasseranstieg

Abbildung 1.2 veranschaulicht die Prozesse, die das unterirdische Hochwasser bestimmen, schematisch. Diese Abbildung wird im Folgenden auch verwendet, um die Inhalte der Kapitel und Abschnitte in den Gesamtzusammenhang einzuordnen. Teile der Beschriftung werden dazu kursiv hervorgehoben.

Den großen Flüssen Mitteleuropas fließt das Grundwasser bei Mittel- und Niedrigwasser meistens zu (Brombach et al. 2001). Diese Art des Austauschs zwischen dem Fluss und dem unterirdischen Raum wird auch Exfiltration genannt (Abbildung 1.2, Zustand 1). Bei Hochwasser steigt der Wasserstand im Fluss und übt Druck auf das Grundwasser aus. Dadurch kann es 
zu einer Umkehrung der Fließrichtung kommen, die Infiltration verursacht einen Anstieg des Grundwasserspiegels in Ufernähe (Abbildung 1.2, Zustand 2). Ein Teil des Wassers aus dem Fluss wird unterirdisch im Bereich des Ufers gespeichert und trägt so zu einer Dämpfung der Hochwasserwelle im Fluss bei (Pinder \& Sauer 1971). Dieser Vorgang wird Uferspeicherung genannt. Bei Niedrigwasser fließt das im Ufer gespeicherte Wasser wieder zurück in den Fluss. Neben dem oben angesprochenen Beispiel von der Elbe werden Uferspeicherungseffekte auch am Rhein zum Beispiel bei Krefeld von Eckert \& Rueber (1992), in Köln von Losen (1984) und in Neuwied von Ubell (1987a, b) beobachtet. Beispiele von der Donau beschreiben Ubell (1964) und Vekerdy \& Meijerink (1998).

Verlässt der Fluss sein Bett und überflutet Vorländer und Teile der Talaue (Abbildung 1.2, Zustand 3), dann dringt zusätzlich Flusswasser von oben durch die ungesättigte Zone in den Grundwasserleiter ein. An der Oberfläche bewegt sich das Wasser sehr viel schneller als im Untergrund, so dass der Auen-Grundwasserleiter über eine wesentlich größere Fläche angereichert wird. Durch die Infiltration aus einer Überflutungsfläche kann sich der Einfluss des unterirdischen Hochwassers deshalb weiter ausdehnen als durch Uferspeicherung allein. Dadurch, dass ein Teil des Wassers in den Untergrund eindringt, ändert sich aber auch die Ausdehnung der überfluteten Fläche, da der Raum unter einer überfluteten Fläche als Speicher wirkt und ein zusätzliches Retentionsvolumen schafft (Monninkhoff \& Kernbach 2006).

Bei Hochwasser können auch Flächen ohne Zuströmen von Oberflächenwasser eingestaut werden, wenn der Grundwasserstand so weit ansteigt, dass das Grundwasser an der Oberfläche austritt. Dieses Wasser wird Qualmwasser oder Drängewasser bezeichnet. Kiesige und sandige Grundwasserleiter, wie sie in Flussauen oft vorkommen, begünstigen eine solche Ausbreitung des unterirdischen Hochwassers (Brombach et al. 2001). Qualmwasser tritt oft hinter Deichen auf, denn durch die Eindeichung entstehen im Fließgewässer höhere Wasserstände als dies ohne Eindeichung der Fall wäre (Abbildung 1.2). Wirksame Hochwasserschutzanlagen wie Deiche und mobile Hochwasserschutzwände schützen zwar die dahinter liegende Bereiche vor Hochwasser an der Oberfläche, können aber das unterirdische Hochwasser verstärken. Der erhöhte Wasserdruck führt zu einem Anstieg des Grundwasserspiegels. In einem ungespannten Grundwasserleiter steigt Wasser dann in den vorher ungesättigten Bereich auf und führt zu einer Vernässung oder sogar zu einer Flutung der Talaue ausschließlich über den Grundwasserpfad (Walther \& Marre 2004). Dies kann die Ausbreitung des Wassers, das später zum Beispiel infolge eines Deichbruchs oder einer gezielten Überflutung seitlich zufließt, beeinflussen (Briechle 2006). Erreicht der Grundwasserstand jedoch eine Auelehmdeckschicht, so bilden sich zunächst gespannte Verhältnisse. Ein Bruch oder das Zerfließen der Auelehmdeckschicht sind möglicherweise die Folge (Lehners \& Sassenhagen 2003), oder der Qualmwasseraustritt konzentriert sich an natürlichen oder künstlichen Fehlstellen der Deckschicht (Brombach et al. 2001; Lehners \& Sassenhagen 2003).

Als mögliche nachteilige Folgen eines unterirdischen Hochwassers werden im Wesentlichen

- Schäden an Bauwerken

- Veränderung der Grundwasserbeschaffenheit 


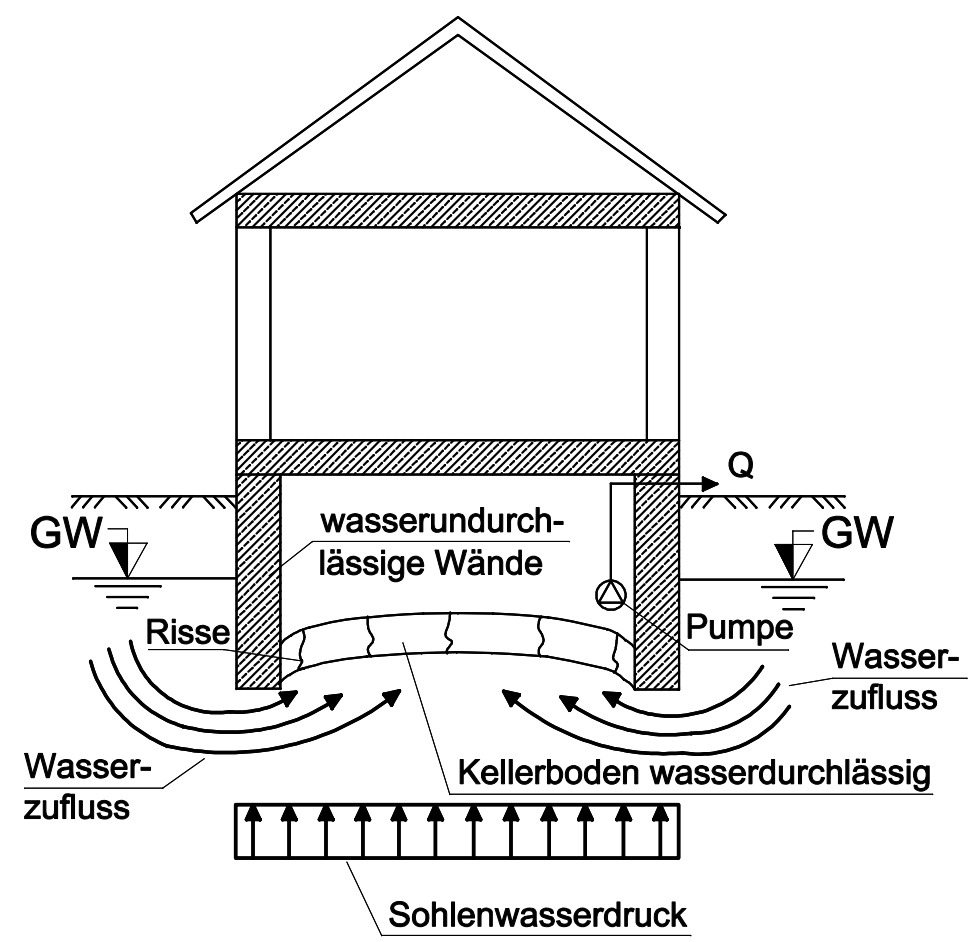

Abbildung 1.3: Wirkung des Auftriebs auf ein Gebäude (Walther \& Marre 2004; Brombach et al. 2001): Das Leerpumpen des Kellers kann durch den Sohlwasserdruck zum Aufbrechen der Bodenplatte führen.

- Schäden an der Kanalisation und

- Einschränkung der Trinkwasserversorgung

genannt.

Durch ansteigendes Grundwasser erhöhte Auftriebskräfte gefährden die Standsicherheit von Wohn- und Geschäftshäusern und öffentlichen Gebäuden (Abbildung 1.3), aber auch von Infrastruktureinrichtungen wie Straßentunnel oder U-Bahnanlagen. Insbesondere historische Gebäude sind oft nicht auf hohe Grundwasserstände ausgelegt und müssen geflutet werden, um größeren Schaden zu bewahren (Kreibich \& Thieken 2008). In Dresden konnte in einigen Fällen nur durch gezielte Belastung oder gar Flutung der Bodenplatten größerer Schaden verhindert werden (Sommer \& Ullrich 2005). Ein solches Beispiel wird von Beyer (2003) beschrieben: die Bodenplatte der tiefliegenden Turnhalle des Sankt-Benno-Gymnasiums in Dresden hob sich durch den Anstieg des Grundwasserspiegels um $20 \mathrm{~cm}$. Nach In-situ-Messungen der Grundwasserdruckhöhe, der Befragung von Fachleuten und der Auswertung alter Baupläne wurden zunächst Sofortmaßnahmen eingeleitet: über 500 Freiwillige brachten Sandsäcke und Behältersysteme (quick dam) als Gegengewichte in die Turnhalle (Abbildung 1.4). Ein im Gebäude untergebrachter Transformator des örtlichen Energieversorgers musste abgeschaltet werden, was einen zeitweisen Ausfall der Stromversorgung in der unmittelbaren Umgebung mit sich brachte. 

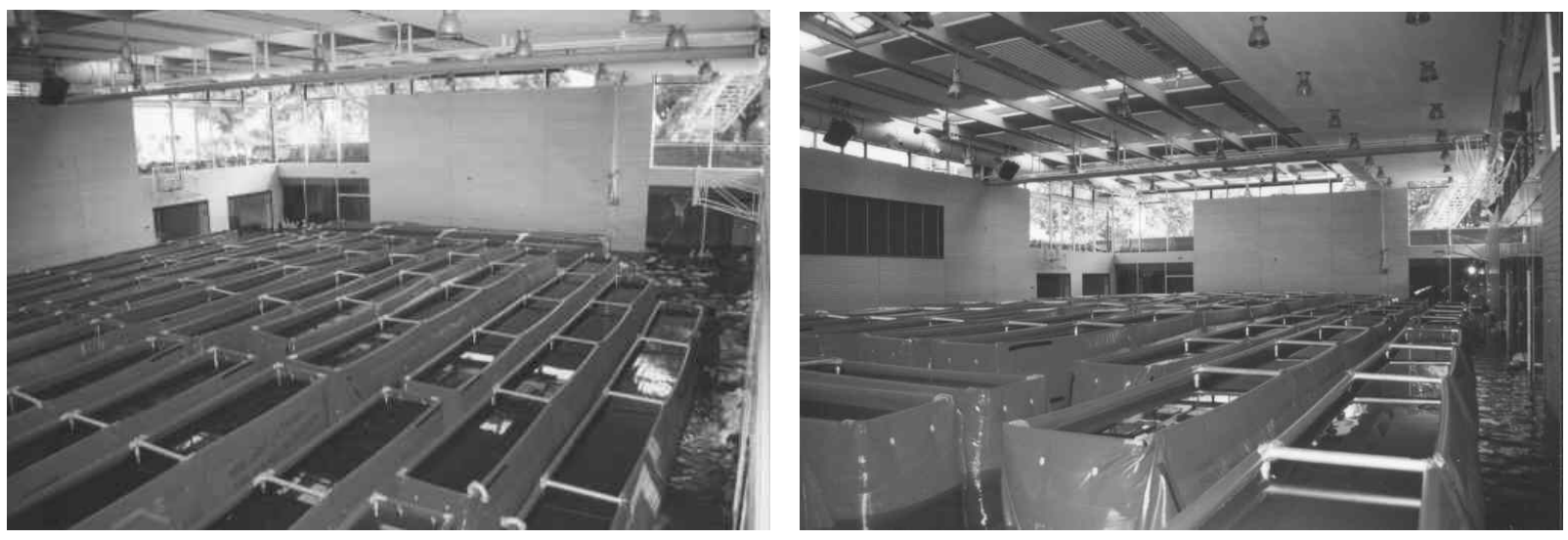

Abbildung 1.4: Gegengewicht aus Behältersystemen und Flutung der Bodenplatte als Sofortmaßnahme gegen das Aufbrechen der Bodenplatte in der Turnhalle des St.-Benno-Gymnasiums durch unterirdisches Hochwasser (St.-Benno-Gymnasium 2002)

Veränderungen der Grundwassersituation können auch geomechanische Prozesse wie Setzungen, Rutschungen oder hydraulischen Grundbruch auslösen. In Gebäude eindringendes Wasser greift Inventar, Putz und Bodenbeläge an (Kreibich \& Thieken 2008). Es begünstigt die Schimmelbildung und führt damit zu hygienischen Problemen. Akute Gefahr für Leib und Leben von Menschen aus dem unterirdischen Hochwasser entsteht dann, wenn Wasser in die Stromversorgung der Gebäude eindringt (Brombach et al. 2001).

Die Beschaffenheit des Grundwassers ändert sich bei unterirdischem Hochwasser zunächst durch das eindringende Oberflächenwasser aus dem Fluss oder einer überfluteten Fläche, welches in der Regel anders beschaffen ist als das Grundwasser. Wasser, das von einer überfluteten Landfläche in das Grundwasser eindringt, kann Stoffe, die bisher in den oberen Bodenschichten gebunden waren, in den Grundwasserleiter transportieren. Andersherum besteht auch die Möglichkeit, dass Grundwasser in Bereiche ansteigt, die bisher in der ungesättigten Zone lagen. Auf diese Weise können Stoffe aus der ungesättigten Zone mobilisiert und transportiert werden (Sommer \& Ullrich 2005).

Nachteilig für die Trinkwasserversorgung ist unterirdisches Hochwasser zunächst wegen der oben beschriebenen potentiellen Änderung der Grundwasserbeschaffenheit. Die technischen Anlagen und Gebäude zur Trinkwassergewinnung sind durch ähnliche Mechanismen gefährdet wie andere Gebäude.

Unterirdisch verlegte Rohrleitungen sind so wie Gebäude Auftriebskräften ausgesetzt, wenn der Grundwasserspiegel entsprechend ansteigt. Im Hochwasserfall können darüber hinaus auch in der Kanalisation extreme Druckverhältnisse entstehen, zum Beispiel, wenn Schachtdeckel und Straßenabläufe wegen einer Überflutung hoch eingestaut sind. Da die Kanalnetze für drucklosen Betrieb ausgelegt sind, muss mit Schäden gerechnet werden. Dringt dagegen Grundwasser über Schadstellen von außen in Abwasserkanäle ein, steigt der Fremdwasseranteil im Kanalsystem und überlastet möglicherweise Kläranlagen, in jedem Fall steigen die Kosten der Abwasserableitung und -behandlung durch den zunehmendem Fremdwasseranteil. Andersherum kann Ab- 
wasser aus schadhaften Leitungen austreten und so zu einem Schadstoffeintrag führen, wenn das Grundwasser höher als normal ansteht (Sommer \& Ullrich 2005).

Für den operativen Hochwasserschutz ist es von Bedeutung, die Austrittsstellen von Qualmwasser zu kennen oder vorhersagen zu können, denn diese Gegenden sind in der Regel nicht begehbar und nicht befahrbar, was zum Beispiel bei der Planung von Evakuierungs- oder Versorgungsmaßnahmen zu berücksichtigen ist. Im Bereich von Bebauung, die oft künstliche Fehlstellen der Auelehmdeckschicht darstellen, sind möglicherweise kleine Qualmwasserdeiche herzustellen, um das austretende Wasser daran zu hindern, in die Gebäude einzudringen. Besonders kritisch ist die Tatsache, dass Qualmwasser in der Regel unerwartet auftritt. So wird weit entfernt vom Fließgewässer oft nicht mit Überflutungen gerechnet und es werden dementsprechend keine baulichen Vorkehrungen getroffen. Beispielsweise kann ein verlandeter Altarm des Flusses als präferenzieller Fließweg wirken (Puffahrt 2007; Tacke 1988), der den Einfluss des Hochwassers weit ins Hinterland bringt. Oder das eigentliche Hochwasserereignis ist bereits abgeklungen, während im Hinterland die Grundwasserstände noch weiter ansteigen (Brombach et al. 2001).

\subsection{Problemstellung}

Methoden und Modelle, mit denen eine Hochwasserwelle und ihre oberirdischen Auswirkungen prognostiziert werden können, stehen zur Verfügung und werden auch zur Hochwasserschutzplanung und im operativen Hochwasserschutz eingesetzt (siehe z. B. Stadtentwässerungsbetriebe Köln 2009a). Dem unterirdischen Raum dagegen wird in vielen Untersuchungen zur Wirkung eines Hochwassers bisher wenig Bedeutung beigemessen (Walther \& Marre 2004; Sommer \& Ullrich 2005). So werden Grundwassermodelle im operativen Hochwasserschutz nach derzeitigem Kenntnisstand nicht eingesetzt, selbst für Planungsaufgaben des Hochwasserschutzes sind modellgestützte Untersuchungen, die das Grundwasser mit einbeziehen, selten. Ein herausragendes Beispiel ist das Projekt „3ZM-GRIMEX“, bei dem ein Drei-Zonen-Modell für den Pilotstandort Dresden entwickelt wurde (Sommer et al. 2008). Für andere Standorte können die Auswirkungen eines Hochwassers auf die Grundwasserverhältnisse momentan oft nur aufgrund von Erfahrungswerten oder aus den Hochwassergefahrenkarten abgeschätzt werden.

Hochwassergefahrenkarten werden meistens auf Basis eindimensionaler Modellrechnungen erstellt. Der für ein bestimmtes Hochwasserszenario, meistens gegeben durch einen Bemessungsabfluss, ermittelte Wasserstand im Fluss wird mit einem digitalen Geländemodell verschnitten (Ministerium f. Umwelt u. Naturschutz, Landwirtschaft und Verbraucherschutz d. Landes Nordrhein-Westfalen 2003; Umweltministerium, Innenministerium und Wirtschaftsministerium Baden-Württemberg 2005). In den Hochwassergefahrenkarten der Stadt Köln wird unterirdisches Hochwasser dadurch berücksichtigt, dass der hydrostatisch ermittelte Wasserstand auch auf Standorte, die keine Verbindung zur Überflutungsfläche haben (Druckwasserbereiche), übertragen wird (Wieczorrek 2007). Ein Ausschnitt aus einer solchen Hochwassergefahrenkarte ist in Abbildung 1.5 gezeigt. Aus den Hochwassergefahrenkarten kann für Bereiche, die direkt hinter einer Hochwasserschutzeinrichtung liegen, sicherlich auf einen Grundwasseranstieg geschlossen 


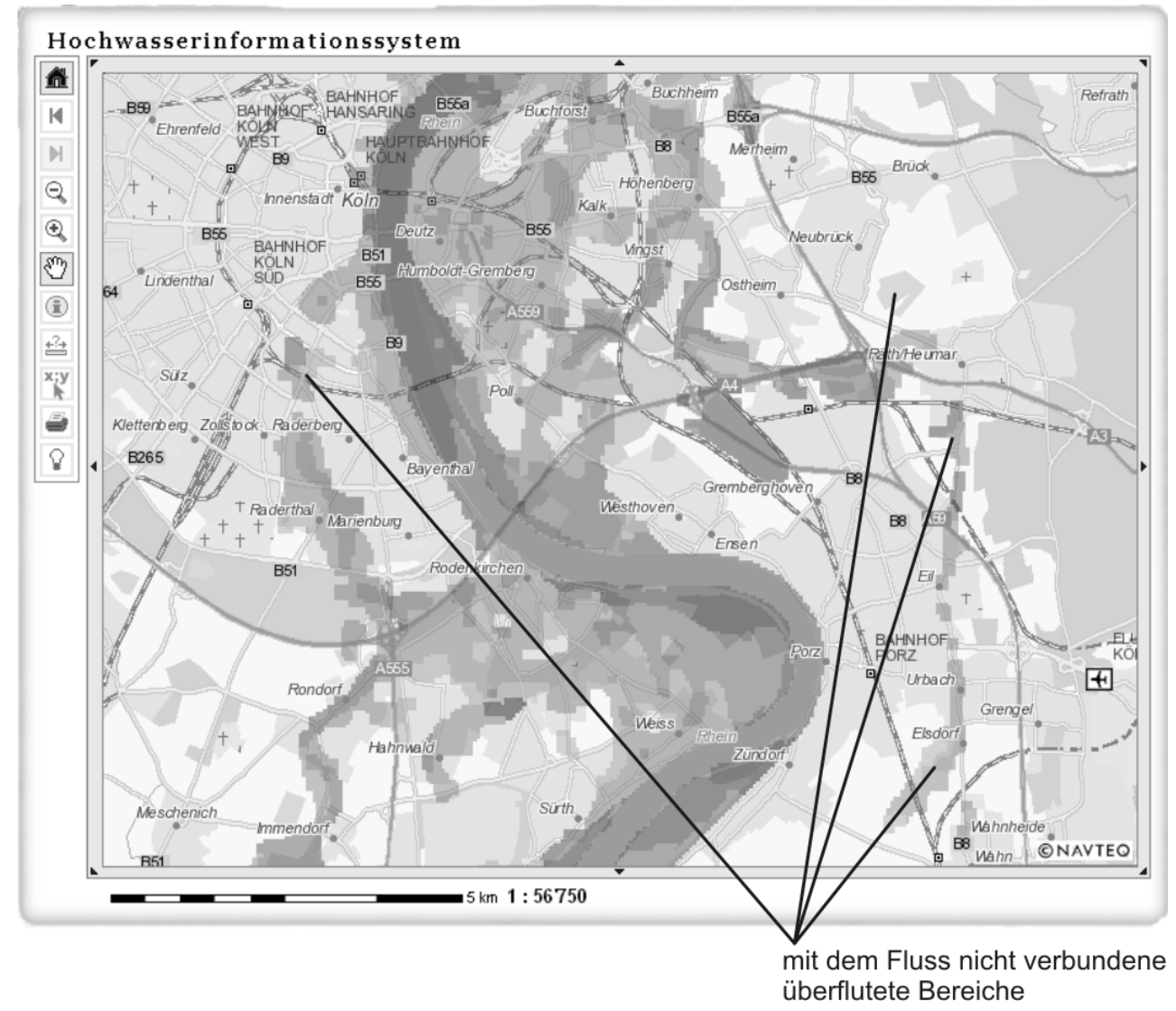

Abbildung 1.5: Ausschnitt aus einer Hochwassergefahrenkarte der Stadt Köln (Geomer \& Infoterra 2009) mit der zu erwartenden hydrostatischen Wasserausbreitung für ein Extremhochwasserereignis. Beispielhaft sind Bereiche markiert, die nicht über die Oberfläche mit dem Fließgewässer in Verbindung stehen.

werden. Für Standorte, die weiter vom Hauptwasserkörper entfernt liegen, ist dies jedoch sehr ungenau, da das unterirdische Hochwasser der Hochwasserwelle an der Oberfläche verzögert nachläuft. Es reicht zudem nicht aus, die Orte, an denen unterirdisches Hochwasser an der Oberfläche austritt, zu kennen, da der Grundwasseranstieg ja schon zu Schäden führen kann, bevor er die Geländeoberkante erreicht.

Forberig (2008) stellt eine „Karte der Gefährdung durch Grundwasseranstieg infolge eines Hochwassers (GWK) “ für die Stadt Torgau an der Elbe vor. In diese Karte flossen Grundwassermessdaten des Bearbeitungsgebietes für vergangene Hochwasserereignisse sowie hydrogeologisches Fachwissen zur Lage von präferenziellen Fließwegen aus Fluss-Altarmen, zu den hydraulischen Wechselwirkungen zwischen Grundwasserleiter und Fluss und zu hydrogeologischen Barrieren ein (Forberig 2009; Flögel 2009). Während eine solche Grundwassergefährdungskarte einen wichtigen Beitrag zur Planung für Stadtplaner, Bauämter und Umweltbehörden darstellt (Flögel 2009), ist es unter Umständen nicht ausreichend, Entscheidungen des operativen Hochwasserschutzes für den Untergrund aus Erfahrungswerten abzuleiten, da jedes Hochwasser eine eigene 
Charakteristik aufweist. Ein lang anhaltendes Hochwasser mit einem vergleichsweise kleinen Pegelstand im Fluss kann zu größeren Problemen führen als ein kurzzeitiges Hochwasserereignis mit hohem Flusspegelstand.

Bedarf an Aussagen über den zu erwartenden Grundwasserstand besteht deshalb, da die Folgen des unterirdischen Hochwassers kostenintensiver sein können als die Folgen des oberirdischen Hochwassers, wenn sie unvorhergesehen eintreten. Eine frühzeitige Warnung der Bevölkerung vor erhöhten Grundwasserständen führt nicht nur zu einer entsprechenden Verhaltensvorsorge, sondern erlaubt auch die rechtzeitige Auslösung von Objektschutzmaßnahmen und schafft somit die Grundlage zur Schadensreduzierung. Eine solche Objektschutzmaßnahme kann das Leerräumen eines Kellers sein, das Aufbringen einer zusätzlichen Auflast auf die Bodenplatte eines gefährdeten Gebäudes oder die Einrichtung von Qualmwasserdeichen.

Großes Potential zur Schadensreduzierung liegt zunächst in Bereichen mit einer hohen Bevölkerungs- oder Industriedichte vor. Oft sind solche Gebiete bereits mit Deichen oder mobilen Hochwasserschutzeinrichtungen vor oberirdischem Hochwasser geschützt. Dieser bauliche Hochwasserschutz vergrößert, wie oben bereits erläutert, potentielle Schäden durch unterirdisches Hochwasser.

Die Grundwasserstände befinden sich im Zustand ständiger Veränderung entsprechend der Speisung aus der Grundwasserneubildung, dem Kontakt zu Fließgewässern in Form einer In- oder Exfiltration und den Entnahmen zum Beispiel zur Trink- und Brauchwassergewinnung. Diese Veränderungen finden jedoch auf einer deutlich größeren Zeitskala statt als ein Hochwasserereignis und Überflutungen am Fluss. Hochwasserereignisse treten daher bei sehr unterschiedlichen Grundwassersituationen auf. Belastbare Daten können somit nur aus einer Analyse der aktuellen Situation abgeleitet werden. Der bereits vor dem Hochwasserereignis hohe Grundwasserstand wird als eine von mehreren Ursachen für die starke Ausbreitung des unterirdischen Hochwassers in Dresden genannt (Sommer \& Ullrich 2005). Auch Flögel (2009) nennt als wichtige Einflussgröße auf die Ausbreitung einer unterirdischen Hochwasserwelle die Ausgangslage im Hinterland. Wegen der klimatischen Veränderung und der damit verbundenen Veränderung der hydrologischen Situation (Kropp et al. 2009) werden in Zukunft möglicherweise auch Bereiche betroffen sein, in denen bisher kein kritischer Grundwasseranstieg auftrat und für die dementsprechend auch keine Erfahrungswerte vorliegen.

\subsection{Zielsetzung und Vorgehensweise}

Eine Prognose der Ausbreitung des unterirdischen Hochwassers ist mit einem gekoppelten numerischen Modell, das die Bewegung des Wassers im Untergrund und auf einer überfluteten Fläche sowie die Wechselwirkungsprozesse abbildet, möglich. Neben den Abflussprognosen für den Fluss und der davon ausgehenden Berechnung der Ausbreitung von Wasser auf Überflutungsflächen kann mit einem numerischen Modell auch die Ausgangssituation im Grundwasser durch Festlegung von Anfangsbedingungen berücksichtigt werden. 


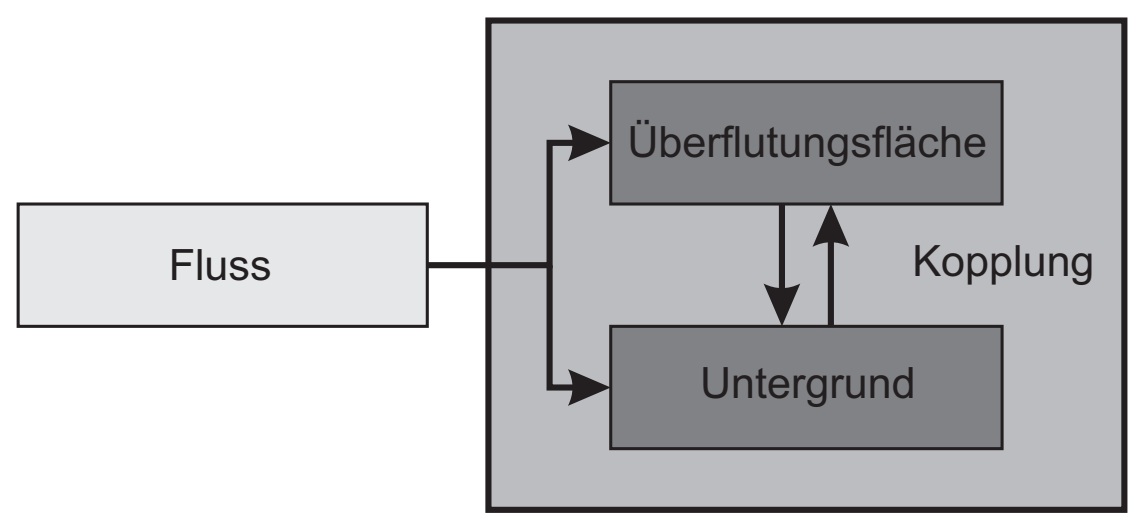

Abbildung 1.6: Schematische Darstellung eines gekoppelten Systems aus Überflutungsflächen- und Grundwassermodell, für das der Fluss die wesentlichen Randbedingungen vorgibt

Ziel dieser Arbeit ist es, einen Weg aufzuzeigen, wie ein Grundwassermodell mit einem Überflutungsflächenmodell gekoppelt werden kann, um damit vorherzusagen, wo und wann im Hochwasserfall ein Grundwasserstandsanstieg zu erwarten ist. Der Fluss liefert für das Grundwassermodell und das Überflutungsflächenmodell die wesentlichen Randbedingungen in Form von Wasserständen oder Zuflüssen. Eine schematische Übersicht des gekoppelten Systems zeigt Abbildung 1.6. Jeder Kasten in Abbildung 1.6 repräsentiert eine Modellierungsaufgabe, für die jeweils ein Lösungsvorschlag vorgestellt wird.

Das Prognosewerkzeug für unterirdisches Hochwasser muss operativen Anforderungen genügen. Ein wesentlicher Aspekt ist dabei die Akzeptanz durch die Anwender. Modelle zur Berechnung von überfluteten Flächen sind oft im Rahmen eines Hochwasserrisikomanagements für die Erstellung von Hochwassergefahrenkarten entwickelt worden. Ein Beispiel ist das Überflutungsflächenmodell der Stadt Köln (Ruiz Rodriguez Zeisler Blank Ingenieurgemeinschaft für Wasserbau und Wasserwirtschaft 2009; Stadtentwässerungsbetriebe Köln 2009b). Grundwassermodelle für den Hochwasserschutz sind allerdings meistens nicht vorhanden. Oft existieren jedoch für ganze Stadtgebiete oder für Teile davon bereits numerische Grundwassermodelle, die zwar für andere Zwecke als den Hochwasserschutz entwickelt wurden, aber grundsätzlich auch mit einem Überflutungsflächenmodell gekoppelt werden können und damit für Fragen des unterirdischen Hochwassers nutzbar werden. Zum Beispiel betreibt der örtliche Wasserversorger der Stadt Köln drei zweidimensional-horizontale Grundwassermodelle, die sich über das gesamte Stadtgebiet erstrecken (Abbildung 1.7). Ein anderes Beispiel ist ein ebenfalls zweidimensionalhorizontales Grundwassermodell für den Bereich am linken Niederrhein zwischen Xanthen und Krefeld (Junghans 2001). Können diese Modelle im Hochwasserschutz eingesetzt werden, ist es nicht nötig, sich in ein weiteres Programm oder Modell einzuarbeiten. Dies wird zur Bereitschaft der Grundwassermodellierer an einer Mitwirkung am operativen Hochwasserschutz beitragen. Sofern die Voraussetzungen für eine Kooperation gegeben sind, werden so das Expertenwissen und die Erfahrungen der Grundwassermodellierer für den Hochwasserschutz verfügbar gemacht. Durch die Verwendung bereits bestehender Grundwassermodelle für den Hochwasserschutz entfallen zudem die arbeitsaufwändigen Phasen des Modellaufbaus, der Kalibrierung und 


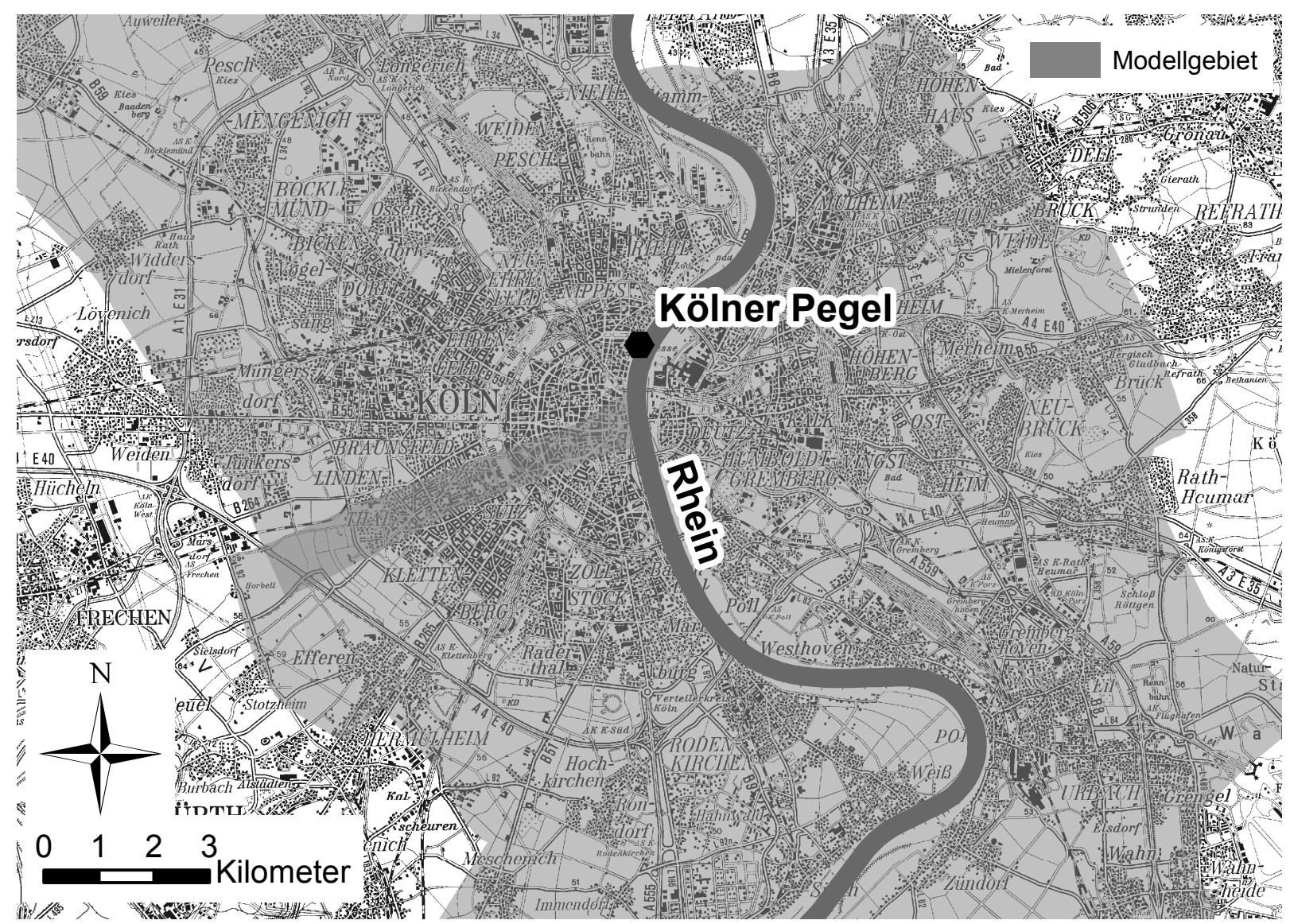

Abbildung 1.7: Abdeckung des Stadtgebietes von Köln mit Grundwassermodellen des örtlichen Wasserversorgers (Ausschnitt), Datengrundlage: Rheinenergie AG (2007)

der Validierung des Grundwassermodells. Im Gegenzug können die Szenarien des Hochwassers als zusätzliche Validierungsdaten genutzt werden, um die Qualität des Grundwassermodells zu bestätigen oder Anpassungsbedarf aufzuzeigen.

Das Besondere der kombinierten Betrachtung von Wasser an der Oberfläche und im Untergrund besteht darin, dass hier zwei unterschiedliche Räume der Hydrodynamik (Kompartimente) verbunden werden, für die unterschiedliche Fließgesetze gelten. Die Prozesse, die für die Ausbreitung von Hochwasser im Untergrund verantwortlich sind, stellt bereits Abbildung 1.2 schematisch dar. Zunächst sind

- die Bewegung des Wassers im Untergrund in der

- gesättigten Zone und der

- ungesättigte Zone sowie

- die Bewegung des Wassers an der Landoberfläche

- im Fließgewässer und 
- auf einer Überflutungsfläche

als die Prozesse der beiden betroffenen Kompartimente zu unterscheiden. Auf der Bewegung des Wassers im Untergrund muss für Fragestellungen des unterirdischen Hochwassers naturgemäß der Schwerpunkt der Untersuchungen liegen. In den Kapiteln 2 und 3 werden Gleichungen zur mathematischen Beschreibung der Strömung wiedergegeben. Es handelt sich dabei um partielle Differenzialgleichungen, die numerisch mit Hilfe von Strömungsprogrammen gelöst werden. Als Beispiel für ein Programm zur Berechnung der Wasserbewegung im Untergrund wird Feflow vorgestellt, für die Berechnung der Bewegung des Wassers an der Landoberfläche dient das Programm Ilmoflood als Beispiel.

In Kapitel 4 werden verschiedene Methoden zur Kopplung von Strömungsprogrammen vorgestellt. Die Kopplung von Modellen ist derzeit Stand der Wissenschaft und Forschung, hat sich aber bisher noch nicht so durchgesetzt, dass gekoppelte Modellrechnungen generell zum Leistungsangebot der meisten Ingenieurbüros gehören. Um gekoppelte Modellrechnungen dem Stand der Technik näher zu bringen, wurde vor dem Hintergrund eines steigenden Bedarfs an integrierter Betrachtung wasserwirtschaftlicher Fragestellungen der OpenMI-Standard entwickelt (Moore et al. 2005). Kapitel 4 enthält eine Beschreibung dieses Standards, mit dem einheitliche Schnittstellen für wasserwirtschaftliche Strömungsprogramme definiert werden, die es erlauben, beliebig unterschiedliche Modellkomponenten zu einem gekoppelten System zusammenzuführen.

Ein Standard wird sich aber nur dann durchsetzen, wenn er auch für die gängigen Strömungsprogramme und damit für bereits existierende Modelle verwendet werden kann. Das Programm Feflow ist ein in Deutschland verbreitetes Programm zur Berechnung der Grundwasserströmung. Das Ziel der Modellkopplung wird deshalb dahingehend konkretisiert, dass das Programm Feflow um eine OpenMI-Schnittstelle erweitert wird, so dass damit Fragen des unterirdischen Hochwassers beantwortet werden können. Die Schwierigkeit bei der Implementierung des OpenMIStandards in Feflow besteht darin, dass der Programmcode für Feflow nicht allgemein verfügbar ist. Damit ist eine wesentliche Voraussetzung für die Implementierung des OpenMI-Standards nicht erfüllt (Gijsbers et al. 2005). Es soll aber möglich sein, ohne eine spezielle Version des Programms zu installieren, gekoppelte Modellsysteme aufzubauen und zu berechnen. Der Weg dorthin führt über die offene Programmierschnittstelle, mit der eigene Programmbausteine innerhalb des Feflow-Programmablaufs implementiert werden können.

Für die Berechnung der Überflutungsflächen hat sich bisher in Deutschland noch kein Programm so etabliert, dass man von einer weiten Verbreitung sprechen kann. Dies liegt vielleicht auch daran, dass zweidimensionale Strömungsberechnungen des Oberflächenwassers im Vergleich zu eindimensionalen Strömungsberechnungen seltener durchgeführt werden. Mit der Implementierung des OpenMI-Standards in ein Überflutungsflächenmodell kann deshalb noch keine große Nutzergemeinschaft erreicht werden. Da zudem der Schwerpunkt bei Fragen des unterirdischen Hochwassers auf dem unterirdischen Raum liegt, geht es zunächst darum, exemplarisch ein geeignetes Überflutungsflächenprogramm für die Kopplung mit dem Grundwasserströmungsprogramm Feflow bereit zu stellen. Ilmoflood wurde ausgewählt, weil für dieses Programm der Programmcode zur Verfügung steht und die OpenMI-Schnittstelle deshalb leicht implementiert wer- 


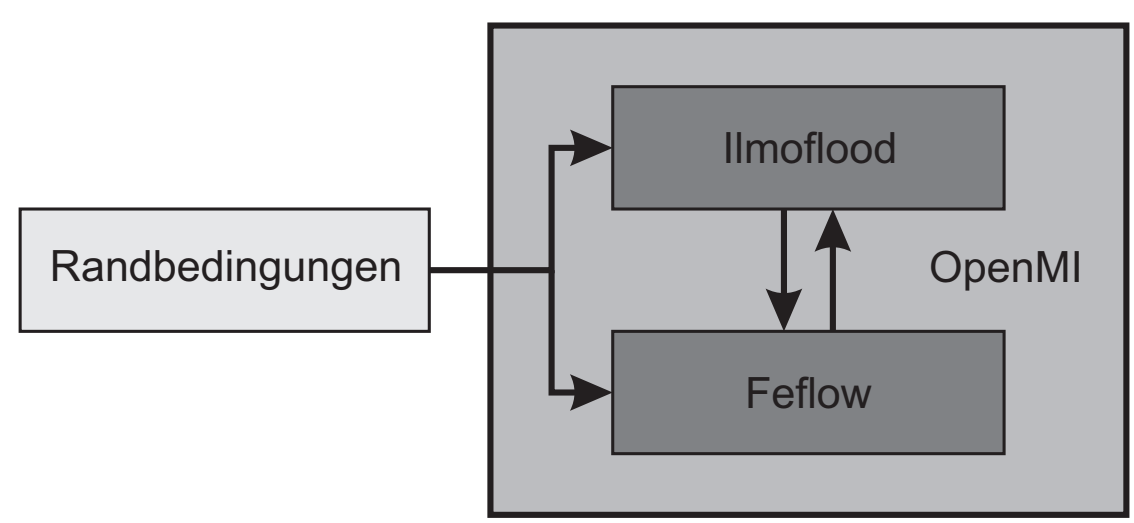

Abbildung 1.8: (Programm-)Komponenten des gekoppelten Systems zur Modellierung des unterirdischen Hochwassers

den kann. Durch die Verwendung des OpenMI-Standards kann in einem gekoppelten System das Ilmoflood-Modell gegen ein anderes Modell mit OpenMI-Schnittstelle ausgetauscht werden.

Wie die Modellkopplung nach dem OpenMI-Standard für die Programme Feflow und Ilmoflood technisch umgesetzt wird, ist in Anhang C dokumentiert. Das in Abbildung 1.6 vorgestellte gekoppelte System konkretisiert sich damit gemäß Abbildung 1.8. Ein Ilmoflood-Modell bildet die Strömung auf einer Überflutungsfläche ab, ein Feflow-Modell die Grundwasserströmung und der Fluss findet als Randbedingung Eingang in die beiden Modellkomponenten.

Ein weiteres Ziel dieser Arbeit ist es, das Prozessverständnis für die Wechselwirkungen zwischen dem Wasser der Landoberfläche und dem Wasser im Untergrund zu vertiefen, um daraus abzuleiten, wie diese Wechselwirkungen geeignet in einem gekoppelten System erfasst werden können. Die Wechselwirkungsprozesse

- Uferspeicherung

- Infiltration von einer Überflutungsfläche und

- Qualmwasseraustritt

(Abbildung 1.2) sind Gegenstand der Kapitel 5, 6 und 7. Zwischen den beiden Komponenten IImoflood und Feflow werden die Wechselwirkungen als Randbedingungen an den Kopplungspunkten der Modelle umgesetzt. Vor diesem Hintergrund werden in diesen Kapiteln geeignete Wege zur modelltechnischen Erfassung dieser Prozesse abgeleitet. Dabei kommt es nicht darauf an, neue Ansätze zu entwickeln, sondern die bestehenden Möglichkeiten auf ihre Eignung für die Fragen des unterirdischen Hochwasserschutzes zu überprüfen. Für jeden der drei Prozesse sind in der Fachliteratur Möglichkeiten zu ihrer mathematischen Beschreibung dokumentiert. Davon ausgehend werden die Prozesse mit Hilfe numerischer Berechnungen und dem Vergleich der Berechnungsergebnisse mit Felddaten oder numerischer Referenzrechnungen weiter untersucht. Wie der Wechselwirkungsprozess mit den bestehenden Programmfunktionen vor dem Hintergrund des unterirdischen Hochwassers geeignet modelliert werden kann, wird am Schluss des jeweiligen Kapitels diskutiert. 
Während in den Kapiteln 2 bis 7 die für das unterirdische Hochwasser entscheidenden Prozesse isoliert betrachtet werden, enthält Kapitel 8 Anwendungsbeispiele der gekoppelten Modellierung. Hier wird auf technische Aspekte der Modellkopplung eingegangen und es werden Wechselwirkungsprozesse im Zusammenhang analysiert. Vergleichsrechnungen zeigen auf, welche Einschränkungen der Modellaussage in Kauf genommen werden müssen, wenn die verfügbaren Programmfunktionen die einzelnen Prozesse vereinfacht abbilden.

Im letzten Kapitel werden die wesentlichen Ergebnisse zusammengefasst und es wird ein Ausblick auf mögliche weitere Untersuchungen gegeben. 


\section{Die Bewegung des Wassers im Untergrund}

\subsection{Einführung}

Gegenstand dieses Kapitels ist die mathematische Beschreibung der Bewegung des Wassers im Untergrund. Wie schon eingangs erwähnt, äußert sich unterirdisches Hochwasser durch den Anstieg des Grundwasserspiegels, so dass die Beschreibung oder Modellierung der Grundwasserströmung (Abbildung 2.1) eine wesentliche Aufgabe bei der Prognose unterirdischen Hochwassers ist.

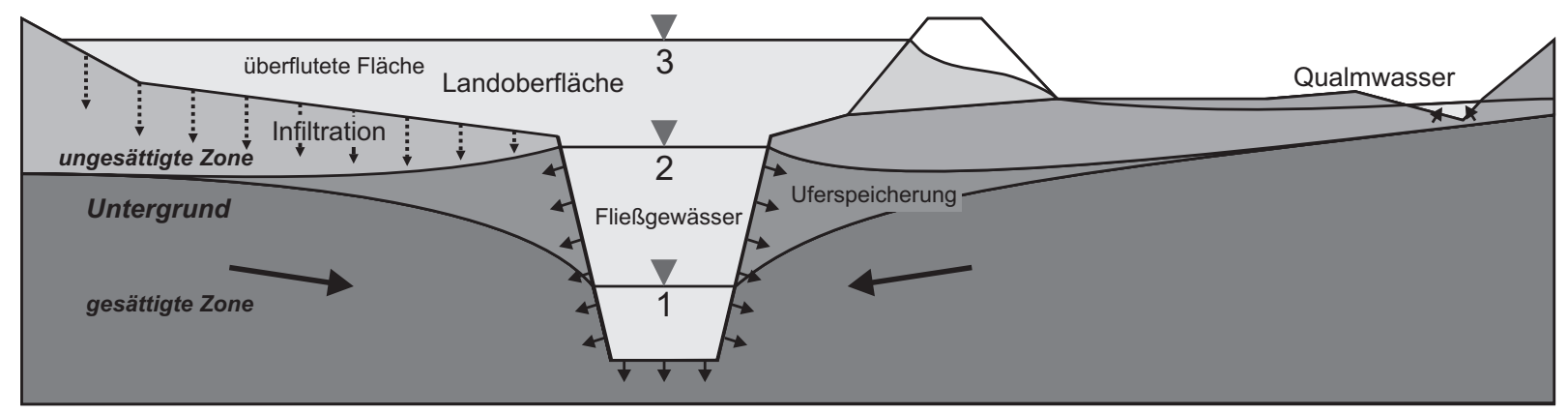

Abbildung 2.1: Einordnung der Bewegung des Wassers im Untergrund (gesättigte Zone und ungesättigte Zone) in die Prozesse der Wasserbewegung beim unterirdischen Hochwasser

Die aus Lockergestein bestehenden Sedimente einer Talaue lassen sich als ein loses Haufwerk von Körnern beschreiben. Selbst bei dichtester Lagerung können die Körner den Raum nicht vollständig ausfüllen. Die Zwischenräume zwischen den Körnern werden Poren genannt. Feststoffpartikel und Porenraum bilden zusammen die Matrix, in der die Prozesse der Wasserbewegung stattfinden (Scheffer 2002). Gesteinskörper, in denen das Wasser fließen kann, bezeichnet man als Grundwasserleiter. Im Gegensatz dazu bilden zum Beispiel Tonschichten oder Festgestein Grundwassergeringleiter oder Grundwasserstauer. Die Abgrenzung des Grundwasserleiters nach unten wird als Unterkante oder Basis des Grundwasserleiters bezeichnet, nach oben hin wird der Grundwasserleiter durch seine Oberkante abgeschlossen. Die obere Grenze kann dabei sowohl die Basis eines Grundwasserstauers als auch die Geländeoberkante sein.

Die Porosität oder nach DIN 4049-3 (1994) der Hohlraumanteil $n_{\mathrm{P}}$ ist ein Maß für das Hohlraumvolumen pro Volumeneinheit des Lockergesteins. Der nutzbare Porenraum $n_{\mathrm{Pn}}$ ist das Verhältnis des frei aus dem Porenraum abfließenden Wassers zum Gesamtvolumen einer gesättigten Bodenprobe (Langguth \& Voigt 1980). Der nicht nutzbare Porenraum wird dagegen von sogenanntem 
Haftwasser ausgefüllt. Der Wassergehalt $\theta$, definiert als Volumen Wasser $V_{\mathrm{W}}$ pro Volumeneinheit Gestein $V_{\mathrm{G}}$

$$
\theta=\frac{V_{\mathrm{W}}}{V_{\mathrm{G}}}
$$

wird aus der Porosität und dem Sättigungsgrad $S$ bestimmt:

$$
\theta=n_{\mathrm{P}} \cdot S
$$

Der Sättigungsgrad $S$ ist das Verhältnis des mit Wasser gefüllten Teils des Hohlraumes zum gesamten Hohlraum (Dracos 1980). Für gesättigte Grundwasserströmung wird $S=1$, für Grundwasserströmung in der ungesättigten Zone dementsprechend $S<1$ (Freeze \& Cherry 1979).

Auf das Grundwasser wirken im wesentlichen die Schwerkraft und Druckkräfte. Die Fließgeschwindigkeiten des Grundwassers sind fast immer so gering, dass die damit verbundene kinetische Energie vernachlässigt werden kann (Freeze \& Cherry 1979). Es wird weiter angenommen, dass keine thermischen und chemischen Einflüsse vorliegen und die Dichte $\rho_{\mathrm{W}}$ des Fluids sich nicht ändert. Die potenzielle Energie pro Masseneinheit $\phi$ ist dann

$$
\phi=\mathrm{g} z+\frac{p-p_{0}}{\rho_{\mathrm{W}}}
$$

mit dem Flüssigkeitsdruck $p$, der geodätischen Höhe $z$ und der Gravitationskonstante g und der Dichte des Wassers $\rho_{\mathrm{W}}$. $p_{0}$ bezeichnet den atmosphärischen Druck oder einen Referenzdruck. Setzt man den atmosphärischen Druck zu $p_{0}=0$, so wird aus Gleichung 2.3

$$
\phi=\mathrm{g} z+\frac{p}{\rho_{\mathrm{W}}}=\mathrm{g} h
$$

$h$ gibt in dieser Gleichung die Standrohrspiegelhöhe an. Anders formuliert gilt

$$
h=z+\frac{p}{\rho_{\mathrm{W} g}} .
$$

Für den Term $\frac{p}{\rho_{\mathrm{W}} \mathrm{g}}$ wird die Flüssigkeitsdruckhöhe $\psi$ eingeführt, so dass die Standrohrspiegelhöhe des Grundwassers auch in der Form

$$
h=z+\psi
$$

notiert werden kann. Die Grundwasserdruckhöhe $h$ drückt hier das hydraulische Gesamtpotenzial aus, das sich aus den Teilpotenzialen Gravitationspotenzial $z$ und Druckpotenzial $\psi$ zusammensetzt.

Erreicht der Grundwasserspiegel die Oberkante des Grundwasserleiters oder steht das Wasser im Grundwasserleiter sogar unter Druck, so spricht man von gespannten Grundwasserverhältnissen. Befindet sich der Grundwasserspiegel hingegen im Grundwasserleiter selber, so kennzeichnet dies ungespannte Grundwasserverhältnisse (Abbildung 2.2). 


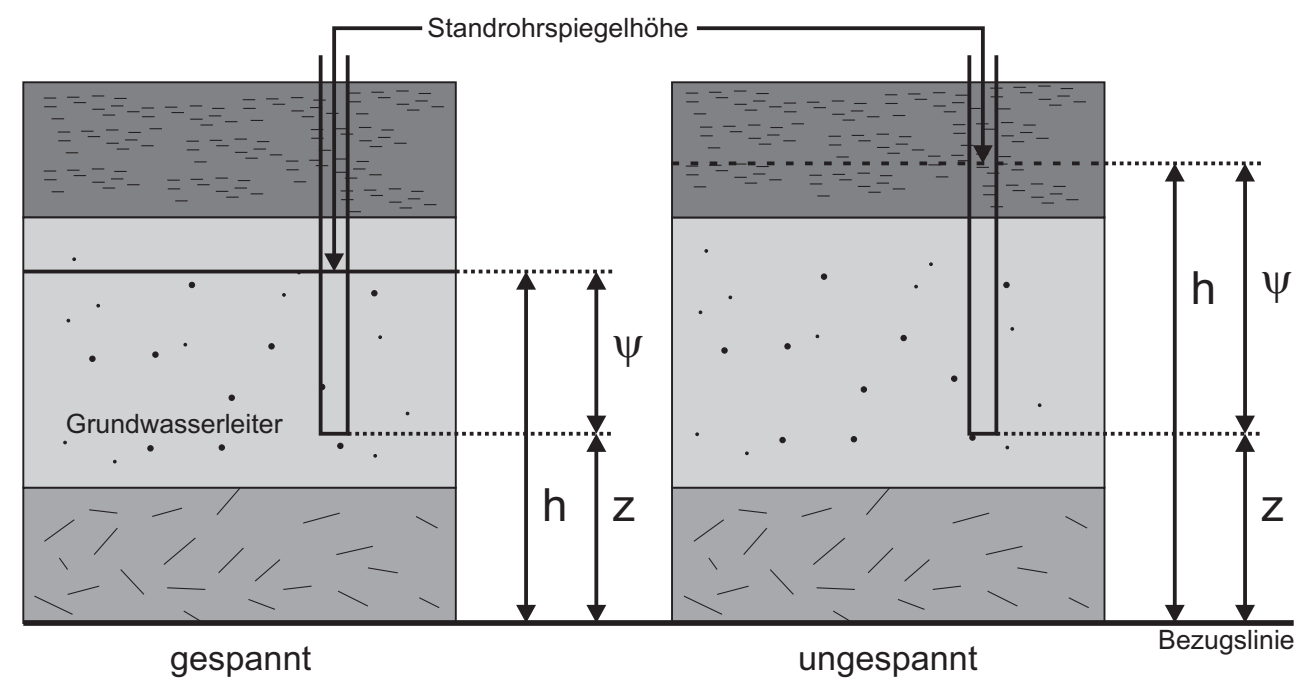

Abbildung 2.2: Definition der Standrohrspiegelhöhe für gespannte und ungespannte Verhältnisse für einen Punkt auf der Höhenlage $z$ über einer Bezugslinie (nach Forkel 2004)

Oberhalb des Grundwasserspiegels schließt sich der Kapillarsaum an, in dem Wasser aufgrund von Kapillarkräften aufsteigen kann. Dieser Bereich und auch der darüberliegende Bereich wird als ungesättigte Bodenzone bezeichnet; durch diesen Bereich gelangt die sogenannte Grundwasserneubildung (im allgemeinen lediglich der Regen) zum Grundwasser. Der Bereich unterhalb des Grundwasserspiegels wird als gesättigter Bereich bezeichnet (Forkel 2004).

\subsection{Die Bewegung des Wassers in der gesättigten Zone}

Grundlage für die Beschreibung der Strömungsprozesse im Untergrund ist das empirische Gesetz von Darcy (1856), das aussagt, dass die Wassermenge pro Zeiteinheit $Q$, die durch eine endliche Fläche $A$ fließt, linear vom Verlust der Druckhöhe $\Delta h$ über eine endliche Strecke $\Delta l$ abhängig ist (Freeze \& Cherry 1979):

$$
\frac{Q}{A}=-k \frac{\Delta h}{\Delta l}
$$

Der Quotient $\frac{Q}{A}=v$ ist der spezifische Fluss und hat die Dimension einer Geschwindigkeit. Er wird auch Darcy-Geschwindigkeit oder Filtergeschwindigkeit genannt. Sie wird dem Schwerpunkt der Fläche $A$ zugeordnet und stellt einen Mittelwert der in einem repräsentativen Elementvolumen auftretenden Geschwindigkeiten dar. Die inhomogene Bodenstruktur wird dabei als Kontinuum aufgefasst. Die durchflossene Fläche $A$ des Kontinuums ist größer als die mit der sogenannten Abstandsgeschwindigkeit $v_{\mathrm{a}}$ tatsächlich durchströmte Porenraumfläche des hetero- 
Heterogenes System

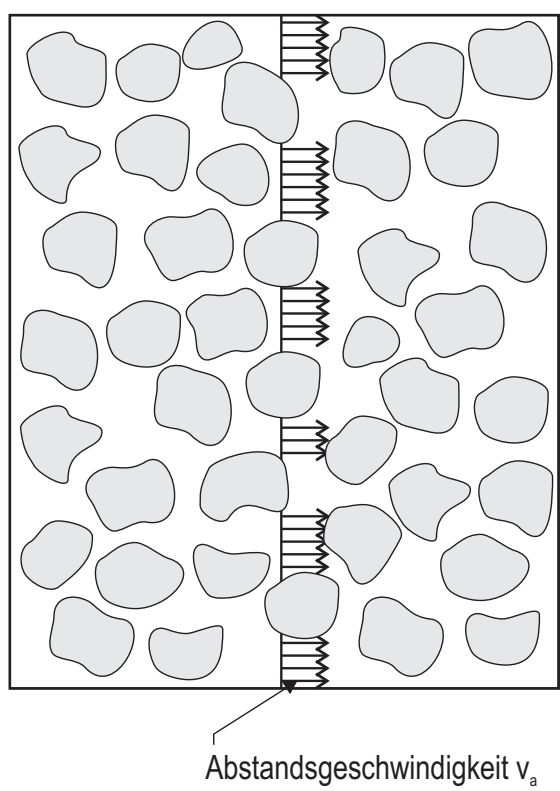

Kontinuumsmodell

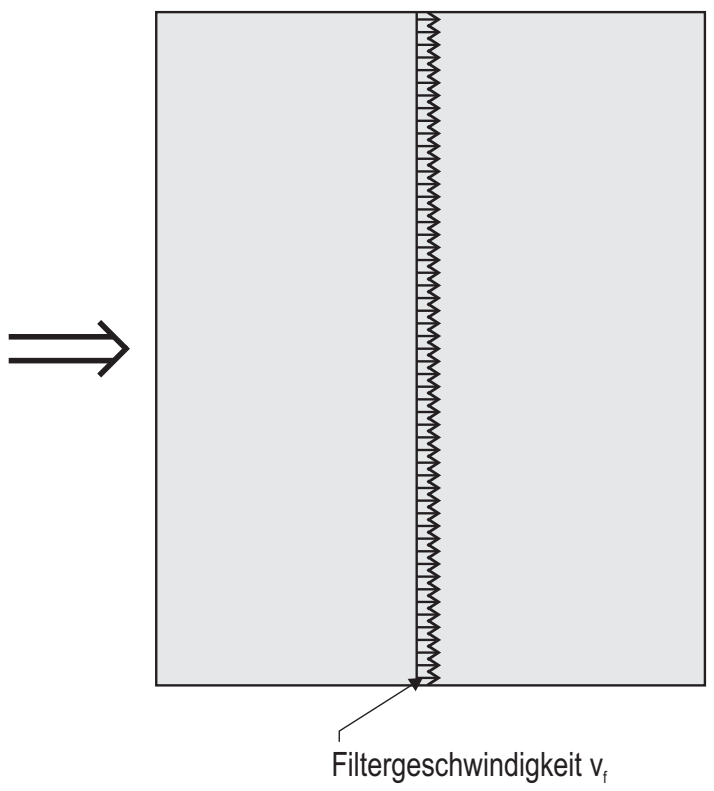

Abbildung 2.3: Abstands- und Filtergeschwindigkeit in porösen Medien nach Forkel (2004)

genen Systems (Abbildung 2.3). Abstands- und Filtergeschwindigkeit hängen damit über den nutzbaren Hohlraumanteil zusammen:

$$
\frac{v}{v_{\mathrm{a}}}=n_{\mathrm{Pn}}
$$

Der Proportionalitätsfaktor $k$ ist der Beiwert der hydraulischen Durchlässigkeit und eine Funktion der Erdbeschleunigung g und der kinematischen Zähigkeit des Fluids $v$. Er charakterisiert den Hohlraum innerhalb des Gesteins in Bezug auf den Strömungswiderstand und bildet damit eine ortsabhängige Kenngröße. Der Quotient $\frac{\Delta h}{\Delta l}$ wird auch hydraulisches Gefälle genannt.

In ihrer Differentialform wird die Darcy-Gleichung zu

$$
v=-k \frac{\partial h}{\partial s}
$$

mit der Koordinate $s$ in Fließrichtung. Das Fließgesetz von Darcy gilt für jede Raumrichtung. Die Verallgemeinerung für den durch $x_{i}=x, y, z$ beschriebenen dreidimensionalen Raum ist

$$
v_{i}=-k_{i j} \frac{\partial h}{\partial x_{i}} \quad i, j=x, y, z
$$

mit dem symmetrischen Durchlässigkeitstensor $k_{i j}$.

Während man in der zweidimensional-horizontalen Betrachtung im Allgemeinen annimmt, dass die Durchlässigkeitseigenschaften isotrop sind, ist dies im dreidimensionalen Raum nur selten 
der Fall. Dies liegt daran, dass Grundwasserleiter vorwiegend durch Ablagerungen von flachem gekörntem Material entstanden sind. Die vertikale Durchlässigkeit ist meist eine Größenordnung niedriger als die horizontale Durchlässigkeit (Forkel 2004):

$$
k_{x}=k_{y} \approx 10 \cdot k_{z}
$$

Im Falle einer horizontalen Lagerung ist nur die Hauptdiagonale des Durchlässigkeitstensors $k_{i j}$ besetzt. Ist dagegen die Lagerung nicht horizontal, sondern zum Beispiel aufgrund von Faltungsvorgängen im Boden schräg, so ist der Durchlässigkeitstensor zwar weiterhin symmetrisch, aber voll besetzt.

Die Gültigkeit des Darcy-Gesetzes beschränkt sich auf die laminare Sickerströmung zwischen den Bodenkörnern. Für die turbulente Sickerströmung, die etwa ab einer Reynolds-Zahl von 1 bis 10 eintritt, müssen Erweiterungsterme berücksichtigt werden (siehe z. B. Forchheimer 1901; Ward 1964; Mattheß \& Ubell 1983). Turbulente Strömungsverhältnisse in porösen Medien liegen in der Regel nur bei besonderen Randbedingungen vor, zum Beispiel in der unmittelbaren Umgebung von Förderbrunnen (Langguth \& Voigt 1980). Die Reynolds-Zahl als Maßzahl für die Turbulenz wird für Grundwasser mit

$$
R e=\frac{v \cdot d_{\mathrm{K}}}{v}
$$

angegeben, wobei $d_{\mathrm{K}}$ der charakteristische Korndurchmesser ist und $v$ die kinematische Viskosität (Forkel 2004). Der charakteristische Korndurchmesser berechnet sich mit der Ungleichförmigkeitszahl $U \mathrm{zu}$

$$
\begin{aligned}
d_{\mathrm{K}} & =U \cdot d_{50} \\
U & =\frac{d_{90}}{d_{10}}
\end{aligned}
$$

(Bollrich 2000) mit $d_{n}$ als dem Korndurchmesser bei $n \%$ der Sieblinie des durchströmten Materials.

Die untere Grenze des Gültigkeitsbereiches des Darcy-Gesetzes lässt sich nicht eindeutig definieren. Langguth \& Voigt (1980) nennen als eine Anwendungsgrenze den Fall toniger Sedimente, bei denen Adsorptionskräfte zwischen den Tonmineralien und den Wassermolekülen wirksam werden und so Schichten von Hydratationswasser entstehen, die den Durchgang von Wasser, das der Schwerkraft folgt, verhindern. Für Kiese und Sande ist eine untere Grenze der Gültigkeit nicht zu definieren (Langguth \& Voigt 1980).

Die Kombination des Darcy-Gesetzes mit der Kontinuitätsbedingung

$$
\frac{\partial v_{x}}{\partial x}+\frac{\partial v_{y}}{\partial y}+\frac{\partial v_{z}}{\partial z}=0
$$


die die Massenerhaltung des Fluids am Kontrollvolumen ausdrückt, liefert eine partielle Differentialgleichung für die dreidimensionale instationäre Strömung in einem wassergesättigten anisotropen porösen Medium, die im Folgenden Grundwasserströmungsgleichung genannt wird:

$$
S_{\mathrm{sp}} \frac{\partial h}{\partial t}=\frac{\partial}{\partial x_{i}}\left(k_{i j} \frac{\partial h}{\partial x_{j}}\right) \quad i, j=1,2,3
$$

Die Herleitung dieser Gleichung 2.16 ist zum Beispiel bei Freeze \& Cherry (1979) oder Forkel (2004) zu finden. $h$ bezeichnet hier die unbekannte Grundwasserdruckhöhe zu einem Zeitpunkt $t, k_{i}$ ist der richtungsabhängige hydraulische Durchlässigkeitsbeiwert und $x, y$ und $z$ oder $x_{1}, x_{2}$ und $x_{3}$ sind die karthesischen Raumkoordinaten.

Der spezifische Speicherkoeffizient $S_{\mathrm{sp}}$ gibt die Volumenänderung über die Zeit an. Für gespannte Strömungsverhältnisse ergibt er sich aus der Elastizität des Korngerüstes und des Wassers. Im ungespannten Aquifer ändert sich das Wasservolumen im betrachteten Bilanzelement durch jede zeitliche Wasserspiegeländerung, die zu einem Entleerungs- und Füllungsvorgang der Poren des Korngerüstes führt. Der hierauf beruhende spezifische Speicherkoeffizient ergibt sich aus dem nutzbaren Porenvolumen $n_{\mathrm{Pn}}$ und ist damit in der Regel um mehrere Zehnerpotenzen größer als der Elastizitätsanteil (Pelka 1988; Langguth \& Voigt 1980).

Oft lassen sich gesättigte Strömungsprozesse im Untergrund ausreichend genau mit einer Vereinfachung der Grundwasserströmungsgleichung auf zwei Dimensionen des Raumes erfassen. Die Reduktion von Gleichung 2.16 kann

- zweidimensional-vertikal oder

- zweidimensional-horizontal

erfolgen. Für eine zweidimensional-vertikale Vereinfachung muss der Vertikalschnitt entlang eines Stromfadens erfolgen, die Geschwindigkeitskomponenten senkrecht zur Schnittebene müssen vernachlässigbar klein sein.

Für praktische Anwendungen von besonderer Bedeutung ist die Integration zur Gleichung für die zweidimensional-horizontale Grundwasserströmung (Forkel 2004)

$$
S_{\mathrm{s}} \frac{\partial h}{\partial t}=\frac{\partial}{x_{i}}\left(T_{i j} \frac{\partial h}{\partial x_{j}}\right) \quad i, j=1,2 .
$$

Die in Abschnitt 1.2 erwähnten Grundwassermodelle für das Stadtgebiet Köln basieren zum Beispiel auf dieser Gleichung, weitere Beispiele beschreiben Bachmann et al. (2007), Becker et al. (2009a), Homann (2006), van Linn (2006) und Junghans (2001).

$T_{i j}$ ist in Gleichung 2.17 die Transmissivität, die sich aus dem Integral des horizontalen Durchlässigkeitsbeiwertes über die wassergefüllte Mächtigkeit des Grundwasserleiters ergibt. Im ungespannten Fall ist die Mächtigkeit vom Grundwasserstand $h$ abhängig, so dass sich eine Nichtlinearität ergibt. $S_{\mathrm{s}}$ ist der Speicherkoeffizient, der sich im gespannten Fall aus der Integration 
des spezifischen Speicherkoeffizienten über die Mächtigkeit des Grundwasserleiters ergibt. Für den ungespannten Fall kann näherungsweise die nutzbare Porosität $n_{\mathrm{Pn}}$ angesetzt werden. Voraussetzungen für die zweidimensional-horizontale Vereinfachung der Grundwasserströmungsgleichung ist, dass

- die Geschwindigkeiten in vertikaler Richtung vernachlässigbar klein sind und

- die horizontalen Geschwindigkeitskomponenten über die Tiefe gleich sind

oder anders ausgedrückt, dass die Standrohrspiegelhöhe über die Tiefe gleich bleibt. Diese Voraussetzungen treffen für viele praktische Anwendungen zu (Forkel 2004). Ein wesentlicher Vorteil der zweidimensional-horizontalen Grundwasserströmungsgleichung ist, dass sich die Lage des Grundwasserspiegels durch die Grundwasserdruckhöhe $h$ ergibt, während für die dreidimensionale und zweidimensional-vertikale Grundwasserströmungsgleichung für die Bestimmung der freien Oberfläche besondere Randbedingungen umgesetzt werden müssen (siehe dazu z. B. Forkel 2004; Diersch 2005e; Scherf 2009).

\subsection{Die Bewegung des Wassers in der ungesättigten Zone}

Nach der einfachsten Modellvorstellung werden die gesättigte und die ungesättigte Zone vom Grundwasserspiegel getrennt. Die Lage des Grundwasserspiegels ist dort, wo der Flüssigkeitsdruck gerade dem atmosphärischen Druck entspricht (Freeze \& Cherry 1979). In der ungesättigten Zone befindet sich im Porenraum des Bodens neben Wasser auch Luft, so dass das Gesetz von Darcy in der im vorangegangenen Abschnitt vorgestellten Form dafür nicht gültig ist. Für die Betrachtung der ungesättigten Strömung wird angenommen, dass die Phasen Luft und Wasser nicht verbunden sind und Luft leicht aus dem Boden entweichen kann, so dass dem Wasser aus der Luftphase nur ein vernachlässigbar kleiner Widerstand entgegengebracht wird. Einflüsse aus der Veränderung des Salzgehaltes und der Temperatur werden ebenfalls nicht berücksichtigt, der Transport von Wasser im gasförmigen Zustand wird vernachlässigt (Rawls et al. 1992).

In der ungesättigten Bodenzone gilt für das Gesamtpotenzial $h$ eine der Gleichung 2.6 entsprechenden Formulierung

$$
h=z+\psi_{\mathrm{m}} .
$$

Das Druckpotenzials $\psi$ ist hier das Matrixpotential $\psi_{\mathrm{m}}$. Das Matrixpotential - auch Saugspannungshöhe - ist ein Maß für den Einfluss der Bodenmatrix auf Wasser. Je weniger Wasser ein Boden enthält, desto stärker halten die matrixbedingten Kräfte, dies sind die Kapillarkräfte des Korngerüsts und die Oberflächenspannung des Fluids, es fest (Scheffer 2002). Da die Auswirkungen des Matrixpotentials in der ungesättigten Zone auf das Wasser dem des Gravitationspotentials entgegengesetzt ist, erhält es ein negatives Vorzeichen (Freeze \& Cherry 1979; Scheffer 2002):

$$
\psi_{\mathrm{m}}<0
$$




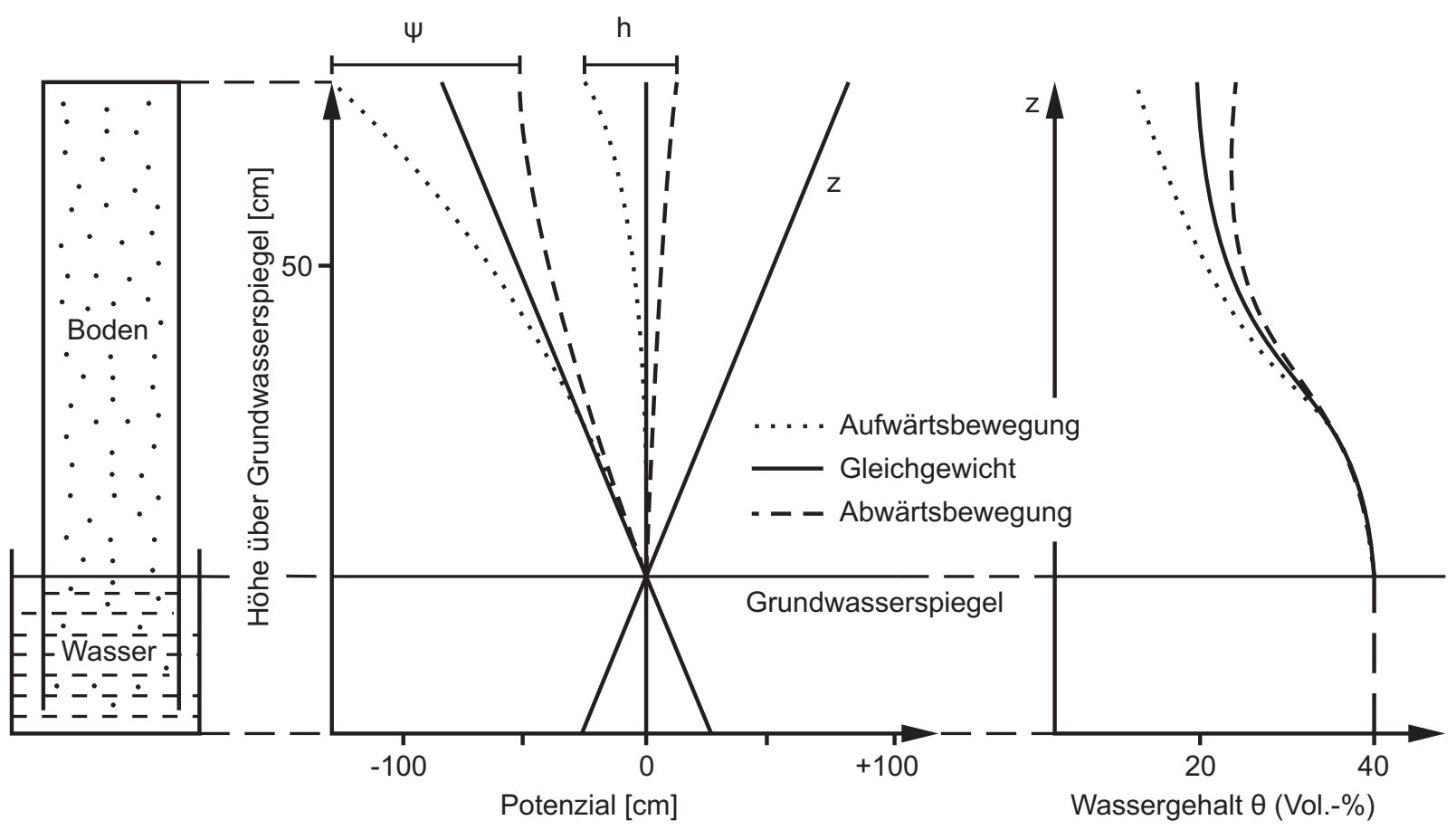

Abbildung 2.4: Hydraulisches Potenzial $h$, Matrixpotenzial $\psi$, Gravitationspotenzial $z$ bezogen auf den Grundwasserstand und Wassergehalt $\theta$ einer Bodensäule im Gleichgewicht, bei Versickerung und bei kapillarem Aufstieg (nach Scheffer 2002)

Bei Betrachtung variabel gesättigter Strömung, die auch den Zustand der vollen Sättigung umfasst, bietet es sich an, vom Druckpotenzial $\psi$ allgemein zu sprechen, das positive Werte bei voller Sättigung und bei Teilsättigung negative Werte annimmt.

In Abbildung 2.4 werden die Komponenten des hydraulischen Potenzials $h$ am Beispiel einer Bodensäule dargestellt. Das Gravitationspotenzial $z$ entspricht der Höhenlage des betrachteten Punktes. Im Gleichgewichtszustand verlaufen die Potentiallinien geradlinig, Gravitationspotential und Matrixpotential sind für jeden Punkt vom Betrag her gleich. Für den gesättigten Bereich nimmt das Gravitationspotential bezogen auf den Wasserspiegel positive Werte, das Matrixpotential negative Werte an. Das Gesamtpotenzial zeigt für jede geodätische Höhenlage auf den Grundwasserspiegel. Der Wassergehalt erreicht unterhalb des Grundwasserspiegels sein Maximum, hier im Beispiel $40 \%$, oberhalb des Grundwasserspiegels liegt er darunter.

Trocknet der Boden von oben her aus, sinkt das Matrixpotenzial $\psi$ an der Oberkante der Bodenprobe. Das Gesamtpotenzial $h$ liegt hier jetzt unter Null, da $|\psi|>|z|$. Die Probe ist damit nicht im Gleichgewicht, deshalb wird eine Aufwärtsbewegung des Wassers ausgelöst. Eine Infiltration von Sickerwasser in die Bodenprobe von oben, wie sie zum Beispiel durch Niederschlag ausgelöst wird, erhöht das Matrixpotenzial und auch den Wassergehalt $\theta$ an der Oberkante der Bodenprobe, das hydraulische Gesamtpotenzial liegt dann dort über Null, es gilt $|\psi|<|z|$. Das Wasser wird sich wegen des abwärts gerichteten Potenzialgradienten in Richtung des Grundwasserspiegels bewegen. 


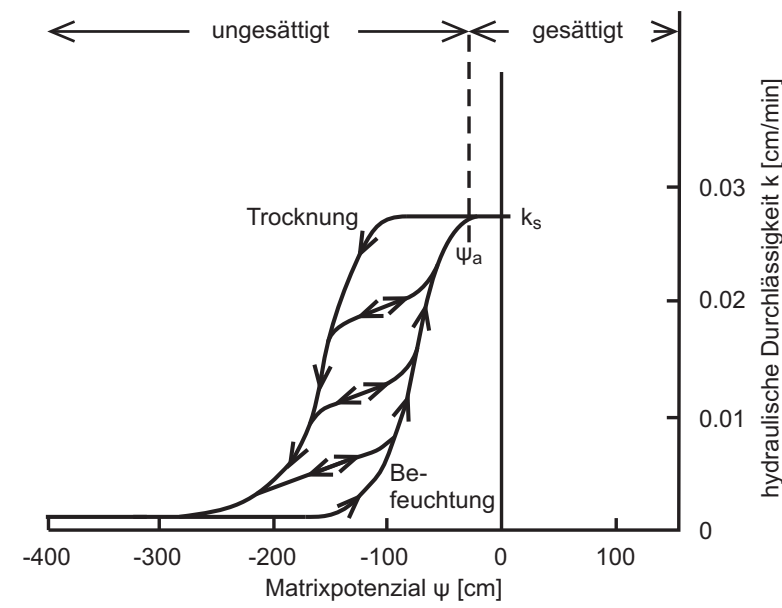

(a) hydraulische Durchlässigkeit $k$

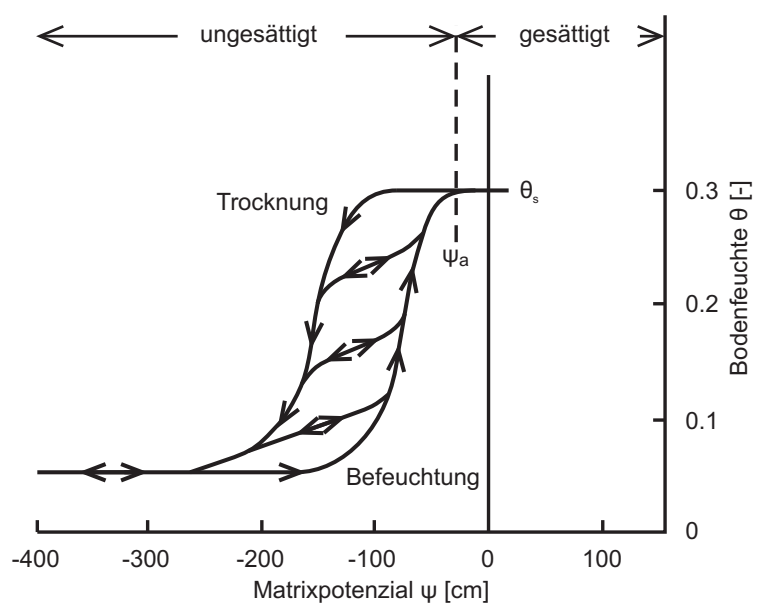

(b) Wassergehalt $\theta$

Abbildung 2.5: Abhängigkeit der hydraulischen Durchlässigkeit $k$ (a) und des Wassergehalts $\theta$ (b) vom Matrixpotential $\psi$ (nach Freeze \& Cherry 1979). Innerhalb der Umhüllenden können alle Zustände erreicht werden, je nach dem, an welcher Stelle eine Befeuchtung bzw. Trocknung einsetzt oder endet. $\psi_{\mathrm{a}}$ kennzeichnet die Saugspannung bei Lufteintritt.

Ausgehend von Gleichung 2.10 lautet das Darcy-Gesetz verallgemeinert für ungesättigte Strömungen in porösen Medien

$$
v_{i}=-k_{i j}(\psi) \frac{\partial h}{\partial x_{i}} \quad i, j=x, y, z .
$$

Die hydraulische Konduktivität $k$, aber auch der Wassergehalt $\theta$ hängen von der Druckhöhe $\psi$ $\mathrm{ab}$ :

$$
\begin{aligned}
& k=k(\psi) \\
& \theta=\theta(\psi)
\end{aligned}
$$

Dementsprechend gilt auch

$$
k=k(\theta) .
$$

Für die hydraulische Durchlässigkeit bei Sättigung wird das Formelzeichen $k_{\mathrm{s}}$ eingeführt, für den Wassergehalt bei Sättigung entsprechend $\theta_{\mathrm{s}}$. Die Beziehungen zwischen $k$ beziehungsweise $\theta$ und $\psi$ sind hysteretischer Natur, das heißt, die Funktion ist für den Fall der Trocknung anders als für den Fall einer Befeuchtung (Abbildung 2.5). Nahe der vollkommenen Sättigung existiert ein Bereich, bei dem sich das Matrixpotential trotz geringfügiger Abnahme der Bodenfeuchte nicht ändert. Die Grenze dieses Bereichs wird durch die Saugspannung $\psi_{\mathrm{a}}$ gekennzeichnet. Erst ab einer Saugspannung von $\psi_{\mathrm{a}}$ beginnt der Boden zu entwässern. $\psi_{\mathrm{a}}$ kennzeichnet damit den Übergang vom gesättigten Bereich zum ungesättigten. 
Die Kombination der Darcy-Gleichung für ungesättigte Strömungen in porösen Medien 2.20 mit dem Gesetz der Massenerhaltung liefert die Richards-Gleichung

$$
C(\psi) \frac{\partial h}{\partial t}=\frac{\partial}{\partial x}\left(k_{\mathrm{x}}(\psi) \frac{\partial h}{\partial x}\right)+\frac{\partial}{\partial y}\left(k_{\mathrm{y}}(\psi) \frac{\partial h}{\partial y}\right)+\frac{\partial}{\partial z}\left(k_{\mathrm{z}}(\psi) \frac{\partial h}{\partial z}\right)
$$

(Freeze \& Cherry 1979; Richards 1931; Forkel 2004) für ungesättigte oder auch teilgesättigte Strömung in porösen Medien mit der spezifischen Feuchtigkeitskapazität $C(\psi)$.

Ein Zusammenhang zwischen $\psi$ und $\theta$ ist nach Mualem (1976) und van Genuchten (1978) über einen dimensionslosen Feuchtigkeitsgehalt $\Theta$ gegeben:

$$
\begin{aligned}
& \Theta=\frac{\theta-\theta_{\mathrm{r}}}{\theta_{\mathrm{s}}-\theta_{\mathrm{r}}} \\
& \Theta=\frac{1}{\left.\left(1+\left|\alpha_{\mathrm{G}} \psi\right|\right)^{n_{\mathrm{G}}}\right)^{m_{\mathrm{G}}}}
\end{aligned}
$$

$\theta_{\mathrm{r}}$ ist der sogenannte Residualwassergehalt, $\alpha_{\mathrm{G}}, n_{\mathrm{G}}$ und

$$
m_{\mathrm{G}}=1-\frac{1}{n_{\mathrm{G}}}
$$

sind sogenannte Van-Genuchten-Parameter. $\alpha_{\mathrm{G}}$ steht im Zusammenhang mit der Saugspannung bei Lufteintritt, während $n$ ein Maß für die Porengrößenverteilung ist (Schaap \& van Genuchten 2006). Die hydraulische Durchlässigkeit für unterschiedliche Befeuchtungsgrade wird aus der Beziehung

$$
k(\Theta)=k_{0} \Theta^{L_{\mathrm{G}}}\left(1-\left(1-\Theta^{\frac{1}{m_{\mathrm{G}}}}\right)^{m_{\mathrm{G}}}\right)^{2}
$$

bestimmt (Schaap \& van Genuchten 2006). $L_{\mathrm{G}}$ ist ein empirischer Parameter zur Berücksichtigung der Porenverbindung (pore-connectivity parameter). Mualem (1976) verwendet einen festen Wert von $L_{\mathrm{G}}=\frac{1}{2}$. Der Parameter $k_{0}$ wird oft mit $k_{0} \approx k_{\mathrm{s}}$ abgeschätzt. Gleichungen 2.26 bis 2.28 sind als Mualem-van-Genuchten-Modell bekannt.

\subsection{Modellierung der Bewegung des Wassers im Untergrund mit Feflow}

\subsubsection{Einführung}

Die oben beschriebenen Differentialgleichungen für die Bewegung des Wassers im Untergrund sind nur für Sonderfälle analytisch lösbar. Im Allgemeinen können die auftretenden Problemstellungen und die dazugehörigen partiellen Differentialgleichungen nur numerisch gelöst werden. Auf dem Markt existiert eine Vielzahl unterschiedlicher Computerprogramme zur numerischen 


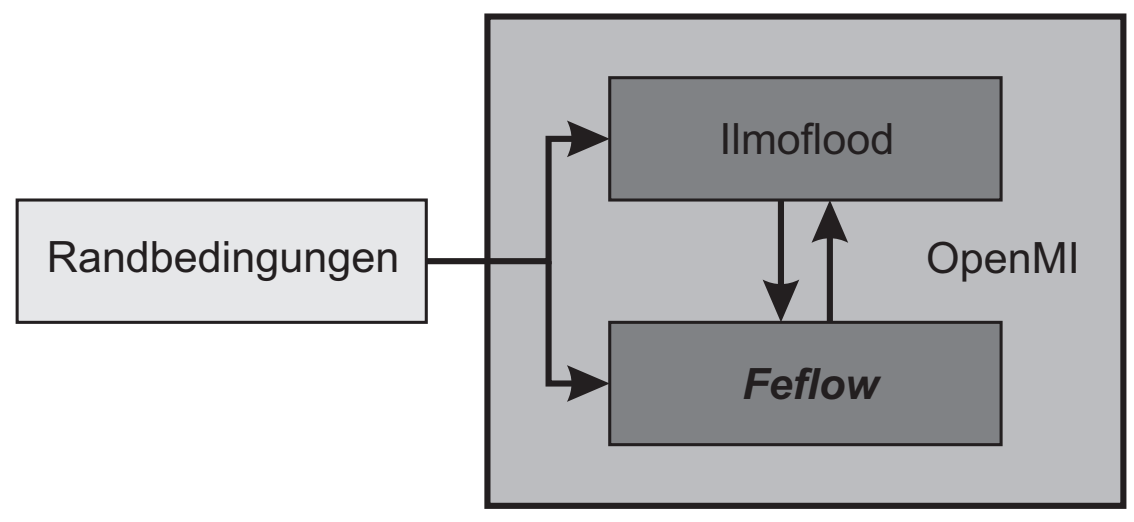

Abbildung 2.6: Einordnung von Feflow in das gekoppelte System zur Modellierung des unterirdischen Hochwassers

Lösung der Bewegungsgleichungen des unterirdischen Wassers. Eine Möglichkeit der numerischen Lösung der Differenzialgleichungen zur Beschreibung der Wasserbewegung in porösen Medien wird anhand des Programms Feflow aufgezeigt. Zur Prognose des unteriridischen Hochwassers deckt Feflow damit die Bewegung des Wassers im Untergrund ab (Abbildung 2.6).

Feflow ist ein kommerzielles Softwarepaket zur Berechnung von Wasserströmung, Massen- und Wärmetransport in porösen Medien nach der Methode der finiten Elemente. Für die Verwendung des Programms Feflow im Rahmen der vorliegenden Problemstellung sprechen folgende Gründe:

- Es ist die Berechnung sowohl gesättigter als auch ungesättigter Strömung im Untergrund möglich. Damit können die für die Fragestellungen des unterirdischen Hochwassers relevanten Prozesse modelliert werden.

- Es können zweidimensional-horizontale, zweidimensional-vertikale und dreidimensionale Modelle aufgebaut werden.

- Zur Implementierung eigener Programmteile dient eine Programmierschnittstelle (siehe Anhang A). Die Kopplung von Strömungsprogrammen erfordert Änderungen im Programmablauf. Feflow erfüllt mit der Programmierschnittstelle somit eine wesentliche Grundvoraussetzung für die Kopplung mit anderen Programmen.

- Die Programmgrundlagen sind wissenschaftlich dokumentiert.

- Durch die weite Verbreitung des Programms in Deutschland kann ein großer Nutzerkreis für gekoppelte Modellrechnungen erschlossen werden, indem das Programm Feflow in ein gekoppeltes System eingebunden wird.

Eine ausführliche Beschreibung des Programms hinsichtlich Funktionalitäten und mathematischer Grundlagen liefern die Benutzerhandbücher, zum Beispiel Diersch (2005c), Diersch (2005d) und Wasy GmbH (2005), die über den Hersteller (Wasy GmbH 2006) zu beziehen sind. Eine positive Bewertung erfährt Feflow von Trefry \& Muffels (2007). 


\subsubsection{Mathematische Grundlagen}

Die in Feflow implementierte Grundgleichung für dreidimensionale vollständig gesättigte Grundwasserströmung ist

$$
S_{\mathrm{sp}} \frac{\partial h}{\partial t}-\frac{\partial}{\partial x_{i}}\left(k_{i j} \frac{\partial h}{\partial x_{j}}\right)=Q_{\mathrm{QS}} \quad i, j=1,2,3
$$

(siehe Diersch 2005d). Gleichung 2.29 entspricht Gleichung 2.16 und ist um einen Quellen-undSenken-Term $Q_{\mathrm{QS}}$ erweitert worden. Der spezifische Speicherkoeffizient $S_{\text {sp }}$ berücksichtigt die Zusammendrückbarkeit des Korngerüsts und das nutzbare Porenvolumen $n_{\mathrm{Pn}}$.

Für die Strömung von Wasser in variabel gesättigten porösen Medien liegt Feflow diese Form der Richards-Gleichung zu Grunde:

$$
\left[S_{\mathrm{S}} S(\psi)+n_{\mathrm{P}} C(\psi)\right] \frac{\partial h}{\partial t}-\nabla\left(k^{\prime}(S) \mathbf{k} \nabla h\right)=Q_{\mathrm{QS}}
$$

(siehe Diersch 2005e). k ist der Tensor der hydraulischen Durchlässigkeit bei Sättigung. Mit der relativen hydraulischen Durchlässigkeit $k^{\prime}$ wird die Abhängigkeit der Durchlässigkeit vom Wassergehalt $\theta$ erfasst.

Zur Beschreibung der Abhängigkeit zwischen dem Wassergehalt $\theta$ und der Druckhöhe $\psi$ steht unter anderen das Mualem-van-Genuchten-Modell (siehe Abschnitt2.3) in der durch die beiden folgenden Gleichungen gegebenen Form zur Verfügung:

$$
\begin{aligned}
& \Theta=\left\{\begin{array}{cc}
\frac{1}{\left(1+\left|\alpha_{\mathrm{G}} \psi\right|^{n} \mathrm{G}\right)^{m_{\mathrm{G}}}} & : \quad \psi<\psi_{\mathrm{a}} \\
1 & : \quad \psi \geq \psi_{\mathrm{a}}
\end{array}\right. \\
& k^{\prime}=\Theta^{\frac{1}{2}}\left(1-\left(1-\Theta^{\frac{1}{m_{\mathrm{G}}}}\right)^{m_{\mathrm{G}}}\right)^{2}
\end{aligned}
$$

Der dimensionslose Feuchtigkeitsgehalt oder auch die effektive Sättigung $\Theta$ ist mit Gleichung 2.26 gegeben. $k^{\prime}$ ist die relative hydraulische Durchlässigkeit

$$
k^{\prime}=\frac{k}{k_{\mathrm{S}}}
$$

(Mualem 1976).

Für eine detaillierte Beschreibung wird auf die Programmdokumentation Diersch (2005d) und Wasy GmbH (2005) verwiesen. Hier sind auch die zweidimensionalen Formulierungen der Bestimmungsgleichungen enthalten. 
Tabelle 2.1: Knotenbasierte Randbedingungsarten des Programms Feflow

\begin{tabular}{cclcc}
\hline Art & Name & Vorgabewert & benannt nach & $\begin{array}{c}\text { Bezeichnung } \\
\text { in Feflow }\end{array}$ \\
\hline 1 & $\mathrm{~h}$ & Grundwasserdruckhöhe & Dirichlet & Head \\
2 & $\mathrm{Q}$ & $\begin{array}{l}\text { Zu- oder Abstromrate senkrecht zur } \\
\text { Modellberandung }\end{array}$ & Neumann & Flux \\
& & $\begin{array}{l}\text { Cauchy } \\
3\end{array}$ & Leakage & $\begin{array}{l}\text { Flusswasserstand } \\
4\end{array}$ \\
\hline
\end{tabular}

\subsubsection{Diskretisierung, Anfangs- und Randbedingungen}

Die räumliche Diskretisierung der partiellen Differentialgleichungen 2.29 und 2.30 erfolgt nach der Methode der finiten Elemente (siehe dazu z. B. Diersch 2005b; Pinder \& Frind 1972). Die Differentialgleichung kann mit dem Programm Feflow zweidimensional oder dreidimensional gelöst werden. Dazu können Finite-Elemente-Netze aus Elementen mit entweder dreieckiger oder viereckiger Grundfläche erstellt werden.

Die Simulationszeit kann entweder in gleichgroße Zeitschritte oder in unterschiedlich große Zeitschritte aufgeteilt werden. Bei letzterer Variante erfolgt die Zeitschrittweitensteuerung entweder automatisch nach Stabilitätskriterien oder die Zeitschritte werden vorab vom Anwender festgelegt. Zur Lösung der Strömungsgleichungssysteme stehen verschiedene numerische Lösungsverfahren zur Verfügung.

Die Differentialgleichungen beschreiben ein Rand- und Anfangswertproblem, für dessen eindeutige Lösung die Vorgabe der Unbekannten zum Zeitpunkt $t=0$ sowie eine lückenlose Beschreibung des Modellrandes durch Randbedingungen für alle Zeitschritte notwendig ist. Anfangsbedingungen müssen für die unbekannte Größe $h$ an jedem Knoten vorgegeben werden. Bei gesättigter Strömung werden die Anfangsbedingungen als Grundwasserstand angegeben, für ungesättigten Strömungsverhältnisse können entweder Druckhöhe, Wassergehalt oder Sättigungsgrad angegeben werden.

Randbedingungen lassen sich grundsätzlich in externe und interne Randbedingungen unterteilen. Externe Randbedingungen beschreiben den Austausch des Modellrandes mit der Umgebung. Ihre Vorgabe ist aus mathematischen Gründen zur Lösung des Rand- und Anfangswertproblems erforderlich. Mit internen Randbedingungen werden Modellobjekte - zum Beispiel ein Brunnen zur Wasserentnahme - innerhalb des Modellgebietes abgebildet. Ihre Vorgabe ist nicht aus mathematischen Gründen erforderlich, sondern sie ergeben sich aus der Problemstellung, der sich mit Hilfe einer numerischen Strömungsberechnung genähert werden soll. Weil es bei der Bearbeitung eines Modells keinen Unterschied macht, ob eine externe oder interne Randbedingung vorliegt, genügt es, den Begriff Randbedingung ohne Zusatz zu verwenden. 


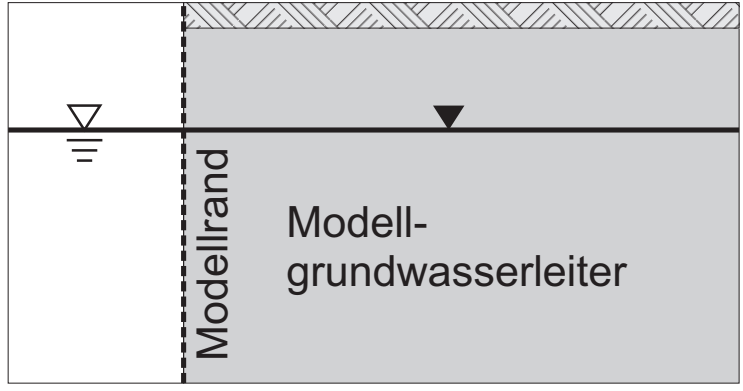

(a) Wasserspiegel eines Oberflächengewässers mit direktem Kontakt zum Grundwasser

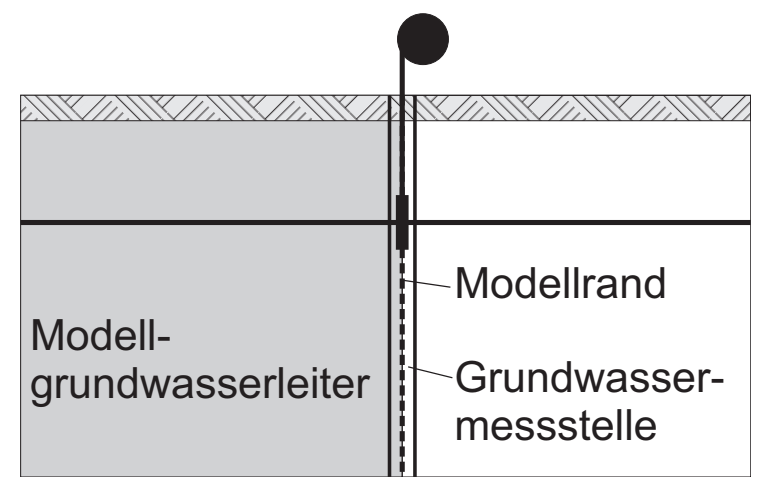

(b) gemessener Grundwasserstand

Abbildung 2.7: Anwendungsbeispiele für h-Randbedingungen in einem Grundwassermodell (nach Spitz \& Moreno 1996)

Die vom Programm Feflow unterstützten Randbedingungsarten für Modellknoten sind in Tabelle 2.1 aufgelistet. Eine Randbedingung erster Art ist die Vorgabe der Unbekannten selbst. Im Falle der Grundwassermodellierung wird für Grundwasserdruckhöhe $h$ für einen Knoten an der Stelle $x_{i}$ für den Zeitpunkt $t$ ein Randbedingungswert $h_{\mathrm{RB}}$ vorgegeben:

$$
h\left(x_{i}, t\right)=h_{\mathrm{RB}}, \quad i=1,2,3
$$

In der Grundwassermodellierung wird wegen der Vorgabe eines Grundwasserstands $h$ die Randbedingung erster Art auch h-Randbedingung genannt. Eine h-Randbedingung wird dort gesetzt, wo der Grundwasserspiegel bekannt ist, also zum Beispiel bei einem direkten Kontakt mit einem Oberflächengewässer (Abbildung 2.7a) oder wenn gemessene Grundwasserspiegel vorliegen (Abbildung 2.7b). Es ist zu beachten, dass der angesetzte Randbedingungswert mit einer Zufluss- oder Abflussmenge korrespondiert. Bei Ansatz einer h-Randbedingung muss es dazu eine Entsprechung in der Realität geben.

Eine Randbedingung zweiter Art ist die Vorgabe einer Ableitung der Unbekannten in Randnormalenrichtung $n_{i}$ durch einen Randbedingungswert $q_{\mathrm{RB}}$ :

$$
k_{i j} \frac{\partial h}{\partial x_{j}} n_{i}\left(x_{i}, t\right)=q_{\mathrm{RB}}\left(x_{i}, t\right), \quad i, j=1,2,3
$$

Dies entspricht der Vorgabe einer $\mathrm{Zu}$ - oder Abstromrate. Typische Anwendungsfälle für eine solche Randbedingung sind zum Beispiel undurchlässige Störungen, die den Modellrand bilden (Abbildung 2.8a), der Zustrom wäre in diesem Fall gleich Null, oder bekannte Zuflüsse, zum Beispiel aus einem Gebirge (Abbildung 2.8b). Für zweidimensional-vertikale oder dreidimensionale Modelle wird die freie Oberfläche des Grundwassers bei ungespannten Verhältnissen als Randbedingung zweiter Art formuliert (siehe z. B. Diersch 2005e). 


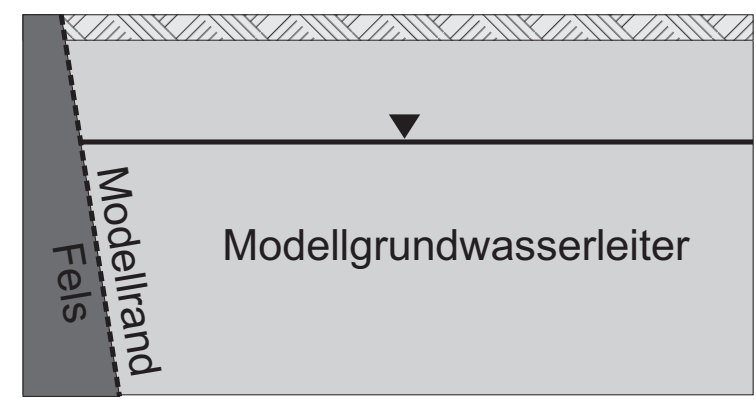

(a) undurchlässige Störung (no flow boundary)

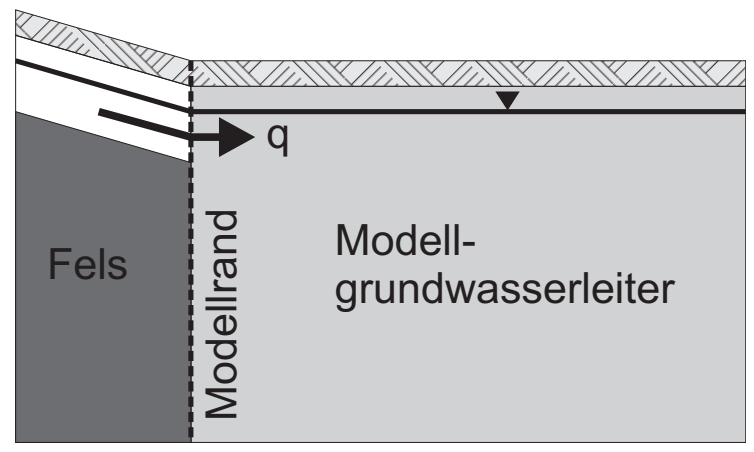

(b) bekannte Zuflussrate

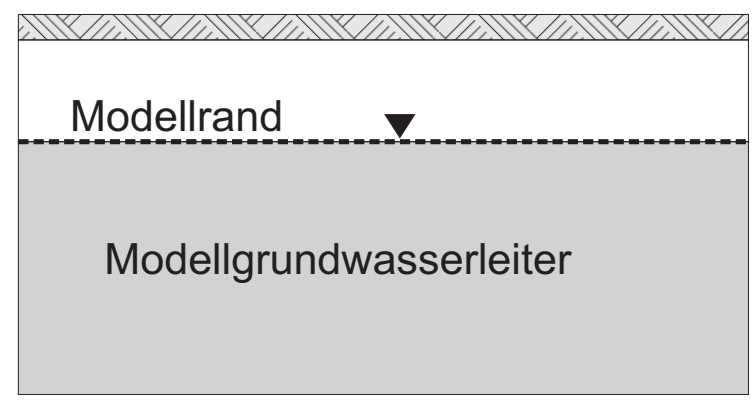

(c) freie Oberfläche (2D-vertikal oder 3D bei ungespannten Verhältnissen)

Abbildung 2.8: Anwendungsbeispiele für Randbedingungen zweiter Art in einem Grundwassermodell (nach Spitz \& Moreno 1996)

Eine Randbedingung dritter Art ist eine Linearkombinationen aus einer Randbedingung erster Art und einer Randbedingung zweiter Art (Forkel 2004). In der Regel wird dabei der Quellenund-Senken-Term aus einem Randbedingungswert $h_{\mathrm{RB}}$ und weiteren Parametern berechnet:

$$
Q_{\mathrm{QS}}\left(x_{i}, t\right)=f\left(h_{\mathrm{RB}}\left(x_{i}, t\right)\right)
$$

Der bekannteste Anwendungsfall ist die Leakage-Randbedingung für den Wasseraustausch mit einem Fließgewässer (Abbildung 2.9p). Der Leakage-Randbedingung widmet sich detaillierter Kapitel 5 .

Als Randbedingung vierter Art stellt das Programm Feflow die Brunnenrandbedingung zur Verfügung (Abbildung 2.10). Dabei wird eine Quelle oder eine Senke direkt vorgegeben:

$$
Q_{\mathrm{QS}}\left(x_{i}, t\right)=Q_{\mathrm{RB}}\left(x_{i}, t\right)
$$

Eine Quelle oder Senke wird im Modell dort angesetzt, wo in der Realität Grundwasser zum Beispiel für Industrie oder öffentliche Wasserversorgung entnommen wird. Randbedingungen vierter und zweiter Art werden programmtechnisch ähnlich umgesetzt und geben $\mathrm{Zu}$ - oder $\mathrm{Ab}$ flüsse für das Modellgebiet an, so dass beide auch als Q-Randbedingung bekannt sind. 


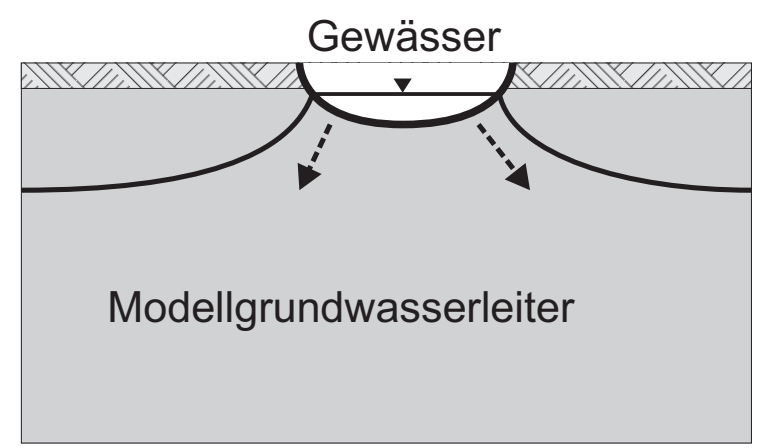

Abbildung 2.9: Anwendungsbeispiel für eine Randbedingung dritter Art: Austausch des Grundwasserleiters mit einem Fließgewässer (nach Spitz \& Moreno 1996)

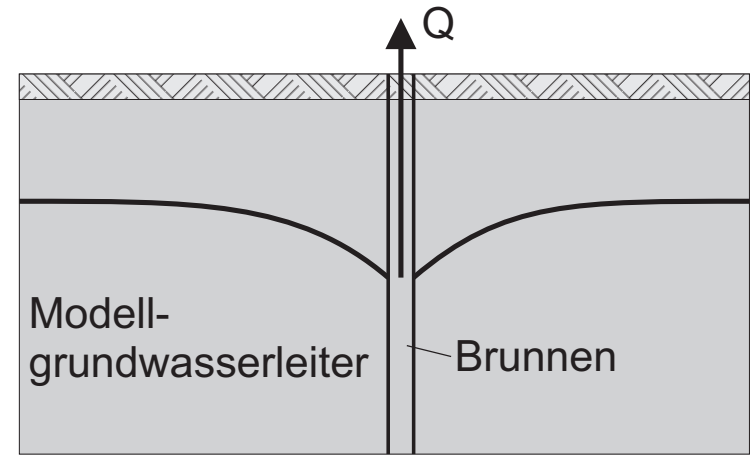

Abbildung 2.10: Anwendungsbeispiel für eine Randbedingung vierter Art: Entnahme aus einem Brunnen im Modellgebiet

Für alle Randbedingungen können im Programm Feflow Nebenbedingungen vorgegeben werden. Darauf wird in den folgenden Kapiteln an geeigneter Stelle eingegangen. Die Nebenbedingungen werden nach der Lösung des Strömungsgleichungssystems überprüft und bei Nichteinhaltung wird eine erneute Berechnung mit veränderten Einstellungen entsprechend der vorgegebenen Nebenbedingung eingeleitet.

Die vorgestellten Randbedingungsarten werden grundsätzlich knotenweise vorgegeben. Eine Randbedingung zweiter Art kann darüber hinaus auch elementweise vorgegeben werden. Anwendungsfall der Elementrandbedingungen sind flächige $\mathrm{Zu}$ - oder Abflüsse wie zum Beispiel die Grundwasserneubildung, die dem System von der Oberfläche zufließt, oder Festgesteinsleakage aus einem liegenden Grundwasserleiter.

\subsection{Zusammenfassung des Kapitels}

Grundgrößen zur Beschreibung der Bewegung des Wassers im Lockergestein sind der Wassergehalt des Bodens und die Standrohrspiegelhöhe. Letztere setzt sich aus der geodätischen Höhe des Messpunktes und der Wasserdruckhöhe zusammen. 
Als Grundlage zur Beschreibung der gesättigten Grundwasserströmung dient das Darcy-Gesetz. Zusammen mit der Kontinuitätsbedingung ergibt sich damit eine Differentialgleichung zur Berechnung der Grundwasserströmung. Wichtige Bodenparameter in dieser Gleichung sind die hydraulische Durchlässigkeit und der Speicherkoeffizient.

Für die Beschreibung des ungesättigten Zustands wird der Begriff Standrohrspiegelhöhe um das Matrixpotential erweitert. Die entsprechende Erweiterung des Darcy-Gesetzes für ungesättigten Zustand führt schließlich zur Richards-Gleichung, mit der sich die Bewegung des Wassers in ungesättigtem porösen Medium mathematisch beschreiben lässt. Auch die Bodenparameter in dieser Richards-Gleichung sind abhängig vom Matrixpotential und damit vom Wassergehalt des Bodens. Als eine Möglichkeit zur Erfassung des Zusammenhangs zwischen Wassergehalt und Matrixpotential wird das Mualem-van-Genuchten-Modell eingeführt.

Sowohl die Gleichung der Grundwasserströmung als auch die Richards-Gleichung sind partielle Differentialgleichungen, deren analytische Lösung nur für Sonderfälle gelingt. Das Grundwasserströmungsprogramm Feflow löst die Grundwasserströmungsgleichung oder die RichardsGleichung nach der Methode der finiten Elemente und stellt damit eine Möglichkeit dar, auch komplexere Fragestellungen der Bewegung des Wassers im Untergrund zu analysieren. 


\section{Die Bewegung des Wassers an der Oberfläche}

\subsection{Einführung}

Die Bewegung des Wassers an der Oberfläche (Abbildung 3.1) stellt, wie bereits in Kapitel 1.2 angesprochen, eine wesentliche Randbedingung für die Ausbreitung des unterirdischen Hochwassers dar.

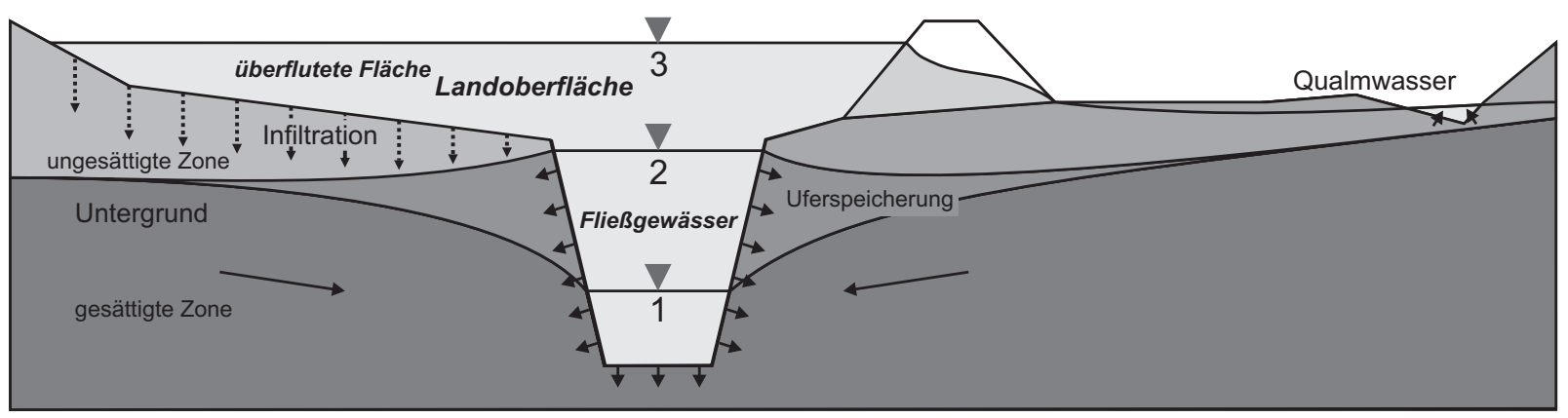

Abbildung 3.1: Einordnung der Bewegung des Wassers an der Landoberfläche (Fließgewässer und überflutete Fläche) in die Prozesse der Wasserbewegung beim unterirdischen Hochwasser

Während die anerkannten Verfahren zur Berechnung von Strömungen im unterirdischen Raum auf dem empirischen Gesetz von Darcy basieren, gelingt für die Beschreibung der Strömung des Wassers an der Erdoberfläche die theoretische Herleitung der Bestimmungsgleichungen, die als Navier-Stokes-Gleichungen in der Hydraulik allgemein bekannt sind. Zur Integration dieser Gleichungen mit dem Ziel, dreidimensionale Strömungsprobleme auf zwei Dimensionen zu reduzieren, ist man jedoch auf verschiedene Annahmen angewiesen, die zu unterschiedlichen Modellklassen führen (Malcherek 2001). Die weitere Integration der zweidimensional-tiefengemittelten Navier-Stokes-Gleichungen über die Breite liefert die SaintVenant-Gleichungen, die eine hohe praktische Bedeutung haben. Die vollständigen Saint-Venant-Gleichungen oder Vereinfachungen davon, wie zum Beispiel das Modell der kinematischen Welle und das Diffusionswellenmodell, sind häufig verwendete Grundgleichungen für die Modellierung des Oberflächenabflusses auf einer beregneten Fläche oder der Gerinneströmung. 


\subsection{Die Navier-Stokes-Gleichungen}

Die Navier-Stokes-Gleichungen

$$
\begin{aligned}
\operatorname{div} \vec{v} & =0 \quad \text { und } \\
\frac{D \vec{v}}{D \vec{t}} & =-\frac{1}{\rho} \operatorname{grad} p+v \operatorname{div} \operatorname{grad} \vec{v}+\vec{f}
\end{aligned}
$$

gelten für inkompressible Flüssigkeiten (Malcherek 2001). Wasser kann als inkompressible Flüssigkeit aufgefasst werden, da sich seine Dichte mit einer Änderung des Drucks nicht ändert. Gleichung 3.1 bezeichnet man als Kontinuitätsgleichung (siehe auch Gleichung 2.15), sie beschreibt die Erhaltung der Masse, Gleichung 3.2 drückt die Impulsbilanz aus. Unbekannt ist der Geschwindigkeitsvektor

$$
\vec{v}=\left(\begin{array}{c}
v_{x} \\
v_{y} \\
v_{z}
\end{array}\right) \text {. }
$$

$p$ steht für den Druck, $\rho$ ist die Dichte des Fluids und $v$ die kinematische Viskosität des Fluids.

Für $v_{x}$, die Geschwindigkeitskomponente in x-Richtung, lautet Gleichung 3.2 ausgeschrieben

$$
\frac{\mathrm{d} v_{x}}{\mathrm{~d} t}+\underbrace{v_{x} \frac{\partial v_{x}}{\partial x}+v_{y} \frac{\partial v_{x}}{\partial y}+v_{z} \frac{\partial v_{x}}{\partial z}}_{\text {Advektion }}=\underbrace{-\frac{1}{\rho} \frac{\partial p}{\partial x}}_{\text {Druck }}+\underbrace{v\left(\frac{\partial^{2} v_{x}}{\partial x^{2}}+\frac{\partial^{2} v_{x}}{\partial y^{2}}+\frac{\partial^{2} v_{x}}{\partial z^{2}}\right)}_{\text {innere Reibung }}+\underbrace{f_{x}}_{\text {Massenkräfte }} .
$$

Der erste Term drückt in dieser Gleichung die zeitlichen Änderung des Impulses am Kontrollvolumen aus. Die Advektionsterme berücksichtigen die zeitlichen Differenz der ein- und austretenden Impulse am Kontrollvolumenelement und beschreiben somit die Impulsänderung infolge einer räumlichen Veränderung des Impulsfeldes. Diese beiden Beschleunigungskomponenten stehen im Gleichgewicht mit Kräften aus Druck, Reibung und Masse (Forkel 2004).

Der Kraftvektor $\vec{f}$ setzt sich im Wesentlichen aus der Schwerkraft und der Coriolis-Kraft

$$
\vec{f}=-\overrightarrow{\mathrm{g}}-2 \vec{\omega} \times \vec{v} \cong\left(\begin{array}{c}
2 \omega v_{y} \sin \Phi \\
-2 \omega v_{x} \sin \Phi \\
\mathrm{g}
\end{array}\right)
$$

zusammen, wobei letztere erst bei ausgedehnten Strömungen relevant wird (Forkel 2004). $\vec{\omega}$ ist die Winkelgeschwindigkeit der Erde, $\Phi$ die geographische Breite.

Die Lage der Gewässeroberfläche ist über eine kinematische ${ }^{1}$ Randbedingung gegeben. Ferner hat die Strömung an der freien Oberfläche die dynamische ${ }^{2}$ Randbedingungen zu erfüllen, über

\footnotetext{
${ }^{1}$ Kinematik: Untersuchung und Beschreibung von Bewegungen ohne Berücksichtigung der sie verursachenden Kräfte (Brockhaus-Enzyklopädie Online 2009b)

${ }^{2}$ Dynamik: Lehre von den Kräften und Wechselwirkungen und den von ihnen hervorgerufenen Bewegungs- oder Zustandsänderungen (Brockhaus-Enzyklopädie Online 2009a)
} 
die an der Oberfläche wirkende Windschubspannungen einbezogen werden. An der unteren Begrenzung des Fließgewässers sind Sohlrandbedingungen zu formulieren (Malcherek 2001).

\subsection{Zweidimensional-tiefenintegrierte Strömungsgleichungen}

Für viele Fragestellungen ist es ausreichend, die Strömung zweidimensional-tiefengemittelt zu beschreiben, weil die vertikale Geschwindigkeitskomponente ausreichend klein ist. Die Herleitung der auch als zweidimensionale Flachwassergleichungen bekannten tiefenintegrierten Strömungsgleichungen lässt sich aus den Navier-Stokes-Gleichungen nicht exakt ausführen, sondern es sind verschiedene Annahmen zu treffen. Malcherek (2001) nennt als wesentliche Grundannahmen die Voraussetzung

- eines hydrostatischen Druckverlaufs (Flachwassernäherung) und

- einer konstanten Dichte über die vertikale Achse.

Die zweidimensional-tiefengemittelte Kontinuitätsgleichung lautet

$$
\frac{\partial H}{\partial t}+\frac{\partial v_{x} H}{\partial x}+\frac{\partial v_{y} H}{\partial y}=0
$$

$v_{x}$ und $v_{y}$ haben hier die Bedeutung der tiefengemittelten Geschwindigkeitskomponenten in xund in y-Richtung.

Für die x-Richtung lautet die Gleichung zur Impluserhaltung

$$
\frac{\partial v_{x}}{\partial t}+\underbrace{v_{x} \frac{\partial v_{x}}{\partial x}+v_{y} \frac{\partial v_{x}}{\partial y}}_{\text {Advektion }}+\underbrace{\mathrm{g} \frac{\partial h}{\partial x}}_{\text {freie Oberfläche }}=\underbrace{\frac{1}{H} \frac{\partial}{\partial x} \frac{\tau_{x x}^{\mathrm{D}} h}{\rho}+\frac{1}{H} \frac{\partial}{\partial y} \frac{\tau_{x y}^{\mathrm{D}} h}{\rho}}_{\text {Impulsdispersion }}-\underbrace{\frac{1}{H} \frac{\tau_{x}^{\mathrm{B}}}{\rho}}_{\text {Sohlreibung }}+\underbrace{\frac{1}{H} \frac{\tau_{x}^{\mathrm{W}}}{\rho}}_{\text {Wind }}+\underbrace{2 \omega v_{y} \sin \Phi}_{\text {Coriolis-Kraft }}
$$

mit der geodätischen Lage der freien Oberfläche $h$, der Sohlschubspannung $\tau^{\mathrm{B}}$ und der Windschubspannung $\tau^{\mathrm{W}}$. Die Wassertiefe $H$ wird als

$$
H(x, y, t):=h(x, y, t)-z_{\mathrm{B}}(x, y)
$$

definiert, $z_{\mathrm{B}}$ bezeichnet die Lage der Gewässersohle (Malcherek 2001).

Der Impulsdispersionstensor enthält Impulsbeiwerte $\tau_{i j}^{\mathrm{D}}$, die Geschwindigkeitsänderungen über die Tiefe berücksichtigen. Die Impulsbeiwerte sind durch weitere Modellannahmen oder empirische Gleichungen zu bestimmen (siehe z. B. Malcherek 2001).

Während in den Navier-Stokes-Gleichungen die Sohl- und Windschubspannungen nicht direkt in der Strömungsgleichung, sondern in Form von Randbedingungen auftauchen, erscheinen sie in den zweidimensional-tiefengemittelten Strömungsgleichungen als Quell- und Senkenterme für den tiefengemittelten Impuls. 


\subsection{Die Saint-Venant-Gleichungen}

Durch die Integration der zweidimensional-tiefengemittelten Strömungsgleichungen über die Breite des Gerinnes erhält man die Saint-Venant-Gleichungen. Weil die eindimensionale Strömungsmodellierung mit dem wenigsten Rechenaufwand verbunden ist und weil viele Fragestellungen wie zum Beispiel die Berechnung von Wasserspiegellagen in einem Fluss mit eindimensionalen Berechnungen beantwortet werden können, haben die Saint-Venant-Gleichungen eine hohe praktische Bedeutung. Hochwassergefahrenkarten werden oft aus Modellergebnissen auf Basis der Saint-Venant-Gleichunggen erstellt (Ministerium f. Umwelt u. Naturschutz, Landwirtschaft und Verbraucherschutz d. Landes Nordrhein-Westfalen 2003; Umweltministerium, Innenministerium und Wirtschaftsministerium Baden-Württemberg 2005).

Die eindimensionale Kontinuitätsgleichung lautet

$$
\frac{\partial A}{\partial t}+\frac{\partial Q}{\partial s}=0
$$

(Malcherek 2001), $s$ ist hier die eindimensionale Koordinate in Fließrichtung, $Q$ beschreibt den über die benetzte Querschnittsfläche $A$ strömenden Durchfluss. Die gemittelten Impulsgleichungen werden $\mathrm{zu}$

$$
\frac{\partial Q}{\partial t}+\frac{\partial v Q}{\partial s}=-\mathrm{g} A \frac{\partial h}{\partial s}-\mathrm{g} A I_{\mathrm{E}}
$$

Die Annahmen, dass keine Strömungen senkrecht zur Hauptachse vorliegen und dass die tiefengemittelten Transversalgeschwindigkeiten Null sind, setzt voraus, dass die Koordinatenachse entlang der Hauptströmung ausgerichtet ist.

Gleichungen 3.9 und 3.10 werden in der Literatur oft in der Form

$$
\frac{\partial H}{\partial t}+v \frac{\partial H}{\partial s}+H \frac{\partial v}{\partial s}=\frac{\partial v}{\partial s}\left(H-\left(\frac{\partial A}{\partial H}\right)^{-1}\right)
$$

und

$$
\frac{\partial v}{\partial t}+v \frac{\partial v}{\partial s}+\mathrm{g} \frac{\partial H}{\partial s}=-\mathrm{g} \frac{\partial z_{\mathrm{B}}}{\partial s}-\mathrm{g} I_{\mathrm{E}}
$$

angegeben. Diese Form der eindimensionalen Strömungsgleichungen werden Saint-VenantGleichungen genannt. Es ist hier zu beachten, dass $v$ und $h$ Verteilungen über Breite und Tiefe aufweisen, daher ist laut Malcherek (2001) die Formulierung gemäß Gleichungen 3.9 und 3.10 vorzuziehen.

Es existieren verschiedene Ansätze zur Beschreibung des Energieliniengefälles $I_{\mathrm{E}}$. Bekannt sind zum Beispiel Ansätze von Manning-Strickler, Darcy-Weisbach oder Colebrook-White (Malcherek 2001). 


\subsection{Das Modell der kinematischen Welle}

Die Vereinfachung der Saint-Venant-Gleichungen nach dem Modell der kinematischen Welle ( $k i$ nematic wave approximation) basiert auf der Annahme, dass der Impuls einer instationären Strömung gleich dem Impuls einer stationär-gleichförmigen Strömung gesetzt werden kann (Fread 1992). Die Impulsgleichung lautet dann

$$
\frac{\partial Q}{\partial t}+c \frac{\partial Q}{\partial s}=0
$$

mit der kinematischen Wellengeschwindigkeit $c$, die eine Funktion des Durchflusses $Q$ ist. Die Anwendung des Modells der kinematischen Welle ist auf eindeutige Wasserstands-DurchflussSchlüsselkurven begrenzt. Weiterhin können Rückstaueffekte und negative Fließgeschwindigkeiten nicht modelliert werden (Fread 1992). Die kinematic wave approximation wird deshalb oft für hydrologische Einzugsgebietsmodelle zur Beschreibung des Oberflächenabflusses verwendet.

\subsection{Das Modell der Diffusionswelle}

Eine andere Vereinfachung der Saint-Venant-Gleichungen ist unter dem Namen diffusive wave approximation oder zero inertia-Modell (,keine Trägheitskräfte“) bekannt. Aus den Impulsgleichungen werden nur der Druck- und Reibungsterm übernommen (Fread 1992):

$$
\frac{\partial h}{\partial s}+I_{\mathrm{E}}=0
$$

Dieser Ansatz geht davon aus, dass die Reibungsterme dominant sind, während die Wassertiefe besonders klein ist. Die diffusive wave approximation wird ebenfalls für den Oberflächenabfluss in hydrologischen Modellen eingesetzt. Rückstaueffekte lassen sich bedingt abbilden, nicht anwendbar ist dieses Modell für hochgradig instationäre Strömungen (Fread 1992).

\subsection{Modellierung der Wasserbewegung an der Landoberfläche mit Ilmoflood}

\subsubsection{Allgemeine Beschreibung des Programms}

Zur Berechnung der Bewegung des Wassers an der Oberfläche wurde das Programm Ilmoflood entwickelt (Niemeyer \& Kamrath 2007). Ilmoflood nutzt ein hybrides Verfahren zur Modellierung von Fließgewässern mit Überflutungsflächen: ein Fließgewässer wird als eindimensionale 


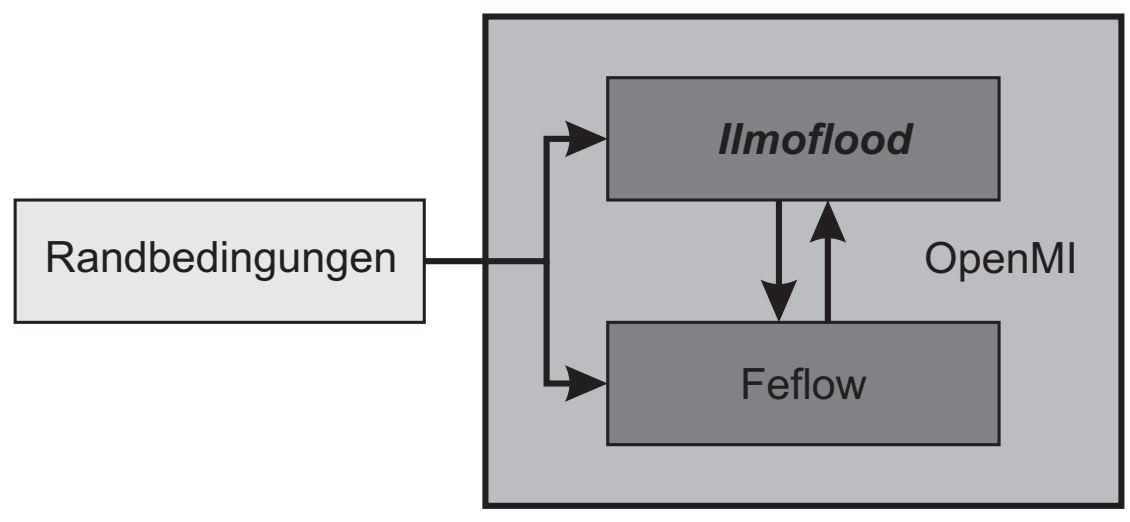

Abbildung 3.2: Einordnung von Ilmoflood in das gekoppelte System zur Modellierung des unterirdischen Hochwassers

Strömung auf Basis des Diffusionswellenmodells (Abschnitt 3.6 modelliert, Überflutungsbereiche werden mit Hilfe des Storage-Cell-Verfahrens berechnet. Nur der Programmteil zur Berechnung von Überflutungsflächen findet Eingang in das gekoppelte Systems zur Modellierung des unterirdischen Hochwassers (Abbildung 3.2). Die Berechnungsgrundlagen des IImoflood zu Grunde liegenden Storage-Cell-Verfahrens werden deshalb im folgenden Abschnitt erläutert. Storage-Cell-Verfahren sind für die Berechnung von Überflutungsflächen verbreitet (Kamrath 2009), auch das in Abschnitt 1.2 erwähnte Überflutungsflächenmodell für die Stadt Köln verwendet einen Storage-Cell-Ansatz.

Das Programm Ilmoflood wurde am Institut für Wasserbau und Wasserwirtschaft (RheinischWestfälische Technische Hochschule Aachen) entwickelt (Hausmann 2005a; Kamrath 2009) und bereits in mehreren Untersuchungen erfolgreich eingesetzt (z. B. Kufeld et al. 2009, Schüttrumpf et al. 2008; Kamrath et al. 2008). Ilmoflood ist als „Forschungscode“ in der Programmiersprache $\mathrm{C}_{++}$objektorientiert entwickelt und als Konsolenanwendung erstellt worden. Alle Eingaben sind deshalb vom Anwender über Textdateien vorzugeben. Der Quellcode des Programms ist für diese Arbeit verfügbar und kann für eine Kopplung mit einem Grundwasserströmungsprogramm angepasst werden. Das Programm zeichnet sich durch eine schnelle Berechnung der Strömung aus. Eine detaillierte Beschreibung des Programms liefert Kamrath (2009).

\subsubsection{Grundlagen des zweidimensionalen Überflutungsflächenmoduls}

Das zweidimensionale Überflutungsflächenmodul ist eine Weiterentwicklung des Storage-CellProgramms polderFlood (Hausmann 2005b, a). Das Modellgebiet wird in ein viereckiges Raster aus gleichgroßen quadratischen Zellen aufgeteilt. Für jede dieser Zellen wird die Kontinuitätsbe- 
dingung als Bilanz der einer Zelle zu- und abfließenden Wassermengen formuliert. Die zeitliche Änderung des Wasservolumens $V$ einer Zelle $i$ ist damit

$$
\frac{\partial V_{i}}{\partial t}=b^{2} \frac{\partial H_{i}}{\partial t}=\sum_{j} Q_{i, j}+Q_{\mathrm{QS}}
$$

(Niemeyer \& Kamrath 2007). $b$ bezeichnet die Kantenlänge der Zelle, $Q_{i, j}$ steht für den Fluss zwischen der Zelle $i$ und einer Nachbarzelle $j, Q_{\mathrm{QS}}$ ist ein als Randbedingung vorgegebener Zuoder Abfluss der Zelle $i$. Der Quotient $\frac{\partial h}{\partial t}$ drückt die Veränderung der Wasserspiegellage einer Zelle aus.

Anstelle der Impulsgleichung tritt die empirische Fließformel nach Manning-Strickler, mit denen die Flüsse $Q_{i, j}$ zwischen den Zellen berechnet werden:

$$
Q_{i, j}=A_{i, j} \cdot k_{\mathrm{St} i, j} \cdot r_{\mathrm{h} i, j}{ }^{\frac{2}{3}} \cdot \sqrt{I_{\mathrm{E} i, j}} .
$$

$A_{i, j}$ ist die Durchflussfläche an der Grenze der benachbarten Zellen $i$ und $j, k_{\mathrm{St} i, j}$ bezeichnet den Strickler-Beiwert an dieser Stelle, $r_{\mathrm{h} i, j}$ den hydraulischen Radius und $I_{\mathrm{E} i, j}$ das Energieliniengefälle zwischen den benachbarten Zellen. Das Energieliniengefälle wird vereinfachend mit der Differenz der Wasserstände zwischen benachbarten Zellen abgeschätzt (Hausmann 2005a):

$$
I_{\mathrm{E} i, j} \approx h_{i}-h_{j}
$$

Somit ist das Ilmoflood zu Grunde liegende Storage-Cell-Verfahren eine vereinfachte zweidimensionale Formulierung des Diffusionswellenmodells mit einem empirischen Reibungsterm.

Es entsteht so für das vollständige Raster mit einer impliziten zeitlichen Diskretisierung ein nichtlineares inhomogenes System von gekoppelten steifen Differentialgleichungen erster Ordnung (Niemeyer \& Kamrath 2007). Damit lässt sich der Wasserspiegel für jedes Zellelement für unterschiedliche Zeitpunkte unter dem Einfluss von $\mathrm{Zu}$ - und Abflüssen von außen sowie dem Ausspiegeln und dem Wasseraustausch zwischen den Zellen untereinander berechnen.

Die zeitliche Diskretisierung erfolgt mit einem impliziten Mehrschrittverfahren. Dadurch wird im Vergleich zu alternativen expliziten Verfahren die Effizienz erhöht (Kamrath 2007). Zur Lösung des Gleichungssystems wird der Gleichungslöser CVODE von SUNDIALS (2008) verwendet. Bei Bedarf wird die Bestimmungsmatrix des bestehenden Gleichungssystems zur Optimierung der Bandbreite transformiert, um weitere Effizienzgewinne zu erreichen.

Das Berechnungsnetz wird vom Anwender durch die Vorgabe der Anzahl von Zellen in x- und in y-Richtung sowie durch Vorgabe der Zellkantenlänge definiert. Für die Einordnung des Rasters in ein lokales Koordinatensystem wird die Lage des Mittelpunktes der linken unteren Zelle benötigt. Weiterhin sind für jede Zelle die Höhenlage (z-Koordinate) und ein Strickler beiwert $k_{\mathrm{St}}$ vorzugeben. Als Randbedingung können Zellzuflüsse vorgegeben werden (Q-Randbedingung). Ilmoflood sieht dabei konstante oder zeitlich veränderliche Randbedingungen vor. Letztere werden in Form einer der Zelle zugeordneten Ganglinie vorgegeben. Über diese Zellzuflüsse wird 
auch der Austausch von Wasser mit benachbarten Kompartimenten realisiert. Schließlich werden Anfangsbedingungen benötigt. Dabei können die Zellen zu Beginn der Berechnung auch trocken liegen. Als Besonderheit können Geradenabschnitte vorgegeben werden, über die kein Wasser fließen kann (Hausmann 2005b).

\subsection{Zusammenfassung des Kapitels}

Zur vollständigen Beschreibung der Strömung eines inkompressiblen Fluids im dreidimensionalen Raum sind die Navier-Stokes-Gleichungen anerkannt. Eine Integration dieser Gleichungen über die Fließtiefe liefert die zweidimensional-tiefengemittelten Flachwassergleichungen, nach einer weiteren Integration über die Breite gelangt man zu den eindimensionalen Saint-Venant-Gleichungen. Diese Gleichungen haben eine große Bedeutung in der Ingenieurpraxis. Zwei gängige Vereinfachungen dieser Saint-Venant-Gleichungen sind das Modell der kinematischen Welle und das Diffusionswellenmodell.

Letzteres ist die Grundlage für die Berechnung eindimensionaler Gerinneströmung im Programm Ilmoflood. Mit Ilmoflood können an ein eindimensionales Fließgewässermodell auch zweidimensionale Überflutungsflächenmodelle angekoppelt werden. Das Programmmodul für Überflutungsflächen wird für das Prognosewerkzeug des unterirdischen Hochwassers verwendet. Die Wasserbewegung auf einer Überflutungsfläche berechnet IImoflood mit Hilfe des StorageCell-Verfahrens. Es handelt sich dabei um eine eine zweidimensionale Formulierung nach dem Vorbild der Diffusionswellengleichung. Die Kontinuitätsbedingung wird als Bilanz der ein- und ausströmenden Wassermengen für jede der quadratischen Rasterzellen formuliert und anstelle der Impulsgleichung tritt die empirische Fließformel nach Manning-Strickler. Ilmoflood erreicht mit den gewählten numerischen Verfahren besonders geringe Rechenzeiten. 


\section{Kopplung von Strömungsmodellen}

\subsection{Einführung}

In den Kapiteln 2 und 3 wurden die Fließgesetze für die Bewegung des Wassers an der Oberfläche und für die Bewegung des Wassers im Untergrund eingeführt. Für beide Kompartimente werden jeweils Programme zur Berechnung der Wasserbewegung innerhalb des Kompartiments vorgestellt. Zur Modellierung des unterirdischen Hochwassers ist es wegen der Wechselwirkungen zwischen den beiden Kompartimenten notwendig, Berechnungsergebnisse des einen Modells an das andere Modell zu übergeben. Erfolgt dies während der Laufzeit der Strömungsberechnung, wird von einer Modellkopplung gesprochen. Nur durch eine Modellkopplung ist eine wechselseitige Beeinflussung der Berechnungsergebnisse möglich. Eine Modellkopplung muss neben den verschiedenen Fließgesetzen in der Regel auch auf unterschiedliche Netze für Raum und Zeit Rücksicht nehmen. Schließlich sind die Wasseraustauschvorgänge zwischen den gekoppelten Kompartimenten modelltechnisch zu erfassen.

Der folgende Abschnitt enthält einen Überblick über verschiedene Arten der Modellkopplungen, über die in der Fachliteratur im Zusammenhang mit den Wechselwirkungen zwischen Oberflächenwasser und Grundwasser berichtet wird. Der Überblick gliedert sich entsprechend der Klassifizierung von Morita \& Yen (2002) in

- vollständig implizite Kopplung

- iterative Kopplung und

- externe Kopplung.

Im Anschluss werden die drei Arten der Modellkopplung hinsichtlich ihrer Eignung für die Modellierung des unterirdischen Hochwassers unter Verwendung bereits bestehender Programme diskutiert. Im letzten Abschnitt wird der OpenMI-Standard vorgestellt, mit dem die Kopplung der Programme Feflow und Ilmoflood realisiert wird. Allgemeine Informationen darüber, wie bestehende Programme mit einer OpenMI-Schnittstelle ausgerüstet werden können, enthält Anhang $B$. Wie dies für die Programme Feflow und Ilmoflood durchgeführt wurde, wird in Anhang C] beschrieben. 


\subsection{Arten der Modellkopplung}

\subsubsection{Vollständig implizite Kopplung}

Eine vollständig implizite Kopplung bedeutet eine simultane Lösung der Differentialgleichungen für beide Kompartimente jeweils für einen Zeitschritt. Es entsteht in der Regel eine globale Matrix, die beide Kompartimente umfasst. Für eine simultane Lösung müssen die Zeitschritte beider Kompartimente daher zusammenfallen und zumindest für die Austauschpunkte in beiden Modellteilen identische Knoten vorliegen. In der Regel muss ein System aus vollständig implizit gekoppelten Strömungsvorgängen als solches konzipiert und entwickelt werden. Damit ist eine Optimierung für ein bestimmtes Problem möglich, die Übertragung eines solchen gekoppelten Systems auf andere Probleme gestaltet sich allerdings oft als schwierig. Eine Verwendung bereits bestehender Modellkomponenten ist nicht möglich. Vollständig implizite Kopplungen werden auch „monolithischer Ansatz“ (monolithic approach) genannt (de Boer 2008), was ein wenig über den Grad der Flexibilität dieser Art der Modellkopplung aussagt. Dafür gelten implizite Kopplungen als robust und rechenzeiteffizient (Fairbanks et al. 2001). Letzteres gilt aber nur dann, wenn die gekoppelten Prozesse für sich betrachtet eine ungefähr gleiche zeitliche Diskretisierung benötigen. Sollen schnell ablaufende Prozesse mit langsam ablaufenden Prozessen gekoppelt werden, würde der langsame Prozess mit einer deutlich feineren Diskretisierung gelöst als aus numerischen Gründen notwendig wäre. Dies kann den Vorteil der Rechenzeiteffizienz wieder zu Nichte machen.

Beispiele für eine vollständig implizite Kopplung sind bei Gunduz \& Aral (2005), Bauer et al. (2006), Panday \& Huyakorn (2004), VanderKwaak (1999) und Weill \& Mouche (2006) zu finden. Die Verbindung der Strömungsgleichungen für Oberflächen- und Grundwasser erfolgt hier über einen Austauschterm, der als Quelle oder Senke Eingang in die Strömungsgleichungen findet. Ohne diesen Austauschterm kommen Thoms \& Johnson (2005) aus. Sie integrieren die Oberflächenströmung in Form der kinematischen Welle (Abschnitt 3.5) in das Gleichungssystem für die dreidimensionale ungesättigte Grundwasserströmung (Gleichung 2.16). Die Reibungsbeiwerte der Landoberfläche werden in horizontale „Durchlässigkeitsbeiwerte“ umgerechnet und der Speicherkoeffizient $S_{\text {sp }}$ zu Eins gesetzt. Damit ist die horizontale Ausdehnung der Berechnungsnetze für beide Kompartimente identisch. Beide Kompartimente werden mit der gleiche Zeitdiskretisierung berechnet.

\subsubsection{Iterative Kopplung}

Eine iterative Kopplung (alternating iterative coupling) bedeutet die wechselseitige Lösung der Gleichungen für die einzelnen Kompartimente in einem Zeitschritt so lange, bis ein Fehlerkriterium erreicht wird. Die Wechselbeziehungen zwischen beiden Kompartimenten werden dabei über interne Randbedingungen modelliert. So kann zum Beispiel das Ergebnis eines Modellknotens für die Oberflächenströmung als h-Randbedingung bei dem entsprechenden Knoten des Grundwassermodells gesetzt werden. Die mit dieser h-Randbedingung korrespondierende 
Lösung des Grundwassermodellknotens wird dem Oberflächenmodellknoten wieder als Randbedingung zugeführt. Dadurch entsteht nach erneuter Berechnung im Oberflächenmodell ein neuer Wasserstand, der wiederum an das Grundwassermodell übergeben wird. Dieser Vorgang wird so lange wiederholt, bis sich die Berechnungsergebnisse nur noch innerhalb einer vorgegebenen Bandbreite bewegen (Abbildung 4.1). Alternativ kann eine Austauschwassermenge aus Schätzwerten für die Wasserstände beider Modelle mit einer Leakage-Funktion (Gleichung 2.36 bestimmt werden (Swain 1994). Diese Austauschwassermenge wird in beiden Modellen als Randbedingung angesetzt und anschließend aus den dadurch resultierenden Wasserständen erneut eine Austauschmenge berechnet. Bleibt die Änderung der Austauschmenge innerhalb der vorgegebenen Fehlertoleranz, wird die Iteration abgebrochen.

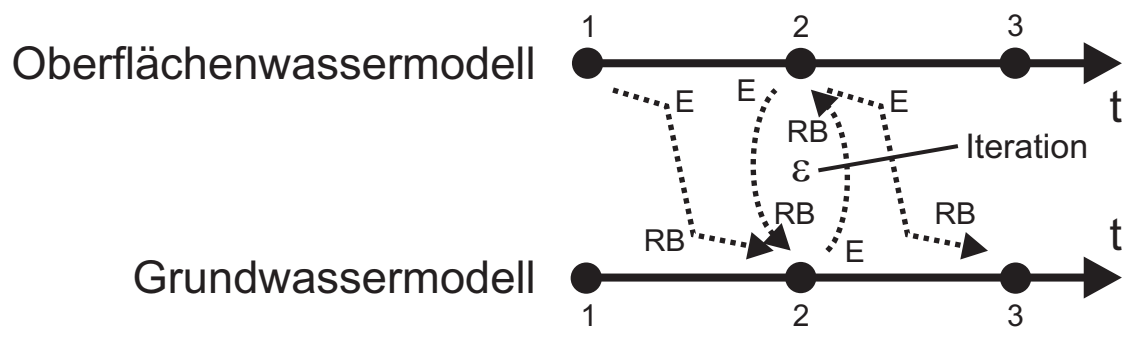

Abbildung 4.1: Funktionsweise der iterativen Kopplung am Beispiel zweier verbundener Modellknoten. E steht für Modellergebnis, RB für Randbedingung, $\mathrm{t}$ für die Simulationszeit und $\varepsilon$ bezeichnet ein Fehlerkriterium, bis zu dessen Einhaltung die Iteration ausgeführt wird.

Grundsätzlich sind für diese Variante zusammenfallende Austauschzeitpunkte notwendig. Es müssen aber nicht zwangsläufig die gleichen Zeitschritte für beide Kompartimentmodelle verwendet werden. So wird oft für die Oberflächenströmung eine feinere Zeitdiskretisierung vorgenommen als für die Grundwasserströmung, weil die Fließprozesse an der Oberfläche in der Regel schneller ablaufen. Die räumlichen Berechnungsnetze müssen bei der iterativen Kopplung nicht exakt zusammenfallen, da die Stützstellen in der Regel beliebig verbunden werden können. Um räumliche Zuordnungsfehler zu vermeiden, können Interpolationen durchgeführt werden, die allerdings wieder zusätzliche Rechenzeit benötigen.

Iterative Kopplungen gelten als weniger robust und benötigen in der Regel mehr Rechenzeit als implizite Kopplungen (Fairbanks et al. 2001), sind dafür aber leichter programmtechnisch umzusetzen. Die Art der iterativen Kopplung erlaubt es, auch bereits bestehende Computerprogramme mit einem in sich geschlossenen Programmablauf, der die Datenverwaltung, die Aufstellung des Strömungsgleichungssystems und dessen Lösung umfasst, zu koppeln. Der Datenaustausch der Programmkomponenten erfolgt dann über interne Schnittstellen.

Beispiele für iterative Modellkopplungen, mit denen der hydrologische Prozess des Oberflächenabflusses untersucht wird, sind bei Morita \& Yen (2000, 2002) und Akan \& Yen (1981) zu finden. Auch für die Beschreibung von Uferspeicherungseffekten werden iterative Modellkopplungen erfolgreich eingesetzt, zum Beispiel von Cunningham \& Sinclair (1979), Pinder \& Sauer (1971), Luckner (1978) oder Swain (1994). 


\subsubsection{Externe Kopplung}

Eine externe Kopplung (externally coupled nach Freeze 1972) meint eine sukzessive Lösung beider Kompartimente für einen Zeitschritt wie bei der iterativen Kopplung, nur dass dabei keine Iteration vorgenommen wird. Für dieses Verfahren ist auch der Begriff time-lagged (Huang \& Yeh 2006; Fairbanks et al. 2001) gebräuchlich. Die Modellergebnisse des einen Kompartiments werden dem anderen als Randbedingung übergeben (Abbildung 4.2). Für eine Kopplung eines Grundwassermodells und eines Oberflächenwassermodellen wird üblicherweise die Oberflächenströmung zuerst berechnet, da die Grundwasserströmung in der Regel langsamer reagiert. Für die Berechnung des unbekannten Zeitschritts der Grundwasserkomponente steht dann ein berechneter Wert des Oberflächenmodells zur Verfügung. Wenn die Zeitpunkte nicht zusammenfallen, können Interpolationen durchgeführt werden (Swain 1994). So ist es auch möglich, für das eine Modell mehr Zeitschritte als für das andere vorzusehen.

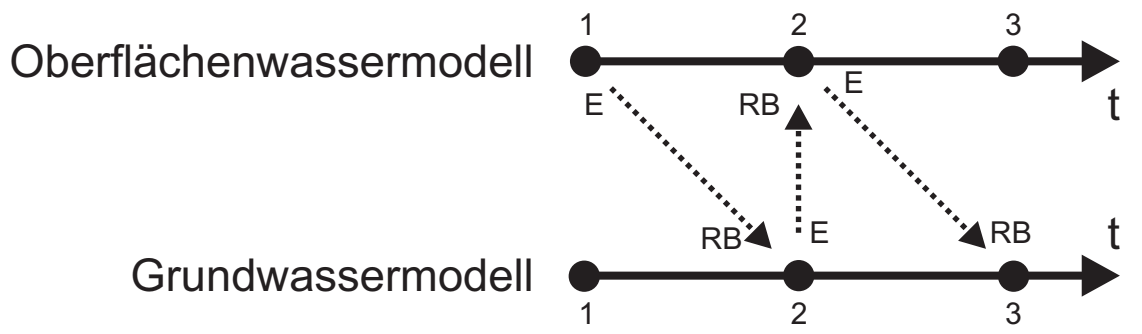

Abbildung 4.2: Funktionsweise der externen Kopplung am Beispiel zweier verbundener Modellknoten. E steht für Modellergebnis, RB für Randbedingung, t für die Simulationszeit.

Beispiele für externe Kopplungen von Grundwasser- und Oberflächenwasserströmungsprogrammen beschreiben Smith \& Woolhiser (1971), Monninkhoff \& Kernbach (2006), Monninkhoff (2004), Singh \& Bhallamudi (1998) und Sommer et al. (2008). Auch die Kopplung der Überflutungsflächenkomponente mit der eindimensionalen Modellkomponente für Flüsse beim Programm Ilmoflood (Abschnitt 3.7) ist eine externe Kopplung, sie unterscheidet sich von der einfachen externen Kopplung aber durch das zweistufige Schätzverfahren (siehe Niemeyer \& Kamrath 2007). Grundsätzlich sind extern gekoppelte Systeme schneller berechnet als iterativ gekoppelte, weil keine Iterationen durchgeführt werden. Sie sind deshalb naturgemäß weniger genau (Gunduz \& Aral 2005). Gijsbers et al. (2005) empfehlen, für den Anwendungsfall zu prüfen, ob es wirklich nötig ist, Teilmodelle iterativ zu koppeln, da oft schon eine Verdichtung der Zeitschritte helfen kann, die Genauigkeit der externen Kopplung ausreichend zu verbessern.

Externe Kopplungen werden gerne verwendet, wenn bereits für eines oder für beide der betrachteten Kompartimente Modelle existieren (Huang \& Yeh 2006). Der Schwerpunkt liegt dann oft nicht darauf, numerisch elegante oder recheneffiziente Lösungen zu erhalten. Vielmehr kommt es darauf an, den vollständigen Neuaufbau eines gekoppelten Systems zu umgehen. Ungenauigkeiten aus einer eingeschränkte Massentreue beim Austausch werden in Kauf genommen. Darüber hinaus kann es zu räumlichen Ungenauigkeiten aus den unterschiedlichen Berechnungsnetzen kommen, wenn die Austauschpunkte nicht exakt zusammenfallen. Dieses Problem besteht bei iterativer Kopplung allerdings auch. 


\subsection{Auswahl einer Kopplungsart}

Zur Auswahl einer Art der Modellkopplung werden die wesentlichen Aspekte aus Abschnitt 4.2 in Tabelle 4.1 zusammengefasst. Es scheint zunächst so, als sei die vollständig implizite Methode den anderen beiden Methoden in fast allen Bereichen überlegen. Lediglich ein höherer Programmieraufwand schlägt hier negativ zu Buche. Allerdings gilt der Vorteil einer geringeren Rechenzeit, wie schon angesprochen, nur dann, wenn die gekoppelten Strömungsprozesse auf ähnlichen Zeitskalen ablaufen. Im vorliegenden Fall des unterirdischen Hochwassers werden Strömungsprozesse gekoppelt, die - je nach örtlichen Gegebenheiten - auch deutlich unterschiedliche Geschwindigkeiten aufweisen können. Bei einer simultanen Berechnung würde die Grundwasserströmungsgleichung deshalb unter Umständen öfter gelöst als eigentlich notwendig, so dass damit der Rechenzeitbedarf wieder steigen wird.

Tabelle 4.1: Zusammenstellung der Vor- und Nachteile der verschiedenen Arten der Modellkopplung auf Basis der zitierten Literatur

\begin{tabular}{lcccc}
\hline & & & & Programmierungs- \\
Methode & Rechenzeitbedarf & Genauigkeit & Robustheit & aufwand \\
\hline vollständig implizit & +++ & +++ & +++ & + \\
iterativ & + & ++ & + & ++ \\
extern & ++ & + & ++ & +++ \\
\hline
\end{tabular}

Die Angaben zur Robustheit sind insgesamt kritisch zu bewerten. Die Robustheit eines gekoppelten Systems hängt nämlich entscheidend von den verwendeten Programmkomponenten im Falle der externen und iterativen Kopplung beziehungsweise der technischen und numerischen Umsetzung des vollständig implizit gekoppelten Systems ab. Während man den Rechenzeitbedarf, die Genauigkeit und den Programmieraufwand recht gut quantifizieren kann, gibt es für den Grad der Robustheit bisher kein allgemein anerkanntes Kriterium. Ein gekoppeltes System aus Programmkomponenten, die professionell gepflegt werden und sich bei einer großen Anzahl von verschiedenen Anwendern bewährt haben, kann durchaus weniger fehleranfällig und damit robuster sein als ein vollständig implizit gekoppeltes System, das zum Beispiel an einer Forschungsinstitution für einen speziellen Anwendungsfall entwickelt worden ist und nur vom Entwicklerteam selbst angewendet wird.

Neben den Entscheidungskriterien aus Tabelle 4.1, die aus der zitierten Literatur zusammengestellt wurden, sind weitere Aspekte bei einer Entscheidung zu berücksichtigen. Trotz der zunächst deutlichen Überlegenheit der vollständig impliziten Kopplung scheidet diese Methode aus, denn das in Abschnitt 1.3 formulierte Ziel beinhaltet die Kopplung bereits bestehender Modelle. Jede dieser Modellkomponenten verwendet ihre eigene Netzgeometrie, aber auch die Umsetzung des Netzes, der Anfangs- und der Randbedingungen in ein Strömungsgleichungssystem und dessen Lösung sind in verschiedenen Programmen umgesetzt. Damit käme eine vollständig implizite Kopplung einer Neuentwicklung gleich und die Modelldaten müssten wesentlich verändert werden. 
Der Aufbau eines gekoppelten Modells aus bereits fertigen Modellkomponenten muss also iterativ oder extern über den Austausch von Randbedingungen beider Modelle erfolgen. Die iterative Kopplung hat gegenüber der externen Kopplung grundsätzlich einen höheren Rechenzeitbedarf, liefert dafür aber eine höhere Genauigkeit bei entsprechender Einstellung der Abbruchkriterien. Dennoch fällt die Entscheidung zugunsten der externen Kopplung. Sie hat sich als Methode bereits für Planungsaufgaben bewährt (Monninkhoff 2004; Monninkhoff \& Kernbach 2006, Huang \& Yeh 2006; Sommer et al. 2008) und wird für praktische Anwendungen empfohlen (Moore et al. 2005). Für den operativen Einsatz ist die externe Kopplung sicher diejenige Variante, die am einfachsten zu bedienen ist und daher im Hinblick auf die Zielsetzung einer hohen Akzeptanz durch die Anwender vorzuziehen. Schließlich fällt die einfachere programmtechnische Umsetzbarkeit ins Gewicht.

Zur technischen Umsetzung der Kopplung werden in beiden Programmkomponenten Schnittstellen definiert. OpenMI ist eine standardisierte Definition für Schnittstellen zur Kopplung von wasserwirtschaftlichen Strömungsprogrammen. Der Standard bezieht sich zunächst auf eine externe Kopplung, erlaubt aber auch die Ausführung von Iterationen. Die Verwendung einer standardisierten OpenMI-Schnittstelle bietet ein hohes Maß an Flexibilität, da die Modellkomponenten beliebig gegen andere Bausteine ausgetauscht werden können und das System um zusätzliche Modellkomponenten erweitert werden kann.

\subsection{OpenMI}

\subsubsection{Allgemeines zum OpenMI-Standard}

OpenMI bedeutet open modeling interface und definiert einen Standard, mit dem Daten zwischen zeitabhängigen Modellkomponenten integrierter Systeme (gekoppelter Systeme) ausgetauscht werden können. OpenMl wurde für wasserwirtschaftliche Fragestellungen entwickelt, um der Forderung nach einem integrierten Wasserresourcenmanagement durch die Wasserrahmenrichtlinie (Das Europäische Parlament und der Rat der Europäischen Union 2000) zu begegnen (Gregersen et al. 2007).

Der OpenMI-Standard ermöglicht die Verbindung von Modellen, die jeweils unterschiedliche Eigenschaften aufweisen, die also zum Beispiel

- verschiedene Strömungsdomänen beschreiben (z. B. Oberflächenwasser, Grundwasser)

- auf unterschiedliche Modellierungsarten basieren (z. B. deterministisch, stochastisch)

- unterschiedliche Dimensionalitäten haben (1D, 2D oder 3D)

- auf unterschiedlichen Skalen arbeiten (z. B. Großraum- oder Kleinraummodell)

- eine unterschiedliche zeitliche Auflösung aufweisen oder 


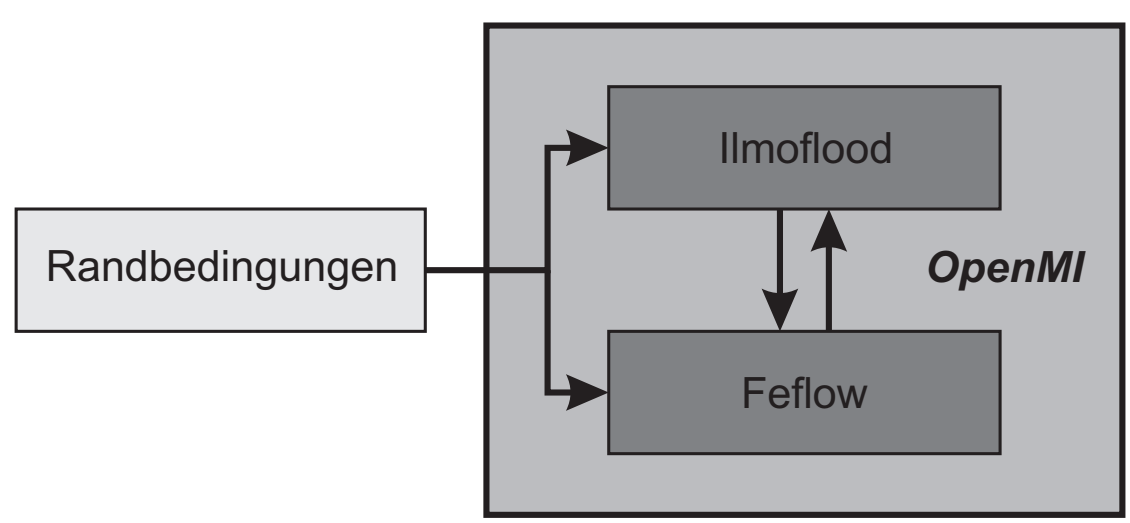

Abbildung 4.3: Einordnung von OpenMl in das gekoppelte System zur Modellierung des unterirdischen Hochwassers

- eine unterschiedliche räumliche Diskretisierung verwenden (z. B. Dreieck- oder Vierecknetze, grobes oder feines Gitter)

(Moore et al. 2005). Derartig gekoppelte Modelle eignen sich nach Moore et al. (2005) für den operativen Einsatz, aber auch für wissenschaftliche Untersuchungen zur Erweiterung des Prozessverständnisses.

OpenMI definiert einerseits einen Standard für Schnittstellen, andererseits wird von der OpenMI Association (2007) Software angeboten, die dem Entwickler die Implementierung des OpenMI-Standards in neue oder bestehende Modelle erleichtert. Die OpenMI-Umgebung umfasst Programmkomponenten, den Quellcode und die entsprechende Dokumentation. Die OpenMIUmgebung ist frei verfügbar. Einige wasserwirtschaftliche Programme sind bereits mit einer OpenMI-Schnittstelle ausgerüstet (siehe OpenMI Association 2007). OpenMI wird deshalb auch im Rahmen dieser Arbeit zur Kopplung der beiden Programme Ilmoflood und Feflow verwendet (Abbildung 4.3).

\subsubsection{Datenaustausch über OpenMI}

Ein OpenMI-System ist ein Software-System, das verschiedene OpenMI-kompatible Komponenten (OpenMl-compliant linkable component) zu einem gekoppelten Gesamtsystem verbindet. Diese Komponenten können Modelle, aber auch andere Bausteine wie zum Beispiel eine Datenbank oder Postprocessing-Werkzeuge sein. Zum Aufbau von OpenMI-Systemen steht der OpenMI-Editor zur Verfügung. Über diesen Editor werden die OpenMI-Komponenten als DLL (dynamic link library) geladen.

Der Datenaustausch des OpenMI-Standards basiert auf einem Frage-und-Antwort-Mechanismus. Eine Komponente, zum Beispiel ein gebietsbezogenes Modell, erfragt die für die Berechnung benötigten Daten bei einer anderen Komponente. Die Komponenten können auf verschiedene Arten verbunden werden: 


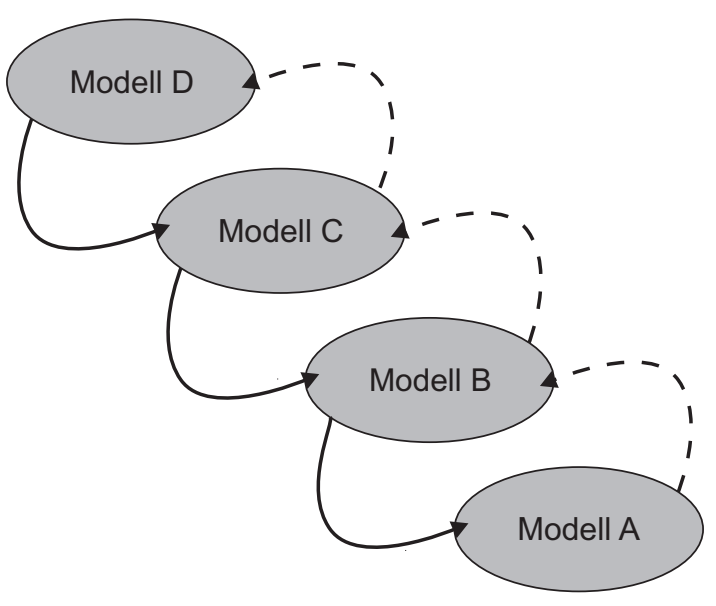

(a) unidirektionale Verbindung

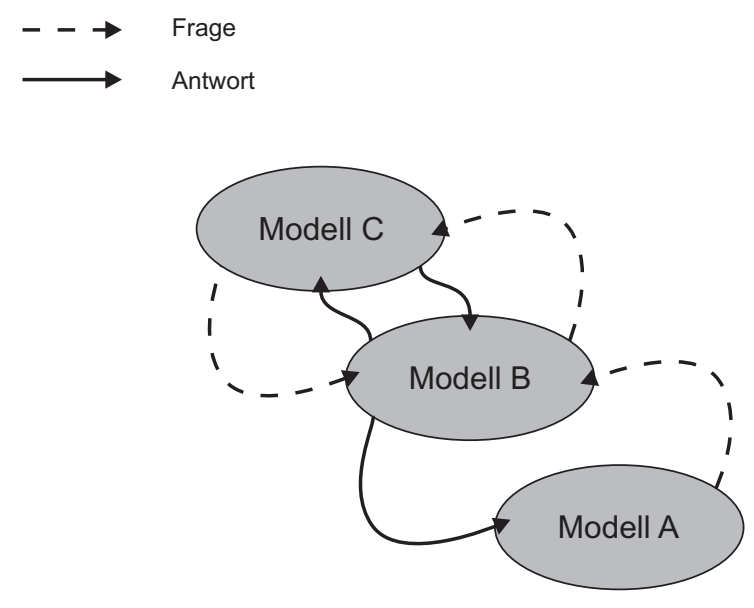

(b) bidirektionale Verbindung

Abbildung 4.4: Frage-Antwort-Mechanismus und Verbindungen zwischen OpenMI-Komponenten (nach Moore \& Tindall 2005)

- lineare Verbindung in einer Richtung (unidirektionale Verbindung)

- lineare Verbindung in zwei Richtungen (bidirektionale Verbindung)

- iterative Verbindung.

Abbildung 4.4 verdeutlicht den Mechanismus eines Frage-und-Antwort-Datenaustauschs mit unidirektionaler und bidirektionaler Verbindung. Für den Fall der unidirektionalen Verbindung (Abbildung 4.4a) fragt Modell A Modell B nach Daten. B richtet selbst eine Anfrage an Modell C, welches wiederum Daten von Modell D erfragt. Dementsprechend wird zunächst Modell D eine Berechnung ausführen, um die Anfrage von Modell C erfüllen zu können. Diese Art des Datenaustauschs ließe sich prinzipiell auch ohne eine Modellkopplung ausführen. Im vorliegenden Beispiel beeinflusst die Berechnung von Modell C nicht die Berechnung von Modell D. Es wäre somit möglich, zunächst den vollständigen Simulationszeitraum von Modell D zu berechnen, um aus den Berechnungsergebnissen zeitlich veränderliche Randbedingungen für Modell $\mathrm{C}$ zu erzeugen. Zum Beispiel gehen Köngeter et al. (2002) und Homann (2006) auf diese Weise vor. Anhand des Beispiels aus Anhang C.3 wird deutlich, dass eine Modellkopplung zur Laufzeit mit unidirektionaler Verbindung die zusammenhängende Betrachtung zweier verschiedener Kompartimente erleichtern kann, da die Übergabe der Daten von einem Modell zum anderen dann über die Modellkopplung umgesetzt wird und nicht vom Modellierer vorgenommen werden muss.

Die Verbindung in zwei Richtungen wird mit Abbildung 4.4b verdeutlicht: Modell A erfragt Daten von Modell B. Dieses benötigt Daten von Modell C, um weiterrechnen zu können, allerdings muss Modell C erst Daten von Modell B erhalten, um dessen Anfrage zu erfüllen. Deshalb gibt $\mathrm{B}$ einen Schätzwert zu C, dieses gibt den damit berechneten Wert zurück zu Modell B, welches 
damit die Anfrage von A erfüllen kann. An dem zweiten Beispiel wird deutlich, dass eine Komponente als Initialisator wirken muss, damit klar ist, welche Kompontente zuerst Berechnungen ausführt. Da Schätzwerte verwendet werden, können sich, je nachdem, welche Komponente die initialisierende ist, die Berechnungsergebnisse ändern. Diese Verbindung entspricht einer externen Kopplung im Sinne von Abschnitt 4.2.3.

Die iterierte Verbindung stellt eine Erweiterung der bidirektionalen Verbindung dar. Im Beispiel von Abbildung 4.4 (rechts) würden die Modelle B und C so lange ihre Rückgabewerte abgleichen, bis die Änderung der zurückgegebenen Werte über den Iterationsverlauf sich innerhalb einer definierten Grenzungenauigkeit bewegt. In Abschnitt 4.3 wurde die Entscheidung zugunsten der externen Kopplung getroffen, so dass die iterierte Verbindung hier nicht weiter behandelt wird.

Die Verbindung von Modellen erfordert, dass das fragende und das gebende Modell die ausgetauschten Werte gleich interpretieren. Ein Wert (value) ist ein Skalar oder ein Vektor. Seine Bedeutung wird durch die drei Angaben

- Was?

- Wo?

- Wann?

konkretisiert. Was ein Wert repräsentiert und in welcher Einheit er angegeben ist, wird durch die Schnittstellenparameter QuantityID, konkretisiert durch Größe (quantity, z. B. flow oder head) und Einheit (unit) festgelegt. Wo ein Wert lokalisiert ist, wird mit Hilfe der Klasse ElementSet definiert. Ein ElementSet können ein oder mehrere Punktkoordinaten, Knotennummern, Linien, Polygone oder Volumenelemente sein. Eine Kombination aus Größe, Einheit und Lokalisierung (QuantityID, unit und ElementSet) heißt Austauschobjekt (exchange item). Welchem Zeitpunkt ein Wert zugeordnet ist, wird über den Schnittstellenparameter time angegeben, entweder als Zeitpunkt (timestep) oder als Zeitspanne (time span).

Es gibt zwei Arten von Austauschobjekten, nämlich

- Eingabeobjekte (input exchange items)

- Ausgabeobjekte (output exchange items).

Für Eingabeobjekte wird der OpenMI-Komponente von außen ein Wert vorgegeben, der die Berechnung beeinflusst. Für Ausgabeobjekte gibt eine OpenMI-Komponente selbst Werte, meistens Berechnungsergebnisse, zurück, die die Berechnung von anderen OpenMI-Komponenten beeinflussen können. 


\subsection{Zusammenfassung des Kapitels}

Zur Kopplung von Modellen des Grundwassers und des Oberflächenwassers werden drei Arten unterschieden (Morita \& Yen 2002):

- vollständig implizite Kopplung

- iterative Kopplung und

- externe Kopplung.

Die drei Methoden unterscheiden sich hinsichtlich ihres Rechenzeitbedarfs, der erreichbaren Genauigkeit, der Robustheit und des Programmieraufwands. Weil für die vorliegende Problemstellung bereits existierende Modelle und damit auch bereits fertige Programme verwendet werden sollen, kommen nur eine iterative Kopplung oder eine externe Kopplung in Frage. Vor dem Hintergrund des geplanten Verwendungszwecks für operative und planerische Aufgaben und aus Gründen einer einfacheren Umsetzbarkeit wird die Wahl zu Gunsten der externen Kopplung getroffen. Die Modellkopplung erfolgt dabei so, dass die Ergebnisse des einen Modells als Randbedingungen in das andere Modell einfließen. Die Programme, die gekoppelt werden sollen, sind dazu mit entsprechenden Schnittstellen auszurüsten.

Eine Möglichkeit, derartige Kopplungen technisch umzusetzen, ist die Verwendung des OpenMI-Standards. Er bringt eine hohe Flexibilität mit sich, da OpenMI-Systeme beliebig erweitert und Systemkomponenten ausgetauscht werden können. Über die Definition von Austauschobjekten wird sichergestellt, dass beide Modellkomponenten einen ausgetauschten Wert korrekt implementieren.

Wie die Programme Feflow und IImoflood mit OpenMI-Schnittstellen ausgerüstet wurden, ist in Anhang C beschrieben. Die wesentliche Herausforderung bestand darin, das Programm Feflow OpenMI-kompatibel zu machen, da der Quellcode dieses Programms nicht verfügbar ist. Über die offene Programmierschnittstelle IFM (Anhang A konnte jedoch mit Methoden der Netzwerkprogrammierung eine Möglichkeit geschaffen werden, die für die OpenMI-Kompatibilität notwendige Ansteuerung des Programms von außen zu realisieren und die vom Standard geforderten Funktionen bereitzustellen. 


\section{Uferspeicherung}

\subsection{Einführung}

Unter Uferspeicherung (bank storage, Abb. 5.1) versteht man den Prozess der Speicherung von Wasser eines Fließgewässers im unterirdischen Bereich des Ufers. Bei steigendem Wasserstand dringt Wasser aus dem Fluss in nicht gesättigte Bereiche des angrenzenden Grundwasserleiters ein, um bei fallendem Wasserstand wieder ganz oder teilweise zurück in das Gewässer zu fließen. Die Uferspeicherung ist von der Dauer, der Höhe und der Form einer Hochwasserwelle und von den Durchlässigkeits- und Speichereigenschaften und der Ausdehnung des angrenzenden Grundwasserleiters abhängig (Ubell 1987a). Bei der Planung von Retentionsräumen ist der Uferspeicherungseffekt durchaus erwünscht (siehe z. B. Monninkhoff \& Kernbach 2006), in städtischen Regionen dagegen ist der Anstieg des Grundwassers in Ufernähe und im Hinterland, wie bereits in Abschnitt 1.1 ausgeführt, problematisch.

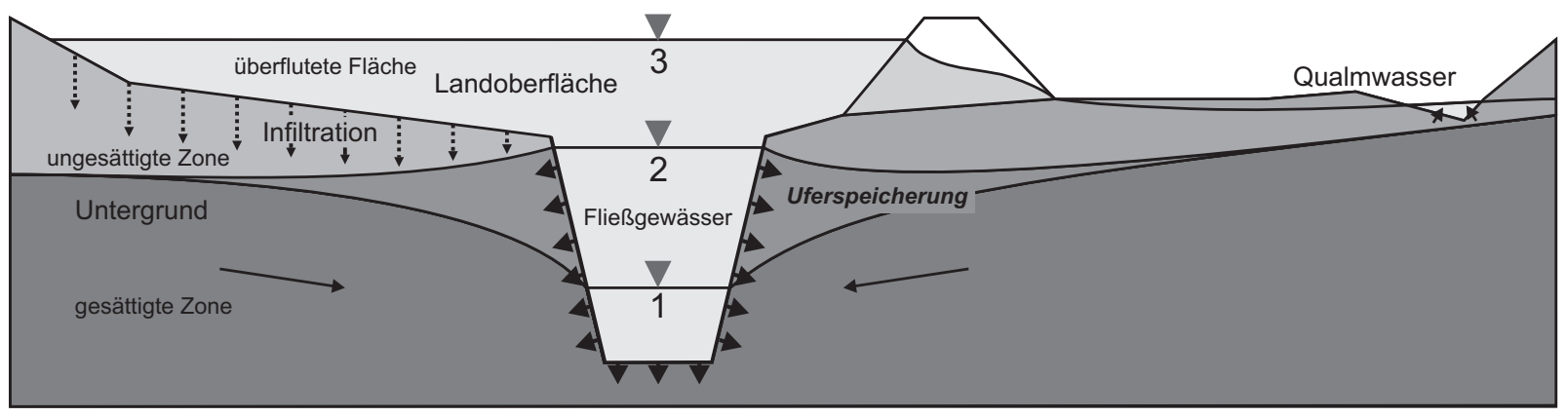

Abbildung 5.1: Einordnung der Uferspeicherung in die Prozesse der Wasserbewegung beim unterirdischen Hochwasser

Da im Rahmen dieser Arbeit der Flusswasserstand als Randbedingung des Grundwassermodells Eingang in das Modellsystem finden soll, nähert sich dieses Kapitel der Frage, auf welche Art sich der Prozess der Uferspeicherung vor dem Hintergrund des unterirdischen Hochwassers in einem Grundwassermodell technisch zweckmäßig abbilden lässt.

Eine Möglichkeit ist es, den Wasserstand eines Fließgewässers als h-Randbedingung anzusetzen (siehe Abschnitt 2.4.3). Durch den Ansatz der Randbedingung wird dem Grundwassermodellgebiet am Randbedingungsknoten Wasser entzogen oder hinzugefügt. Diese Austauschmenge hängt von den Strömungsverhältnissen, die in der Umgebung des Randbedingungsknotens vorherrschen, und natürlich vom Wert der Randbedingung ab. 
Freeze (1972) begrenzt die Anwendbarkeit der h-Randbedingung auf flache Flüsse, deren Tiefe nur schwach variiert und wo gleichzeitig steile Gradienten im Grundwasser und große Sickerflächen vorliegen. Für Fragen des unterirdischen Hochwassers treffen diese Rahmenbedingungen in der Regel jedoch nicht zu. Cunningham \& Sinclair (1979) empfehlen die h-Randbedingung für Flüsse, die den untersuchten Grundwasserleiter vollständig durchschneiden und somit als Grundwasserscheide wirken. Diese Gegebenheiten werden für viele Flüsse, in denen unterirdisches Hochwasser relevant wird, zutreffen. Bachmann et al. (2007) setzen dementsprechend in ihrem Großraum-Grundwassermodell die h-Randbedingung für den Maasrand ein, gemessene Wasserstände der Maas bilden den Randbedingungswert.

Neben der Randbedingung erster Art kommt, wie in Abschnitt 2.4.3 skizziert, auch eine Leakage-Randbedingung in Frage, um den Prozess der Uferspeicherung in einem Grundwassermodell abzubilden. Es gibt mehrere Varianten dieses Randbedingungstyps. Zunächst werden im nächsten Abschnitt deshalb verschiedene Leakage-Ansätze vorgestellt. Abschnitt 5.3 geht auf Methoden ein, mit denen der Leakage-Fluss in der Natur bestimmt werden kann.

Ubell (1987a, b) dokumentiert einen Fall der Uferspeicherung, der sich während des Rheinhochwassers von 1983 im Neuwieder Becken ereignete. Für dieses Beispiel wird ein numerisches Modell aufgebaut. Der Rhein wird auf zwei verschiedene Arten modelliert: einmal mit einer h-Randbedingung und einmal mit einer Leakage-Randbedingung. Anhand eines Vergleichs der Berechnungsergebnisse werden die beiden Varianten zur Modellierung der Uferspeicherung für den Einsatz im Hochwasserfall diskutiert.

\subsection{Leakage-Ansätze}

Nach der Vorstellung des Leakage-Modells bestimmt die Druckhöhendifferenz zwischen dem Wasserstand im Fluss $h_{\mathrm{F}}$ und dem Grundwasserstand $h_{\mathrm{G}}$

$$
\Delta h=h_{\mathrm{F}}-h_{\mathrm{G}}
$$

die Höhe einer Austauschrate $q$. Je größer die Druckhöhendifferenz, desto größer ist auch die Wassermenge, die pro Zeit- und Flächeneinheit zwischen Fließgewässer und Untergrund ausgetauscht wird.

Sicher der bekannteste Leakage-Ansatz ist der nach dem ihm zu Grunde liegenden Fließgesetz (Gleichung 2.2) benannte Darcy-Ansatz

$$
q=c_{1} \cdot \Delta h
$$

Die Austauschrate $q=f(\Delta h)$ ist zunächst auf die Kontaktfläche, die zwischen Fließgewässer und Grundwasserleiter für den Wasseraustausch zur Verfügung steht, bezogen und hat die Dimension der Darcy-Filtergeschwindigkeit m/s. Positive Werte stehen für eine Infiltration des Wassers aus dem Fluss in den Grundwasserleiter, negative für eine Exfiltration. 


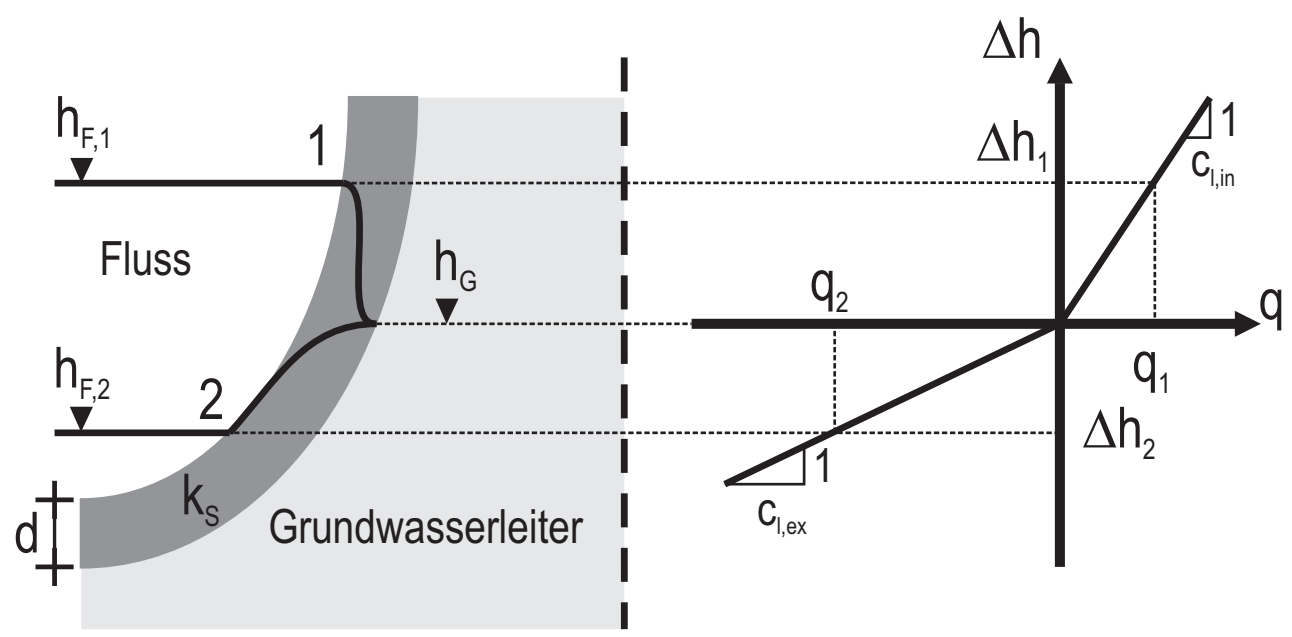

Abbildung 5.2: Querschnitt eines idealisierten Fließgewässers mit Druckhöhendifferenz $\Delta h$ und Austauschrate $q$ für zwei verschiedene Fließzustände. Zustand 1 kennzeichnet die Infiltration bei hohem Wasserstand im Fließgewässer, Zustand 2 zeigt die Exfiltration (Normalzustand) bei niedrigem Flusswasserstand (nach Diersch 2005a).

$c_{1}$ ist ein sogenannter Leakage-Koeffizient und nimmt positive Werte an. Die physikalische Entsprechung des Leakage-Koeffizienten ist der Quotient aus der hydraulischen Durchlässigkeit einer den Fluss und den Grundwasserleiter trennenden halbdurchlässigen Schicht $k_{\mathrm{S}}$ und ihrer Dicke $d$

$$
c_{1}=\frac{k_{\mathrm{S}}}{d}
$$

(Forkel 2004; Sophocleous 2002; Kinzelbach \& Rausch 1995; Rushton \& Tomlinson 1979). Der Quotient $\frac{\Delta h}{d}$ entspricht dem hydraulischen Gefälle $\frac{\Delta h}{\Delta l}$ aus Gleichung 2.7, an die Stelle von $k$ tritt $k_{\mathrm{S}}$. Die trennende Schicht besteht an der Flusssohle aus Sediment (siehe z. B. Schubert 2002; Gölz et al. 1991), im Bereich des Ufers kann sie auch durch eine geschüttete oder gepackte Uferbefestigung oder eine Kaimauer gebildet werden.

Wird Gleichung 5.2 sowohl für influente (Infiltration) als auch für effluente Verhältnisse (Exfiltration) verwendet, so setzt dies voraus, dass für beide Fließrichtungen der gleiche Widerstand vorliegt. Das Gewässerbett kann jedoch wie ein mechanischer Filter wirken. Abhängig von den Fließverhältnissen und der Partikelfracht im Fließgewässer kommt es zur Kolmation der Gewässersohle, die zu einer reduzierten Infiltrationsrate führt (Schubert 2002; Brunke \& Gonser 1997; Schälchli 1992). Deshalb werden für Infiltration und Exfiltration oft unterschiedliche Koeffizienten vorgegeben (siehe z. B. Diersch 2005d; Schüttrumpf \& Becker 2008):

$$
c_{1}=\left\{\begin{array}{lll}
c_{1, \text { in }} & \text { für } & h_{\mathrm{F}}>h_{\mathrm{G}} \\
c_{1, \mathrm{ex}} & \text { für } & h_{\mathrm{F}}<h_{\mathrm{G}}
\end{array}\right.
$$

In Abbildung 5.2 ist der Zusammenhang zwischen dem Leakage-Fluss und der Wasserstandsdifferenz nach dem Darcy-Leakage-Ansatz mit unterschiedlichen Leakage-Koeffizienten für jeweils unterschiedliche Fließrichtungen dargestellt. Zustand 1 zeigt den Prozess der Infiltration. 


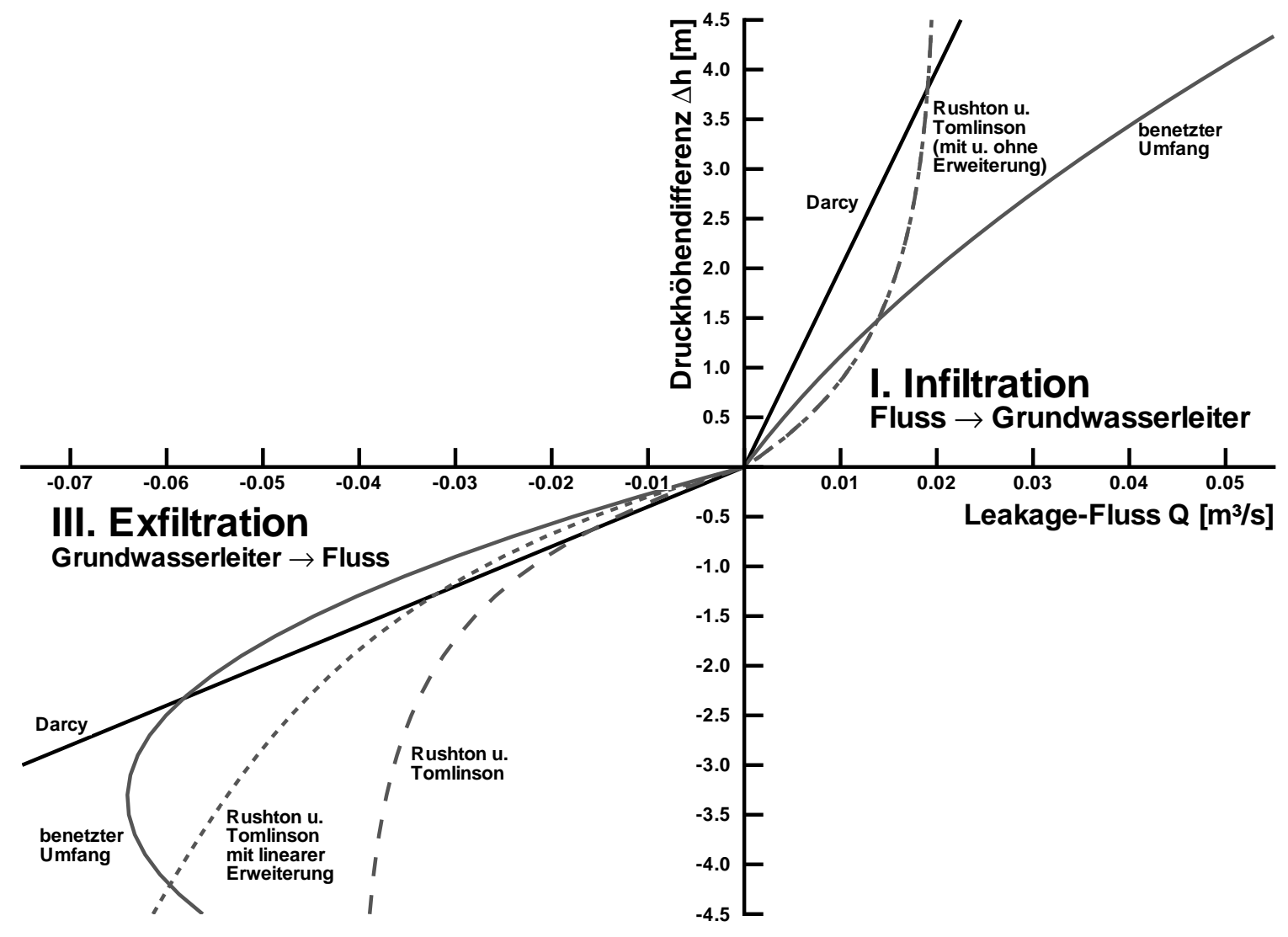

Abbildung 5.3: Leakage-Fluss $Q$ in Abhängigkeit von der Wasserstandsdifferenz $\Delta h$ für den DarcyLeakage-Ansatz (Gleichungen 5.2 und 5.4), den Ansatz nach Rushton \& Tomlinson (1979) (Gleichung 5.7), den kombinierten Ansatz nach Rushton \& Tomlinson (1979) (Gleichung 5.9) und den LeakageAnsatz mit Berücksichtigung des benetzten Umfangs (Gleichung 5.6, hier unter Ansatz eines Trapezquerschnitts). Die den Kurven zu Grunde liegenden Werte enthält Tabelle 5.1 .

Wasserstandsdifferenz und Leakage-Fluss sind positiv und in Richtung des Grundwasserleiters gerichtet. Zustand 2 ist die Exfiltration von Grundwasser in Richtung Fließgewässer mit negativen Vorzeichen für Wasserstandsdifferenz und Austauschrate. Die Steigung der LeakageFunktion wird jeweils durch den Leakage-Koeffizienten bestimmt.

Für eine Leakage-Randbedingung in einem Grundwassermodell ist der Flusswasserstand $h_{\mathrm{F}}$ der vorgegebene Randbedingungswert, während der Grundwasserstand $h_{\mathrm{G}}$ und die Austauschrate $q$ Modellergebnisse sind. Der einem Modellknoten zugeordnete Leakage-Fluss $Q$ ist das Produkt aus Austauschrate und der Kontaktfläche $A$, über die der Wasseraustausch stattfindet:

$$
Q=q \cdot A
$$

Die Kontaktfläche $A$ kann fest vorgegeben werden (Schüttrumpf \& Becker 2008) oder programmintern aus der Netzgeometrie berechnet werden (Nowack 2007). Der Darcy-Leakage-Ansatz 
Tabelle 5.1: Zur Berechnung der Leakage-Flüsse in Abbildung 5.3 verwendete Werte. Der Grundwasserstand $h_{\mathrm{G}}$ liegt konstant auf 4,5 m, der Flusswasserstand $h_{\mathrm{F}}$ variiert zwischen $0 \mathrm{~m}$ und $9 \mathrm{~m}$, die Flusssohle liegt auf $0 \mathrm{~m}$. Die Parameter und Koeffizienten wurden so gewählt, dass die Kurvenverläufe in Abbildung 5.3 qualitativ gut vergleichbar sind.

\begin{tabular}{|c|c|c|c|}
\hline Ansatz & Variable & Wert & Einheit \\
\hline \multirow[t]{3}{*}{ Darcy } & Leakage-Koeffizient $c_{1, \text { in }}$ & $5,0 \cdot 10^{-6}$ & $1 / \mathrm{s}$ \\
\hline & Leakage-Koeffizient $c_{1, \mathrm{ex}}$ & $2,5 \cdot 10^{-5}$ & $1 / \mathrm{s}$ \\
\hline & Kontaktfläche $A$ & 1000 & $\mathrm{~m}^{2}$ \\
\hline \multirow{5}{*}{$\begin{array}{l}\text { benetzter Umfang } \\
\text { (Trapezquerschnitt) }\end{array}$} & Leakage-Koeffizient $c_{1, \text { in }}$ & $5,0 \cdot 10^{-6}$ & $1 / \mathrm{s}$ \\
\hline & Leakage-Koeffizient $c_{1, \mathrm{ex}}$ & $2,5 \cdot 10^{-5}$ & $1 / \mathrm{s}$ \\
\hline & Sohlbreite am Boden & 8,0 & $\mathrm{~m}$ \\
\hline & Neigung der Gerinnewand & $1: 1,55$ & \\
\hline & Länge des Gewässerabschnitts $l$ & 62,5 & $\mathrm{~m}$ \\
\hline \multirow[t]{4}{*}{ Rushton \& Tomlinson } & Leakage-Parameter $C_{1}$ & 0,02 & \\
\hline & Leakage-Parameter $C_{2}$ & 0,8 & \\
\hline & Leakage-Parameter $C_{3}$ & 0.04 & \\
\hline & Länge des Gewässerabschnitts $l$ & 1 & \\
\hline Rushton \& Tomlinson & Leakage-Parameter $C_{1}$ & 0,02 & \\
\hline \multirow[t]{4}{*}{ kombiniert } & Leakage-Parameter $C_{2}$ & 0,8 & \\
\hline & Leakage-Parameter $C_{3}$ & 0,04 & \\
\hline & Leakage-Parameter $C_{4}$ & 0,005 & \\
\hline & Länge des Gewässerabschnitts $l$ & 1 & \\
\hline
\end{tabular}

beschreibt so mit Gleichung 5.5 einen linearen Zusammenhang zwischen $Q$ und $\Delta h$, wenn sich die Kontaktfläche nicht ändert. Daneben gibt es auch nichtlineare Leakage-Funktionen (Abbildung 5.3). So kombinieren Cunningham \& Sinclair (1979) die Darcy-Leakage-Rate mit einer vom benetzten Umfang $l_{\mathrm{U}}$ und damit vom Wasserstand im Fließgewässer abhängenden Kontaktfläche

$$
A=l \cdot l_{\mathrm{U}}
$$

$l$ steht in dieser Gleichung für die Länge des betrachteten Gewässerabschnitts. Panday \& Huyakorn (2004) berechnen den benetzten Umfang je nach dem, in welche Richtung der Austausch fließt, auf Basis des Grundwasserstands für Exfiltration beziehungsweise auf Basis des Flusswasserstands für Infiltration.

Krom \& Graham (2006) verwenden einen Leakage-Ansatz gemäß Gleichung 5.6 für einen etwa $300 \mathrm{~m}$ breiten und unregelmäßig geformten Flussquerschnitt, in dem ein kleiner Wasseranstieg dadurch, dass zuvor trockene Bereiche des Gewässerbetts überflutet werden, zu einer deutlichen Erhöhung des Fließquerschnitts führen kann. Bei dem von Pinder \& Sauer (1971) verwendeten Rechteckquerschnitt ist der Zuwachs der Kontaktfläche mit steigendem Wasserstand im 
Fluss nicht so deutlich ausgeprägt. Der in Abbildung 5.3 gezeigten Leakage-Kurve ,benetzter Umfang“ liegt ein Trapezquerschnitt zu Grunde. Der Leakage-Fluss $Q$ steigt für große positive Druckhöhendifferenzen überproportional stark mit der Wasserstandsdifferenz $\Delta h$ an. Im negativen Bereich wächst der Betrag des Leakage-Flusses mit steigendem Betrag der Druckhöhendifferenz unterproportional mit der Wasserstandsdifferenz $\Delta h$. Auffällig ist für dieses Zahlenbeispiel der Funktionsverlauf für Wasserstandsdifferenzen kleiner als -3,3 m. Diese fallen im Beispiel mit kleinen Wasserständen im Fluss $h_{\mathrm{F}}$ zusammen. Entsprechend stellt sich ein kleiner benetzter Umfang $l_{\mathrm{U}}$ ein, so dass eine kleine Kontaktfläche berechnet wird. Mit dem relativ hohen Wasserstandsdifferenzbetrag kann sich so der gleiche Leakage-Fluss einstellen wie für einen kleinen Betrag der Wasserstandsdifferenz, der dann aber wegen des höheren Flusswasserstands mit einer entsprechend größeren Kontaktfläche verbunden ist. Bei der Anwendung dieses Ansatzes ist deshalb zu überprüfen, in welchem Bereich sich der Wasseraustausch abspielt.

Nach Kollet \& Maxwell (2006) kann nicht davon ausgegangen werden, dass Fließgewässer und Grundwasserleiter generell durch eine abdichtende Sohlschicht getrennt sind, wie es die Modellvorstellung der auf dem Darcy-Gesetz basierenden Leakage-Ansätze vorsieht. Werden in solchen Fällen dennoch die oben beschriebenen Leakage-Formeln verwendet, so werden die Leakage-Koeffizienten zu Parametern (Cunningham \& Sinclair 1979; Kollet \& Maxwell 2006). Im Zuge der Modellkalibrierung werden diese Parameter so eingestellt, dass die Modellergebnisse die verfügbaren Messwerte möglichst gut wiedergeben (siehe z. B. Bachmann et al. 2007; Crebas et al. 1984).

Eine empirische nichtlineare Beziehung zwischen Austauschrate und Druckhöhendifferenz schlagen Rushton \& Tomlinson (1979) vor:

$$
q=\left\{\begin{array}{lll}
C_{1}\left(1-\mathrm{e}^{-C_{2} \Delta h}\right) & \text { für } & \Delta h>0 \\
C_{3}\left(\mathrm{e}^{C_{2} \Delta h}-1\right) & \text { für } & \Delta h<0
\end{array}\right.
$$

$C_{1}, C_{2}$ und $C_{3}$ sind die Leakage-Parameter, die positive Werte annehmen. Für kleine Grundwasserspiegeländerungen wird bei entsprechender Einstellung der Parameter $C_{1}$ und $C_{3}$ ein starker Anstieg der Austauschmenge erreicht, jedoch wird mit $C_{2}$ gleichsam ein Grenzwert der Austauschmenge festgelegt, der auch bei großen $\Delta h$ nicht überschritten wird (Abbildung 5.3).

Die Leakage-Rate $q$ bezieht sich auf die Länge des Flussabschnittes. Damit berechnet sich der Leakage-Fluss zu

$$
Q=q \cdot l
$$

Im I. Quadranten des $Q$ - $\Delta h$-Diagramms (Abbildung 5.3 laufen die Kurven des Ansatzes mit benetztem Umfang (Gleichung 5.6) und der Rushton-Tomlinson-Ansatz stark auseinander. Steigt bei ersterem der Leakage-Fluss mit steigender Druckhöhendifferenz, also bei steigendem Flusswasserstand, stark an, so wird bei letzterem für große $\Delta h$ der Leakage-Fluss begrenzt. 
Die Erweiterung des nichtlinearen Ansatzes (Gleichung 5.7) um eine lineare Komponente für negative $\Delta h$ nach dem Vorbild des Darcy-Ansatzes (Gleichung 5.2) liefert die Austauchmenge $\mathrm{zu}$

$$
q=\left\{\begin{array}{lll}
C_{1}\left(1-\mathrm{e}^{-C_{2} \Delta h}\right) & \text { für } & \Delta h>0 \\
C_{4} \Delta h+C_{3}\left(\mathrm{e}^{-C_{2} \Delta h}-1\right) & \text { für } & \Delta h<0
\end{array}\right.
$$

mit der zusätzlichen Konstanten $C_{4}$. Für kleine $\Delta h$ wird nun der Exponentialterm dominant, während der lineare Anteil für große $\Delta h$ dominiert (Abbildung 5.3). Rushton \& Tomlinson (1979) stellen für diesen Ansatz die beste Übereinstimmung mit ihren Felddaten fest. Schwerpunkt ihrer Untersuchung ist aber die Analyse des Basisabflusses vom Grundwasser in den Fluss. Die untersuchten Zustände bewegen sich deshalb hauptsächlich im III. Quadranten aus dem Diagramm in Abbildung 5.3. Der Wasserstand im Fluss blieb in den Untersuchungen weitgehend konstant. Für Fragen des unterirdischen Hochwassers gelten derartige Rahmenbedingungen nicht.

Für die Parameter $C_{1}$ bis $C_{4}$ geben Rushton \& Tomlinson keine physikalische Entsprechung an. Die Ansätze kommen damit ohne die Modellvorstellung einer Grenzfläche, die zwischen zwei Kompartimenten existiert, aus und werden durch Vergleich berechneter Ergebnisse mit gemessenen Werten eingestellt.

Grundsätzliches Problem der Verwendung von Leakage-Ansätzen als Randbedingung dritter Art in einem numerischen Grundwasserströmungsmodell ist, dass der unbekannte Grundwasserstand zur Bestimmung der Leakage-Menge bereits bekannt sein muss, sich aber erst als Modellergebnis aus der Leakage-Menge ergibt (Forkel 2004). Eine Randbedingung dritter Art mit linearer Leakage-Formel kann in das algebraische Gleichungssystem eines Strömungsprogramms durch eine entsprechende Umformung integriert werden (siehe z. B. Diersch 2005d). Dies ist für die Leakage-Beziehungen von Rushton \& Tomlinson (1979) wegen des Exponentialterms nicht möglich. Becker et al. (2009b) implementieren deshalb für den nichtlinearen Ansatz nach Gleichung 5.9 eine eigene Iterationsschleife zur Bestimmung des unbekannten Grundwasserwertes $h_{\mathrm{G}}$ aus Gleichung (5.1) nach dem Vorbild eines Prädiktor-Korrektor-Verfahrens. Für die Verwendung der mit Gleichungen 5.4, 5.7 oder 5.9 beschriebenen Fallunterscheidung muss zumindest die Richtung, in der der Austausch stattfindet, bekannt sein, um den richtigen LeakageKoeffizienten zu wählen. Dies erfordert gegebenenfalls eine wiederholte Berechnung des Strömungsgleichungssystems (Kinzelbach \& Rausch 1995).

\subsection{Zur Bestimmung des Leakage-Flusses}

Um für den Einzelfall zu prüfen, welcher Leakage-Ansatz geeignet ist, um die vorliegende Situation zu erfassen, werden die Leakage-Menge und die zugehörige Druckhöhendifferenz benötigt. Während die Druckhöhendifferenz aus Pegeldaten des Flusses und flussnaher Grundwassermessstellen relativ einfach abgeleitet werden kann, ist die Bestimmung der Wassermenge, die zwischen Fließgewässer und Grundwasserleiter ausgetauscht wird, vergleichsweise aufwändig. 
Eine direkte Messung der Leakage-Menge ist mit einem sogenannten Seepage-Meter möglich. Ein Seepage-Meter besteht aus einem offenen zylindrischen Behälter, zum Beispiel mit 2001 Fassungsvermögen, der mit der Öffnung nach unten unter Wasser in das Sediment eingebracht und entlüftet wird. Mit einem Schlauch ist an diesen Behälter ein Beutel zur Sammlung des Leakage-Wassers angeschlossen (Australian Government 2008b). Fließt Wasser durch die vom Behälter abgedeckte Sedimentoberfläche, so verändert sich das Volumen im Beutel. Aus dem Messintervall und der Volumenänderung in der Blase kann damit auf die Austauschraten geschlossen werden. Eine wesentliche Einschränkung der Messungen mit dem Seepage-Meter ist, dass nur die Verhältnisse eines Punktes wiedergegeben werden (Australian Government 2008a). Es besteht dadurch die Gefahr, dass die am Ort der Messung beobachteten Fließverhältnisse nicht den gesamten Fließgewässerabschnitt repräsentieren. Schubert (2002) berichtet von Zonen verschiedener Durchlässigkeit der Flusssohle in einem Abschnitt des Rheins. Das Seepage-Meter eignet sich also zunächst nur für die Messung der Austauschmengen auf der Flussbettskala, während für Fragen des unterirdischen Hochwassers in der Regel größere Bereiche abgedeckt werden sollen.

Rushton \& Tomlinson (1979) bestimmen die Leakage-Mengen durch Abtrennung des Basisabflusses vom Gesamtabfluss (base flow separation). Methoden zur Trennung der einzelnen Abflusskomponenten beschreiben zum Beispiel Mattheß \& Ubell (1983). Die Verwendung solcher Trennungsverfahren setzt voraus, „, dass - auch bei höherem Wasserstand im Vorfluter - stets ein hydraulischer Gradient vom Grundwasser zum Vorfluter erhalten bleibt, also ständig effluente Verhältnisse herrschen. Diese hypothetischen Annahmen sind in der Regel allenfalls in gebirgigen Gebieten erfüllt, im Allgemeinen wechseln effluente und influente Verhältnisse einander $a b$ “. Ein wesentliches Merkmal des unterirdischen Hochwassers ist aber gerade der Vorzeichenwechsel des hydraulischen Gradienten, so dass Verfahren der Trennung der Abflusskomponenten grundsätzlich nicht geeignet sind, um die Austauschmengen bei unterirdischem Hochwasser zu bestimmen.

Die Abschätzung der Leakage-Mengen durch Vergleich der Durchflüsse am Einlauf und am Auslauf eines Flussabschnittes (Bilanzierung) ist laut Rushton (2007) in ihrer Genauigkeit begrenzt, weil die Leakage-Mengen im Vergleich zu den Durchflüssen des Fließgewässers oft klein sind. Hinzu kommt, dass die genaue Bestimmung des Durchflusses bei Hochwasser ein Problem darstellt, da aufgrund von Instationaritäten eine eindeutige Beziehung zwischen dem vergleichsweise leicht zu messenden Flusswasserstand und dem Durchfluss nicht gegeben ist (Dyck \& Peschke 1983).

Ubell (1987a) stellt ein Verfahren zur Quantifizierung der Uferspeicherung vor, bei dem Daten von Grundwassermessstellen im Zusammenhang mit Flusspegeldaten ausgewertet werden. An einem Profil senkrecht zum Fließgewässer sind dazu auf einer Linie mehrere Grundwassermessstellen in unterschiedlicher Entfernung zum Fluss anzuordnen (Abbildung 5.4). Aus den gemessenen Grundwasserstandsänderungen wird auf die Volumenänderung des Grundwasservorrates geschlossen. Im Beispiel aus Abbildung 5.4 ist die Ausgangslage zum Zeitpunkt $t_{1}$ mit dem Wasserstand im Fluss $h_{01}$ und den Grundwasserständen $h_{11}$ bis $h_{41}$ gegeben. Der zum Zeitpunkt $t_{2}$ angestiegene Wasserstand im Fluss führt zu einer Einspeisung von Flusswasser in den 


\section{Grundwassermessstellen}

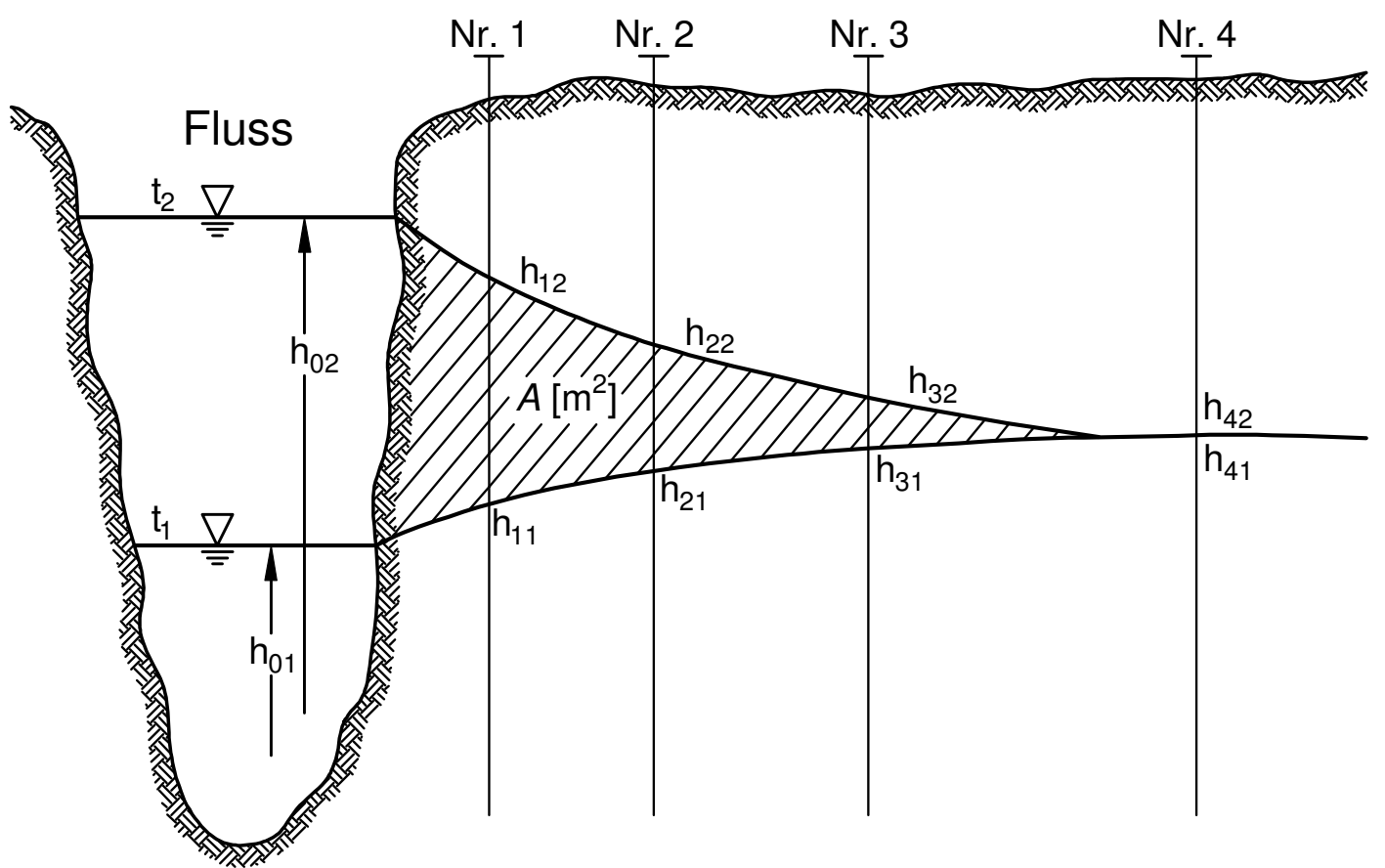

Abbildung 5.4: Prinzipskizze zur Methode der Volumenbetrachtung nach Ubell (1987a, 1964)

angrenzenden Grundwasserleiter, so dass der Grundwasserstand an den Messstellen auf $h_{12}, h_{22}$ und $h_{32}$ ansteigt, während sich an der Messstelle 4 der Wasserstand nicht ändert. Die zwischen $t_{1}$ und $t_{2}$ eingetretene Grundwasservorratsänderung ist durch die Fläche $A$ gegeben. Die auf die Profilbreite bezogene Volumenänderung $V^{\prime}$ ergibt sich zu

$$
V_{t_{1} \rightarrow t_{2}}^{\prime}=A \cdot n_{\mathrm{Pn}}
$$

mit dem nutzbaren Porenvolumen $n_{\mathrm{Pn}}$. Die Volumenänderung pro Zeiteinheit liefert den Leakage-Fluss

$$
Q_{t_{1} \rightarrow t_{2}}=\frac{V_{t_{1} \rightarrow t_{2}}^{\prime}}{t_{1}-t_{2}} \cdot l
$$

mit der Länge des betrachteten Flussabschnittes $l$.

Örtlicher Niederschlag und Rückstau des vor dem Wasseranstieg vorhandenen Grundwasserzuflusses lassen sich mit diesem Verfahren nicht abtrennen. Im Idealfall ist der Grundwasserspiegel vor Beginn des Wasseranstiegs im Fluss horizontal und es regnet nicht. Mit dieser Methode lassen sich die großräumigen Effekte der Uferspeicherung auf das Grundwasser erfassen. Damit ist die Methode von Ubell gut geeignet, um die Austauschmenge zwischen einem Fließgewässer und dem angrenzenden Grundwasserleiter zu quantifizieren. Der Bodenkennwert $n_{\mathrm{Pn}}$ ist für die meisten Bodenarten gut schätzbar und liegt „,ür die wichtigsten Grundwasserleiter zwischen 
den Grenzen 0,17 und 0,25“ (Ubell 1987a). Nachteilig ist, dass entsprechende Grundwassermessstellen vorhanden sein müssen. Das Verfahren setzt darüber hinaus eine Orientierung der Grundwasserströmung in Richtung des Messprofils sowohl für den Normalzustand als auch bei Hochwasser voraus.

\subsection{Beispiel: Rheinhochwasser von 1983 im Neuwieder Becken}

\subsubsection{Zur Geologie des Neuwieder Beckens}

Das Neuwieder Becken ist eine Talweitung am Rhein und erstreckt sich zwischen den Städten Koblenz und Andernach. Entstanden ist es aus einem tektonischen Senkungsgebiet innerhalb des Rheinischen Schiefergebirges. Durch Ablagerungen und Erosion vom Rhein finden sich heute verschiedene terrassenartige Schichtungen. Der Ausbruch des Laacher-See-Vulkans führte zu den großflächig vorzufindenden Ablagerungen von Bimstuffen (Gränitz \& Grundmann 2003). Die Niederterrasse nimmt eine Fläche von etwa $7 \mathrm{~km}$ Breite und $15 \mathrm{~km}$ Länge in der Talweitung von Koblenz bis Neuwied ein. Wegen des gut abgrenzbaren Hauptgrundwasserleiters ist die Niederterrasse besonders gut für die Untersuchung der Interaktion von Rhein und angrenzendem Grundwasserleiter geeignet (Giebel et al. 1990). In der Nähe der Ortschaft Urmitz bei Rheinkilometer 602,4 wurde ein Messprofil aus sieben Grundwassermessstellen senkrecht zur Achse des Rheins eingerichtet (Ubell 1987a; Giebel \& Hommes 1988). Eine Übersichtskarte des Neuwieder Beckens und die Lage des Messprofils Urmitz enthält Abbildung 5.5, ein Profilschnitt und die Lage der Grundwassermessstellen ist in Abbildung 5.6 gegeben.

\subsubsection{Das Hochwasserereignis von 1983}

Im oberen Teil der Abbildung 5.7 sind die Ganglinien des Rheins bei Urmitz und dreier Grundwassermessstellen, die in unterschiedlicher Entfernung zum Ufer (Tab. 5.2) angeordnet sind, für das Hochwasserereignis vom April 1983 dargestellt. Der Einfluss des ansteigenden Wasserstands im Rhein auf die Grundwasserstände der Messstellen ist deutlich zu erkennen. Es fällt allerdings auf, dass der maximale Grundwasserstand für keine der Messstellen mit dem Hochwasserscheitel zusammenfällt, sondern je nach Abstand zum Ufer verzögert eintritt. Auch der Leakage-Fluss, der von Ubell (1987a) nach dem oben beschriebenen Verfahren der Volumenbetrachtung berechnet wurde, fällt weder mit dem Rheinwasserstand noch mit der Wasserstandsganglinie einer Grundwassermessstelle zusammen. Der Maximalwert des Austauschflusses wird schon am 12. Tag erreicht, während der größte Rheinwasserstand erst am 13. Tag eintritt. Die Grundwassermessstelle U01, die dem Ufer am nächsten liegt, erreicht ihren Maximalwert erst am 14. Tag.

Aus den Wasserstandsganglinien des Rheins und der entsprechenden Grundwasserstandsganglinie lassen sich Ganglinien der Druckhöhendifferenzen für die Grundwassermessstellen berechnen. Diese Druckhöhendifferenzen (Gleichung 5.1) zeigt das untere Diagramm in Abbildung 5.7. Für Grundwassermessstellen, die weiter vom Ufer entfernt liegen, ergeben sich größere 


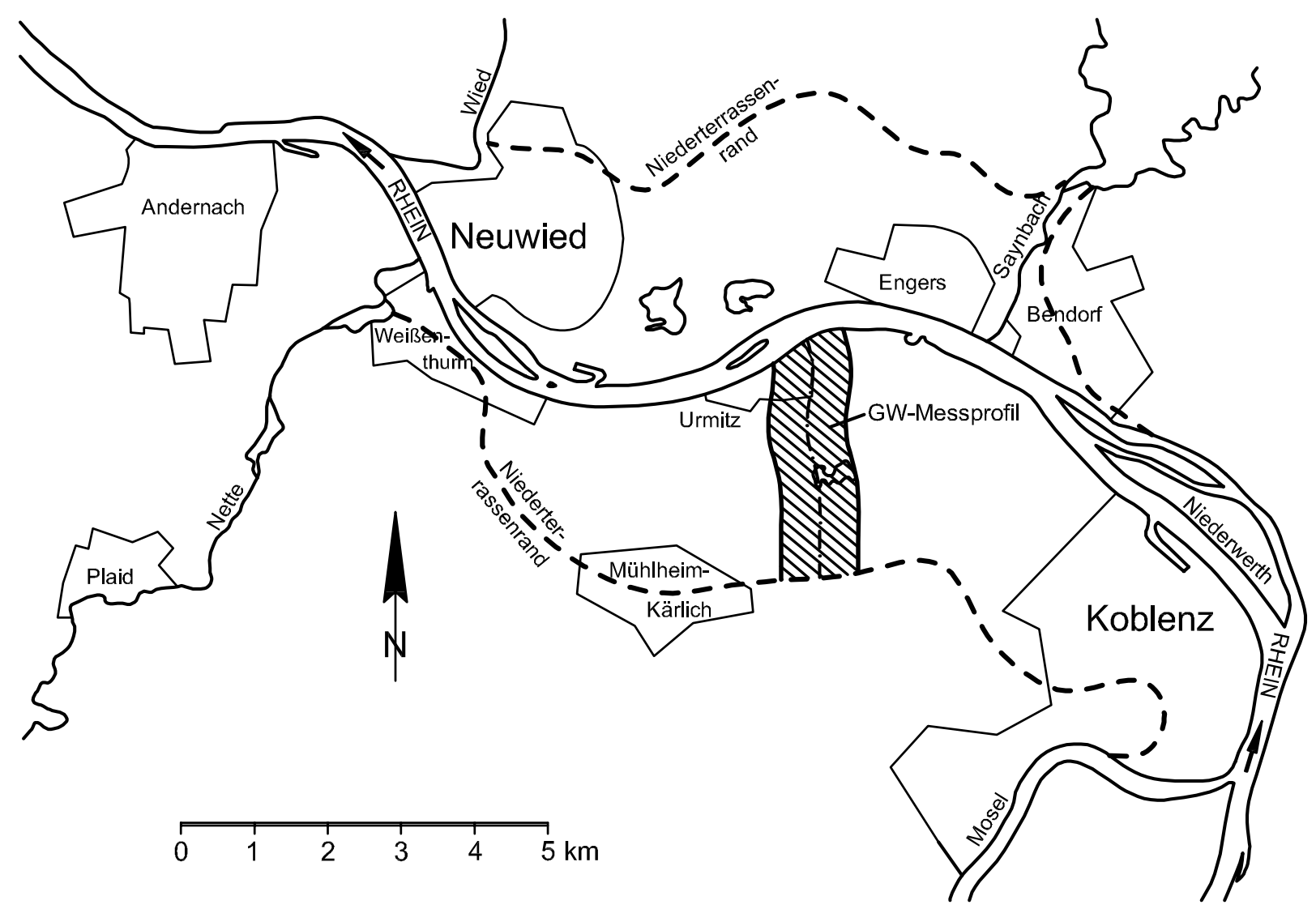

Abbildung 5.5: Übersichtskarte des Neuwieder Beckens mit dem Grundwassermessprofil bei Urmitz (nach Ubell 1987a)

Tabelle 5.2: Entfernung der Grundwassermessstellen des Messprofils Urmitz zum Ufer (nach Ubell 1987a)

\begin{tabular}{cc}
\hline Messstelle & Entfernung zum Ufer \\
\hline U01 & - \\
U03 & ca. $500 \mathrm{~m}$ \\
U05 & ca. $1600 \mathrm{~m}$ \\
\hline
\end{tabular}

Druckhöhendifferenzen, weil der durch den Anstieg des Rheinwasserstands verursachte Grundwasserstandsanstieg mit zunehmender Entfernung vom Ufer abnimmt und somit niedrige Grundwasserstände mit den hohen Flusswasserständen kombiniert werden.

\subsubsection{Leakage-Beziehung}

Mit den Austauschflüssen liegen damit die notwendigen Angaben zur Ableitung einer LeakageFunktion für das vorliegende Hochwasserereignis vor. Als Bezugspunkt für die Ableitung einer 


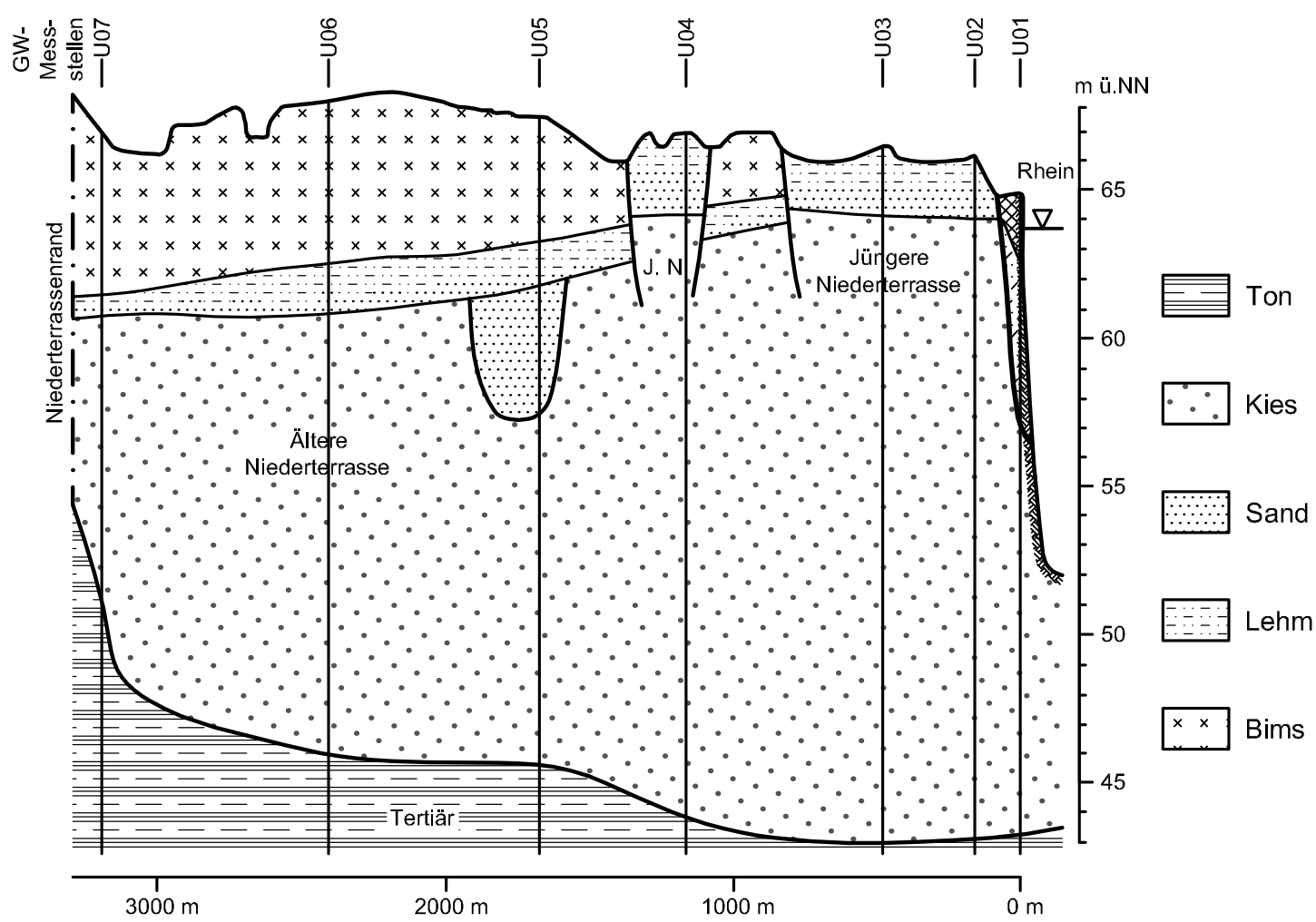

Abbildung 5.6: Profilschnitt durch die Rheinniederung bei Urmitz (Jansen 2009, geändert nach Ubell 1987a)

Leakage-Funktion muss die Messstelle U01 verwendet werden, da sie von allen Messstellen am nächsten am Ufer liegt. Die aus den angegebenen Messwerten der Grundwassermessstelle U01 berechneten Werte der Wasserstandsdifferenzen $\Delta h$ sind in Abbildung 5.8 über dem LeakageFluss $Q$ aufgetragen. Für positive Werte scheint demnach eine lineare Leakage-Funktion den Vorgang der Infiltration verhältnismäßig gut wieder zu geben, wenn auch die Regressionsgerade nicht exakt den Koordinatenursprung trifft. Ursache hierfür ist vermutlich, dass die Wasserstände im Grundwasser nicht direkt unter der Sedimentschicht aufgenommen wurden. Darüber hinaus bezieht sich der Rheinwasserstand nicht auf den Flusskilometer des Grundwassermessprofils (Ubell 1987a). Weitere mögliche Quellen für Ungenauigkeiten sind die Datenherkunft (Anhang D) und der zur Berechnung der Leakage-Flüsse verwendete Speicherkoeffizient (siehe dazu Jansen 2009).

Für den Rückfluss des im Ufer gespeicherten Wassers (Exfiltration) ergibt sich aus den Messwerten eine Regressionsgerade mit etwas geringerer Steigung. Der Achsenabschnitt ist deutlich größer als bei der Infiltration, während das Bestimmtheitsmaß $R^{2}$ (siehe Anhang E.1) etwas unter dem der Regressionsgeraden für die Infiltration liegt. Eine augenscheinlich geeignete Anpassung würde in diesem Fall auch eine Exponentialfunktion liefern. Die Daten der Exfiltration lassen sich aber auch so interpretieren, dass die Austauschmenge unabhängig von der Wasser- 

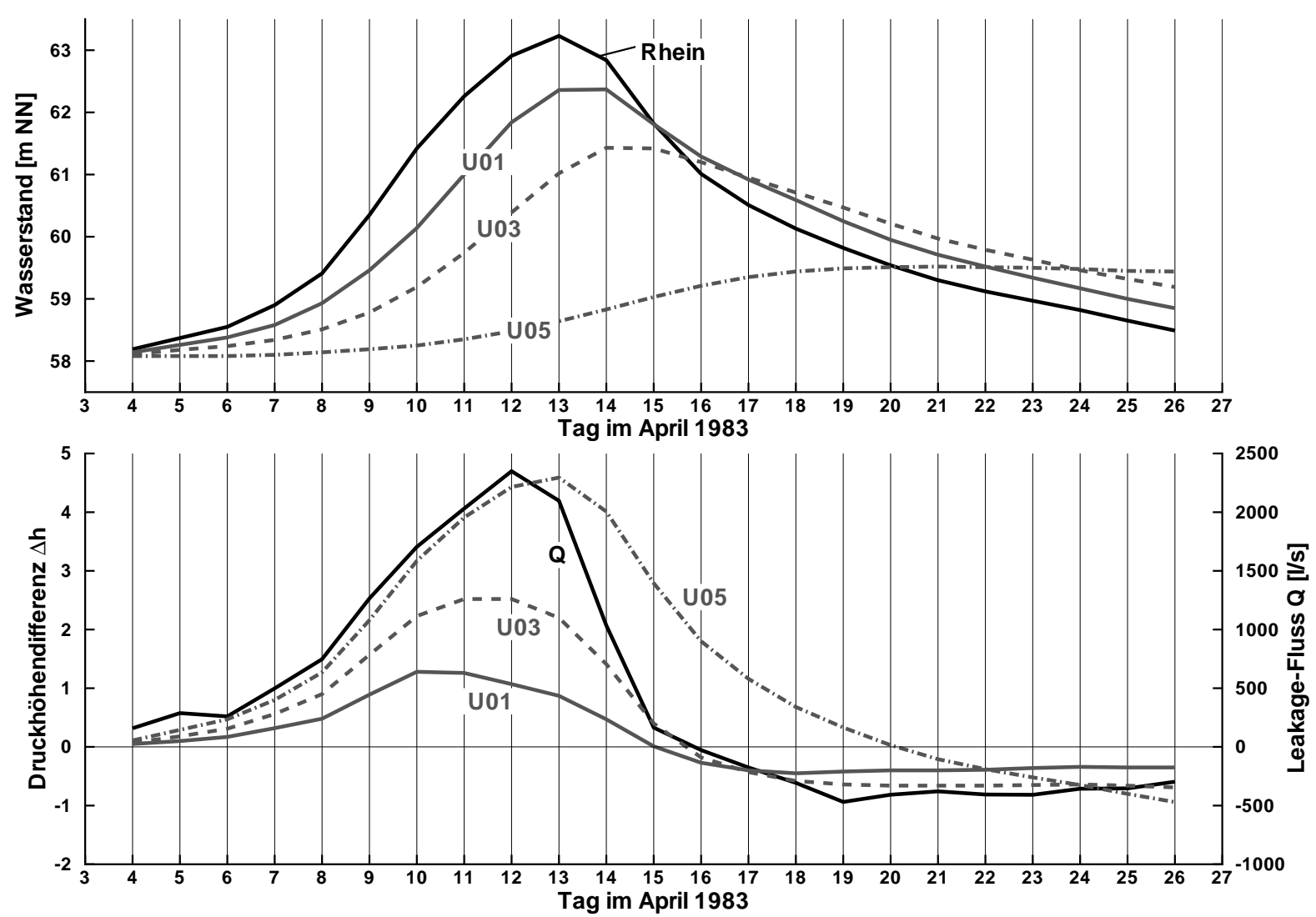

Abbildung 5.7: Ganglinen des Rheins und dreier Grundwassermessstellen mit unterschiedlicher Entfernung zum Ufer (oben) sowie Leakage-Fluss für das betrachtete $1 \mathrm{~km}$ breite Profil „Urmitz“ als Tagesmittelwerte (nach Ubell 1987a, siehe Anhang D) und berechnete Druckhöhendifferenz (unten). Die vertikalen Gitternetzlinien liegen jeweils auf der Mitte des Tages.

standsdifferenz ist. Das Leakage-Modell wäre vor diesem Hintergrund nicht geeignet, um den Austauschprozess zu beschreiben.

Insgesamt erreichen positive Leakage-Flüsse, die also vom Fließgewässer in Richtung des Grundwasserleiters gerichtet sind, im Vergleich zu negativen Leakage-Flüssen betragsmäßig deutlich höhere Werte. Auch werden für den Fall der Infiltration deutlich höhere Wasserstandsdifferenzen erreicht als für den Rückfluss. Dies entspricht der Anschauung dahingehend, dass der Grundwasseranstieg in relativ kurzer Zeit vonstatten geht (siehe Abschnitt 1.1), die unterirdische Hochwasserwelle aber lange Zeit braucht, um wieder abzuklingen. Bei Hochwasser steigt der Wasserstand im Fluss sehr stark an und liefert hohe Wasserstandsdifferenzen. Einen starken Abfall des Wasserspiegels nach dem Hochwasserereignis, der zu betragsmäßig entsprechenden Wasserstandsdifferenzen in negativer Richtung führt, gibt es im vorliegenden Fall jedoch nicht. 


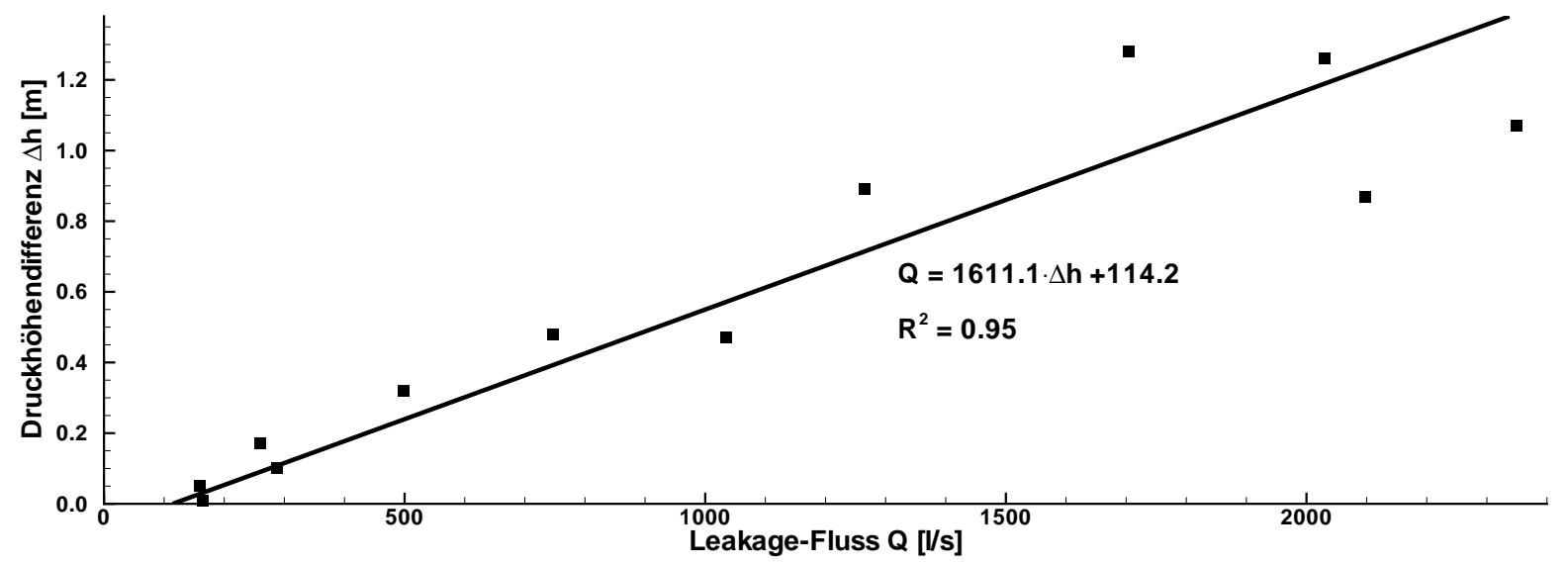

(a) Zufluss zum Grundwasserleiter

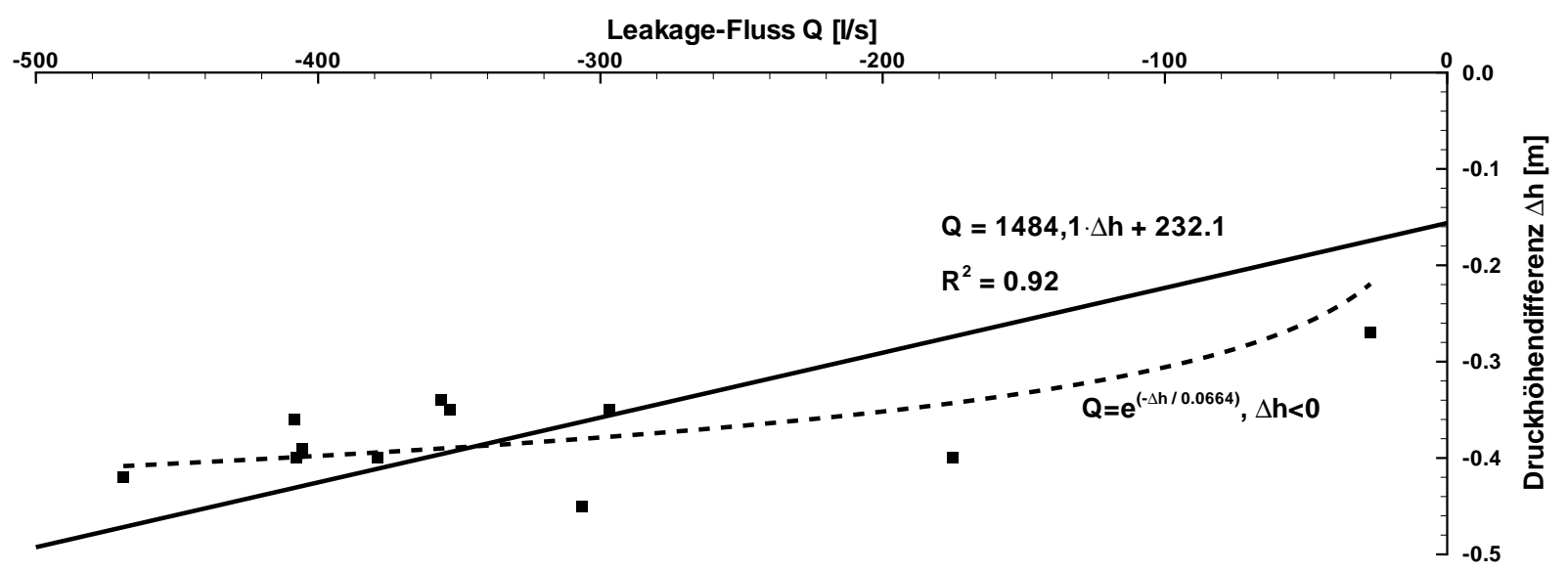

(b) Rückfluss zum Rhein

Abbildung 5.8: Druckhöhendifferenz $\Delta h$ über Leakage-Fluss $Q$ für die Grundwassermessstelle U01 (Datengrundlage nach Ubell 1987a) und Ausgleichsfunktionen für den Zufluss zum Grundwasserleiter und den Rückfluss zum Rhein (jeweils unterschiedliche Skalen)

Dass bei betragsmäßig gleichen Druckhöhendifferenzen wegen der Kolmation der Gewässersohle für die Infiltration verhältnismäßig kleinere Flüsse auftreten als bei umgekehrter Fließrichtung, wie in Abschnitt 5.2 beschrieben, wird für den hier vorliegenden Fall nicht bestätigt.

Wertet man die $Q-\Delta h$-Beziehung im zeitlichen Verlauf des Hochwasserereignisses aus, so zeigt sich ein hysteretischer Verlauf (Abbildung 5.9). Bis zum Tag 10 steigt die Kurve der Messstelle U01 nahezu linear an. Am Tag 11 steigt die Wasserstandsdifferenz nur noch wenig, trotzdem nimmt die Austauschmenge zu. Bei Tag 12 sinkt die Wasserstandsdifferenz schon wieder um einen kleinen Betrag, der Leakage-Fluss steigt jedoch weiter. Für Messstelle U03 erfährt die Hysterese einen anderen Drehsinn als bei Messstelle U01. Bei Messstelle U05 liegen einige Wertepaare sogar im II. Quadranten des $Q-\Delta h$-Diagramms. Die Wasserstandsdifferenz ist also noch positiv, obwohl das im Ufer gespeicherte Wasser schon zurückfließt. Besonders an dieser 


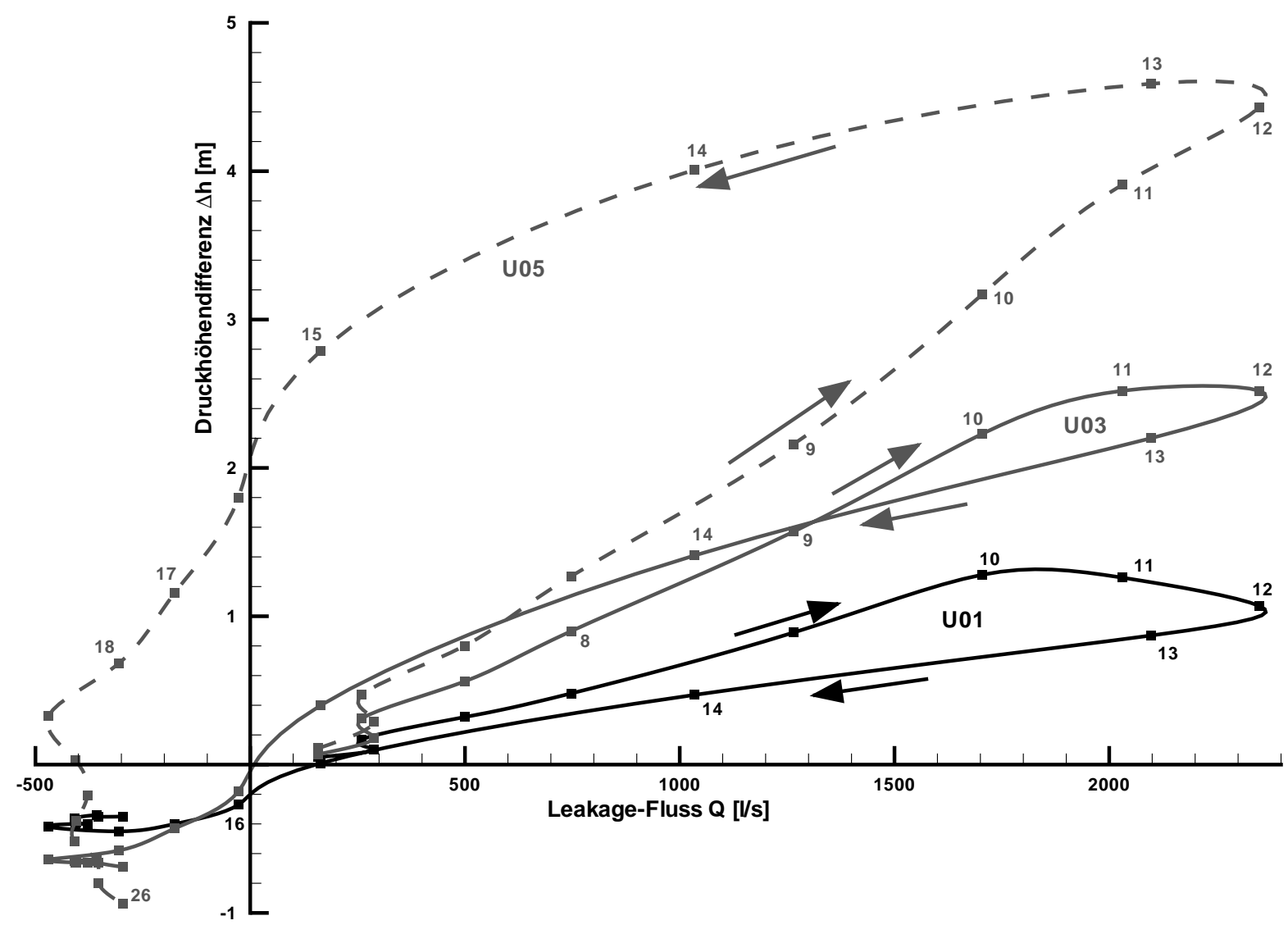

Abbildung 5.9: Druckhöhendifferenz $\Delta h$ über Leakage-Fluss $Q$ für die Grundwassermessstellen U01, U03 und U05 (Datengrundlage nach Ubell 1987a). Die Messspunkte sind entsprechend dem Verlauf des Hochwasserereignisses verbunden, die Zahlen an den Messpunkten geben den Tag des April 1983 an.

Kurve wird das verzögerte Nachlaufen der unterirdischen Hochwasserwelle hinter der Welle im Fluss deutlich.

Eine Begrenzung des Leakage-Flusses für hohe Wasserstandsdifferenzen $\Delta h$, wie es zum Beispiel der Ansatz aus Gleichung 5.9 oder Gleichung 5.7 umsetzen, erscheint für das vorliegende Beispiel nicht zweckmäßig. Der überproportionale Anstieg des Leakage-Flusses mit der Wasserstandsdifferenz würde die Verwendung eines Leakage-Ansatzes unter Berücksichtigung des benetzten Umfangs rechtfertigen. Der hysteretische Verlauf würde dann dadurch entstehen, dass sich, anders als im Beispiel, für das in Abbildung 5.3 die Leakage-Funktion gezeigt wird, sowohl der Grundwasserspiegel als auch der Wasserstand im Fluss verändern. Für zwei gleiche Wasserstandsdifferenzen $\Delta h$ können daher unterschiedlich große Kontaktflächen und damit auch unterschiedliche Leakage-Flüsse berechnet werden. So ändert sich die Wasserstandsdifferenz vom 10. auf den 11. Tag nur unwesentlich, der Wasserstand des Rheins steigt jedoch bis zum 12. Tag noch weiter an (Abbildung 5.7) und erhöht damit den benetzten Umfang. Den Rückgang der 

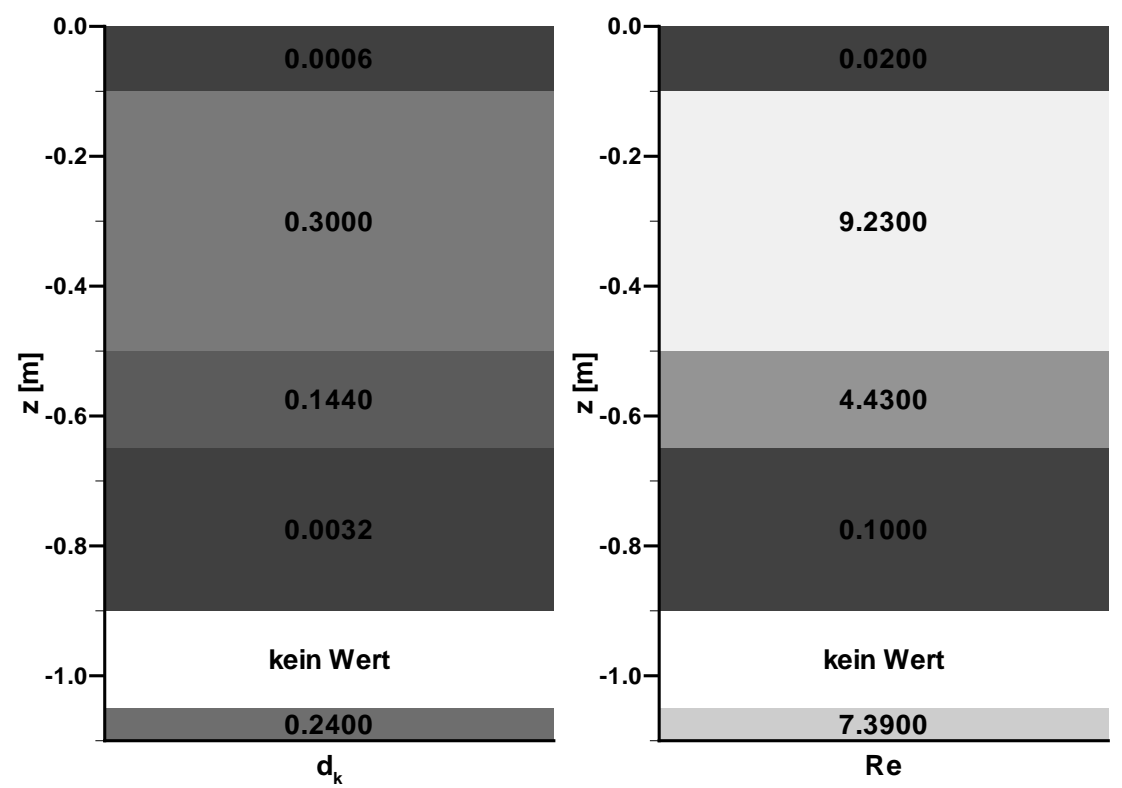

Abbildung 5.10: Charakteristische Korndurchmesser $d_{\mathrm{k}}$ für das von Schubert (2002) beschriebene Sedimentprofil des Rheins bei Rheinkilometer 731,5 (Entfernung vom rechten Ufer: $78 \mathrm{~m}$ ) und überschläglich berechnete Reynolds-Zahlen $R e$

Austauschmenge am 13. Tag, an dem der Rhein seinen maximalen Wasserstand erreicht, wäre dann der reduzierten Wasserstandsdifferenz geschuldet.

Eine andere mögliche Ursache für den hysteretischen Verlauf ist, dass sich Gegebenheiten, die den Austausch zwischen dem Fließgewässer und dem Grundwasserleiter bestimmen, über die Dauer des Hochwasserereignisses verändern (siehe z. B. Blaschke 2002). So entsteht eine abdichtende Sohlschicht, die das Fließgewässer vom Grundwasserleiter trennt, durch Sedimentation von Feinmaterial und biogene Stabilisierung. Eine Hochwasserwelle kann diese Sedimentschicht kurzzeitig aufwühlen und so die Dicke der Sedimentschicht reduzieren oder die Sedimentschicht vollständig erodieren. Eine Auflockerung führt zu zudem zu einer Veränderung der hydraulischen Durchlässigkeitseigenschaften. Mit Abklingen der Hochwassererscheinung werden Sedimentations- und Stabilisierungsprozesse wiederum für eine Abdichtung sorgen, so dass die Leakage-Menge wieder langsam abnimmt (Vázquez-Suñé et al. 2007; Schälchli 1992, 1993). Durch Erosions- und Sedimentationsprozesse ändert sich also die Mächtigkeit der abdichtenden Sohlschicht und auch ihr Gefüge.

Schließlich kann die im Bereich der hohen Leakage-Flüsse auftauchende Hysterese oder Nichtlinearität auch dadurch verursacht sein, dass der Anwendungsbereich des dem Leakage-Modell zu Grunde liegenden Darcy-Gesetzes verlassen wird, weil zu hohe Reynolds-Zahlen erreicht werden (Abschnitt 2.2p. Es liegen keine genauen Angaben zur Beschaffenheit der Flusssohle 
am Messprofil Urmitz vor. Deshalb wird eine überschlägliche Berechnung der Reynolds-Zahlen nach Gleichungen 2.12, 2.13 und 2.14 und dem maximalen Leakage-Fluss (Tabelle D.1) über

$$
v=\frac{Q}{A}=\frac{Q}{l \cdot l_{\mathrm{U}^{*}}}=\frac{2,349 \mathrm{~m}^{3} / \mathrm{s}}{1000 \mathrm{~m} \cdot 58,7 \mathrm{~m}}=4,00 \cdot 10^{-5} \mathrm{~m} / \mathrm{s}
$$

und $v=1,31 \cdot 10^{-6} \mathrm{~m}^{2} / \mathrm{s}$ für das von Schubert (2002) veröffentliche Sedimentprofil durchgeführt. Allerdings liegt dieses 129,1 km weiter flussabwärts in einer Flussbiegung. Weiterhin berücksichtigt die Überschlagsrechnung keinen Potentialabbau innerhalb der Sedimentschicht, keine Geschwindigkeitsschwankungen und auch keine Unterschiede in der Flussbettbeschaffenheit entlang des Querprofils. Der benetzte Umfang $l_{\mathrm{U}}$ wird anhand Abbildung 5.6 abgeschätzt. Das Ergebnis der Überschlagsrechnungen zeigt Abbildung 5.10. Es wird deutlich, dass die Strömungsverhältnisse in der von oben gesehen zweiten und dritten Schicht die Obergrenze des Gültigkeitsbereichs für die Anwendung des Darcy-Gesetzes $(R e \leq 1 \ldots 10$, Abschnitt 2.2) erreicht, aber nicht überschritten wird.

\subsubsection{Numerische Modellrechnungen}

\subsubsection{Einführung und Vorgehensweise}

Nachdem im vorangegangenen Abschnitt der Prozess der Uferspeicherung im Neuwieder Becken während des Hochwassers von 1983 anhand von veröffentlichten Felddaten untersucht wurde, soll nun der Frage nachgegangen werden, wie dieser Prozess in einem numerischen Modell erfasst werden kann. Für das vorliegende Beispiel wird dazu ein Grundwassermodell nach dem Vorbild von Ubell (1987b) aufgebaut. Bei dem Vorbild handelt es sich um ein eindimensionalhorizontales Grundwassermodell, das den Vertikalschnitt entlang des Messprofils (Abbildung 5.6) abbildet. Dieses Modell setzt Jansen (2009) mit dem Programm Feflow (siehe Abschnitt 2.4) als dreidimensionales Streifenmodell für gesättigte Grundwasserströmung um, wobei die Grundwasserströmung der Achse des Messprofils folgt. Die Netzgeometrie zeigt Abbildung 5.11 .

Für die modelltechnische Umsetzung des Flusses steht zunächst die h-Randbedingung, deren Randbedingungswert der Wasserstand im Fluss ist (Abbildung 2.7) und die auch von Ubell (1987b) angewendet wurde, zur Auswahl. Die Sedimentschicht des Uferbereichs wird direkt modelliert, das heißt, Elemente des Uferbereichs und der Sohle bilden die Sedimentschicht nach und erhalten Durchlässigkeitsbeiwerte $\left(k_{\mathrm{F}}\right.$-Werte), die sich vom Rest des Grundwasserleiters unterscheiden. Daher muss für den Einsatz einer h-Randbedingung eine entsprechend feine Modelldiskretisierung vorliegen. Die Modellgeometrie im Bereich des Ufers, den Ansatz der Randbedingungen und die Modellierung der Sedimentschicht über die Durchlässigkeitsbeiwerte der ufernahen Elemente zeigt Abbildung 5.11. Bei Ansatz einer h-Randbedingung ergibt sich der Austauschfluss $Q$ korrespondierend zum vorgegebenen Flusswasserstand $h_{\mathrm{F}}$.

Die Leakage-Randbedingung kommt ohne eine direkte Abbildung der Sedimentschicht aus. Die Elemente im Bereich des Ufers und der Sohle haben die gleichen Durchlässigkeitseigenschaften 


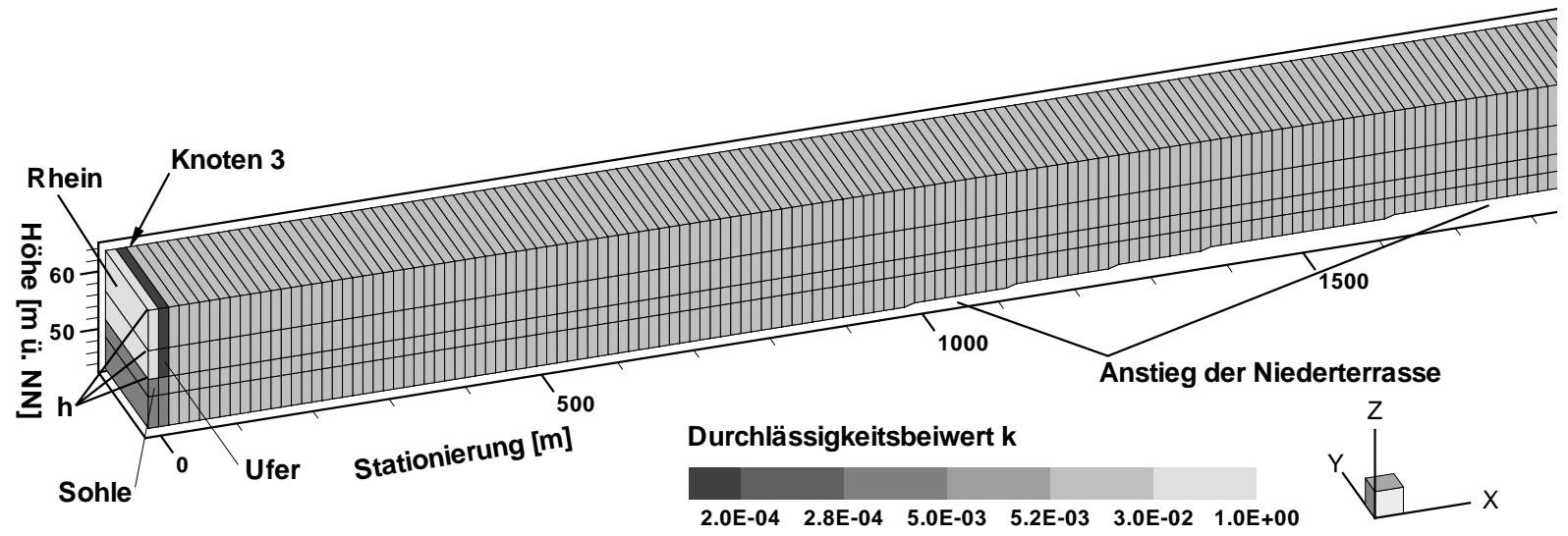

(a) Ergebnis der manuellen Kalibrierung

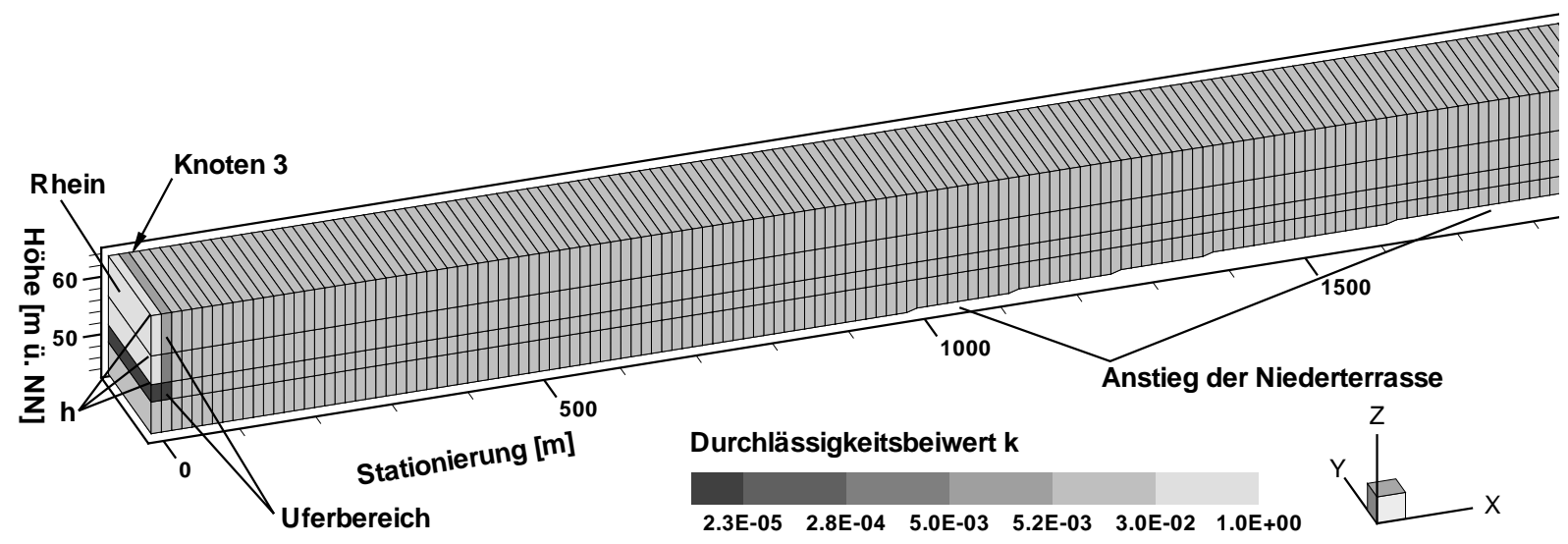

(b) Ergebnis der automatischen Kalibrierung

Abbildung 5.11: 3D-Grundwassermodell (Ausschnitt) des Messprofils Urmitz mit dem Fluss als hRandbedingung und Kalibrierungsergebnis der Durchlässigkeitsbeiwerte aus manueller Kalibrierung (a) und automatischer Kalibrierung (b) von Jansen (2009)

wie der Grundwasserleiter. Zu- und Abfluss werden über die Leakage-Parameter derjenigen Elemente gesteuert, die die Knoten mit Leakage-Randbedingung verbinden (Abbildung 5.12). Die verwendete Leakage-Funktion entspricht von der Modellvorstellung her dem Darcy-LeakageAnsatz (Gleichung 5.2), für den unterschiedliche Leakage-Parameter für beide Fließrichtung vorgegeben werden können (Gleichung 5.3). Die Kontaktfläche $A$ für den Leakage-Fluss $Q$ wird programmintern in Abhängigkeit vom Grundwasserstand $h_{\mathrm{G}}$ berechnet. Damit ähnelt die Leakage-Randbedingung dem mit Gleichung 5.6 gegebenen Ansatz, wobei bei der im Programm Feflow verwendeten Randbedingungsformulierung allerdings die Kontaktfläche nicht über den benetzten Umfang vom Wasserstand im Fluss, sondern vom Grundwasserstand abhängt:

$$
\begin{aligned}
A & =l \cdot l_{\mathrm{U}}{ }^{*} \\
l_{\mathrm{U}}{ }^{*} & =f\left(h_{\mathrm{G}}\right)
\end{aligned}
$$


Je nach Wasserstandsdifferenz $\Delta h$ kann der Unterschied zwischen $l_{\mathrm{U}}$ und $l_{\mathrm{U}}{ }^{*}$ durchaus beträchtlich sein.

In einem dreidimensionalen Feflow-Grundwassermodell müssen die Modellknoten jeweils vertikal übereinander liegen (siehe Abbildung 5.12). Damit sind einer naturgetreuen Abbildung des Ufers Grenzen gesetzt, denn ein Flussquerprofil mit einer geneigten Böschung kann so nicht exakt wiedergegeben werden.

Die verschiedenen Grundwassermodellvarianten wurden auf zwei Arten, nämlich manuell und mit einem mathematischen Optimierungsalgorithmus (PEST, Doherty et al. 1994) automatisch kalibriert. Als Kalibrierungsdaten wurden die von Ubell (1987a) veröffentlichten aus den Messwerten konstruierten Grundwasseroberflächen und die Ganglinien der Grundwassermessstellen U01 bis U07 verwendet. Kalibrierungszielgrößen waren die Durchlässigkeitsbeiwerte der finiten Elemente im gesamten Modellgebiet. Die Elemente wurden in fünf $k$-Wert-Zonen aufgeteilt (Jansen 2009). Der Speicherkoeffizient ist keine Kalibrierungsgröße, er wird im Wesentlichen mit $S_{\mathrm{sp}}=17,5 \%$ für die Elemente, die Leitermaterial abbilden, festgelegt und mit $S_{\mathrm{sp}}=0$ für die Elemente, die für den Fluss im Grundwassermodell stehen.

Für die Modellvariante, bei denen der Fluss über eine Leakage-Randbedingung abgebildet wird, kamen als Kalibrierungsparameter die Leakage-Parameter hinzu, bei der Modellvariante mit hRandbedingung die Durchlässigkeitsbeiwerte der ufernahen Elemente. Die Kalibrierungsparameter wurden nun so angepasst, dass sich eine möglichst gute Übereinstimmung zwischen den Modellergebnissen, also den berechneten Werten, und den gemessenen Werten ergab. Die Kalibrierungsgüte wurde visuell und mit Hilfe des Korrelationskoeffizienten $R$ sowie der Nash-Sutcliffe-Effizienz NSE beurteilt. Die Kennzahlen $R$ und NSE wurden für jede Messstelle berechnet. Anhang $\mathrm{E}$ enthält eine kurze Beschreibung zur Bedeutung und zur Berechnung der Kennzahlen.

Die Kalibrierung lieferte für alle Varianten gute bis sehr gute Übereinstimmungen zwischen berechneten und gemessenen Werten. Abbildungen 5.11 und 5.12 zeigen jeweils einen Ausschnitt aus dem dreidimensionalen Grundwassermodell mit einer Modellvariante und den entsprechenden Kalibrierungsergebnissen.

\subsubsection{Auswertung der Modellrechnungen}

In Tabelle 5.3 sind die Kennzahlen für die Bewertung der Kalibrierungsergebnisse aus der manuellen Kalibrierung für jede Messstelle aufgeführt. Aus der Tabelle geht hervor, dass mit der Leakage-Randbedingung für das vorliegende Beispiel bessere Kalibrierungsergebnisse erreicht wurden als mit der h-Randbedingung. Dies verdeutlicht auch Abbildung 5.13a, wo die konstruierten Grundwasseroberflächen den Berechnungsergebnissen des dreidimensionalen Modells gegenübergestellt werden. Für das Modell mit h-Randbedingung liegt am 13. April, also an dem Tag, an dem der Wasserstand im Rhein seinen größten Wert erreicht, der berechnete Grundwasserstand bis etwa $1400 \mathrm{~m}$ vom Ufer entfernt über der entsprechenden konstruierten Linie. Für den 15. April stimmen die berechnete und die gemessene Kurve sehr gut überein. Für den 26. 


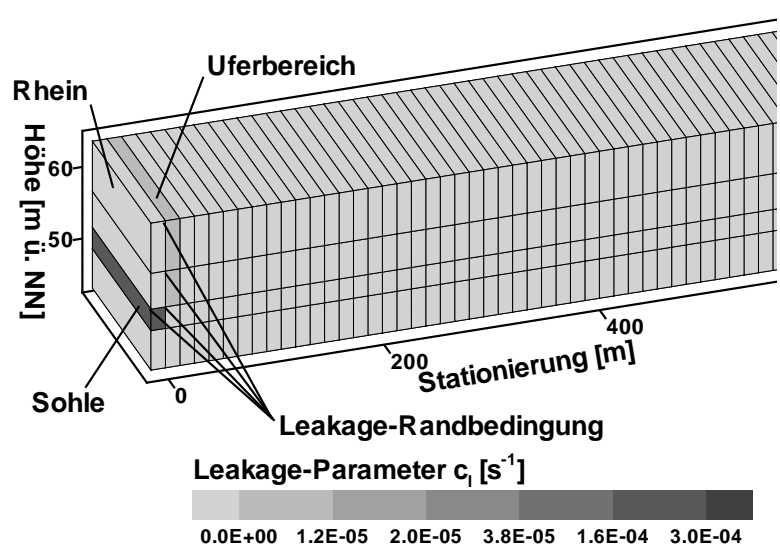

(a) Ergebnis der manuellen Kalibrierung

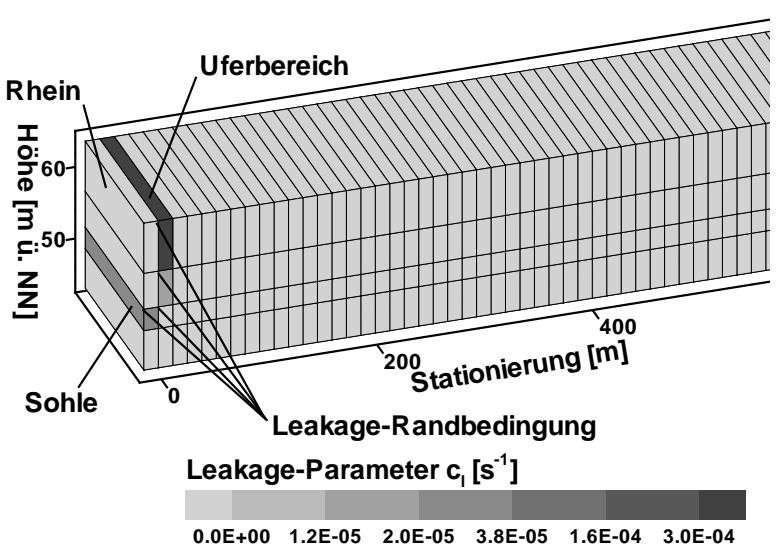

(b) Ergebnis der automatischen Kalibrierung

Abbildung 5.12: Ausschnitt aus dem 3D-Grundwassermodell des Messprofils Urmitz mit dem Fluss als Leakage-Randbedingung und Kalibrierungsergebnis der Leakage-Parameter $c_{1, \text { in }}$ aus manueller Kalibrierung (a) und automatischer Kalibrierung (b) von Jansen (2009)

Tabelle 5.3: Kennzahlen zur Kalibrierungsgüte der manuell kalibrierten Modelle für die verschiedenen Grundwassermessstellen (Werte übernommen von Jansen 2009)

\begin{tabular}{llccccccc}
\hline \multirow{2}{*}{ Kennzahl } & Randbedingung & \multicolumn{7}{c}{ Grundwassermessstelle } \\
& für den Rhein & $\mathrm{U} 01$ & $\mathrm{U} 02$ & $\mathrm{U} 03$ & $\mathrm{U} 04$ & $\mathrm{U} 05$ & U06 & U07 \\
\hline \multirow{2}{*}{$R$} & $\mathrm{~h}$ & 0,984 & 0,975 & 0,975 & 0,997 & 0,997 & 0,998 & 0,986 \\
& Leakage & 0,994 & 0,988 & 0,990 & 0,998 & 0,999 & 0,999 & 0,985 \\
\hline \multirow{2}{*}{$N S E$} & $\mathrm{~h}$ & 0,962 & 0,942 & 0,947 & 0,976 & 0,981 & 0,984 & 0,737 \\
& Leakage & 0,987 & 0,976 & 0,977 & 0,977 & 0,980 & 0,975 & 0,786 \\
\hline
\end{tabular}

April liegt der berechnete Wasserstand dagegen unterhalb der Linie, die aus Messwerten konstruiert wurde. Eine Reduzierung der Durchlässigkeitsbeiwerte im Uferbereich würde zwar für den 13. April eine Verbesserung liefern, dann aber die Übereinstimmung der Kurvenverläufe für die Tage 15 und 26 verschlechtern.

Die Variante mit Leakage-Randbedingung (Abbildung 5.13b) zeigt dagegen eine bessere Übereinstimmung zwischen den konstruierten Grundwasseroberflächen und den Rechenwerten, da hier die Leakage-Parameter $c_{1}$ für den Zufluss und den Abfluss des Wassers getrennt voneinander eingestellt werden können. In der Variante mit h-Randbedingung ist dies für die Durchlässigkeitsbeiwerte $k$ so nicht möglich. Einige Grundwasserströmungsprogramme, darunter auch Feflow, bieten zwar die Möglichkeit, zeitlich veränderliche Durchlässigkeitsbeiwerte für einzelne Elemente vorzugeben (siehe z. B. Becker et al. 2009a; Wasy GmbH 2008), aber es ist dann die Vorgabe fester Zeitpunkte für den Wechsel der Parameter erforderlich. Ein Anwendungsfall für zeitlich veränderliche Materialeigenschaften ist ein wandernder Tagebau, bei dem das 


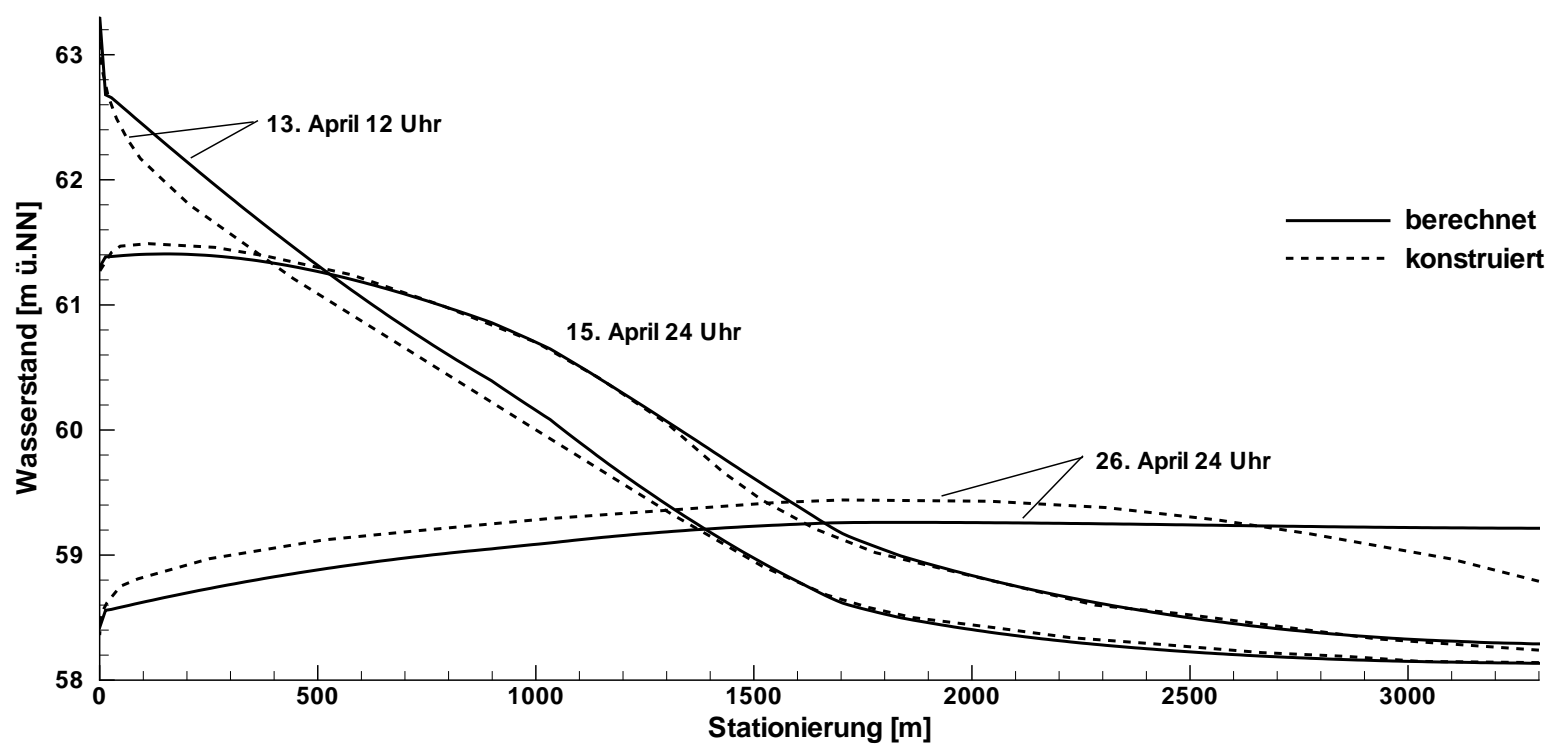

(a) Ergebnisse des Modells mit h-Randbedingung

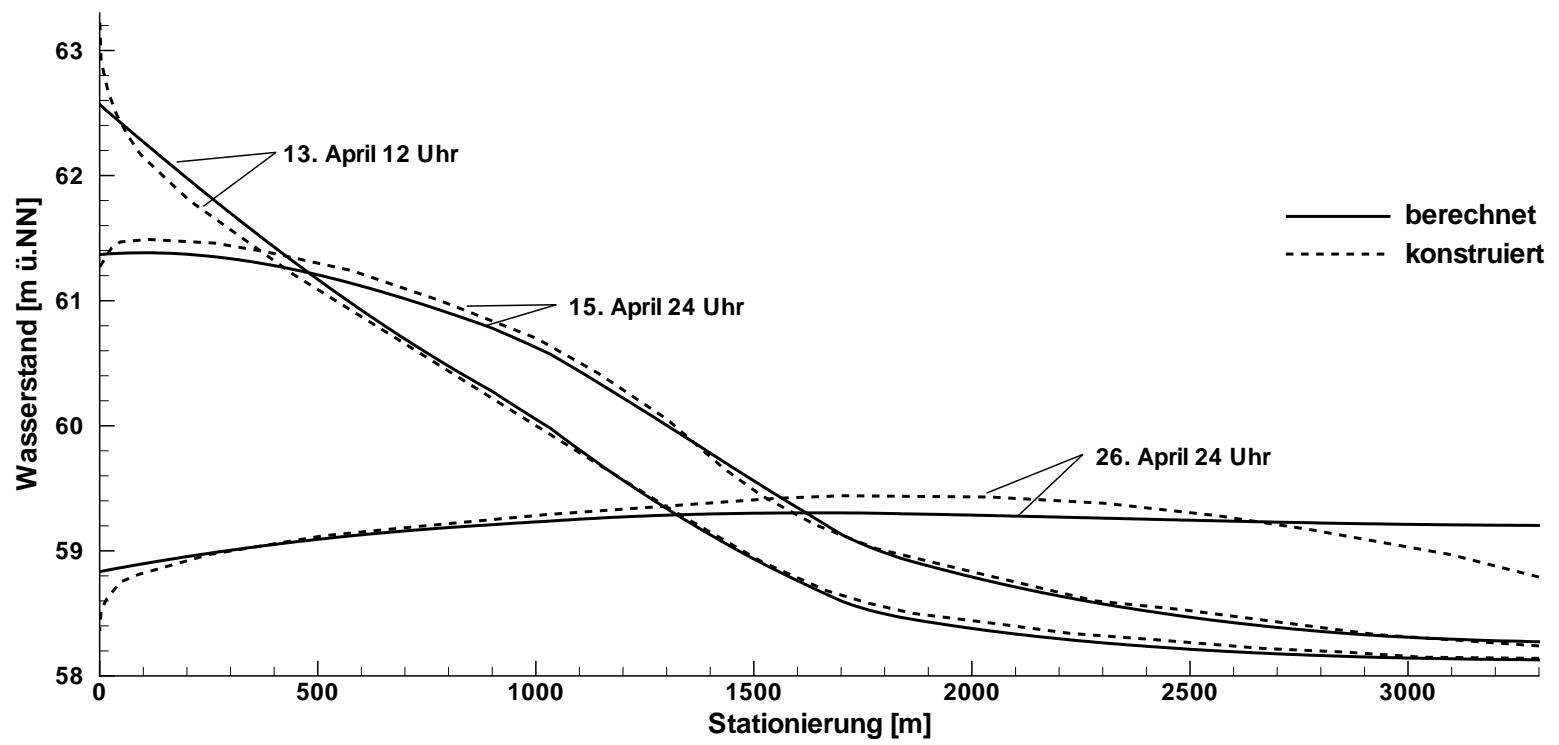

(b) Ergebnisse des Modells mit Leakage-Randbedingung

Abbildung 5.13: Berechnete Grundwasseroberflächen des manuell kalibrierten 3D-Modells mit hRandbedingung (a) und mit Leakage-Randbedingung (b) sowie die von Ubell (1987a) konstruierten Grundwasseroberflächen für drei verschiedene Zeitpunkte (nach Jansen 2009) 
Tabelle 5.4: Kennzahlen zur Kalibrierungsgüte der automatisch kalibrierten Modelle für die verschiedenen Grundwassermessstellen (Werte zum Teil übernommen aus Jansen 2009)

\begin{tabular}{llccccccc}
\hline \multirow{2}{*}{ Kennzahl } & Randbedingung & \multicolumn{6}{c}{ Grundwassermessstelle } \\
& für den Rhein & U01 & U02 & U03 & U04 & U05 & U06 & U07 \\
\hline \multirow{2}{*}{$R$} & h & 0,996 & 0,992 & 0,994 & 0,996 & 0,996 & 0,998 & 0,984 \\
& Leakage & 0,998 & 0,993 & 0,993 & 0,997 & 0,991 & 0,993 & 0,987 \\
\hline \multirow{2}{*}{$N S E$} & h & 0,988 & 0,978 & 0,954 & 0,927 & 0,986 & 0,988 & 0,709 \\
& Leakage & 0,980 & 0,971 & 0,945 & 0,957 & 0,976 & 0,952 & 0,103 \\
\hline
\end{tabular}

Tabelle 5.5: Summe der Kennzahlen zur Kalibrierungsgüte über die Messstellen U01 bis U07 aus Tabellen 5.3 und 5.4

\begin{tabular}{llcc}
\hline Kalibrierungsmethode & Randbedingung für den Rhein & $R$ & $N S E$ \\
\hline \multirow{2}{*}{ manuell } & $\mathrm{h}$ & 6,912 & 6,529 \\
& Leakage & 6,953 & 6,659 \\
\hline \multirow{2}{*}{ automatisch } & $\mathrm{h}$ & 6,956 & 6,528 \\
& Leakage & 6,953 & 5,884 \\
\hline
\end{tabular}

ungestörte, geschichtete Gebirgsmaterial im Laufe des Abbauprozesses durch Kippenmaterial ohne ausgeprägte Schichtung ersetzt wird (Bachmann et al. 2007). Für den vorliegenden Fall, bei dem eine Änderung der Durchlässigkeitseigenschaften mit einem Wechsel zwischen Infiltration und Exfiltration zusammenfällt, führt diese Programmoption deshalb nicht weiter. Schon die Auswertung der Messwerte in Form von $Q$ - $\Delta h$-Diagrammen deutet darauf hin, dass für den Zustrom zum Grundwasserleiter andere Leakage-Eigenschaften vorliegen als für den Abstrom in Richtung Fluss. Die hier vorgestellten numerischen Untersuchen untermauern dies.

Bei beiden Varianten konnte für die Grundwasseroberfläche des 26. Aprils die Abweichung ab einer Stationierung von etwa $2600 \mathrm{~m}$ nicht durch eine Variation der Durchlässigkeitsparameter behoben werden. Jansen (2009) nennt als mögliche Ursache für die Abweichungen Strömungen, die von der Hauptstromrichtung parallel zum Messprofil abweichen und die nicht im Modell erfasst werden.

Für die Ergebnisse der automatischen Kalibrierung zeigt die Tabelle 5.4 auf den ersten Blick nicht, welche Variante die bessere Übereinstimmung liefert. Zwar deutet der Korrelationskoeffizient $R$ darauf hin, dass die Strömungssituation mit einer Leakage-Randbedingung für die meisten Messstellen besser wiedergegeben wird, die Nash-Sutcliffe-Effizienzen zeigen dies jedoch nicht. Zur besseren Übersicht wurden die Kennzahlen zur Kalibrierungsgüte aus den Tabellen 5.3 und 5.4 über die Messstellen aufsummiert (Tabelle 5.5).

Bei dem Vergleich der Kalibrierungsergebnisse aus Abbildungen 5.11a und 5.11b fällt auf, dass bei der manuellen Kalibrierung die höhere Durchlässigkeit im Bereich der Sohle liegt, bei der 
automatischen Kalibrierung wurde die höhere Durchlässigkeit für den oberen Bereich des Ufers bestimmt. Für die Leakage-Randbedingung (Abbildung 5.12) ergibt sich ein ähnliches Bild: hohe Leakage-Parameter für die Infiltration ins Grundwasser wurden bei der manuellen Kalibrierung in den Bereich der Sohle gesetzt, während an der Uferböschung der Bereich mit kleineren Leakage-Parametern liegt. Das Ergebnis der automatischen Kalibrierung ist entgegengesetzt. Hier sind die hohen Leakage-Parameter im Bereich der Uferböschung. Die manuelle und die automatische Kalibrierung liefern also voneinander deutlich unterschiedliche Ergebnisse. Beide Ergebnisse zeigen aber eine gute Übereinstimmung von Mess- und Rechenwerten sowohl anhand der Kennzahlen als auch anhand eines visuellen Vergleichs (siehe Jansen 2009), so dass nicht davon ausgegangen wird, dass eine der beiden Methoden versagt hat.

Als Vorteil der automatischen Kalibrierung wird genannt, dass diese Methode die Kalibrierungsaufgabe unbefangen löst. So wurde im vorliegenden Beispiel bei der Handkalibrierung davon ausgegangen, dass der der Hauptanteil der Austauschmenge wie bei Giebel \& Hommes (1988) und Ubell (1987b) beschrieben über den ufernahen Bereich der Sohle geleitet wird und dass dort höhere Durchlässigkeitseigenschaften vorliegen als im Uferbereich. Da die Handkalibrierung gute Ergebnisse lieferte, wurde gar nicht ausprobiert, ob eine vollkommen andere Verteilung der Durchlässigkeitseigenschaften auch zum Ziel führt (Jansen 2009). Der Optimierungsalgorithmus der automatischen Kalibrierung sucht dagegen das Minimum einer Zielfunktion und erlaubt damit auch, dass weniger naheliegende oder sogar physikalisch nicht sinnvolle Ergebnisse gefunden werden. Grundsätzlich lassen die Kalibrierungsergebnisse nur bedingt Rückschlüsse auf die in der Realität vorhandenen Verhältnisse zu (Forkel 2004). Dennoch kann das Ergebnis einer automatischen Kalibrierung zu einer Erweiterung des Prozessverständnisses beitragen, indem man über Lösungen nachdenkt, die zunächst nicht naheliegend sind. Das Ergebnis der automatischen Kalibrierung wirft zum Beispiel die Frage auf, ob nicht doch im Uferbereich höhere Durchlässigkeiten vorliegen als an der Flusssohle. Müller (1999) beschreibt zum Beispiel präferenzielle Fließwege, die an der Uferböschung durch Wühltierbaue oder durch Eisbruch entstehen. Für das vorliegende Beispiel finden sich in der untersuchten Literatur weder Hinweise auf derartige präferenzielle Fließwege, noch detaillierte Beschreibungen der Uferbeschaffenheit, so dass diese Frage nicht abschließend geklärt werden kann. Wären solche hohen Durchlässigkeiten im oberen Uferbereich vorhanden, so könnten sie dennoch nicht ursächlich für das Kalibrierungsergebnis sein, denn die Datengrundlage, die für die automatische Kalibrierung verwendet wurde, enthält keine Informationen über den Weg, den das Wasser entlang des Flussufers nimmt.

Abbildung 5.14 zeigt die $Q-\Delta h$-Beziehungen der unterschiedlichen Modellvarianten. Es wird deutlich, dass bei den manuell kalibrierten Modellen ein nicht oder kaum hysteretischer Verlauf vorliegt. Bei den automatisch kalibrierten Modellen ist die Hysterese dagegen ausgeprägt, der Verlauf der aus Messwerten abgeleiteten Kurve ist wiederzuerkennen. Hier liegt auch die Ursache für die gänzlich unterschiedlichen Kalibrierungsergebnisse. Der Wasserstand im Fluss und der Grundwasserstand variieren in dem Grundwassermodell bei dem untersuchten Szenario nur innerhalb der obersten Elementschicht. Zu Beginn des Hochwassers und zum Ende hin trägt die obere Elementschicht nur wenig zum gesamten Leakage-Fluss bei. Mit steigendem Grundwasserstand nimmt der Anteil der oberen Elementlage am gesamten Leakage-Fluss zu: für die Leakage-Randbedingung erhöht sich die Kontaktfläche, die zusammen mit dem Leakage- 


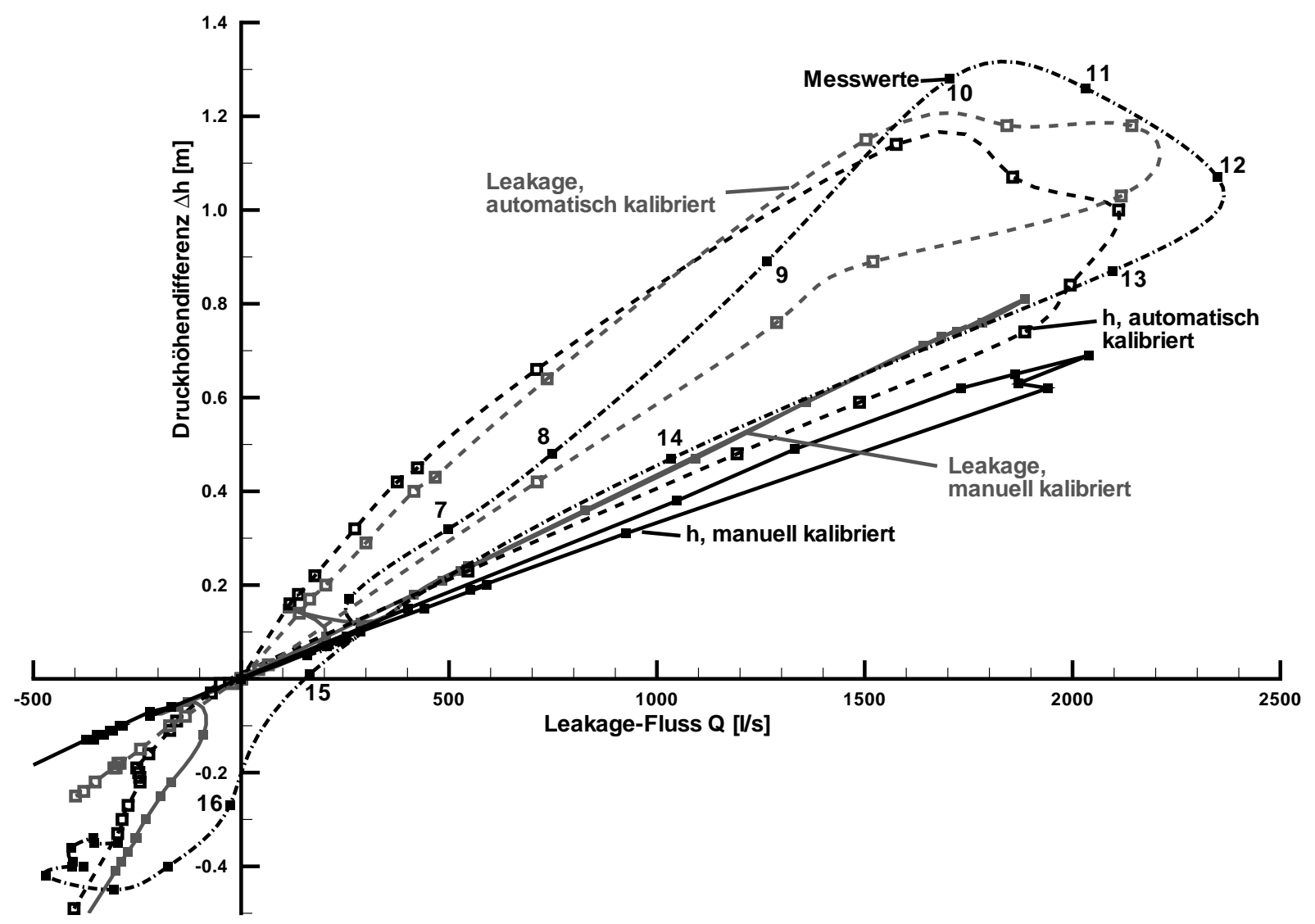

Abbildung 5.14: $Q$ - $\Delta h$-Diagramme aus den automatisch und manuell kalibrierten Modellvarianten mit Leakage- und h-Randbedingung für den Rhein. Die Zahlen an den aus Messwerten abgeleiteten Punkten geben den Tag im April 1983 an. Für die Berechnung der Wasserstandsdifferenzen bei den Modellen mit h-Randbedingung wurden die angesetzten Flusswasserstände jeweils mit den Grundwasserständen der Knoten auf Höhe des Knotens 3 (siehe Abbildung 5.11) in Beziehung gesetzt.

Koeffizienten die Austauschmenge bestimmt. So liegt in dem skizzierten Beispiel aus Abbildung 5.15 bei beiden Zuständen die gleiche Wasserstandsdifferenz $\Delta h$ vor. Bei insgesamt höheren Wasserständen (Abbildung 5.15b) wird jedoch, weil der benetzte Umfang größer und der Leakage-Koeffizient $c_{1}$ der obersten Schicht durchlässiger ist, dem Grundwassermodell mehr Wasser zugeführt als bei dem in Abbildung 5.15a skizzierten Fall.

Die Wahl von im Vergleich zur Sohle hohen Durchlässigkeiten beziehungsweise LeakageParametern erlaubt dem Algorithmus also, die Zuflüsse für große Wasserstände oder Wasserstandsdifferenzen überproportional zu steigern, bei der Einstellung der Parameter der unteren Schichten kann dagegen mehr auf die kleinen Durchflüsse Rücksicht genommen werden. Bei dem Ergebnis der Handkalibrierung fällt der nichtlineare Anteil nicht ins Gewicht, weil hier in der oberen Elementlage vergleichsweise niedrige Parameter gesetzt wurden. Während bei der automatischen Kalibrierung die modelltechnischen Möglichkeiten das Ergebnis bestimmen, 


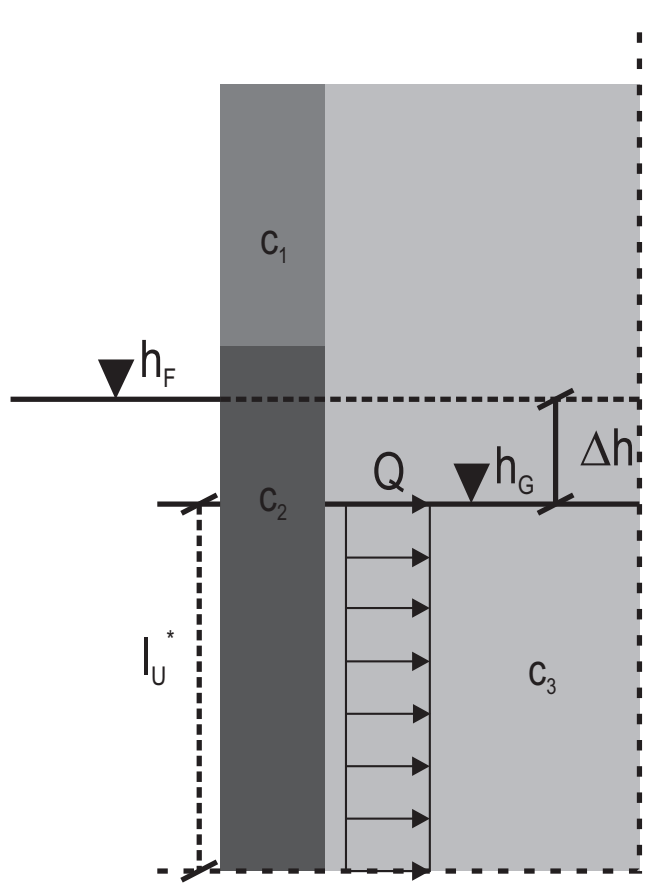

(a) Niedriger Flusswasserstand und niedriger Grundwasserstand

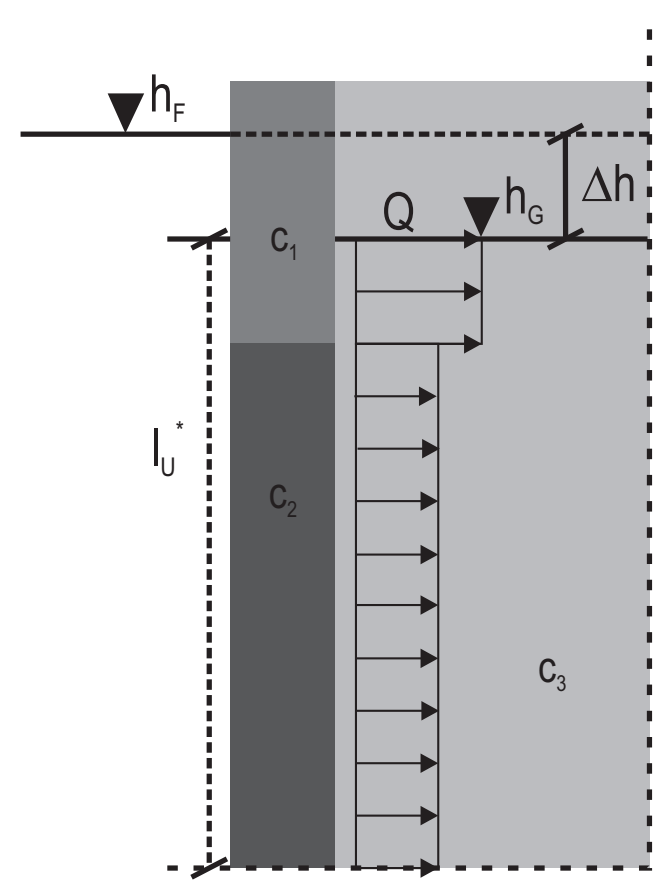

(b) Hoher Flusswasserstand und hoher Grundwasserstand

Abbildung 5.15: Benetzter Umfang $l_{\mathrm{U}}{ }^{*}$ und Leakage-Fluss $Q$ bei unterschiedlichen Wasserständen $h_{\mathrm{F}}$ und $h_{\mathrm{G}}$ und schichtweise unterschiedlichen Durchlässigkeitseigenschaften $\left(c_{3}>c_{1}>c_{2}\right)$, aber gleicher Wasserstandsdifferenz $\Delta h$

wurden bei der manuellen Kalibrierung dagegen zusätzliche Informationen über die Sohl- und Uferbeschaffenheit und die daraus resultierenden Strömungsverhältnisse einbezogen.

Deutlich wird in Abbildung 5.14 auch die für positive und negative Werte gleiche Steigung der Leakage-Funktion, die sich aus der h-Randbedingung bei manueller Kalibrierung ergibt, während die Leakage-Funktion des handkalibrierten Modells für positive und negative Werte unterschiedliche Steigungen aufweist. Die auffällige Abweichung einzelner Werte um den Nullpunkt herum resultieren aus dem bereits angesprochenen Problem, dass der Grundwasserstand zum Zeitpunkt der Berechnung nicht bekannt ist, aber der Leakage-Parameter bereits festgelegt werden muss. Eine Wiederholung der Berechnung wird vom Programm Feflow nicht vorgenommen, so dass für die Zeitschritte, die auf einen Wechsel der Fließrichtung folgen, der falsche LeakageParameter verwendet werden kann.

Leider geben alle vier vorgestellten Modellrechnungen die Verhältnisse für negative LeakageFlüsse nur recht grob wieder. Aus Abbildung 5.7 kann entnommen werden, dass ab Tag 16 ähnlich wie beim Zustrom bei nahezu gleichbleibender Wasserstandsdifferenz verschiedene Leakage-Flüsse auftreten. Anders als für den Prozess der Infiltration und den damit verbundenen hohen Wasserständen können für die Exfiltration keine Elementbereiche über die Parameter angesteuert werden, die nur bei niedrigen Wasserständen greifen. Insgesamt treten bei der Ex- 
filtration vergleichsweise kleine Beträge des Leakage-Flusses auf, so dass der Einfluss auf die Grundwassersituation anders als bei der Infiltration eher gering sein dürfte.

\subsubsection{Schlussfolgerungen und Diskussion}

Es ist für das Beispiel des Rheinhochwassers von 1983 möglich, den Austausch zwischen Fluss und Grundwasserleiter sowohl über Leakage-Ansätze als auch mit einer h-Randbedingung zu modellieren. Es konnte mit beiden Varianten eine gute Übereinstimmung der gemessenen und der berechneten Werte für das Hinterland erreicht werden. Für die vorliegende Zielsetzung ist dieses Ergebnis ausreichend, zu einer detaillierten Beschreibung der Prozesse in unmittelbarer Umgebung des Flussbettes besteht allerdings noch Forschungsbedarf. So kann die Ursache für den hysteretischen Verlauf der Leakage-Beziehung mit den vorgenommenen Untersuchungen nicht abschließend geklärt werden.

Zur Frage, welcher Randbedingungstyp besser zur Modellierung der Wechselwirkungen des Grundwasserleiters mit einem Fluss geeignet ist, wird zunächst als Vorteil der Leakage-Randbedingung festgehalten, dass mit ihr für den Prozess der Infiltration und der Exfiltration jeweils unterschiedliche Durchlässigkeitseigenschaften vorgegeben werden können. Im Rahmen der Handkalibrierung konnte damit eine bessere Übereinstimmung zwischen den gemessenen und den berechneten Grundwasserständen erreicht werden als mit der h-Randbedingung. Es entsteht so allerdings der Eindruck, die Leakage-Eigenschaft des Ufers ändere sich mit der Fließrichtung des Leakage-Flusses. Untersuchungen verschiedener Autoren (Blaschke 2002; Schälchli 1992; Schubert 2002) deuten darauf hin, dass dies vermutlich nur mittelbar der Fall ist. Vielmehr ändern sich die Durchlässigkeitseigenschaften der Sohle, die Mächtigkeit der Sohlschicht und die Kontaktfläche, über die ein Wasseraustausch stattfindet, in Abhängigkeit von den Strömungsverhältnissen im Fluss. Bei großen Wasserständen kommt es zu verstärkter Erosion, damit werden Sohle und Uferbereich durchlässiger, die Dicke der Sedimentschicht nimmt - zumindest bereichsweise - ab, aber der benetzte Umfang steigt. Sinkt der Wasserspiegel, dann sorgen Sedimentationsprozesse, möglicherweise auch biogene Stabilisierung, für eine Kolmation, die die Durchlässigkeit reduziert und gleichzeitig die Mächtigkeit der Sohlschicht vergrößert, und der benetzte Umfang des Fließquerschnitts sinkt. Insbesondere während eines Hochwassers wird der Schwebstofftransport hoch sein, so dass mit abklingender Hochwasserwelle verstärkte Sedimentation eintritt. Die Sedimentation fällt damit ungefähr mit dem Wechsel der Fließrichtung zusammen, das Sinken des Wasserspiegels, das zu niedrigeren Fließgeschwindigkeiten führt und eine Sedimentation begünstigt, wird aber, wie die Ganglinien des untersuchten Beispiels zeigen (Abbildung 5.7), unter Umständen schon früher eintreten als der Fließrichtungswechsel.

Besonders für großräumigere Modelle wird es ein weiterer Vorteil für die Anwendung sein, dass bei Verwendung einer Leakage-Randbedingung eine den Fluss und den Grundwasserleiter trennende Schicht nicht, wie bei der h-Randbedingung der Fall, mit eigenen Uferelementen modelliert werden muss. 
Obwohl ein hysteretischer Verlauf der Leakage-Funktion den aus Messwerten abgeleiteten Austausch zwischen Fluss und Grundwasserleiter besser wiedergibt, reicht eine lineare LeakageFunktion aus, um für das Beispiel eine gute Übereinstimmung zwischen gemessenen und berechneten Grundwasserdruckhöhen zu erreichen. Eine Hysterese der Leakage-Funktion kann in einem dreidimensionalen Modell sowohl über eine Leakage-Randbedingung als auch über eine h-Randbedingung in Kombination mit einer Schichtung der Modellparameter, die den Wasseraustausch bestimmen, abgebildet werden. Bei beiden Varianten kommt dem Modellparameter der obersten Schicht, in der sich der Grundwasserstand bewegt, eine große Bedeutung zu. Die vergleichsweise hohen Durchlässigkeiten beziehungsweise Leakage-Koeffizienten, die für die Elementlage im oberen Uferbereich ermittelt wurden, deuten darauf hin, dass der angesetzte $\mathrm{Zu}$ wachs des benetzten Umfangs allein nicht ausreicht, um den erhöhten Leakage-Fluss bei steigenden Wasserständen zu erreichen, denn sonst wäre eine homogene Sedimentschicht entlang des benetzten Umfangs des Fließquerschnitts ausreichend gewesen, um den Verlauf der aus Messwerten abgeleiteten Leakage-Funktion herzustellen. Allerdings ist zur Berechnung der Kontaktfläche nicht der benetzte Umfang des Flusses, sondern eine vom Grundwasserstand abgeleitete Kontaktfläche als Einflussgröße verwendet worden. Weiterhin ist die Uferböschung entsprechend dem Vorbildmodell von Ubell (1987b) und wegen der Anwendungsgrenzen des verwendeten Strömungsprogramms senkrecht modelliert worden. Bei einem solchen Rechteckquerschnitt steigt die Kontaktfläche mit steigendem Grundwasserstand nahezu linear und nicht überproportional an und kann damit nur wenig zu einem überproportionalen Anstieg des Leakage-Flusses bei hohen Wasserständen beitragen.

Die Leakage-Flüsse von Ubell (1987a) basieren auf gemessenen Grundwasserständen. Die $Q$ $\Delta h$-Beziehung ist damit quasi nur eine andere Darstellung der Messwerte, die für die Kalibrierung herangezogen wurden. Ungenauigkeiten entstehen über den Speicherkoeffizient, für den im Rahmen der Kalibrierung ein anderer Wert ermittelt wurde (Jansen 2009) als zur Berechnung der Leakage-Flüsse verwendet (Ubell 1987a).

Für den Prozess der Exfiltration ließ sich mit den verschiedenen Modellen für das vorliegende Beispiel der Verlauf der aus Messwerten abgeleiteten Leakage-Funktion nicht gut wiedergeben. Dies kann aber in Kauf genommen werden, da die Exfiltrationsmengen pro Zeit vom Betrag her deutlich unter den Infiltrationsmengen liegen. Somit ergibt sich ein vergleichsweise geringer Einfluss auf die Grundwassersituation, so dass zumindest die Ganglinien der einzelnen Grundwassermessstellen dennoch gut mit den berechneten Werten übereinstimmen.

Eine zweidimensional-horizontale Modellierung, bei der eine schichtweise Abstufung der Modellparameter im Bereich des Ufers naturgemäß nicht möglich ist, liefert dementsprechend einen weniger ausgeprägten hysteretischen Verlauf der $Q-\Delta h$-Beziehung, denn hier trägt nur der benetzte Umfang $l_{\mathrm{U}}{ }^{*}$ zur Hysterese bei. Abbildung 5.16 zeigt die Leakage-Funktionen für zwei Modellvarianten des zweidimensional-horizontalen Streifenmodells von Jansen (2009). Trotz der deutlichen Unterschiede zur aus Messwerten abgeleiteten $Q-\Delta h$-Beziehung wurden für beide Varianten gute Übereinstimmungen zwischen gemessenen und berechneten Werten erreicht. Tabellen 5.6 und 5.7 zeigen die Kennzahlen der Kalibrierungsgüte, zur Gegenüberstellung der Ganglinien aus Mess- und Rechenwerten wird auf Jansen (2009) verwiesen. 


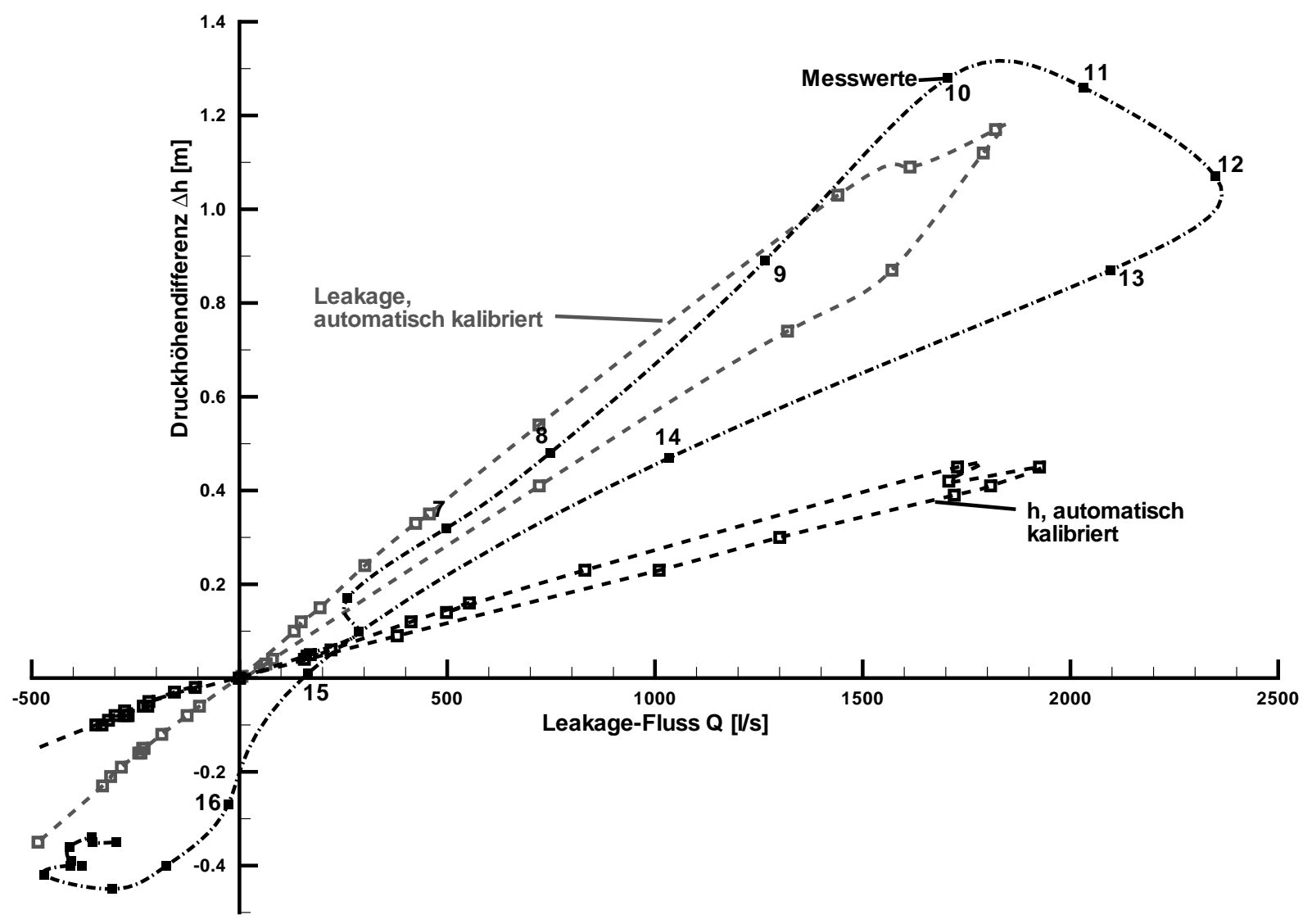

Abbildung 5.16: $Q-\Delta h$-Diagramme aus den automatisch kalibrierten zweidimensional-horizontalen Modellvarianten mit Leakage- und h-Randbedingung für den Rhein. Die Zahlen an den aus Messwerten abgeleiteten Punkten geben den Tag im April 1983 an.

Die Eignung der verschiedenen Modellvarianten wurde auf Basis der erreichten Kalibrierungsgüte festgestellt. Allerdings ist sowohl für die automatische als auch für die manuelle Kalibrierung nicht abschließend sichergestellt, dass die so bestimmten Modellparameter das Optimum einer Übereinstimmung zwischen gemessenen und berechneten Werten liefern. Dass vier verschiedene Modelle zu einer guten Übereinstimmung zwischen Mess- und Rechenwerten führen, zeigt die Grenzen einer Modellkalibrierung auf. In der Regel hat die Kalibrierungsaufgabe eines Modells keine eindeutige Lösung (Konikow \& Bredehoeft 1992). Das Ergebnis unterstreicht die Bedeutung einer Modellvalidierung ${ }^{1}$ mit Daten, die nicht zur Kalibrierung herangezogen wurden, und eines Post-Audit (Anderson \& Woessner 1992), wenn ein solches Modell zu Prognoserechnungen verwendet werden soll.

\footnotetext{
${ }^{1}$ Die Validierung eines Modells zeigt, dass das Modell bestimmte Prozesse für ein bestimmtes Gebiet repräsentiert. Mit der Verifikation dagegen wird für ein Computerprogramm nachgewiesen, dass es die Bestimmungsgleichungen korrekt löst. (Siehe auch Konikow \& Bredehoeft 1992)
} 
Tabelle 5.6: Kennzahlen zur Kalibrierungsgüte der automatisch kalibrierten zweidimensional-horizontalen Modelle für die verschiedenen Grundwassermessstellen (Werte zum Teil übernommen aus Jansen 2009)

\begin{tabular}{llccccccc}
\hline \multirow{2}{*}{ Kennzahl } & Randbedingung & \multicolumn{7}{c}{ Grundwassermessstelle } \\
& für den Rhein & $\mathrm{U} 01$ & $\mathrm{U} 02$ & $\mathrm{U} 03$ & $\mathrm{U} 04$ & $\mathrm{U} 05$ & U06 & U07 \\
\hline \multirow{2}{*}{$R$} & $\mathrm{~h}$ & 0,976 & 0,965 & 0,972 & 0,998 & 0,998 & 0,999 & 0,986 \\
& Leakage & 0,997 & 0,990 & 0,992 & 0,995 & 0,999 & 0,998 & 0,989 \\
\hline \multirow{2}{*}{$N S E$} & $\mathrm{~h}$ & 0,937 & 0,914 & 0,934 & 0,906 & 0,955 & 0,946 & 0,847 \\
& Leakage & 0,978 & 0,962 & 0,920 & 0,855 & 0,958 & 0,979 & 0,688 \\
\hline
\end{tabular}

Tabelle 5.7: Summe der Kennzahlen zur Kalibrierungsgüte über die Messstellen U01 bis U07 aus Tabelle 5.6

\begin{tabular}{llcc}
\hline Kalibrierungsmethode & Randbedingung für den Rhein & $R$ & NSE \\
\hline \multirow{2}{*}{ automatisch } & $\mathrm{h}$ & 6,893 & 6,439 \\
& Leakage & 6,960 & 6,339 \\
\hline
\end{tabular}

Bei dem Vergleich der Modellergebnisse mit Messwerten ist zu beachten, dass auch die Messwerte und die Übertragung der Daten Ungenauigkeiten und Unsicherheiten unterliegen. Auch die Ausdehnung der gemäß der Leakage-Modellvorstellung angenommenen Sedimentschicht ist nicht bekannt. Möglicherweise weist sie eine große Variabilität oder sogar Fehlstellen auf. Schließlich wurde die Leakage-Beziehung über eine ufernahe Messstelle ermittelt, die Messwerte beziehen sich also nicht auf den Rand der Sedimentschicht.

Bei der Übertragbarkeit der vorgestellten Ergebnisse auf andere Bereiche sind die örtlichen $\mathrm{Ge}$ gebenheiten zu berücksichtigen. Dazu zählen zum Beispiel Maßnahmen des Flussbaus. Buhnen, Leitwerke und die Art einer Uferbefestigung prägen die Gestalt des Ufers und die Durchlässigkeiten. Auf die Bedeutung der Situation im Flussbett und das Transportregime wurde bereits hingewiesen. Auch die Art, wie der Fluss in den angrenzenden Grundwasserleiter einschneidet, ist von großer Bedeutung. So wird unterirdisches Hochwasser ein Problem der großen Flüsse sein, die großflächig in den Grundwasserleiter einschneiden, hohe Schwankungen des Wasserstands haben und damit große Uferspeicherungseffekte bewirken. Bei kleineren Flüssen ist der Anteil des Zustroms im Vergleich zu anderen Quellen wie zum Beispiel der Grundwasserneubildung eher gering. Eine Rolle wird auch der angrenzenden Bebauung zukommen. Eine Hochwasserschutzwand mit unterirdischer Verbauwand, Kaimauern, Gebäude mit tiefliegenden Kellern und Infrastrukturbauwerke wie parallel zum Fluss laufende Tunnel (Rheinufertunnel in Köln) haben einen Einfluss auf die Strömungssituation im Grundwasser (Brombach et al. 2001) und sind im Modell entsprechend zu berücksichtigen. Ebenso ist die Wassernutzung in der Nähe des Ufers entscheidend: wird Uferfiltrat gewonnen, so liegt im Bereich der Wasserentnahme auch im Normalzustand eine Fließrichtung vom Fluss in den Grundwasserleiter vor, so dass dort eine 


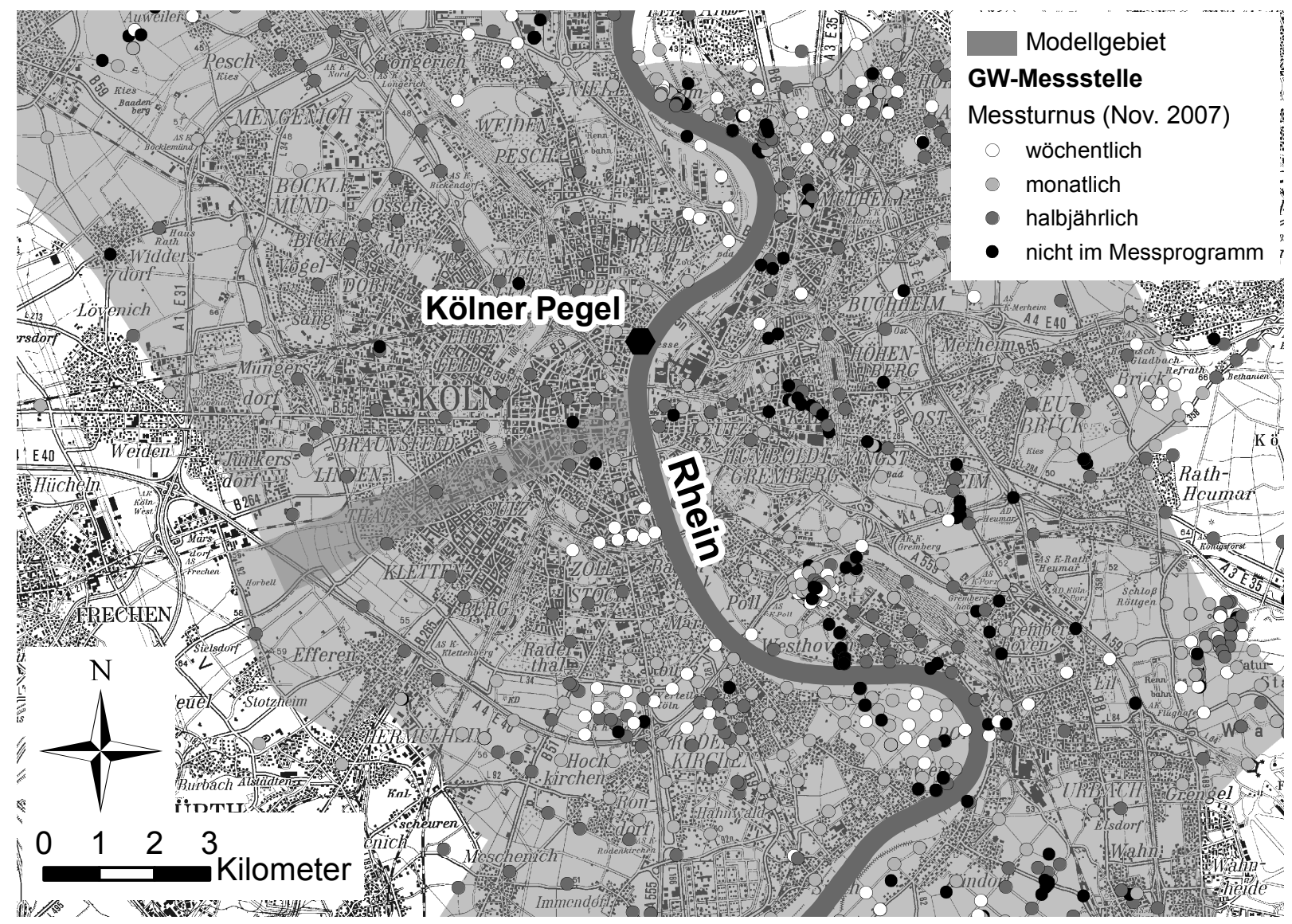

Abbildung 5.17: Messstellen im Stadtgebiet von Köln, die vom örtlichen Wasserversorger betrieben werden, und der Turnus der Messwerterfassung zum Stand November 2007 (Datengrundlage: Klatt 2007)

größere Kolmation im Uferbereich zu erwarten ist (Schubert 2002). Der Zufluss zum Grundwasser hängt zusätzlich auch von der Entnahmemenge und dem Ort der Entnahme ab (Eckert \& Irmscher 2006). Zur Grundwasserströmungssituation allgemein ist im vorliegenden Beispiel der Idealfall einer senkrecht zum Rhein verlaufenden Strömung angenommen worden. Bei Biegungen des Flusses und einem anderen geologischen Aufbau kann die Strömungssituation deutlich komplexer werden, so dass es schwieriger sein dürfte, aus Messungen Aussagen über die $\mathrm{Zu}-$ und Abströme zwischen Fluss und Grundwasserleiter abzuleiten. Eine Rolle spielen möglicherweise präferenzielle Fließwege, wie sie zum Beispiel aus Abwasserkanälen aus der Römerzeit (Meißner 2008) oder nicht mehr abflusswirksamen Altarmen eines Flusses (Tacke 1988) entstehen können. Im Zuge der Modellkalibrierung kann es helfen, gezielt verschiedene LeakageFunktionen auszuprobieren, wenn komplexe Austauschvorgänge erwartet werden. Ideal ist es natürlich, wenn dazu Feldmessungen zur Unterstützung vorliegen. In einem Stadtgebiet ist dazu oft ein dichtes Messstellennetz vorhanden, wie Abbildung 5.17 für das Beispiel der Stadt Köln zeigt. Einige Messstellen sind hier so angeordnet, dass Messprofile senkrecht zum Fluss zusammengestellt werden können. Der Flusswasserstand könnte in diesem Fall vom Kölner Pe- 
gel übertragen werden. Allerdings wäre es notwendig, die Häufigkeit der Messstellenablesung zu erhöhen, damit das unterirdische Hochwasser gut erfasst werden kann.

\subsection{Zusammenfassung des Kapitels}

Unter Uferspeicherung versteht man den Prozess der Speicherung von Wasser eines Fließgewässers im Bereich des Flussufers. Die Uferspeicherung entsteht dann, wenn der Wasserstand im Fluss und der Grundwasserstand im angrenzenden Grundwasserleiter nicht gleich sind. LeakageAnsätze drücken eine Beziehung zwischen dieser Druckhöhendifferenz $\Delta h$ und der Wassermenge $Q$, die pro Zeiteinheit zwischen dem Fließgewässer und dem Untergrund ausgetauscht werden, aus. Der Leakage-Funktion liegt die Modellvorstellung zu Grunde, dass zwischen dem Fluss und dem Grundwasserleiter eine halbdurchlässige Schicht vorhanden ist. Es gibt lineare und nichtlineare Leakage-Funktionen.

Für das Beispiel des Rheinhochwassers im April 1983 am Rhein im Neuwieder Becken wurde bei der Auswertung der von Ubell (1987a) und Ubell (1987b) publizierten Daten festgestellt, dass für den Vorgang der Infiltration bei gleicher Druckhöhendifferenz betragsmäßig deutlich größere Austauschmengen je Zeiteinheit erreicht werden als für die Exfiltration. Über die Dauer des Hochwasserereignisses ändert sich die $Q$ - $\Delta h$-Beziehung, so dass die Leakage-Funktion im vorliegenden Beispiel hysteretischer Natur ist. Ursachen dafür können eine Veränderung der Kontaktfläche, über die der Wasseraustausch zwischen Fluss und Grundwasserleiter stattfindet, sein oder eine zeitliche Veränderung der Durchlässigkeitseigenschaften durch Erosions- und Sedimentationsprozesse.

Sowohl mit einer h-Randbedingung als auch mit einer Leakage-Randbedingung ließ sich ein dreidimensionales Streifen-Grundwassermodell des Beispiels mit gutem Ergebnis kalibrieren. Bei der h-Randbedingung wird die Schicht, die Fluss und Grundwasserleiter trennt, direkt über Elemente im Modell abgebildet, während bei der Leakage-Randbedingung für jede Fließrichtung unterschiedliche Leakage-Parameter vorgegeben werden können. Mit letzterer Variante ließ sich zumindest für die Handkalibrierung ein besseres Kalibrierungsergebnis erreichen. Vergleiche mit den Ergebnissen einer automatischen Kalibrierung zeigen die Bedeutung der Modellvalidierung auf, denn insgesamt wurden vier verschiedene Modelle so kalibriert, dass sich eine gute Übereinstimmung zwischen Mess- und Rechenwerten ergab. Allerdings unterscheiden sich die Kalibrierungsergebnisse, also die Verteilung der Durchlässigkeitsbeiwerte beziehungsweise der Leakage-Parameter im Uferbereich, bei den Varianten deutlich. Die automatische Kalibrierung lieferte hohe Durchlässigkeiten beziehungsweise Leakage-Koeffizienten im Bereich der oberen Uferzone. Dieses Kalibrierungsergebnis deckt sich nicht mit den Informationen, die aus der Literatur für das natürliche Vorbild des Anwendungsbeispiels vorliegen und die bei der manuellen Kalibrierung umgesetzt wurden, es liefert jedoch einen hysteretischen Verlauf der LeakageFunktion, der dem aus Messwerten abgeleiteten Verlauf sehr ähnlich ist.

Auch mit einem zweidimensional-horizontalen Grundwassermodell wird das Beispiel gut repräsentiert. Der hysteretische Verlauf der Leakage-Funktion kann jedoch nur mit Einschränkung 
wiedergegeben werden, da eine vertikale Schichtung naturgemäß in einem zweidimensional-horizontalen Modell nicht abgebildet werden kann.

Bei der Übertragung der Erkenntnisse aus dem vorgestellten Beispiel muss auf die örtlichen Gegebenheiten und Strömungsverhältnisse Rücksicht genommen werden. Letzlich kommt es für die Prognose des unterirdischen Hochwassers darauf an, die Strömungssituation im Hinterland gut wiederzugeben. Das Beispiel zeigt, dass dazu auch die weit verbreiteten linearen LeakageBeziehungen verwendet werden können. Möglicherweise kann es aber für eine Modellkalibrierung hilfreich sein, auch die Eignung alternativer nichtlineare Ansätze zu prüfen. Nach Möglichkeit sollte dies mit Feldmessungen unterstützt werden. 


\section{Infiltration aus einer Überflutungsfläche}

\subsection{Einführung}

Im Zusammenhang mit Wechselwirkungen zwischen einem Oberflächengewässer und dem Grundwasserkörper wird mit dem Begriff Infiltration das Eindringen von Wasser in den unterirdischen Raum bezeichnet. Dabei ist die Bewegungsrichtung des Wassers nicht genau festgelegt. Während in Kapitel 5 die Infiltration als das Eindringen von Wasser in den Grundwasserleiter über das Ufer eines Flusses verstanden wurde, geht es in diesem Kapitel um die nach DIN 40493 (1994) zweite Art der Infiltration, die von Scheffer (2002) als „Bewegung des Sickerwassers von oben her in den Boden, wenn das Matrixpotential höher ist, als dem Gleichgewicht mit dem freien Grund- oder Stauwasserspiegel entspricht" definiert wird. Diese Infiltration ist eine Folge von Niederschlägen, Beregnung oder Überstauung (Abbildung 6.1).

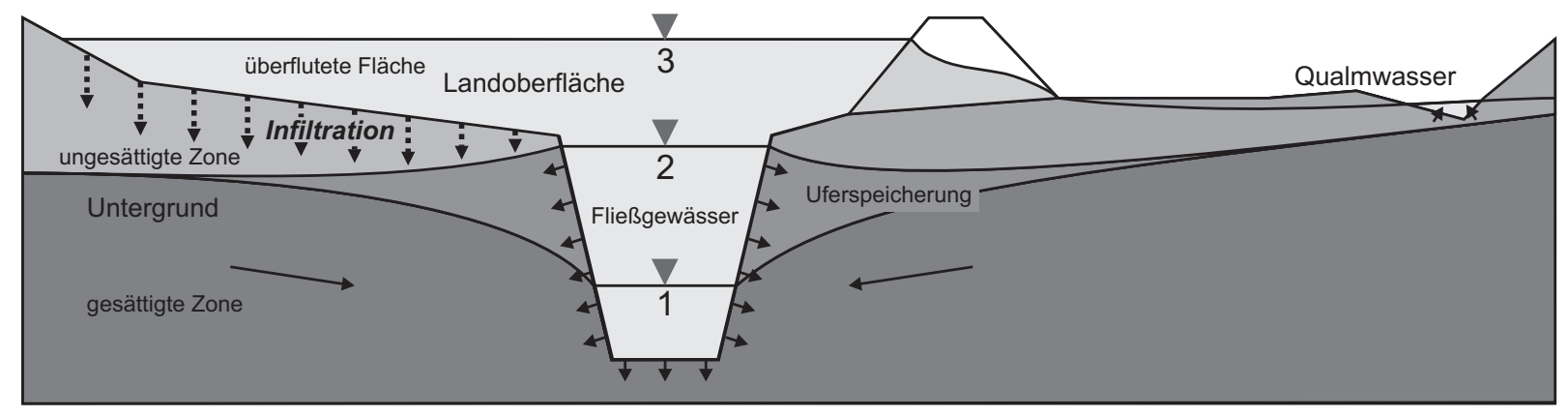

Abbildung 6.1: Einordnung der Infiltration aus einer überfluteten Fläche in die Prozesse der Wasserbewegung beim unterirdischen Hochwasser

Mathematische Ansätze zur Beschreibung der Infiltration werden verwendet, um zum Beispiel im Rahmen der Niederschlag-Abfluss-Modellierung zu erfassen, welcher Anteil des Niederschlags an der Oberfläche abfließt und welcher in den Untergrund versickert. Infiltration durch Niederschlag beeinflusst das unterirdische Hochwasser mittelbar. So erhöht vor einem Hochwasserereignis niedergehender Regen den Wassergehalt des Bodens und bestimmt damit die Anfangsbedingungen für die Ausbreitung des Wassers im Untergrund während des Hochwasserereignisses. Mit zunehmender Feuchte des Untergrundes sinkt seine Fähigkeit, Hochwasser aus dem Fluss aufzunehmen. Kommt es zu größerer Versickerung, so wird der Grundwasserspiegel ansteigen.

Während der Niederschlag nicht zu einer Sättigung des Bodens von oben her führen muss, dringt bei einer Überflutung eine Sättigungsfront vertikal in den Boden ein. Eine Überflutung entsteht, 
weil an der Oberfläche mehr Wasser zufließt, als in den Boden eindringt. Ein solcher Zufluss kann seine Ursache in sehr starken Niederschlägen oder seitlichen Zuflüssen haben. Seitliche Zuflüsse, die zu unterirdischem Hochwasser führen, entstehen dadurch, dass ein Fluss über die Ufer tritt oder Hochwasserschutzeinrichtungen versagen. Je nach Art des Versagens können hier besonders hohe Einstauhöhen auftreten. Auch eine Überlastung der Kanalisation kann eine Überflutung hervorrufen.

Für die Fragestellung des unterirdischen Hochwassers spielt die Infiltration des Wassers von einer überfluteten Fläche in den Grundwasserleiter eine wesentliche Rolle, denn das Wasser breitet sich an der überfluteten Landoberfläche deutlich schneller aus als im Untergrund. Von oben infiltrierendes Hochwasser aus einer Überflutungsfläche trägt somit zu einer Ausbreitung des unterirdischen Hochwassers im Hinterland bei.

Für den Prozess der Uferspeicherung bestimmt entsprechend der gängigen Modellvorstellung eine trennende Sedimentschicht den Wasseraustausch zwischen Fluss und unterirdischem Raum. Bei der Infiltration wäre eine solche Trennfläche die ungesättigte Bodenzone zwischen dem Grundwasserspiegel und der Landoberfläche, die mit Überflutungswasser eingestaut ist. Vor dem Hintergrund einer numerischen Modellierung stellt sich die Frage, ob diese ungesättigte Zone in einem Vorhersagewerkzeug berücksichtigt werden muss oder ob vereinfachte Ansätze über gängige Randbedingungen hier weiter helfen können. Dazu werden zunächst analytische Infiltrationsmodelle ausgewertet, um festzustellen, mit welchen Zeiträumen gerechnet werden muss, bis der Bereich unterhalb einer Überflutungsfläche gesättigt wird. Ausgewählte Infiltrationsformeln, die eine Einstauhöhe berücksichtigen, werden dazu im folgenden Abschnitt vorgestellt.

Anschließend werden verschiedene Möglichkeiten zur Modellierung des Wasseraustauschs zwischen einer Überflutungsfläche und dem Grundwasserleiter anhand numerischer Berechnungen diskutiert. Für eine gesättigte Modellierung stehen wieder mehrere Varianten zur Auswahl, die unterschiedliche Grade der Vereinfachung darstellen. Zum Vergleich werden Modellrechnungen auf Basis der Richards-Gleichung herangezogen, denn diese Gleichung beschreibt die Bewegung des Wassers in der ungesättigten Bodenzone und kann damit auch für den Infiltrationsprozess verwendet werden.

\subsection{Mathematische Modelle für die Infiltration unter Einstau}

\subsubsection{Einführung}

Die übliche Kenngröße für den Verlauf einer Infiltration ist die zeitlich veränderliche Infiltrationsrate $q$. Sie gibt die auf die Fläche bezogene Wassermenge an, die je Zeiteinheit versickert (Scheffer 2002). Eine ebenfalls gebräuchliche Größe ist die kumulative Infiltration $I$, die die insgesamt versickerte Wassermenge für einen Zeitpunkt nach Beginn der Infiltration wiedergibt.

Während für die Hydrologie der Oberfläche oft die Kenngrößen $q$ und $I$ im Vordergrund stehen, ist für die Grundwasserhydrologie auch von Bedeutung, wie sich das Wasser im Boden bewegt. 


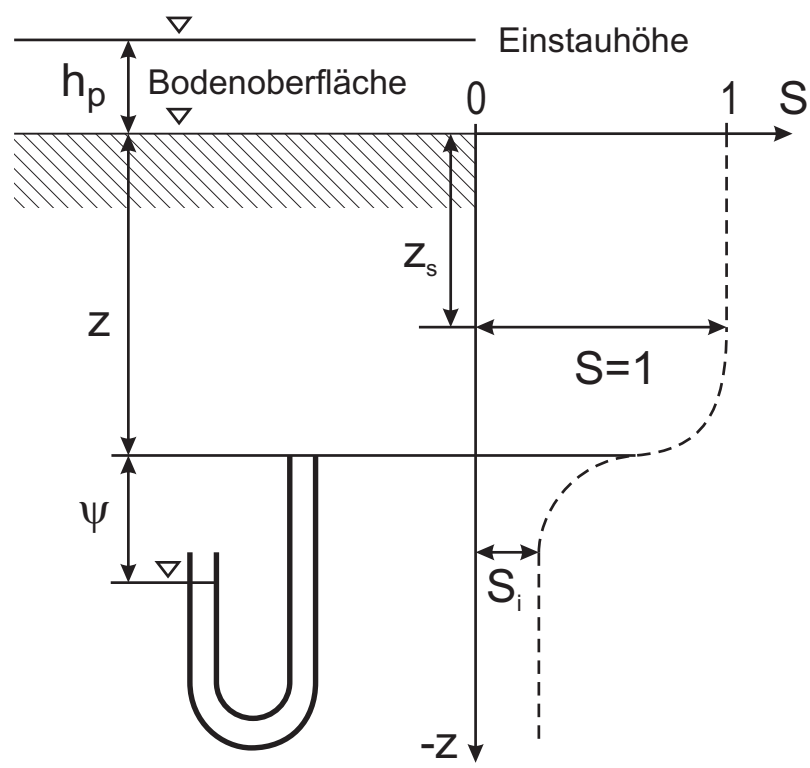

Abbildung 6.2: Exemplarisches Sättigungssprofil einer Bodenprobe nach Infiltration aus eingestauter Bodenoberfläche (nach Dracos 1980)

In Abbildung 6.2 ist das Sättigungsprofil eines Bodens nach einer Infiltration mit eingestauter Bodenoberfläche skizziert. Anders als in dem mit Abbildung 2.4 gezeigten Beispiel erreicht die Bodenprobe in diesem Fall an der Oberfläche die volle Wassersättigung $S=1$ durch die Einstauung. Für den speziellen Fall der eingestauten Bodenoberfläche kann daher der Infiltrationsverlauf mit der Lage der Sättigungsfront $z_{\mathrm{s}}$ beschrieben werden. Der Sättigungsfront voraus läuft ein teilgesättigter Bereich, in dem die Saugspannung $\psi$ wirkt. An dem von der Infiltration unbeeinflussten Bereich liegt der Anfangssättigungsgrad $S_{\mathrm{i}}$ vor.

\subsubsection{Die Richards-Gleichung}

Infiltration ist eine Bewegung des Wassers in teilgesättigten porösen Medien und kann deshalb mit der Richards-Gleichung (2.24) beschrieben werden. Bei entsprechenden Voraussetzungen wird ihre eindimensional-vertikale Komponente zur Beschreibung der Infiltration ausreichen:

$$
C(h) \frac{\partial h}{\partial t}=\frac{\partial}{\partial z}\left(k(h) \frac{\partial h}{\partial z}-1\right)
$$

Eine analytische Lösung der Richards-Gleichung ist nur für Sonderfälle möglich (Rawls et al. 1992). Eine numerische Lösung ermöglicht dagegen, auch komplexere Anfangs- und Randbedingungen zu erfassen. Ein Niederschlag der Intensität $i_{\mathrm{R}}$ an der Geländeoberkante $(z=0)$ lässt sich zum Beispiel durch

$$
k(h) \frac{\partial h}{\partial z}-1=-i_{\mathrm{R}}(t)
$$


ausdrücken, eine Überstauung der Höhe $h_{\mathrm{p}}$ geht über

$$
h=h_{\mathrm{p}}(t)
$$

in die Berechnung ein (Smith \& Woolhiser 1971; Rawls et al. 1992). Die Infiltrationsrate $q$ ergibt sich dann als sekundäres Modellergebnis. Gesättigte Bereiche $z_{\mathrm{s}}$ liegen dort vor, wo das Matrixpotenzial $\psi>0$ ist.

\subsubsection{Das Infiltrationsmodell von Green und Ampt}

Green \& Ampt (1911) entwickeln für die vertikale Infiltration in $z$-Richtung mit zeitlich konstanter Einstauung $h_{\mathrm{p}}$ folgendes mathematisches Modell:

$$
q=k \frac{z_{\mathrm{s}}+h_{\mathrm{p}}+C_{\mathrm{K}}}{z_{\mathrm{s}}} .
$$

Dem Green-Ampt-Modell liegt die Vorstellung einer scharfen Sättigungsfront an der Stelle $z_{\mathrm{s}}$ zu Grunde, die sich kolbenförmig im Boden bewegt. Einen teilgesättigten Übergangsbereich zwischen dem vom Infiltrationsvorgang unbeeinflussten Bereich und dem gesättigten Bereich, wie in Abbildung 6.2 angedeutet, kennt das Konzeptmodell nach Green \& Ampt nicht.

$C_{\mathrm{K}}$ ist der Kapillaritätskoeffizient (capillarity coefficient) des Bodens. Laut Morel-Seytoux \& Khanji (1974) drückt dieser Wert den Kapillardruck am Ort der Sättigungsfront $z_{\mathrm{s}}$ aus und wird als Druckhöhe angegeben. Einige Autoren nähern sich diesem Wert theoretisch über hydraulische Bodeneigenschaften (Bouwer 1966) oder der Verteilung der Poren (Rawls \& Brakensiek 1983). Haverkamp et al. (1988) zeigen die Zeitabhängigkeit des Kapillaritätskoeffizienten und halten eine physikalische oder statistische Interpretation aufgrund ihrer Analysen nicht für anwendbar.

Laut Bouwer (1966) ist in Gleichung 6.4 anstelle der hydraulischen Durchlässigkeit $k$ die Durchlässigkeit nach Wiederbefeuchtung $k_{\mathrm{r}}$ (resaturated hydraulic conductivity) mit einem Wert von etwa

$$
k_{\mathrm{r}}=0,5 k
$$

zu verwenden, um die Wirkung im Boden eingeschlossener Luft zu berücksichtigen (MorelSeytoux \& Khanji 1974).

Die kumulative Infiltration hängt mit $z_{\mathrm{s}}$ unter der Annahme einer scharfen Sättigungsfront über die Differenz der überall als gleich angenommenen Anfangsfeuchte und der Sättigungsfeuchte zusammen:

$$
I=z_{\mathrm{s}}\left(\theta_{\mathrm{s}}-\theta_{\mathrm{i}}\right)
$$




\subsubsection{Die Infiltrationsformeln von Haverkamp}

Haverkamp et al. (1990) stellen eindimensionale Infiltrationsformeln vor, die von der RichardsGleichung abgeleitet sind und ausschließlich auf zeitlich konstanten physikalischen Parametern beruhen. Infiltrationsrate und kumulative Infiltration werden dimensionslos angegeben, was eine leichte Umsetzung der Formeln in Programmcode ermöglicht.

Die dimensionslose kumulative Infiltration $I^{*}$ berechnet sich nach Barry et al. (1995) zu

$$
I^{*}=\frac{\gamma}{q^{*}-1}+(1-\gamma) \ln \left(1+\frac{1}{q^{*}-1}\right)
$$

aus der dimensionslosen Infiltrationsrate $q^{*}$. Diese ist implizit mit

$$
t^{*}=(1-2 \gamma) \ln \left(1+\frac{1}{q^{*}-1}\right)+\frac{\gamma}{q^{*}-1}-\frac{1-\gamma}{q^{*}} .
$$

gegeben. $t^{*}$ ist die dimensionslose Infiltrationszeit. Der Parameter $\gamma$ liegt zwischen 0 und 1 und fasst Bodeneigenschaften, Anfangs- und Randbedingungen zusammen:

$$
\gamma=\frac{2 k_{\mathrm{s}}\left(h_{\mathrm{p}}-\psi_{\mathrm{a}}\right) \Delta \theta}{S_{\mathrm{P}}^{2}+2 k_{\mathrm{s}} h_{\mathrm{p}} \Delta \theta} .
$$

$S_{\mathrm{P}}$ steht in dieser Gleichung für die Sorptivität. Die Sorptivität charakterisiert die Fähigkeit eines Bodens, Feuchtigkeit nur durch Kapillarkräfte zu absorbieren (Schmitz et al. 1989) und nimmt die Einheit $\mathrm{m} / \sqrt{\mathrm{s}}$ an. Es wird weiterhin die Differenz $\Delta \theta$ zwischen Anfangswassergehalt und Sättigungswassergehalt eingeführt:

$$
\Delta \theta=\theta_{\mathrm{s}}-\theta_{\mathrm{i}} .
$$

$\psi_{\mathrm{a}}$ steht in Gleichung 6.9 für die Saugspannung bei Lufteintritt (air entry pressure, siehe auch Abschnitt 2.3).

Die Zusammenhänge zwischen den dimensionslosen Größen $I^{*}, t^{*}$ und $q^{*}$ und den entsprechenden dimensionsbehafteten Größen $I, t$ und $q$ sind über die drei Gleichungen

$$
\begin{array}{r}
I^{*}=\left(I-k_{\mathrm{i}} t\right) \frac{2 \Delta k}{S_{\mathrm{P}}^{2}+2 k_{\mathrm{s}} h_{\mathrm{p}} \Delta \theta} \\
t^{*}=\frac{2 t \Delta k^{2}}{S_{\mathrm{P}}^{2}+2 k_{\mathrm{s}} h_{\mathrm{p}} \Delta \theta} \\
q^{*}=\frac{q-k_{\mathrm{i}}}{\Delta k}
\end{array}
$$

mit

$$
\Delta k=k_{\mathrm{s}}-k_{\mathrm{i}}
$$


gegeben (Barry et al. 1995). $k_{\mathrm{i}}$ ist eine Anfangsbedingung und bezeichnet den hydraulischen Durchlässigkeitsbeiwert $k$ zu Beginn des Infiltrationsvorgangs.

Ein wesentlicher Unterschied zum Infiltrationsmodell von Green \& Ampt ist die Berücksichtigung eines teilgesättigten Übergangsbereiches vor der Sättigungsfront. Die Lage der Sättigungsfront kann daher nicht mit Gleichung 6.6 bestimmt werden. Nach $S$ Schmitz et al. (1989) gilt aber folgende Gleichung:

$$
z_{\mathrm{s}}(t)=k_{\mathrm{s}} \frac{h_{\mathrm{p}}-\psi_{\mathrm{a}}}{q(t)-k_{\mathrm{s}}}
$$

\subsubsection{Zur Berechnung der Sorptivität}

Zur Berechnung der Sorptivität $S_{\mathrm{P}}$ geben Chong \& Green (1983) folgende Formel an:

$$
S_{\mathrm{P}}\left(\theta_{\mathrm{i}}\right)^{2}=-2\left(\theta_{\mathrm{s}}-\theta_{\mathrm{i}}\right) k_{\mathrm{s}} C_{\mathrm{K}} .
$$

$C_{\mathrm{K}}$ ist die Druckhöhe am Ort der Sättigungsfront aus dem Green-Ampt-Modell (Gleichung 6.4). Sinaba (2007) schätzt den Wert für $C_{\mathrm{K}}$ in Gleichung $6.16 \mathrm{mit}$

$$
C_{\mathrm{K}} \approx \psi_{\mathrm{i}}\left(\theta_{\mathrm{i}}\right)
$$

ab und erhält folgende Gleichung für die einem initialen Feuchtigkeitszustand eines Bodens zugeordnete Sorptivität:

$$
S_{\mathrm{P}}\left(\theta_{\mathrm{i}}\right)=\sqrt{\left(\theta_{\mathrm{s}}-\theta_{\mathrm{i}}\right) k_{\mathrm{s}}\left|\psi_{\mathrm{i}}\right|}
$$

In dieser Gleichung ist die von Bouwer (1966) vorgeschlagene Reduktion des Durchlässigkeitsbeiwertes nach Gleichung 6.5 berïcksichtigt.

\subsection{Auswertung des Haverkamp-Modells zur Frage des Fortschritts einer Sättigungsfront}

Zur Frage, wie schnell sich ein gesättigter Bereich unterhalb einer Überflutungsfläche ausbildet, wertet Sinaba (2007) das von Barry et al. (1995) beschriebene Haverkamp-Infiltrationsmodell (siehe Abschnitt 6.2.4) für zwei verschiedene Beispiel-Böden (Tabelle 6.1) und verschiedene Anfangsbedingungen und Überstauhöhen aus. Die Wahl fiel zu Gunsten dieses mathematischen Infiltrationsmodells, weil es ausschließlich auf physikalischen Parametern beruht. Zudem erfasst es auch den teilgesättigten Übergangsbereich, der der Sättigungsfront vorausläuft. Das Modell kann also ohne eine Kalibrierung an Felddaten angewendet werden und erreicht eine hohe Qualität der Prozessabbildung.

Die Sorptivität wurde mit Gleichung 6.18 berechnet, die Anfangsfeuchte $\theta_{\mathrm{i}}$ ist jeweils eine Festlegung, die Mualem-van-Genuchten-Bodenparameter stammen aus dem Rosetta-Programm 


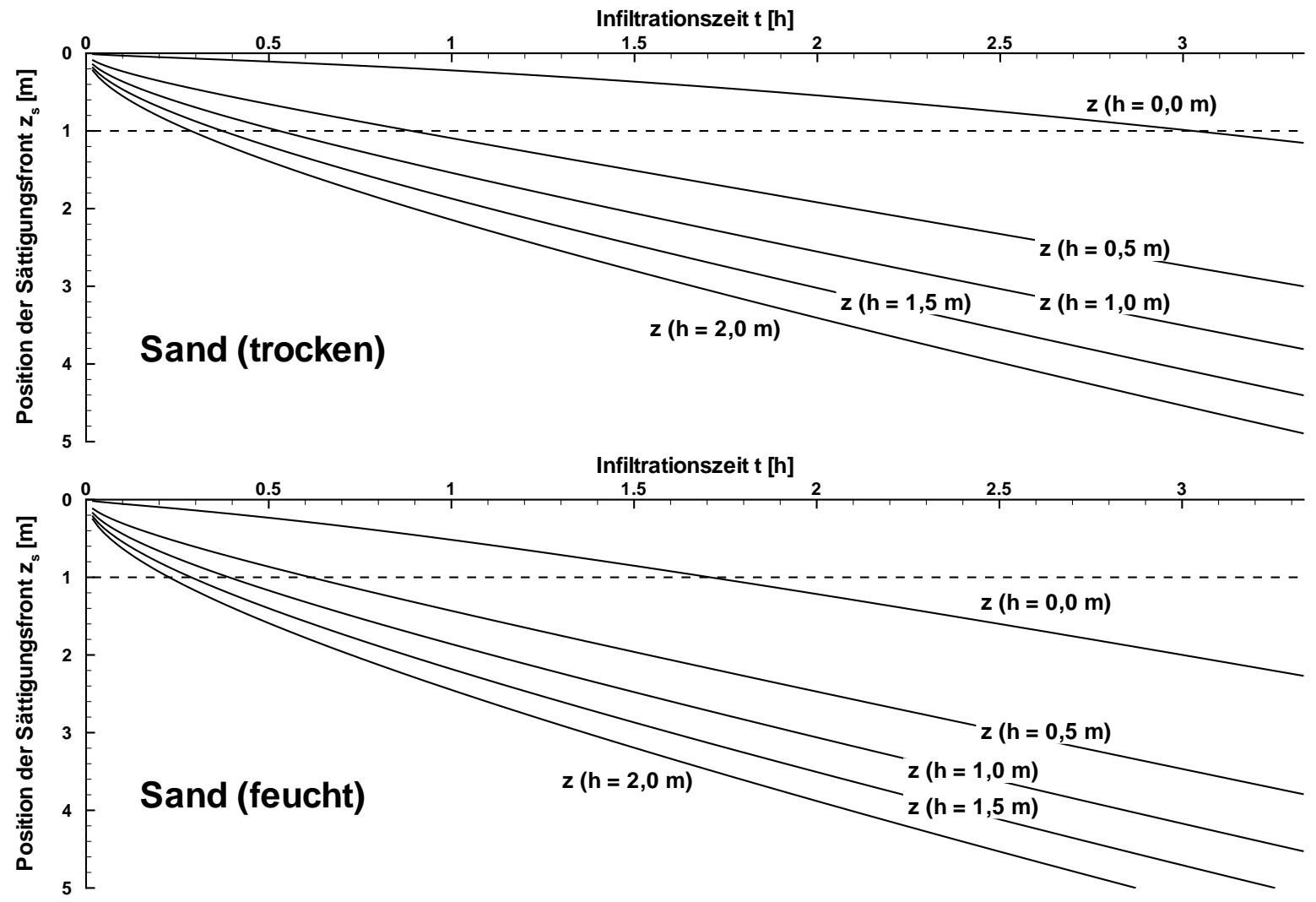

Abbildung 6.3: Fortschritt einer Sättigungsfront über die Infiltrationszeit für einen Sandboden (Bodeneigenschaften siehe Tabelle 6.1 bei zwei verschiedenen Anfangsbedingungen und jeweils unterschiedlichen Einstauhöhen (nach Sinaba 2007)

(Schaap et al. 2001). Die zeitabhängige Eindringtiefe der Sättigungsfront $z$ schließlich wird aus der Infiltrationsrate $q$ mit Gleichung 6.15 berechnet.

Abbildung 6.3 zeigt den Fortschritt einer Sättigungsfront über den Verlauf der Infiltrationszeit jeweils für anfänglich trockenen und feuchten Sand bei verschiedenen Einstauhöhen. Der Fall einer konstanten Einstauhöhe von 0 charakterisiert den Zustand, dass an der Oberfläche gerade so viel Wasser zufließt, wie in den Boden infiltrieren kann. Ein solcher Zustand kann bei einer geneigten Oberfläche und hohem Niederschlag vorliegen, wenn der Anteil des Niederschlags, der nicht in den Boden eindringt, an der Oberfläche abfließt. Für diesen Fall ohne Einstau ist ein nahezu linearer Verlauf der Kurve zu beobachten. Um einen Meter Boden von oben her zu sättigen, dauert es für trockenen Sand etwa drei Stunden, bei Sand mit Anfangsfeuchte nur 13/4 Stunden. Der Sättigungsvorgang spielt sich also in der Größenordnung von Stunden ab. Mit zunehmender Einstauhöhe steigt auch die Bewegungsgeschwindigkeit der Sättigungsfront. Allerdings zeigen die Diagramme auch, dass mit größerer Einstauhöhe der Einfluss der Anfangsbedingung zurückgeht. 


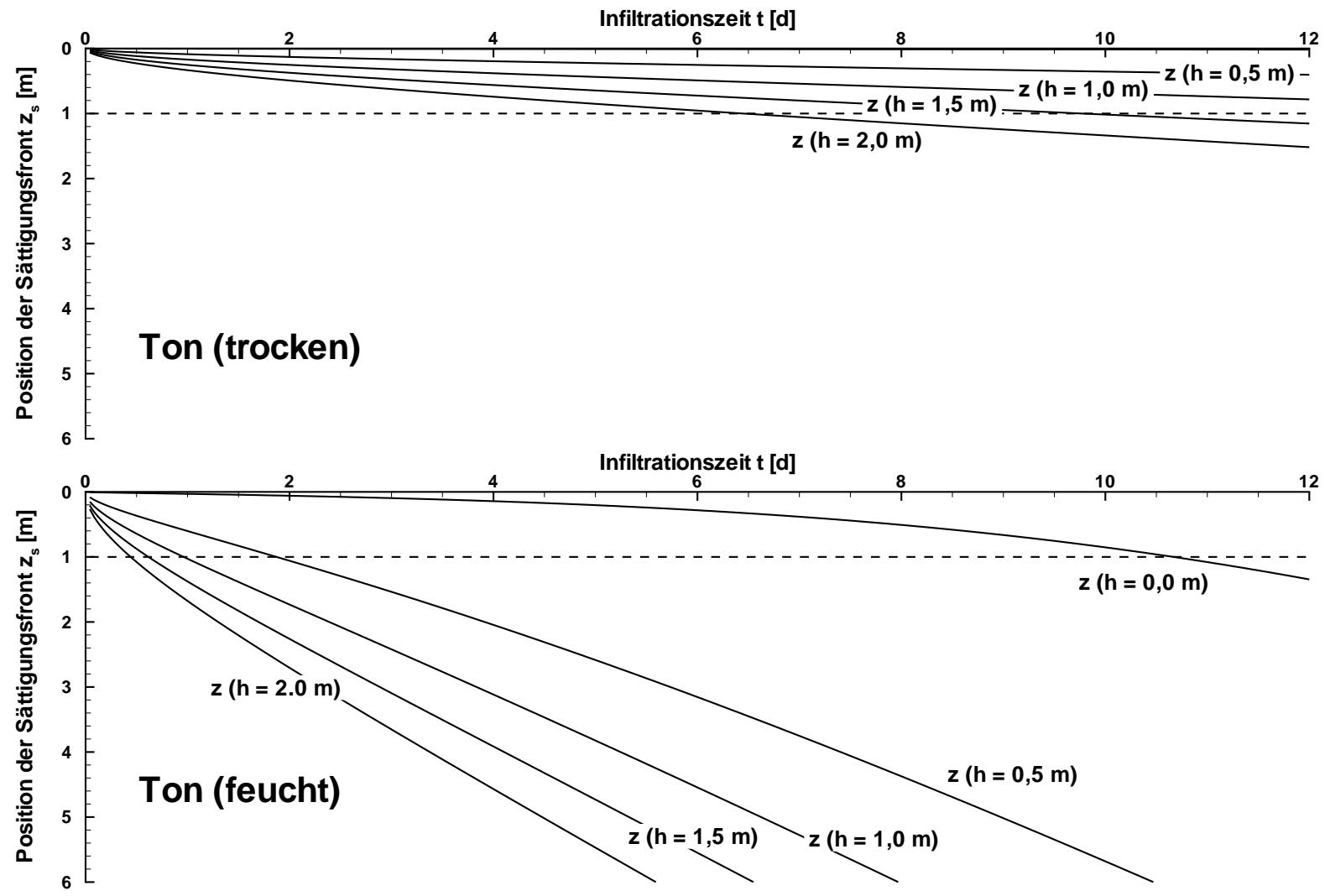

Abbildung 6.4: Fortschritt einer Sättigungsfront über die Infiltrationszeit für einen Tonboden (Bodeneigenschaften siehe Tabelle6.1) bei zwei verschiedenen Anfangsbedingungen und jeweils unterschiedlichen Einstauhöhen (nach Sinaba 2007)

Völlig andere Größenordungen ergeben sich für das andere Bodenbeispiel mit deutlich kleinerer Durchlässigkeit (Abbildung 6.4). Die Sättigung von einem Meter Boden kann hier mehrere Tage dauern, wenn der Boden eine Anfangsfeuchte aufweist. Große Einstauhöhen beschleunigen den Infiltrationsprozess bis zu Größenordnungen von mehreren Stunden. Für einen trockenen Ton ergeben die Modellrechnungen für einen Meter Sättigungstiefe eine Zeitspanne von etwa sechs Tagen bei einer besonders großen Einstauhöhe von zwei Metern. Mit abnehmender Überstauhöhe erhöht sich die Infiltrationsdauer.

Den zugehörigen Verlauf der Infiltrationsrate über die Zeit für verschiedene zeitlich konstante Einstauhöhen bei zwei unterschiedlichen Anfangszuständen für den Beispielboden Sand zeigt Abbildung 6.5. Zu Beginn ist der Boden nicht wassergesättigt, die Infiltrationsrate ist deshalb hoch. Sie konvergiert gegen die hydraulische Durchlässigkeit bei Sättigung $k_{\mathrm{s}}$. Für Sand, der anfänglich trocken ist, werden höhere Infiltrationsraten erreicht als für Sand, der vor Beginn des Infiltrationsprozesses feucht ist, weil ein feuchter Boden ein niedrigeres Matrixpotential aufweist als der gleiche Boden im trockenen Zustand. Damit entstehen kleinere Sorptivitätswerte und entsprechend kleinere Infiltrationsraten. Mit größerer Einstauhöhe nimmt die Infiltrationsrate generell zu, aber mit zunehmender Einstauhöhe geht ihr Einfluss auf die Infiltrationsrate zurück. 
Tabelle 6.1: Eigenschaften der Beispielböden und Anfangsbedingungen (nach Sinaba 2007)

\begin{tabular}{cccccc}
\hline & \multicolumn{3}{c}{ Sand } & \multicolumn{2}{c}{ Ton } \\
Parameter & Einheit & trocken & feucht & trocken & feucht \\
\hline$\theta_{\mathrm{s}}\left(n_{\mathrm{P}}\right)$ & - & 0,375 & 0,459 & \\
$\theta_{\mathrm{i}}$ & - & 0,059 & 0,085 & 0,200 & 0,300 \\
$S_{\mathrm{i}}$ & - & 0,157 & 0,226 & 0,436 & 0,653 \\
$\theta_{\mathrm{r}}$ & - & 0,053 & 0,098 \\
$S_{\mathrm{r}}$ & - & 0,141 & 0,214 \\
$k_{\mathrm{s}}$ & $\mathrm{m} / \mathrm{s}$ & $7,439 \cdot 10^{-5}$ & $1,708 \cdot 10^{-6}$ \\
$k_{\mathrm{i}}$ & $\mathrm{m} / \mathrm{s}$ & $1,163 \cdot 10^{-8} 1,364 \cdot 10^{-10}$ & $3,684 \cdot 10^{-13}$ & $1,152 \cdot 10^{-13}$ \\
$k_{0}$ & $\mathrm{~m} / \mathrm{s}$ & $2,836 \cdot 10^{-6}$ & $3,432 \cdot 10^{-7}$ \\
$\psi_{\mathrm{i}}$ & $\mathrm{m}$ & 1,7772 & 0,8115 & 98,3645 & 6.3162 \\
$\psi_{\mathrm{a}}$ & $\mathrm{m}$ & 0,0514 & 0,0219 \\
$S_{\mathrm{P}}$ & $\mathrm{m} / \mathrm{s}^{-0,5}$ & $6,4640 \cdot 10^{-3} 4,1840 \cdot 10^{-3}$ & $6,5965 \cdot 10^{-3}$ & $1,3097 \cdot 10^{-3}$ \\
$\alpha_{\mathrm{G}}$ & $1 / \mathrm{m}$ & 3,52 & \multicolumn{2}{c}{1,50} \\
$n_{\mathrm{G}}$ & - & 3,1767 & 1,2530 \\
$L_{\mathrm{G}}$ & - & $-0,930$ & $-1,561$ \\
\hline
\end{tabular}

Zwar bewirkt eine große Einstauhöhe einen hohen Druckgradienten an der Oberfläche, aber gleichzeitig reduziert das infiltrierende Wasser das Matrixpotential.

Anders als bei Sand verändern sich die Infiltrationsraten für den anfänglich feuchten BeispielTon nur marginal, für anfänglich trockenen Ton lassen sich die Infiltrationskurven für die unterschiedlichen Einstauhöhen kaum unterscheiden (Sinaba 2007). Deshalb wird an dieser Stelle auf eine Darstellung verzichtet. Obwohl Ton wesentlich höhere Saugspannungen als Sandboden erreicht, sind die berechneten Infiltrationsraten generell kleiner, weil ein höherer Feuchtigkeitsgehalt und eine geringere hydraulische Durchlässigkeit die hohen Saugspannungswerte kompensieren (Sinaba 2007).

\subsection{Möglichkeiten zur Abbildung der Infiltration unter Einstau in numerischen Modellen}

\subsubsection{Einführung}

Wenn die Sättigung des Bodens unter einer Überflutungsfläche insgesamt im Zeitraum von Stunden vonstatten geht, wie im vorangegangenen Abschnitt 6.3 für den Fall des Sandbodens gezeigt, so könnte in einem numerischen Modell sicherlich die Vereinfachung getroffen werden, dass sich unterhalb der Überflutungsfläche der Bereich im Zustand der Sättigung befindet, da die Zeit, die zur Sättigung benötigt wird, im Vergleich zur Dauer eines Hochwasserereignisses eher 

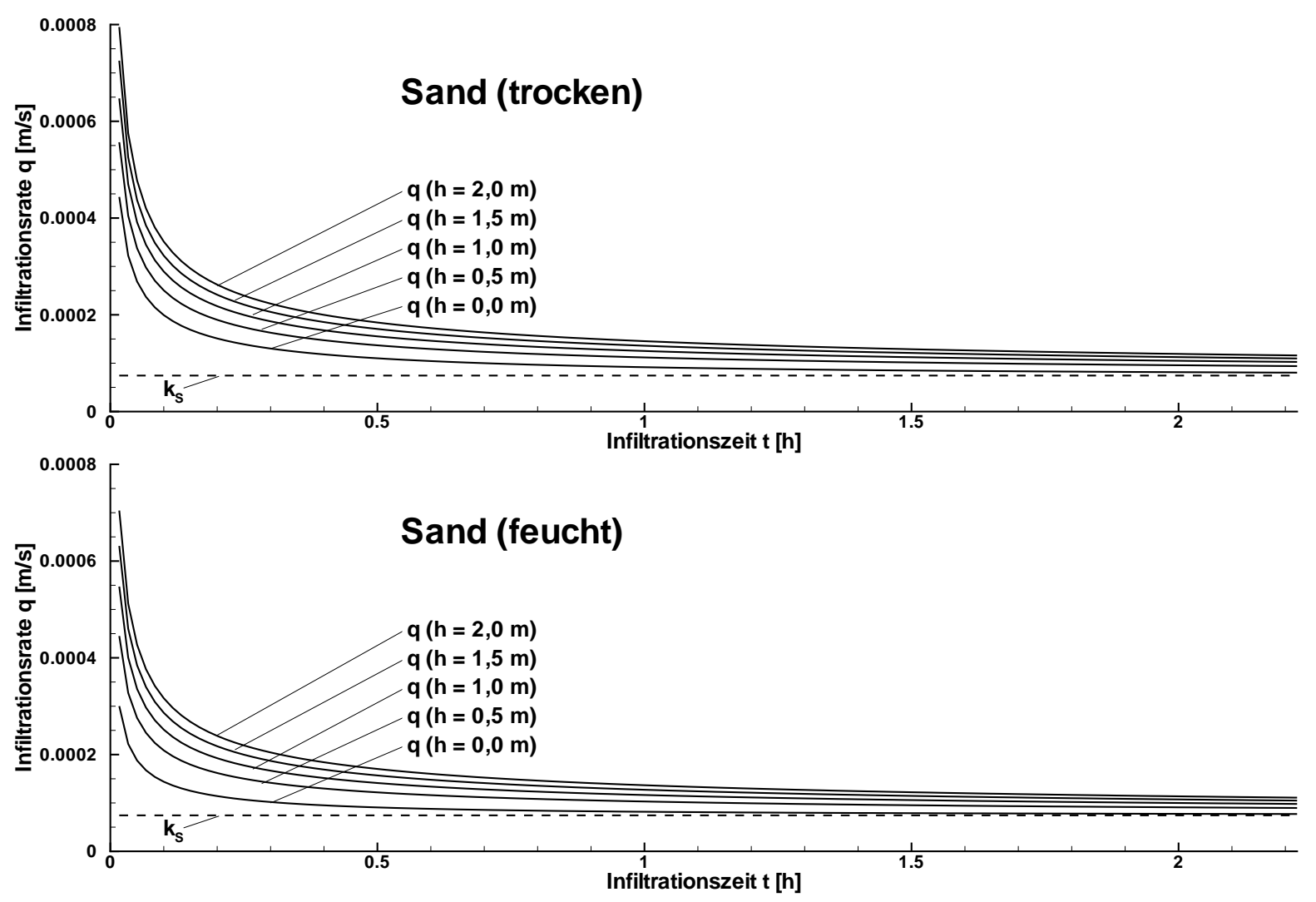

Abbildung 6.5: Verlauf der Infiltrationsrate über die Infiltrationszeit für einen Sandboden bei verschiedenen Anfangsbedingungen und jeweils unterschiedlichen Einstauhöhen (nach Sinaba 2007)

kurz ist. Oft ist der Grundwasserleiter in der Nachbarschaft eines großen Flusses aber durch eine Auelehm-Deckschicht abgeschlossen. Die Auswertung des Haverkamp-Infiltrationsmodells für Tonboden, der sicherlich die untere Grenze der Durchlässigkeit üblicher Deckschichten darstellt, zeigt jedoch, dass in diesem Fall die Annahme einer schnellen Sättigung des Bodens unterhalb der überfluteten Fläche nicht gerechtfertigt ist.

In diesem Abschnitt soll daher der Frage nachgegangen werden, wie sich der Prozess der Infiltration in einem Grundwassermodell geeignet abbilden lässt. Es kommt für die Fragen des unterirdischen Hochwassers besonders darauf an, zu wissen, wie sich der Grundwasserspiegel infolge einer Infiltration verhält. Diese Frage lässt sich mit den im vorangegangenen Abschnitt vorgestellten mathematischen Infiltrationsformeln von Green \& Ampt und Haverkamp nicht beantworten. Es wird deshalb auf die numerische Lösung der Richards-Gleichung mit Feflow zurückgegriffen. Zunächst wird anhand einfacher Beispiele eine nähere Beschreibung des Infiltrationsprozesses geliefert.

Die Lösung der Richards-Gleichung erfordert im Allgemeinen eine sehr feine räumliche Auflösung und bringt deshalb hohe Rechenzeiten mit sich (Forkel 2004; Trübger 2006). Darüber hinaus ist die Datenbeschaffung für die Bodenparameter, Anfangsbedingungen, Kalibrierungs- 
und Validierungsdaten recht aufwändig. Deshalb werden anschließend Berechnungen durchgeführt, in denen die Infiltration auf verschiedene Art ohne Berücksichtigung der ungesättigten Zone modelliert wird. Der Vergleich der Ergebnisse von Berechnungen mit und ohne ungesättigter Zone vermittelt ein Gefühl dafür, wie sich eine Vernachlässigung der ungesättigten Zone auf die Berechnungsergebnisse auswirken wird.

\subsubsection{Ungesättigte Modellierung des Infiltrationsvorgangs mit Lösung der Richards-Gleichung}

Die Anwendung einer numerischer Lösung der Richards-Gleichung für Fragen der Infiltration in den Boden ist allgemein anerkannt, stellt aber dennoch eine Vereinfachung der Realität dar. So gilt die Richards-Gleichung unter der Annahme, dass die Bodenluft ungehindert entweichen kann. Solche Bedingungen sind in der Realität nicht zwangsläufig gegeben. Nach Al Masri \& Sharif (1985) trifft die Annahme für Regen eher zu als für den Fall der Überstauung, um den es hier geht. Auch zeigen Al Masri \& Sharif (1985), dass unterschiedlicher Bewuchs einen Einfluss auf den Infiltrationsvorgang haben kann. Weitere Einschränkungen ergeben sich durch Makroporen aus Bodenflora und -fauna, chemische und biologische Verwitterungsprozesse und Inhomogenitäten der Bodenparameter (Beven \& Germann 1982; Demuth \& Hiltpold 1993), die in der Realität oft vorhanden sind, in einem numerischen Modell nicht oder nur schwer erfasst werden können. Zudem ist der Hystereseeffekt bei wechselnder Feuchtigkeit (Abschnitt 2.3) in den folgenden Untersuchungen nicht angesetzt worden. Dennoch werden die numerischen Lösungen der Richards-Gleichung aus dem Programm Feflow hier als Referenz verwendet, mit denen die unter vereinfachten Annahmen berechneten Ergebnisse verglichen werden.

Für eine Infiltration unter Oberflächeneinstau ist nach Abschnitt 6.2.2 (Gleichung 6.2) eine hRandbedingung zu setzen. Ihr Wert stellt den Wasserstand auf der Oberfläche $h_{\mathrm{p}}$ dar. Smith \& Woolhiser (1971), Akan \& Yen (1981) und Singh \& Bhallamudi (1998) modellieren den Infiltrationsprozess in ihren gekoppelten Modellen auf diese Weise.

Sättigungsprofile, die sich aus dem Ansatz einer h-Randbedingung mit dem Wert von 0,5 m für zwei verschiedene Anfangsbedingungen einer zweidimensional-vertikalen Bodensäule aus dem Beispielboden „Sand“ (Tabelle 6.1) ergeben, zeigt Abbildung 6.6 für verschiedene Zeitpunkte. Das Einsickern des Infiltrationswassers von oben her in den Boden ist hier gut zu erkennen, ebenso die Übergangszone, die sich zwischen dem gesättigten und dem von der Infiltration unbeeinflussten Bereich ausbildet. Abbildung 6.6a zeigt den Fall der homogenen Anfangsbedingung, bei dem an jeder Stelle der Bodenprobe zu Beginn die gleiche Sättigung vorliegt. Die Sättigungskurven aus Abbildung 6.6b gehören zu einer Bodensäule, bei der an der Unterkante auf Höhe -2 m der Grundwasserspiegel ansteht und sich ein Kapillarsaum ausgebildet hat. Für diesen Fall ist auch der Ausschnitt aus dem Berechnungsnetz (Abbildung 6.6c) dargestellt. Nach etwa 3700 s trifft die Sättigungsfront des infiltrierenden Wassers auf den Grundwasserspiegel. Einige Sekunden später ist die gesamte Probe gesättigt. 


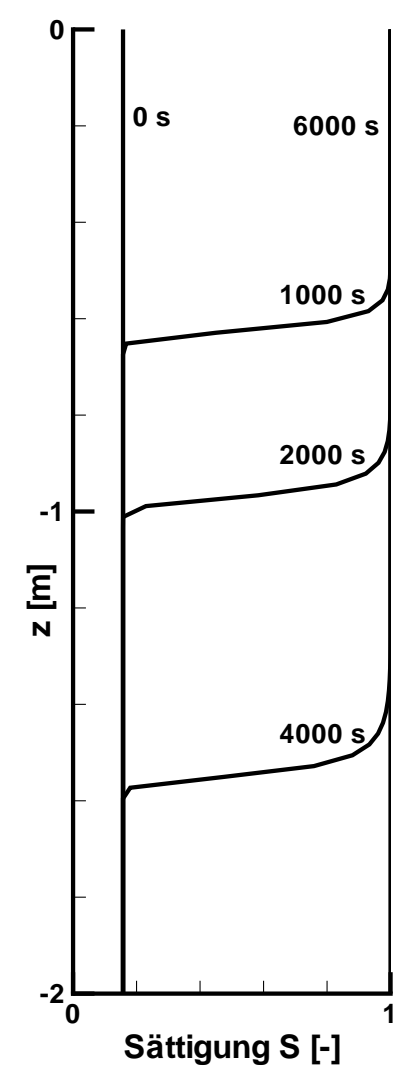

(a) Sättigungsprofil für homogene Anfangsbedingungen

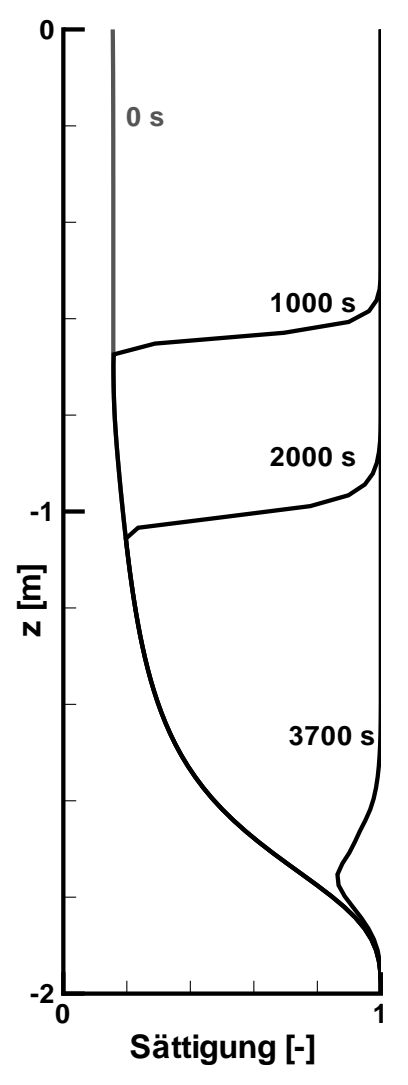

(b) Sättigungsprofil für Anfangsbedingung mit Kapillarsaum

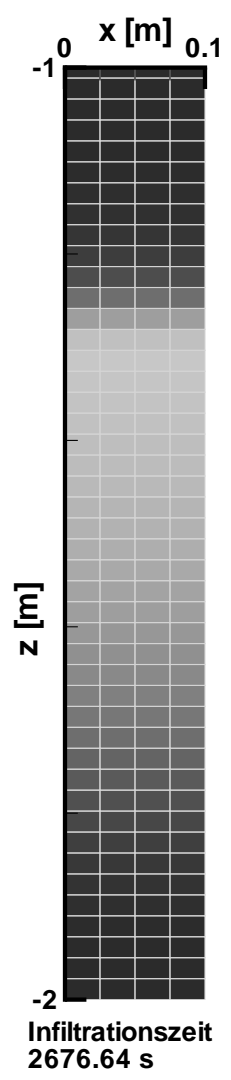

(c) Ausschnitt aus dem Berechnungsnetz

Abbildung 6.6: Berechnete Sättigungsprofile eines Sandbodens (Bodenparameter: Tabelle 6.1) bei konstantem Einstau von 0,5 m für zwei unterschiedliche Anfangsbedingungen zu jeweils unterschiedlichen Infiltrationszeitpunkten sowie das verwendete Berechnungsnetz. Die Schattierung des Berechnungsnetzes entspricht der Bodenfeuchte zur angegebenen Infiltrationszeit (schwarz: vollständig gesättigt, weiß: ungesättigt).

Die zu der an der Oberkante der Bodensäule angesetzten h-Randbedingung korrespondierenden Infiltrationskurven sind in Abbildung 6.7 zusammengefasst. Die Infiltrationskurve sinkt dann auf den Wert Null ab, wenn die Bodenprobe vollständig mit Wasser gefüllt ist. Dies wird beim Beispiel mit Kapillarsaum schneller erreicht, da hier zu Beginn des Infiltrationsvorgangs mehr Wasser im Boden gespeichert ist als im Fall der homogenen Anfangsbedingung. Mit zunehmender Infiltrationszeit liegt die Infiltrationsmenge für diesen Fall deshalb auch leicht unter der Infiltrationskurve für homogene Anfangsbedingungen. Der Grundwasserstand bleibt zu Beginn des Infiltrationsvorgangs unverändert und steigt nach etwas mehr als einer Stunde sprunghaft an, wenn die Sättigungsfront den Grundwasserspiegel erreicht. 


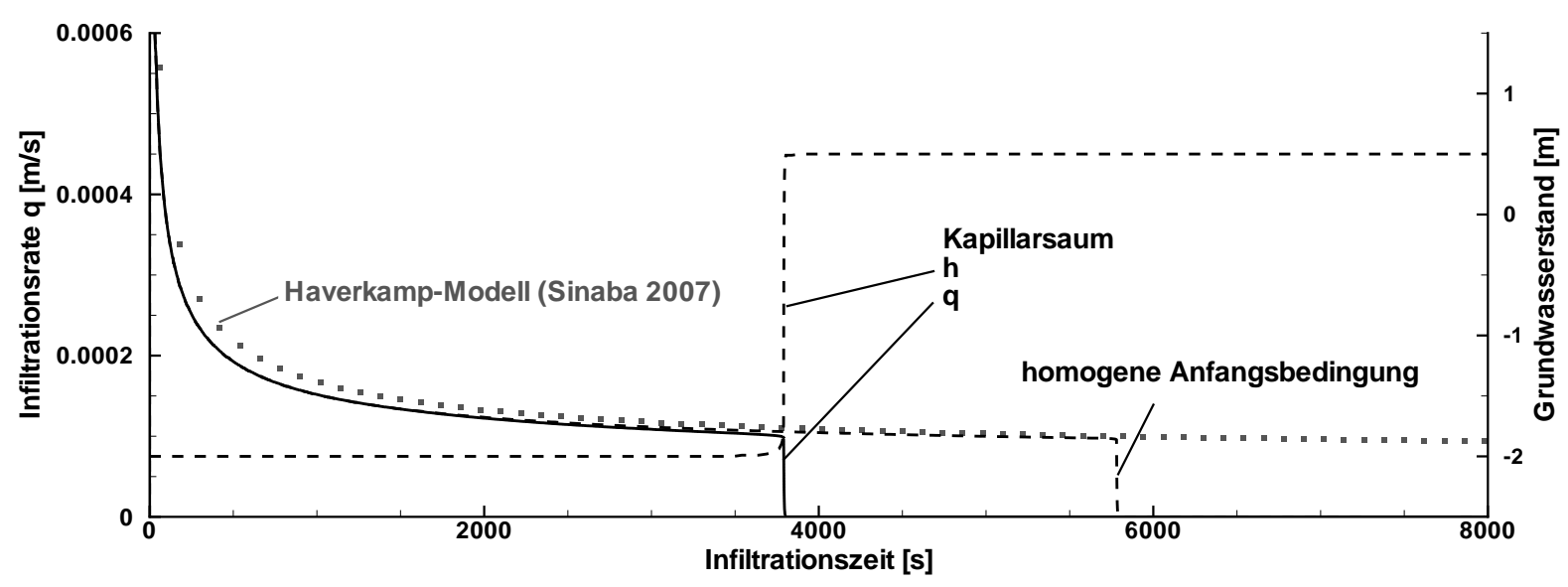

Abbildung 6.7: Infiltrationsraten ungesättigter Strömungsberechnungen mit Feflow für die Bodensäule mit homogenen Anfangsbedingungen und mit Kapillarsaum (Abbildung 6.6, Entwicklung des Grundwasserstands für das Beispiel mit Kapillarsaum sowie Infiltrationsraten des Haverkamp-Modells (Sinaba 2007)

Zum Vergleich ist die entsprechende Infiltrationskurve der Berechnung mit dem HaverkampModell aus Abbildung 6.5 (Sinaba 2007) in das Diagramm eingetragen. Hier wird wegen der Annahme eines nach unten unbegrenzten Raumes niemals eine vollständige Füllung der Bodenprobe erreicht, so dass die Infiltrationskurve auch nicht abknickt. Die Kurve des Wasserstands kann für das Haverkamp-Modell nicht angegeben werden, da die Vorstellung eines nach unten unendlich ausgedehnten Halbraums das Auftreffen der Sättigungsfront auf einen Grundwasserspiegel nicht hergibt. Entsprechendes gilt für das Beispiel mit einer homogenen Anfangsbedingung.

Der trotz Ansatz der gleichen Bodeneigenschaften, Anfangsbedingungen und Randbedingungen in vorhandene Unterschied zwischen den Feflow-Berechnungsergebnissen und der Auswertung des Haverkamp-Modells wird auf die Sorptivität zurückgeführt, die als physikalische Bodenkonstante des Haverkamp-Modells nicht in der Richards-Gleichung, die der Feflow-Lösung zu Grunde liegt, auftaucht. Darüber hinaus unterscheidet sich das von Sinaba verwendete Mualemvan-Genuchten-Modell (Gleichung 2.28) von der in Feflow implementierten Form (Gleichung 2.33) durch die Parameter $L_{\mathrm{G}}$ und $k_{0}$.

So wie ein Fluss und ein Grundwasserleiter durch eine Sedimentschicht getrennt sein können, kann zwischen einer Überflutungsfläche und dem Grundwasserleiter eine weniger durchlässige Schicht liegen. Diese Schicht kann aus natürlichem Auelehm bestehen, aber auch eine künstliche Versiegelung kann vergleichbare Effekte hervorrufen. Eine Infiltration durch eine Deckschicht, die mit den Bodeneigenschaften des Beispielbodens Ton (Tabelle 6.1) im Modellprofil umgesetzt wurde, ist in Abbildung 6.8a gezeigt. Nach 4,5 Stunden ist Wasser durch die nunmehr vollständig gesättigte und etwa 0,2 m dicke Tonschicht in den Sandboden eingedrungen. Anders als in dem Beispiel ohne Deckschicht wird im Sand hier die vollständige Sättigung durch das infiltrierende Wasser nicht erreicht. Der Ton hemmt die vertikale Wasserbewegung, so dass die Infiltrationsrate 


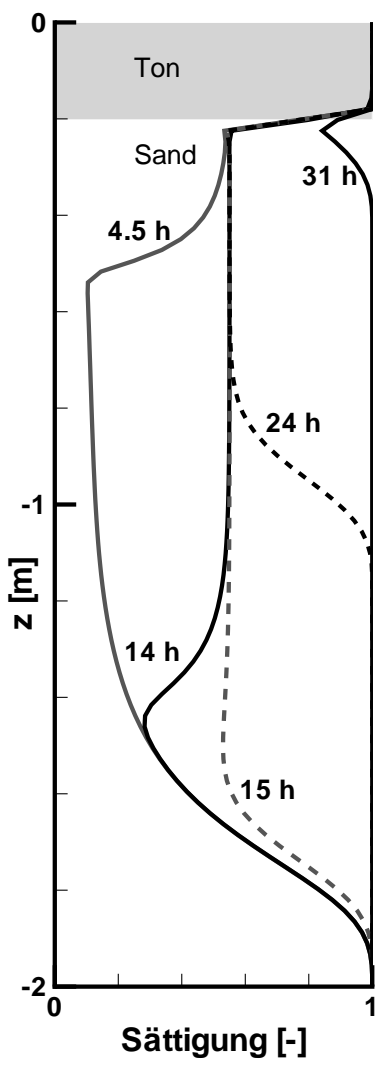

(a) Ton-Deckschicht mit hRandbedingung

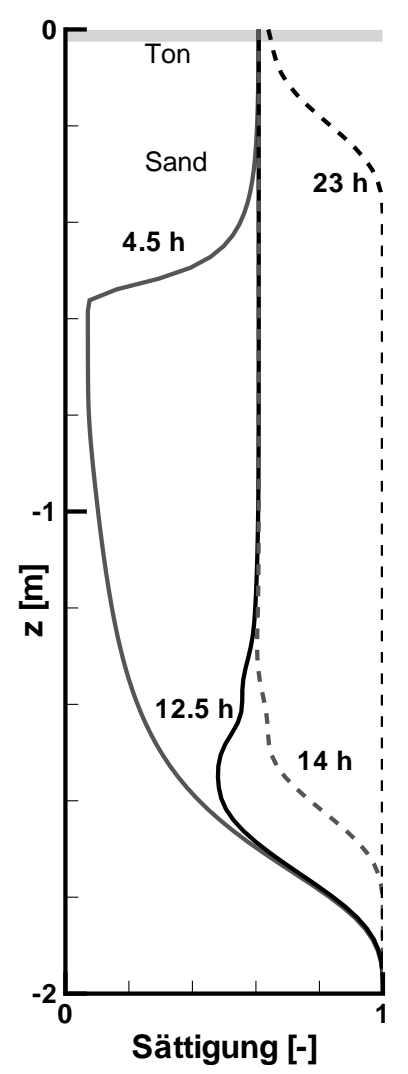

(b) Leakage-Randbedingung

Abbildung 6.8: Sättigungsprofile für eine Infiltration unter Einstau in eine Sand-Bodensäule mit Deckschicht mit verschiedenen Randbedingungsarten modelliert

(Abbildung 6.9a) hier deutlich niedriger ist als im Beispiel ohne Deckschicht (Abbildung 6.7). Erst, wenn das Infiltrationswasser so weit durch den Boden gesickert ist, dass es den Grundwasserspiegel erreicht, steigt dieser über die Zeit fast linear an, da die Infiltrationsmenge, die die Tonschicht bis zum Sand-Grundwasserleiter durchlässt, sich über die Zeit kaum ändert. Die durch die Deckschicht verursachte Verzögerung des Grundwasseranstiegs beträgt in diesem Beispiel etwa einen halben Tag. Dieser Vorgang ähnelt dem Prozess der Grundwasserneubildung, bei der Wasser infolge Niederschlags von oben in den Untergrund eindringt, ohne den Boden von oben her zu sättigen.

Mit Blick auf Kapitel 5 liegt es nahe, einen Leakage-Ansatz für die Modellierung des Wasseraustauschs zu verwenden und das Modell dahingehend zu vereinfachen, dass auf eine direkte Abbildung der Deckschicht verzichtet wird. Panday \& Huyakorn (2004) und Gunduz \& Aral (2005) setzen Leakage-Ansätze für den Austausch zwischen Wasser auf der Landoberfläche und dem teilgesättigt modellierten Untergrund an. Die Infiltrationsmenge pro Zeiteinheit bestimmt 


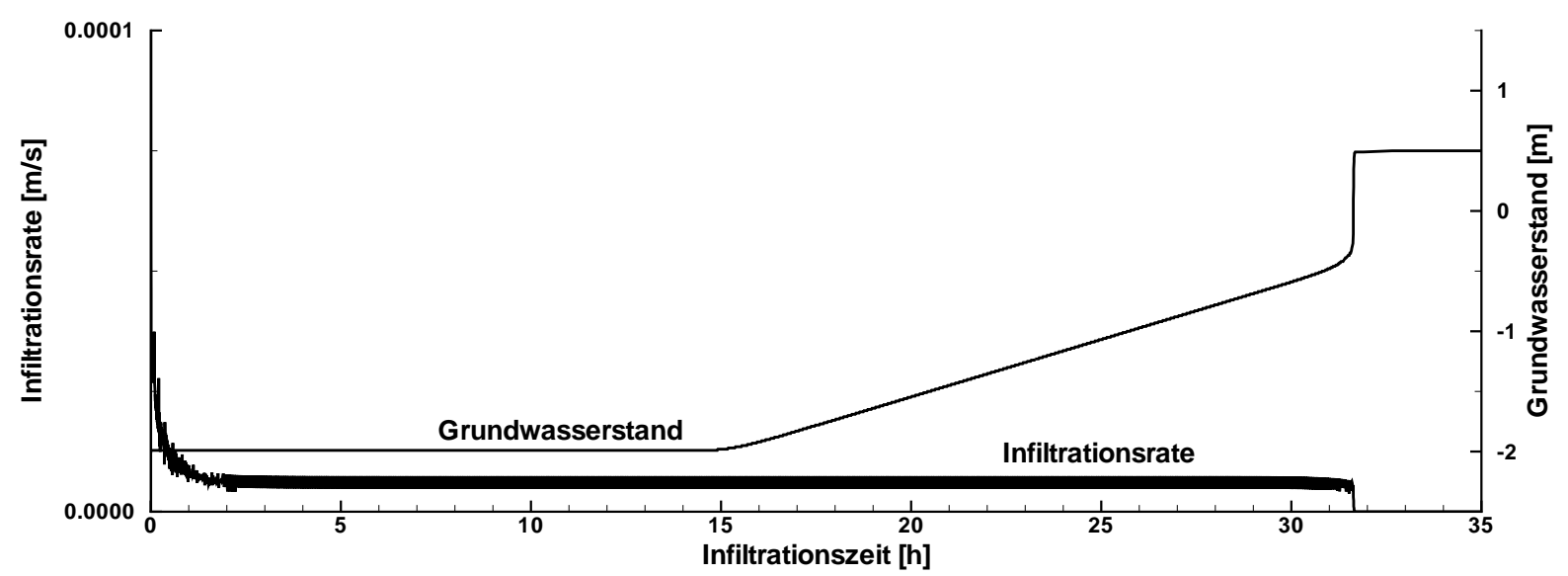

(a) Ton-Deckschicht als Elemente und h-Randbedingung

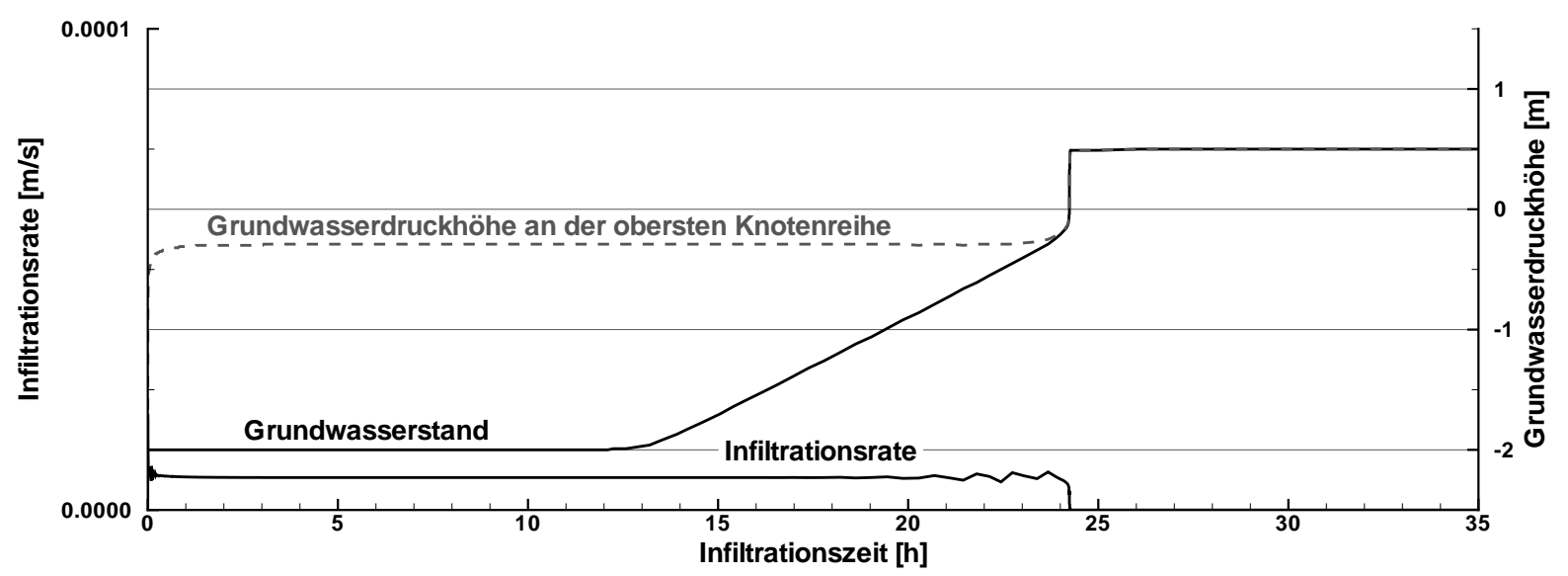

(b) Leakage-Randbedingung

Abbildung 6.9: Infiltrationskurven und Entwicklung des Grundwasserstands für einen Infiltrationsvorgang in einem Sandboden mit Deckschicht auf zwei verschiedene Arten modelliert

sich dann nach Gleichung 5.2 mit der Druckhöhendifferenz nach Gleichung 5.1, wobei der Randbedingungswert die Einstauhöhe ist:

$$
h_{\mathrm{F}}=h_{\mathrm{p}}
$$

Die Grundwasserdruckhöhe $h_{\mathrm{G}}$ ist zunächst unbekannt, bei ungesättigten Verhältnissen enthält sie einen negativen Beitrag aus dem Matrixpotenzial $\psi$.

Setzt man für die Berechnung des Leakage-Koeffizienten in Gleichung 5.3 eine Schichtdicke von 0,2 $\mathrm{m}$ und den Durchlässigkeitsbeiwert des Tons aus Tabelle 6.1 ein, also einen LeakageKoeffizienten, der der Tonschicht aus Abbildung 6.8a entspricht, so entwickelt sich das Sättigungsprofil durchaus ähnlich (Abbildung 6.8b). Allerdings läuft der Infiltrationsprozess schneller $\mathrm{ab}$, entsprechend steigt der Grundwasserstand, nachdem die Sickerwasserfront ihn erreicht hat, auch schneller an (Abbildung 6.9). Auch der Kurvenverlauf der Infiltrationsrate ist bei der 
Leakage-Variante (Abbildung 6.9b) ähnlich wie bei der Variante mit direkter Modellierung der Deckschicht und h-Randbedingung (Abbildung 6.9a). Allerdings liegt die Infiltrationsrate zu Beginn des Infiltrationsvorgangs bei der Variante mit Leakage-Randbedingung deutlich niedriger. Der Grund dafür ist, dass die Strömung durch die Deckschicht mit der Leakage-Randbedingung nicht als ungesättigte, sondern als gesättigte Strömung behandelt wird. Für die Sättigung der Tonschicht zu Beginn des Infiltrationsvorgangs wird deshalb kein Infiltrationswasser verbraucht.

Die Kurven der Infiltrationsraten weisen Schwingungen auf. Da es sich bei der Infiltrationsrate um Ergebnisse einer nachgeordneten Berechnung auf Basis der numerischen Näherungslösung der Richards-Gleichung handelt, werden für die Schwingungen numerische Gründe verantwortlich gemacht. Sie liegen innerhalb einer begrenzten Bandbreite (Abbildungen 6.9a und 6.9b) oder treten zusätzlich nur temporär auf (Abbildung 6.9b). Da sie keine Stabilitätsprobleme auslösen, werden sie als nicht problematisch eingestuft und ihrer Entstehung wird nicht weiter nachgegangen.

Die Infiltrationsrate, die sich für den Zeitraum bis zur vollständigen Sättigung der Probe quasistationär einstellt, liegt bei der Variante mit Leakage-Randbedingung geringfügig über der Infiltrationsrate aus der Variante mit h-Randbedingung. Dies liegt daran, dass das negative Matrixpotenzial für die oberste Knotenlage im vorliegenden Beispiel ein negatives Druckpotenzial liefert (Abbildung 6.9b). Für den Leakage-Fluss wird diese Grundwasserdruckhöhe bei der Berechnung der Wasserstandsdifferenz angesetzt und liefert damit eine entsprechend große Leakage-Menge. Das Matrixpotenzial des Sandes beeinflusst damit die Infiltration durch die Tonschicht. Tatsächlich hat der Sand unterhalb der Deckschicht unmittelbar keinen Einfluss auf die Infiltration durch die Deckschicht.

Sykes et al. (2006) und VanderKwaak (1999) berechnen die Leakage-Menge, die durch die Deckschicht fließt, deshalb unabhängig von den Verhältnissen im Grundwasserleiter, indem

$$
h_{\mathrm{G}}=z
$$

wird, also die geodätische Höhe der Geländeoberkante anstelle der Grundwasserdruckhöhe angesetzt wird.

\subsubsection{Vereinfachung durch gesättigte Modellierung der Infiltration}

In diesem Abschnitt werden Möglichkeiten diskutiert, die Infiltrationsvorgänge unter einer Überflutungsfläche ohne die gesättigte Bodenzone abzubilden. Die Ausführungen stützen sich auf Berechnungen mit einem zweidimensional-horizontalen Feflow-Testmodell mit quadratischer Grundfläche, das mit 16 Quadratelementen einer Kantenlänge von 0,1 m diskretisiert wird. Die Testrechnungen werden so ausgeführt, dass sich die Wasserstände an allen Knoten gleich entwickeln. Damit sind die Modellergebnisse mit denen der Bodensäulen aus Abschnitt 6.4.2 vergleichbar.

Der Ansatz einer h-Randbedingung liefert für Wasserstand und Infiltrationsmenge die Kurven aus Abbildung 6.10. Zu Beginn der Berechnung steht der Wasserstand bei $-2 \mathrm{~m}$, um direkt im 


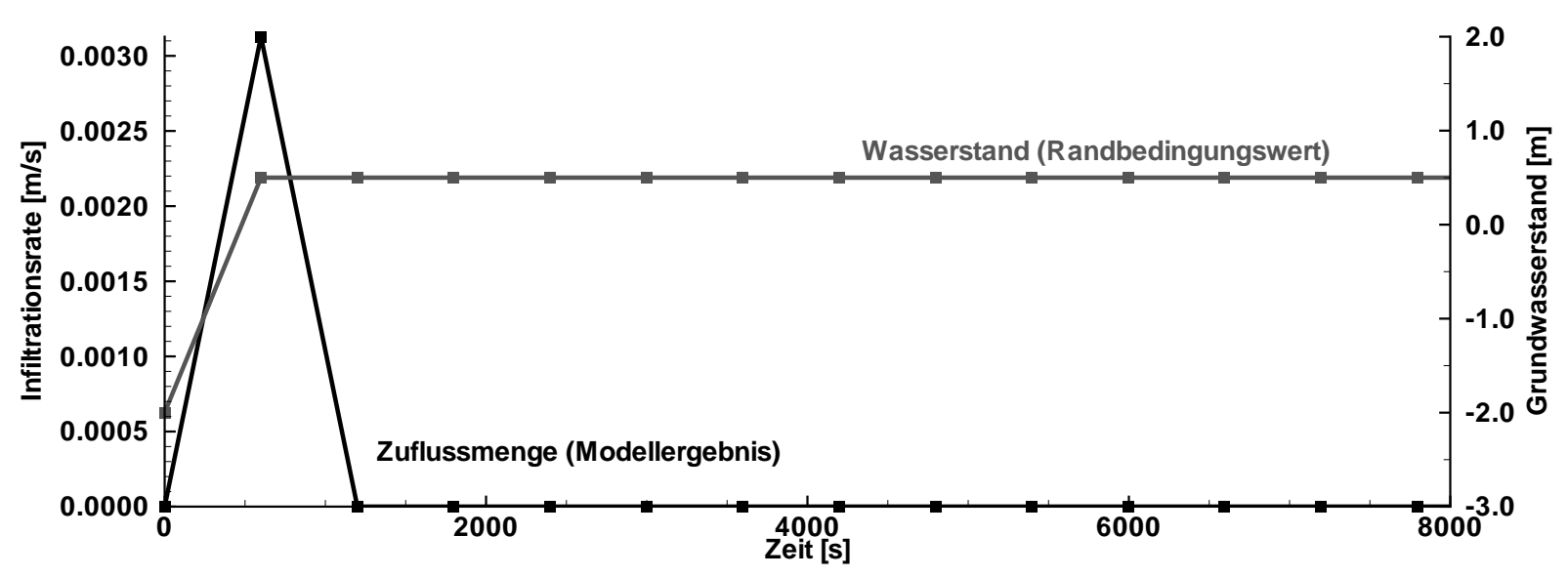

Abbildung 6.10: Angesetzter h-Randbedingungswert und mit Feflow als gesättigte Strömung berechnete Zuflussmenge über die Simulationszeit

ersten Zeitschritt auf den für alle weiteren Zeitschritte vorgegebenen Wert von 0,5 m anzusteigen. Entsprechend steigt der mit der h-Randbedingung korrespondierende Zufluss deshalb im ersten Zeitschritt sprunghaft auf einen Maximalwert an und fällt auf Null zurück, nachdem der Randbedingungswert erreicht ist. Eine Ähnlichkeit mit der Infiltrationskurve aus Abbildung 6.5 oder 6.7 ist allein dahingehend gegeben, dass die Infiltrationsmenge auf Null fällt, wenn die Bodenprobe vollständig gesättigt ist. Der typische Infiltrationskurvenverlauf ist jedoch nicht zu erkennen. Allerdings wird der sprunghafte Anstieg des Grundwasserstands wiedergegeben. Die Verzögerung, die sich durch die Bewegung der Sättigungsfront von oben herab einstellt, kann nicht wiedergegeben werden, da der Infiltrationsprozess selbst nicht abgebildet wird. Wie schon angesprochen, ist eine Modellierung des Wasseraustauschs auf diese Art nur dann sinnvoll, wenn die Sättigung besonders schnell abläuft und innerhalb weniger Modellzeitschritte passiert. Monninkhoff \& Kernbach (2006) zum Beispiel übergeben die Wasserstände einer Überflutungsflächenberechnung direkt als h-Randbedingung an ein Grundwassermodell.

Der Ansatz der Infiltrationskurve aus Abbildung 6.7 als flächige Q-Randbedingung bewirkt in dem horizontal-ebenen Modell den in Abbildung 6.11 gezeigten Anstieg des Wasserstands. Anders als im Fall der ungesättigten Berechnung beginnt der Wasserstand mit Beginn der Infiltration anzusteigen. Die Bodensäule wird quasi von unten her aufgefüllt. Mit der angesetzten Infiltrationsmenge wird jedoch nicht der Wasserstand von 0,5 m erreicht, weil eine Anfangsfeuchte des Bodens anders als bei teilgesättigter Modellierung nicht berücksichtigt wird. Naturgemäß verläuft bei Ansatz der Infiltrationsmenge in Form einer Q-Randbedingung die Infiltrationskurve exakt so wie gewünscht. Für praktische Anwendungen ist diese Variante nur in Ausnahmefällen möglich, weil die Infiltrationsmenge in der Regel nicht bekannt ist. Allerdings wird die Grundwasserneubildung aufgrund Niederschlags in vielen Grundwassermodellen, die nur die gesättigte Strömung umfassen, in Form einer Q-Randbedingung angesetzt (siehe z. B. Bachmann et al. 2007; Becker \& Köngeter 2005). Die Grundwasserneubildungsrandbedingung stellt dann aber das Ergebnis der Infiltration aufgrund Niederschlags dar und bildet nicht den Infiltrationsprozess selbst ab. 


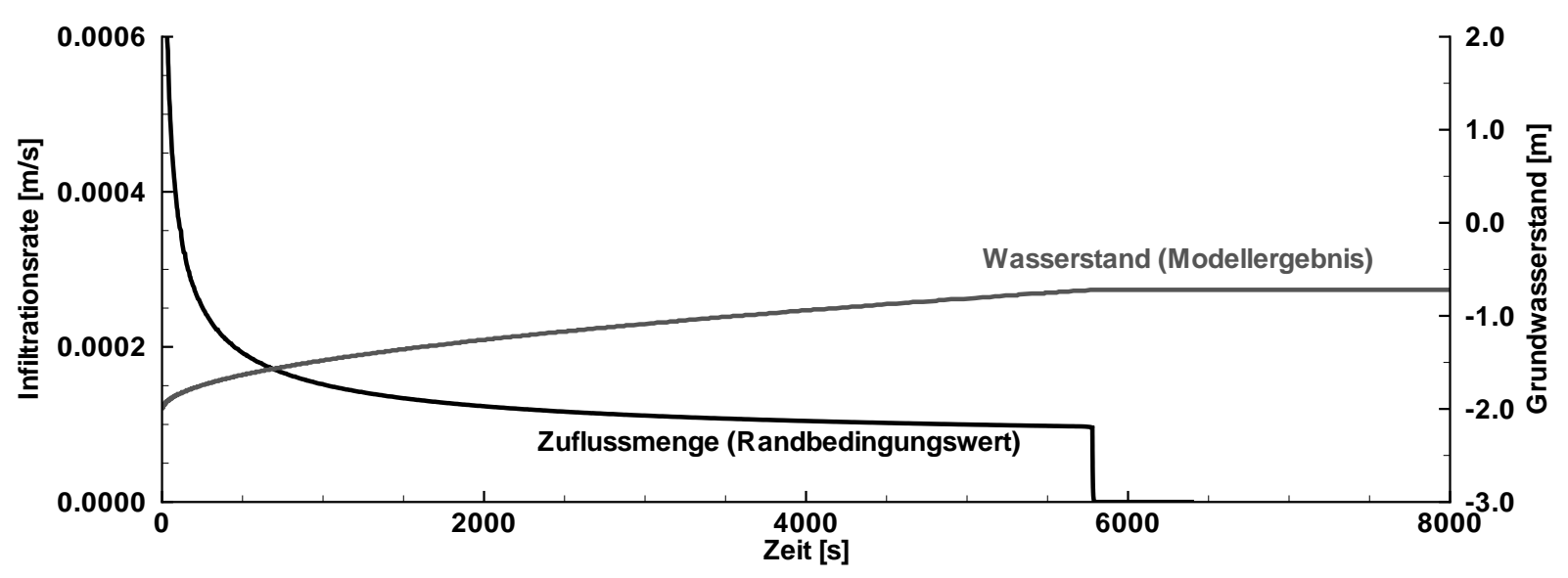

Abbildung 6.11: Angesetzte Q-Randbedingungsmenge und berechneter Wasserstand über die Simulationszeit

Bei Ansatz einer Leakage-Randbedingung mit der $Q$ - $\Delta h$-Beziehung aus Gleichung 5.2 wird für einen über die Zeit konstanten Randbedingungswert $h$ in einem zweidimensional-horizontalen Modell erreicht, dass zu Beginn des Infiltrationsvorgangs viel und zu seinem Ende hin weniger Wasser infiltriert. Damit kommt zumindestens der qualitative Verlauf der Zuflussmenge einer Infiltrationskurve recht nahe (Abbildung 6.12a). Der Grundwasserspiegel steigt mit Beginn des Infiltrationsvorgangs an, eine Verzögerung des Wasseranstiegs, wie er von der ungesättigten Zone verursacht wird, findet nicht statt.

Der Leakage-Parameter kann hier nur zur Steuerung der Infiltrationsrate und des Grundwasseranstiegs verwendet werden, er übernimmt dann keine physikalische Bedeutung für den Infiltrationsvorgang. In dem Beispiel aus Abbildung 6.12a wurde allerdings ein Leakage-Parameter für eine 0,2 m dicke Deckschicht aus Ton (Tabelle 6.1) gewählt, der somit dem Beispiel aus Abbildung 6.9 entspricht. Der Vergleich der beiden Abbildungen zeigt, dass bei Ansatz der LeakageRandbedingung in einer rein gesättigten Modellierung der Infiltrationsprozess deutlich schneller abläuft als im Richards-Modell und somit überschätzt wird. Eine Ursache dafür ist die große Wasserstandsdifferenz $\Delta h$, die zur Berechnung der Leakage-Menge angesetzt wird. Im Beispiel beträgt sie zu Beginn des Infiltrationsvorgangs 2,5 m und liefert damit eine hohe Infiltrationsmenge. Darüber hinaus wirkt die Infiltratiosmenge nach Passage der Deckschicht im rein gesättigten Modell direkt auf den Grundwasserspiegel, während sie im ungesättigten Modell noch die ungesättigte Zone zwischen Deckschicht und Grundwasserspiegel passieren muss. Hier hängt die Durchlässigkeit vom Matrixpotenzial ab (Abschnitt 2.3, Abbildung 2.5), die ungesättigte Bodenzone stellt damit einen erheblichen Widerstand für das eindringende Wasser dar.

Wie in Abschnitt 6.4.2 gezeigt wurde, hat der Grundwasserspiegel keinen Einfluss auf den Infiltrationsvorgang, solange sich zwischen Überflutungsfläche und Grundwasserstand eine unge- 


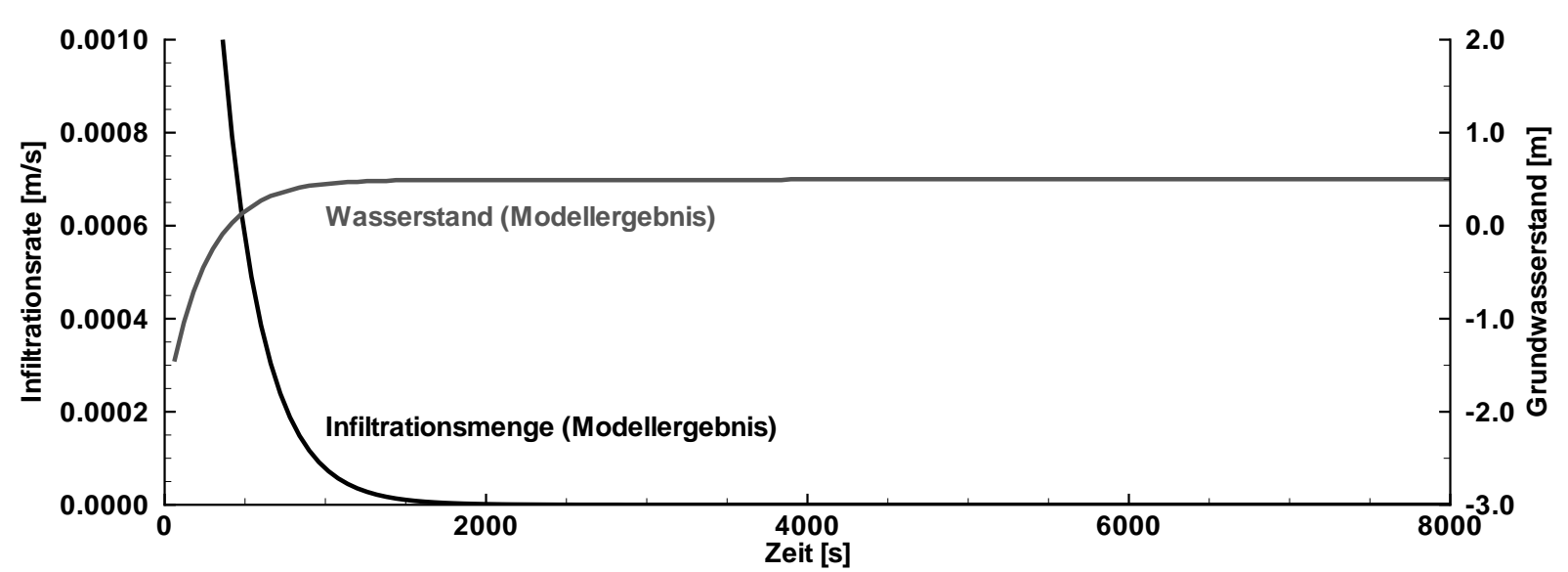

(a) Leakage-Randbedingung

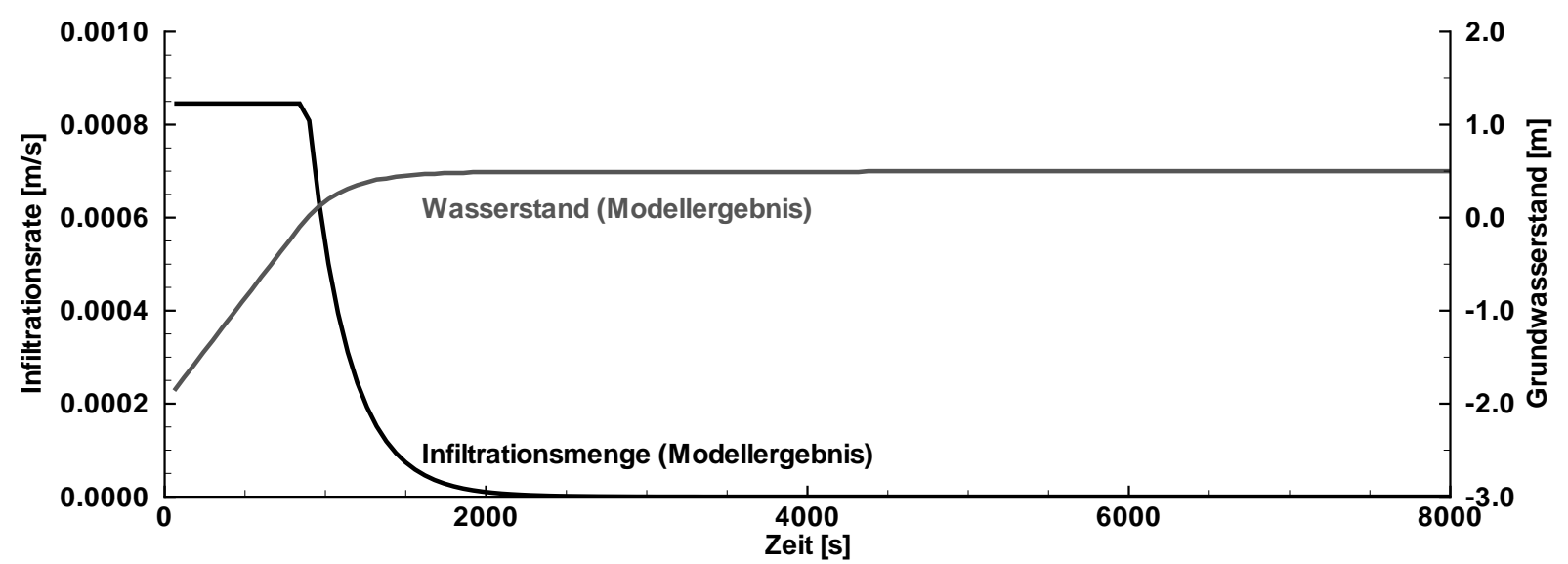

(b) Leakage-Randbedingung mit Nebenbedingung (wenn $h_{\mathrm{G}} \leq z_{\mathrm{B}}$, dann $h_{\mathrm{G}}=z_{\mathrm{B}}$ )

Abbildung 6.12: Mit der Leakage-Randbedingung berechnete Zuflussmenge und berechneter Wasserstand (Leakage-Parameter: $c=8,45545 \cdot 10^{-6} 1 / \mathrm{s}$, Randbedingungswert $h=0,5 \mathrm{~m}$ )

sättigte Zone befindet. Es liegt nahe, die Wasserstandsdifferenz $\Delta h$ durch Ansatz einer Nebenbedingung zu begrenzen. Gunduz \& Aral (2005) verwenden folgende Fallunterscheidung:

$$
\Delta h=\left\{\begin{array}{lll}
h_{\mathrm{F}}-h_{\mathrm{G}} & \text { für } & h_{\mathrm{F}}<h_{\mathrm{G}} \\
h_{\mathrm{F}}-z_{\mathrm{B}} & \text { für } & h_{\mathrm{F}}>h_{\mathrm{G}}
\end{array}\right.
$$

Der Anwendungsfall von Gleichung 6.21 ist ein Fluss ohne Grundwasseranschluss, bei dem im Falle der Infiltration, also für positive Wasserstandsdifferenzen, das Wasser vom Fluss aus nach unten in Richtung Grundwasserspiegel sickert. Die Ausbildung einer Sättigungsfront wird durch eine Sedimentschicht an der Sohle verhindert. Für den Fall einer Infiltration tritt an die Stelle der Lage der Flusssohle $z_{\mathrm{B}}$ die Lage der Geländeoberkante. Ein Nachteil beim Ansatz von Nebenbedingungen ist es, dass nicht im Voraus bekannt ist, ob die gesetzte Nebenbedingung 
greift oder nicht. Berechnungsschritte müssen deshalb gegebenenfalls wiederholt werden, was eine längere Rechenzeit mit sich bringt.

In Abbildung 6.12b ist das Berechnungsergebnis für das zweidimensional-horizontale Beispielmodell bei Ansatz einer derartigen Nebenbedingung dargestellt. Solange der Wasserstand die Geländeoberkante von $0 \mathrm{~m}$ noch nicht erreicht, bleibt die zugeführte Menge konstant über die Zeit, der Wasserstand steigt linear an. Erreicht der Wasserstand die Geländeoberfläche von $z_{\mathrm{B}}=0 \mathrm{~m}$, dann ändert sich die Wasserstandsdifferenz und damit geht die zugeführte Menge zurück. Im Vergleich zum Beispiel ohne Nebenbedingung reduziert sich die Infiltrationsmenge zu Beginn des Infiltrationsvorgangs nicht. Die Infiltrationskurve beginnt bedingt durch die geringere Wasserstandsdifferenz $\Delta h$ mit einem vergleichsweise geringeren Wert als im Fall ohne Nebenbedingungen. Sie verbleibt anfangs auf einem konstanten Niveau solange, bis der Grundwasserstand über den Wert der Nebenbedingung steigt und so die Wasserstandsdifferenz und damit auch die Leakage-Menge reduziert. Der Wasserstand steigt daher etwas langsamer an, der lineare Verlauf erinnert an die Vorbilder aus Abbildung 6.9.

\subsubsection{Schlussfolgerungen und Diskussion}

Die numerische Lösung der Richards-Gleichung mit Ansatz einer Überflutungsrandbedingung erster Art (h-Randbedingung) bildet den Infiltrationsverlauf am Besten ab. Erst nachdem die Infiltrationsfront den Grundwasserspiegel erreicht, wirkt sich die Überflutung auf den Grundwasserspiegel aus und er steigt mit einer Verzögerung schlagartig an. Diese Verzögerung kann nicht berücksichtigt werden, wenn der Prozess als reine gesättigte Strömung aufgefasst wird. Die Verzögerung ist bei gut durchlässigen Bodenarten jedoch kurz, so dass eine derartige Einschränkung der Modellaussage - je nach Zielsetzung der Modelluntersuchung - in Kauf genommen werden kann.

Sind die Überflutungsfläche und der unter ihr liegende Grundwasserleiter durch eine Deckschicht getrennt, kann bei Berücksichtigung der ungesättigten Bodenzone eine Leakage-Randbedingung mit oder ohne Nebenbedingung verwendet werden, um die Infiltration durch diese Deckschicht zu erfassen, da sich so nur geringfügige Unterschiede gegenüber einer direkten Modellierung der Deckschicht ergeben. Die Deckschicht wirkt als Grundwassergeringleiter und verzögert den Anstieg des Grundwasserspiegels. Nebenbedingungen sind in der Regel numerisch aufwändiger und erhöhen die Rechenzeit. Oft ist es deshalb wünschenswert, auf den Ansatz von Nebenbedingungen zu verzichten.

Wird ein Grundwassermodell mit rein gesättigter Strömung verwendet, kann der Prozess der Infiltration aus einer Überflutungsfläche näherungsweise mit einer Leakage-Randbedingung modelliert werden. Die numerischen Untersuchungen zeigen, dass der Infiltrationsvorgang hinsichtlich Geschwindigkeit und Infiltrationsmenge überschätzt wird, wenn auch der Verlauf der Infiltrationsmenge über die Zeit qualitativ wiedergegeben wird. Es besteht die Möglichkeit, den Leakage-Koeffizienten als Parameter aufzufassen und im Rahmen einer Modellkalibrierung geeignet einzustellen. Bei einer rein gesättigten Modellierung kann Wasser, das vor Beginn des 
Infiltrationsvorgangs im Boden innerhalb der ungesättigten Bodenzone gespeichert ist, nicht berücksichtigt werden. Für das August-Hochwasser 2002 wird der hohe Wassergehalt des Bodens zu Beginn des Hochwasserereignisses wegen zuvor lang anhaltender Starkniederschläge als eine wesentliche Ursache für die dort beobachteten Ausmaße des unterirdischen Hochwassers genannt (Sächsisches Landesamt für Umwelt und Geologie 2003). Zudem wirkt sich die Infiltration sofort auf den Grundwasserspiegel aus, die Verzögerung durch den Sickerungsprozess wird nicht abgebildet. Der Ansatz einer Nebenbedingung verbessert das Bild nicht wesentlich. Mit einem Verzicht auf Modellierung der ungesättigten Zone muss also in Kauf genommen werden, dass der Infiltrationsprozess auch bei einer Kalibrierung nur grob wiedergegeben wird und sein Einfluss auf den Grundwasserstand zumindest was den Beginn seines Anstiegs angeht eher überschätzt wird.

Trotz dieser Unzulänglichkeiten ist die Leakage-Randbedingung für Infiltrationsprobleme weit verbreitet. Bauer et al. (2006) und Koch et al. (2007) verwenden Leakage-Ansätze zur Modellierung des Wasseraustauschs in einem gekoppelten Modell für Feuchtgebiete. Anwendungsbeispiele, die den Bereich des unterirdischen Hochwassers berühren, sind zum Beispiel Köngeter et al. (2002), Homann (2006), Köngeter et al. (2002), Köngeter et al. (1997), van Linn (2006) sowie Sommer \& Eulitz (2007) und Peetz \& Steckel (2007).

Für eine praktische Anwendung im operativen oder planerischen Hochwasserschutz sind Modelle auf Basis der rein gesättigten Grundwasserströmung deutlich besser geeignet als Modelle, die die numerische Lösung der Richards-Gleichung beinhalten, da für die Lösung der RichardsGleichung ein hoher räumlicher Auflösungsgrad erforderlich ist. Eine Überschätzung der Infiltration bedeutet zudem, dass die Modellaussagen, was den unterirdischen Raum angeht, ,,auf der sicheren Seite liegen“. Beim operativen und planerischen Modelleinsatz steht eher im Vordergrund, Entscheidungen zu unterstützen und Tendenzen aufzuzeigen, als eine möglichst genaue Wiedergabe der realen Verhältnisse zu erreichen. Darüber hinaus ist es ein wesentliches Ziel dieser Arbeit, bereits bestehende Grundwassermodelle für die Planung von Schutzmaßnahmen gegen unterirdisches Hochwasser einzusetzen. In der Regel basieren solche Grundwassermodelle auf der rein gesättigten Modellierung der Grundwasserströmung.

So wird vorgeschlagen, in einem Grundwassermodell, das allein die gesättigte Strömung in porösen Medien abbildet, die Leakage-Randbedingung zur Abbildung der Infiltration aus einer Überflutungsfläche zu verwenden. Da der Ansatz von Nebenbedingungen nicht zu einer wesentlichen Verbesserung der Abbildungsgüte verhilft, kann darauf verzichtet werden. In besonderen Fällen kann auch eine h-Randbedingung mit dem Wert der Überstauung angesetzt werden. Insgesamt wird es ohne die Berücksichtigung der ungesättigten Zone aber nicht gelingen, sowohl den Grundwasserstand als auch die infiltrierende Menge genau zu treffen. Der Verzicht auf die ungesättigte Zone stellt somit eine wesentliche Vereinfachung des Infiltrationsprozesses dar. 


\subsection{Zusammenfassung des Kapitels}

Infiltration ist eine Bewegung des Sickerwassers von oben her in den Boden, wenn das Matrixpotential höher ist, als es dem Gleichgewicht mit dem freien Grund- oder Stauwasserspiegel entspricht. Infiltration entsteht durch Niederschläge, Beregnung oder Überstauung. Für die Fragestellung des unterirdischen Hochwassers spielt die Infiltration des Wassers von einer überfluteten Fläche in den Grundwasserleiter eine wesentliche Rolle, weil sich das Wasser auf einer überfluteten Landoberfläche deutlich schneller ausbreitet als im Untergrund. Von oben infiltrierendes Hochwasser aus einer Überflutungsfläche trägt somit zu einer Verbreitung des unterirdischen Hochwassers im Hinterland bei. Wegen der Einstauung bildet sich eine Sättigungsfront unterhalb der Überflutungsfläche aus, wenn keine Deckschicht die Landoberfläche und die grundwasserführende Schicht trennt.

Mit dem mathematischen Infiltrationsmodell von Haverkamp wurde gezeigt, dass nicht in jedem Fall davon ausgegangen werden kann, dass sich unter einer Überflutungsfläche sofort vollständig gesättigte Verhältnisse einstellen. Je nach Bodenart und Anfangszustand schreitet eine Sättigungsfront unterschiedlich schnell voran.

Zur Frage, wie sich eine solche Infiltration in einem Grundwassermodell abbilden lässt, wurden numerische Testmodelle ausgewertet. Mit einer numerischen Lösung der Richards-Gleichung kann das Eindringen des Wassers in den Boden realitätsnah modelliert werden. Für praktische Anwendungen ist ein Modell auf Basis der Richards-Gleichung jedoch nicht geeignet. Allerdings bringt der Verzicht auf die Modellierung der ungesättigten Bodenzone Einschränkungen der Modellaussagefähigkeit mit sich. Mit Hilfe von h-Randbedingungen und LeakageRandbedingungen können die Auswirkungen einer Infiltration auf den Grundwasserspiegel wiedergegeben werden. Für beide Varianten sind in der Fachliteratur Anwendungsbeispiele dokumentiert. Die Ergebnisse der Modellrechnungen deuten allerdings darauf hin, dass die Infiltrationsmengen in der Regel mit diesen Vereinfachungen nicht genau zu ermitteln sind. Vor dem Hintergrund eines Einsatzes für eine operative oder planerische Entscheidung kann dies in Kauf genommen werden, da nicht die genaue Wiedergabe der tatsächlichen Verhältnisse im Vordergrund steht, sondern es vielmehr darum geht, Entscheidungen zu unterstützen und Tendenzen aufzuzeigen. 


\section{Qualmwasseranstieg}

\subsection{Einführung}

Qualmwasser (Abbildung 7.1) oder Drängewasser ist an der Oberfläche austretendes Grundwasser. Voraussetzung für seine Entstehung ist, dass die Grundwasserdruckhöhe über die Geländeoberfläche ansteigt und die Durchlässigkeitseigenschaften des anstehenden Materials einen Austritt an der Oberfläche zulassen, dass also zumindest bereichsweise keine gespannten Verhältnisse entstehen. Oft wird Qualmwasser in Senken oder Niederungen hinter eingedeichten Flussabschnitten bei Hochwasser beobachtet, da die Eindeichung des Flusses bewirkt, dass der Wasserstand höher steigt als ohne Eindeichung. Der Begriff „Qualmwasser“ kommt daher, dass das Wasser manchmal brodelnd oder qualmend an der Oberfläche austritt, weil im Boden eingelagerte Luft hochgedrückt wird. Geschieht dies bei niedrigen Lufttemperaturen, kommt es zusätzlich zu einer sichtbaren Dampfentwicklung (Qualm), da das aufsteigende Wasser im Verhältnis zur Lufttemperatur deutlich wärmer ist (Lehners \& Sassenhagen 2003).

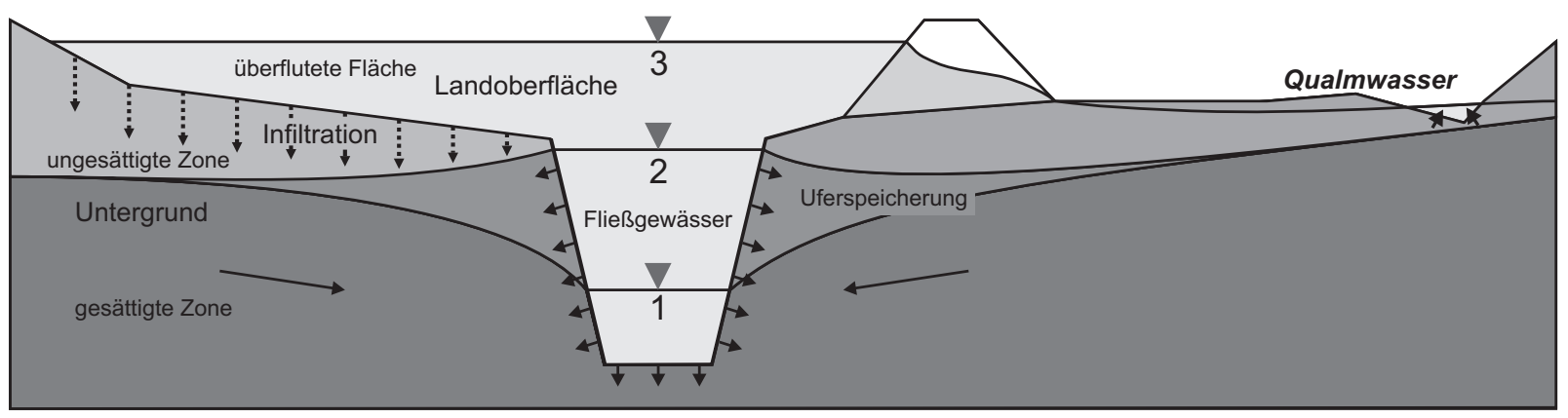

Abbildung 7.1: Einordnung der Bildung von Qualmwasser in die Prozesse der Wasserbewegung beim unterirdischen Hochwasser

Die DIN 4047-2 (1988) definiert Drängewasser als Wasser, ,das durch einen Deich und/oder dessen Untergrund in eine Niederung eintritt“" und unterteilt das Drängewasser in Kuver- und Qualmwasser. Der Unterschied zwischen Kuverwasser und Qualmwasser besteht darin, dass Kuverwasser durch den Deichkörper sickerndes Drängewasser ist, wohingegen durch den Untergrund des Deiches sickerndes Drängewasser als Qualmwasser bezeichnet wird. Kuverwasser tritt demnach auf der Luftseite des Deichkörpers aus, Qualmwasser dagegen tritt in den Niederungen im Deichhinterland an die Erdoberfläche.

In der Literatur wird die nach DIN 4047-2 (1988) normierte Bezeichnung Qualmwasser nicht einheitlich verwendet. So wird bei Lehners \& Sassenhagen (2003) Qualmwasser dem Dränge- 
wasser gleichgesetzt und Tacke (1988) verwendet anstelle des Begriffs Qualmwasser auch die Bezeichnung Quellwasser, da das Wasser aus dem Boden quillt.

Qualmwasseraustritt in unmittelbarer Nähe eines Deiches wird auch als ein Mechanismus des Deichversagens genannt, denn mit dem Qualmwasser wird Bodenmaterial ausgespült (piping). Damit verändert sich die Untergrundstruktur, was zu einer Reduzierung seiner Tragfähigkeit führen kann (Lehners \& Sassenhagen 2003). Dieses Problem ist allerdings nicht Gegenstand der vorliegenden Arbeit. Im Rahmen des Schutzes vor unterirdischem Hochwasser interessiert das Wasser, das im Hinterland zum Beispiel in Senken zu Tage tritt.

Während für die Wechselwirkungsprozesse der Uferspeicherung und der Infiltration die Trennflächen Sohlschicht und ungesättigte Bodenzone definiert werden, gibt es eine solche Trennfläche bei Qualmwasseraustritt an der Oberfläche nicht zwangsläufig. Die Bewegung des Wassers im Untergrund gehorcht den Gesetzmäßigkeiten, die in Kapitel 2 angesprochen wurden, und wird bei Hochwasser wesentlich durch die Uferspeicherungs- und Infiltrationsprozesse gesteuert. Damit ist der Qualmwasseranstieg eine Folge dieser beiden Wechselwirkungsprozesse.

Erreicht das Grundwasser die Landoberfläche, so gelten die Fließgesetze aus Kapitel 3, Die Übergabe des Wassers von dem Kompartiment des Untergrundes an das Kompartiment der Landoberfläche in einem gekoppelten System aus numerischen Modellen ist damit eher eine programmtechnische Aufgabe. In Abschnitt 7.3 wird dazu eine Möglichkeit vorgestellt. Zuvor wird aber auf den Wasseranstieg selbst eingegangen, da ein Grundwasserstandsanstieg vor dem Anstieg des Wasserstands im Fluss als eine Ursache für die weitläufige Ausbreitung des unterirdischen Hochwassers im August 2002 im Einzugsgebiet der Elbe genannt wird (Sächsisches Landesamt für Umwelt und Geologie 2003).

\subsection{Wasseranstieg im Untergrund}

Abbildung 7.2a zeigt das Sättigungsprofil des aus Abschnitt 6.4.2 bekannten Bodenbeispiels mit dem Kapillarsaum über einem Grundwasserspiegel auf der Höhe von $-2 \mathrm{~m}$. Im oberen Bereich ist der Boden trocken. Es infiltriert hier jedoch nicht von oben Wasser in die Bodenprobe, sondern am unteren Rand ist ein Zufluss von 0,5 m/d angesetzt. Dieser Zufluss bewirkt einen Anstieg des Wasserstands, so wie er zum Beispiel durch einen Uferspeicherungsprozess ausgelöst werden kann. Der Kapillarsaum verschiebt sich quasi nach oben und bildet eine Übergangszone zu dem vom Zufluss unbeeinflussten Bereich. Den Anstieg des Wasserstands über die Zeit gibt die mittlere Kurve aus dem Diagramm in Abbildung 7.3 wieder. Der über die Zeit konstante Zufluss bewirkt einen fast linearen Wasserstandsanstieg. Zu Beginn ist jedoch ein leicht beschleunigter Anstieg zu beobachten, weil bereits ein Teil der ungesättigten Bodenzone mit Wasser gefüllt ist und die Zuflussmenge deshalb zur Sättigung weiter oben liegender Bereiche zur Verfügung steht.

Im zweiten Beispiel (Abbildung 7.2b) ist die Ausgangslage ein Sättigungsgrad von 0,6 im oberen Bereich der Bodensäule, wie er sich nach einer anhaltenden Niederschlagsinfiltration von 


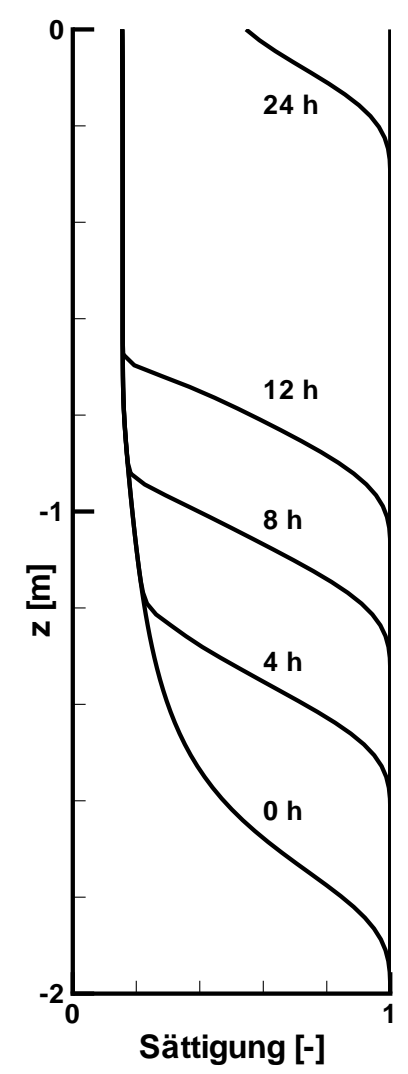

(a) trockener Anfangszustand mit Kapillarsaum

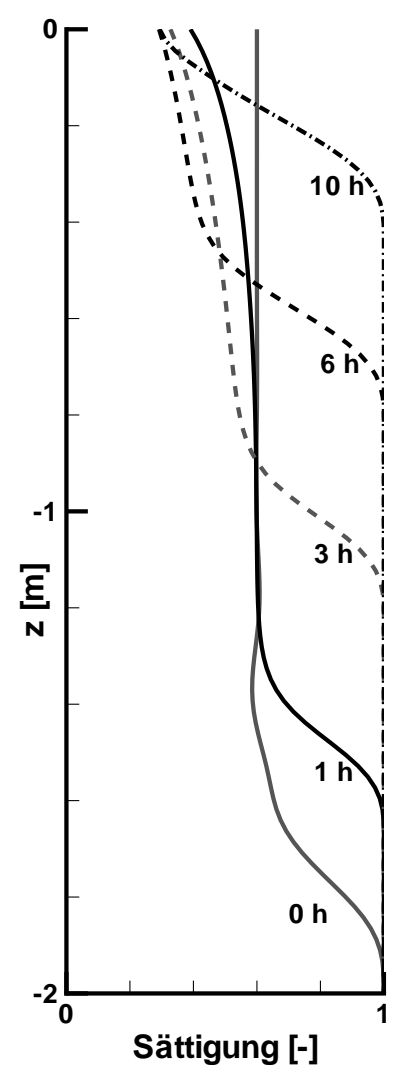

(b) nach Niederschlag

Abbildung 7.2: Sättigungsprofile für Wasseranstieg infolge Zufluss von unten bei unterschiedlichen Anfangsbedingungen

etwa $17 \mathrm{~mm} / \mathrm{h}$ einstellen könnte. Diese Infiltrationsrate entspricht von der Intensität her einem mittelstarken bis starken Regenschauer. Es wird zur besseren Vergleichbarkeit angenommen, dass dieser Niederschlag ebenfalls zu einem Grundwasserstand von -2 m geführt hat. Am zeitlichen Verlauf des Sättigungsprofils wird deutlich, dass dieser Anfangszustand nicht stabil ist. Der Boden kann diese Wassermenge nicht gegen die Schwerkraft halten und hat damit seine Feldkapazität überschritten. Der Sättigungsgrad nimmt im oberen Bereich der Bodenprobe ab, denn es fließt kein Wasser von oben nach. Das Wasser bewegt sich also wegen der Schwerkraft nach unten und trägt zusammen mit dem an der Unterkante angesetzten Zufluss von 0,5 m/d zum Anstieg des Grundwasserspiegels bei. Dass bereits viel Wasser im teilgesättigten Bereich des Bodens vorhanden war, führt bei diesem Beispiel zu einem deutlich schnelleren Anstieg des Grundwasserspiegels, wie in der linken Kurve von Abbildung $7.3 \mathrm{zu}$ sehen ist.

Mit einer rein gesättigten Modellierung lässt sich dieser Effekt nicht erfassen, da dabei nur zwischen einem vollständig gesättigten Bereich unterhalb des Grundwasserspiegels und einem vollständig ungesättigten Bereich oberhalb des Grundwasserspiegels unterschieden wird. Oberhalb 


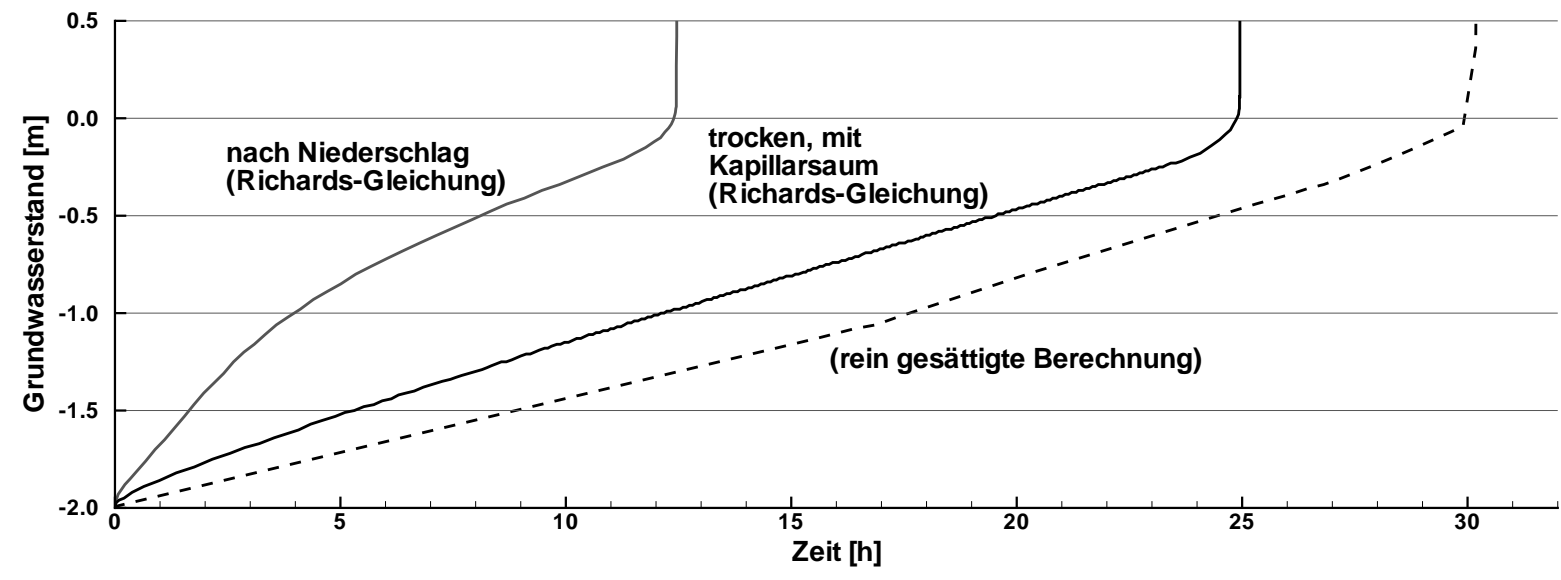

Abbildung 7.3: Grundwasseranstieg für verschiedene Anfangszustände

des Grundwasserspiegels steht deshalb immer der gesamte nutzbare Porenraum $n_{\mathrm{Pn}}$ für ansteigendes Wasser zur Verfügung. Entsprechend dauert es deutlich länger, bis der Wasserstand die Oberkante der Bodenprobe erreicht. Die rechte Kurve aus Abbildung 7.3 ist das Ergebnis einer Berechnung mit dem zweidimensional-horizontalen Testmodell, das in Abschnitt 6.4.3 beschrieben wird. In einem Grundwassermodell, das die rein gesättigte Strömung abbildet, wird der durch Niederschlag verursachte Grundwasseranstieg über die Grundwasserneubildung erfasst. Damit werden die Prozesse der Wasserbewegung und der Wasserspeicherung in der ungesättigten Bodenzone selbst nicht im Modell abgebildet, sondern nur deren Effekte auf den Grundwasserspiegel. Der zeitliche Verlauf der Grundwasserneubildung kann zum Beispiel aus Lysimeteranalysen bestimmt werden. Um unterschiedlich langen Sickerwegen durch unterschiedliche Flurabstände im Modell Rechnung zu tragen, können zum Beispiel Flurabstandsklassen gebildet werden, für welche die Grundwasserneubildungsganglinie dann geeignet zeitlich versetzt wird. Hinweise auf das Versatzmaß liefern die Daten der Grundwassermessstellen (Becker \& Köngeter 2005).

Bei allen drei Beispielen steigt der Wasserstand mit Erreichen der Geländeoberkante bei $0 \mathrm{~m}$ deutlich stärker an. Dies ist dem Übergang von ungespannten zu gespannten Strömungsverhältnissen geschuldet. Ab hier kann Wasser nicht mehr durch das Auffüllen des Porenraums, sondern nur noch über die Zusammendrückbarkeit des Korngerüstes im Bodengefüge gespeichert werden. Der Speicheranteil für gespannte Grundwasserverhältnisse liegt in der Regel mehrere Zehnerpotenzen unter den Werten des nutzbaren Porenraums (Langguth \& Voigt 1980), so dass ein Zufluss bei gleicher Zuflussmenge pro Zeiteinheit wesentlich stärker ansteigt.

\subsection{Modellierung des Wasseraustritts an der Landoberfläche}

Nachdem in den vorangegangenen Abschnitten auf den Wasseranstieg im unterirdischen Raum eingegangen wurde, wird in diesem Abschnitt als Möglichkeit zur Modellierung des Was- 
seraustritts an der Landoberfläche, die mit der Oberkante des Leiters zusammenfällt, die Sickerflächenrandbedingung (seepage face-Randbedingung, Diersch 2005c) vorgestellt. Eine Sickerflächenrandbedingung ist eine h-Randbedingung mit Nebenbedingung:

$$
\begin{gathered}
h=z \\
Q<0
\end{gathered}
$$

$Q$ ist hierbei positiv für einen Zufluss ins Modellgebiet definiert.

Der Wert der Randbedingung wird mit der $z$-Koordinate der Geländeoberkante belegt und die Nebenbedingung sorgt dafür, dass die Randbedingung nur greift, wenn sich Wasser aus dem Modellgebiet weg bewegt, also ein entsprechender Gradient vorliegt. Der Ansatz der hRandbedingung mit dem Wert der geodätischen Höhe des betreffenden Knotens bewirkt dann, dass dem Modell genau so viel Wasser entzogen wird, wie notwendig ist, um die Grundwasserdruckhöhe gerade auf Höhe der Geländeoberkante zu halten. Diese der h-Randbedingung korrespondierende Wassermenge steht dann als Übergabewert für ein Oberflächenströmungsmodell zur Verfügung. Der klassische Anwendungsfall dieser Randbedingung ist eine leicht geneigte Oberfläche, bei der austretendes Wasser sofort als Oberflächenabfluss wirksam wird. In dem Beispiel aus Anhang C.4 wird diese Randbedingung zur Modellierung des Wasseraustritts an der landseitigen Deichböschung verwendet.

\subsection{Zusammenfassung des Kapitels}

Qualmwasser ist Grundwasser, das bei Hochwasser an der Landoberfläche hinter eingedeichten Flussabschnitten in Senken oder Niederungen aufsteigt, weil Grundwasserdruckhöhe wegen des Deiches über der Geländeoberkante liegt. Anders als bei der Uferspeicherung und der Infiltration aus einer Überflutungsfläche existiert beim Prozess des Qualmwasseraustritts keine Trennfläche, die modelltechnisch besonders behandelt werden muss.

Die Speicherung von Wasser in der ungesättigten Bodenzone aus Niederschlägen kann zu einem schnelleren Anstieg des Grundwasserstands führen. Mit einer rein gesättigten Strömungsberechnung lässt sich dieser Effekt nicht abbilden, da hier der gesamte nutzbare Porenraum des Korngerüsts speicherwirksam angesetzt wird.

Um den Austritt des Grundwassers an der Oberfläche in einem Grundwassermodell zu erfassen, bietet sich die sogenannte Sickerflächen-Randbedingung an, eine Randbedingung erster Art, die den Wert der geodätischen Höhe der Landoberfläche annimmt und für die eine Nebenbedingung so gesetzt wird, dass nur ein Abfluss aus dem Modellgebiet zugelassen ist. Die korrespondierende Wassermenge steht dann für ein numerisches Modell der Oberflächenströmung zur Verfügung. 


\section{Anwendungsbeispiele der Modellkopplung}

\subsection{Einführung}

In den Kapiteln 2 und 3 werden die für das unterirdischen Hochwasser relevanten Grundgleichungen für

- die Bewegung des Wassers im Untergrund und

- die Bewegung des Wassers an der Landoberfläche

eingeführt und als Möglichkeit zu ihrer Modellierung die Programme Feflow und Ilmoflood vorgestellt. Kapitel 4 widmet sich der Kopplung von Strömungsprogrammen allgemein. Für die beiden vorgestellten Programme wurde eine Modellkopplung unter Verwendung des OpenMIStandards technisch umgesetzt und verifiziert (Anhang C). Anschließend wird in den Kapiteln 5,6 und 7) auf die Wechselwirkungsprozesse

- Uferspeicherung

- Infiltration aus einer Überflutungsfläche und

- Qualmwasseranstieg

eingegangen. Dabei werden die drei Wechselwirkungsprozesse für sich isoliert und zum Teil unter einfachen Randbedingungen betrachtet. In diesem Kapitel geht es nun darum, die Wechselwirkungsprozesse zusammenzuführen und das Verständnis für diese Prozesse zu vertiefen. Es werden aber auch technische Aspekte der Modellkopplung angesprochen. Alle Anwendungsbeispiele werden mit Hilfe der nach dem OpenMI-Standard gekoppelten Programme Feflow und Ilmoflood durchgeführt.

\subsection{Uferspeicherung und Infiltration}

\subsubsection{Einführung}

Die Prozesse Uferspeicherung und Infiltration aus einer Überflutungsfläche (siehe Abbildung 8.1) werden mit Hilfe eines Modells analysiert, das einen vertikalen Schnitt durch einen idealisierten Grundwasserleiter, der rechtwinklig zur Achse eines idealisierten Flusses liegt, darstellt. Das Modellgebiet ähnelt dem aus Abschnitt 5.4.4 und dehnt sich $3600 \mathrm{~m}$ vom Fluss weg ins 


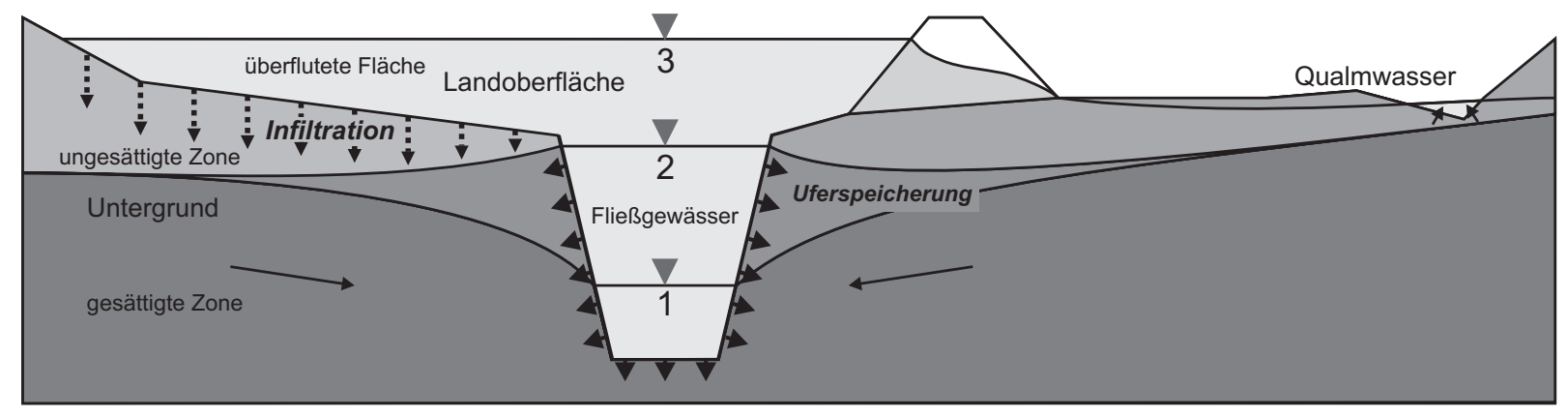

Abbildung 8.1: Die Wechselwirkungsprozesse Uferspeicherung und Infiltration im Zusammenhang mit dem unterirdischem Hochwasser

Hinterland aus. An den Fluss schließt sich auf 63 m Höhenlage eine Überflutungsfläche mit einer Steigung von $2 \%$ an.

Die Bewegung des Wassers an der Landoberfläche wird mit dem Ilmoflood-Modell von Jansen (2009) realisiert. In diesem Modell bildet eine Kette aus quadratischen Zellen einer Kantenlänge von 13,41 m und jeweils unterschiedlicher Höhenlage den Fluss und die Überflutungsfläche ab (Abbildung 8.2a). Für alle Zellen wird ein Strickler-Rauheitsbeiwert von $k_{\mathrm{St}}=50 \mathrm{~m}^{\frac{1}{3}} / \mathrm{s}$ angesetzt. Eine Hochwasserganglinie wird über eine Zuflussrandbedingung zu einer Zelle im Fluss erzeugt. Das Hochwasser dauert zehn Tage, sein Scheitel wird zu Beginn des fünften Tages erreicht. Der Wasserstand steigt so stark an, dass der Fluss über sein Ufer tritt. Im Bereich des Flussquerschnitts ist ständig Wasser vorhanden, während auf der Überflutungsfläche nur zeitweise Wasser steht.

Die Geländeoberfläche besteht aus einer 0,1 m dicken Deckschicht. Diese wird im Grundwassermodell über eine Leakage-Randbedingung mit einem Leakage-Koeffizienten $c_{1}$ (siehe Gleichung 5.3 mit einem Schicht-Durchlässigkeitsbeiwert $k_{\mathrm{S}}$ von $1 \cdot 10^{-6} \mathrm{~m} / \mathrm{s}$ abgebildet. Eine Leakage-Randbedingung wird auch für den Prozess der Uferspeicherung verwendet. Werte für die Leakage-Parameter des Uferbereichs stammen aus den Ergebnissen der automatischen Kalibrierung des Modells aus Abschnitt 5.4.4 (Abbildung 5.12b).

In Kapitel 6 wurde gezeigt, dass die Vernachlässigung der ungesättigten Zone beim Infiltrationsprozess möglicherweise zu deutlich anderen Ergebnissen hinsichtlich der Infiltrationsmenge und der Entwicklung des Wasserstands führen kann. Der idealisierte Grundwasserleiter dieses Anwendungsbeispiels wird deshalb auf zwei Arten modelliert. Einmal wird die dreidimensionale rein gesättigte Grundwasserströmung zu Grunde gelegt. Die Lage der freien Grundwasseroberfläche wird mit Hilfe des Bewegliche-Netze-Ansatzes (Diersch 2005e) erfasst. Die Höhenlage der Modellknoten passt sich dabei dem Wasserstand an. Das Netz wird in vertikaler Richtung so gestaucht oder ausgedehnt, dass an allen Modellknoten eine vollständige Wassersättigung vorliegt. Einen Ausschnitt dieses Grundwassermodells zeigt Abbildung 8.2b.

In der zweiten Modellvariante wird die Richards-Gleichung zweidimensional-vertikal gelöst und damit auch ein ungesättigter Bereich zwischen dem Grundwasserspiegel und der Überflutungs- 


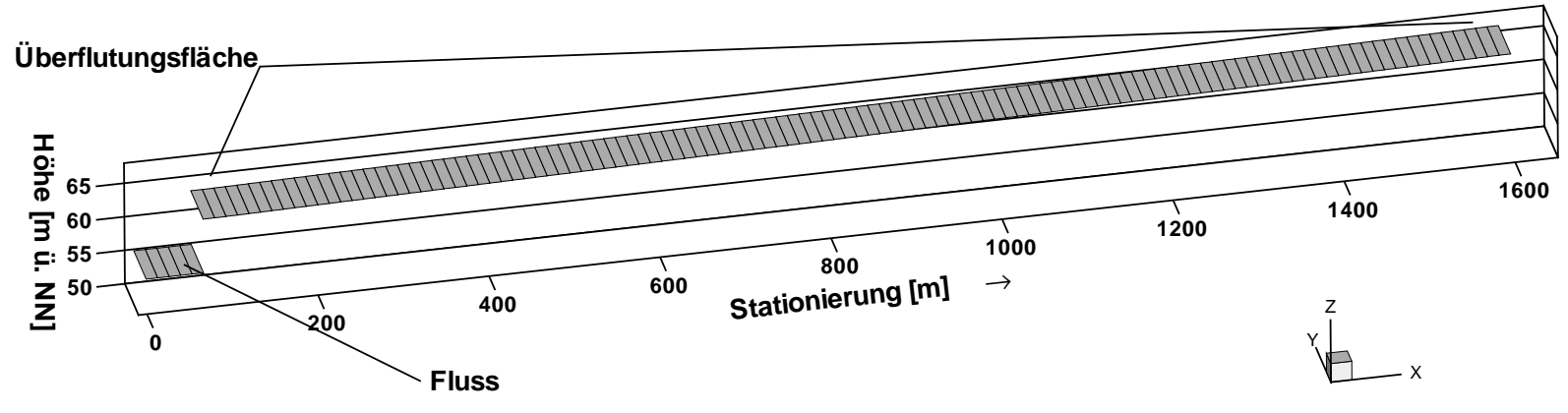

(a) Überflutungsflächenmodell

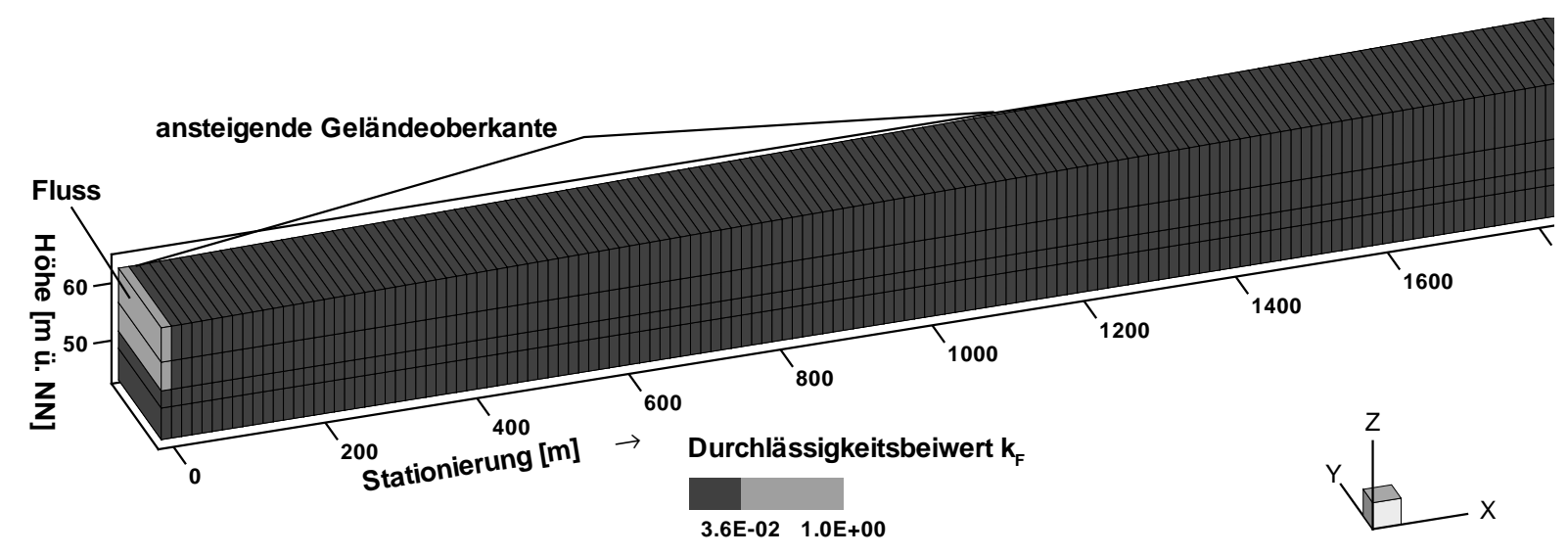

(b) Grundwassermodell

Abbildung 8.2: Modellkomponenten des gekoppelten Testmodells für die Prozesse Uferspeicherung und Infiltration

fläche modelltechnisch ermöglicht. Für die zweite Modellvariante werden erhöhte Anforderungen an die räumliche Diskretisierung gestellt. Das Modellgebiet wird im Vergleich zur ersten Modellvariante deshalb mit deutlich mehr Knoten und Elementen abgebildet (siehe Tabelle 8.1). Die Zeitschrittweite liegt bei 0,04d, beide Modellkomponenten verwenden die gleichen zeitlichen Stützstellen, wobei IImoflood die Zeitschritte noch intern unterteilt.

Wenn Wasser von der Oberfläche in den Grundwasserleiter eindringt, dann ändert dies die Strömungssituation sowohl im Grundwasserleiter als auch im Oberflächengewässer. Damit beide Modellvarianten hinsichtlich des Einflusses dieser Wechselwirkungen auf den unterirdischen Raum verglichen werden können, müssen jeweils gleiche Randbedingungen im Oberflächenwasser vorliegen. Die Wechselwirkungen dürfen die Strömungssituation auf der Oberfläche für einen Vergleich deshalb nicht zu sehr beeinflussen. Dies wird dadurch erreicht, dass das IImoflood-Modell in y-Richtung deutlich breiter als die Grundwassermodelle gestaltet wird. Im IImoflood-Modell ist so ein deutlich größeres Wasservolumen gespeichert als in den Grundwassermodellen. Der Infiltrationsprozess wirkt deshalb stark auf die Grundwassersituation, beeinflusst die Situation an der Oberfläche jedoch nur wenig. 
Tabelle 8.1: Eigenschaften der Grundwassermodellvarianten

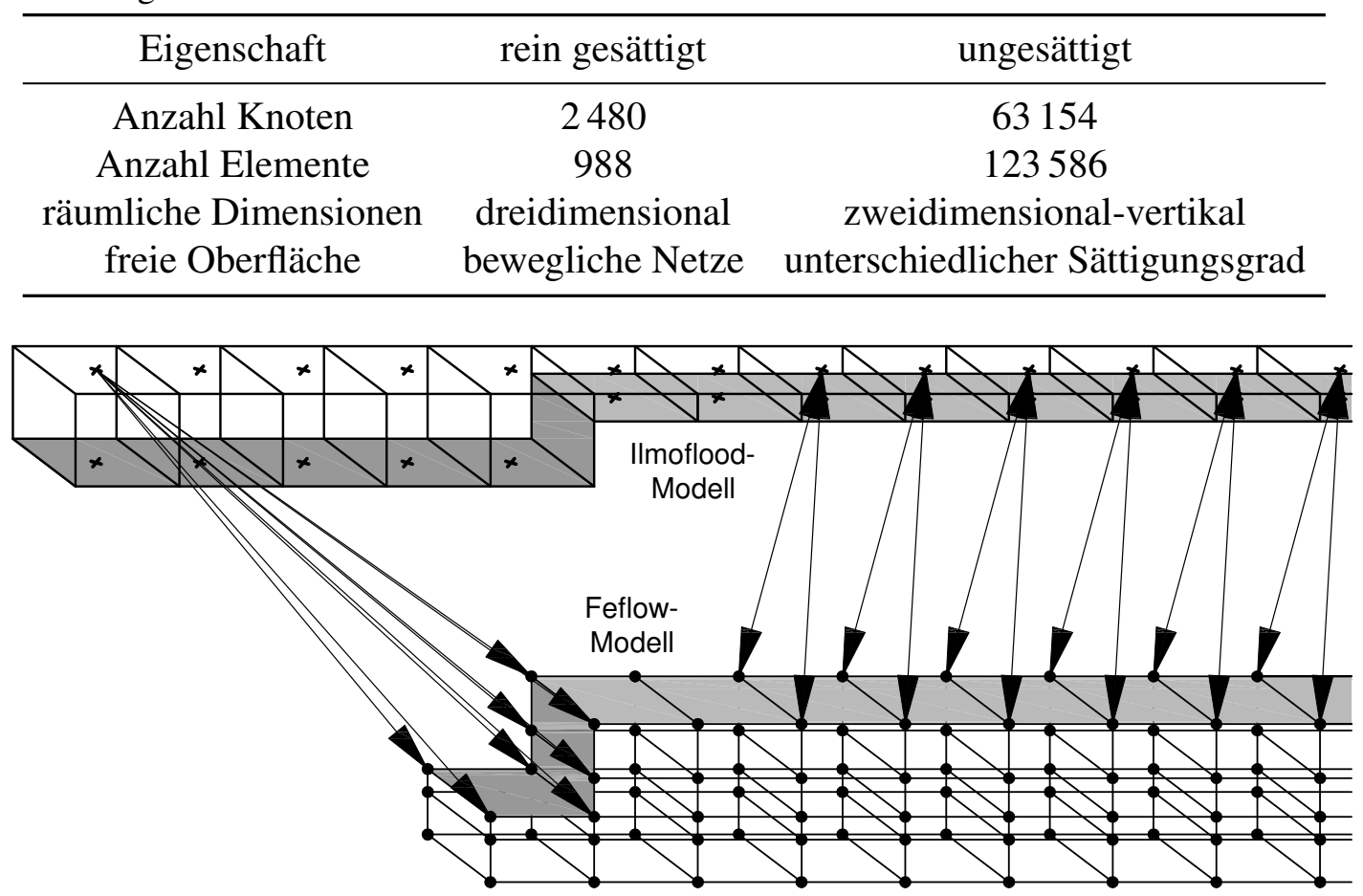

Abbildung 8.3: Kopplung der IImoflood-Zellen mit den Feflow-Knoten (nach Jansen 2009). Von IImoflood wird der Wasserstand als Randbedingungswert einer Leakage-Randbedingung an das Feflow-Modell übergeben, die korrespondierende Austauschmenge wird auf der Überflutungsfläche von Feflow an IImoflood zurückgegeben.

\subsubsection{Rein gesättigte Modellierung der Grundwasserströmung}

\subsubsection{Grundwassermodell und Modellkopplung}

Die Knoten des rein gesättigten Grundwassermodells (Abbildung 8.2b) sind jeweils als Doppelreihe auf fünf Ebenen angeordnet, die fünf Ebenen schließen vier Elementschichten ein. Anders als in dem in Abschnitt 5.4.4 beschriebenen Streifenmodell wurden für alle Elemente, die nicht im Fluss liegen, die Durchlässigkeitsbeiwerte mit $k=3,6 \cdot 10^{-2} \mathrm{~m} / \mathrm{s}$ und die Speicherkoeffizienten mit $S_{\mathrm{sp}}=17,5 \%$ angesetzt. Das Modell entspricht damit einem der Testmodelle von Jansen (2009). Die Breite des Modells wurde jedoch auf $1 \mathrm{~m}$ reduziert, um eine Vergleichbarkeit mit einem zweidimensional-vertikalen Richards-Modell mit Einheitsbreite herstellen zu können.

Die Kopplung des Grundwassermodells mit dem Überflutungsflächenmodell erfolgt so, dass das Überflutungsflächenmodell die Wasserstände einer Modellzelle an die entsprechenden Knoten des Grundwassermodells übergibt (Abbildung 8.3 und Tabelle 8.2). Das Grundwassermodell setzt diese Wasserstände gemäß Gleichungen 5.2 und 5.13 in eine Leakage-Randbedingung 
Tabelle 8.2: Übersicht der Austauschobjekte zur Verbindung zwischen IImoflood-Zellen und FeflowKnoten

\begin{tabular}{lcccc}
\hline Ilmoflood-Modell & & \multicolumn{2}{c}{ Feflow-Modell } \\
\hline Ausgabe $\mathrm{h}$ & $\rightarrow$ & Eingabe & Leakage $1^{a}$ \\
Eingabe & $\mathrm{Q}$ & $\leftarrow$ & Ausgabe & $\mathrm{Q}$ \\
\hline
\end{tabular}

${ }^{a}$ Kontaktfläche abhängig vom Grundwasserstand

um. Die sich daraus ergebende korrespondierende Zufluss- oder Abflussmenge wird dem Überflutungsflächenmodell im nächsten Berechnungsschritt entzogen beziehungsweise hinzugefügt. Diese Rückkopplung wird allerdings nur für Zellen, die die Überflutungsfläche repräsentieren, durchgeführt, um den Einfluss der Überflutungsfläche auf den Wasserstand zu isolieren. Um einen gegenseitigen Einfluss des Uferbereichs und des Bereichs aus der Überflutungsfläche bei der programminternen Berechnung der Kontaktfläche zu vermeiden, wird zwischen Ufer und Überflutungsfläche eine Knotenreihe bei der Kopplung ausgelassen.

\subsubsection{Berechnungsergebnisse}

Die Wirkung des Hochwassers auf den Grundwasserstand im an den Fluss angrenzenden Grundwasserleiter zeigt Abbildung 8.4. Am ersten und am zweiten Tag steigt der Wasserstand innerhalb des Flussquerschnittes an. Der Grundwasserstand folgt dem Flusswasserstand, erreicht aber wegen der Leakage-Funktion, die einen Fließwiderstand verursacht, am Ufer-Modellrand nicht dessen Höhe.

Sobald der Flusswasserstand $63 \mathrm{~m}$ erreicht, macht sich die Infiltration aus der Überflutungsfläche bemerkbar. Auf der Überflutungsfläche ist der Leakage-Parameter kleiner als im Bereich des Ufers, der Widerstand gegen Eindringen von Wasser ist also größer. Dennoch infiltriert wegen der zusätzlich aktivierten Kontaktfläche deutlich mehr Wasser in den Untergrund, was sich im Ergebnis darin äußert, dass der Unterschied zwischen Flusswasserstand und Grundwasserstand kleiner wird. Durch die zusätzliche Infiltrationsmenge breitet sich die Grundwasserwelle vom Ufer des Flusses nun schneller zum Hinterland aus. Trotz des relativ hohen Durchlässigkeitsbeiwertes, der für den Grundwasserleiter angesetzt wurde, beschränken sich in den ersten fünf Tagen des Hochwasserereignisses die besonders starken Grundwasseranstiege auf den Nahbereich der Überflutungsfläche. Am fünften Tag erreicht die Überflutung die Stationierung $1400 \mathrm{~m}$, der Grundwasserstand liegt hier auf etwa 62,8 m. Bei $2000 \mathrm{~m}$ ist schon ein deutlicher Anstieg gegenüber dem Anfangszustand (58 m) mit einem Grundwasserstand von 59,5 m zu verzeichnen. Weiter im Hinterland bei Stationierung $2400 \mathrm{~m}$ beträgt der Anstieg noch 0,5 m gegenüber dem Anfangszustand.

Bei abklingender Hochwasserwelle (Abbildung 8.4b) liegt der Grundwasserstand über dem Wasserstand an der Oberfläche. Das Grundwasser breitet sich dem hydraulischen Gefälle folgend 


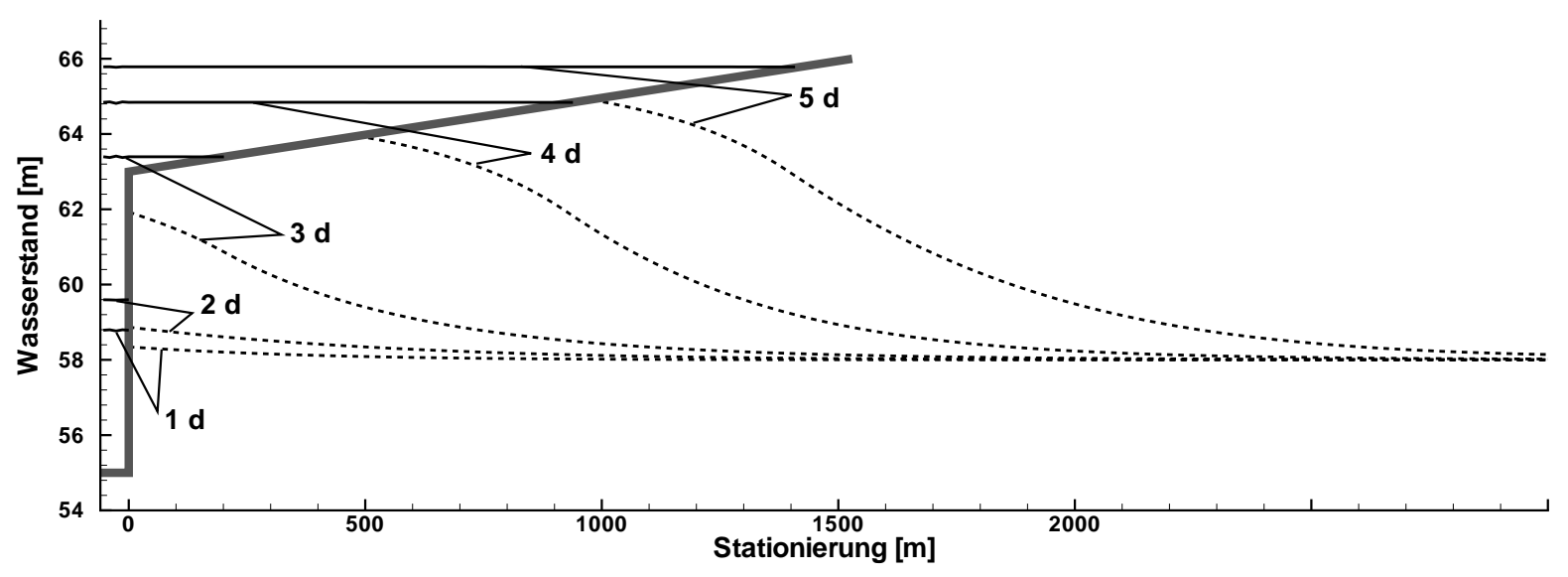

(a) auflaufende Hochwasserwelle

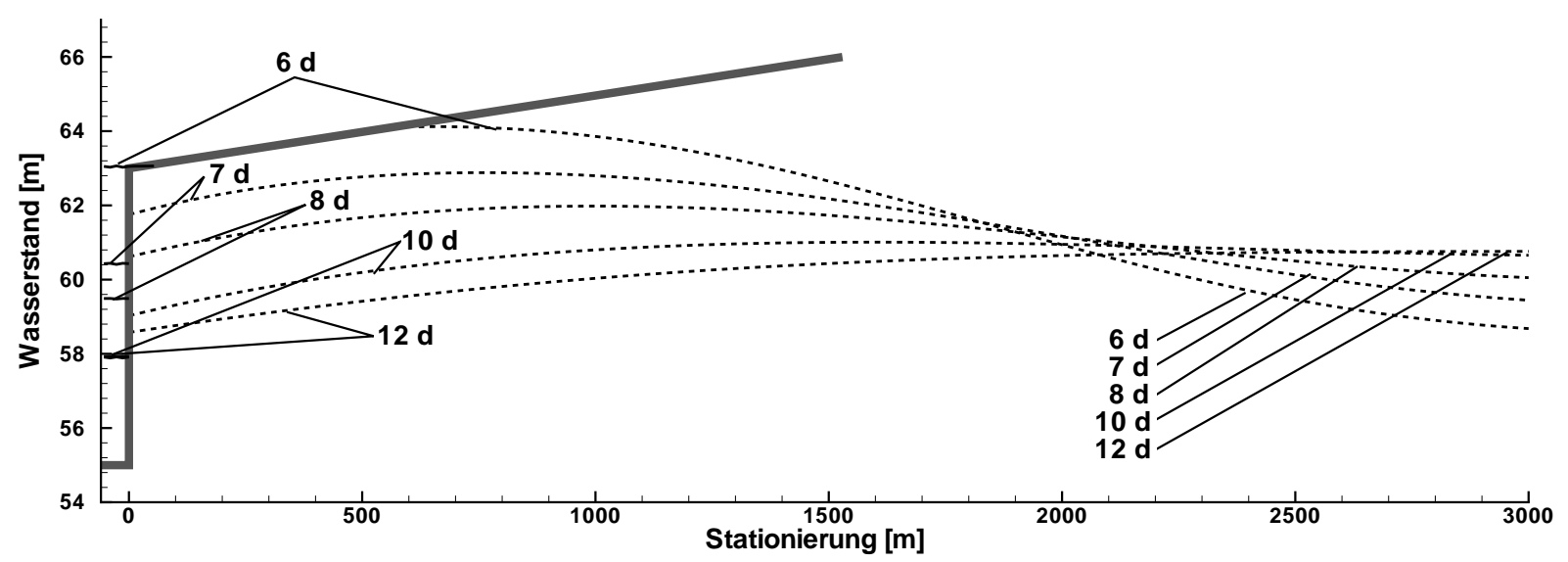

(b) ablaufende Hochwasserwelle

Abbildung 8.4: Gekoppelt berechneter Wasserstand im Fluss und im Untergrund für verschiedene Zeitpunkte (Hochwasserganglinie siehe Abb. 8.5)

sowohl in Richtung des Flusses als auch ins Hinterland aus. An Punkten, die weit weg vom Ufer liegen, beginnt der Anstieg des Grundwassers deshalb erst dann, wenn im Fluss die Hochwasserwelle schon längst abgeklungen ist.

Abbildung 8.5 zeigt die $\mathrm{Zu}$ - und Abflüsse des Modellgebietes insgesamt und aufgeteilt nach der Herkunft aus Ufer oder Überflutungsfläche als Bilanz des Grundwasservorrats. Der Wasserstand des Flusses ist ebenfalls in das Diagramm eingetragen, um den Bezug der Mengen zum Verlauf des Hochwasserereignisses zu erleichtern. Zum Zeitpunkt 2,8 d erreicht der Wasserstand die Marke $63 \mathrm{~m}$. Bis zu diesem Zeitpunkt findet keine Infiltration von der Überflutungsfläche statt, da der Wasserstand diese noch nicht erreicht hat. Bereits wenige Stunden nach Beginn der Überflutung überschreitet die Infiltrationsmenge aus der Überflutung jedoch den Wert der Infiltration über das Flussufer deutlich. Kurz nach Beginn des fünften Tages hat der Flusspegel seinen Maximalwert erreicht und fällt wieder ab. Entsprechend geht die Grundwasservorratsänderung aus 


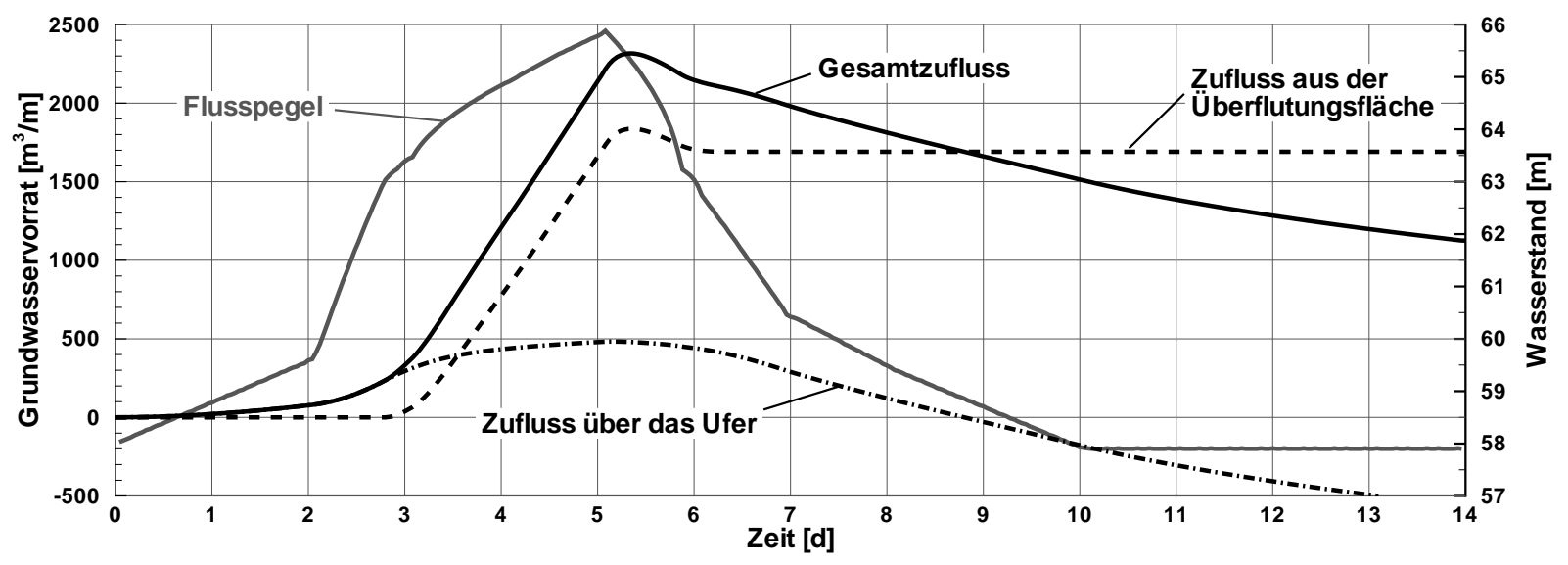

Abbildung 8.5: Gesamter Grundwasservorrat, kumulierte $\mathrm{Zu}$ - und Abflüsse getrennt nach Überflutungsfläche und Ufer sowie Verlauf des Flusspegels über die Simulationszeit

der Überflutungsfläche zurück, da Wasser auch über die geneigte Überflutungsfläche exfiltriert. Insgesamt ist diese Exfiltrationsmenge jedoch vergleichsweise gering und beträgt insgesamt weniger als $200 \mathrm{~m}^{3} / \mathrm{m}$. Nach sechs Tagen fällt der Flusswasserstand wieder auf $63 \mathrm{~m}$ zurück. Die Überflutungsfläche ist nun trocken, so dass keine Wasserstandsdifferenz zwischen Grundwasserleiter und Überflutungsfläche mehr berechnet wird und somit kein Wasseraustausch mehr stattfindet. Die Wassermenge, die über die Überflutungsfläche insgesamt in den unterirdischen Raum einfließt, ändert sich deshalb nicht mehr, sie beträgt etwa 1700 m³. Der Rest des aus der Überflutungsfläche infiltrierten Wassers verbleibt entweder im Grundwasserleiter und fließt weiter ab ins Hinterland oder sie exiltriert über das Ufer in den Fluss. Deshalb zeigt der Anteil der Grundwasservorratsänderung des Ufers am Ende des Simulationszeitraumes einen Fehlbetrag von $560 \mathrm{~m}^{3} / \mathrm{m}$, da mehr Wasser aus dem Grundwasserleiter den Weg über das Ufer zum Fluss hin genommen hat als umgekehrt. Der Gesamtzufluss liegt am Ende des betrachteten Zeitraums um diesen Betrag unter dem Teilzufluss aus der Überflutungsfläche.

\subsubsection{Modelltechnische Aspekte der Kopplung}

Nachdem die Berechnungsergebnisse vorgestellt wurden, soll nun in diesem Abschnitt der Ablauf der Modellkopplung erläutert werden. Die Funktionsweise der Modellkopplung in zwei Richtungen veranschaulicht Abbildung 8.6. Ausgangslage für die Erläuterung ist, dass beide Modellkomponenten für den Zeitpunkt 5,08 d die Berechnungen abgeschlossen haben. Der nächste Arbeitsschritt für das Feflow-Modell ist es nun, den folgenden Zeitschritt 5,12 d zu berechnen. Es benötigt dazu für diejenigen Modellknoten, die mit Ilmoflood-Zellen verbunden sind, Randbedingungswerte aus der Überflutungsflächenberechnung des IImoflood-Modells und fragt diese über die OpenMI-Schnittstelle an. Ilmoflood kann diese Anfrage nicht für den Zeitpunkt 5,12 d erfüllen, da es erst den Zeitpunkt 5,08 d erreicht hat, und muss daher zunächst selbst eine Berechnung ausführen. Es benötigt aber für die selben Zellen, für die bereits das Feflow-Modell Werte 


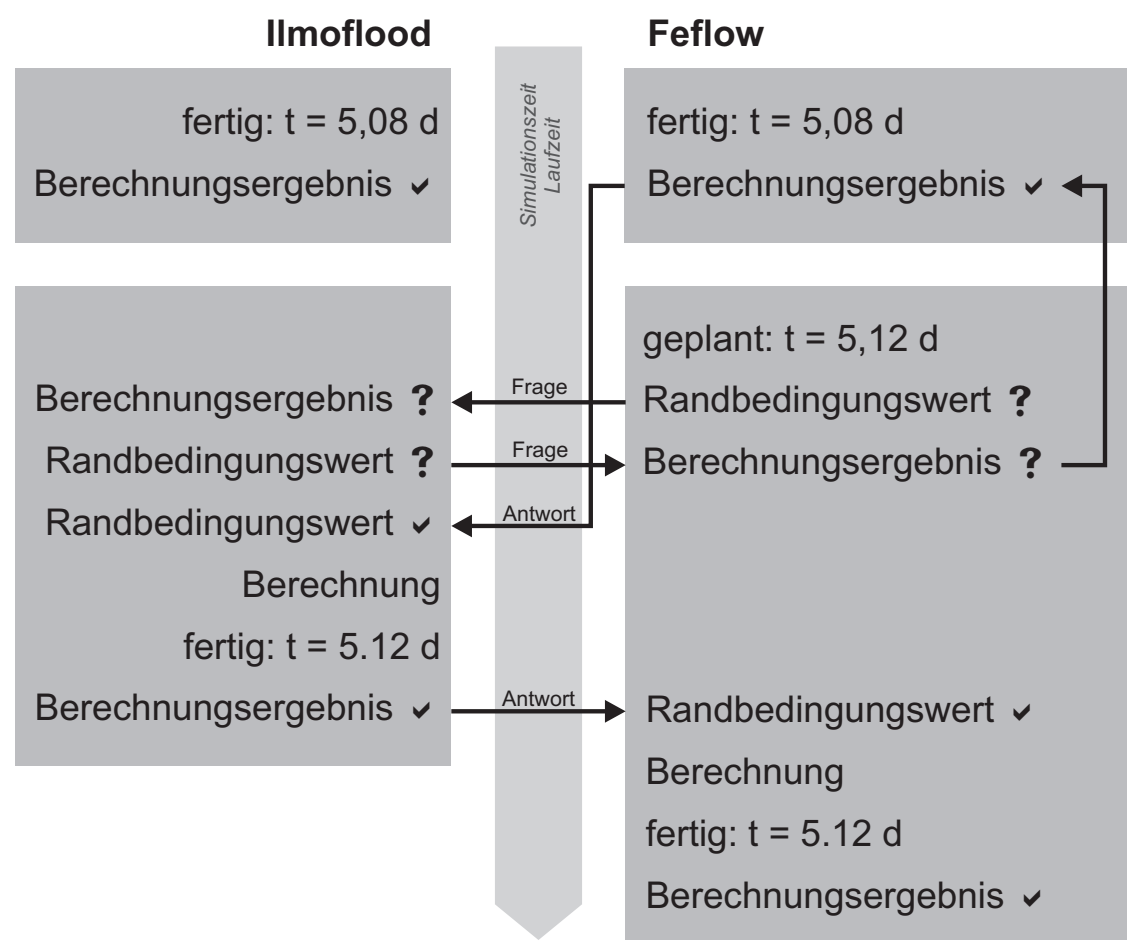

Abbildung 8.6: Funktionsweise des Frage-Antwort-Mechanismus für die bidirektionale Kopplung für gleiche Zeitschritte

für die entsprechenden Knoten angefragt hat, für das Eingabe-Austauschobjekt Q Berechnungsergebnisse der entsprechenden Knoten aus dem Feflow-Modell, die ebenfalls noch nicht vorliegen. Das entsprechende Feflow-Ausgabe-Austauschobjekt Q ist deshalb so programmiert, dass es als Schätzwerte die Berechnungswerte des vorangegangenen Zeitschritts zurückgibt. Mit diesen Schätzwerten ist es dem Ilmoflood-Modell nun möglich, die Berechnung des Zeitschrittes 5,12 d durchzuführen. Die Berechnungsergebnisse stehen nun als Ilmoflood-Ausgabe-Austauschobjekte $\mathrm{h}$ zur Verfügung. Das Feflow-Modell übernimmt diese Berechnungsergebnisse nun als EingabeAustauschobjekt Leakage 1 und führt damit seine geplante Berechnung aus.

Das Ilmoflood-Modell erhält also, weil es mit dem Feflow-Modell extern gekoppelt wurde (siehe Abschnitt 4.2.3), die Werte aus der Rückkopplung immer einen Zeitschritt verzögert. Die Auswirkungen dieses time lag wird für ein Paar gekoppelter räumlicher Stützstellen, das in etwa $500 \mathrm{~m}$ weit vom Ufer entfernt liegt, anhand Abbildung 8.7 verdeutlicht. Es sind hier die berechneten Wasserstände und die sich daraus ergebende Wasserstandsdifferenz $\Delta h$ zwischen der Ilmoflood-Zelle und dem Feflow-Knoten über einen Ausschnitt aus der Simulationszeit von Tag 4 bis zum Tag 6,12 dargestellt. Zusätzlich sind die in den beiden Modellkomponenten angesetzten Austauschmengen pro Zeiteinheit über den Zeitabschnitt und die Unterschiede der jeweiligen Werte aufgeführt.

$\mathrm{Zu}$ Beginn des vierten Tages besteht eine große Differenz zwischen dem Wasserstand auf der Überflutungsfläche und dem Grundwasserstand. Diese Differenz reduziert sich bis zum Tag 5,08. 

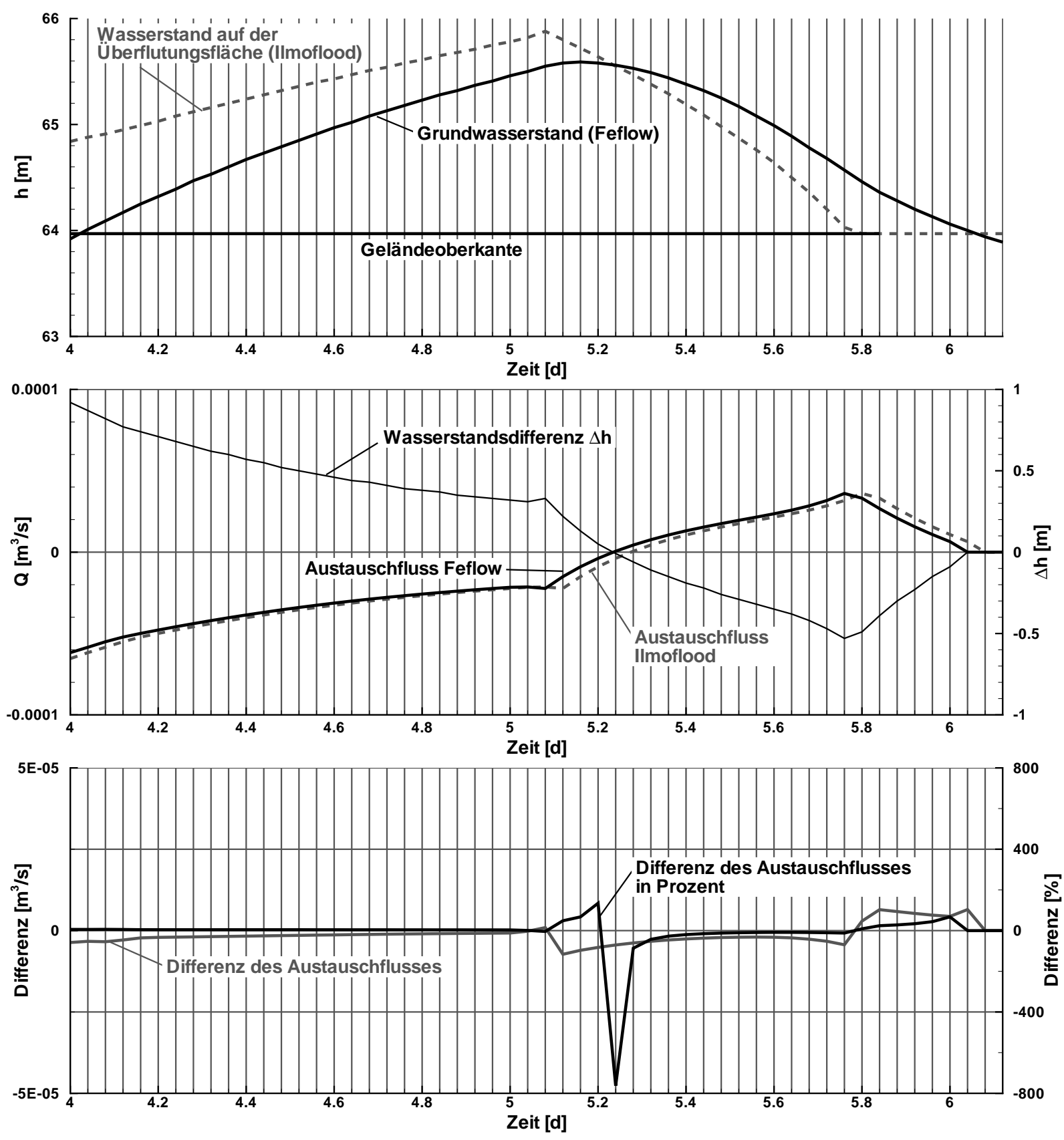

Abbildung 8.7: Verlauf des Wasserstands auf der Überflutungsfläche und des Grundwasserstands (oben), der Wasserstandsdifferenz $\Delta h$ und der Austauschmengen der jeweiligen Modellkomponente (Mitte) und Unterschiede der Austauschmengen (unten) über die Zeit für das Zellen-Knoten-Paar 43-39 (Entfernung vom Ufer etwa $500 \mathrm{~m}$ ). Negative Austauschmengen zeigen entgegen der sonst verwendeten Konvention einen Zufluss zum Grundwasserleiter an. Eine vertikale Gitternetzlinie kennzeichnet eine zeitliche Stützstelle. Die Differenz wurde nach $\Delta Q=Q_{\mathrm{F}}-Q_{\mathrm{G}}$ berechnet, die Prozentangabe der Differenz nach $\Delta Q_{\%}=\frac{\Delta Q}{Q_{\mathrm{F}}}$ bestimmt. 
Von einem Zeitpunkt zum nächsten ändert sich die ausgetauschte Wassermenge nur geringfügig. Der Fehler, der dadurch entsteht, dass im Ilmoflood-Modell die Menge des Feflow-Modells immer zeitverzögert angesetzt wird, nimmt deshalb ab. Bei 5,08 d erreichen die Differenzen besonders kleine Werte, da hier die Wasserstandsdifferenz für 5,04 d und 5,08d fast gleich ist und der Zeitverzug damit keinen Unterschied in der Berechnung verursacht.

Der Knick im Verlauf der Wasserstandsganglinie im Fluss bei 5,08 d verursacht jedoch eine deutlich größere Ungenauigkeit: die Wasserstandsdifferenz sinkt stark, da der Wasserstand im Fluss stark abfällt, während der Grundwasserstand auf seinem Niveau verbleibt. Mit der Wasserstandsdifferenz reduziert sich auch die im Feflow-Modell berechnete Austauschmenge. Dem Ilmoflood-Modell wird jedoch weiterhin die vergleichsweise hohe Menge des vorangegangenen Zeitschritts zugeführt. Entsprechend hoch ist die Differenz zwischen dem, was in diesem Zeitschritt hätte angesetzt werden sollen und dem, was in diesem Zeitschritt angesetzt wurde. Mit fortschreitender Simulationszeit sinkt jedoch diese Differenz.

Beim Wechsel der Fließrichtung, der zwischen 5,2d und 5,24d stattfindet, werden nur geringe Mengen ausgetauscht, so dass die absoluten Unterschiede in den angesetzten Mengen hier nicht so stark ins Gewicht fallen. In der prozentualen Differenz macht sich hier jedoch der Wechsel des Vorzeichens bemerkbar. Nach dem Fließrichtungswechsel steigt der Betrag der Wasserstandsdifferenz $\Delta h$ an, denn der Wasserstand auf der Überflutungsfläche fällt schneller als der Grundwasserstand. Entsprechend steigt auch der Betrag der exfiltrierten Menge. Die Kurve des Austauschflusses von Ilmoflood folgt der entsprechenden Kurve von Feflow weiterhin zeitversetzt. Bei geringen Änderungen über die Zeit gleicht sich die Ilmoflood-Kurve der Feflow-Kurve an.

Am Zeitpunkt 5,8 d erreicht der Wasserstand auf der Überflutungsfläche die Geländeoberkante. Auf der Oberfläche ist nur noch ein dünner Wasserfilm vorhanden. Der Grundwasserspiegel sinkt weiter, am Tag 6,04 erreicht die Wasserstandsdifferenz den Wert Null. Am Zeitschritt danach erhält das IImoflood-Modell ein letztes Mal einen Zufluss aus dem Grundwassermodell.

Es treten zum Teil sehr hohe relative Abweichungen der ausgetauschten Mengen auf (Abbildung 8.7 unten). Maximalwerte liegen hier jedoch im Bereich der Fließrichtungswechsel, wo insgesamt naturgemäß kleine Austauschmengen vorliegen, bei denen schon geringe absolute Unterschiede (Abbildung 8.7 Mitte) prozentuale Werte in die Höhe schnellen lassen. Vor diesem Hintergrund relativiert sich die Bedeutung der Prozentangaben. Bei den absoluten Differenzwerten ist zu berücksichtigen, dass es sich hier lediglich um eine Zeitverschiebung der angesetzten Mengen handelt. Wie bereits im Kapitel 4 erwähnt, ist die externe Kopplung trotz der Ungenauigkeiten derzeit gängige Praxis in der Modellierung. 


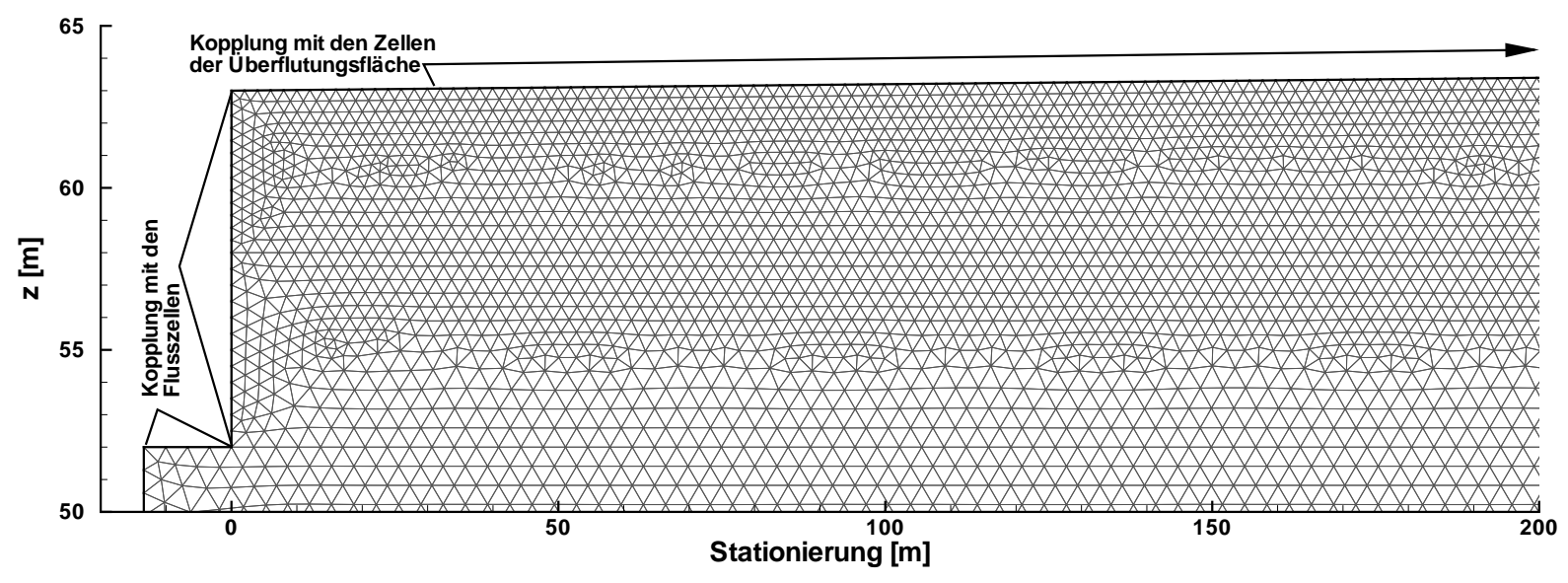

Abbildung 8.8: Ausschnitt aus dem Finite-Elemente-Netz des Richards-Modells und Bereiche der Kopplung

\subsubsection{Ungesättigte Grundwasserströmung}

\subsubsection{Grundwassermodell und Modellkopplung}

Ein Ausschnitt des zweidimensional-vertikalen Finite-Elemente-Netzes, das für die ungesättigte Strömungsberechnung verwendet wird, zeigt Abbildung 8.8. Im Unterschied zur rein gesättigten Modellvariante besteht es aus dreieckigen Elementen. Das Netz ist oben feiner als unten, weil von oben her die Infiltration aus der Überflutungsfläche erwartet wird, während unten rein gesättigte Strömung stattfindet.

Die Modellparameter Durchlässigkeitsbeiwert $k$ und Leakage-Parameter $c_{1}$ sind die gleichen wie beim Grundwassermodell mit gesättigter Strömungsberechnung. Der Speicherkoeffizient beträgt

$$
S_{\mathrm{sp}}= \begin{cases}0,175 & \text { für ungespannte Verhältnisse } \\ 3,0 \cdot 10^{-6} & \text { für gespannte Verhältnisse }\end{cases}
$$

und entspricht damit für den Fall ungespanner Verhältnisse ebenfalls dem Modell mit gesättigter Strömungsberechnung. Ein Unterschied liegt jedoch für gespannte Verhältnisse vor, denn im rein gesättigten Modell wird bedingt durch den Bewegliche-Netze-Ansatz nicht zwischen gespannten und ungespannten Verhältnissen unterschieden (Scherf 2009). Ebenfalls keine Entsprechung im gesättigten Modell gibt es für die Van-Genuchten-Parameter, die die Bewegung des Wassers in der ungesättigten Bodenzone bestimmen. Die Van-Genuchten-Parameter sind zu $\alpha_{\mathrm{G}}=4,11 / \mathrm{m}$, $n_{\mathrm{G}}=1,96$ und $L_{\mathrm{G}}=\frac{1}{2}$ gewählt worden, es handelt sich dabei um Standardwerte des Programms Feflow.

Die Kopplung mit dem Ilmoflood-Modell (Abbildung 8.2a) erfolgt im Prinzip wie beim Modell mit gesättigter Strömungsberechnung, nur dass jetzt einer Ilmoflood-Zelle wegen der feineren 


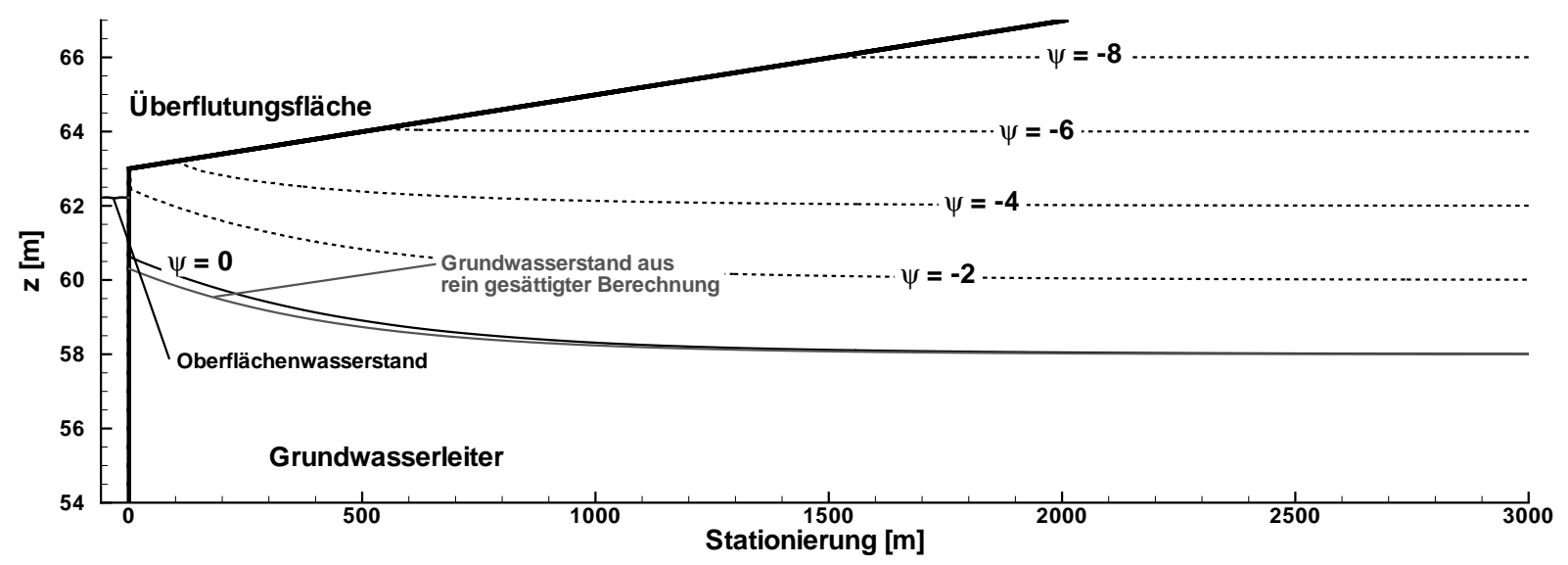

Abbildung 8.9: Ergebnisse der gekoppelten Berechnung mit dem Richards-Grundwassermodell (Isolinien des Matrixpotenzials $\psi$ und Wasserspiegellage an der Oberfläche) für Tag 2,6 (steigender Oberflächenwasserstand) sowie Grundwasserstand aus der gekoppelten Berechnung mit dem rein gesättigten Grundwassermodell aus Abbildung 8.4

Diskretisierung mehrere Feflow-Knoten zugewiesen werden. Das Ilmoflood-Modell übergibt den Wasserstand einer Zelle an mehrere Modellknoten, die korrespondierende Wassermenge der einzelnen Knoten des Grundwassermodells wird aufsummiert und in der betreffenden Zelle angesetzt. Auch beim ungesättigten Modell wird ein Bereich zwischen Ufer und Überflutungsfläche nicht gekoppelt, um eine klare Trennung zwischen beiden Bereichen herzustellen. Um die Vergleichbarkeit mit dem rein gesättigten Modell zu gewährleisten, wird dieser Bereich genau so groß gewählt wie beim rein gesättigten Modell, wegen der feineren Modelldiskretisierung des ungesättigten Modells könnte dieser Abstand jedoch auch kleiner gewählt werden.

\subsubsection{Berechnungsergebnisse}

In Abbildung 8.9 sind die Ergebnisse der gekoppelten Berechnung mit dem Richards-Grundwassermodell und die Wasserspiegellage an der Oberfläche für einen Simulationszeitpunkt 2,6 Tage nach Beginn des Hochwasserereignisses gegeben. Für den Grundwasserleiter sind die Ergebnisse als Isolinien des Matrixpotenzials dargestellt. Ein Matrixpotenzial von Null zeigt die Lage des Grundwasserspiegels an, negative Werte beschreiben den ungesättigten Bereich oberhalb des Wasserspiegels. Während beim dreidimensionalen Streifenmodell aus Abschnitt 8.2.2 das Finite-Elemente-Netz die Bewegung des Wasserstands mitmacht, wird die Bewegung der Grundwasseroberfläche im ungesättigten Modell über eine Veränderung des Druckpotenzials, das über das Mualem-van-Genuchten-Modell mit dem Sättigungsgrad zusammenhängt (siehe Abschnitt 2.3 , erfasst. Zum Vergleich sind die Grundwasserstände aus der gekoppelten Modellrechnung mit rein gesättigter Grundwasserströmung in die Ergebnisdarstellung mit eingetragen. 


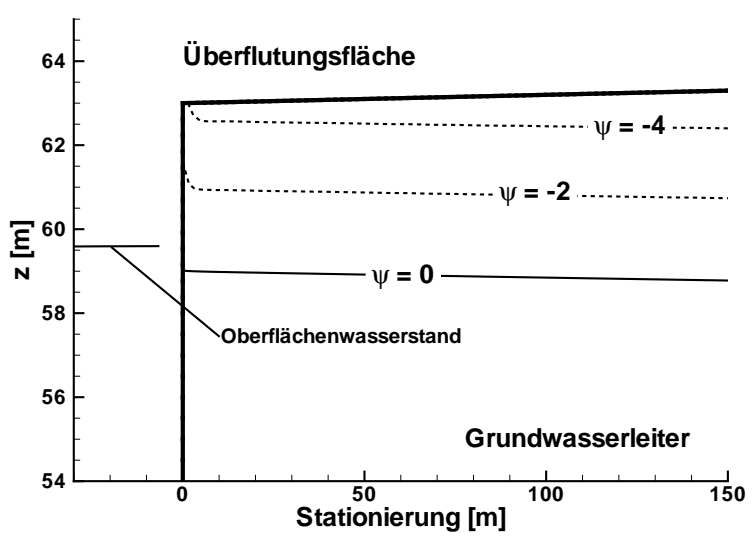

(a) 2 Tage

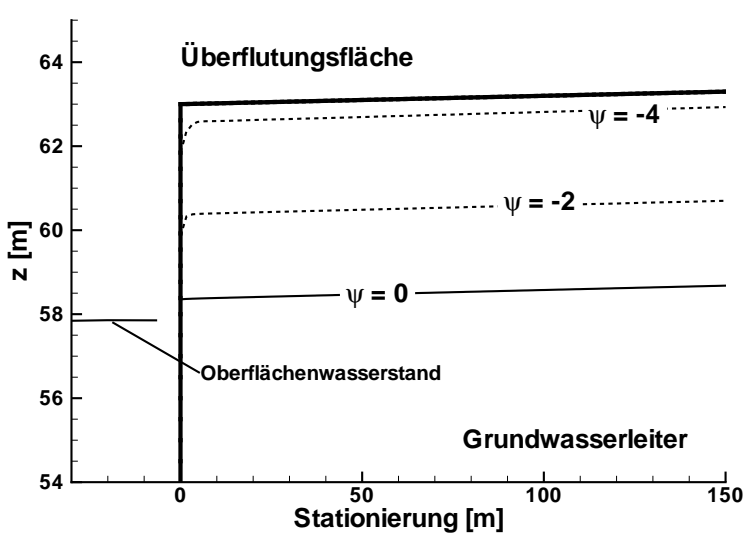

(b) 11 Tage

Abbildung 8.10: Detail des Uferbereich mit Isolinien des Druckpotenzials und dem Wasserstand im Fluss für die Simulationstage 2 und 11

2,6 Tage nach Beginn des Hochwasserereignisses ist der Fluss noch nicht über das Ufer getreten. Der Grundwasserstand aus der ungesättigten Berechnung liegt etwas über dem aus der gesättigten Berechnung. Die Ursache liegt hier in der unterschiedlichen Handhabung der LeakageRandbedingung im Bereich des Ufers. Bei der ungesättigten Variante wird der Uferbereich mit festen Knoten modelliert, die alle mit einer entsprechenden Zelle aus dem Ilmoflood-Modell verbunden sind. Wegen des negativen Matrixpotenzials im ungesättigten Bereich kann die für die Berechnung des Leakage-Flusses $q$ verwendete Grundwasserdruckhöhe $h_{\mathrm{G}}$ unterhalb des Flusswasserstands $h_{\mathrm{F}}$ liegen. Es entsteht damit auch für Knoten, die oberhalb des Grundwasserspiegels liegen, eine Infiltration. An der Uferlinie steigt deshalb zu Beginn des Hochwasserereignisses das Druckpotenzial $\psi$ im Nahbereich des Modellrands oberhalb des Wasserspiegels an, wie in Abbildung 8.10a gezeigt. Bei ablaufender Welle (Abbildung 8.10b) ergibt sich ein entsprechendes Bild. Beim gesättigten Modell gibt es dagegen keinen Knoten, der oberhalb des Grundwasserspiegels liegt, weil das Berechnungsnetz dem Grundwasserstand angepasst wird.

Am fünften Tag (Abbildung 8.11a) ist der Wasserstand im Fluss weiter angestiegen, so dass auch auf der Überflutungsfläche Wasser steht. Unterhalb der überfluteten Fläche kommt es zu einer Infiltration, die im Richards-Modell das Matrixpotenzial reduziert, aber nicht zu einer vollständigen Sättigung führt. Diese Sickerwasserbewegung ist gut zu sehen anhand der $-2 \mathrm{~m}$-Linie, die den Oberflächenwasserspiegel zusammen mit der -4 m- und der -6 m-Linie trifft und in der Nähe der Geländeoberkante vertikal verläuft. Das bedeutet, dass sich das Sickerwasser der Anschauung entsprechend vertikal nach unten bewegt, sich aber nicht nennenswert horizontal ausbreitet. Der Grundwasserstand stimmt gut mit dem entsprechenden Verlauf aus der rein gesättigten Berechnung überein.

Bis zum Tag 5,72 liegt der Oberflächenwasserspiegel über dem Grundwasserstand. Es infiltriert also immer noch Wasser in den Untergrund, obwohl der Wasserstand an der Oberfläche bereits kurz nach dem fünften Tag schon wieder abnimmt. Anhand Abbildung 8.11blässt sich die in den 


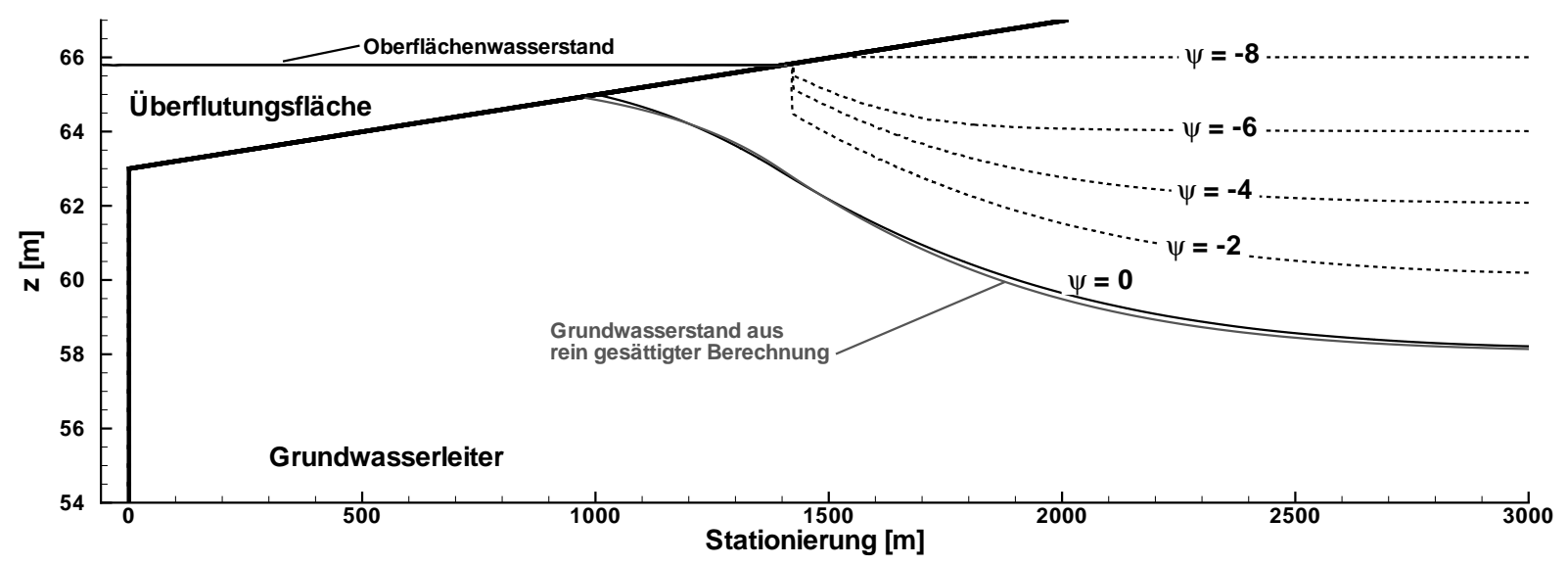

(a) Tag 5, steigender Oberflächenwasserstand

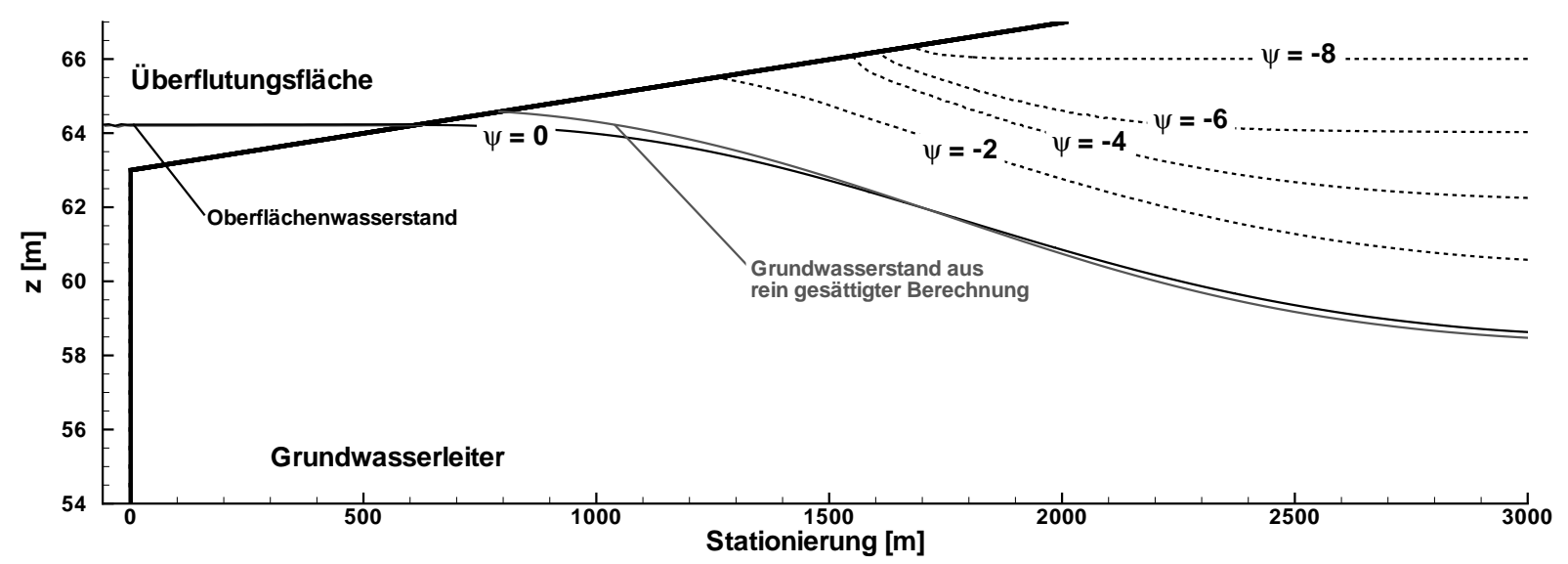

(b) Tag 5,72, fallender Oberflächenwasserstand, Zeitpunkt der maximalen Infiltration über die Überflutungsfläche

Abbildung 8.11: Ergebnisse der gekoppelten Berechnung mit dem Richards-Grundwassermodell (Isolinien des Matrixpotenzials $\psi$ und die Wasserspiegellage an der Oberfläche) für die Tage 5 und 5,72 sowie Grundwasserstand aus der gekoppelten Berechnung mit dem Grundwassermodell mit rein gesättigter Strömung aus Abbildung 8.4

beiden Modellvarianten unterschiedliche Handhabung eines Wechsels zwischen gespannten und ungespannten Verhältnissen nachvollziehen. Bei der rein gesättigten Berechnung bewegt sich das Berechnungsnetz mit dem Grundwasserstand mit, und zwar auch über die gedachte Geländeoberkante hinaus. Es wird dabei nicht zwischen gespannten und ungespannten Verhältnissen unterschieden. Das Porenvolumen des Leitermaterials steht somit auch zur Wasserspeicherung oberhalb der gedachten Geländeoberkante zur Verfügung. Bei der ungesättigten Berechnung wechselt der Speicherkoeffizient bei Erreichen gespannter Verhältnisse, es kann deutlich weniger Wasser gespeichert werden, wenn der Grundwasserspiegel den oberen Modellrand erreicht. Entsprechend schnell reagiert der Grundwasserspiegel auf einen Abfall des Oberflächenwasserstands, der durch die gesetzte Kopplungsrandbedingung eine Wasserentnahme hervorruft. Der ungesättigt berechnete Grundwasserstand $(\psi=0)$ folgt dem Wasserstand auf der Überflutungs- 


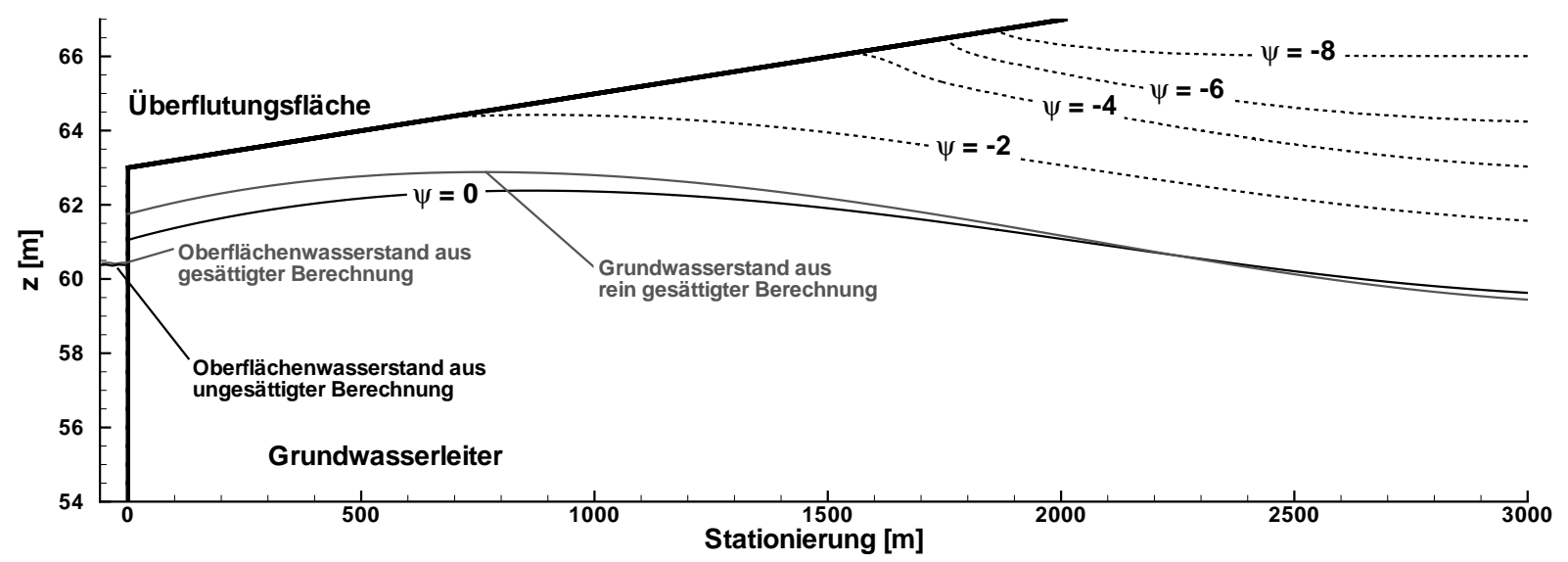

Abbildung 8.12: Ergebnisse der gekoppelten Berechnung mit dem Richards-Grundwassermodell (Isolinien des Matrixpotenzials $\psi$ und die Wasserspiegellage an der Oberfläche) für Tag 7 sowie Grundwasserstand aus der gekoppelten Berechnung mit dem Grundwassermodell mit rein gesättigter Strömung aus Abbildung 8.4

fläche, während der entsprechende Grundwasserstand aus der gesättigten Berechnung langsamer auf eine Entnahme reagiert hat und zum Zeitpunkt 5,72 d noch über dem Grundwasserstand der ungesättigten Berechnung liegt.

Am siebten Tag (Abbildung 8.12) bewegt sich der Fluss wieder in seinem Flussbett. Der Grundwasserspiegel der ungesättigten Berechnung liegt niedriger als bei der rein gesättigten Berechnung. Anhand der Isolinien des Matrixpotenzials wird deutlich, dass noch Wasser in der ungesättigten Bodenzone gespeichert ist. Dieses Wasser sickert langsam nach unten und kann erst verzögert für den Anstieg des Grundwasserspiegels wirksam werden. Darüber hinaus wirkt sich die oben beschriebene in beiden Modellen unterschiedliche Handhabung des Wechsels zwischen gespannten und ungespannten Verhältnissen aus. Bei einer größeren Entfernung vom Ufer sind die Unterschiede zwischen den beiden Modellvarianten geringer als im Nahbereich des Flussufers.

Der Anteil des Grundwasservorrats, der durch den Zufluss über das Ufer entsteht, verläuft bei der ungesättigten Berechnung ungefähr so wie bei der gesättigten Berechnung (Abbildung 8.13). Der Maximalwert des Grundwasservorrats aus Uferspeicherung bei fünf Tagen liegt in der rein gesättigten Berechnung etwas über dem der ungesättigten Berechnung, zum Ende des Hochwasserereignisses liegt der Grundwasservorrat aus Uferspeicherung bei der ungesättigten Berechnung über dem der gesättigten Berechnung. Das bedeutet, dass mit den gewählten Parametern die Leakage-Flüsse der ungesättigten Berechnung betragsmäßig leicht unter denen der gesättigten Berechnung liegen.

Ein Zufluss aus einer Überflutung setzt in beiden Berechnungen nach 2,6 Tagen ein. Mit steigendem Wasserstand werden immer mehr Knoten aktiviert, in denen Wasser in der zweiten Modellvariante zunächst über die ungesättigte Zone versickert. Im Verlauf des Hochwasserereignisses steigt der Grundwasservorrat in beiden Varianten linear an. Allerdings ist die Steigung 

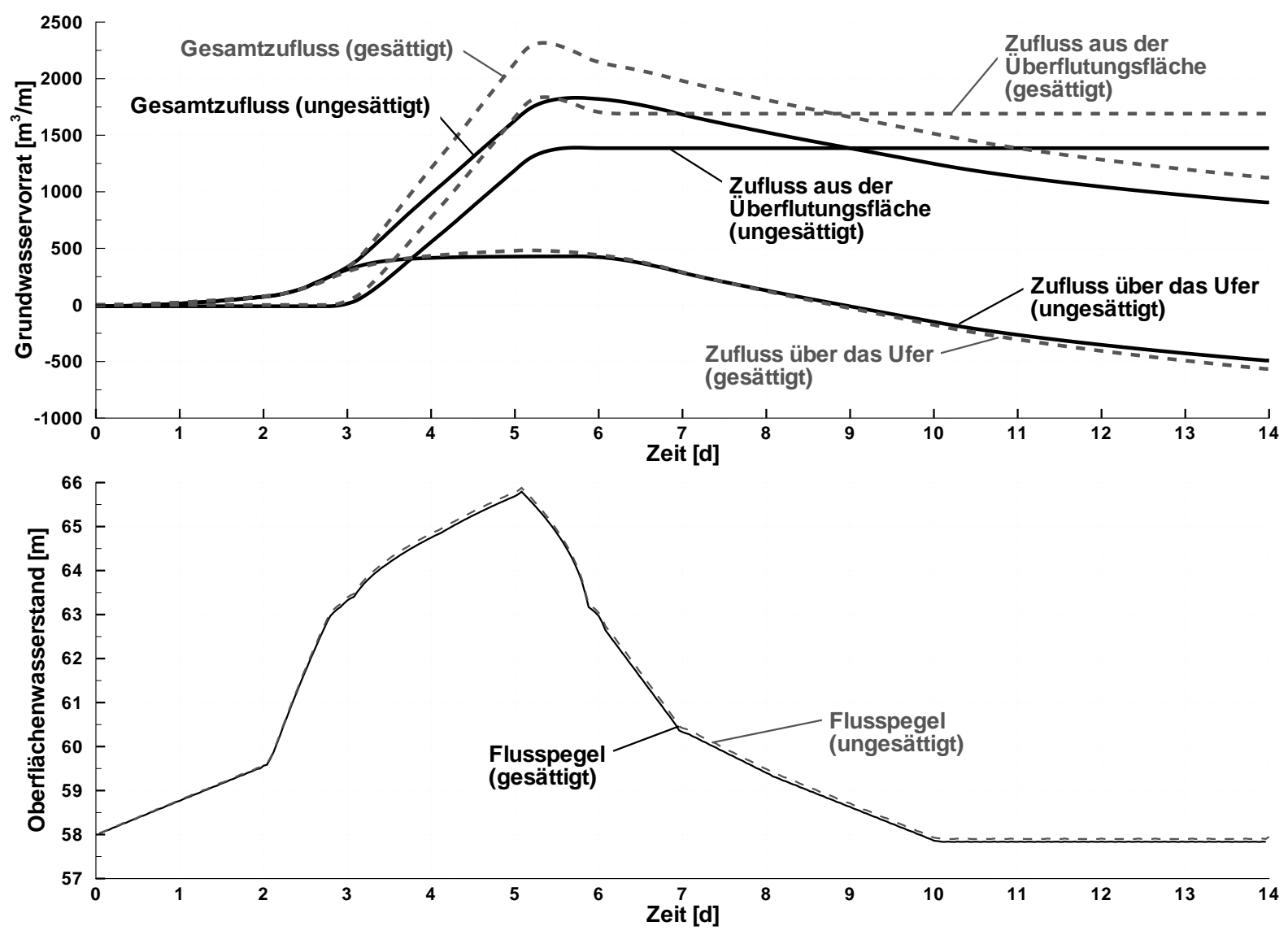

Abbildung 8.13: Gesamte Grundwasservorratsänderung gegenüber dem Ausgangszustand und kumulierte $\mathrm{Zu}$ - und Abflüsse getrennt nach Überflutungsfläche und Ufer sowie Verlauf des Flusspegels über die Simulationszeit aus der gekoppelten Berechnung mit dem Richards-Modell (ungesättigt) und dem rein gesättigten Grundwassermodell

der Grundwasservorratsentwicklung über die Zeit in der gesättigten Berechnung deutlich höher. Wie in Abschnitt 6.4 erläutert, ergeben sich bei rein gesättigter Betrachtung deutlich höhere Infiltrationsraten.

Anders als bei der rein gesättigten Berechnung kommt es bei der ungesättigten Variante nur in ganz geringem Umfang zu einem Rückfluss vom Grundwasserleiter auf die Überflutungsfläche, Im Diagramm ist dies kaum erkennbar. Bei der gesättigten Variante ist der Rückfluss deutlich daran zu erkennen, dass der Grundwasservorrat aus der Überflutungsfläche wieder zurückgeht, nachdem er einen Maximalwerts bei 5,68 d erreicht hat. Hier wirkt sich aus, dass es bei der gesättigten Berechnung keinen Wechsel zwischen gespannten und ungespannten Verhältnissen gibt. Trotz niedrigerer Infiltrationsmenge kann bei steigendem Wasserstand im Fluss der Grundwasserstand aus der ungesättigten Modellierung deshalb auch ungefähr die Höhe des entsprechenden Grundwasserspiegels aus dem rein gesättigten Modell erreichen. 
Die unterschiedlichen Infiltrationsmengen aus der Überflutungsfläche beeinflussen den Wasserstand im Fluss, wie aus dem unteren Teil der Abbildung hervorgeht. Dieser Einfluss ist wie geplant nur sehr gering, so dass die Berechnungsergebnisse beider Varianten hinsichtlich ihrer Wirkung auf den Oberflächenwasserstand vergleichbar sind.

\subsubsection{Schlussfolgerungen und Diskussion}

Die Auswertung der berechneten Grundwasserstände aus den Varianten der rein gesättigten und der ungesättigten Strömungsberechnung zeigt, dass beide Varianten durchaus ähnliche Ergebnisse liefern, obwohl die Bewegung des Wassers in der ungesättigten Zone unterhalb der Überflutungsfläche ganz anders modelliert wird. Im vorliegenden Beispiel steigt der Grundwasserstand durch den Prozess der Uferspeicherung bereits vor der Überflutung an, so dass mit fortschreitender Überflutung weite Teile unterhalb der Überflutungsfläche schnell gesättigt oder nahezu gesättigt werden.

Dennoch ergeben sich in den Berechnungen Unterschiede, wenn auf die Abbildung der ungesättigten Bodenzone im Modell verzichtet und die Strömung als rein gesättigt aufgefasst wird. Allerdings kommt im gezeigten Beispiel noch hinzu, dass ein Wechsel zwischen gespannten und ungespannten Verhältnissen im rein gesättigten Modell nicht erfasst wird.

An der Entwicklung des Grundwasservorrats über die Simulationszeit wird deutlich, dass die Überflutung in den gezeigten Beispielen im Vergleich zur Uferspeicherung den größeren Anteil der Zuflussmenge stellt, da die Kontaktfläche zwischen dem Oberflächenwasser und dem Grundwasser wesentlich größer ist als im Falle der Uferspeicherung. Abflüsse aus dem Grundwasserleiter über die Überflutungsfläche sind jedoch vergleichsweise klein. Das über die Überflutungsfläche von oben in den Grundwasserleiter eindringende Wasser bewegt sich im Grundwasserleiter entweder in Richtung Hinterland oder es verlässt ihn über das Ufer.

Insbesondere der hohe numerische Aufwand und die zusätzlichen Parameter, die bei einer numerischen Lösung der Richards-Gleichung in Kauf genommen werden müssen, rechtfertigen vor diesem Hintergrund eine rein gesättigte Betrachtung für praktische und planerische Anwendungen im Zusammenhang mit unterirdischem Hochwasser. So lag die Berechnungsdauer für das gekoppelte Modell mit rein gesättigter Grundwasserströmung in der Größenordnung von acht Minuten, während für das gekoppelte Modell mit Richards-Gleichung etwa sieben Stunden benötigt wurden.

Bedingt durch die externe Kopplung stimmen die in einem Zeitschritt angesetzten Austauschraten der beiden Modelle nicht überein, da das Überflutungsflächenmodell die Berechnungsergebnisse des Grundwassermodells zeitversetzt erhält. Diese zeitliche Verschiebung (time lag) ist im vorliegenden Beispiel unkritisch, da sie nicht zu numerischen Schwingungen führt. Für andere Systeme ist nicht auszuschließen, dass die externe Kopplung Ungenauigkeiten mit sich bringt, die numerische Schwingungen und Instabilitäten hervorrufen können. Die externe Kopplung ist jedoch nicht massentreu, die berechneten Wasserstände des Oberflächen- und des Grundwassermodells weichen deshalb von einer unbekannten exakten Lösung ab. An den Ergebnissen wird 
deutlich, dass für die vorliegende Art der Modellkopplung die Ungenauigkeiten durch diesen Zeitversatz umso kleiner werden, je geringer sich die Austauschraten über die Zeit verändern. Bei einem Wechsel der Fließrichtung kommt es wegen des Vorzeichenwechsels zu besonders großen relativen Abweichungen, die absolut gesehen jedoch vernachlässigbar sind, weil vor und nach dem Fließrichtungswechsel nur besonders geringe Mengen ausgetauscht werden. Die Zeitschrittweite wurde für das Beispiel relativ klein gewählt. Je kleiner die Zeitschrittweite ist, desto geringer wird auch die Differenz zwischen dem Schätzwert eines Austauschflusses, der vor der Berechnung eines Zeitschrittes zur Verfügung steht, und dem Wert, der sich nach vollständiger Berechnung des Zeitschrittes ergibt. Eine geringe Zeitschrittweite kann damit zur Reduzierung der Ungenauigkeiten beitragen (Gijsbers et al. 2005).

\subsection{Qualmwasser und Infiltration}

\subsubsection{Einführung}

Während im Beispiel aus Abschnitt 8.2 das Zusammenwirken der Prozesse Uferspeicherung und Infiltration aus einer Überflutungsfläche im Vordergrund steht, wird in diesem Abschnitt ein Beispiel vorgestellt, bei dem die Infiltration mit Qualmwasseranstieg zusammenwirkt (Abbildung 8.14). Grundwasser tritt an einer Stelle an der Oberfläche aus und fließt an der Oberfläche über ein unterirdisches Hindernis hinweg, um an einer anderen Stelle wieder von der Oberfläche her in den Grundwasserleiter einzudringen. Während im vorangegangenen Abschnitt das Oberflächenwassermodell im Wesentlichen die Randbedingung für die Grundwasserströmung geliefert hat, der Einfluss der Rückkopplung jedoch bewusst klein gehalten wurde, ist die Kopplung in beide Richtungen für dieses Beispiel von besonderer Bedeutung.

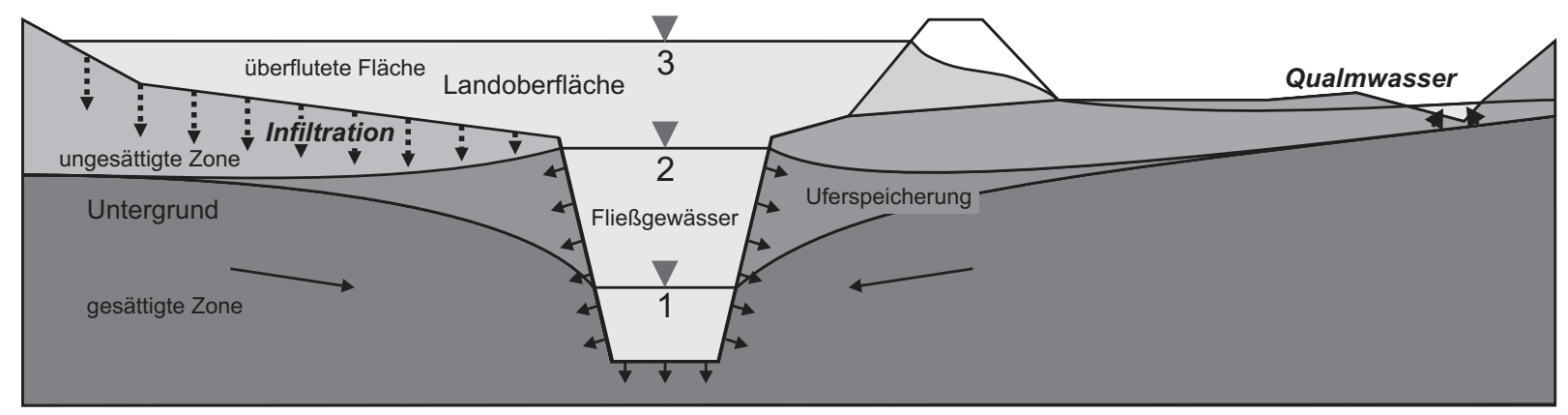

Abbildung 8.14: Die Wechselwirkungsprozesse Qualmwasser und Infiltration im Zusammenhang mit dem unterirdischem Hochwasser

Vor dem Hintergrund, dass in der praktischen Anwendung meistens zweidimensional-horizontale rein gesättigte Grundwassermodelle eingesetzt werden (Abschnitt 1.2), wird das Grundwassermodell dieses Beispiels entsprechend aufgebaut. Dennoch verläuft die Hauptströmung in x-Richtung, um die ablaufenden Prozesse gut veranschaulichen zu können. Die Modellgebiete 


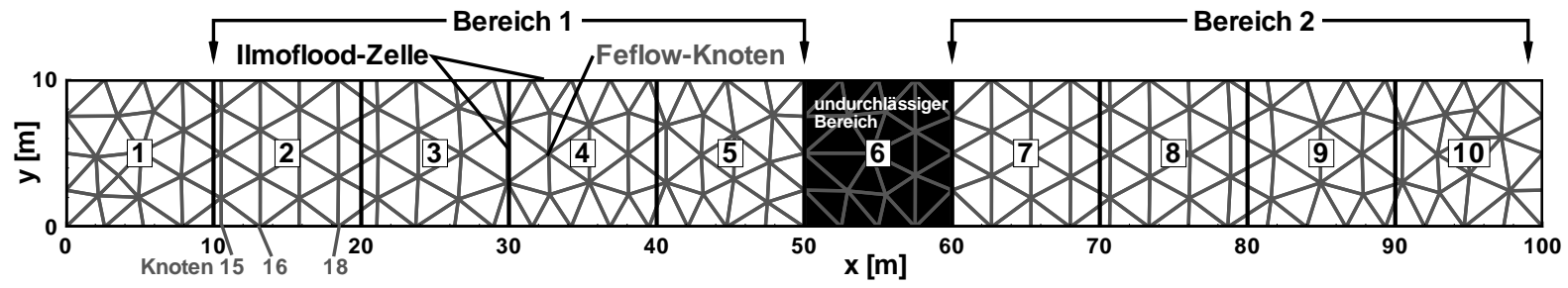

Abbildung 8.15: Berechnungsnetze des Ilmoflood- und des Feflow-Modells mit Zellennummerierung und Einteilung von Kopplungsbereichen (Draufsicht)

des Oberflächenwasser- und des Grundwassermodells stimmen überein, nicht jedoch die Netzgeometrie. Ähnlich wie beim Beispiel aus Abschnitt 8.2 werden hier mehrere Grundwassermodellknoten einer Zelle des Oberflächenmodells zugewiesen. Die Berechnungsnetze stammen aus der Arbeit von Scherf (2009).

\subsubsection{Modellaufbau}

Eine Draufsicht über die Berechnungsnetze des Feflow-Grundwassermodells und des IImofloodOberflächenwassermodells zeigt Abbildung 8.15. In der horizontalen Ebene schließen die Modellgebiete ein Rechteck der Grundfläche $100 \cdot 10 \mathrm{~m}$ ein. Das Storage-Cell-Modell besteht entsprechend des Modellkonzeptes aus quadratischen Zellen, das Grundwassermodell ist aus dreieckigen finiten Elementen aufgebaut. Die Kopplung zwischen den beiden Netzen wird so umgesetzt, dass alle Feflow-Knoten mit derjenigen Ilmoflood-Zelle verbunden werden, die sie einschließt. Von der Kopplung ausgenommen sind jedoch Grundwassermodellknoten, die innerhalb der Zellen 1 und 6 liegen, da hier kein Austausch mit der Landoberfläche beabsichtigt ist, und die Knoten am rechten Modellrand, weil hier eine Randbedingung angesetzt wird. Die Bereiche 1 und 2, in denen beide Modelle gekoppelt werden, sind in Abbildung 8.15 eingezeichnet.

Die Durchlässigkeitseigenschaften des Grundwassermodells sind so gewählt, dass das Modellgebiet in der Mitte durch einen undurchlässigen Bereich in zwei Teile aufgeteilt wird. Die Mächtigkeit des Grundwasserleiters ist jedoch mit $2 \mathrm{~m}$ überall gleich. Die Geländeoberkante verläuft waagerecht. Im Ilmoflood-Modell ist deshalb für alle Zellen die gleiche Höhenlage von $1 \mathrm{~cm}$ zugewiesen, nur über dem undurchlässigen Bereich ist eine Erhöhung von $31 \mathrm{~cm}$ eingerichtet. Beim Ilmoflood-Modell sind keine seitlichen Zuflüsse vorgesehen, Wasser kann allein aus dem Grundwasserleiter über die Modellkopplung in das Oberflächenmodellgebiet einfließen. Auf der linken Seite des Grundwassermodells sind die vier Knoten am Modellrand mit einer Zuflussrandbedingung belegt, die von Beginn der Simulation an bis zum Tag 0,5 einen konstanten Zufluss $Q_{\mathrm{RB}}$ von $250 \mathrm{~m}^{3} / \mathrm{d}$ je Knoten zufließen lässt:

$$
Q_{\mathrm{RB}}= \begin{cases}250 \mathrm{~m}^{3} / \mathrm{d} & \text { für } \quad 0<t \leq 0,5 \mathrm{~d} \\ 0 \mathrm{~m}^{3} / \mathrm{d} & \text { für } t>0,5 \mathrm{~d}\end{cases}
$$




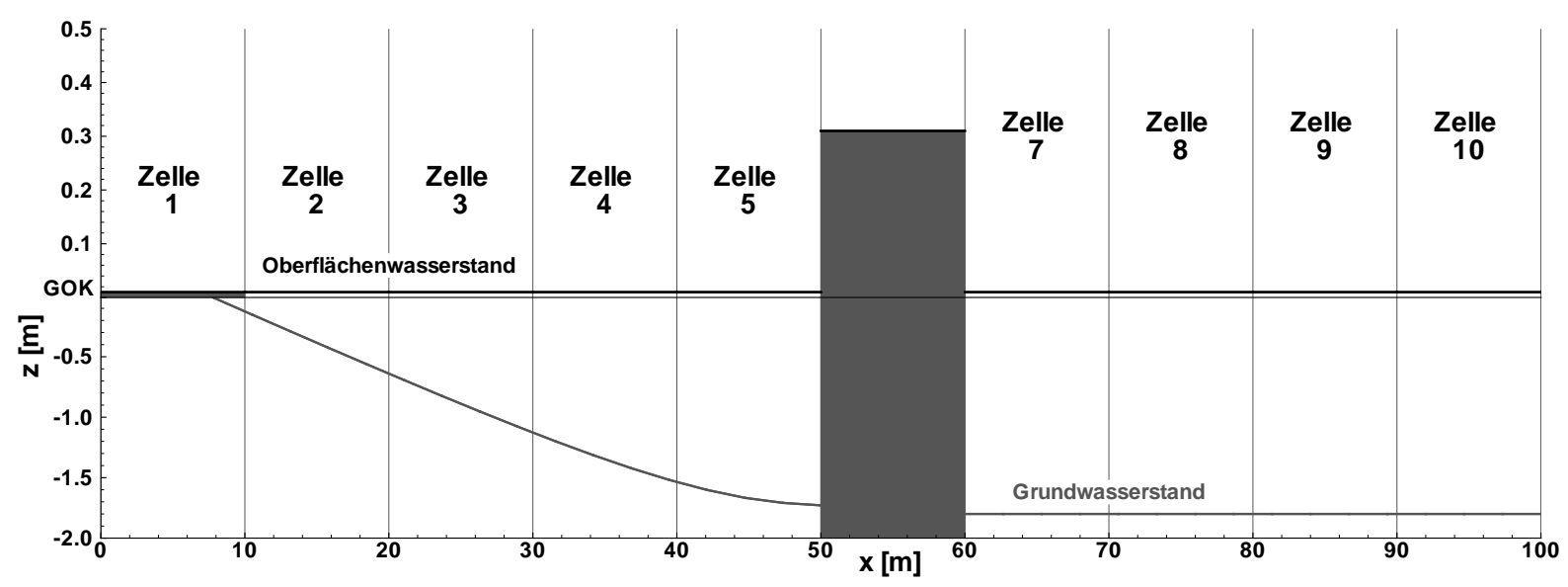

Abbildung 8.16: Grundwasserstand (unterhalb der Geländeoberkante GOK) und Oberflächenwasserstand (oberhalb der Geländeoberkante) zur Simulationszeit 0,15d (unterschiedliche Skaleneinteilung auf der Ordinate)

Die Wirkung dieser Randbedingung zeigt Abbildung 8.16. Am linken Modellrand ist durch den Zufluss bereits ein Anstieg des Grundwasserstands eingetreten, unterhalb der Zelle 1 erreicht der Grundwasserstand die Geländeoberkante (GOK) und es bilden sich gespannte Grundwasserverhältnisse. Die Abbildung zeigt auch die vertikale Ausdehnung des Grundwasserleiters, der aus einem Sandboden mit einem Durchlässigkeitsbeiwert von $k=0,74 \cdot 10^{-4} \mathrm{~m} / \mathrm{s}$ besteht. Der undurchlässige Bereich wird mit einem $k$-Wert von $1,0 \cdot 10^{-20} \mathrm{~m} / \mathrm{s}$ modelliert. Die Speicherkoeffizienten sind

$$
S_{\mathrm{s}}= \begin{cases}0,4 & \text { für ungespannte Verhältnisse } \\ 1,0 \cdot 10^{-4} & \text { für gespannte Verhältnisse. }\end{cases}
$$

$\mathrm{Zu}$ beachten ist, dass oberhalb der Geländeoberkante die Skaleneinteilung der Höhendarstellung nicht derjenigen unterhalb der Geländeoberkante entspricht. Das Modell gibt vereinfacht die Situation wieder, dass ein Zufluss aus einem größeren Fließgewässer durch ein parallel zum Flusslauf liegendes unterirdisches Bauwerk wie zum Beispiel einen Ufertunnel gehemmt wird. Anfänglich steht auf der Landoberfläche kein Wasser. Vor dem unterirdischen Bauwerk kann Wasser aus dem Untergrund austreten, dann über das Bauwerk fließen und dahinter wieder versickern. Für die Oberfläche wird ein Rauheitsbeiwert von $k_{\mathrm{St}}=70 \mathrm{~m}^{\frac{1}{3}} / \mathrm{s}$ für den Bereich des Bauwerks angesetzt, alle anderen Zellen erhalten Rauheitsbeiwerte von $k_{\mathrm{St}}=35 \mathrm{~m}^{\frac{1}{3}} / \mathrm{s}$.

Die Austauschobjekte, die über die Modellverbindungen angesteuert werden, fasst Tabelle 8.3 zusammen. In Bereich 1 gibt es keine Deckschicht über dem Grundwasserleiter. Das Wasser soll hier ohne Widerstand auch dann an der Oberfläche austreten können, wenn die entsprechende Zelle des Oberflächenmodells trocken ist. Steht an der Oberfläche jedoch Wasser, muss sich dies auch im Grundwassermodell auswirken. Dies ist mit dem Eingabe-Austauschobjekt Sickerfläche möglich. Wenn vom Oberflächenmodell ein Wasserstand übergeben wird, dann fließt er als h-Randbedingung in das Grundwassermodell ein. Ist die Oberfläche aber trocken, 
Tabelle 8.3: Übersicht der Austauschobjekte zur Verbindung zwischen IImoflood-Zellen und FeflowKnoten

\begin{tabular}{cllllc}
\hline Bereich & \multicolumn{2}{l}{ Ilmoflood-Modell } & & \multicolumn{2}{c}{ Feflow-Modell } \\
\hline \multirow{2}{*}{1} & Ausgabe & $\mathrm{h}$ & $\rightarrow$ & Eingabe & $\mathrm{h}$ \\
& Eingabe & $\mathrm{Q}$ & $\leftarrow$ & Ausgabe & Sickerfläche \\
\hline \multirow{2}{*}{2} & Ausgabe & $\mathrm{h}$ & $\rightarrow$ & Eingabe & Leakage $1^{a}$ \\
& Eingabe & $\mathrm{Q}$ & $\leftarrow$ & Ausgabe & $\mathrm{Q}$ \\
\hline
\end{tabular}

${ }^{a}$ Kontaktfläche abhängig vom Grundwasserstand

dann wird dem Grundwassermodell das Wasser, das an der Oberfläche austritt, entzogen und dem Oberflächenmodell als Q-Randbedingung zugefügt. Liegt der Grundwasserstand bei trockener Oberfläche unterhalb der Geländeoberkante, kommt es zu keinem Austausch. Anders als bei Leakage-Austauschobjekten ist es so möglich, dass auch Wasser an das Oberflächenmodell übergeben wird, wenn dort kein Wasser vorhanden ist. Die Behandlung einer möglichen Infiltration als h-Randbedingung eignet sich nach den Überlegungen aus Kapitel 6jjedoch nur für gut durchlässiges Material und stellt eine Vereinfachung des Infiltrationsprozesses dar.

Im Bereich 2 wird eine Deckschicht angenommen, die in bewährter Weise (siehe Abschnitt 8.2) im Grundwassermodell über das Eingabe-Austauschobjekt Leakage 1 umgesetzt wird. Vom Ilmoflood-Modell wird dafür das Ausgabe-Austauschobjekt h benötigt. Die korrespondierende Randbedingungsmenge dieser Leakage-Randbedingung wird aus der Budgetierung des Grundwassermodells übernommen und als Q-Randbedingung im Ilmoflood-Modell umgesetzt.

Die zeitliche Abfolge der Berechnung erfolgt wie in den Beispielen aus Abschnitt 8.2 so, dass zunächst das Feflow-Modell beim Ilmoflood-Modell Daten für die Berechnung eines unbekannten Zeitschritts anfragt. Das IImoflood-Modell führt die Berechnung mit Schätzwerten des Grundwassermodells zuerst aus und übergibt seine Berechnungsergebnisse dann an das Grundwassermodell.

Damit der Grundwassermodellraum nicht vollläuft, ist auf der rechten Seite für die Randknoten eine Sickerflächenrandbedingung (siehe Abschnitt 7.3) angesetzt, die für den Fall, dass der Grundwasserstand dort auf $-1,8 \mathrm{~m}$ ansteigt, den Abstrom aus dem Modellgebiet so einstellt, dass ein Wasserstand von -1,8 m gehalten wird. Die Zeitschritte sind in beiden Modellen gleich. Die Zeitschrittweite beträgt $0,01 \mathrm{~d}$.

\subsubsection{Berechnungsergebnisse}

In Abbildung 8.16 erreicht der Grundwasserstand im Bereich der Zelle 1 schon die Oberfläche. Allerdings ist in diesem Bereich keine Modellkopplung vorgesehen, so dass sich dort gespannte Verhältnisse ausbilden, ohne dass eine Wechselwirkung mit dem Wasser auf der Landoberfläche 


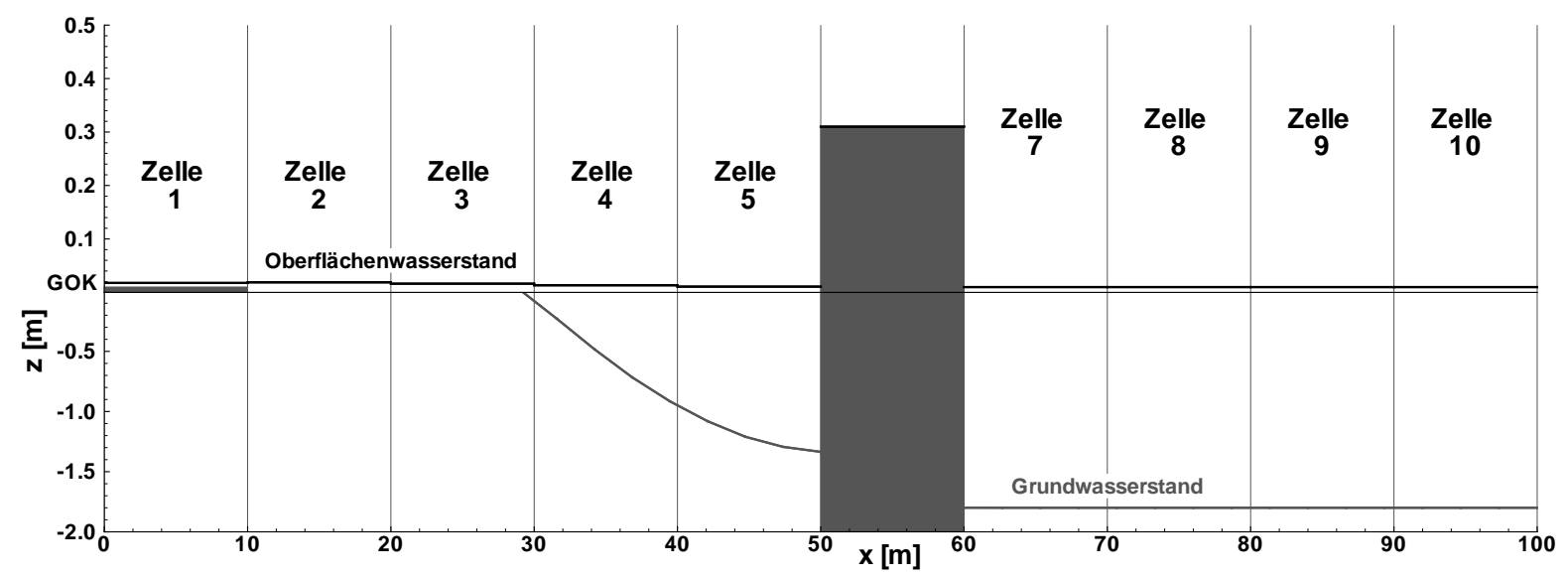

(a) $0,20 \mathrm{~d}$

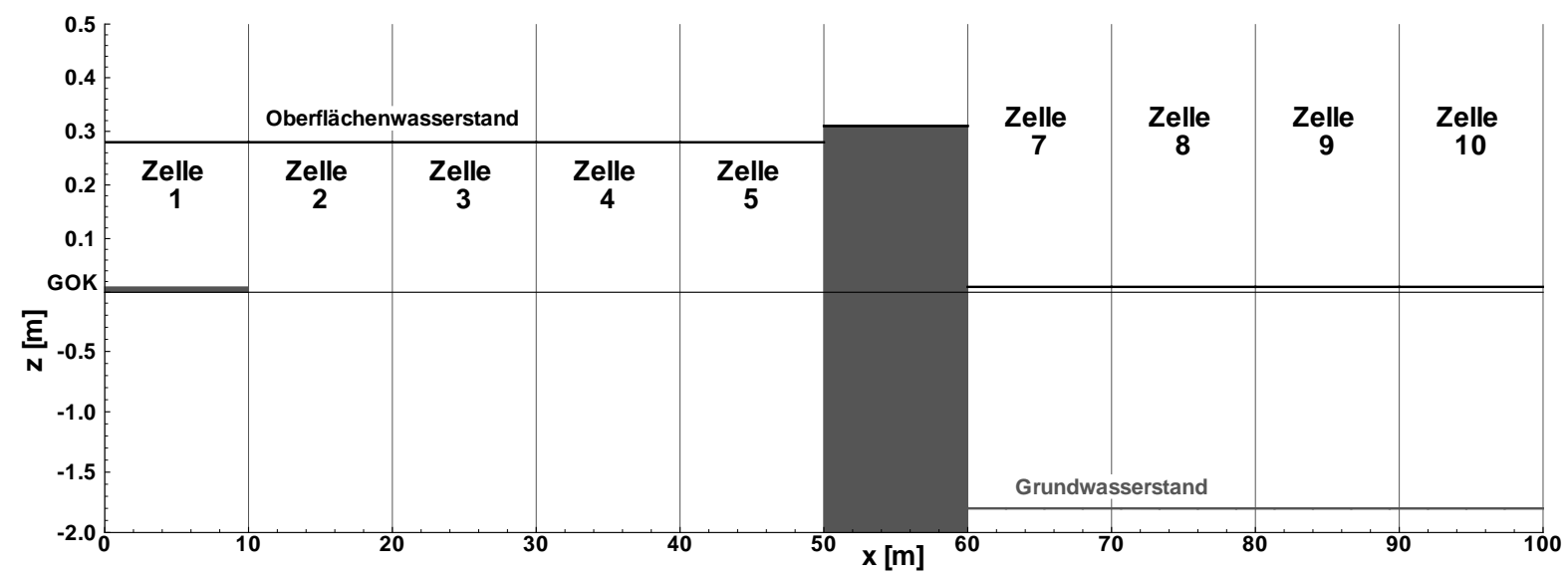

(b) $0,32 \mathrm{~d}$

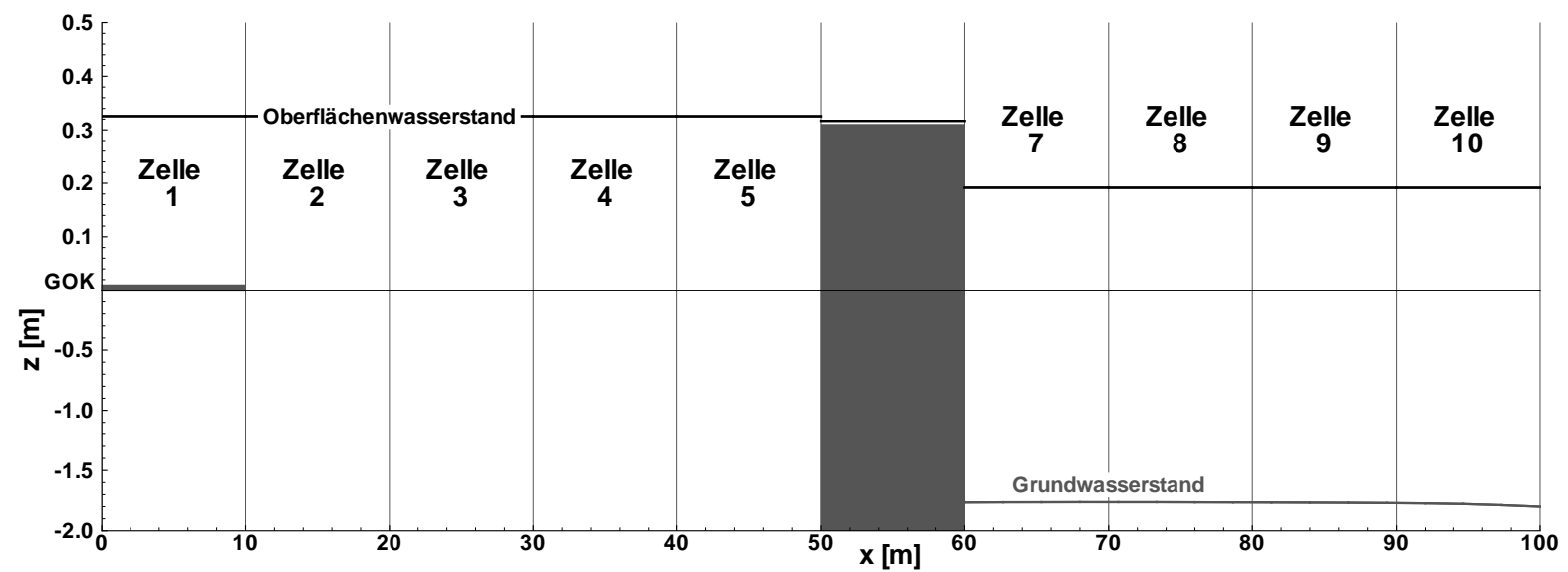

(c) $0,40 \mathrm{~d}$

Abbildung 8.17: Grundwasserstand und Oberflächenwasserstand für die Simulationszeitpunkte 0,20 d, 0,32 d und 0,40 d (unterschiedliche Skaleneinteilung auf der Ordinate) 
stattfindet. Erst 0,05 d später erreicht der Grundwasserspiegel im Bereich der Zellen 2 und 3 die Geländeoberfläche. Für das IImoflood-Modell entsteht deshalb ein Zufluss, der sich in Form eines leicht angestiegenen Wasserspiegels an der Oberfläche in Abbildung 8.17a äußert. Das Wasser breitet sich im Vergleich zum Grundwasser nun schnell nach links und nach rechts aus. Dort, wo der Grundwasserstand bereits die Geländeoberkante erreicht hat und eine Modellkopplung vorgesehen ist, sind der Wasserstand an der Oberfläche und im Grundwasser gleich, denn der Oberflächenwasserstand stellt hier eine h-Randbedingung für das Grundwassermodell. Wegen der gespannten Verhältnisse ist der Speicherkoeffizient relativ klein, so dass die der h-Randbedingung im Grundwassermodell korrespondierende Austauschwassermenge ebenfalls klein ist und den Wasserstand im Oberflächenmodell kaum verändert.

Der anhaltende Zufluss ins Grundwassermodellgebiet sorgt in Bereich 1 für einen weiteren Anstieg des Wassers an der Oberfläche (Abbildung 8.17b). Zum Zeitpunkt 0,40 d (Abbildung 8.17c) erreicht der Oberflächenwasserstand die Oberkante des unterirdischen Bauwerks. Wasser gelangt nun von oben in den rechten Bereich, der mit den Zellen 7 bis 10 abgedeckt wird. Im bisher vom Zufluss auf der linken Seite wegen des unterirdischen Bauwerks unbeeinflusst gebliebenen rechten Bereich des Grundwassermodells steigt der Grundwasserstand nun an, weil das Wasser von der Oberfläche her infiltriert.

Zum Zeitpunkt 0,55 d ist der Zufluss zum Grundwasser entsprechend der angesetzten Ganglinie (Gleichung 8.2) bereits abgeklungen. An der Oberfläche ist der maximale Wasserstand erreicht (Abbildung 8.18a). Durch die Infiltration im rechten Teil des Modellgebietes steigt hier der Grundwasserstand weiter an, gleichzeitig sinkt der Wasserstand an der Oberfläche im linken und im rechten Bereich ab. Am rechten Modellrand verbleibt der Grundwasserstand wegen der dort gesetzten Randbedingung bei $-1,8 \mathrm{~m}$. Die Abbildung $8.18 \mathrm{~b}$ zeigt die Situation, nachdem der Wasserstand bis auf die Bauwerksoberkante auf Höhe von 0,31 $\mathrm{m}$ abgesunken ist. Da eine Infiltration nur im rechten Bereich stattfindet, sinkt der Wasserstand hier weiter, während er im linken Bereich auf Höhe der Bauwerksoberkante verbleibt.

Für die Knoten 15 und 18 zeigt Abbildung 8.19 jeweils die Ganglinie des Wasserstands, des Randbedingungswertes, der aus der Kopplung gesetzt wird, und der korrespondierenden Durchflussmenge für den ersten Teil der Simulationszeit. Die Lage dieser beiden Knoten ist in Abbildung 8.15 eingezeichnet. Knoten 15 liegt von links aus gesehen direkt hinter dem nicht gekoppelten Bereich, wo sich gespannte Grundwasserverhältnisse ausbilden. An Knoten 15 kommt es deshalb zum Qualmwasseraustritt. Knoten 18 liegt etwas weiter entfernt von dieser Stelle.

Die Verbindung des Knotens 15 mit Zelle 2 über das Ausgabe-Austauschobjekt „Sickerfläche“ (Gleichungen 7.1 und 7.2) bewirkt, dass ein Randbedingungswert auf der Höhenlage des Knotens von $0 \mathrm{~m}$ gesetzt wird. Der Ansatz der Nebenbedingung nach Gleichung 7.2 lässt nur einen Abstrom aus dem Modellgebiet heraus zu. Solange der Grundwasserstand noch nicht die Geländeoberkante erreicht hat, ist dieser Abstrom Null. Ohne die Nebenbedingung würde in diesem Fall ein Zustrom entstehen, da der Wert der Randbedingung höher als der Grundwasserstand ist. 


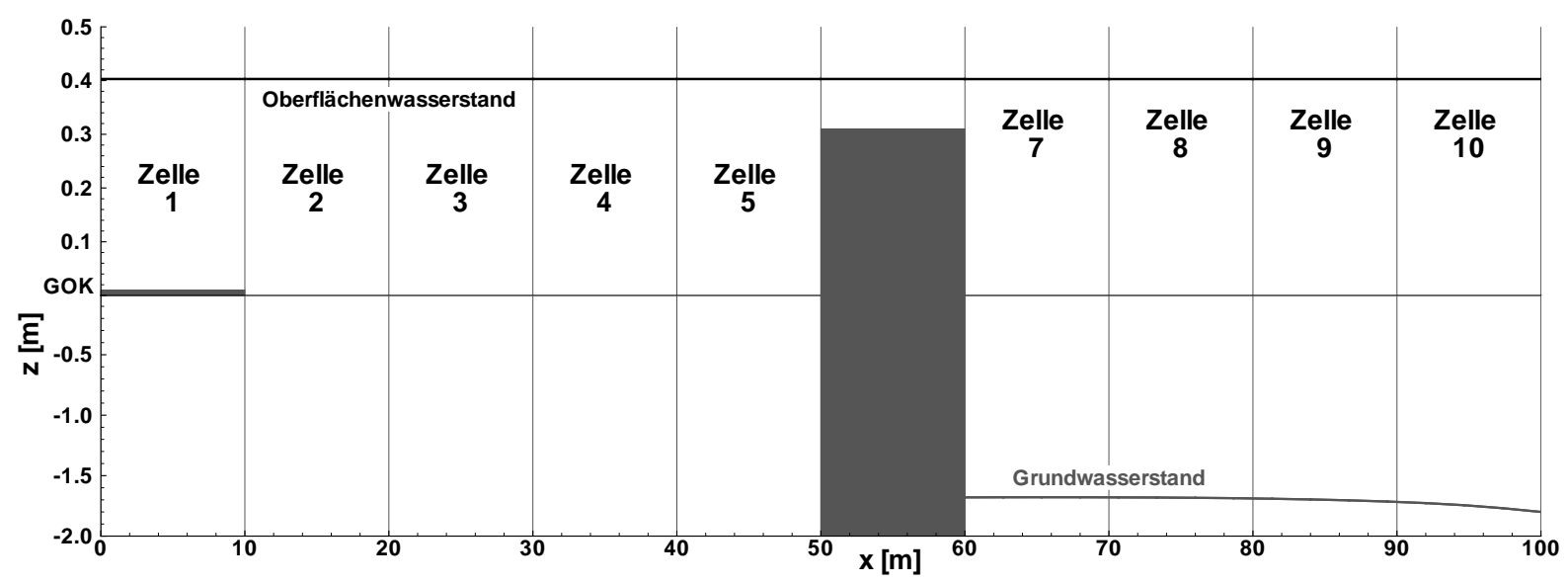

(a) $0,55 \mathrm{~d}$

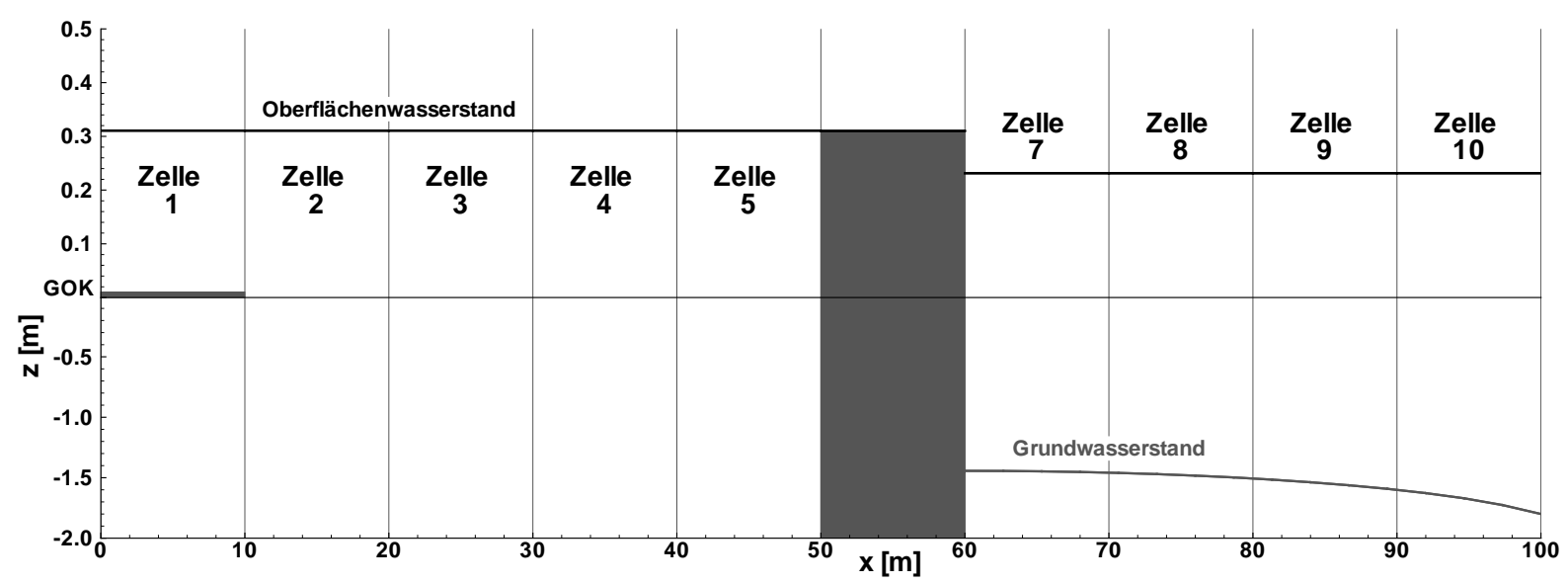

(b) $2,0 \mathrm{~d}$

Abbildung 8.18: Grundwasserstand und Oberflächenwasserstand für die Simulationszeitpunkte $0,55 \mathrm{~d}$ und 2,0 d (unterschiedliche Skaleneinteilung auf der Ordinate)

Bei 0,17 d erreicht der Grundwasserstand die Geländeoberfläche. Der Randbedingungswert bewirkt, dass Wasser aus dem Modellgebiet ausströmt. Dieses Wasser wird dem Oberflächenströmungsmodell über das Ilmoflood-Eingabe-Austauschobjekt übergeben und führt hier zu einem Anstieg des Oberflächenwasserspiegels. Dieser Oberflächenwasserstand steht nun im nächsten Zeitschritt als Ilmoflood-Ausgabe-Austauschobjekt zur Verfügung und wird über das FeflowEingabe-Austauschobjekt als Randbedingungswert angesetzt. Die bisher bestehende Sickerflächenrandbedingung wird mit einer h-Randbedingung ohne Nebenbedingung überschrieben. Damit kann jetzt auch Wasser dem Grundwassermodell zufließen.

Dies passiert auch bei Knoten 18 (Abbildung 8.19b). An diesem Knoten liegt der Grundwasserstand zum Zeitpunkt 0,19 d noch bei -0,31 m, er hat also die obere Begrenzung des Modellleiters noch nicht erreicht. Einen Zeitschritt weiter steigt er auf $0,02 \mathrm{~m}$ an, weil dieser Wert als h-Randbedingung vom Ilmoflood-Modell übergeben wird. Der Anstieg des Grundwassers an 


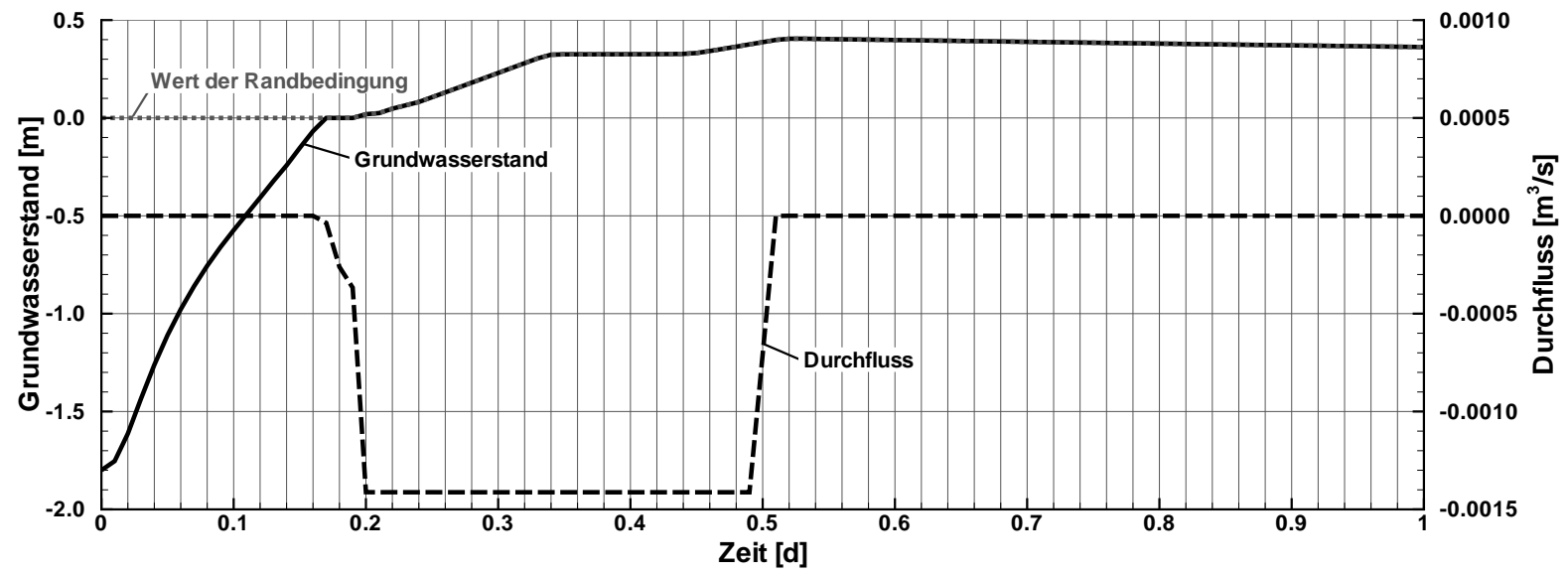

(a) Knoten 15

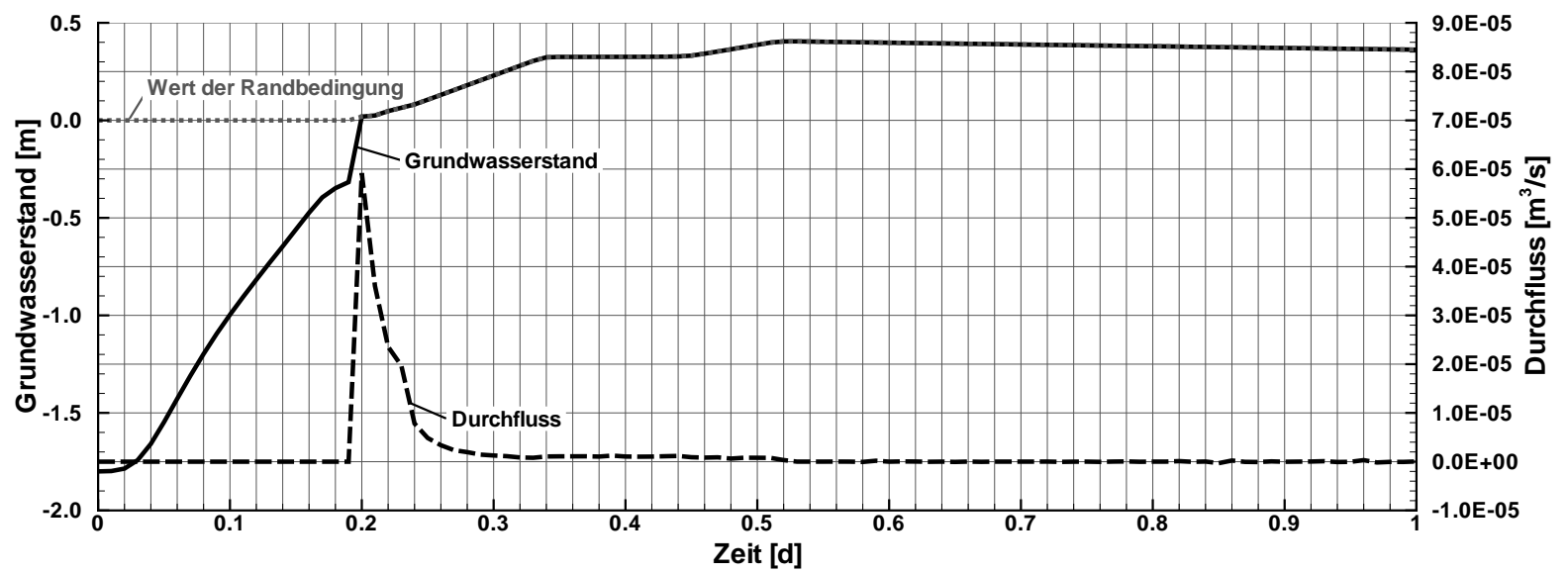

(b) Knoten 18

Abbildung 8.19: Grundwasserstand, Randbedingungswert und korrespondierender Durchfluss über einen Teil der Simulationszeit für die Knoten 15 und 18 des Grundwassermodells (Lage siehe Abbildung 8.15). Eine negative Zuflussmenge zeigt einen Abstrom aus dem Grundwassermodellgebiet an.

diesem Knoten kommt also nicht von unten, sondern ist durch eine „schlagartige Infiltration“ von oben her verursacht. Entsprechend wird eine korrespondierende Randbedingungsmenge berechnet, die dem Grundwassermodell hinzugefügt wird, um den Wert der Randbedingung zu erreichen. Da die Menge vergleichsweise klein ist, bewirkt ihr Abzug im nächsten Zeitschritt beim Ilmoflood-Modell keine wesentliche Änderung des Wasserspiegels, zumal sie vom Zufluss aus den Knoten mit Qualmwasserbildung überlagert wird.

Der seitliche Zufluss in das Grundwassermodell endet nach dem Zeitpunkt 0,5 d. An der Oberfläche tritt daher zu diesem Zeitpunkt bei Knoten 15 auch kein Wasser mehr aus, so dass die Durchflussganglinie auf Null zurückfällt (Abbildung 8.19a).

Die Kurve für Zuflüsse aus dem Grundwasser von Zelle 2 (Abbildung 8.20) verläuft qualitativ entsprechend der Kurve von Knoten 15. Die absoluten Werte sind nicht direkt vergleichbar, da 


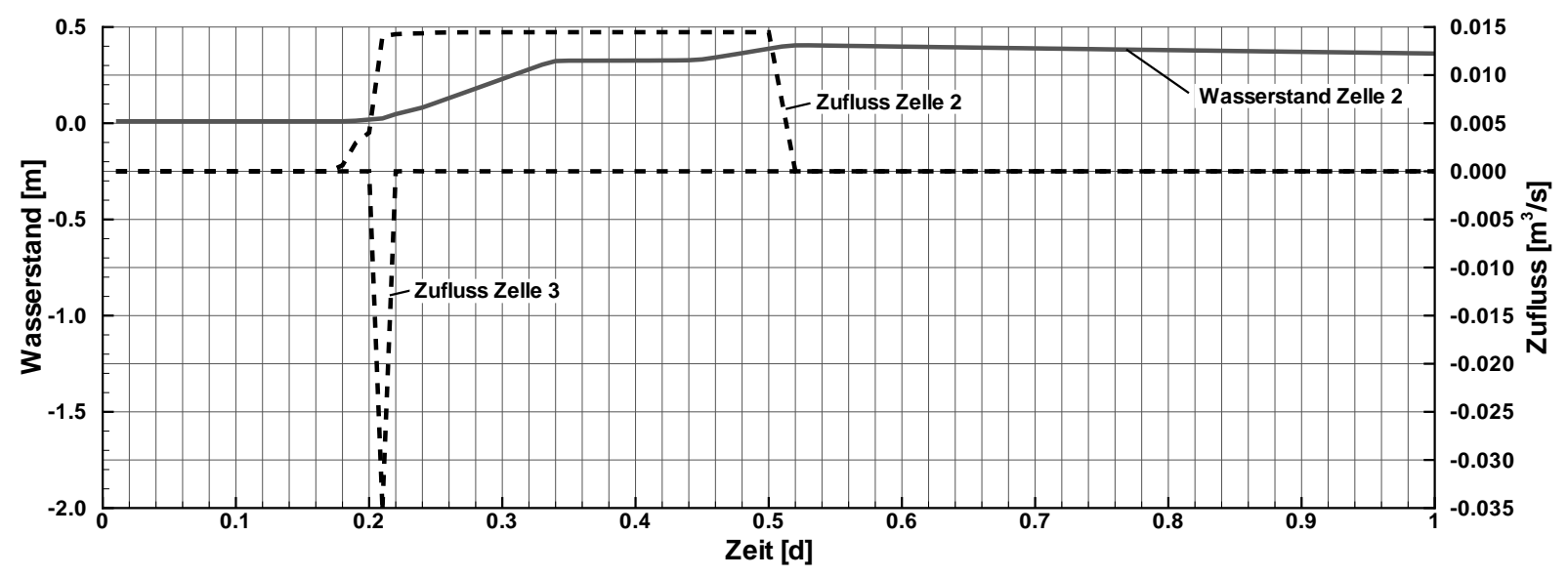

Abbildung 8.20: Zufluss der Zellen 2 und 3 und Wasserstand der Zelle 2

der Zufluss von Zelle 2 sich aus mehreren Knoten zusammensetzt und auch zum Beispiel der Abstrom zu Knoten 18 hier mitbilanziert wird. Der in Abschnitt 8.2.2.3 beschriebene Zeitversatz durch die externe Kopplung wird in diesem Beispiel aber dennoch daran deutlich, denn bei Zelle 2 erreicht der Zufluss erst zum Zeitpunkt 0,52 d den Wert Null, während bei Knoten 15 schon zum Zeitpunkt 0,51 d kein Abstrom mehr erfolgt.

Bei den Grundwassermodellknoten unterhalb von Zelle 3 dagegen findet kein Austritt von Qualmwasser, sondern nur eine Infiltration statt, deshalb sind die Zuflüsse für Zelle 3 alle negativ. Die Infiltration läuft, weil sie mit einer h-Randbedingung modelliert wird, schlagartig ab (siehe Abschnitt 6.4.3, Abbildung 6.10). Deshalb erreicht der Ausfluss aus der Zelle für nur einen Zeitschritt einen hohen Betrag, um dann wieder auf Null zurückzugehen.

\subsubsection{Schlussfolgerungen und Diskussion}

Das Beispiel mit Qualmwasseraustritt und Infiltration zeigt besonders deutlich den Mehrwert einer wechselseitigen Kopplung gegenüber ungekoppelten Modellen oder Modellen, die nur in einer Richtung gekoppelt werden. So hat das Ilmoflood-Modell keine eigenen Randbedingungen, die überhaupt eine Strömung verursachen. Es stellt hier eine Verbindung zwischen dem linken und dem rechten Modellteil des Grundwassermodells über einen undurchlässigen Bereich in der Mitte her. Wasser aus dem linken Grundwassermodellteil tritt an der Oberfläche aus und bewegt sich dann nach den Fließgesetzen des Oberflächenwassers über den undurchlässigen Bereich hinweg, um im rechten Teil des Modells wieder in den Untergrund zu infiltrieren. Ohne Kopplung oder mit einer Kopplung nur in einer Richtung ließe sich ein solches Zusammenwirken der Strömungsprozesse nicht realisieren. Das Grundwassermodell stellt mit dem Wasseraustritt an der Oberfläche Randbedingungen für das Überflutungsflächenmodell, nimmt aber Berechnungsergebnisse des Überflutungsflächenmodells auch selbst als Randbedingungen entgegen. 


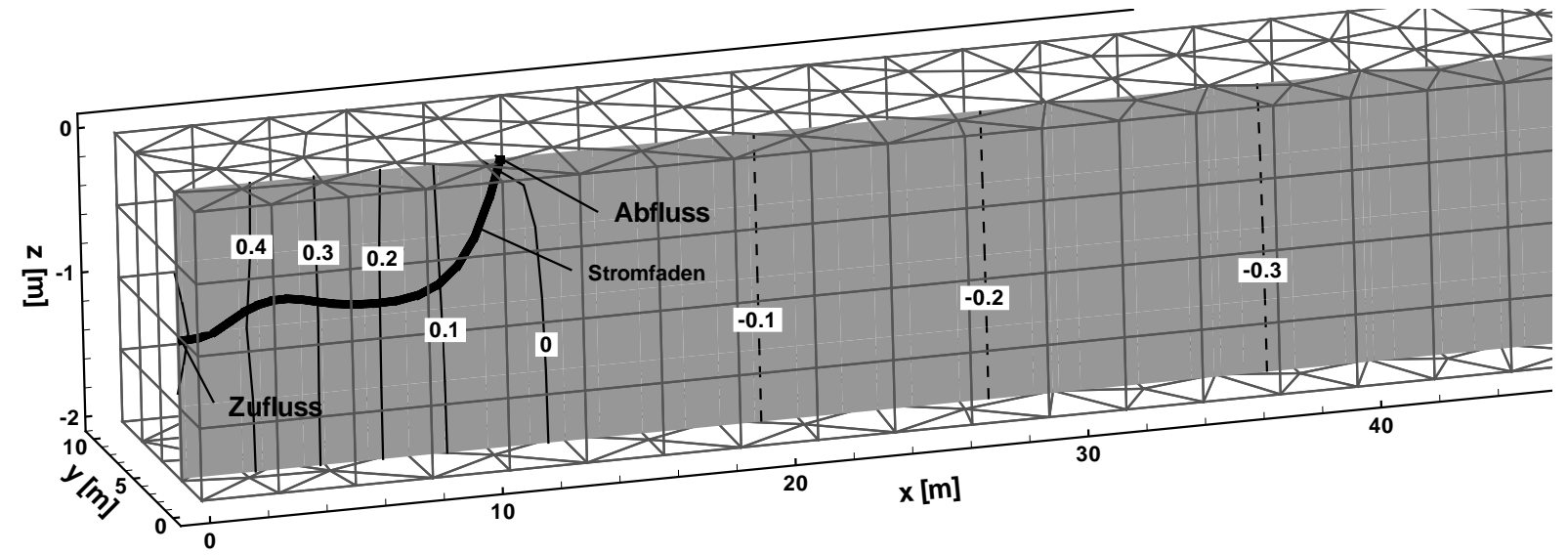

Abbildung 8.21: Ausschnitt aus dem Finite-Elemente-Netz des dreidimensionalen Vergleichsmodells mit Isolinien gleichen Grundwasserstands und einem Stromfaden in einem vertikalen Schnitt durch das Modellgebiet für den Simulationszeitpunkt 0,37 d

Das Grundwassermodell ist zweidimensional-horizontal aufgebaut. Derartige Modellkonzepte werden häufig in der Praxis verwendet. In dem vorliegenden Beispiel wurde quasi eine Erweiterung um die dritte Dimension erreicht, da eine Überströmung des unterirdischen Fließhindernisses möglich ist. Der zweidimensional-horizontale Ansatz stellt aber eine Vereinfachung der Strömungssituation dar.

Eine realitätsnähere Darstellung mit vertikaler Strömungskomponente liefert eine dreidimensionale Berechnung der Grundwasserströmung. Abbildung 8.21 zeigt für einen Vertikalschnitt durch das dreidimensionale Finite-Elemente-Netz eines solchen 3D-Modells die Linien gleicher Grundwasserdruckhöhe und einen Stromfaden vom Punkt des Zuflusses bis zur Austrittsstelle des Wassers an der Oberfläche. Der Stromfaden verläuft zunächst ungefähr horizontal und knickt dann nach oben hin ab. Die Isolinien der Grundwasserdruckhöhe verlaufen jedoch weitgehend vertikal, so dass die Annahme einer grundsätzlich horizontalen Strömung durchaus gerechtfertigt ist, vor allem, wenn es nicht darauf ankommt, die Grundwasserströmung genau wiederzugeben, sondern lediglich die Austrittsstellen von Qualmwasser im Rahmen von Planungsaufgaben ungefähr zu erfassen. Lediglich die Isolinie mit dem Wert 0 knickt im Bereich der Wasseraustrittsstelle in ihrem oberen Bereich ab. Der Bereich des Austritts ist in diesem Beispiel also begrenzt.

Bei der Vergleichsrechnung wurde gegenüber dem in Abschnitt 8.3.2 beschriebenen gekoppelten Modell bei der Grundwassermodellkomponente das zweidimensional-horizontale Netz um die dritte Dimension erweitert, indem das Netz auf vier weitere Ebenen kopiert wurde, so dass insgesamt vier Schichten vertikal übereinander liegen. Das Überflutungsflächenmodell unterscheidet sich nicht von dem aus Abschnitt 8.3.2. Die Verbindungen zwischen Knoten und Zellen sind im Prinzip auch identisch, nur dass im linken Bereich keine Rückkopplung stattfindet (Tabelle 8.4), um den Wasseraustritt an der Oberfläche nicht von einem Infiltrationsprozess zu überlagern. Es wird nur der Grundwasseraustritt an der Oberfläche ans Oberflächenmodell über- 
Tabelle 8.4: Übersicht der Austauschobjekte zur Verbindung zwischen Ilmoflood-Zellen und FeflowKnoten des 3D-Modells

\begin{tabular}{clcccc}
\hline Bereich & \multicolumn{2}{l}{ Ilmoflood-Modell } & & \multicolumn{2}{c}{ Feflow-Modell } \\
\hline 1 & Eingabe & $\mathrm{Q}$ & $\leftarrow$ & Ausgabe & Sickerfläche \\
\hline \multirow{2}{*}{2} & Ausgabe & $\mathrm{h}$ & $\rightarrow$ & Eingabe & Leakage $1^{a}$ \\
& Eingabe & $\mathrm{Q}$ & $\leftarrow$ & Ausgabe & $\mathrm{Q}$ \\
\hline
\end{tabular}

${ }^{a}$ Kontaktfläche abhängig vom Grundwasserstand

geben, die Druckhöhe aus dem Oberflächenmodell wird jedoch nicht an das Grundwassermodell übertragen.

Anhand der dreidimensionalen Vergleichsrechnung kann deshalb auch gut die Wirkung der Rückkopplung verdeutlicht werden. Im gekoppelten Modell mit zweidimensionaler Grundwassermodellkomponente überträgt die Rückkopplung die Druckhöhe des Oberflächenmodells zurück ins Grundwassermodell. Ohne diese Rückkopplung verbleibt der Grundwasserstand mit der Sickerflächenrandbedingung auf Höhe der Knotenlage und der Wert der Randbedingung ändert sich nicht. Dies kann anhand der Knotenganglinien des gekoppelten Modells mit dreidimensionaler Grundwasserkomponente in Abbildung 8.22 nachvollzogen werden. Der Wasserstand im Untergrund steigt bei Knoten 15 an (Abbildung 8.22a), erreicht allerdings nicht wie im zweidimensional-horizontalen Grundwassermodell bei 0,17 d (Abbildung 8.19), sondern erst bei $0,19 \mathrm{~d}$ die Oberfläche. Dies wird auf die zur zweidimensional-horizontalen Berechnung unterschiedliche Handhabung der gespannten Verhältnisse im dreidimensionalen Modell, auf die bereits in Abschnitt 8.2 eingegangen wurde, zurückgeführt. Bei Knoten 16 (Abbildung 8.22b) nimmt die Steigung der Grundwasserstandskurve zu diesem Zeitpunkt ab, weil ein Teil des zuströmenden Wassers nun an der Oberfläche abgezogen wird und somit nicht mehr zur Füllung des unterirdischen Raums zur Verfügung steht. Auch bei Knoten 16 erreicht der Grundwasserstand die Oberkante des Grundwasserleiters, so dass ab dem Simulationszeitpunkt 0,38d auch dieser Knoten zum Wasseraustritt an der Oberfläche beiträgt. Knoten 18 trägt, wie im zweidimensionalhorizontalen Modell, nicht zum Wasseraustritt bei. Bei allen drei Knoten sinkt der Grundwasserstand ab einem Zeitpunkt von etwa 0,5 d geringfügig wieder ab. Da ab diesem Zeitpunkt der seitliche Zufluss versiegt und von der Oberfläche kein Rückfluss ins Modell erfolgt, spiegelt sich der Grundwasserstand aus, denn im hinteren Bereich hat der Grundwasserstand noch nicht überall die Geländeoberkante erreicht.

Die Kombination der Austauschobjekte Sickerfläche und Q-Zuflussrandbedingung von einem Feflow-Modell zu einem Ilmoflood-Modell bei gleichzeitiger Rückkopplung der Wasserstände eignet sich also dazu, den Wasseraustritt an einer Oberfläche zu modellieren, wenn beide Kompartimente nicht durch eine Deckschicht getrennt sind. Im Oberflächenwasser und im Untergrund kann so erreicht werden, dass jeweils der gleiche Wasserstand vorliegt. Die Eignung eines Feflow-Eingabe-Austauschobjekts mit Leakage-Randbedingung für eine Infiltration durch eine Deckschicht wurde bereits in Abschnitt 8.2 diskutiert. 


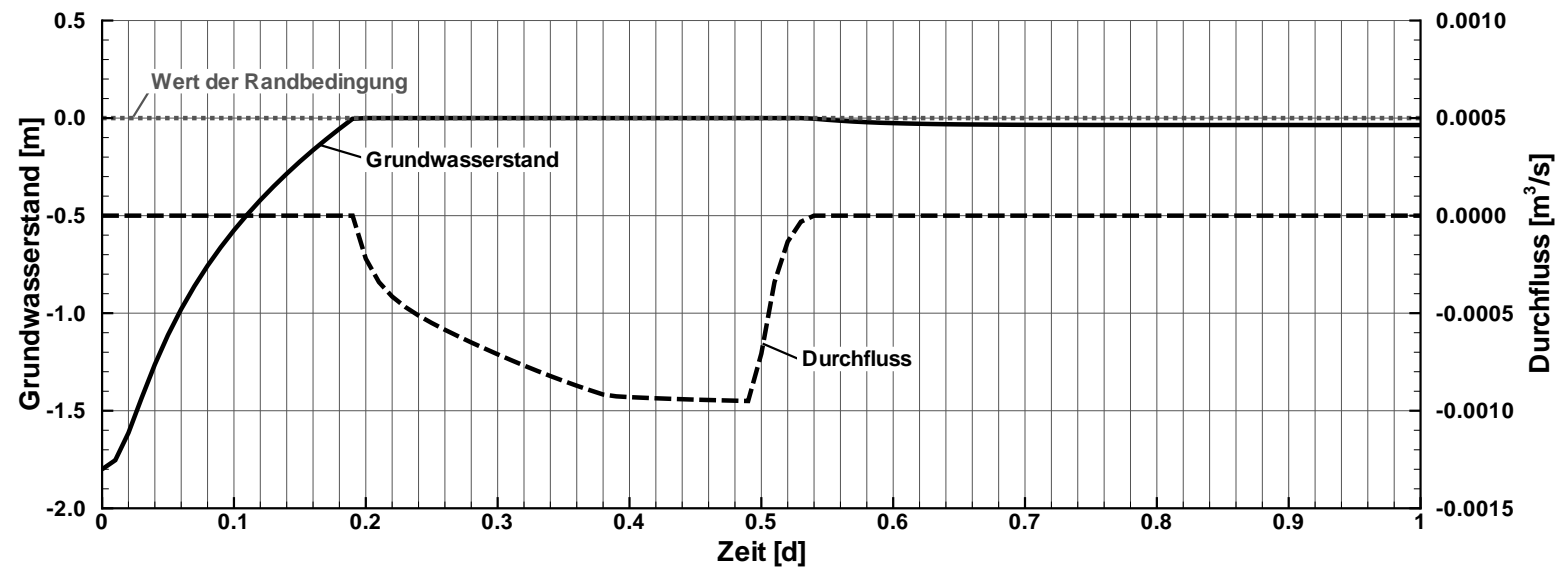

(a) Knoten 15

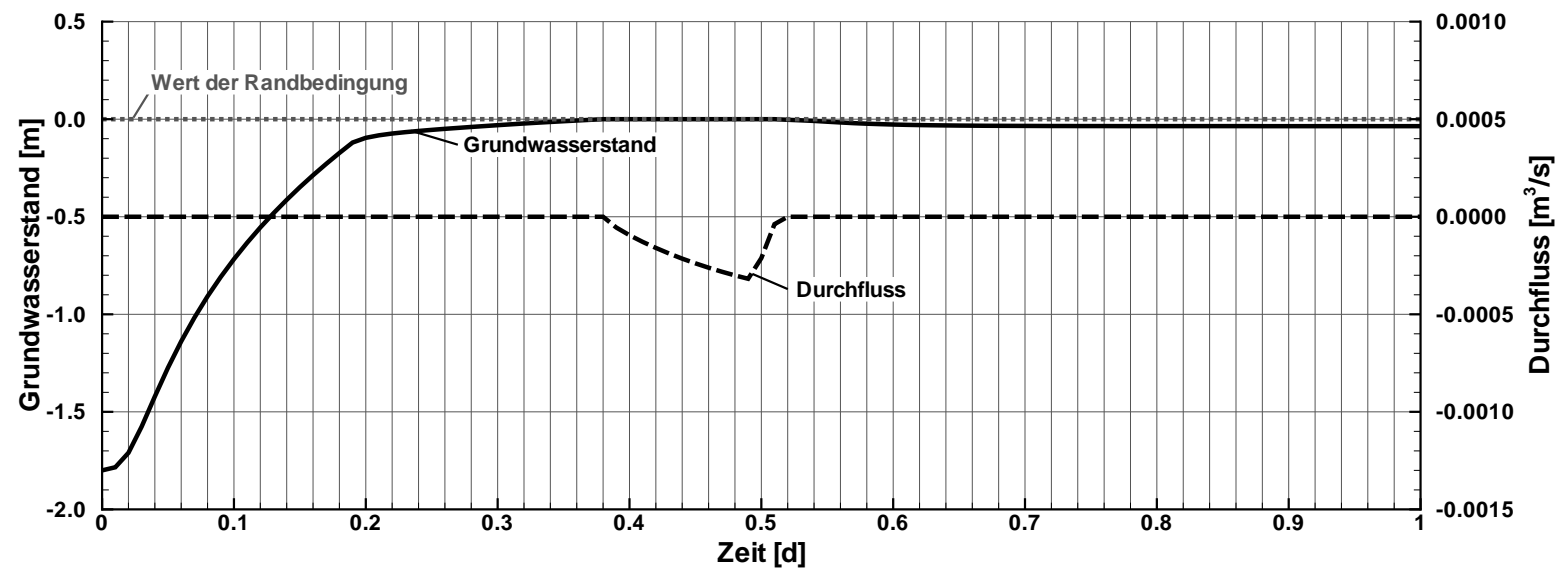

(b) Knoten 16

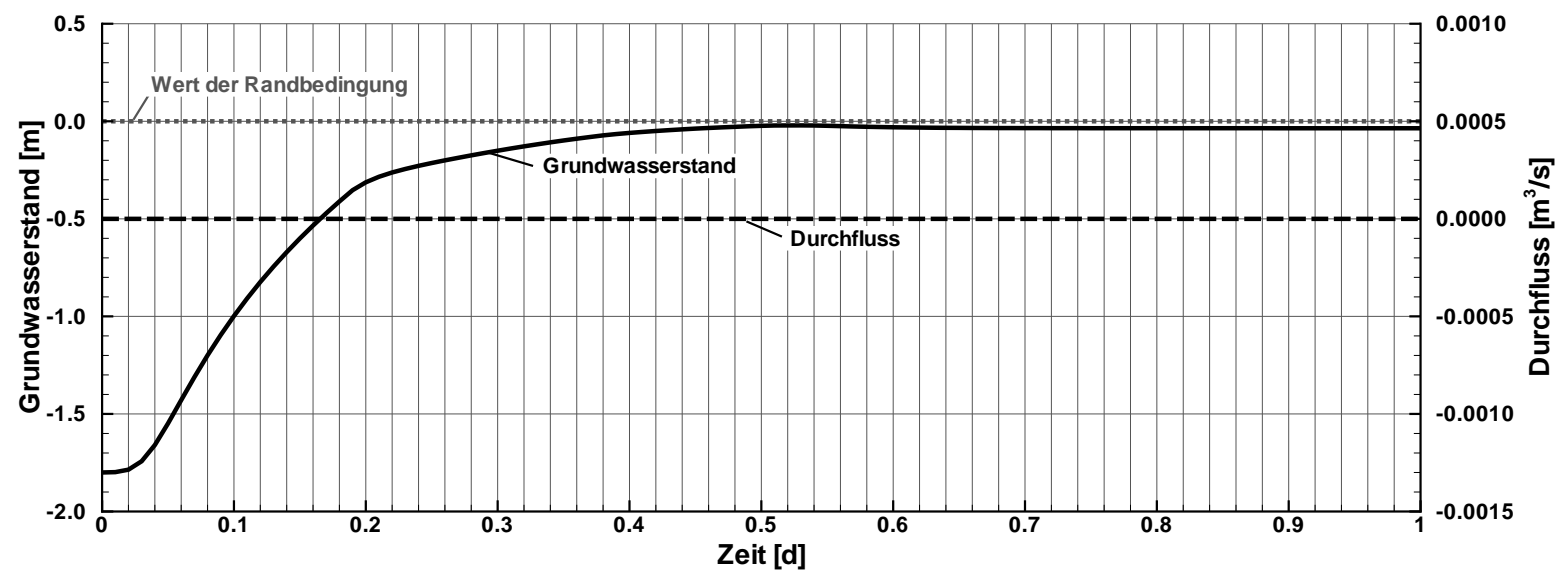

(c) Knoten 18

Abbildung 8.22: Grundwasserstand, Randbedingungswert und korrespondierender Durchfluss für die Knoten 15, 16 und 18 und einen Teil der Simulationszeit 


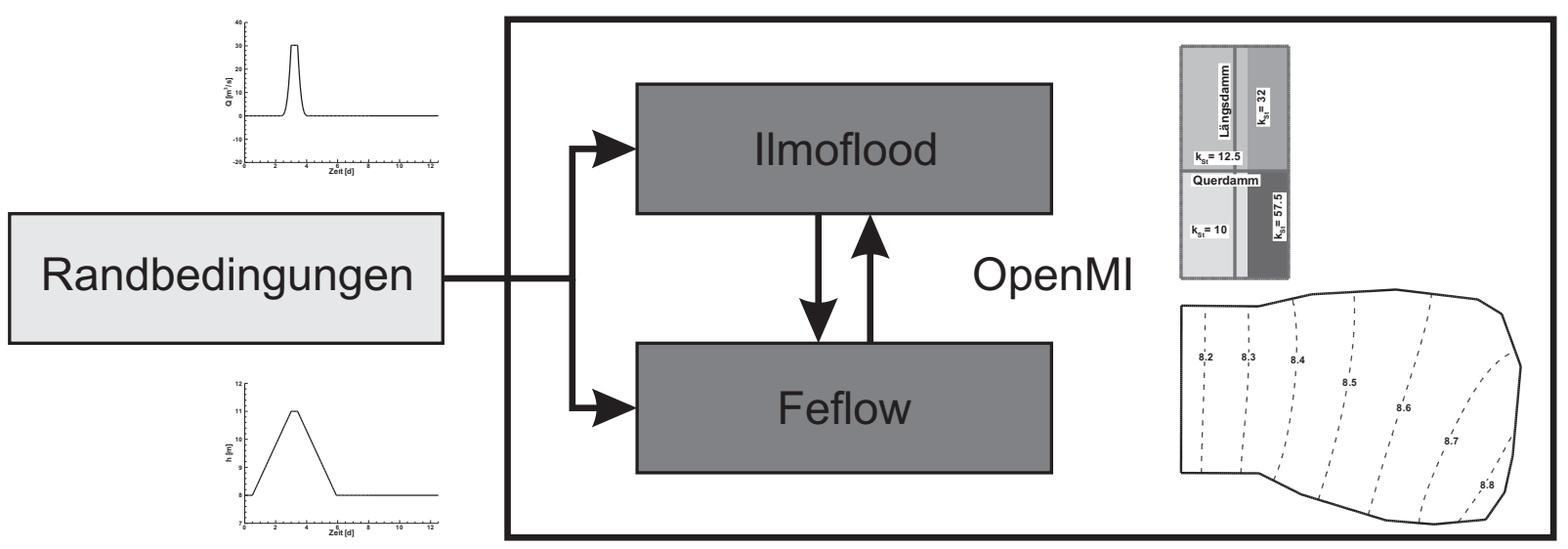

Abbildung 8.23: Modellkopplung für das Anwendungsbeispiel

Ungenauigkeiten beim Austausch ergeben sich durch Einschränkungen der Modellkomponenten. So wird im Ilmoflood-Modell die Wasserentnahme einer Zelle bei kleinen Wasserständen (hier $h \leq 0,05 \mathrm{~m}$ ) über der Höhenlage nicht umgesetzt, um negative Wasserstände zu vermeiden. Auch die räumliche Diskretisierung spielt eine Rolle. So bewirkt austretendes Grundwasser sofort in der gesamten Zelle einen Wasseranstieg, der dann für alle darunter liegenden Grundwassermodellknoten wirksam wird. Das Modell mit der räumlich gröberen Auflösung bestimmt somit die räumliche Ausdehnung des Austauschprozesses.

\subsection{Fallbeispiel}

\subsubsection{Einführung}

Das Fallbeispiel dient dazu, Prozesse des unterirdischen Hochwassers für ein größeres Modellgebiet darzustellen und zu analysieren, aber auch die Anwendbarkeit der im Rahmen dieser Arbeit vorgestellten Modellkopplung für größere Modelle nachzuweisen. Das Fallbeispiel ist fiktiv, da für die vorliegende Arbeit keine Daten und Modelle eines realen Szenarios vorliegen. Das fiktive Anwendungsbeispiel ist jedoch so gestaltet, dass die ablaufenden Prozesse des unterirdischen Hochwassers isoliert von anderen Prozessen betrachtet und damit gut veranschaulicht werden können. Geometrisch einfache Modellgebiete erleichtern zudem die Ergebnisdarstellung und die Interpretation. Für Fragen des unterirdischen Hochwassers ist dies zweckmäßig, da derzeit die Prozesse des unterirdischen Hochwassers noch nicht umfassend dokumentiert sind.

Das Anwendungsbeispiel wird als gekoppeltes System gemäß Abbildung 1.8 aus einem IImoflood-Überflutungsflächenmodell, einem zweidimensional-horizontalen Feflow-Grundwassermodell und Randbedingungen, die den Fluss beschreiben, realisiert (Abbildung 8.23). Der Aufbau des Überflutungsflächen- und des Grundwassermodells wird im folgenden Abschnitt beschrieben. 


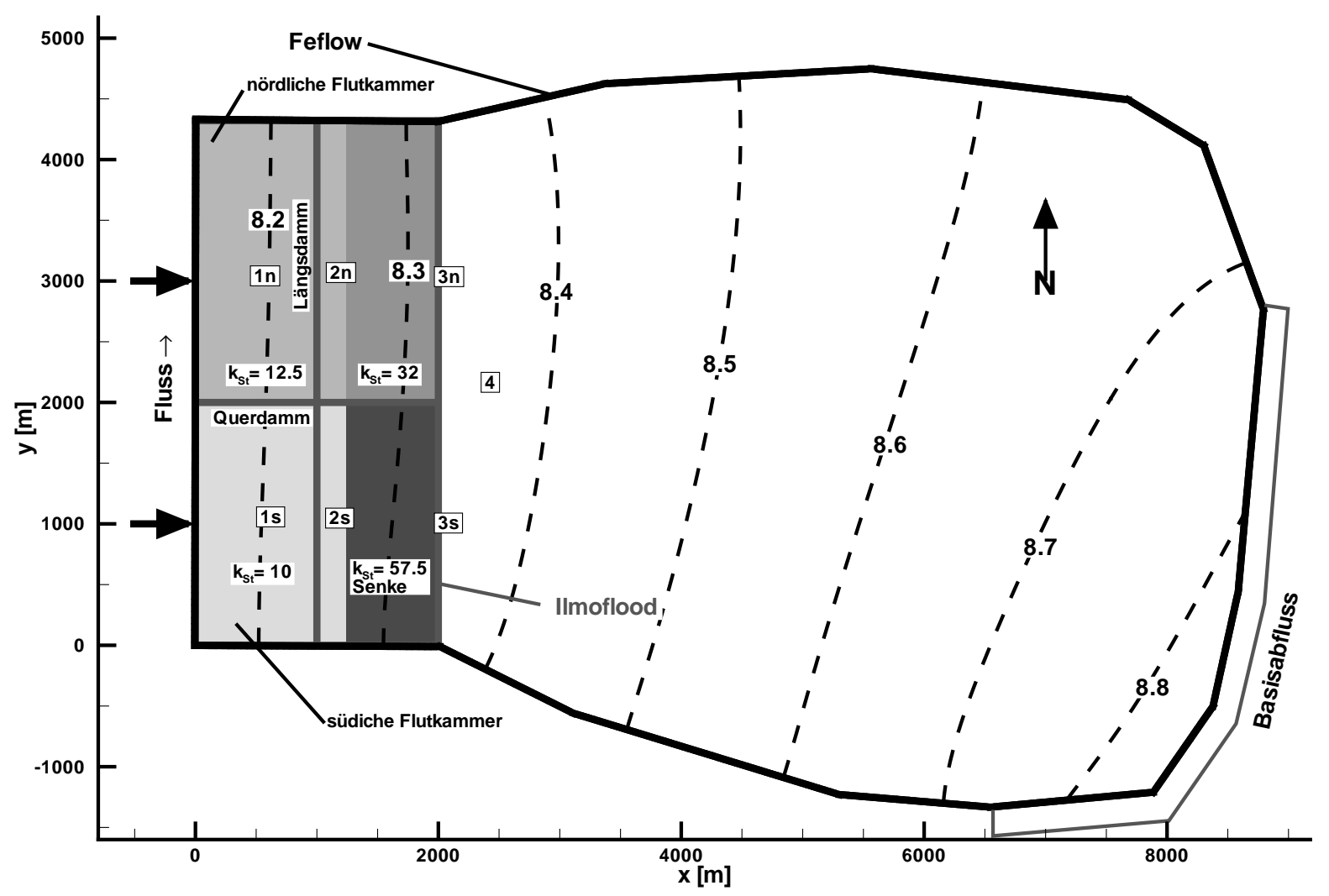

Abbildung 8.24: Modellgebiete des gekoppelten Modellsystems, Anfangsbedingungen des Grundwassermodells als Linien gleicher Grundwasserdruckhöhe, Ansatz der Randbedingungen des Grundwassermodells, Rauheitsbeiwerte und Begrenzungen der Überflutungsfläche des IImoflood-Modells, Zuflusspunkte und Lage der Beobachtungspunkte (1n bis 3n, 1s bis 3s und 4; s steht für südlich, n steht für nördlich)

Sowohl Sommer et al. (2008) als auch Flögel (2009) nennen als wesentliche Einflussgröße für das unterirdische Hochwasser die Dauer des Hochwasserereignisses im Fluss und den Anfangszustand, auf den eine unterirdische Hochwasserwelle auftritt. Entsprechend werden im Folgenden ein Szenario „kurzes Hochwasser“, ein „langes Hochwasser“ und ein „langes Hochwasser mit hohem Anfangswasserstand" beschrieben. Zum Abschluss des Fallbeispiels folgt eine Zusammenfassung der wesentlichen Erkenntnisse und eine Diskussion der Ergebnisse.

\subsubsection{Modellaufbau und Modellkopplung}

Abbildung 8.24 zeigt das fiktive Untersuchungsgebiet als Draufsicht in Form einer Lagekarte mit den wichtigsten Modellobjekten. Im linken Berech verläuft ein Fluss von Süden nach Norden, er bildet für das Überflutungsflächenmodell und das Grundwassermodell den westlichen Modellrand und wird über Randbedingungen in beiden Modellkomponenten modelliert. 
Tabelle 8.5: Eigenschaften und Parameter des Ilmoflood-Überflutungsflächenmodells

\begin{tabular}{lc}
\hline Anzahl Zellen & $50 \cdot 108=5400$ \\
Kantenlänge der Zellen & $40 \mathrm{~m}$ \\
Fläche des Modellgebiets & $8,64 \mathrm{~km}^{2}$ \\
Geodätische Höhe der Überflutungsfläche & $10 \mathrm{~m}$ (Senke: $9,5 \mathrm{~m})$ \\
\hline Ausgabezeitschrittweite & $3600 \mathrm{~s}$ \\
\hline
\end{tabular}

Tabelle 8.6: Eigenschaften und Parameter des Feflow-Grundwassermodells

\begin{tabular}{lc}
\hline Dimension und Ausrichtung & $\begin{array}{c}\text { zweidimensional-horizontal } \\
\text { Grundwasserströmung }\end{array}$ \\
Fläche des Modellgebiets & $44,89 \mathrm{~km}^{2}$ \\
Zeitschrittweite & $0,041666 \mathrm{~d}(3600 \mathrm{~s})$ \\
Anzahl Knoten & 29994 \\
Anzahl Dreieckelemente & 59465 \\
\hline Anzahl Grundwasserleiter & 1 \\
Grundwasserleiteroberkante & $10 \mathrm{~m}($ Senke: $9,5 \mathrm{~m})$ \\
Grundwasserleiterunterkante & $0 \mathrm{~m}$ \\
Durchlässigkeit $k$ & $1,968 \cdot 10^{-2} \mathrm{~m} / \mathrm{s}$ \\
Speicherkoeffizient $S_{\mathrm{sp}}$ & $0,2\left(\right.$ ungespannt) $1,0 \cdot 10^{-4}$ (gespannt) \\
Leakage-Parameter des Flusses & $5,0 \cdot 10^{-2} 1 / \mathrm{s}$ (Infiltration und Exfiltration) \\
\hline
\end{tabular}

Das Überflutungsflächenmodell hat, bedingt durch den Storage-Cell-Ansatz, eine rechteckige Grundfläche. Mit Ausnahme einer Senke und zweier Dämme, die das Modellgebiet in vier Kammern einteilen, liegt überall im Modellgebiet die gleiche Geländehöhe vor. Der Längsdamm und der Querdamm können zum Beispiel einen Bahndamm längs des Flusses und den Damm einer Straße, die den Fluss quert, repräsentieren. Die Rauheitsparameter stehen für bebaute Fläche $\left(k_{\mathrm{St}}=10 \mathrm{~m}^{\frac{1}{3}} / \mathrm{s}\right.$ oder $\left.12,5 \mathrm{~m}^{\frac{1}{3}} / \mathrm{s}\right)$, Ackerland $\left(k_{\mathrm{St}}=32 \mathrm{~m}^{\frac{1}{3}} / \mathrm{s}\right)$ oder Wiese $\left(k_{\mathrm{St}}=57,5 \mathrm{~m}^{\frac{1}{3}} / \mathrm{s}\right)$. Die Senke liegt im südöstlichen Teil des Modells. Ihre Ausdehnung deckt sich mit der des Rauheitsbereichs der Wiese. Eine solche großflächige Absenkung kann in der Realität zum Beispiel durch unterirdischen Steinkohlebergbau als sogenannte Bergsenkung entstanden sein. Für die westlichen Überflutungskammern ist jeweils ein Zuflusspunkt vorgesehen. In Abbildung 8.24 sind die Zuflusspunkte mit einem Pfeil markiert. Als Anfangsbedingung wird für alle Zellen eine Wassertiefe von $H=0$ angesetzt, das bedeutet, dass kein Wasser im Modellgebiet vorhanden ist. Weitere Angaben zum Modell enthält Tabelle 8.5 .

Das Grundwassermodell dehnt sich nach Osten hin weiter aus als das Überflutungsflächenmodell. In Flussnähe decken beide Modellgebiete aber den selben Bereich ab. Das Grundwassermodell umfasst einen Modellleiter, der bis auf den Bereich der Senke überall die gleiche Mächtigkeit von $10 \mathrm{~m}$ aufweist. Modellparameter und Angaben zur Diskretisierung des Modells ent- 
Tabelle 8.7: Horizontale Entfernung der Beobachtungspunkte vom Fluss und vom Längsdamm

\begin{tabular}{crr}
\hline Beobachtungspunkt & Entfernung zum Fluss & Entfernung zum Längsdamm \\
\hline 1n und 1s & ca. $500 \mathrm{~m}$ & ca. $-500 \mathrm{~m}$ \\
2n und 2s & ca. $1100 \mathrm{~m}$ & ca. $100 \mathrm{~m}$ \\
3n und 3s & ca. $2000 \mathrm{~m}$ & ca. $1000 \mathrm{~m}$ \\
4 & ca. $2400 \mathrm{~m}$ & ca. $1400 \mathrm{~m}$ \\
\hline
\end{tabular}

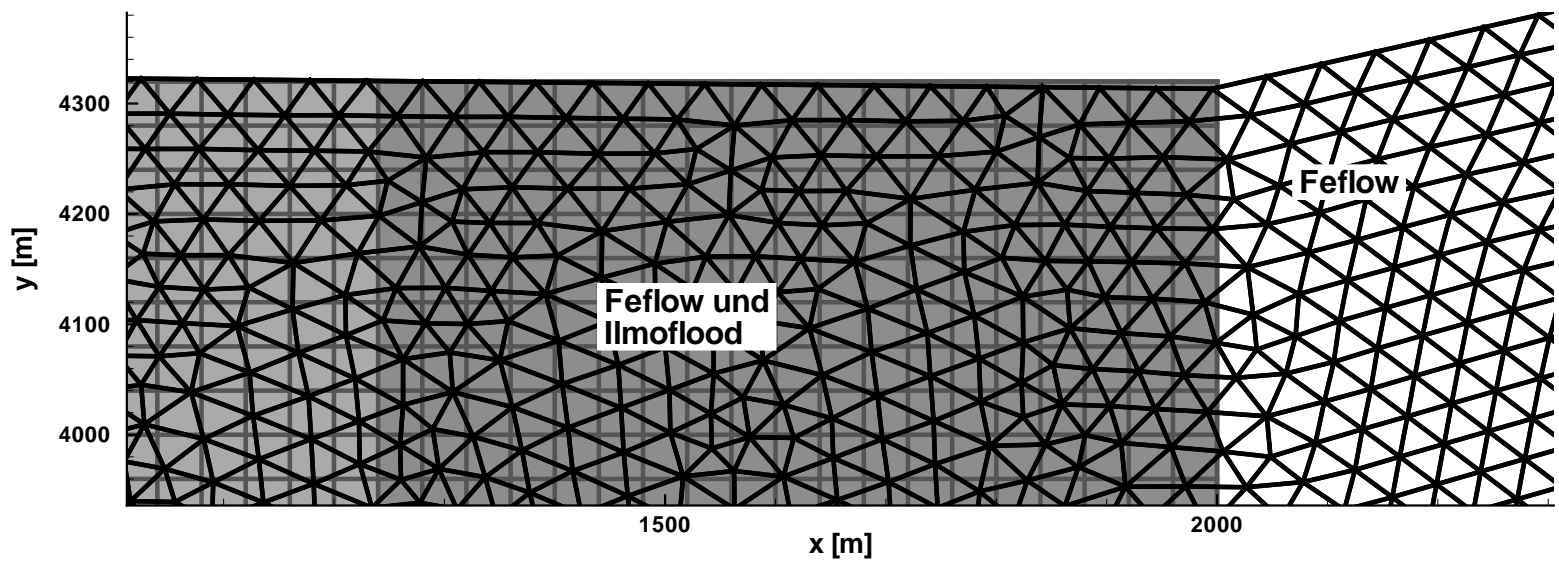

Abbildung 8.25: Detailausschnitt der Netze an der Nordostecke des Ilmoflood-Modellgebiets mit den quadratischen Zellen des Ilmoflood-Überflutungsflächenmodells und den dreieckigen finiten Elementen des Feflow-Grundwassermodells

hält Tabelle 8.6. Der Fluss wird im Grundwassermodell gemäß den Ergebnissen aus Kapitel 5 durch eine Kette von Leakage-Randbedingungsknoten modelliert, denen eine lineare LeakageBeziehung nach Gleichungen 5.2 und 5.5 zu Grunde liegt. Eine Auelehm-Deckschicht, die den Wasseraustausch mit dem Überflutungsflächemodell westlich des Längsdamms bestimmt, wird entsprechend der Ergebnisse aus Kapitel 6 ebenfalls mit Hilfe der Leakage-Randbedingung und entsprechenden Leakage-Parametern abgebildet. Am südöstlichen Modellrand ist eine über die Simulationszeit konstante Q-Randbedingung angesetzt, die einen Basisabfluss zum Fluss hin darstellt. Der Wert des Basisabflusses ist für die verschiedenen Szenarien unterschiedlich. In den Abschnitten des Modellrandes wo weder eine Flussrandbedingung noch ein Basisabfluss angesetzt ist, greift die natürliche Randbedingung des undurchlässigen Modellrandes.

Im Modellgebiet sind mehrere Beobachtungspunkte angeordnet. Ihre Lage ist in Abbildung 8.24 eingetragen. Tabelle 8.7 enthält die horizontale Entfernung der Beobachtungspunkte zum Fluss und zum Längsdamm.

Abbildung 8.25 zeigt einen Detailausschnitt der übereinander liegenden Modellnetze. Die Netze sind, wie für die meisten praktischen Anwendungen, bei denen bereits bestehende Modelle gekoppelt werden sollen, nicht deckungsgleich. Für die Modellkopplung wird eine IlmofloodZelle deshalb jeweils mit allen Knoten verbunden, die innerhalb der Zelle liegen. Für Ilmoflood- 


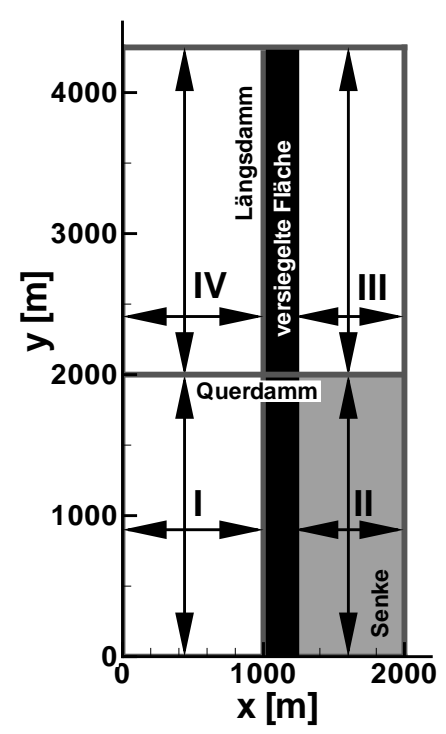

Abbildung 8.26: Einteilung der Kopplungsbereiche

Tabelle 8.8: Übersicht der Austauschobjekte

\begin{tabular}{cllllcc}
\hline Bereich & \multicolumn{1}{l}{ Ilmoflood-Modell } & & \multicolumn{2}{c}{ Feflow-Modell } & Leakage-Parameter \\
\hline \multirow{2}{*}{$\mathrm{I}$} & Ausgabe & $\mathrm{h}$ & $\rightarrow$ & Eingabe & Leakage $2^{a}$ & $2,0 \cdot 1^{-4} 1 / \mathrm{s}$ \\
& Eingabe & $\mathrm{Q}$ & $\leftarrow$ & Ausgabe & $\mathrm{Q}$ & \\
\hline \multirow{2}{*}{$\mathrm{II}$} & Ausgabe & $\mathrm{h}$ & $\rightarrow$ & Eingabe & $\mathrm{h}$ & \\
& Eingabe & $\mathrm{Q}$ & $\leftarrow$ & Ausgabe & Sickerfläche & \\
\hline \multirow{2}{*}{ III und IV } & Ausgabe & $\mathrm{h}$ & $\rightarrow$ & Eingabe & Leakage $2^{a}$ & $2,0 \cdot 1^{-5} 1 / \mathrm{s}$ \\
& Eingabe & $\mathrm{Q}$ & $\leftarrow$ & Ausgabe & $\mathrm{Q}$ & \\
\hline
\end{tabular}

${ }^{a}$ Kontaktfläche nicht abhängig vom Grundwasserstand

Zellen, die keinen Feflow-Knoten enthalten, wird keine Kopplung vorgesehen. Die Modellkopplung erfolgt bereichsweise als bidirektionale Kopplung gemäß Tabelle 8.8. Daten werden zu jeder Stunde der Simulationszeit ausgetauscht. Dieses Intervall entspricht der Zeitschrittweite des Grundwassermodells und den Ausgabezeitpunkten des Überflutungsflächenmodells. Die vier Kopplungsbereiche sind in Abbildung 8.26 eingetragen. Zwischen den Bereichen II und III und dem Längsdamm liegt ein Streifen, an dem nicht gekoppelt wird und in dem keine Wechselwirkung zwischen Grundwasserleiter und Landoberfläche stattfinden kann. Auf diese Weise wird eine vollständig versiegelte Fläche dargestellt, die im realen Vorbild als entsprechende Bebauung oder als eine mächtige Auelehm-Deckschicht aus undurchlässigem Material vorliegen kann. 


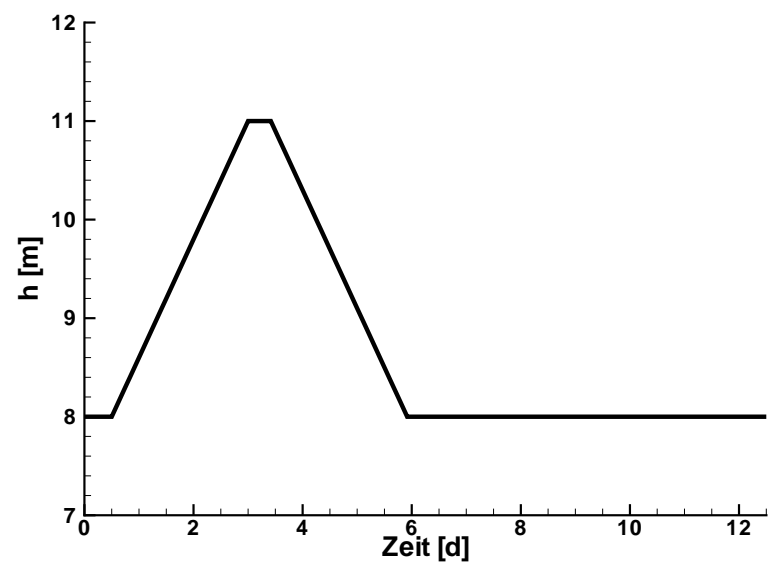

(a) h-Randbedingung für das Feflow-Grundwassermodell

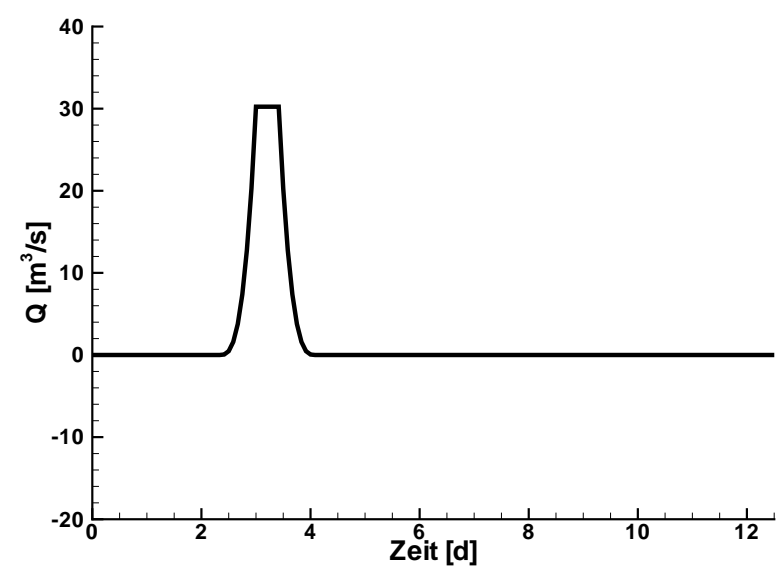

(b) Q-Randbedingung für das Ilmoflood-Überflutungsflächenmodell

Abbildung 8.27: Randbedingungsganglinien des Flusses

In den Bereichen I, III und IV wird die Infiltration über das Leakage-Modell umgesetzt, indem der Wasserstand des Überflutungsflächenmodells als Leakage-Randbedingungswert in das Grundwassermodell übertragen wird. Der korrespondierende Rückfluss findet als Q-AusgabeAustauschobjekt Eingang in das IImoflood-Modell. Für den Bereich I wird eine höhere Durchlässigkeit der Auelehm-Deckschicht angenommen als für die Bereiche III und IV. Im Bereich II dagegen ist keine Auelehm-Deckschicht vorhanden, so dass das Grundwasser hier als Qualmwasser an der Landoberfläche austreten kann. In Bereich II werden die Modelle deshalb über die Austauschobjektkombination Sickerfläche $\leftarrow \mathrm{Q}$ und $\mathrm{h} \rightarrow \mathrm{h}$ gekoppelt.

\subsubsection{Szenario „kurzes Hochwasser“}

\subsubsection{Rand- und Anfangsbedingungen}

In Abbildung 8.27 sind die Ganglinien der Randbedingungen für das Grundwassermodell und das Überflutungsflächenmodell gezeigt. Abbildung 8.27a gibt den Wasserstand im Fluss wieder, der als $h_{\mathrm{F}}$ für alle Grundwassermodellknoten am westlichen Rand in Form einer linearen Leakage-Randbedingung angesetzt wird. Es steht dahinter die Annahme, dass der Flusswasserstand für einen Pegel im Untersuchungsgebiet aus einer Hochwasservorhersage bekannt ist. Der Basisabfluss, der dem Grundwassermodellgebiet am südöstlichen Modellrand zufließt, hat einen Wert von $q=1,157 \cdot 10^{-5} \mathrm{~m} / \mathrm{s}$. Die Anfangsbedingungen, in Abbildung 8.24 als Linien gleichen Grundwasserstands dargestellt, ergeben sich aus einer stationären Berechnung mit diesem Basisabfluss und einem Flusswasserstand von $8 \mathrm{~m}$.

Für das Überflutungsflächenmodell wird die Zuflussrandbedingung aus Abbildung $8.27 \mathrm{~b}$ an den beiden in Abbildung 8.24 mit Pfeilen gekennzeichneten Stellen angesetzt, so dass sich die nörd- 


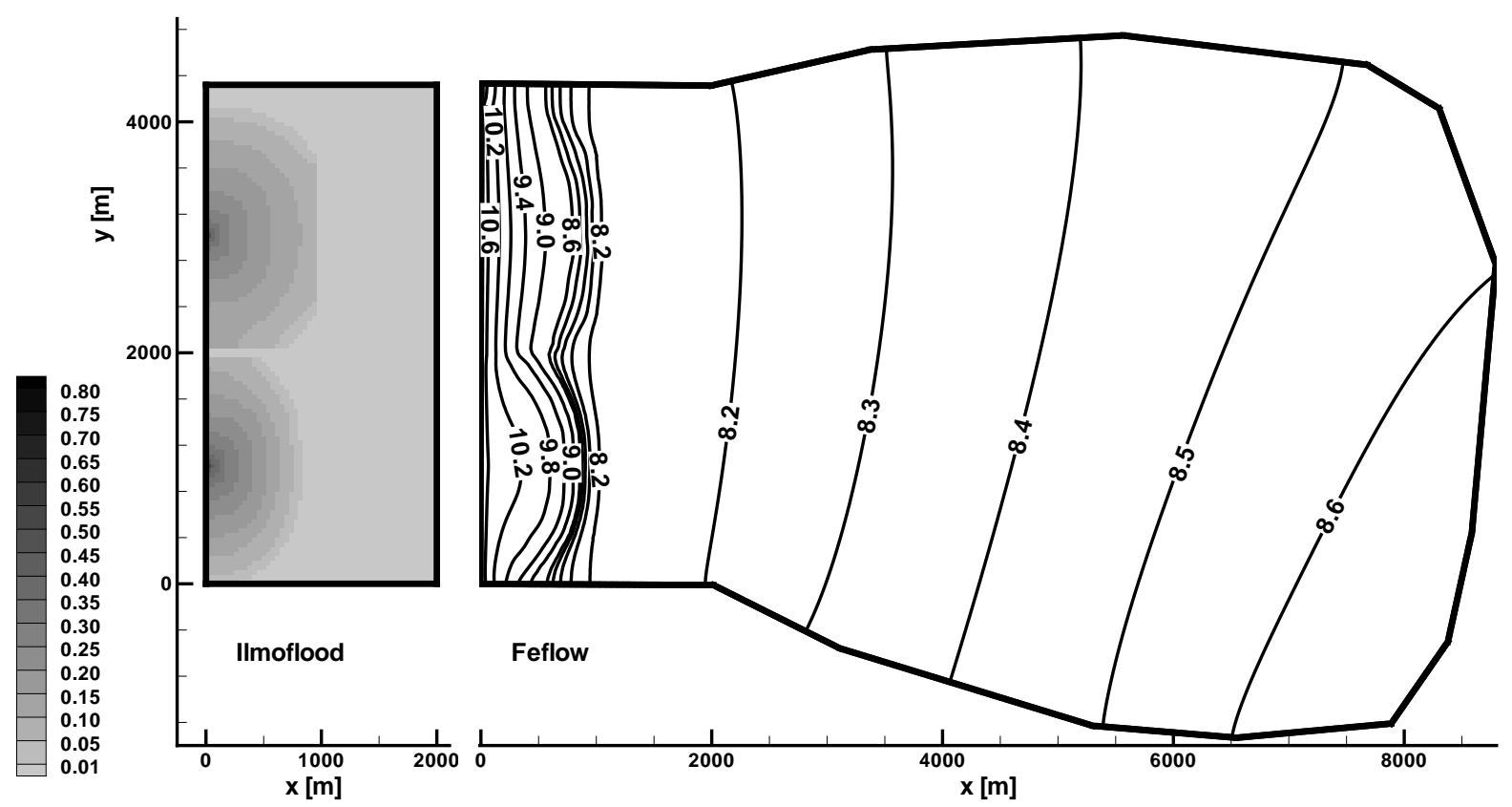

Abbildung 8.28: Wasserausbreitung auf der Überflutungsfläche und Isolinien des Grundwasserstands für den Simulationszeitpunkt 3 d. Die Beschriftung der Gleichen zeigt den Grundwasserstand $h$ an, die Farbskala des Überflutungsflächenmodells bezieht sich auf die Wassertiefe $H$ über der geodätischen Höhe.

liche und die südliche Flutkammer bilden. Der Zufluss setzt jeweils in dem Moment ein, in dem der Flusswasserstand die Lage der Geländehöhe von $10 \mathrm{~m}$ überschreitet. Ein solcher Zufluss kann zum Beispiel durch eine Öffnung in einem Deich oder einer Hochwasserschutzmauer entstehen. Die Zuflusskurve wurde aus der Flussganglinie mit Hilfe der Wehrüberfallformel nach Poleni (siehe z. B. Bollrich 2000) abgeleitet. Das Szenario deckt damit also den Fall des Versagens einer Hochwasserschutzeinrichtung ab oder den Fall, dass keine Hochwasserschutzeinrichtung vorhanden ist. Denkbar ist auch eine gesteuerte Polderflutung über ein Einlassbauwerk. Ein oberflächiger Ausfluss von der Überflutungsfläche ist nicht vorgesehen, die Entleerung der Überflutungskammern findet allein durch die Versickerung, also den numerischen Wasseraustausch mit dem Grundwassermodell, statt.

Die beiden Randbedingungsganglinien aus Abbildung 8.27 werden in den beiden Modellkomponenten vollständig unabhängig voneinander angesetzt, der Zufluss zur Überflutungsfläche beeinflusst also nicht den Flusswasserstand, der im Grundwassermodell als LeakageRandbedingungswert angesetzt wird. Umgekehrt beeinflussen Uferspeicherungseffekte auch nicht den Zufluss zur Überflutungsfläche. 


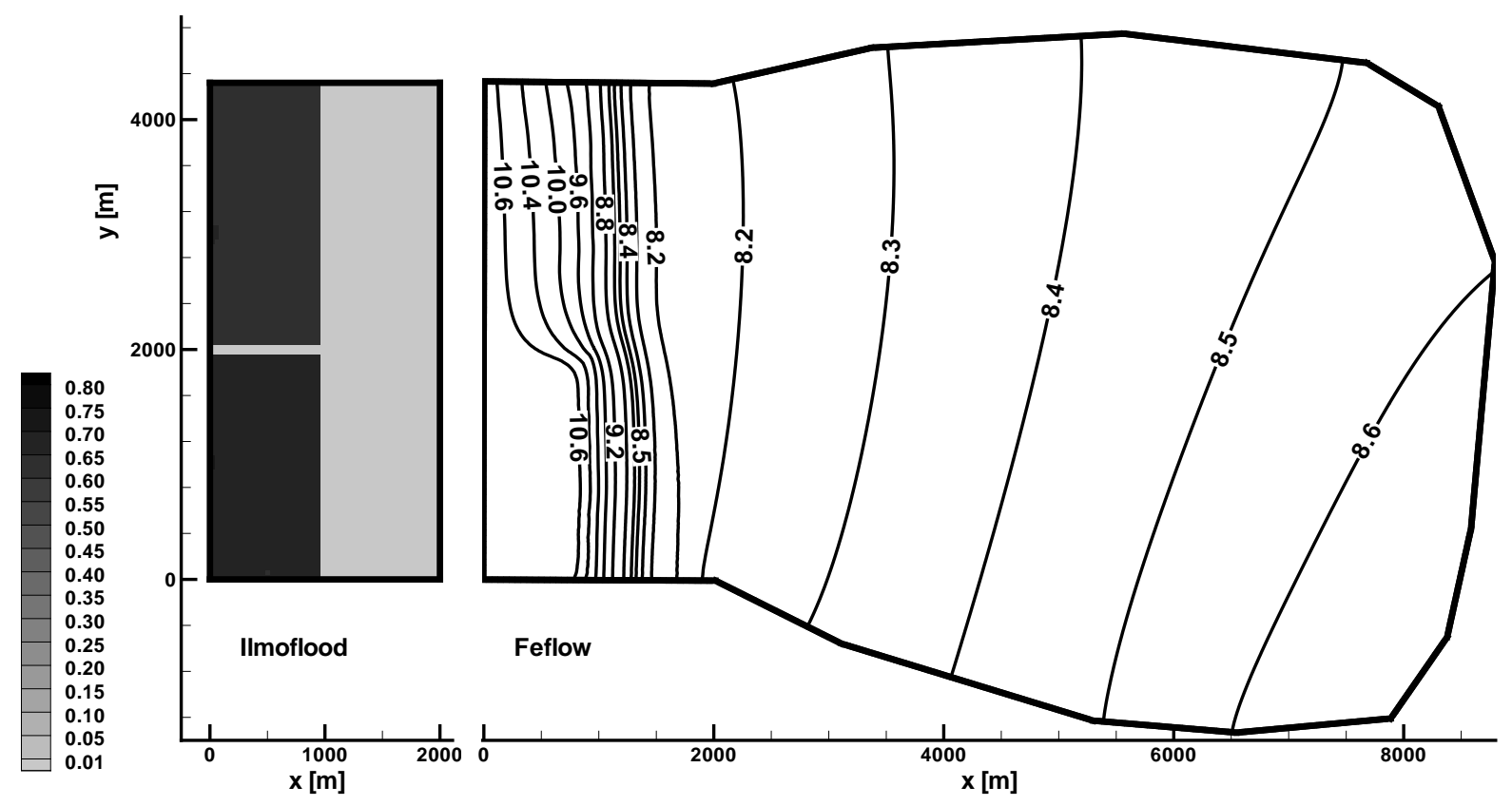

Abbildung 8.29: Wasserausbreitung auf der Überflutungsfläche und Isolinien des Grundwasserstands für den Simulationszeitpunkt 3,75d. Die Beschriftung der Gleichen zeigt den Grundwasserstand $h$ an, die Farbskala des Überflutungsflächenmodells bezieht sich auf die Wassertiefe $H$ über der geodätischen Höhe. Der Wasserstand in der nördlichen Flutkammer beträgt 0,64 m, in der südlichen Flutkammer beträgt er $0,68 \mathrm{~m}$.

\subsubsection{Ergebnisse}

Ein Berechnungsergebnis des Szenarios „kurzes Hochwasser“ zeigt Abbildung 8.28 als Gleichenplan für das Grundwassermodell und als Konturdarstellung für das Überflutungsflächenmodell. Aus Gründen einer übersichtlicheren Darstellung ist das Berechnungsergebnis des Überflutungsflächenmodells vom Grundwassermodellergebnis abgesetzt. Zum gezeigten Zeitpunkt von drei Tagen hat gerade der Zufluss in die beiden Überflutungskammern eingesetzt. Nördlich des Querdamms machen sich die niedrigere Rauheit (höherer $k_{\mathrm{St}}$-Wert) und die größere Kammer dahingehend bemerkbar, dass die Welle flacher verläuft als in der südlichen Kammer und nicht der gleiche maximale Wasserstand erreicht wird.

Am westlichen Rand des Grundwassermodells wirkt die Fluss-Leakage-Randbedingung. Es kommt zu einem seitlichen Zufluss, der einen Anstieg des Grundwasserspiegels auf 10,6 m parallel zum Modellrand hervorruft und ein hydraulisches Gefälle ins Hinterland verursacht. Der Verlauf der Grundwassergleichen unterhalb der Überflutungsfläche wird im südlichen Bereich durch die sehr große Infiltrationsrate, die über einen hohen Leakage-Parameter erreicht wird, bestimmt, so dass die Ausbreitung des an der Oberfläche einströmenden Wassers auch im Gleichenplan erkennbar ist. Im nördlichen Bereich sind Überflutungsfläche und Grundwasserleiter über einen kleineren Leakage-Parameter gekoppelt, so dass zwischen den beiden Kompartimen- 


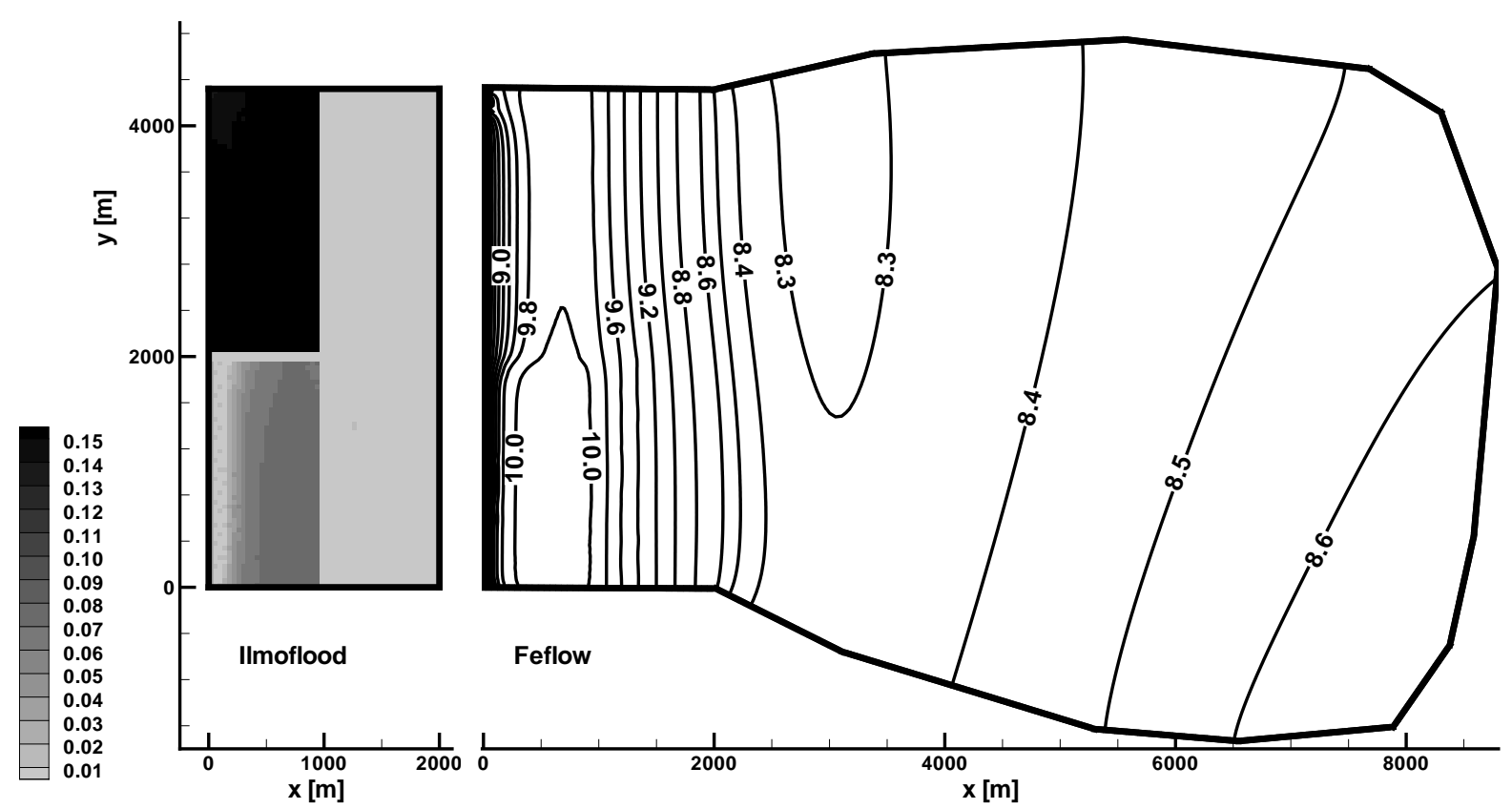

Abbildung 8.30: Wasserausbreitung auf der Überflutungsfläche und Isolinien des Grundwasserstands für den Simulationszeitpunkt 8 d. Die Beschriftung der Gleichen zeigt den Grundwasserstand $h$ an, die Farbskala des Überflutungsflächenmodells bezieht sich auf die Wassertiefe $H$ über der geodätischen Höhe.

ten ein größerer Fließwiderstand wirkt. Die Bewegung des Wassers an der Oberfläche macht sich deshalb deutlich gedämpfter im Grundwassergleichenplan bemerkbar. An der 8,6-Meter-Linie deutet sich jedoch auch hier der Verlauf der Flutwelle an. Wegen der niedrigeren Infiltrationsrate ergeben sich im nördlichen Bereich generell deutlich niedrigere Grundwasserstände als unterhalb der südlichen Kammer.

In Abbildung 8.29 ist der Zustand dargestellt, in dem auf der Überflutungsfläche jeweils der maximale Wasserstand erreicht wird. Wegen des größeren Kammervolumens erreicht die nördliche Kammer trotz niedrigerer Infiltrationsrate nicht den gleichen Wasserstand wie die südliche, obwohl in beiden Kammern der gleiche Zufluss angesetzt wurde. Unterhalb der südlichen Kammer liegt der Grundwasserstand höher als im Bereich der nördlichen Flutkammer, so dass sich nicht nur ein Gefälle in Richtung des Hinterlandes ausbildet, sondern auch der Bereich unter der Nordkammer von dem hohen Grundwasserstand unterhalb der südlichen Kammer beeinflusst wird.

Nach Versiegen des Zuflusses zur Überflutungsfläche sinkt der Wasserstand innerhalb der Überflutungskammern, weil das Wasser im Boden versickert. Es liegt auf der Hand, dass dieser Prozess der Versickerung nur mit einer wechselseitig gekoppelten Modellrechnung abgebildet werden kann. Nach acht Tagen entstehen an der Uferseite erste trockene Bereiche in der südlichen Überflutungskammer (Abbildung 8.30). Im noch feuchten Bereich liegt ein geringes Gefälle vor, so dass bei dem hohen Rauheitswert das Wasser nur langsam in die trockenen Bereiche nachläuft. Fast vollständig entleert sind beide Kammern nach 9,75 Tagen. Diesen Zustand zeigt 


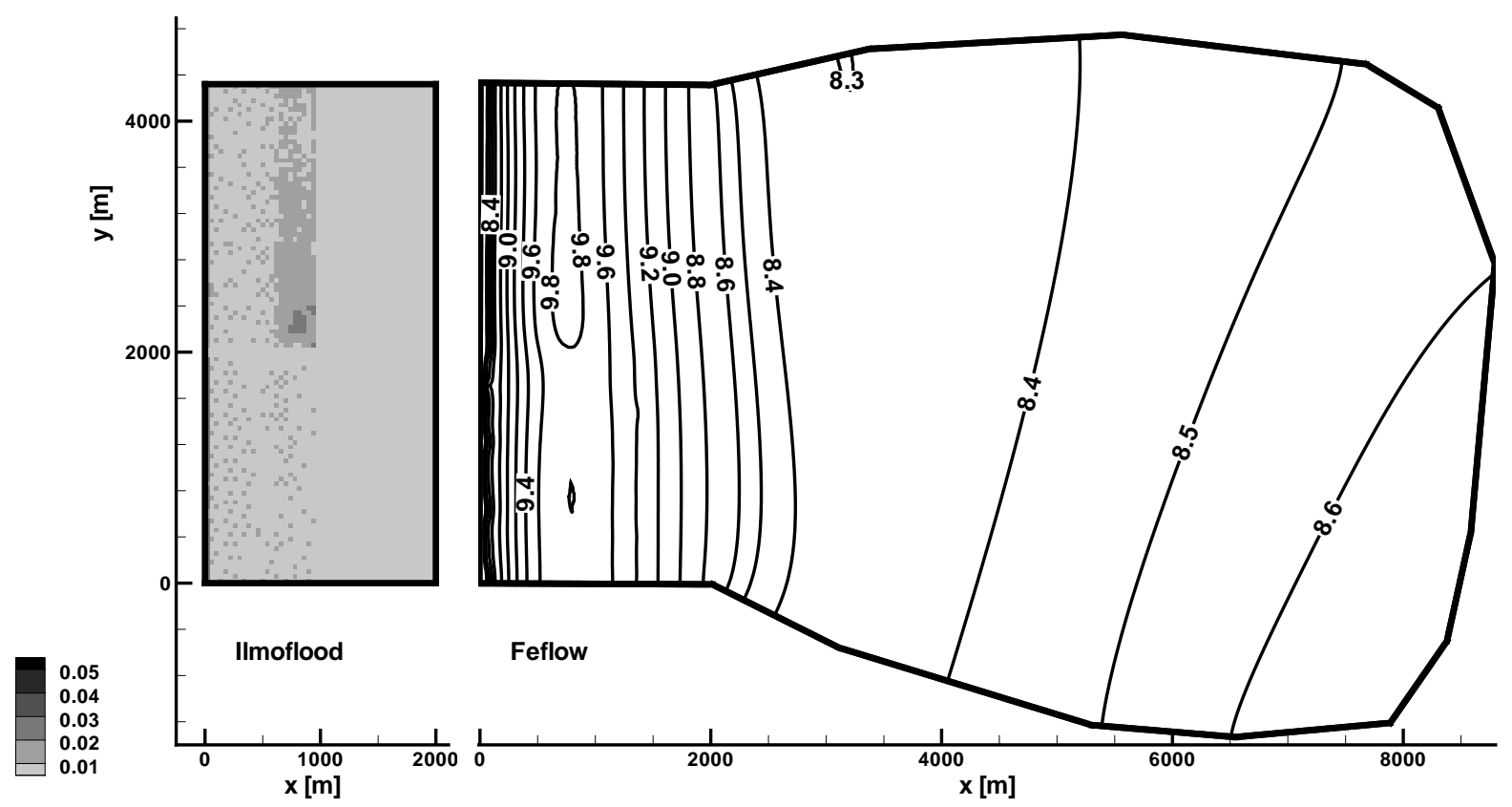

Abbildung 8.31: Wasserausbreitung auf der Überflutungsfläche und Isolinien des Grundwasserstands für den Simulationszeitpunkt 9,75d. Die Beschriftung der Gleichen zeigt den Grundwasserstand $h$ an, die Farbskala des Überflutungsflächenmodells bezieht sich auf die Wassertiefe $H$ über der geodätischen Höhe.

Abbildung 8.31. In der Südkammer steht nur noch auf vereinzelten Zellen Wasser. Dies sind diejenigen Zellen, die nicht mit dem Grundwassermodell gekoppelt sind. In der Nordkammer ist noch ein kleiner Bereich flächig mit Wasser benetzt. Die Nordkammer läuft wegen der kleineren Infiltrationsrate langsamer leer als die Südkammer. Im Untergrund hat sich nun etwa auf Höhe von $x=1000 \mathrm{~m}$ ein in Nord-Süd-Richtung ausgerichteter Grundwasserrücken gebildet, von dem sich das Wasser nach Westen in Richtung des Flusses und nach Osten ins Hinterland bewegt.

In Abbildung 8.32 sind die Zuflüsse ins Grundwassermodell getrennt nach Fluss und Kopplungsbereichen über die Simulationszeit aufgetragen. Zum Vergleich enthält die Abbildung ein Diagramm mit den Oberflächenwasserständen des Flusses und der beiden Beobachtungspunkte, die innerhalb der Flutkammern liegen. Für diese Beobachtungspunkte sind auch Berechnungsergebnisse einer Vergleichsrechnung ohne Modellkopplung in das Diagramm eingetragen. Es wird daran deutlich, dass im vorliegenden Beispiel auch die Entwicklung der maximalen Wasserstände auf der Überflutungsfläche durch die Wechselwirkung mit dem Untergrund beeinflusst werden. Die bidirektionale Kopplung ist deshalb nicht nur für die Ausbreitung des unterirdischen Hochwassers wichtig, sondern kann auch für Fragen der Überflutungsflächenausbreitung an der Oberfläche, die hier allerdings nicht im Vordergrund stehen, von Bedeutung sein.

Mit dem Anstieg des Flusswasserstands steigt zunächst der Zustrom aus dem Fluss ins Grundwasser an. Zum Zeitpunkt 2,5 d stagniert der Zufluss aus dem Fluss, weil hier gleichzeitig der Zufluss aus der Überflutungsfläche einsetzt. Durch die damit verbundene Infiltration erhöhen 

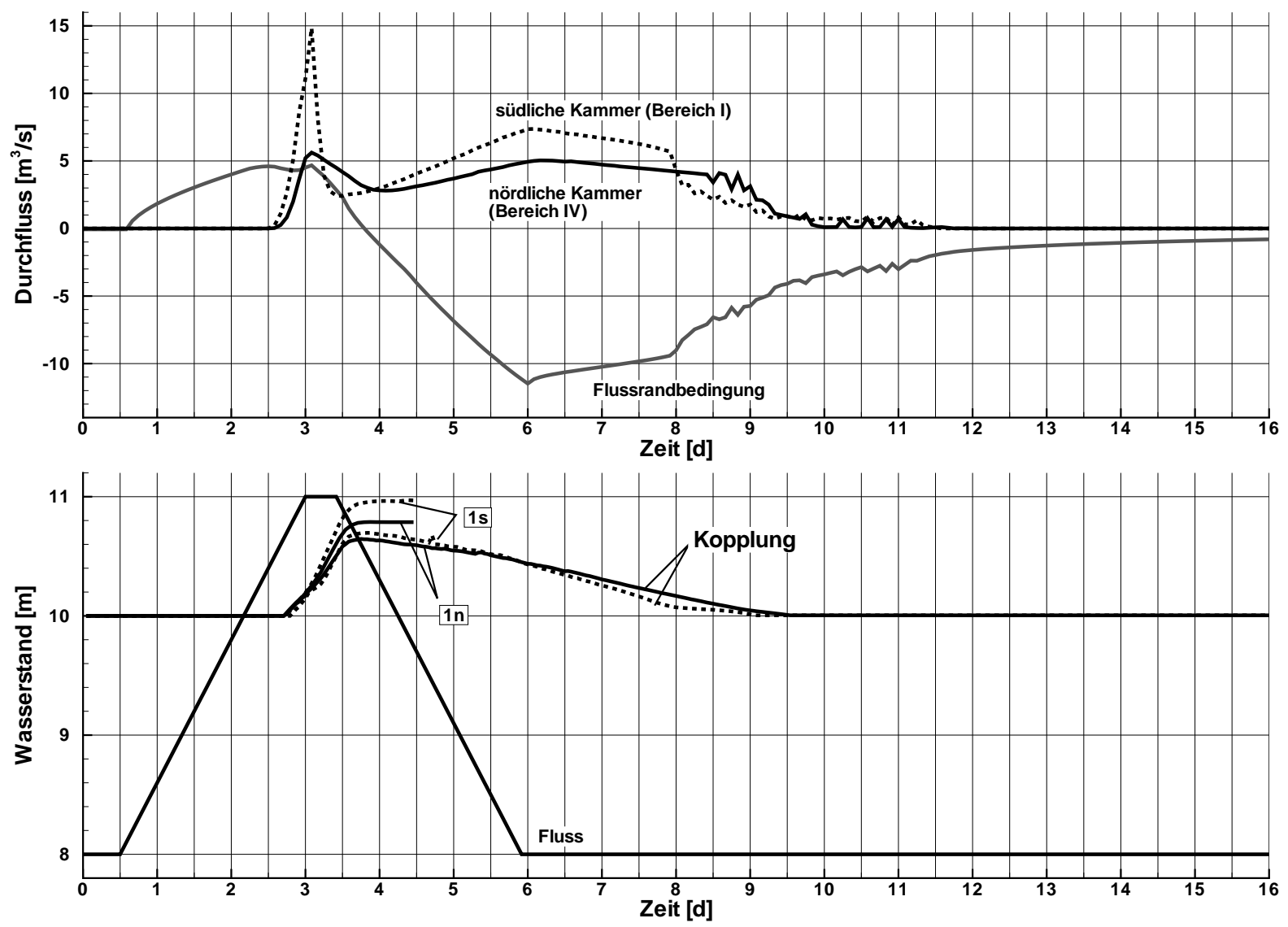

Abbildung 8.32: $\mathrm{Zu}$ - und Abflüsse des Grundwassermodells getrennt nach Kopplungsbereichen (oben) und Wasserstand im Fluss und auf der Überflutungsfläche für die Beobachtungspunkte 1s und 1n aus gekoppelter und ungekoppelter Berechnung (unten). Die Lage der Beobachtungspunkte zeigt Abbildung 8.24. Eine Entnahme aus dem Grundwasserleiter hat ein negatives Vorzeichen.

sich die Grundwasserstände im Nahbereich des Flusses, so dass die Wasserstandsdifferenz nicht mehr weiter ansteigt. Den Maximalwert erreicht die Kurve des Zustroms aus dem Fluss nach etwa 3,1 d, danach sinkt der Zustrom. Der Flusswasserstand geht zurück, gleichzeitig steigt der Grundwasserstand in der Nähe des Flusses durch die Infiltration aus dem Fluss und aus der Überflutungsfläche weiter an, so dass die Wasserstandsdifferenz zwischen Fluss und Grundwasserleiter sinkt und sich eine niedrigere Infiltrationsrate ergibt. Nach etwa 3,75 d erreicht die Zuflusskurve die Nulllinie, ab jetzt fließt Wasser vom Grundwasserleiter zurück in den Fluss. Da der Wasserstand im Fluss bis zum Simulationszeitpunkt $6 \mathrm{~d}$ sinkt und der Grundwasserstand nicht in gleichem Maße folgt, wird die Differenz zwischen den beiden Wasserständen immer größer, so dass auch der Betrag der Exfiltrationsmenge bis zum sechsten Tag weiter ansteigt. Nach sechs Tagen verbleibt der Wasserstand im Fluss, bedingt durch die angesetzte Randbedingungsganglinie, konstant auf einem Niveau von $8 \mathrm{~m}$. Die Exfiltrationsmenge wird nun allein durch den Grundwasserspiegel bestimmt. 
Der Zufluss aus der südlichen Überflutungskammer steigt mit Beginn der Überflutung wegen des hohen Leakage-Parameters sprunghaft an. Daraufhin steigt der Grundwasserstand wegen der großen Infiltrationsmenge, so dass die Wasserstandsdifferenz zurückgeht und die Infiltrationsmenge abnimmt. Unterhalb der nördlichen Flutkammer verläuft die Infiltration langsamer. Bemerkenswert ist der erneute Anstieg der Zuflussmenge aus der südlichen Flutkammer zum Zeitpunkt 3,3 d, der bis zum Tag 6 anhält und dann zurückggeht. Dieser Kurvenverlauf hängt mit dem Rückgang des Flusswasserstands zusammen. Durch das Wasser aus der Überflutungsfläche bleibt der Grundwasserstand am westlichen Modellrand auf einem hohen Niveau, so dass sich für die Exfiltration mit sinkendem Flusswasserstand große Wasserstandsdifferenzen berechnen. Die über die Flussrandbedingung aus dem Modellgebiet abfließende Wassermenge wird durch die Infiltration von der Überflutungsfläche ausgeglichen, so lange dort noch Wasser vorhanden ist. Die Zuflussmenge aus dem Kopplungsbereich I bestimmt damit wesentlich die Exfiltrationsmenge, die dem Fluss zuströmt. Für den Bereich IV ist dieser Effekt ebenfalls zu beobachten, er fällt jedoch etwas weniger ins Gewicht.

Nach acht Tagen bricht die Infiltrationsmenge aus der südlichen Flutkammer stark ein, weil jetzt der Teilbereich in der Nähe des Flusses trocken gefallen ist, wie Abbildung 8.30 zeigt. Dieses Ereignis spiegelt sich auch in der Zuflusskurve der Flussrandbedingung wieder. Für die Entleerung der Flutkammer steht jetzt eine kleinere Sickerfläche zur Verfügung, so dass der Rückgang des ohnehin schon niedrigen Wasserspiegels in der Südkammer sich nun weiter verlangsamt.

In den Infiltrationskurven der Flutkammern sind zum Ende des Infiltrationsvorgangs hin Schwankungen zu beobachten. Die Ursache dieser Schwankungen sind die niedrigen Wassertiefen, die sich einstellen, wenn die Flutkammer bereichsweise trockenfällt. Programmtechnisch wird dieser Zustand von Ilmoflood so gelöst, dass eine Zelle als trockene Zelle behandelt wird, wenn ein vom Anwender vorgegebener Schwellenwert der Wassertiefe unterschritten wird. Im vorliegenden Fall ist dieser Schwellenwert mit $H_{\text {tr }}=0,005 \mathrm{~m}$ recht niedrig angesetzt. Von einer trockenen Zelle wird kein Randbedingungswert an das Grundwassermodell übergeben, entsprechend wird für diesen Zeitschritt keine Zuflussmenge bilanziert. Durch seitliche Zuflüsse von anderen Zellen kann sich die Wassertiefe der Zelle in einem der nächsten Zeitschritte so ändern, dass der Schwellenwert überschritten und dann wieder ein Randbedingungswert angesetzt wird. Diese Randbedingung führt zu einer bilanzwirksamen Wasserentnahme der Zelle. Darauf kann diese Zelle modelltechnisch wieder trockenfallen, so dass im nächsten Zeitschritt diesmal keine Versickerungsmenge bilanziert wird. In der nördlichen Flutkammer setzen die Schwankungen später ein, weil diese Kammer später trockenfällt. Die Schwankungen äußern sich, wie anhand der Wasserstandsganglinien in Abbildung 8.33 und 8.32 unten zu sehen, nicht im primären Berechnungsergebnis des Wasserstands und führen im vorliegenden Fall auch nicht zu numerischen Stabilitätsproblemen. Nach etwa elf Tagen treten die Schwankungen nicht mehr auf, denn nun sind nur noch vereinzelte Zellen mit Wasser benetzt. Dadurch kommt es nicht mehr so häufig zum Wechsel zwischen trockenem und nicht trockenem Zustand.

Für in Abbildung 8.24 eingezeichneten Beobachtungspunkte gibt Abbildung 8.33 den Grundwasserstand über die Zeit wieder. Die Punkte 1n und 1s liegen im Gebiet der Überflutungsfläche. Der Grundwasseranstieg hängt deshalb hier sehr stark mit der Flutung der Kammern zusammen. 


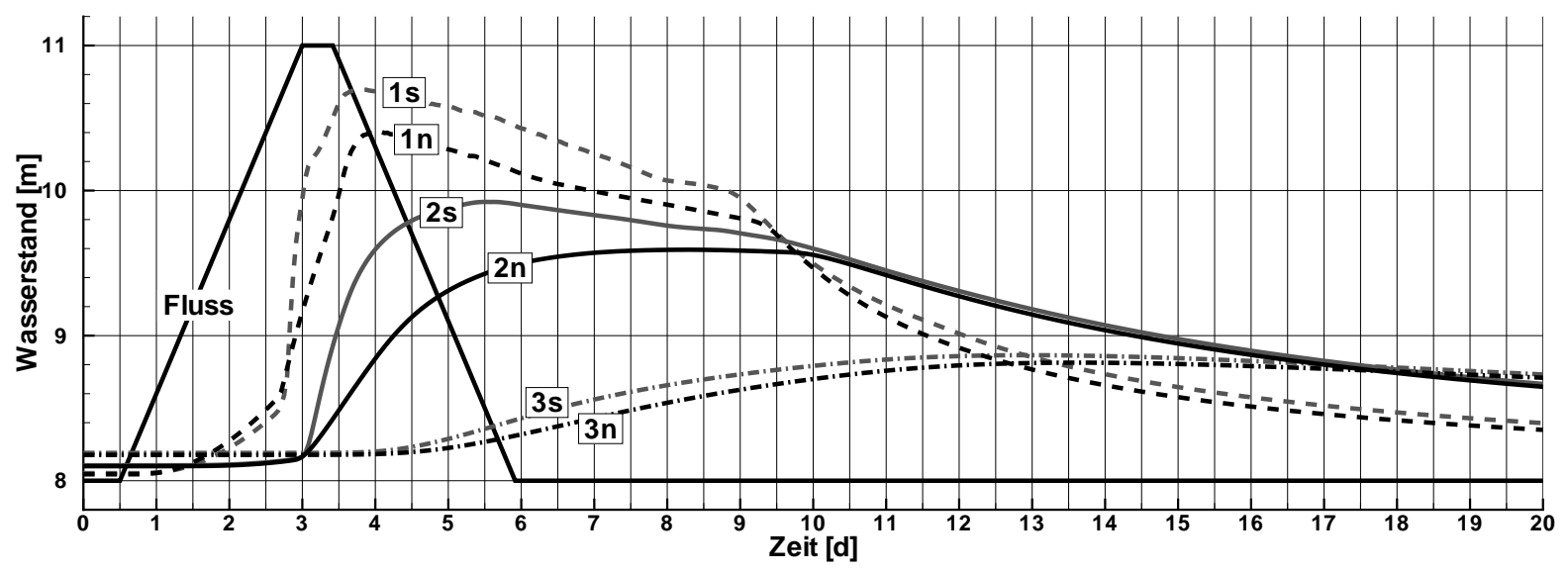

Abbildung 8.33: Verlauf des Grundwasserstands über die Zeit an verschiedenen Beobachtungspunkten sowie die Pegelganglinie des Flusses. Die Lage der Beobachtungspunkte ist in Abbildung 8.24 eingetragen

Beobachtungspunkt 1s erreicht höhere Maximalwerte als Beobachtungspunkt 1n, weil die Infiltrationsrate im Süden höher ist. Ein leichter Wasseranstieg ist bei beiden Beobachtungspunkten bereits ab etwa einem Tag wahrnehmbar, da die Wechselbeziehung mit dem Fluss, die über die Leakage-Randbedingung Eingang in das Grundwassermodell findet, eine seitliche Infiltration verursacht. Ihre Maximalwerte erreichen die Kurven 1n und 1s erst nachdem der Wasserstand im Fluss bereits wieder zurückgegangen ist. Unter beiden Flutkammern bilden sich gespannte Grundwasserverhältnisse. Das durch das teilweise Trockenfallen der südlichen Kammer verursachte langsamere Sinken des Oberflächenwasserspiegels ab Simulationstag 8 wird auch in den Grundwasserstand der Kurve 1s übertragen.

Beobachtungspunkte $2 \mathrm{~s}$ und $2 \mathrm{n}$ liegen außerhalb der Überflutungsfläche und vom Fluss aus gesehen hinter dem Längsdamm. Bei Punkt 2s steigt der Grundwasserstand schneller an als bei Punkt 2n, da in der benachbarten Kammer eine schnelle Infiltration einsetzt. Der Vergleich mit der Flussganglinie zeigt, dass hier die Überflutungsfläche den wesentlichen Einfluss ausübt, denn im Fluss klingt die Hochwasserwelle bereits ab, bevor an den Beobachtungspunkten $2 \mathrm{~s}$ und $2 \mathrm{n}$ der maximale Wasserstand erreicht wird.

Noch langsamer reagieren die Beobachtungspunkte 3s und 3n, beide liegen etwa $2000 \mathrm{~m}$ entfernt vom Fluss und $1000 \mathrm{~m}$ entfernt von der Überflutungsfläche. Während nach etwa neun Tagen beide Überflutungskammern nahezu trocken sind und damit das Hochwasser an der Oberfläche endgültig abgeklungen ist, setzt sich hier der Grundwasseranstieg noch fort bis etwa zum Tag 13. Die Grundwasserstände sind jedoch deutlich niedriger als bei den Beobachtungspunkten, die näher am Fluss liegen. 

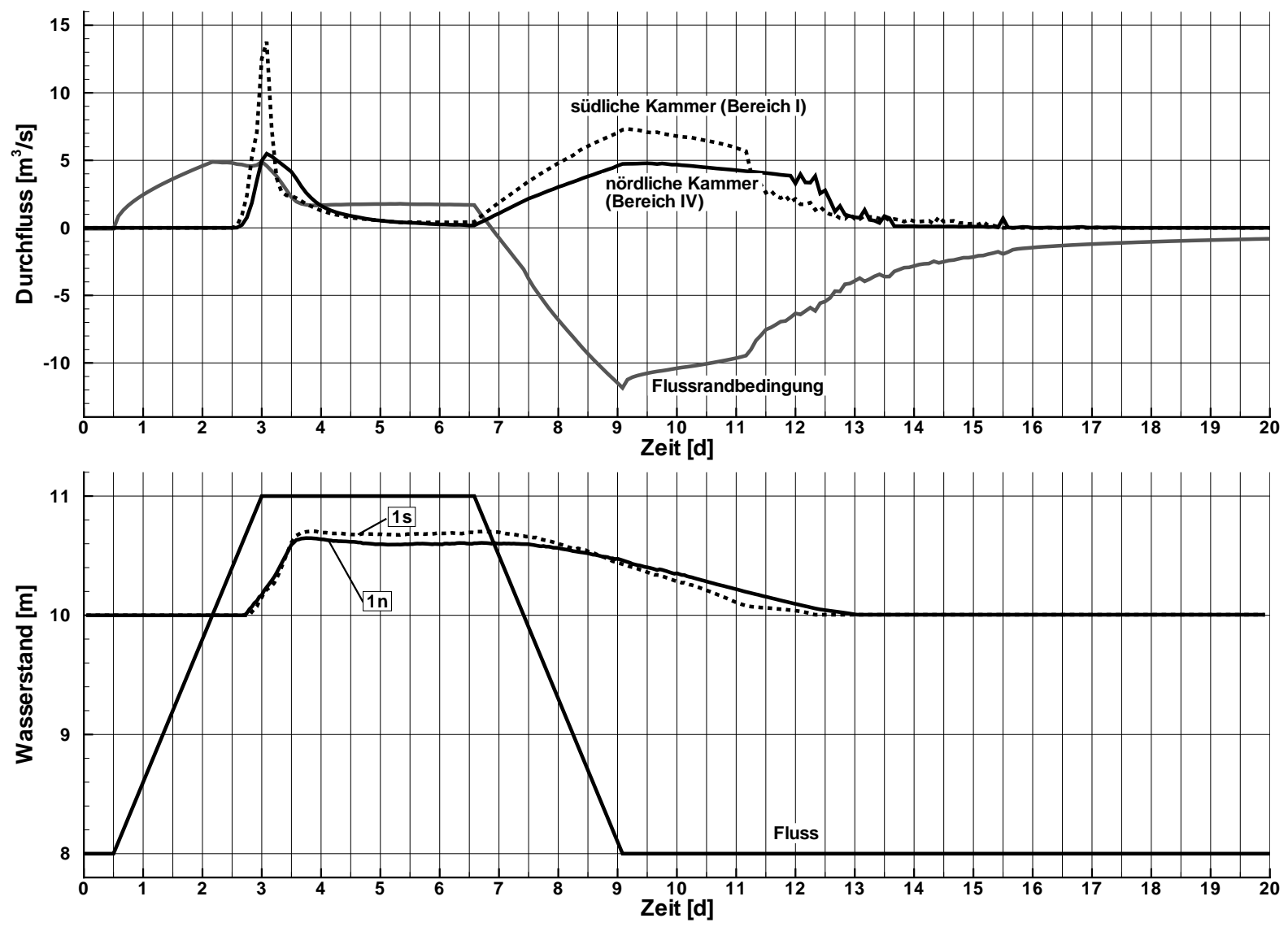

Abbildung 8.34: Zu- und Abflüsse des Grundwassermodells nach Kopplungsbereichen (oben) und Wasserstand im Fluss und auf der Überflutungsfläche für die Beobachtungspunkte 1s und 1n (unten) für das Szenario „langes Hochwasser“. Die Lage der Beobachtungspunkte zeigt Abbildung 8.24.

\subsubsection{Szenario „langes Hochwasser“}

Das Szenario „langes Hochwasser“ unterscheidet sich vom Szenario „kurzes Hochwasser“ dadurch, dass der maximale Wasserstand im Fluss länger anhält (Abbildung 8.34 unten). Der Zufluss zu den Überflutungsflächen wird dagegen zur besseren Vergleichbarkeit der Szenarien nicht verändert, für die Anfangsbedingungen und die Basisabfluss-Randbedingungen wurden ebenfalls keine Änderungen vorgenommen.

Wie beim kurzen Hochwasser kommt es mit dem Anstieg des Flusswasserstandes zu einer Infiltration über das Ufer. Die Infiltrationsmenge steigt mit dem Flusswasserstand an und stagniert mit dem Beginn der Infiltration aus den Überflutungsflächen (Abbildung 8.34). Diese Infiltrationsflüsse nehmen zunächst, je nach den Infiltrationseigenschaften des Bodens (LeakageParameter), große Werte an, um anschließend mit dem Anstieg des Grundwasserspiegels zurückzufallen. Während beim kurzen Hochwasser der Flusswasserstand nach kurzer Zeit wieder abfällt, stützt der in diesem Fall lang anhaltend hohe Flusswasserstand den Grundwasserspiegel 


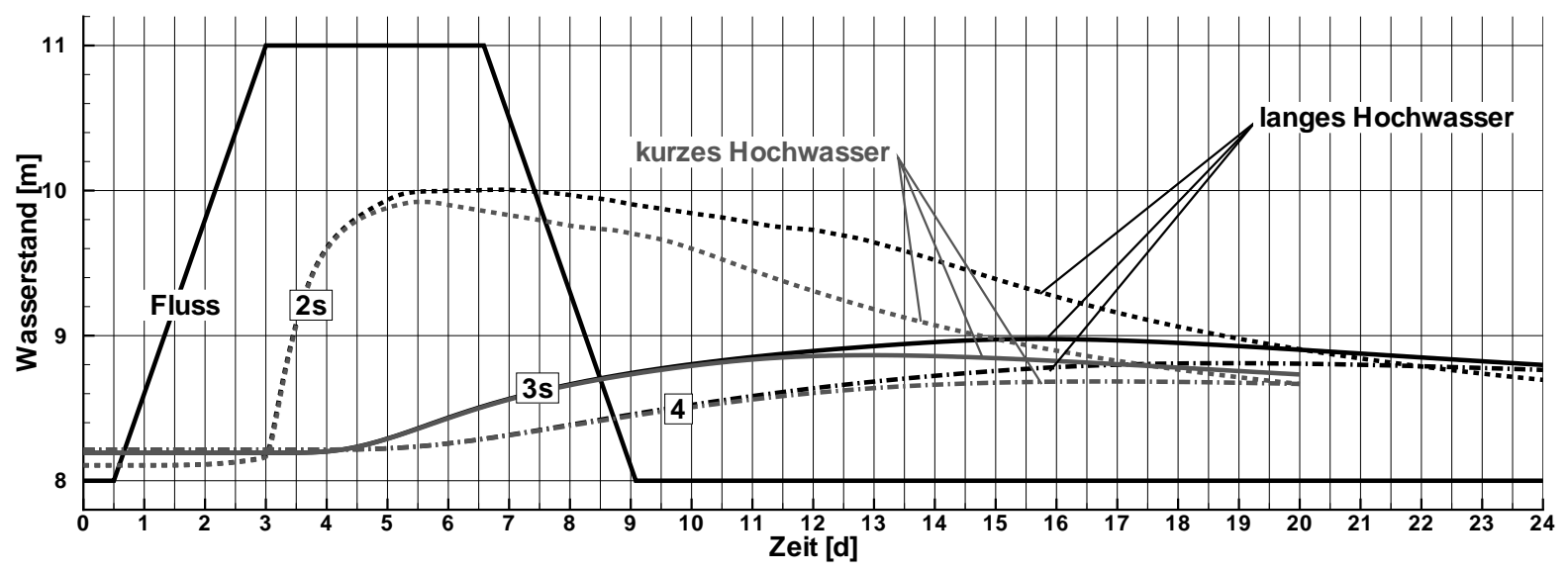

Abbildung 8.35: Vergleich der Grundwasserstände an den Beobachtungspunkten 2s, 3s und 4 für die Szenarien „langes“ und „kurzes Hochwasserereignis“ und die Ganglinie des Flusses für das lange Hochwasserereignis. Die Lage der Beobachtungspunkte zeigt Abbildung 8.24.

durch Infiltration. Gleichzeitig nimmt die Infiltration aus den Überflutungskammern langsam ab. Auch die Wasserstände in den Flutkammern gehen, bedingt durch die niedrigen Infiltrationsraten, nur langsam zurück. Die Zuflüsse aus den Kammern ins Grundwasser steigen erst wieder an, nachdem der Wasserstand im Fluss zu fallen beginnt und Grundwasser aus dem Leiter herauszieht, so dass unterhalb der Überflutungsfläche wieder die Voraussetzungen für das Versickern gegeben ist. Ein Teil der Exfiltration in den Fluss wird so wieder ausgeglichen. Auch die Einbrüche in den Infiltratonsmengen durch das Trockenfallen von Teilbereichen der Flutkammern mit den Schwankungen durch den Wechsel des Zustands sind wie beim Szenario des kurzen Hochwassers in den Bilanzkurven zu sehen.

An den Beobachtungspunkten im Hinterland werden im Szenario „langes Hochwasser“ höhere Grundwasserstände erreicht als beim kurzen Hochwasserereignis (Abbildung 8.35), da durch die länger andauernde Infiltration aus dem Fluss insgesamt eine größere Wassermenge in den Grundwasserleiter infiltriert und das Wasser aus der Überflutungsfläche zunächst nicht auf kurzem Wege zurück in den Fluss strömen kann. Zudem halten die hohen Grundwasserstände beim langen Hochwasserereignis länger an. Beim Beobachtungspunkt 3s zum Beispiel beginnt der Rückgang des Grundwasserspiegels für das lange Hochwasserereignis erst nach 16 Tagen, während ein Rückgang des Grundwasserstands beim kurzen Hochwasserereignis bereits nach 13 Tagen stattfindet. Auch weiter im Hinterland sind am Beispiel der Gangline von Beobachtungspunkt 4 deutliche Unterschiede für beide Szenarien auszumachen.

Beim langen Hochwasserereignis kommt es sogar zu einem geringen Austritt von Qualmwasser an der Oberfläche im Bereich der Senke (Abbildung 8.36). Der Qualmwasseraustritt ist jedoch so gering, dass sich nur Wassertiefen bis zu einem Zentimeter an der Oberfläche einstellen. Das Qualmwasser breitet sich deshalb nicht flächig an der Oberfläche aus. 


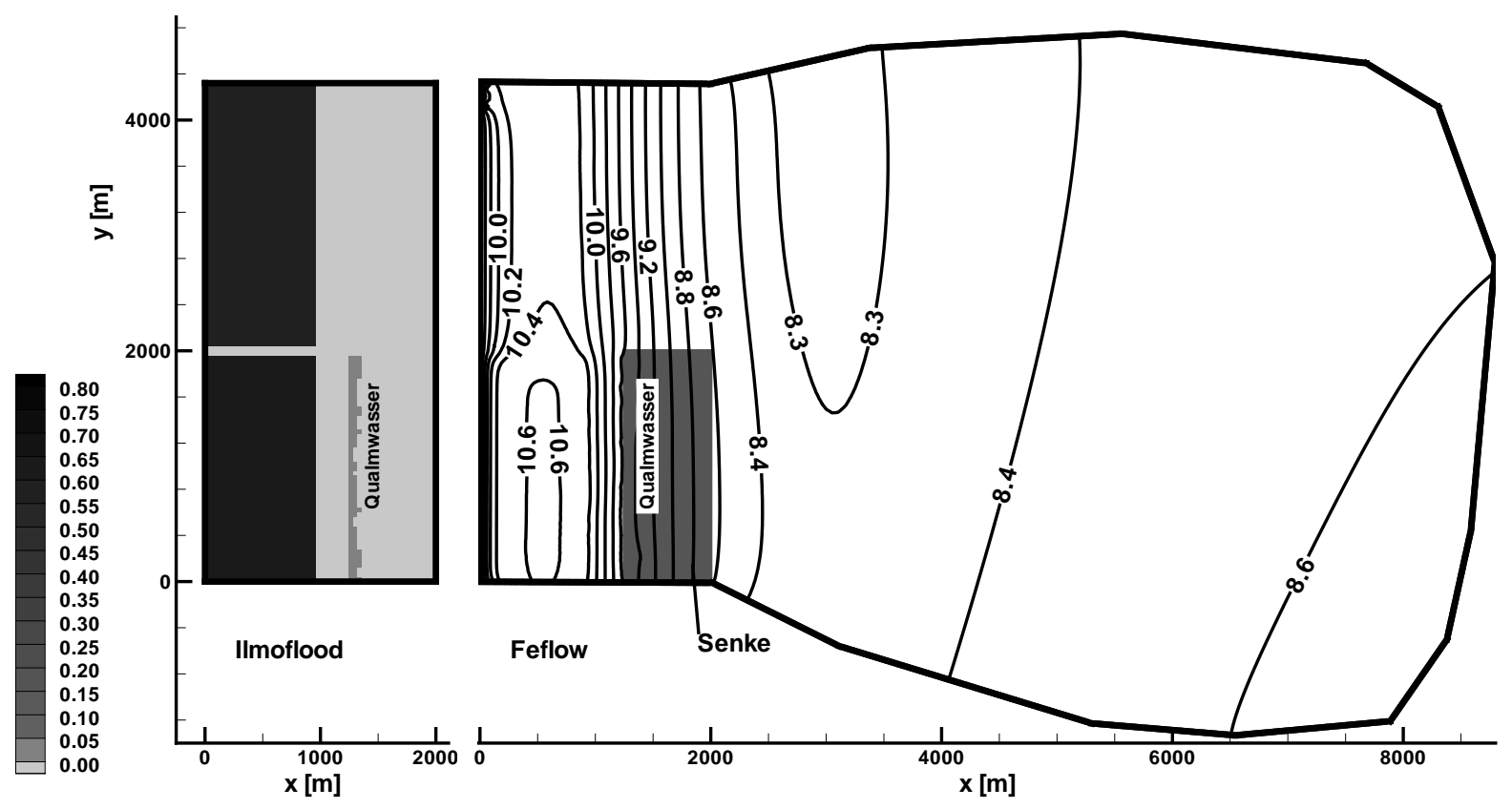

Abbildung 8.36: Qualmwasseraustritt nach acht Tagen Simulationszeit. Die Beschriftung der Gleichen zeigt den Grundwasserstand $h$ an. In der Senke liegt die geodätische Höhe der Geländeoberkante bei 9,5 m. Die Farbskala des Überflutungsflächenmodells bezieht sich auf die Wassertiefe $H$ über der geodätischen Höhe.

\subsubsection{Szenario „Ianges Hochwasser und hoher Anfangswasserstand“}

Das Szenario „langes Hochwasser“ wird nun durch erhöhte Anfangswasserstände ergänzt. Es soll mit diesem Szenario der Zustand eines durch Niederschlag erhöhten Grundwasserstands im Modellgebiet dargestellt werden. Modelltechnisch werden diese höheren Anfangswasserstände aus einer stationären Berechnung, bei der ein erhöhter Basisabfluss und ein höherer Flusswasserstand eingestellt werden, erzeugt. Der Anfangszustand ist in Abbildung 8.37 als Gleichenplan gezeigt. Die Grundwasserstände liegen etwa 20 bis $40 \mathrm{~cm}$ über den Anfangswasserständen, die den Szenarien aus den Abschnitten 8.4.3 und 8.4.4 zu Grunde liegen. Zusätzlich wird im Szenario „langes Hochwasser und hoher Anfangswasserstand“ der Basisabfluss auf $q=2,314 \cdot 10^{-5} \mathrm{~m} / \mathrm{s}$ erhöht. Die Ganglinie des Flusswasserstands und der Zufluss zu den Überflutungskammern werden jedoch nicht verändert.

Am Verlauf der Grundwasserstandsganglinien dieses Szenarios wird deutlich, dass sich das unterirdische Hochwasser anders entwickelt, wenn das Hochwasser im Fluss mit einer ungünstigen Ausgangssituation zusammenfällt. Abbildung $8.38 \mathrm{~b}$ zeigt, dass an Beobachtungspunkt 4 die Marke von $9 \mathrm{~m}$ deutlich und über längere Zeit überschritten wird. Beim Szenario „langes Hochwasser" (Abbildung 8.38a) wird diese Marke nicht erreicht.

Während beim Szenario „langes Hochwasser“ gegenüber dem Szenario „kurzes Hochwasser“ die höheren Grundwasserstände damit zu erklären sind, dass beim langen Hochwasser eine grö- 


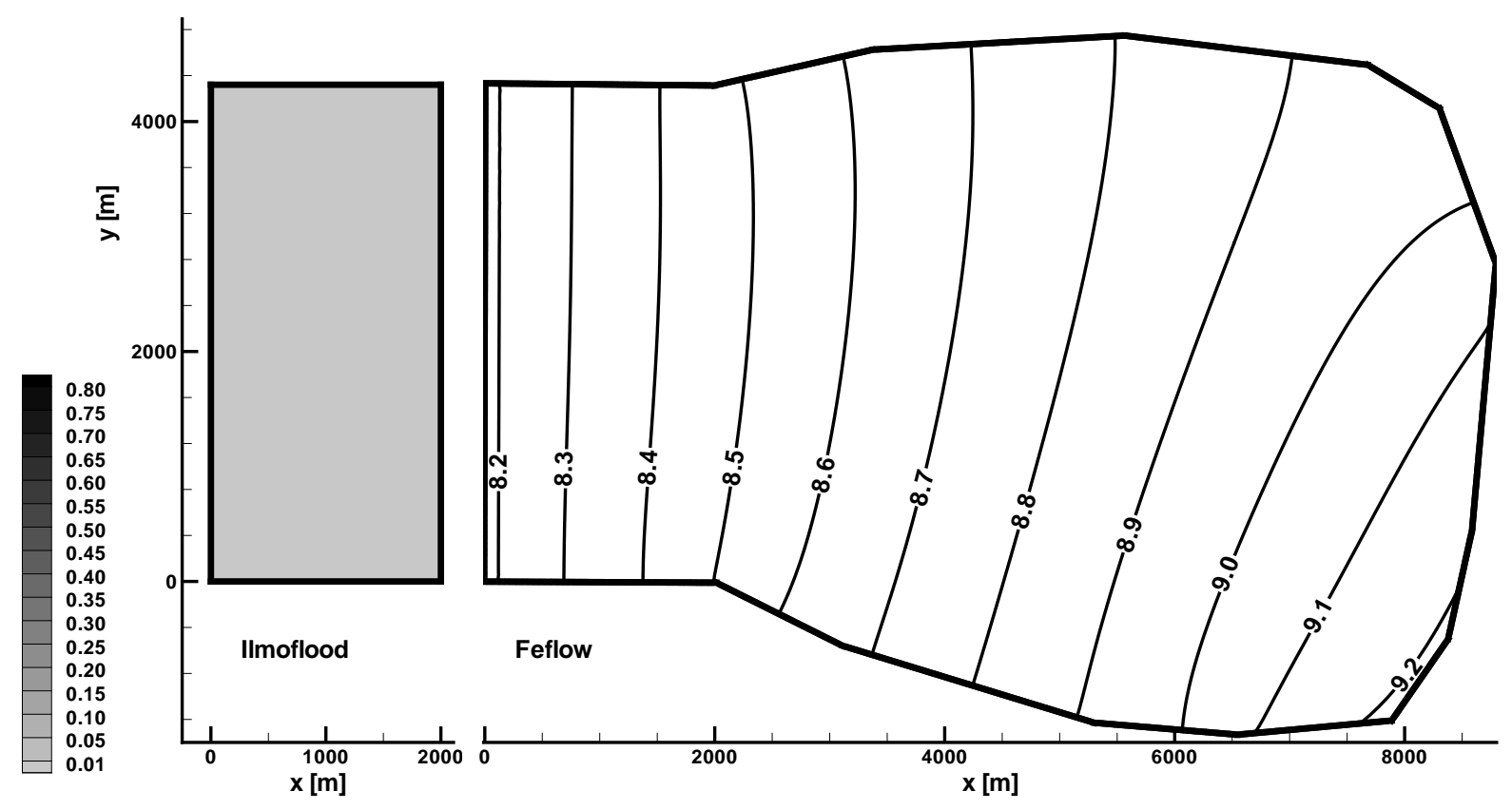

Abbildung 8.37: Anfangsbedingungen des Szenarios „langes Hochwasser und hoher Anfangswasserstand“. Die Beschriftung der Gleichen zeigt den Grundwasserstand $h$ an, die Farbskala des Überflutungsflächenmodells bezieht sich auf die Wassertiefe $H$ über der geodätischen Höhe.

Bere Wassermenge aus dem Fluss infiltriert, sind die noch höheren Grundwasserstände beim dritten Szenario „langes Hochwasser und hoher Anfangswasserstand“ eher auf das Wasser, das vor dem Hochwasserereignis bereits im Untergrund vorhanden ist, zurückzuführen. Abbildung 8.39 zeigt die Grundwasservorratsänderung der ersten 20 Simulationstage für die drei Szenarien. Beim kurzen Hochwasser (Abbildung 8.39a) klingt die Grundwasservorratsänderung aus dem Fluss schnell wieder ab, so dass der Weg frei für die Versickerung aus den beiden Überflutungskammern ist. Beim langen Hochwasser (Abbildung 8.39b) entsteht eine länger anhaltende Infiltration aus dem Fluss, die die Infiltration aus den beiden Überflutungskammern verzögert. Die gesamte Grundwasservorratsänderung ist größer als im Fall des kurzen Hochwassers und erklärt die höheren Grundwasserstände an den Beobachtungspunkten, die in Abbildung 8.35 vergleichend gezeigt sind. Beim langen Hochwasser mit hohen Anfangsgrundwasserständen (Abbildung 8.39c liegt die kumulierte Infiltrationsmenge des Flusses etwas unter dem Szenario „langes Hochwasser“, denn die höheren Grundwasserstände liefern zusammen mit dem angesetzten Flusswasserstand für die Berechnung des Leakage-Flusses eine kleinere Wasserstandsdifferenz. Aus dem gleichen Grund dauert die Infiltration aus den Überflutungsflächen bis zu deren Entleerung länger. Die maximal erreichte gesamte Infiltrationsmenge ist also bedingt durch die Anfangswasserstände etwas niedriger als im Szenario des langen Hochwassers, liefert aber trotzdem die höheren Wasserstände. 


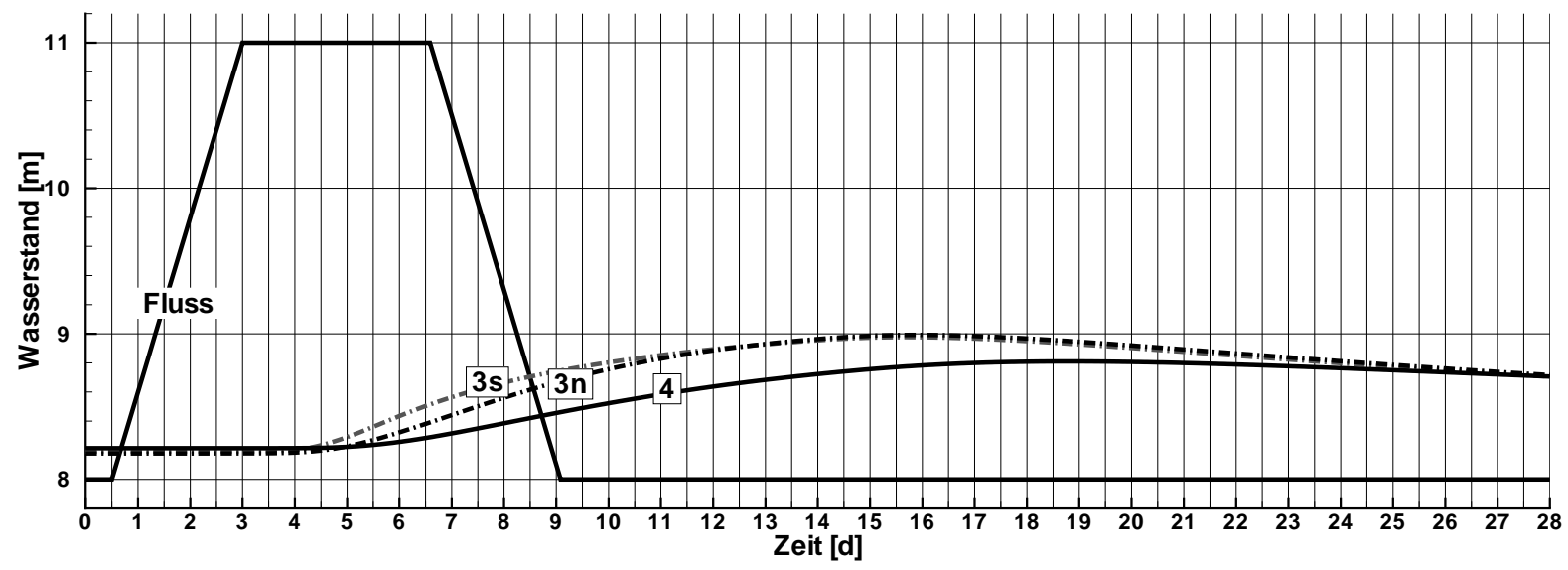

(a) langes Hochwasser

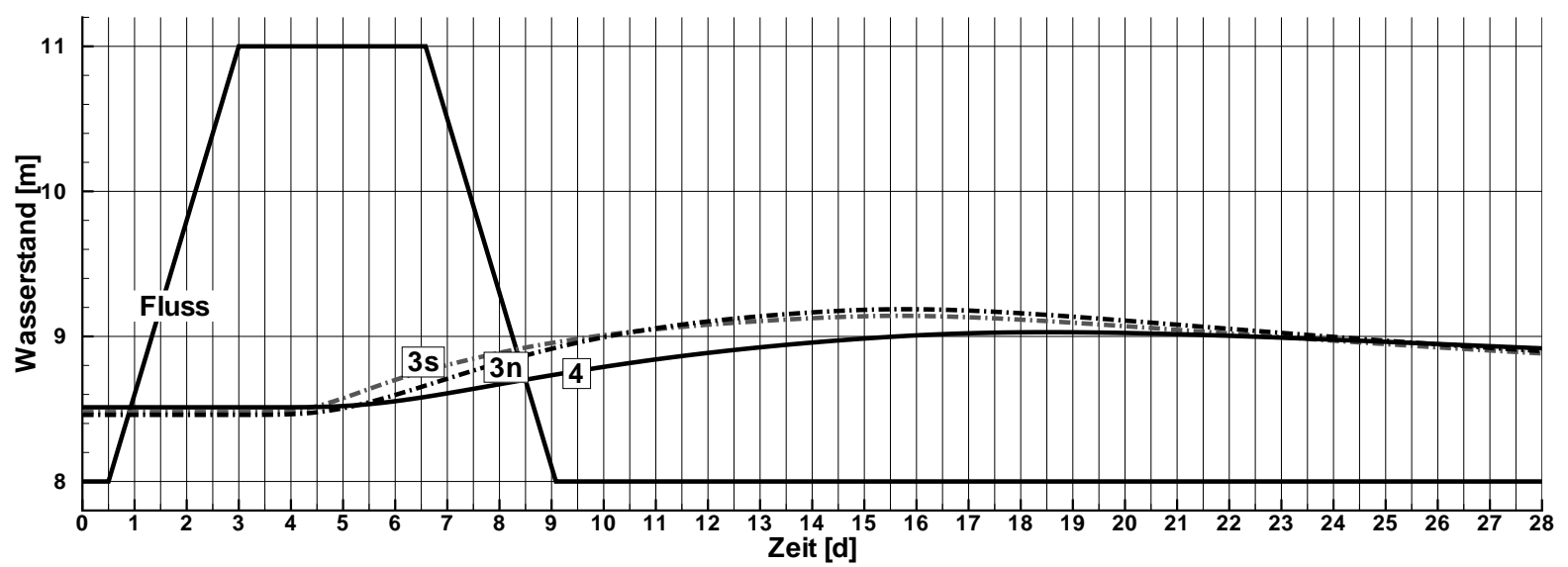

(b) langes Hochwasser und hoher Anfangswasserstand

Abbildung 8.38: Verlauf des Grundwasserstands über die Zeit an verschiedenen Beobachtungspunkten und die Pegelganglinie des Flusses. Die Lage der Beobachtungspunkte ist in Abbildung 8.24 eingetragen.

Aus den Diagrammen geht auch hervor, dass der Grundwasservorrat durch den Austritt von Qualmwasser an der Oberfläche und den Basisabfluss bei diesem Beispiel nur eine untergeordnete Rolle spielen.

Der Vergleich der Grundwasservorratsänderung durch die Infiltration aus der Südkammer (Abbildungen $8.39 \mathrm{a}$, bund c) zeigt, dass die gekoppelte Berechnung mit Ungenauigkeiten behaftet ist. Für alle drei Szenarien wurde für die Überflutungskammer die gleiche Zuflussmenge angesetzt, die vollständig ins Grundwasser infiltriert, da keine Entnahme über die Oberfläche vorgenommen wird. Der Maximalwert des Grundwasservorratsänderungsanteils aus der Südkammer, der je nach Szenario nach 11,5 bis 15 Tagen erreicht wird, müsste deshalb in allen Fällen gleich sein. Die Ursache für diese Ungenauigkeit ist, wie bereits in Abschnitt 8.2 erläutert wurde, der Zeitversatz, der durch die Methode der externen Kopplung erreicht wird. Numerische Fehler der Modellkomponenten des Ilmoflood- und des Feflow-Modells können ebenfalls dazu beitragen. 


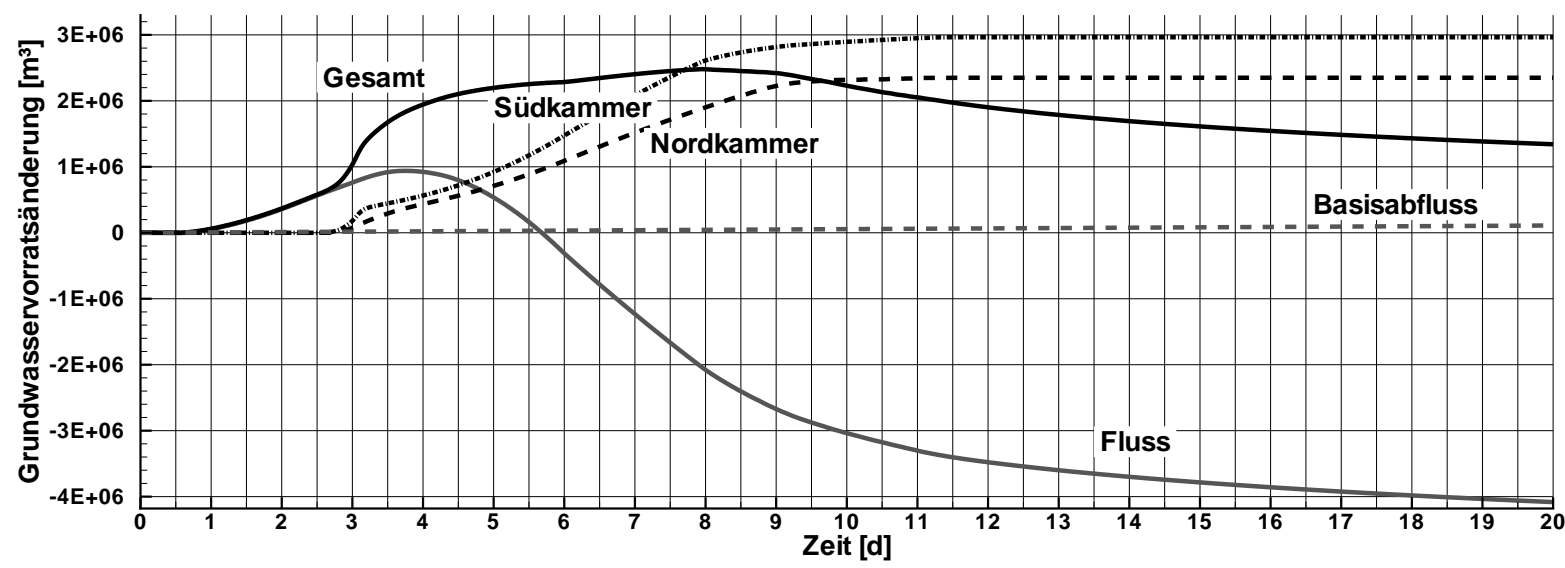

(a) Szenario ,kurzes Hochwasser“

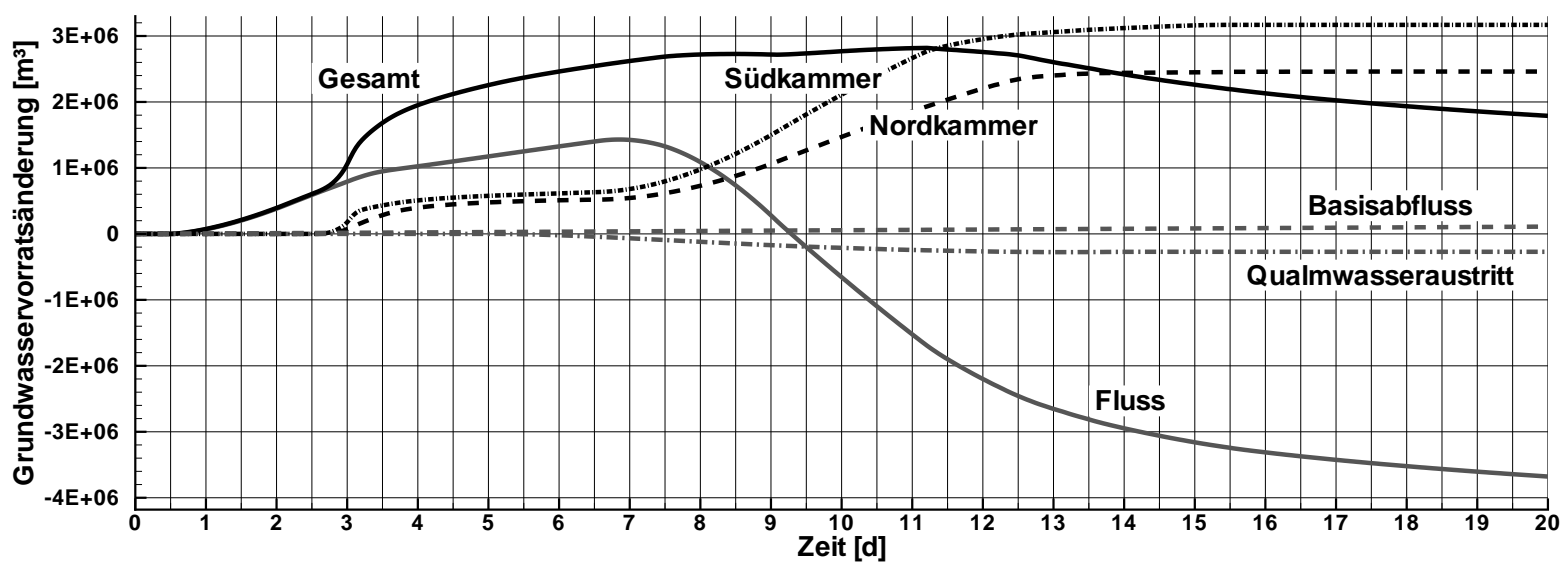

(b) Szenario „langes Hochwasser“

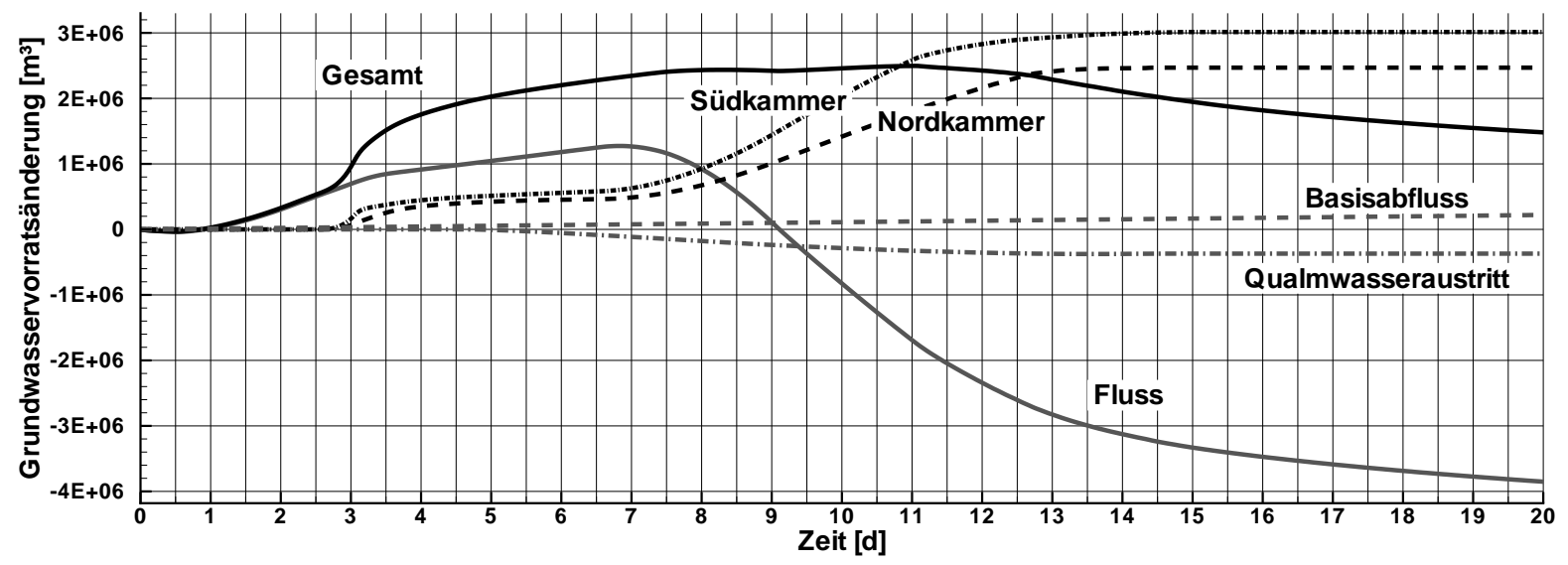

(c) Szenario „langes Hochwasser und hoher Anfangswasserstand“

Abbildung 8.39: Grundwasservorratsänderung über die Simulationszeit für die drei Szenarien des Fallbeispiels 


\subsubsection{Schlussfolgerungen und Diskussion}

Das Anwendungsbeispiel zeigt, dass die Ausbreitung eines unterirdischen Hochwassers durch

- die Dauer des Hochwassers im Fluss und

- die Anfangssituation im Grundwasserleiter

beeinflusst wird. Diese Ergebnisse decken sich qualitativ mit den Beobachtungen von Sommer et al. (2008) und Flögel (2009). Gekoppelte Modellrechnungen eignen sich also dazu, die Ausbreitung des unterirdischen Hochwassers unter Berücksichtigung der Wechselwirkungen mit dem Oberflächenwasser für verschiedene Szenarien und Anfangszustände zu berechnen. Durch das Anwendungsbeispiel wird weiterhin deutlich, dass der Prozess der Infiltration aber nicht nur die Ausbreitung des unterirdischen Hochwassers, sondern auch die Wasserspiegelentwicklung auf der Überflutungsfläche beeinflusst. Das von einer Überflutungsfläche infiltrierende Wasser kann über den Untergrund sowohl auf kurzem Wege als auch nach längerer Zwischenspeicherung im Grundwasserleiter wieder seinen Weg in den Fluss finden. Somit liefert die bidirektionale Kopplung eines Überflutungsflächenmodells und eines Grundwassermodells einen Mehrwert gegenüber ungekoppelten Berechnungen sowohl für die Oberfläche als auch für den unterirdischen Raum. Im vorliegenden Beispiel würden die Flutkammern ohne die Wechselwirkung zwischen Überflutungsfläche und Grundwasserleiter niemals leerlaufen, zudem würden die Wasserstände der Überflutungsfläche überschätzt. Ein gekoppeltes Modellsystem, das die Kompartimente Überflutungsfläche und Untergrund umfasst, kann also auch einen Beitrag zur Prognose der Hochwasserausbreitung auf der Landoberfläche leisten. Dies wird besonders dann relevant, wenn - anders als im vorliegenden Fallbeispiel - Fluss und Überflutungsfläche zusammenhängend modelliert werden (siehe z. B. Kamrath 2009).

Das Szenario, dass einer Hochwassergefahrenkarte zu Grunde liegt, wird oft mit einem Abflusswert, zum Beispiel dem HQ 100 ${ }^{1}$, beschrieben (Ministerium f. Umwelt u. Naturschutz, Landwirtschaft und Verbraucherschutz d. Landes Nordrhein-Westfalen 2003; Umweltministerium, Innenministerium und Wirtschaftsministerium Baden-Württemberg 2005). Über eine Schlüsselkurve lässt sich dieser Bemessungsdurchfluss oft genau genug mit einem Wasserstand in Beziehung setzen. Erst für die Analyse flächiger Überflutungen müssen Szenarien näher beschrieben werden, wie etwa durch die Angabe von Ort und Zeitpunkt einer Breschenbildung in einer Hochwasserschutzeinrichtung (siehe z. B. Harms 2004). Auch das Bemessungsszenario eines unterirdischen Hochwassers erfordert eine nähere Beschreibung, denn den drei hier betrachteten Szenarien liegt der gleiche Flusswasserstand zu Grunde, die Auswirkungen auf den unterirdischen Raum sind jedoch unterschiedlich. Die Gefahrenkarte des unterirdischen Hochwassers von Forberig (2009) bezieht sich deshalb auf einen Pegelstand des Flusses, der über einen bestimmten Zeitraum überschritten wird. Während bei der Hochwassergefahrenkarte die Wassertiefe auf der Landoberfläche dargestellt ist, zeigt die Gefahrenkarte des unterirdischen Hochwassers die Flurabstände. Eine gekoppelte Modellrechnung kann als Grundlage zur Erstellung derartiger Karten verwendet werden. Abbildung 8.40 zeigt die Flurabstandskarte des Fallbeispiels für das Szenario

\footnotetext{
${ }^{1}$ Hochwasser, das statistisch einmal in 100 Jahren auftritt
} 


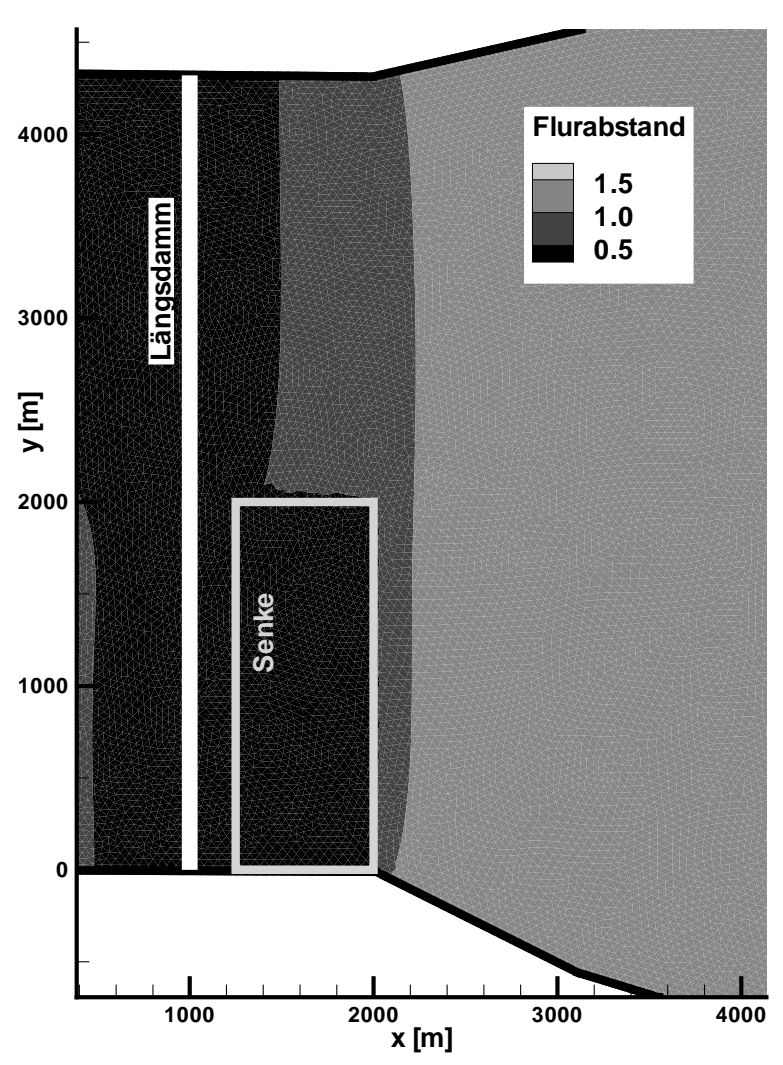

(a) 13 Tage

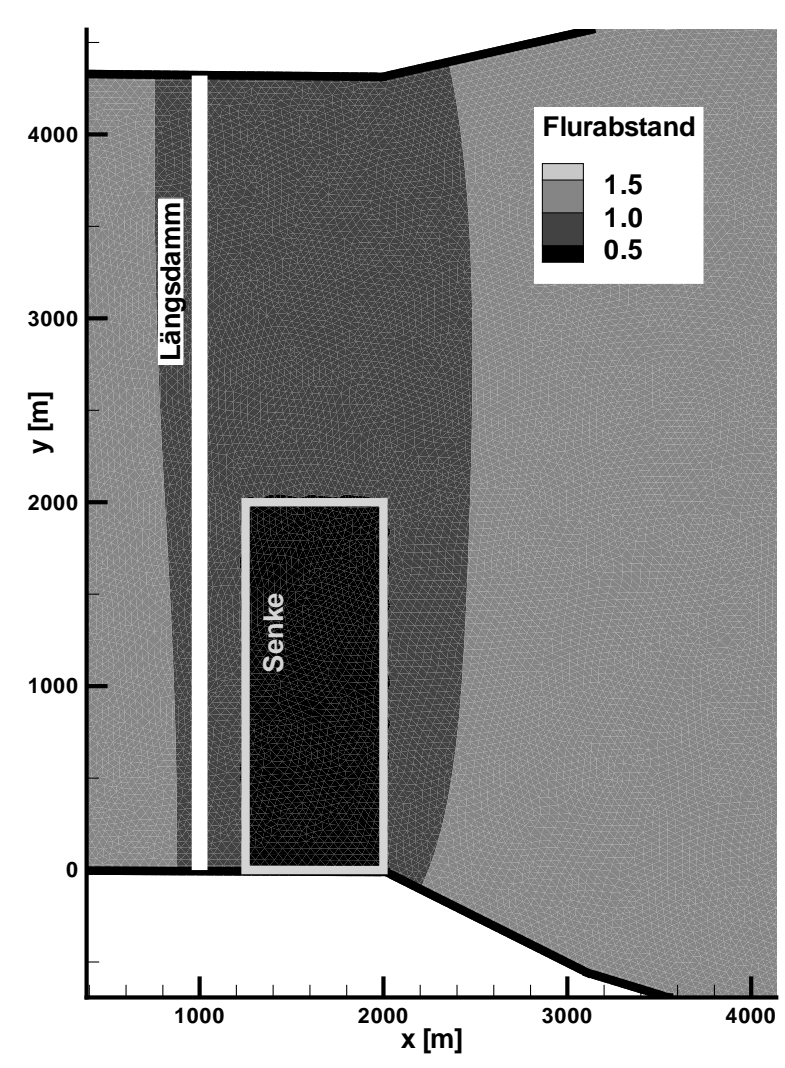

(b) 23 Tage

Abbildung 8.40: Flurabstände aus dem Szenario „langes Hochwasser mit hohem Anfangswasserstand“ für die Simulationszeitpunkte 13 Tage und 18 Tage als Detailansicht

„langes Hochwasser und hoher Anfangswasserstand“ zu zwei verschiedenen Zeitpunkten. Der Vergleich der beiden Karten zeigt, dass auch hier unterschiedliche Schwerpunkte gewählt werden können. So hat sich die unterirdische Hochwasserwelle nach 13 Tagen (Abbildung 8.40a) noch nicht so weit ausgebreitet wie nach 18 Tagen (Abbildung 8.40b), dafür sind im ersteren Fall aber die Flurabstände in weiten Bereichen deutlich geringer.

Das Anwendungsbeispiel ist in allen Punkten sehr einfach gehalten. Der einzelne Grundwasserleiter ist in seinen Durchlässigkeitseigenschaften homogen, es gibt also weder präferenzielle Fließwege, in denen sich das Wasser schneller als anderswo bewegt, noch Bereiche, die gering durchlässig sind und so einen Widerstand für die unterirdische Hochwasserwelle darstellen. Flussbiegungen oder Flussverzweigungen, in deren Innenkurve sich erfahrungsgemäß vorzugsweise Probleme des unterirdischen Hochwassers einstellen können (Köngeter et al. 2002, 1997), enthält das Modell nicht. Auch entstehen in dem Anwendungsbeispiel keine weitläufigen gespannten Bereiche, die nach Vekerdy \& Meijerink (1998) zur Beschleunigung der Ausbreitung des unterirdischen Hochwassers führen können. Es gibt keine Prozesse wie Grundwasserentnahmen, die das unterirdische Hochwasser auffangen oder bei ihrem Ausfall verstärken können. 
Deshalb können die Ergebnisse nur qualitativ auf reale Untersuchungsgebiete übertragen werden, sie zeigen aber auf, welche Aspekte für den Einsatz von gekoppelten Modellen zu beachten sind.

Auch die Überflutungsfläche ist mit ihrer einfachen Geometrie und den flächig verteilten Rauheitsbeiwerten einfach modelliert. Das Hochwasserereignis selbst ist durch eine idealisierte Ganglinie charakterisiert, bei der zum Beispiel keine Verstärkung durch aufeinander folgende Hochwasserereignisse auftritt. Der Zufluss zur Überflutungsfläche wurde vor Beginn der Berechnung ermittelt, so dass er keine Rückstaueffekte durch den Wasserstand auf der Überflutungsfläche berücksichtigt. Wünschenswert wäre hier die Vorgabemöglichkeit einer h-Randbedingung für das Programm Ilmoflood, so dass für beide Modellkomponenten die gleiche Randbedingungskurve verwendet werden kann und die Modelle dahingehend synchronisiert sind. Da der Fluss im vorliegenden Fallbeispiel als Randbedingung in die gekoppelte Berechnung einfließt, können keine Aussagen hinsichtlich der Retentionswirkung aus dem seitlichen Abstrom in die Flutkammern und den unterirdischen Raum auf die Hochwasserwelle im Fluss getroffen werden.

Technische Aspekte und konzeptionelle Fehler der Kopplung durch vereinfachte Modellansätze wurden bereits in den Abschnitten 6 und 7 und bei den beiden anderen Anwendungsbeispielen dieses Kapitels (Abschnitte 8.2 und 8.3 angesprochen. Ein neuer Aspekt dieses Anwendungsbeispiels sind die Schwankungen des sekundären Berechnungsergebnisses, die beim Trockenfallen der Überflutungsfläche auftreten.

Die Rechenzeit des gekoppelten Modells wird im Wesentlichen von den Modellkomponenten bestimmt. Der Datenaustausch stellt keine besonders rechenzeitintensive Operation dar und ist im Vergleich zum Rechenzeitbedarf der Modellkomponenten verschwindend gering. Lediglich die Initialisierungsphase zu Anfang dauert einige Minuten. Auf einem handelsüblichen Rechner ${ }^{2}$ wurden Rechenzeiten von vier (16 Tage Simulationszeit) bis zwölf (28 Tage Simulationszeit) Stunden benötigt.

\subsection{Zusammenfassung des Kapitels}

Verschiedene Anwendungsbeispiele in diesem Kapitel zeigen zunächst Grenzen und Möglichkeiten der Modellkopplung auf. So entsteht zum Beispiel bedingt durch die hier umgesetzten Variante der externen Modellkopplung ein Zeitversatz bei der Übergabe der Austauschmengen, der zu Massenbilanzfehlern der Kopplung führt. Zu numerischen Stabilitätsproblemen führte diese Ungenauigkeit bei den vorgestellten Beispielen jedoch nicht.

Der Mehrwert einer Modellkopplung wird besonders deutlich anhand des Beispiels, bei dem Qualmwasser an der Oberfläche austritt, sich nach den dort gültigen Fließgesetzen bewegt und

\footnotetext{
${ }^{2}$ Prozessoreinheit: Intel ${ }^{\circledR}$ Core $^{\mathrm{TM}} 2$ Duo CPU E6550, 2,33 GHz, 2,0 GB RAM; Betriebssystem: Microsoft Windows XP Professional
} 
an einer anderen Stelle infiltriert. Diese Kombination der Fließwege ist nur mit einer Modellkopplung in beide Richtungen realisierbar. Die Kopplung mit einem Überflutungsflächenmodell erweitert das zweidimensional-horizontale Grundwassermodell quasi um eine dritte Dimension, da eine Überströmung des Grundwasserraums möglich wird. An dem Beispiel wird auch deutlich, dass die verwendeten Modellkomponenten wesentlich die Genauigkeit der Prozessmodellierung bestimmen.

Eine gekoppelte Modellrechnung kann auch zur Erweiterung des Prozessverständnisses beitragen. So geht aus einer anderen Beispielrechnung hervor, dass die Infiltration aus einer überfluteten Fläche im Vergleich zur Uferspeicherung den größeren Anteil am Zufluss zum Grundwasser stellen kann, wenn durch die Infiltration eine große Kontaktfläche aktiviert wird. Die Grundwasservorratsbilanz des Beispiels zeigt auch, dass der größte Teil des Wassers, das über die Überflutungsfläche in den Grundwasserleiter infiltriert, nicht den gleichen Weg zurück nimmt, sondern über das Ufer in den Fluss zurück fließt oder aber sich ins Hinterland ausbreitet. Weit weg vom Ufer steigt der Grundwasserstand deshalb erst dann, wenn im Fluss die Hochwasserwelle schon längst abgeklungen ist.

Je nach Modellkonzept ergeben sich aber deutliche Unterschiede in den Infiltrationsraten, wenn die Infiltration mit einer Leakage-Randbedingung modelliert wird. So wird bei gleichen Leakage-Parametern mit der Richards-Gleichung eine geringere Infiltrationsrate erreicht als bei rein gesättigter Grundwasserströmung. Andere Aspekte der Modellierung, wie zum Beispiel der unterschiedliche Modellansatz beim Wechsel zwischen ungespannten und gespannten Verhältnissen, beeinflussen ebenfalls die Entwicklung des Grundwasserstands. Wegen des hohen numerischen Aufwands, der sich in langen Rechenzeiten und einer notwendigerweise feineren Diskretisierung äußert, und des Bedarfs an weiteren Modellparametern, wird sich die Lösung der Richards-Gleichung für Planungsaufgaben und den operativen Einsatz voraussichtlich in nächster Zeit nicht durchsetzen. In einem rein gesättigten Grundwassermodell müssen die LeakageParameter dann als Kalibrierungsparameter aufgefasst werden, mit denen die Infiltrationsmenge gesteuert wird.

Drei verschiedene Szenarien eines Anwendungsbeispiels zeigen, dass die Dauer des Hochwasserereignisses und der Anfangszustand im Grundwasser, auf den das Hochwasser trifft, die Ausbreitung der Hochwasserwelle im Untergrund beeinflusst. Die Modellkomponenten des Anwendungsbeispiels sind zwar einfach gehalten, orientieren sich aber hinsichtlich ihrer Größe an Modellen, die derzeit eingesetzt werden. Die vorgestellte Modellkopplung eignet sich also nicht nur für kleine Testbeispiele, sondern kann auch zur Kopplung größerer Modelle verwendet werden.

Während Hochwassergefahrenkarten als eine wesentliche Größe die Wassertiefe über Gelände für einen bestimmten Durchfluss zeigen, gibt die Gefahrenkarte des unterirdischen Hochwassers Flurabstände an. Weil Anfangsbedingungen und Dauer des Ereignisses die Entwicklung der Flurabstände wesentlich beeinflussen, muss für Gefahrenkarten des unterirdischen Hochwassers der Bezugsabfluss um die Dauer des Ereignisses erweitert werden. 


\section{Zusammenfassung der wesentlichen Ergebnisse, Bewertung und Ausblick}

\subsection{Einführung}

Das Problem des unterirdischen Hochwassers und seiner möglichen unerwünschten und schädlichen Auswirkungen für urbane Räumen wird am Beispiel des August-Hochwassers 2002 aufgezeigt. Während die Stadt Dresden als Pilotstandort mittlerweile gut mit Modellwerkzeugen für Planungsaufgaben des unterirdischen Hochwassers ausgerüstet ist, besteht für andere Städte, die an größeren Flüssen gelegen sind, weiterhin Bedarf an Methoden und Modellen zur Erfassung des unterirdischen Hochwassers für den operativen und den planerischen Einsatz. Da zwischen dem Wasser an der Oberfläche und im Untergrund Wechselwirkungen bestehen, ist eine zusammenhängende Betrachtung beider Kompartimente durch eine Kopplung numerischer Modelle erforderlich. Diese Arbeit trägt dazu bei, diese Lücke zu schließen, indem zwei Ziele erreicht werden:

- es wird eine Möglichkeit der Kopplung eines Überflutungsflächenmodells mit einem Grundwassermodell zur Prognose des unterirdischen Hochwassers vorgestellt und

- das Verständnis für die Wechselwirkungsprozesse zwischen Wasser auf der Landoberfläche und in einem Grundwasserleiter wird vertieft.

Die Modellkopplung nimmt Rücksicht darauf, dass für mögliche Einsatzgebiete bereits Modelle vorhanden sind, sie wird deshalb mit Hilfe des OpenMI-Standards umgesetzt. Die Kopplung bezieht sich auf Modelle, die auf Basis der Programme Feflow und Ilmoflood aufgebaut worden sind. Sie kann aber auf Modelle, die andere Programme verwenden, übertragen werden. Für beide Modellkomponenten stellt der Fluss wesentliche Randbedingungen.

Die Wechselwirkungsprozesse werden vor dem Hintergrund der Modellkopplung betrachtet. Mit Blick auf die bestehenden Programmfunktionalitäten werden Möglichkeiten zur modelltechnischen Erfassung der Wechselwirkungsprozesse in einem gekoppelten System erarbeitet.

\subsection{Wechselwirkungsprozesse}

Für den Wasseraustausch zwischen einem Fluss und dem angrenzenden Grundwasserleiter, die sogenannte Uferspeicherung, haben sich Leakage-Ansätze etabliert. Nach dem Leakage-Modell berechnet sich die ausgetauschte Wassermenge pro Zeiteinheit aus der Differenz zwischen den 
Wasserständen der beiden Kompartimente. Die Beziehung zwischen Wasserstandsdifferenz $\Delta h$ und Austauschfluss $Q$ kann auf verschiedene Arten berechnet werden. Zur Beantwortung der Frage, welche Art von Leakage-Ansatz für den Prozess der Uferspeicherung in Frage kommt, wurde für das von Ubell (1987a) dokumentierte Hochwasserereignis eine $Q$ - $\Delta h$-Beziehung aufgestellt.

Die wesentlichen Erkenntnisse aus der Analyse des Uferspeicherungsereignisses von Ubell (1987a) werden wie folgt zusammengefasst:

- die Beziehung zwischen Wasserstandsdifferenz und Leakage-Fluss eines Hochwasserereignisses kann hysteretischer Natur sein

- eine lineare Leakage-Funktion eignet sich zur Modellierung der Uferspeicherung

- der hysteretische Verlauf einer Leakage-Funktion kann in einem numerischen Modell durch Ansatz des sich mit dem Wasserspiegel im Fluss verändernden benetzten Umfangs in Kombination mit einer Schichtung der Leakage-Parameter erreicht werden

- mehrere Modellvarianten führen zu einer guten Übereinstimmung zwischen gemessenen und berechneten Grundwasserständen.

Forschungsbedarf besteht noch dahingehend, zu untersuchen, in wie weit die Veränderung der Kontaktfläche zwischen Fluss und Grundwasserleiter die Uferspeicherung beeinflusst und welche Rolle Erosions- und Sedimentationsprozesse spielen. Hängt der Wasseraustausch im Wesentlichen vom benetzten Umfang ab, dann würde die Eingangsgröße des Wasserstands ausreichen. Wenn Erosions- und Sedimentationsprozesse eine bedeutende Rolle spielen, ergibt sich möglicherweise der Bedarf einer Modellkopplung mit numerischen flussmorphologischen Modellen.

Ganz allgemein ist deshalb auch zu untersuchen, in welchem Teil des Flussquerschnittes der Wasseraustausch bei großen Flüssen hauptsächlich stattfindet, also zum Beispiel, ob Ufersicherungen und Wühltiergänge eine besondere Rolle beim Prozess der Uferspeicherung spielen, oder ob der Wasseraustausch hauptsächlich über die Sohle erfolgt. Sowohl Feldmessungen als auch physikalische Modellversuche können zur Beantwortung derartiger Fragen beitragen.

Die Infiltration von einer Überflutungsfläche wird von der Passage der ungesättigten Bodenzone geprägt. Durch die Einstauung an der Bodenoberfläche dringt, anders als zum Beispiel bei leichtem Niederschlag, eine Sättigungsfront von oben her in den Boden ein. Die Auswertung analytischer Infiltrationsformeln für verschiedene Bodenbeispiele zeigt, dass bei gut durchlässigem Boden diese Sättigungsfront im Zeitraum von Stunden um einen Meter in den Boden eindringt, bei weniger gut durchlässigen Böden kann der Infiltrationsprozess deutlich länger dauern. Generell kann deshalb nicht davon ausgegangen werden, dass der Boden unterhalb einer Überflutungsfläche wassergesättigt ist.

Die Infiltration aus einer Überflutungsfläche ist eine Wasserbewegung durch ein ungesättigtes poröses Medium und gehorcht damit der Richards-Gleichung. Da die numerische Lösung der 
Richards-Gleichung sehr aufwändig ist, ist es zweckmäßig, den Prozess der Infiltration von einer Überflutungsfläche für Planungsaufgaben und den operativen Hochwasserschutz mit einer Leakage-Randbedingung zu modellieren. Ein Vergleich mit numerischen Lösungen der Richards-Gleichung zeigt, dass dieser Ansatz qualitativ durchaus akzeptabel ist. Allerdings können ein durch die Versickerung verzögerter Anstieg des Grundwassers und im ungesättigten Bereich gespeichertes Wasser so nicht erfasst werden. Die Infiltrationsrate wird zudem deutlich überschätzt.

Forschungsbedarf besteht deshalb dahingehend, zur Modellierung der Infiltration von Wasser aus einer Überflutungsfläche eine Möglichkeit zu finden, mit der der Sickerprozess in einem Grundwassermodell abgebildet werden kann, ohne dass für dieses Modell die Richards-Gleichung gelöst werden muss. Eine Idee dazu ist, zwischen das Grundwassermodell und das Oberflächenmodell ein einfaches Richards-Modell für die ungesättigte Zone zu koppeln.

So wie beim Prozess der Infiltration kann auch beim Anstieg des Grundwasserspiegels im Boden gespeichertes Wasser nicht erfasst werden, wenn die Grundwasserströmung rein gesättigt modelliert wird. Erreicht der Wasserstand die obere Leiterbegrenzung, entstehen gespannte Strömungsverhältnisse. Ein Austritt des Grundwassers an der Oberfläche, der sogenannte Qualmwasseraustritt, kann über eine Sickerflächenrandbedingung modelliert werden. Es wird in diesem Fall dem Grundwassermodell gerade so viel Wasser entzogen, dass der Wasserstand am Ort der Randbedingung die obere Leiterbegrenzung erreicht.

\subsection{Modellkopplung}

Wesentliche technische Merkmale der in dieser Arbeit vorgestellten Modellkopplung sind

- die Verwendung des OpenMI-Standards und die

- Anwendung von Methoden der Netzwerkprogrammierung.

Die Verwendung des OpenMI-Standards hat folgende Vorteile:

- Es können bereits bestehende Modelle gekoppelt werden, wenn die entsprechenden Programme mit OpenMI-Schnittstellen ausgerüstet sind.

- Eine Modellkopplung wird einfacher, wenn auch Modelle auf Basis gängiger Programme gekoppelt werden können, denn es müssen nicht mehr aufwändige Einzellösungen entwickelt werden.

- Es wird eine hohe Flexibilität erreicht, denn

- ein gekoppeltes System kann um zusätzliche Modellkomponenten erweitert werden, und

- der Austausch von Modellkomponenten ist möglich. 
Die Anwendung von Methoden der Netzwerkprogrammierung wurde erforderlich, weil für das kommerzielle Programm Feflow der Quellcode nicht verfügbar ist. Der Vorteil der vorgestellten Lösung ist nun jedoch, dass Feflow-Modelle in eine Modellkopplung eingebunden werden können, ohne dass dazu eine neue Programmversion entwickelt werden muss. Möglicherweise trägt dieses Ergebnis dazu bei, den OpenMI-Standard weiter zu verbreiten und so zukünftige Modellkopplungen zu erleichtern. Für das Programm Ilmoflood steht dagegen der Programmcode für diese Arbeit zur Verfügung, so dass hier keine besonderen Schwierigkeiten auftraten.

Die Kopplung der beiden Programme erfolgt extern über den Austausch von primären und sekundären Modellergebnissen, die über Randbedingungen Eingang in das jeweils andere Modell finden. Folgende Wechselwirkungsprozesse können so modelliert werden:

- Uferspeicherung

- Infiltration von einer Überflutungsfläche

- Qualmwasseraustritt.

Anhand verschiedener Anwendungsbeispiele wird der Mehrwert einer Modellkopplung gegenüber den ungekoppelten Modellkomponenten aufgezeigt:

- Es ist möglich, die Versickerung von einer Überflutungsfläche zu modellieren.

- Auch kann der Einfluss der Versickerung auf den Wasserstand der Überflutungsfläche erfasst werden.

- An der Oberfläche austretendes Qualmwasser kann an das Überflutungsflächenmodell übergeben werden. Die Druckhöhe des Oberflächenmodells wird an das Grundwassermodell übergeben.

- Wasser kann Hindernisse im Grundwasserleiter an der Oberfläche umströmen (Kombination der Fließwege).

Anwendungsbeispiele der Modellkopplung erweitern das Verständnis der Prozesse, die ein unterirdisches Hochwasser erzeugen. Die wesentlichen Ergebnisse sind in folgender Liste zusammengefasst:

- Über eine Überflutungsfläche kann, auch wenn eine vergleichsweise wenig durchlässige Auelehm-Deckschicht vorhanden ist, eine deutlich größere Wassermenge infiltrieren als über das Flussufer.

- Die Versickerung von Wasser aus der Überflutungsfläche ins Grundwasser beeinflusst nicht nur die Grundwassersituation, sondern auch die Verhältnisse auf der Überflutungsfläche. 
- Das Wasser, das über die Überflutungsfläche in den Grundwasserleiter eingedrungen ist, bewegt sich größtenteils über das Ufer zurück in den Fluss oder breitet sich in das Hinterland aus. Nur ein geringer Teil fließt bei abnehmendem Wasserstand über die Überflutungsfläche zurück.

- Die vereinfachte Modellierung des Infiltrationsvorgangs als gesättigte Strömung mit Leakage-Ansätzen stellt einen konzeptionellen Fehler dar, kann aber für Planung und operativen Einsatz ausreichen, wenn das Modell entsprechend kalibriert wird.

- Es treten gespannte und ungespannte Verhältnisse im Grundwasser auf, die das Grundwassermodell berücksichtigen muss.

- Die Dauer eines Hochwasserereignisses im Fluss beeinflusst die Ausbreitung des unterirdischen Hochwassers.

- Der Grundwasserspiegel steigt höher, wenn das unterirdische Hochwasser auf eine ungünstige Ausgangssituation trifft. Dies war zum Beispiel beim August-Hochwasser im Jahr 2002 in Dresden der Fall (Sächsisches Landesamt für Umwelt und Geologie 2003).

- Für Planungsaufgaben im Zusammenhang mit unterirdischem Hochwasser, zum Beispiel die Erstellung einer Gefährdungskarte des unterirdischen Hochwassers, reicht es nicht aus, das Ereignis durch ein Bemessungshochwasser, wie für Hochwassergefahrenkarten üblich, zu beschreiben.

Diese Ergebnisse wurden aus fiktiven Anwendungsbeispielen abgeleitet und sind deshalb nicht uneingeschränkt auf andere Untersuchungsgebiete übertragbar. Sie zeigen aber auf, welche Aspekte bei einer Modellierung des unterirdischen Hochwassers besondere Beachtung erfahren sollten.

An den Anwendungsbeispielen wird die Funktionsweise der Modellkopplung erläutert. Es wird gezeigt, dass auch größere Modelle gekoppelt werden können. Es werden aber auch Grenzen und Ungenauigkeiten der vorgestellten Modellkopplung aufgezeigt. Die wesentlichen Einschränkungen, die mit der vorgestellte Modellkopplung in Kauf genommen werden müssen, sind

- räumliche Ungenauigkeiten des Austauschs durch unterschiedliche Netze der Modellkomponenten

- ein Zeitversatz (time lag), der bei der Übergabe der Austauschgrößen zwischen den Modellkomponenten entsteht (externe Kopplung)

- Ungenauigkeiten in der Massenbilanz durch den Zeitversatz.

Tabelle 9.1 enthält eine Übersicht über die Methoden, die zur Erstellung von Hochwassergefahrenkarten eingesetzt werden können, und ordnet die in dieser Arbeit vorgestellte Methode darin ein. 
Tabelle 9.1: Verfahren zur Erstellung von Hochwassergefahrenkarten und Gefahrenkarten des unterirdischen Hochwassers

\begin{tabular}{ccccc}
\hline Zeile & Oberfläche & Wechselwirkung & Untergrund & Mengenbilanz \\
\hline 1 & 1D-Modell & keine & Druckwasserbereiche & - \\
2 & 2D-Modell & keine & Druckwasserbereiche & + \\
\hline 3 & Wasserstand & Expertenwissen & Messwerte & entfällt \\
\hline 4 & Wasserstand & Randbedingungen & Modell & - \\
$\mathbf{5}$ & Modell & unidirektional gekoppelt & Modell & - \\
$\mathbf{6}$ & 2D-Modell & bidirektional gekoppelt & Modell & ++ \\
7 & 2D-Modell & iterativ gekoppelt & Modell & +++ \\
\hline
\end{tabular}

Die eindimensionale Modellierung (1D-Modell, Zeile 1) ist gängige Praxis zur Erstellung von Hochwassergefahrenkarten. Das Ergebnis einer solchen Modellrechnung sind Wasserspiegellagen entlang der Gewässerachse. Die Ermittlung der Überflutungsflächen außerhalb der Hochwasserschutzlinie erfolgt durch eine horizontale Verschneidung der Wasserspiegellage mit dem Geländemodell (Umweltministerium, Innenministerium und Wirtschaftsministerium Baden-Württemberg 2005). Die Retentionswirkung einer Überflutung wird so vollständig vernachlässigt. Von einer ausgeglichenen Mengenbilanz für das Kompartiment der Landoberfläche kann daher nicht gesprochen werden. Eine zweidimensionale Modellierung (Zeile 2) berücksichtigt den Abstrom aus dem Hauptgerinne in die Fläche und stellt damit gegenüber der eindimensionalen Modellrechnung eine deutliche Verbesserung hinsichtlich der Mengenbilanz dar. Da ein solcher Abstrom an verschiedenen Stellen und zu unterschiedlichen Zeitpunkten stattfinden kann, sind in der Regel Szenarien zu definieren.

Die Erstellung von Hochwassergefahrenkarten mit 1D- und 2D-Modellen der Oberflächenströmung ist in Regelwerken umgesetzt (siehe z. B. Ministerium f. Umwelt u. Naturschutz, Landwirtschaft und Verbraucherschutz d. Landes Nordrhein-Westfalen 2003; Umweltministerium, Innenministerium und Wirtschaftsministerium Baden-Württemberg 2005) und deshalb den anerkannten Regeln der Technik zuzuordnen. Die Auswirkung des Hochwassers auf den Untergrund werden dabei jedoch höchstens in Form einer Ausweisung von Druckwasserbereichen angegeben. Druckwasserbereiche sind Senken und Mulden ohne direkte Verbindung zum Gewässer, die aufgrund ihrer Höhenlage unterhalb des Wasserspiegels überflutet werden können (Umweltministerium, Innenministerium und Wirtschaftsministerium Baden-Württemberg 2005). Über die Entwicklung der Grundwasserstände infolge eines Hochwasserereignisses erlauben die Methoden gemäß Zeilen 1 und 2 aus Tabelle 9.1 keine Aussage.

Forberig (2008) und Flögel (2009) erstellen für die Stadt Torgau Gefahrenkarten des unterirdischen Hochwassers für einen Pegelstand der Elbe bei Torgau von $7 \mathrm{~m}$, der über einen Zeitraum von zehn Tagen nicht unterschritten wird. Der Zustand im Untergrund wird auf der Basis gemessener Werte ermittelt, Wechselwirkungen mit dem Fluss und weitere Effekte wie Infiltrationen, der Aufstau des Basisabflusses oder präferenzielle Fließwege wie Flussaltarme werden über 
Expertenwissen berücksichtigt (Tabelle 9.1, Zeile 3). Aussagen zur Mengenbilanztreue können nicht getroffen werden. Dieses Verfahren setzt besondere Fachkenntnisse, Ortskenntnisse und Erfahrungen voraus und kann deshalb unter Umständen nicht allgemein übertragen und standardisiert werden. Eine Prognose für bestimmte Szenarien ist mit der Einschränkung möglich, dass sich die Szenarien nicht zu weit von den historischen Ereignissen entfernen. Sie wird aber mit dieser Methode zum Beispiel dann problematisch, wenn Fragen des unterirdische Hochwassers für Untersuchungsgebiete untersucht werden sollen, in denen bisher noch kein solches Ereignis auftrat oder dokumentiert wurde, oder wenn Extremszenarien betrachtet werden sollen.

Hier kann ein Grundwassermodell weiterhelfen, in dem entsprechende Randbedingungen angesetzt werden, die den Fließzustand auf der Landoberfläche beschreiben (Zeile 4). Die Randbedingungen können zum Beispiel aus Modellrechnungen des Oberflächenwassers gemäß Zeilen 1 und 2 aus Tabelle 9.1 abgeleitet werden. Es werden für solch eine Untersuchung keine besonderen Anforderungen an die Modellierung gestellt, deshalb können sie ebenfalls zum Stand der Technik gezählt werden. Die Methode erlaubt es jedoch nicht, den Einfluss der Wechselwirkungsprozesse auf den Wasserstand an der Landoberfläche zu berücksichtigen, da die Randbedingungen vor Beginn der Berechnung feststehen müssen. Das Leerlaufen einer Flutkammer durch Versickerung kann zum Beispiel auf diese Art nicht modelliert werden.

Auch die unidirektionale Kopplung (Zeile 5) löst dieses Problem nicht, stellt gegenüber dem Verfahren aus Zeile 4 aber eine Erleichterung dar, wenn die Randbedingungen für das Grundwassermodell aus einem anderen numerischen Modell entnommen werden. Die Modellkopplung übernimmt hier den für den Anwender in der Regel recht aufwändigen Datentransfer. Sie setzt aber bestimmte programmtechnische Bedingungen voraus, es müssen nämlich die Strömungsprogramme zur Laufzeit kommunizieren.

Eine bidirektionale Kopplung (Zeile 6) eines Überflutungsflächen- und eines Grundwassermodells erlaubt dagegen, die Wechselwirkungen zwischen den Kompartimenten zu erfassen. Dadurch, dass ein Infiltrationsprozess in beiden Modellteilen berïcksichtigt wird, stellt diese Methode eine wesentliche Verbesserung gegenüber den bisher genannten Methoden dar. Dennoch sind auch hier noch Ungenauigkeiten in der Mengenbilanz vorhanden. Sie hängen davon ab, wie stark sich die Modellkomponenten gegenseitig beeinflussen.

Auf die Zeilen 5 und 6 der Tabelle 9.1 bezieht sich diese Arbeit. Es ist mit der vorgestellten Kopplungsmethode möglich, ein Überflutungsflächen- und ein Grundwassermodell unidirektional oder bidirektional zu koppeln. Derzeit zählen diese Methoden noch nicht zum Stand der Technik. Durch Verwendung eines Schnittstellenstandards soll diese Arbeit aber einen Beitrag dazu leisten, diese Verfahren dem Stand der Technik näher zu bringen. Ein wichtiger Aspekt dabei ist, dass es gelang, das in Deutschland weit verbreitete kommerzielle Programm Feflow mit einer solchen Schnittstelle auszurüsten.

Die in Zeile 7 genannte Methode der iterativen Kopplung ist hinsichtlich der Mengenbilanz deutlich exakter, aber auch rechenzeitintensiver. Sie ist derzeitig noch dem Stand der Forschung zuzuordnen. Da der OpenMI-Standard aber auch iterative Kopplungen grundsätzlich zulässt, wird 
sich möglicherweise auch diese Methode in Zukunft für Modelluntersuchungen zum unterirdischen Hochwasser durchsetzen, insbesondere dann, wenn zukünftig größere Rechnerkapazitäten zur Verfügung stehen.

Viele Programme, so auch Feflow, bringen eine Möglichkeit zur grafischen Darstellung der Berechnungsergebnisse mit oder sie geben, wie zum Beispiel das Programm Ilmoflood, die Berechnungsergebnisse in einem gängigen Datenformat aus, so dass sie mit Hilfe von anderen Programmen ausgewertet werden können. Eine zusammenhängende Betrachtung der Berechnungsergebnisse zweier gekoppelter Modelle ist so aber nicht ohne Weiteres möglich. Für die Auswertung gekoppelter Berechnungen besteht deshalb Bedarf an Auswertungswerkzeugen, mit denen nicht nur die Berechnungsergebnisse kohärent ausgewertet werden können, sondern auch der Verlauf des Datenaustauschs nachvollzogen und kontrolliert werden kann. Für diese Arbeit wurden dazu im Wesentlichen Datenbanken verwendet. Datenbanken bieten die notwendige Flexibilität, um die Modelldaten zum Beispiel in Form von Zeitreihen einzelner Stützstellen aufzubereiten, aber auch zu Bilanzen zusammenzufassen (siehe z. B. Becker et al. 2006; Bachmann et al. 2009). Der Import der Berechnungsergebnisse in eine Datenbank stellte bei beiden verwendeten Programmen jedoch einen nicht unerheblichen Aufwand dar. Die programmeigene Datenausgabe von Feflow kam gar nicht zum Einsatz, stattdessen wurde ein eigenes Modul entwickelt (Scherf 2008), das die Berechnungsergebnisse in Tabellenform ausgibt und so den Import in die Datenbank erleichtert. Für die Ergebnisse von Ilmoflood wurde ein VisualBasic-Modul zum Datenimport in die Datenbank entwickelt. Nachdem mit OpenMI nun ein Kopplungsstandard zur Verfügung steht, sollte der nächste Schritt sein, auch die Ergebnisausgabe zu standardisieren. Möglicherweise können auch hier OpenMI-Komponenten helfen. Schade (2007) setzt zum Beispiel den Import von Modelldaten und die Ausgabe der Ergebnisse in ein geographisches Informationssystem über die OpenMI-Schnittstelle um.

\subsection{Einsatz gekoppelter Modelle zum Schutz gegen unterirdisches Hochwasser}

Die vorgestellte Modellkopplung wurde zur Unterstützung des operativen Einsatzes im Hochwasserschutz und für Planungsaufgaben, zum Beispiel die Raumordnung und Bauleitplanung, entwickelt. Die Ergebnisse einer gekoppelten Modellrechnung können dann auch zur Information der Öffentlichkeit genutzt werden, indem die Berechnungsergebnisse zu Gefahrenkarten des unterirdischen Hochwassers aufbereitet werden. Im Rahmen dieser Arbeit wurde anhand eines fiktiven Anwendungsbeispiels gezeigt, dass die Modellkopplung Ergebnisse liefern kann, die für derartige Aufgaben benötigt werden. Der Einsatz mit echten gebietsbezogenen Modellen steht jedoch noch aus. Es wurde anhand des Anwendungsbeispiels aber deutlich, dass die vorgestellte Methode zur Modellkopplung auch für größere Untersuchungsgebiete tauglich ist.

Für Hochwassergefahrenkarten bestehen zumeist regionale Standards über das, was eine Hochwassergefahrenkarte aussagen soll (Ministerium f. Umwelt u. Naturschutz, Landwirtschaft und 
Verbraucherschutz d. Landes Nordrhein-Westfalen 2003; Umweltministerium, Innenministerium und Wirtschaftsministerium Baden-Württemberg 2005). Für den unterirdischen Raum sind bisher wenig entsprechende Untersuchungen durchgeführt worden. Es liegt jedoch auf der Hand, dass die Vorgaben für Hochwassergefahrenkarten nicht direkt auf das Problem des unterirdischen Hochwassers übertragbar sind. Neben der Bebauung, die auf topographischen Karten eingetragen ist, sind auch unterirdische Bauwerke wie U-Bahn- und Straßentunnel, Kanäle, Tiefgaragen und Fundamente potenziell gefährdeter Gebäude von Bedeutung und sollten in einer Gefahrenkarte des unterirdischen Hochwassers gesondert ausgewiesen werden. Aber auch technische Hochwasserschutzmaßnahmen (Deiche, mobile und feste Hochwasserschutzwände, Drainagen und Entlastungsbrunnen) müssen berücksichtigt werden. Weniger relevant ist dagegen zum Beispiel die Nutzbarkeit von Brücken, die üblicherweise aus Hochwassergefahrenkarten der Landoberfläche hervorgeht. Es sollte deshalb angestrebt werden, auch für Gefahrenkarten des unterirdischen Hochwassers ein entsprechendes Regelwerk zu entwerfen. Der Entwurf kann sich zum Beispiel an den Gefahrenkarten von Forberig (2008) orientieren.

Für den Hochwasserschutz kann eine gekoppelte Modellierung aus einem Überflutungsflächemodell und einem Grundwassermodell auch bei der Planung von einzelnen Maßnahmen eingesetzt werden, zum Beispiel, wenn es darum geht, die Wirksamkeit von vertikalen Abdichtungen, Brunnenreihen oder Drainagen zum Schutz gegen ansteigendes Grundwasser nachzuweisen. Auch bei der Abschätzung der Wirkung von Retentionsräumen an der Oberfläche kann die Modellkopplung helfen, da so auch die unterirdische Speicherwirkung ermittelt werden kann. Hier wäre gegebenenfalls der Fluss als eindimensionales Modell mit in das gekoppelte System einzubinden. Eindimensionale Strömungsprogramme, die den OpenMI-Standard unterstützen, gibt es bereits (OpenMI Association 2007). Selbstverständlich kann auch das in dieser Arbeit verwendete Programm Ilmoflood für solche Untersuchungen verwendet werden. Es ist dazu lediglich die Programmkomponente für die eindimensionale Berechnung von Fließgewässern in die OpenMl-Schnittstelle mit einzubeziehen.

In einem weiteren Schritt sollte das unterirdische Hochwasser in Risk-Assessment-Verfahren zum Hochwasserschutz (siehe zum Beispiel Ernst et al. 2009 und Kutschera et al. 2008) eingebunden werden. Es besteht zur Bewertung des Risikos für Wohn- und Geschäftsbebauung hier noch Bedarf an geeigneten Grundwasserschadensfunktionen, die analog zu Schadensfunktionen aus Überflutung, bei denen der Schaden in Abhängigkeit von der Wassertiefe angegeben wird, zum Beispiel in Abhängigkeit vom Flurabstand formuliert werden können. Es wird daher notwendig sein, bei der Erfassung von Hochwasserschäden stärker auf eine Trennung zwischen Schäden aus unterirdischem und oberirdischem Hochwasser zu achten. Ein gekoppeltes System, dass Oberflächenwasser- und Grundwassermodelle enthält, würde dann die Gefährdungssituation durch ein gegebenes Hochwasserereignis in Form von Flurabständen liefern. Bedeutend wird auch die Risikobetrachtung einzelner Infrastrukturbauwerke wie zum Beispiel Straßen- und UBahn-Tunnel sein. Für derartige Fragestellungen ist es in der Regel sinnvoll, gesonderte Untersuchungen durchzuführen. 


\subsection{Zusammenhängende Beschreibung von Hochwasserereignissen}

Im Zuge des Aufbaus eines gekoppelten Modellsystems für das unterirdische Hochwasser sollte parallel ein Messprogramm vorbereitet werden, so dass aus zukünftigen - aus Sicht der Betroffenen wünschenswerterweise kleinen - Hochwasserereignissen genügend Daten zur Kalibrierung und zur Validierung des Modellsystems zur Verfügung stehen. Es kommt dabei besonders auf eine zusammenhängende Beschreibung des Hochwassereignisses im Untergrund und an der Oberfläche an. Neben der Entwicklung des Wasserstands im Fluss gehört dazu auch die Erfassung von Grundwasserständen in räumlich und zeitlich geeigneter Auflösung. Zudem ist die Ausdehnung der Überflutungsflächen zu erfassen. Dies kann zum Beispiel über eine photogrammetrische Auswertung von Luftbildaufnahmen (Ernst et al. 2009) erfolgen. Besondere Ereignisse, zum Beispiel ein Qualmwasseraustritt, werden am Besten zusätzlich im Rahmen von Ortsbegehungen dokumentiert.

Aus einer solchen zusammenhängenden Betrachtung des Hochwasserereignisses kann das Prozessverständnis über den Ablauf eines Hochwasserereignisses für ein bestimmtes Untersuchungsgebiet erweitert werden. Offene Fragen können zum Beispiel sein:

- Welche Rolle spielt die Grundwasserneubildung?

- Welche Ausgangssituation liegt vor und wie lässt sie sich geeignet im Modell erfassen?

- Welchen Einfluss hat der Aufstau des Basisabflusses auf die Ausbreitung des unterirdischen Hochwassers?

Für die zusammenhängende Auswertung sollte nach Möglichkeit Expertenwissen über die geologischen Verhältnisse allgemein, geologische Besonderheiten (verlandete Altarme von Flüssen), unterirdische Bauwerke (Ufertunnel, Kanalisation, Tiefgaragen) und archäologische Besonderheiten (historische Abwasserkanäle) eingebunden werden.

Die Parameter, die die Wechselwirkungen bestimmen, also insbesondere die Leakage-Parameter, sind nur schwer messbar, und es wurde in dieser Arbeit einmal mehr gezeigt, dass die Kalibrierung eines Modells in der Regel keine eindeutige Lösung hat. Deshalb ist es sinnvoll, diese Parameter im Rahmen von Sensitivitätsanalysen zu variieren und daraus ihren Einfluss auf das Modellergebnis zu quantifizeren. Aus einer Sensitivitätsanalyse können dann auch Schwerpunkte weitergehender Untersuchungen und Feldmessungen abgeleitet werden.

\subsection{Andere Einsatzmöglichkeiten für OpenMI-Modellkopplungen}

Die vorgestellte Modellkopplung mit Hilfe des OpenMI-Standards kann selbstverständlich auch für Problemstellungen, die nicht direkt mit dem unterirdischen Hochwasser zusammenhängen, eingesetzt werden. Immer dann, wenn Modelle bereits existieren, bietet sich die Verwendung standardisierter Schnittstellen an. Im Idealfall basieren bereits eines oder mehrere der zu koppelnden Modelle auf Programmen, die bereits mit einer OpenMI-Schnittstelle ausgerüstet sind. 
Ein denkbarer Anwendungsfall wäre zum Beispiel die Kopplung der drei Kölner Grundwassermodelle (Abbildung 1.7), die alle drei auf dem Programm Feflow basieren, für das jetzt eine OpenMI-Schnittstelle zur Verfügung steht. Die Kopplung könnte ähnlich wie die Kopplung der Großraum-Grundwassermodelle Erftscholle, Rurscholle und Venloer Scholle erfolgen (Becker et al. 2008a, 2009a). Eine gekoppelte Modellrechnung erweitert oft das Wissen über die Strömungsverhältnisse an den Kopplungsbereichen. Hier liegen den zu koppelnden Modelle allerdings die gleichen Fließgesetze zu Grunde, so dass nicht wie im Fall des unterirdischen Hochwassers der Schwerpunkt auf eine Modellkomponente gelegt werden kann. Deshalb sollte dann die Feflow-OpenMI-Schnittstelle für die iterative Kopplung erweitert werden.

Ein zweiter möglicher Anwendungsfall sind große eindimensionale Flussgebietsmodelle wie das eindimensionale Strömungsmodell des Rheins der Bundesanstalt für Gewässerkunde (2009). Seitliche Zuflüsse aus dem Grundwasser werden in derartigen Modellen meistens in Form von Zuflussrandbedingungen erfasst, deren Werte aus Abschätzungen aus Bilanzbetrachtungen der Einzugsgebiete basieren. Wenn zum Beispiel bei ausgeprägten Uferspeicherungseffekten eine solche Abschätzung nicht ausreicht, kann es helfen, ein Grundwassermodell anzukoppeln. Die aus einer Modellkopplung ermittelten Austauschwassermengen kommen dann sowohl dem Flussgebietsmodell als auch dem Grundwassermodell zu Gute. 


\section{Literaturverzeichnis}

Akan \& Yen 1981

Akan, A. O. ; Yen, B. C.: Mathematical model of shallow water flow over porous media. In: Journal of the Hydraulics Division 107 (1981), Nr. HY4, S. 479-494

Al Masri \& Sharif 1985

Al Masri, N. ; Sharif, T.: Water infiltration under rain and ponded conditions. In: Problems of regional hydrology - Methodology, Case Studies - 5th Report of the IGU Working Group on the IHP Bd. 2. Kirchzarten : Verl. Beiträge zur Hydrologie, 1985, S. 775-790

Anderson \& Woessner 1992

Anderson, M. P. ; Woessner, W. W.: The role of the postaudit in model validation. In: Advances in Water Resources 15 (1992), S. 167-173

Australian Government 2008a

Australian Government: Seepage Measurement Methods. 2008. http://www. connectedwater.gov. $\mathrm{au} /$ framework/seepage_measurement.html, Abruf: 15.10.2008

Australian Government 2008b

Australian Government: Seepage Meter Design. 2008. http://www.connectedwater.gov.au/ framework/seepage_design.html, Abruf: 15.10.2008

Bachmann et al. 2007

Bachmann, D. ; Becker, B. ; van Linn, A. ; Köngeter, J.: Das Großraum-Grundwassermodell Rurscholle. In: Grundwasser 12 (2007), Nr. 1, S. 26-36. http://dx.doi.org/10.1007/s00767-007-0020-2

Bachmann et al. 2009

Bachmann, D. ; Huber, N. P. ; Schüttrumpf, H.: REISE: Multikriterielle Entscheidungsunterstützung zur Planung von Hochwasserschutzkonzepten. In: 39. Internationales Wasserbau-Symposium Aachen (IWASA) : Wasserwirtschaft und Klimawandel - Fakten, Folgen und Aufgaben : 6. und 7. Januar 2009. Aachen, 2009, http://www.iww.rwth-aachen.de/fileadmin/internet/iwasa/ vortraege2009/IWASA2009_Bachmann.pdf

Barry et al. 1995

Barry, D. A. ; Parlange, J. Y. ; Haverkamp, R. ; Ross, P. J.: Infiltration under ponded conditions: 4. an explicit predictive infiltration formula. In: Soil Science 160 (1995), Nr. 1, S. 8-17

Bauer et al. 2006

Bauer, P. ; Gumbricht, T. ; Kinzelbach, W.: A regional coupled surface water/groundwater model of the Okavango Delta, Botswana. In: Water Resources Research 42 (2006), Nr. W04403. http://dx.doi.org/10. $1029 / 2005$ WR004234

Becker et al. 2009a

Becker, B. ; Homann, C. ; Köngeter, J.: Coupling of Large Scale Groundwater Models. In: Computing and Visualization in Science 12 (2009), Nr. 2, S. 71-76. http://dx.doi.org/10.1007/s00791-0070079-4 
Becker et al. 2008a

Becker, B. ; Klauder, W. S. ; Reuter, C. ; Köngeter, J.: Modellierung der Randüberströme zwischen Erftscholle, Rurscholle und Venloer Scholle durch Kopplung von Großraum-Grundwassermodellen. In: Grundwasser 13 (2008), Nr. 1, S. 15-26. http://dx.doi.org/10.1007/s00767-007-0056-3

Becker \& Köngeter 2005

Becker, B. ; Köngeter, J.: Instationärer Ansatz der Grundwasserneubildung. In: 7. JuWi-Treffen: Beiträge zum Treffen junger Wissenschaftlerinnen und Wissenschaftler deutschsprachiger Wasserbauinstitute. Graz : Institut für Wasserbau u. Wasserwirtschaft, Technische Universität Graz, 2005 (Schriftenreihe zur Wasserwirtschaft 43). - ISBN 3-902465-22-0, S. 37-42

Becker et al. 2006

Becker, B. ; Notermanns, F. ; Köngeter, J.: Three Large Scale Groundwater Models at the Lower Rhine Coupling and Data Management. In: Binning, P. J. (Hrsg.) ; Engesgaard, P. K. (Hrsg.) ; Dahle, H. K. (Hrsg.) ; Pinder, G. F. (Hrsg.) ; Gray, W. G. (Hrsg.): Proceedings of the XVI International Conference on Computational Methods in Water Resources. Copenhagen, Denmark, June 2006, http://proceedings. cmwr-xvi. org/contributionDisplay.py?contribId=184\&s\%essionId=13\&confId=a0 51

Becker et al. 2009b

Becker, B. ; Nowack, L. ; Klauder, W. S. ; Köngeter, J. ; Schüttrumpf, H.: Eine nichtlineare LeakageRandbedingung für die Modellierung von hochwasserbeeinflusstem Grundwasseranstieg. In: Wasserwirtschaft (2009), Nr. 1-2

Becker et al. 2008b

Becker, B. ; Reuter, C. ; Schüttrumpf, H.: An openMI-connected surface flow - subsurface flow simulation model system to forecast subsurface flood. 2008. http: / / www. iww.rwth-aachen.de/research/papers/ Becker_CMWR2008.pdf.en. Poster presented at the XVII International Conference on Computational Methods in Water Resources, 06.-10.07.2008, San Francisco, USA

Beven \& Germann 1982

Beven, K. ; Germann, P.: Macropore and Water Flow in Soils. In: Water Resources Research 18 (1982), Nr. 5, S. 1311-1325. - ISSN 0043-1397

Beyer 2003

Beyer, K.-D.: Erhalt der Gebäudestandsicherheit - Sofortmaßnahmen und Dauerlösung / Beispiel des St. Benno-Gymnasiums Dresden. In: Landeshauptstadt Dresden (Hrsg.) ; Dresdner Grundwasserforschungszentrum (Hrsg.): Tagungsband zum BMBF-Status-Seminar am 8. Oktober 2003, Rathaus Dresden, 2003, S. 63-68

Blaschke 2002

Blaschke, A. P.: Auswirkungen der Selbstdichtung auf das Grundwasser in staugeregelten Flüssen. In: Wittenberg, H. (Hrsg.) ; Schöniger, M. (Hrsg.): Wechselwirkungen zwischen Grundwasserleitern und Oberflächengewässern : Beiträge zum Tag der Hydrologie 2002 20. bis 22. März 2002 in Suderburg, Lüneburger Heide, Fachgemeinschaft Hydrologische Wissenschaften in der ATV-DVWK, 2002 (Forum für Hydrologie und Wasserbewirtschaftung). - ISBN 3-936514-22-4, S. 165-171

Bollrich 2000

Bollrich, G.: Technische Hydromechanik. Bd. 1. 5. Aufl. Berlin : Verlag Bauwesen, 2000. - ISBN 3-345$00744-4$

Bouwer 1966

Bouwer, H.: Rapid field measurements of air entry value and hydraulic conductivity of soil as significant parameters in flow system analysis. In: Water Resources Research 2 (1966), Nr. 4, S. 729-738 
Briechle 2006

Briechle, S. R.: Die flächenhafte Ausbreitung der Flutwelle nach Versagen von Hochwasserschutzeinrichtungen an Fließgewässern, Fakultät für Bauingenieurwesen der Rheinisch-Westfälischen Technischen Hochschule Aachen, Dissertation, 2006. http://deposit.ddb.de/cgi-bin/dokserv?idn=98060897x\&dok_ var $=$ d1\&dok_ext $\%=p d f \& f i l e n a m e=98060897 \mathrm{x} . \mathrm{pdf}$

Brinkmann et al. 2005

Brinkmann, R. ; Gregersen, J. B. ; Hummel, S. ; Westen, S. J. P.: OpenMI Document Series: Part C - the org.openMI.Standard interface specification. Version 1.0. IT Frameworks (HarmonIT), 2005. http://www . openmi.org/openminew/documents/C_org.openmi.standard_specification.pdf

Brockhaus-Enzyklopädie Online 2008

Brockhaus-Enzyklopädie Online: RPC. 2008. http://www.brockhaus-enzyklopaedie.de, Abruf: 29.01.2008

Brockhaus-Enzyklopädie Online 2009a

Brockhaus-Enzyklopädie Online: Dynamik. 2009. http://www.brockhaus-enzyklopaedie.de, Abruf: 21.06.2009

Brockhaus-Enzyklopädie Online 2009b

Brockhaus-Enzyklopädie Online: Kinematik. 2009. http://www.brockhaus-enzyklopaedie.de, Abruf: 21.06.2009

Brombach et al. 2001

Brombach, H. ; Dillmann, R. ; Patt, H. ; Richwien, W. ; Vogt, R.: Hochwasserschutzmaßnahmen. In: Patt, H. (Hrsg.): Hochwasser-Handbuch. Berlin u. a. : Springer, 2001. - ISBN 3-540-67737-2, Kapitel 7

Bronstein et al. 1997

Bronstein, I. N. ; Semendjajew, K. A. ; Musiol, G. ; Mühlig, H.: Taschenbuch der Mathematik. Thun und Frankfurt am Main : Harri Deutsch, 1997. - ISBN 3-8171-2003-6

Brunke \& Gonser 1997

Brunke, M. ; Gonser, T.: The ecological significance of exchange processes between rivers and groundwater. In: Freshwater biology 37 (1997), Nr. 1, S. 1-33

Bundesanstalt für Gewässerkunde 2009

Bundesanstalt für Gewässerkunde: KLIWAS-Projekt 4.01 — „Hydrologie und Binnenschifffahrt“. 2009. http: //wWw.bafg.de/nn_265022/M2/DE/05_KLIWAS/kliwas__node.html?_nnn=true, Abruf: 12.08.2009

Chong \& Green 1983

Chong, S. K. ; Green, R. E.: Sorptivity measurement and its application. In: American Society of Agricultural Engineers (Hrsg.): Proceedings of the national conference on advances in infiltration, 12.-13. Dec. 1983, Chicago, Illinois. Michigan : American Society of Agricultural Engineers, 1983, S. 82-91

Crebas et al. 1984

Crebas, J. I. ; Gilding, B. H. ; Wesseling, J. W.: Coupling of groundwater and open channel flow. In: Journal of Hydrology 72 (1984), S. 307-330

Cunningham \& Sinclair 1979

Cunningham, A. B. ; Sinclair, P. J.: Application and analysis for a coupled surface and groundwater model. In: Journal of Hydrology 43 (1979), S. 129-148 
Darcy 1856

Appendice Note D. In: Darcy, H.: Les fontaines publiques de la ville de Dijon / exposition et application des principes à suivre et des formules à employer dans les questions de distribution d'eau; ouvrage terminé par un appendice relatif aux fournitures d'eau de plusieurs villes au filtrage des eaux à la fabrication des tuyaux de fonte, de plomb, de tole et de bitume. Batignolles : Typographie Hennuyer, 1856 (Libraire des Corps Imperiaux des ports et chausses et des mines)

Das Europäische Parlament und der Rat der Europäischen Union 2000

Das Europäische Parlament und der Rat der Europäischen Union: Richtlinie zur Schaffung eines Ordnungsrahmens für Maßnahmen der Gemeinschaft im Bereich der Wasserpolitik. 2000 (Richtlinie 2000/60/EG). EU-Wasserrahmenrichtlinie

de Boer 2008

de Boer, A.: Computational fluid-structure interaction / Spatial coupling, coupling shell and mesh deformation, Technische Universiteit Delft, Proefschrift ter verkrijging van de graad van doctor, 2008. http:// repository.tudelft.nl/view/ir/uuido3A7e4cfc71-57eb-4a6e-9e90-38854de21ce2

Demuth \& Hiltpold 1993

Demuth, N. ; Hiltpold, A.: „Preferential flow“: Eine Übersicht über den heutigen Kenntnisstand. In: Zeitschrift für Pflanzenernährung und Bodenkunde 156 (1993), S. 479-484

Diersch 2005a

Diersch, H.-J. G.: Extended formulations of constraints for Cauchy-type (3rd kind) boundary conditions in FEFLOW. In: Wasy GmbH (Hrsg.): FEFLOW $5.2{ }^{\circledR}$ White Papers Vol. 1. Berlin, 2005, Kapitel 14, S. 269-273

Diersch 2005b

Diersch, H.-J. G.: On the primary variable switching technique for simulating unsaturated-saturated flows. In: Wasy GmbH (Hrsg.): FEFLOW 5.2 ${ }^{\circledR}$ White Papers Vol. 1. Berlin, 2005, Kapitel 1, S. 9-66

Diersch 2005c

Diersch, H.-J. G.: FEFLOW 5.3 ${ }^{\circledR}$ User's Manual. Berlin: Wasy GmbH, 2005

Diersch 2005d

Diersch, H.-J. G.: FEFLOW Reference Manual. Berlin: Wasy GmbH, 2005

Diersch 2005e

Diersch, H.-J. G.: Treatment of free surfaces in 2D and 3D groundwater modeling. In: Wasy GmbH (Hrsg.): FEFLOW 5.2 ${ }^{\circledR}$ White Papers Vol. 1. Berlin, 2005, Kapitel 2, S. 67-100

DIN 4047-2 1988

DIN 4047-2: Landwirtschaftlicher Wasserbau Teil 2 : Begriffe Hochwasserschutz, Küstenschutz, Schöpfwerke. Deutsches Institut für Normung e. V., 1988

DIN 4049-3 1994

DIN 4049-3: Hydrologie Teil 3 : Begriffe zur quantitativen Hydrologie. Berlin: Deutsches Institut für Normung e. V., 1994

Doherty et al. 1994

Doherty, J. ; Brebber, L. ; Whyte, P.: PEST - model independent parameter estimation. Corinda, Australia : Watermark Computing, 1994

Dracos 1980

Dracos, T. A.: Hydrologie: eine Einführung für Ingenieure. Wien, New York : Springer, 1980. - ISBN 3-21181574-0 
Dyck \& Peschke 1983

Dyck, S. ; Peschke, G.: Grundlagen der Hydrologie. Berlin u. a. : Ernst \& Sohn, 1983. - ISBN 3-433-00947-3

Eckert \& Irmscher 2006

Eckert, P. ; Irmscher, R.: Over 130 years of experience with Riverbank Filtration in Düsseldorf, Germany. In: Journal of Water Supply: Research and Technology - aqua 55 (2006), Nr. 4, S. 283-291. http: // dx . doi . org/10.2166/aqua.2006.040

Eckert \& Rueber 1992

Eckert, P. ; Rueber, O.: Hydrochemische Untersuchungen und Stofftransport-Modellrechnungen zur Uferfiltration von Rheinwasser im Bereich der Wasserfassung Rheinfähre der Städtischen Werke Krefeld AG. Krefeld : Städtische Werke Krefeld AG, 1992 (Besondere Mitteilungen zum Deutschen Gewässerkundlichen Jahrbuch 56)

Ernst et al. 2009

Ernst, J. ; Dewals, B. J. ; Detrembleur, S. ; Archeambeau, P. ; Erpicum, S. ; Pirotton, M.: Detailed 2D hydraulic modelling as a support for selecting adaptation measures. In: 39. Internationales WasserbauSymposium Aachen (IWASA) : Wasserwirtschaft und Klimawandel - Fakten, Folgen und Aufgaben : 6. und 7. Januar 2009. Aachen, 2009, http: / / www. iww.rwth-aachen. de/fileadmin/internet/iwasa/ vortraege2009/IWASA2009_Pirotton_Dewals.pdf

Fairbanks et al. 2001

Fairbanks, J. ; Panday, S. ; Huyakorn, P. S.: Comparisons of linked and fully coupled approaches to simulating conjunctive surface/subsurface flow and their interactions. In: MODFLOW 2001 and other Modeling Odysseys - Conference Proceedings. Golden, CO, 2001, S. 356-361

Flögel 2009

Flögel, R.: Re: GWK Grundwasseranstieg infolge Hochwassers. E-Mail vom 02.09.2009 von r.floegel@hgnonline.de an b.becker@iww.rwth-aachen.de, 2009

Forberig 2008

Forberig, S.: Informationskarten zur Hochwasserproblematik für Kommunen / HGN Hydrogeologie GmbH, Niederlassung Torgau. Torgau, 2008. - Informationsmaterial der HGN

Forberig 2009

Forberig, S.: Gefahrenkarten für Grundwasseranstieg infolge Hochwasser. Telefongespräch am 18.09.2009, B. Becker und S. Forberig, 2009

Forchheimer 1901

Forchheimer, P.: Wasserbewegung durch Boden. In: Zeitschrift des Vereins deutscher Ingenieure 49 (1901), S. 1736-1741 u. 1781-1788

Forkel 2004

Forkel, C.: Numerische Modelle für die Wasserbaupraxis. Grundlagen, Anwendungen, Qualitätsaspekte. Aachen : Shaker, 2004 (Mitteilungen / Lehrst. u. Inst. f. Wasserbau u. Wasserwirtschaft, Rheinisch-Westfälische Technische Hochschule Aachen 130). - ISBN 3-8322-3082-3

Fread 1992

Fread, D. L.: Flow Routing. In: Maidment, D. R. (Hrsg.): Handbook of Hydrology. New York u. a. : McGrawHill, 1992. - ISBN 0-07-039732-5, Kapitel 10, S. 22.1-22.34

Freeze 1972

Freeze, R. A.: Role of Subsurface Flow in Generating Surface Runoff. 1. Base Flow Contributions to Channel Flow. In: Water Resources Research 8 (1972), Nr. 3, S. 609-623 
Freeze \& Cherry 1979

Freeze, R. A. ; Cherry, J. A.: Groundwater. Englewood Cliffs, NJ, USA : Prentice Hall, 1979. - ISBN 0-13365312-9

Geomer \& Infoterra 2009

Geomer ; Infoterra: Hochwasserinformationssystem. 2009. http://www.floodmaps.de/ FloodServer/, Abruf: 31.08.2009

Giebel et al. 1990

Giebel, H. ; Gölz, E. ; Theis, H.-J. ; Ubell, K.: Hydrogeologie und Grundwasserhaushalt im Neuwieder Becken. Bundesanstalt für Gewässerkunde, 1990 (Besondere Mitteilungen zum Deutschen Gewässerkundlichen Jahrbuch 54)

Giebel \& Hommes 1988

Giebel, H. ; Hommes, A.: Zum Austausch zwischen Fluß- und Grundwasser - Weitergehende Auswertung aus dem Neuwieder Becken. In: Deutsche Gewässerkundliche Mitteilungen 32 (1988), Nr. 1/2, S. 18-27

Gijsbers et al. 2005

Gijsbers, P. ; Gregersen, J. ; Westen, S. ; Dirksen, F. ; Gavardinas, C. ; Blind, M.: OpenMI Document Series: Part B - Guidlines for the OpenMI. Version 1.0. IT Frameworks (HarmonIT), 2005. http: / / www . openmi . org/openminew/documents/B_Guidelines.pdf

Gölz et al. 1991

Gölz, E. ; Schubert, J. ; Liebich, D.: Sohlenkolmation und Uferfiltration im Bereich des Wasserwerks Flehe (Düsseldorf). In: gwf-Wasser/Abwasser 132 (1991), Nr. 2, S. 69-76

Gränitz \& Grundmann 2003

Gränitz, F. ; Grundmann, L.: Das Mittelrheinische Becken. Köln : Böhlau Verlag, 2003. - ISBN 3-412-10102-8

Green \& Ampt 1911

Green, W. H. ; Ampt, G. A.: Studies on Soil Physics. Part I. - The Flow of Air and Water through Soils. In: The journal of Agricultural Science (1911), Nr. IV, S. 1-24

Gregersen et al. 2007

Gregersen, J. P. ; Gijsbers, P. J. A. ; Westen, S. J. P.: OpenMI: Open modelling Interface. In: Journal of Hydroinformatics 9 (2007), Nr. 3, S. 175-191. http://dx.doi.org/10.2166/hydro.2007.023

Gründler 2007

Gründler, R.: RE: support/\#07-0013. E-Mail vom 23.11.2007 von R.Gruendler@wasy.de an b.becker@iww.rwth-aachen.de, 2007

Gunduz \& Aral 2005

Gunduz, O. ; Aral, M. M.: River networks and groundwater flow: a simultaneous solution of a coupled system. In: Journal of Hydrology (2005), Nr. 301, S. 216-234. http://dx.doi.org/10.1016/j.jhydrol. 2004.06 .034

Harms 2004

Harms, M.: Die Kammerung als Maßnahme zur Risikominderung. In: Köngeter, J. (Hrsg.): 34. IWASA, Internationales Wasserbau-Symposium Aachen 2004 : Hochwasserschutz-eindeichen oder ausweichen. Aachen : Shaker, 2004 (Mitteilungen / Lehrst. u. Inst. f. Wasserbau u. Wasserwirtschaft, Rheinisch-Westfälische Technische Hochschule Aachen 136). - ISBN 3-8322-3592-2

Hausmann 2005a

Hausmann, B.: Ein numerisches Verfahren zur Bestimmung von Überflutungsfächen und -dauern auf Basis physikalischer Strömungsgleichungen, Lehrst. u. Inst. f. Wasserbau u. Wasserwirtschaft, RWTH Aachen, Diplomarbeit, 2005 
Hausmann 2005b

Hausmann, B.: Programmbeschreibung Polderflood. Aachen: Lehrst. u. Inst. f. Wasserbau u. Wasserwirtschaft, RWTH Aachen, 2005

Haverkamp et al. 1988

Haverkamp, R. ; Kutilek, M. ; Parlange, J. Y. ; Rendon, L. ; Krejca, M.: Infiltration under ponded conditions. 2. Infiltration equations tested for parameter time-dependence and predictive use. In: Soil Science 145 (1988), Nr. 5, S. 317-329

Haverkamp et al. 1990

Haverkamp, R. ; Parlange, J. Y. ; Starr, J. L. ; Schmitz, G. ; Fuentes, C.: Infiltration under ponded conditions: 3. a predictive equation based on physical parameters. In: Soil Science 149 (1990), Nr. 5, S. 292-300

Homann 2006

Homann, C.: Automatische Optimierung von Grundwasserhaltungen in von Hochwasser beeinflussten Aquiferen, Fakultät für Bauingenieurwesen der Rheinisch-Westfälischen Technischen Hochschule Aachen, Dissertation, 2006. http://deposit.d-nb.de/cgi-bin/dokserv?idn=980190134\&dok_var= d1\&dok_exot=pdf\&filename=980190134.pdf

Huang \& Yeh 2006

Huang, G. ; Yeh, G.-T.: An integrated media, integrated processes watershed model - WASH123D: part 3 - a comparative study on different surface water/groundwater coupling approaches. In: Binning, P. J. (Hrsg.) ; Engesgaard, P. K. (Hrsg.) ; Dahle, H. K. (Hrsg.) ; Pinder, G. F. (Hrsg.) ; Gray, W. G. (Hrsg.): Proceedings of the XVI International Conference on Computational Methods in Water Resources. Copenhagen, Denmark, June 2006, http: / / proceedings . cmwr-xvi .org/ contributionDisplay •py? contribId=88\&se\%ssion $I d=10 \& \operatorname{confId}=\mathrm{a} 051$

Huber et al. 2003

Huber, G. ; Hiller, G. ; Braune, A.: Konzepte des Hochwasserschutzes für die Bauten des Freistaates Sachsen im Historischen Stadtkern von Dresden. In: Landeshauptstadt Dresden (Hrsg.) ; Dresdner Grundwasserforschungszentrum (Hrsg.): Tagungsband zum BMBF-Status-Seminar am 8. Oktober 2003, Rathaus Dresden, 2003, S. $57-62$

Jansen 2009

Jansen, M.: Numerische Untersuchungen zum unterirdischen Hochwasser, Lehrst. u. Inst. f. Wasserbau u. Wasserwirtschaft, RWTH Aachen, Diplomarbeit, 2009

Junghans 2001

Junghans, U.: Grundwassermodell LINEG - Aufbau eines anspruchsvollen Regionalmodells. In: WASY Aktuell 7 (2001), Nr. 3. http://www.wasy.de/Supportwasy/ /media/ 6ECF56388B5F 443A97612F86FFF58B2B.ashx

Kamrath 2007

Kamrath, P.: Grundlagen des gekoppelten Modells Ilmoflood . Lehrst. u. Inst. f. Wasserbau u. Wasserwirtschaft, RWTH Aachen, 2007. - internes Papier

Kamrath 2009

Kamrath, P.: Über die gekoppelte Modellierung von Fließgewässern und Überflutungsfächen, Fakultät für Bauingenieurwesen der Rheinisch-Westfälischen Technischen Hochschule Aachen, Dissertation, 2009

Kamrath et al. 2008

Kamrath, P. ; Huber, N. P. ; Kufeld, M. ; Schüttrumpf, H. ; Köngeter, J.: Interlinked modelling of large floods by combining one and two-dimensional diffusive wave approaches. In: Simonovic, S. P. (Hrsg.) ; Bourget, P. G. (Hrsg.) ; Blanchard, S. F. (Hrsg.): Managing flood risk, reliability and vulnerability: 4th International 
Symposium on Flood Defence, Toronto, Canada, May 6-8 / Proceedings. Toronto : Institute for Catastrophic Loss Reduction, 2008, S. 109,1-109,8

Kinzelbach \& Rausch 1995

Kinzelbach, W. ; Rausch, R.: Grundwassermodellierung : eine Einführung mit Übungen. Berlin, Stuttgart : Gebr. Borntraeger, 1995. - ISBN 978-3-443-01032-4

Klatt 2007

Klatt, C.: Grundwassermessstellen der RheinEnergie AG. $\quad$ E-Mail vom 30.11 .2007 von c.klatt@rheinenergie.com an b.becker@iww.rwth-aachen.de, 2007

Köngeter et al. 1997

Köngeter, J. ; Demny, G. ; Forkel, C.: Vorstudie Rückhalteraum Worringen / Teil C (Grundwasser) / Lehrst. u. Inst. f. Wasserbau u. Wasserwirtschaft, RWTH Aachen. 1997. - [im Auftrag des] Ministerium[s] für Umwelt, Raumordnung und Landwirtschaft Nordrhein-Westfalen

Köngeter et al. 2002

Köngeter, J. ; Vogel, T. ; Kutschera, G.: RIPARIA Retentionsoptimierung in priorisierten Abschnitten der RurInde-Auen im deutsch-niederländischen Grenzraum. Teilprojekt 1: Retentionsraumplanung Untere Rur. Teil 3: Grundwasser / Lehrst. u. Inst. f. Wasserbau u. Wasserwirtschaft, RWTH Aachen. Aachen, 2002. - Projektbericht

Köngeter et al. 2002

Köngeter, J. ; Vogel, T. ; Schmidtke, T.: Wissenschaftliche Modelluntersuchungen zum Grundwasserverhalten bei der Einrichtung eines Hochwasserschutzes am Weißer Bogen, Köln / Lehrst. u. Inst. f. Wasserbau u. Wasserwirtschaft, RWTH Aachen. 2002. - [im Auftrag der] Bezirksregierung Köln

Koch et al. 2007

Koch, A. ; Meon, G. ; Leibundgut, C.: Die gekoppelte Simulation der 2D-Oberflächenwasser- und 3DGrundwasserströmung im Rahmen eines Expertensystems zur Modellierung der hydrodynamischen Wechselwirkungen einer Flusslandschaft. In: Meon, G. (Hrsg.) ; Sauter, M. (Hrsg.) ; Führböter, J. F. (Hrsg.) ; Wolf, J. (Hrsg.): Niedersächsisches Grundwasserkolloquium 21.-22. Februar 2007 in Braunschweig : Grundwasser, Hochwasser und Salzwasser / Konzepte zur Risikominimierung. Stuttgart : E. Schweizerbart'sche Verlagsbuchhandlung (Nägele u. Obermiller), 2007 (Zentralblatt für Geologie und Paläontologie / Teil I Allgemeine, Angewandte, Regionale und Historische Geologie Jg. 2007, H. 1/2), S. 99-114

Kollet \& Maxwell 2006

Kollet, S. J. ; Maxwell, R. M.: Integrated surface-groundwater flow modeling: A free-surface overland flow boundary condition in a parallel groundwater flow model. In: Advances in Water Resources 29 (2006), Nr. 7, S. 945-958. http://dx.doi.org/10.1016/j.advwatres.2005.08.006

Konikow \& Bredehoeft 1992

Konikow, L. F. ; Bredehoeft, J. D.: Ground-water models cannot be validated. In: Advances in Water Resources 15 (1992), S. 75-83

Kreibich \& Thieken 2008

Kreibich, H. ; Thieken, A.: Assessment of damage caused by high groundwater inundation. In: Water Resources Research 44 (2008), Nr. W09409. http: / /dx.doi.org/10.1029/2007WR0 06621

Krom \& Graham 2006

Krom, T. D. ; Graham, D.: Accurate simulation methods for braided rivers in numerical groundwater models. In: Binning, P. J. (Hrsg.) ; Engesgaard, P. K. (Hrsg.) ; Dahle, H. K. (Hrsg.) ; Pinder, G. F. (Hrsg.) ; Gray, W. G. (Hrsg.): Proceedings of the XVI International Conference on Computational Methods in Water Resources. Copenhagen, Denmark, June 2006, http: / / proceedings . cmwr-xvi.org/contributionDisplay. py?contribId=342\&s\%ession Id=10\&conf Id=a 051 
Kropp et al. 2009

Kropp, J. ; Holsten, A. ; Lissner, T. ; Roithmeier, O. ; Hattermann, F. ; Huang, S. ; Rock, J. ; Wechsung, F. ; Lüttger, A. ; Pompe, S. ; Kühn, I. ; Costa, L. ; Steinhäuser, M. ; Walther, C. ; Klaus, M. ; Ritchie, S. ; Metzger, M.: Klimawandel in Nordrhein-Westfalen - Regionale Abschätzung der Anfälligkeit ausgewählter Sektoren / Potsdam-Institut für Klimafolgenforschung (PIK). 2009. - Abschlussbericht für das Ministerium für Umwelt und Naturschutz, Landwirtschaft und Verbraucherschutz Nordrhein-Westfalen (MUNLV). http: //www.umwelt.nrw.de/umwelt/pdf/abschluss_pik_0904.pdf

Kufeld et al. 2009

Kufeld, M. ; Schüttrumpf, H. ; Bachmann, D.: Assessment of flood retention in polders using an interlinked one-two-dimensional hydraulic model. In: Samuels, P. (Hrsg.) ; Huntington, S. (Hrsg.) ; Allsop, W. (Hrsg.) ; Harrop, J. (Hrsg.): Flood risk management: research and practice : proceedings of the European conference on flood risk management research into practice (Floodrisk 2008), Oxford, UK, 30 september - 2 october 2008. Boca Raton, Fla. : CRC Press, Taylor \& Francis Group, 2009, S. 75,667-75,675

Kutschera et al. 2008

Kutschera, G. ; Bachmann, D. ; Huber, N. P. ; Niemeyer, M. ; Köngeter, J.: RAPID - Ein Risk-AssessmentVerfahren für den technischen Hochwasserschutz. In: Wasserwirtschaft 98 (2008), Nr. 1-2, S. 43-48

Langguth \& Voigt 1980

Langguth, H.-R. ; Voigt, R.: Hydrogeologische Methoden. Berlin, Heidelberg, New York : Springer, 1980. ISBN 3-540-10174-8

Lehners \& Sassenhagen 2003

Lehners, C. ; Sassenhagen, A.: Gefährdung von Hochwasserschutzbauwerken durch Qualmwasserbildung am Beispiel des Elbdeiches bei Lauenburg im Jahre 2002. In: Herrmann, R. A. (Hrsg.) ; Jensen, J. (Hrsg.): Sicherung von Dämmen und Deichen : Handbuch für Theorie und Praxis. Siegen : Universitätsverlag Siegen - universi, 2003. - ISBN 3-936533-09-1, S. 371-383

Losen 1984

Losen, H. ; Heitfeld, K.-H. (Hrsg.): Grundwasserstände und Grundwasserbeschaffenheit im südlichen Teil der linksrheinischen Kölner Scholle : Eine hydrogeologische und statistische Analyse. Aachen : Lehrstuhl für Ingenieurgeologie und Hydrogeologie der RWTH Aachen, 1984 (Mitteilungen zur Ingenieurgeologie und Hydrogeologie 20)

Luckner 1978

Luckner, L.: Gekoppelte Grundwasser-Oberflächen-Wassermodelle. In: Wasserwirtschaft-Wassertechnik(WWT) 29 (1978), Nr. 8, S. 276-278

Malcherek 2001

Malcherek, A.: Hydromechanik der Oberfächengewässer. 2001. http://www.baw.de/vip/ abteilungen/wbk/Methoden/hnm/nummeth/nummeth1.pdf, Abruf: 11.10.2007. - Version 5.0

Mattheß \& Ubell 1983

Mattheß, G. ; Ubell, K.: Lehrbuch der Hydrogeologie. Bd. 1: Allgemeine Hydrogeologie - Grundwasserhaushalt. Berlin, Stuttgart : Gebr. Borntraeger, 1983. - ISBN 3-443-01005-9

Meißner 2008

Meißner, O.: Eine kurze Geschichte der Stadt Köln. 2008. http://www.cologneweb.com/koeln1.htm, Abruf: 28.10.2008

Ministerium f. Umwelt u. Naturschutz, Landwirtschaft und Verbraucherschutz d. Landes Nordrhein-Westfalen 2003 Ministerium f. Umwelt u. Naturschutz, Landwirtschaft und Verbraucherschutz d. Landes Nordrhein-Westfalen: Leitfaden Hochwassergefahrenkarten. 2003. http://www.stua-ha.nrw.de/download/texte/ hochwassergefahrenkarten.pdf, Abruf: 14.08.2009 
Münchener Rück 2003

Münchener Rück: Jahresrückblick Naturkatastrophen 2002. 2003 (topics 10). http://www.munichre. de/publications/302-03630_de.pdf

Monninkhoff 2004

Monninkhoff, B.: Kopplung von FEFLOW mit dem 1D-hydrodynamischen Modell MIKE11 (DHI). In: Fachtagung „Grafikgestützte Grundwassermodellierung“, Köln im Juni 2004. Berlin : Wasy GmbH und IDCED Umweltinstitut IWU e. V., 2004 (IWU Tagungsberichte), S. 55-68

Monninkhoff \& Kernbach 2006

Monninkhoff, B. ; Kernbach, K.: Coupled surface water - groundwater modeling for planning of flood retention in the Lower Havel area. In: International FEFLOW User Conference, September 10-15, 2006 Berlin, Germany. Berlin : Wasy GmbH, 2006 (Conference Proceedings), S. 115-124

Moore et al. 2005

Moore, R. ; Gijsbers, P. ; Fortune, D. ; Gregersen, J. ; Blind, M.: OpenMI Document Series: Part A - Scope for the OpenMI. Version 1.0. IT Frameworks (HarmonIT), 2005. http://www.openmi.org/openminew/ documents/A_OpenMI_Scope.pdf

Moore \& Tindall 2005

Moore, R. V. ; Tindall, C. I.: An overview of the open modelling interface and environment (the OpenMI). In: Environmental Science \& Policy 8 (2005), Nr. 3, S. 279-286. http://dx.doi.org/10.1016/j. envsci.2005.03.009

Morel-Seytoux \& Khanji 1974

Morel-Seytoux, H. J. ; Khanji, J.: Derivation of an Equation of Infiltration. In: Water Resources Research 10 (1974), Nr. 4, S. 795-800

Morita \& Yen 2000

Morita, M. ; Yen, B. C.: Numerical methods for conjunctive two-dimensional surface and three-dimensional sub-surface flows. In: International Journal for Numerical Methods in Fluids 32 (2000), S. 921-957

Morita \& Yen 2002

Morita, M. ; Yen, B. C.: Modeling of Conjunctive Two-Dimensional Surface-Three-Dimensional Subsurface Flows. In: Journal of Hydraulic Engineering 128 (2002), Nr. 2, S. 184-200. http://dx.doi.org/10. 1061/(ASCE) 0733-9429(2002) 128:2(184)

MSDN 2007

MSDN: Remote Procedure Call. 2007. http://msdn2.microsoft.com/en-us/library/ aa378651.aspx, Abruf: 29.01.2007

MSDN 2008

MSDN: Interoperating with Unmanaged Code.

library/sd10k43k.aspx, Abruf: 28.01.2008

2008. http://msdn2.microsoft.com/en-us/

Mualem 1976

Mualem, Y:: A new model for predicting the hydraulic conductivity of unsaturated porous media. In: Water Resources Research 12 (1976), Nr. 3, S. 513-522

Müller 1999

Müller, T.: Quantifizierung der Uferfiltration bei hohen Flurabständen am Beispiel des Modau-SandbachSystems. Wiesbaden : Hessische Landesanstalt für Umwelt, 1999 (Umweltplanung, Arbeits- und Umweltschutz / Schriftenreihe der Hessischen Landesanstalt für Umwelt 271). - ISBN 3-89026-307-0 
Nash \& Sutcliffe 1970

Nash, J. E. ; Sutcliffe, J. V.: River flow forecasting through conceptional models. In: Journal of Hydrology 10 (1970), S. 282-290

Niemeyer \& Kamrath 2007

Niemeyer, M. ; Kamrath, P.: Modellierung der Breschenbildung und Überflutung - vom Versagen zu den Folgen. In: Köngeter, J. (Hrsg.): 37. IWASA, Internationales Wasserbau-Symposium Aachen 2007 : Sicherheit und Risiko wasserbaulicher Anlagen. Herzogenrath : Shaker, 2007 (Mitteilungen / Lehrst. u. Inst. f. Wasserbau u. Wasserwirtschaft, Rheinisch-Westfälische Technische Hochschule Aachen 148). - ISBN 3-8322-6665-8, S. I1-I29

Nowack 2007

Nowack, L.: Implementierung alternativer Leakage-Ansätze in das Grundwasserströmungsprogramm FE$F L O W$, Lehrst. u. Inst. f. Wasserbau u. Wasserwirtschaft, RWTH Aachen, Diplomarbeit, 2007

OpenMI Association 2007

OpenMI Association: OpenMI A new era in integrated water management. 2007. http://www.openmi. org, Abruf: 05.11.2007

Panday \& Huyakorn 2004

Panday, S. ; Huyakorn, P. S.: A fully coupled physically-based spatially-distributed model for evaluating surface/subsurface flow. In: Advances in Water Resources 27 (2004), S. 361-382. http://dx. doi.org/10. $1016 / j$.advwatres.2004.02.016

Peetz \& Steckel 2007

Peetz, J.-V. ; Steckel, B.: 3-code coupling in flood simulation. In: MpCCI 8th user forum 2007 proceedings, february 13 and 14, 2007, 2007, 108-116. http://www.mpcci.de/fileadmin/mpcci/Userforum/ MpCCI_8th_UserForum.pdf

Pelka 1988

Pelka, B.: Modelle zur Berechnung mehrschichtiger Grundwasserleiter auf der Basis von Finiten Elementen. Aachen : Lehrst. u. Inst. f. Wasserbau u. Wasserwirtschaft, RWTH Aachen, 1988 (Mitteilungen / Lehrst. u. Inst. f. Wasserbau u. Wasserwirtschaft, Rheinisch-Westfälische Technische Hochschule Aachen 70). ISSN 0343-1045

Pinder \& Frind 1972

Pinder, G. F. ; Frind, E. O.: Application of Galerkin's Procedure to Aquifer Analysis. In: Water Resources Research 8 (1972), Nr. 1, S. 108-120

Pinder \& Sauer 1971

Pinder, G. F. ; Sauer, S. P.: Numerical Simulation of Flood Wave Modification Due to Bank Storage Effects. In: Water Resources Research 7 (1971), Nr. 1, S. 63-70

Puffahrt 2007

Puffahrt, O. ; Niedersächsischer Landesbetrieb für Wasserwirtschaft, Küsten- und Naturschutz, Betriebsstelle Lüneburg für den Jeetzeldeichverband (Hrsg.): Zum Hochwasserschutz für Hitzacker und die Jeetzelniederung. Bd. 1: Deichlexikon für den Landkreis Lüchow-Dannenberg - Erläuterung der Fachbegriffe. Dannenberg : Buchdruckerei Otto Dennstedt, 2007 http://cdl.niedersachsen.de/blob/images/ C49671973_L20.pdf

Rawls \& Brakensiek 1983

Rawls, J. W. ; Brakensiek, D. L.: A procedure to predict Green and Ampt infiltration parameters. In: Proceedings of the national conference on advances in infiltration, 12.-13. Dec. 1983, Chicago, Illinois, American Society of Agricultural Engineers, 1983, S. 102-112 
Rawls et al. 1992

Rawls, W. J. ; Ahuja, L. R. ; Brakensiek, D. L. ; Shirmohammadi, A.: Infiltration and soil water movement. In: Maidment, D. R. (Hrsg.): Handbook of Hydrology. New York u. a. : McGraw-Hill, 1992. - ISBN 0-07039732-5, Kapitel 5, S. 5.1-5.51

Rheinenergie AG 2007

Rheinenergie AG: Daten auf CD-ROM, 22.11.2007. per Post von Dr. Matthias Schmitt (Rheinenergie) an Christian Reuter (IWW), 2007

Richards 1931

Richards, L. A.: Capillary conduction of liquids through porous mediums. In: Physics 1 (1931), S. 318-333

Ruiz Rodriguez Zeisler Blank Ingenieurgemeinschaft für Wasserbau und Wasserwirtschaft 2009

Ruiz Rodriguez Zeisler Blank Ingenieurgemeinschaft für Wasserbau und Wasserwirtschaft: Hochwasserschutzkonzept der Stadt Köln: Risikoanalyse und Risikomanagement für die überflutungsgefährdeten Gebiete der Stadt Köln. 2009. http://www.rzb-gbr.de/html/refs/hydro_sec/proj13.htm, Abruf: 11.06.2009

Rushton 2007

Rushton, K.: Representation in regional models of saturated river-aquifer interaction for gaining/losing rivers. In: Journal of Hydrology 334 (2007), Nr. 1-2, S. 262-281. http://dx.doi.org/10.1016/j.jhydrol. 2006.10 .008

Rushton \& Tomlinson 1979

Rushton, K. R. ; Tomlinson, L. M.: Possible mechanisms for leakage between aquifers and rivers. In: Journal of Hydrology 40 (1979), S. 49-65

Sächsisches Landesamt für Umwelt und Geologie 2003

Sächsisches Landesamt für Umwelt und Geologie (Hrsg.): Einfluss des August Hochwassers 2002 auf das Grundwasser. 2003 (Materialien zur Wasserwirtschaft)

Schaap et al. 2001

Schaap, M. G. ; Leij, F. J. ; van Genuchten, M. T.: ROSETTA: a computer program for estimating soil hydraulic parameters with hierarchical pedotransfer functions. In: Journal of Hydrology 251 (2001), Nr. 3-4, S. 163-176. http://dx.doi.org/10.1016/S0022-1694(01)00466-8

Schaap \& van Genuchten 2006

Schaap, M. G. ; van Genuchten, M. T.: A modified Mualem-van Genuchten formulation for improved description of the hydraulic conductivity near saturation. In: Vadose Zone Journal 5 (2006), Nr. 1, S. 27-34. http: //dx.doi.org/10.2136/vzj2005.0005

Schade 2007

Schade, P.: OpenMI at the Federal Waterways Engineering and Research Institute (BAW). 2007. http:// WWW.openmi.org/reloaded/about/publications-documents/OpenMI_at_BAW.pdf. Vortrag beim 2nd OpenMI Life Workshop and Associated Meetings, 20.-21. November 2007, Wallingford, UK

Schälchli 1992

Schälchli, U.: The clogging of coarse gravel river beds by fine sediment. In: Hydrobiologia 235 (1992), S. 189-197

Schälchli 1993

Schälchli, U. ; Vischer, D. (Hrsg.): Die Kolmation von Fliessgewässersohlen: Prozesse und Berechnungsgrundlagen. 1993 (Mitteilungen der Versuchsanstalt für Wasserbau, Hydrologie und Glaziologie / Eidgenössische Technische Hochschule Zürich 124) 
Scheffer 2002

Scheffer, F.: Lehrbuch der Bodenkunde. 15. neub. u. erw. Aufl. Heidelberg, Berlin : Spektrum, 2002. - ISBN 3-8274-1324-9

Scherf 2008

Scherf, T.: Zur Modellierung der Infiltration in die ungesättigte Bodenzone mit dem Grundwassersimulationsprogramm Feflow, UROP RWTH Aachen / Undergraduate Research Opportunities Program, Lehrstuhl und Institut für Wasserbau und Wasserwirtschaft, Abschlussbericht zum UROP-Projekt, 2008

Scherf 2009

Scherf, T.: Zur numerischen Modellierung von Qualmwasser, Lehrst. u. Inst. f. Wasserbau u. Wasserwirtschaft, RWTH Aachen, Diplomarbeit, 2009

Schmitz et al. 1989

Schmitz, G. ; Haverkamp, R. ; Parlange, J. Y. ; Liedl, R.: Infiltration bei eingestauter Bodenoberfläche. 1. Analytische Lösung der Infiltrationsgleichung und praktische Berechnung der Versickerung. In: Zeitschrift für Kulturtechnik und Landentwicklung 30 (1989), Nr. 2, S. 121-131

Schubert 2002

Schubert, J.: Hydraulic aspects of riverbank filtration - field studies. In: Journal of Hydrology 266 (2002), S. $145-161$

Schüttrumpf \& Becker 2008

Schüttrumpf, H. ; Becker, B.: Eine Leakage-Randbedingung für Großraum-Grundwassermodelle auf Basis des Grundwasserströmungsprogramms FEFLOW / Lehrst. u. Inst. f. Wasserbau u. Wasserwirtschaft, RWTH Aachen. 2008. - Abschlussbericht über die Software-Entwicklung des FEFLOW-Moduls leakGRM im Auftrag des Landesamtes für Natur, Umwelt und Verbraucherschutz Nordrhein-Westfalen. http://www. feflow. info/62+M5c50842c46a.html

Schüttrumpf et al. 2008

Schüttrumpf, H. ; Felder, S. ; Reuter, C.: Oberflächenabflüsse bei Extremniederschlägen : Analyse der Gefährdung des Firmengeländes der Vodafone Group Services GmbH in Ratingen / Lehrst. u. Inst. f. Wasserbau u. Wasserwirtschaft, RWTH Aachen. 2008. - [im Auftrag von] Ingenieurbüro Heitfeld-Schetelig GmbH

Sinaba 2007

Sinaba, B.: Zur Infiltration bei eingestauter Bodenoberfläche, Lehrst. u. Inst. f. Wasserbau u. Wasserwirtschaft, RWTH Aachen, Diplomarbeit, 2007

Singh \& Bhallamudi 1998

Singh, V. ; Bhallamudi, S. M.: Conjunctive surface-subsurface modeling of overland flow. In: Advances in Water Resources (1998), S. 567-579

Smith \& Woolhiser 1971

Smith, R. E. ; Woolhiser, D. A.: Overland flow on an infiltrating surface. In: Water Resources Research 7 (1971), Nr. 4, S. 899-913

Sommer et al. 2008

Sommer, T. ; Ettrich, N. ; Eulitz, K. ; Haase, D. ; Karpf, C. ; Peetz, J.-V. ; Steckel, B. ; Weichel, T.: Entwicklung eines 3-Zonen-Modells für Grundwasser- und Infrastrukturmanagement nach extremen Hochwasserereignissen in urbanen Räumen (,3ZM-GRIMEX“) / Dresdner Grundwasserforschungszentrum e. V. (DGFZ); Fraunhofer Institut für Algorithmen und Wissenschaftliches Rechnen (SCAI); Fraunhofer Institut für Techno- und Wirtschaftsmathematik (ITWM); Technische Universität Dresden, Institut für Siedlungs- und Industriewasserwirtschaft (ISI); Helmholtz-Zentrum für Umfeltforschung GmbH - UFZ, Department Angewandte Landschaftsökologie. 2008. - Abschlussbericht / BMBF-Verbund-Projekt / FKZ: 02WH0557. http://edok01.tib.unihannover.de/edoks/e01fb09/594029457.pdf 
Sommer \& Eulitz 2007

Sommer, T. ; Eulitz, K.: Auswirkungen des Hochwassers auf das Grundwasser / Phänomene und Modellansätze. In: Meon, G. (Hrsg.) ; Sauter, M. (Hrsg.) ; Führböter, J. F. (Hrsg.) ; Wolf, J. (Hrsg.): Niedersächsisches Grundwasserkolloquium 21.-22. Februar 2007 in Braunschweig : Grundwasser, Hochwasser und Salzwasser / Konzepte zur Risikominimierung. Stuttgart : E. Schweizerbart'sche Verlagsbuchhandlung (Nägele u. Obermiller), 2007 (Zentralblatt für Geologie und Paläontologie / Teil I Allgemeine, Angewandte, Regionale und Historische Geologie Jg. 2007, H. 1/2), S. 7-15

Sommer \& Ullrich 2004

Sommer, T. ; Ullrich, K.: Das unsichtbare Hochwasser - Auswirkungen des August-Hochwassers 2002 auf das Grundwasser im Stadtgebiet von Dresden. In: Köngeter, J. (Hrsg.): 34. IWASA, Internationales WasserbauSymposium Aachen 2004 : Hochwasserschutz - eindeichen oder ausweichen. Aachen : Shaker, 2004 (Mitteilungen / Lehrst. u. Inst. f. Wasserbau u. Wasserwirtschaft, Rheinisch-Westfälische Technische Hochschule Aachen 136). - ISBN 3-8322-3592-2, S. 203-224

Sommer \& Ullrich 2005

Sommer, T. ; Ullrich, K. ; Landeshauptstadt Dresden (Hrsg.): Auswirkungen des Hochwassers 2002 auf das Grundwasser. Dresden : Druckerei und Verlag Hille, 2005. - Forschungsbericht: Hochwasser : Nachsorge : Grundwasser : Dresden. http://edok01.tib.uni-hannover.de/edoks/e01fb06/ $507079361 . \mathrm{pdf}$. - ISBN 3-00-016631-9

Sophocleous 2002

Sophocleous, M.: Interactions between groundwater and surface water: the state of the science. In: Hydrogeology Journal 10 (2002), S. 52-67. http://dx.doi.org/10.1007/s10040-001-0170-8

Spitz \& Moreno 1996

Spitz, K. ; Moreno, J.: A practical guide to groundwater and solute transport modeling. New York u. a. : Wiley, 1996. - ISBN 978-0-471-13687-3

St.-Benno-Gymnasium 2002

St.-Benno-Gymnasium: Kurzbericht über die Ereignisse am St. Benno-Gymnasium. 2002. http://www. benno-gym.de/Sites/About/Archives/Flood/, Abruf: 11.08.2009

Stadtentwässerungsbetriebe Köln 2009a

Stadtentwässerungsbetriebe Köln: Hochwasser : Risikomanagement. 2009. http://www.steb-koeln. de/risikomanagement.html, Abruf: 22.07.2009

Stadtentwässerungsbetriebe Köln 2009b

Stadtentwässerungsbetriebe Köln: Hochwasserschutzzentrale Köln. 2009. http://www.steb-koeln. de/hochwasser.html, Abruf: 11.06.2009

SUNDIALS 2008

SUNDIALS: SUite of Nonlinear and DIfferential/ALgebraic equation Solvers. 2008. https:// computation.llnl.gov/casc/sundials/main.html, Abruf: 11.03.2008

Swain 1994

Swain, E. D.: Implementation and use of direct-flow connections in a coupled ground-water and surface-water model. In: Ground Water 32 (1994), Nr. 1, S. 139-144

Sykes et al. 2006

Sykes, J. F. ; Randall, J. E. ; Normani, S. D.: The analysis of seasonally varying flow in a christalline rock watershed using an integrated surface water and groundwater model. In: Binning, P. J. (Hrsg.) ; Engesgaard, P. K. (Hrsg.) ; Dahle, H. K. (Hrsg.) ; Pinder, G. F. (Hrsg.) ; Gray, W. G. (Hrsg.): Proceedings of the 
XVI International Conference on Computational Methods in Water Resources. Copenhagen, Denmark, June 2006, http: / / proceedings. cmwr-xvi.org/getFile.py/access? contribId=234\&amp; ses sion Id=10\&amp; res Id=0 \&amp; material Id=paper\&amp; conf Id=a0 1

Tacke 1988

Tacke, D.: Qualmwasser in der Gartower Elbmarsch, Universität Hannover, Diplomarbeit, 1988

Thoms \& Johnson 2005

Thoms, R. B. ; Johnson, R. L.: Modelling groundwater/surface water interactions using MODFLOW-WhaT. In: Bringing groundwater quality research to the watershed scale, GQ2004 (4th International Groundwater Quality Conference). Wallingford : IAHS Press, Centre for Ecology and Hydrology, 2005, S. 466-472

Trefry \& Muffels 2007

Trefry, M. G. ; Muffels, C.: Feflow: A finite-element ground water flow and transport modeling tool. In: Ground water 45 (2007), Nr. 5, S. 525-528. http: / / dx.doi .org/10.1111/j.1745-6584.2007.00358.x

Trübger 2006

Trübger, E.-R.: Entwicklung eines Ansatzes zur Berücksichtigung der ungesättigten Zone bei der Grundwassersimulation von Feuchtgebieten, Agrar- und Umweltwissenschaftliche Fakultät der Universität Rostock, Dissertation, 2006. http://deposit.d-nb.de/cgi-bin/dokserv?idn=98749564x\&dok_var= d1\&dok_ext=pdf\&filename=98749564x.pdf

Ubell 1964

Ubell, K.: Surface- and groundwater relationships along the Hungarian reach of the Danube river. In: Symposium surface waters hold at the occasion of general assembly of Berkeley of I.U.G.G. 19.-31.8.1963 Bd. 63, 1964 (Publication de l'association internationale d'hydrologie scientifique), 502-512. http://www. iahs. info/

Ubell 1987a

Ubell, K.: Austauschvorgänge zwischen Fluß und Grundwasser - Teil I. In: Deutsche Gewässerkundliche Mitteilungen 31 (1987), Nr. 4, S. 119-125

Ubell 1987b

Ubell, K.: Austauschvorgänge zwischen Fluß und Grundwasser - Teil II. In: Deutsche Gewässerkundliche Mitteilungen 31 (1987), Nr. 5, S. 142-148

Umweltministerium, Innenministerium und Wirtschaftsministerium Baden-Württemberg 2005

Umweltministerium, Innenministerium und Wirtschaftsministerium Baden-Württemberg: Hochwassergefahrenkarten in Baden-Württemberg. 2005. http://www.hochwasseronline.de/vm_allgemein/ Leitfaden_Hochwassergefahrenkarten.pdf, Abruf: 14.08.2009

VanderKwaak 1999

VanderKwaak, J. E.: Numerical simulation of flow and chemical transport in integrated surface-subsurface hydrologic systems. Waterloo, Ont., Canada, University of Waterloo, PhD-thesis, 1999

van Genuchten 1978

van Genuchten, R.: Calculating the unsaturated hydraulic conductivity with a new closed-form analytical model. Princeton, NJ, Water Resources Program : Department of Civil Engineering, Princeton University, Ph. D. thesis, 1978

van Linn 2006

van Linn, A.: Automatische Optimierung zur Bewertung und Risikoanalyse einer Hochwasserschutzmaßnahme, Fakultät für Bauingenieurwesen der Rheinisch-Westfälischen Technischen Hochschule Aachen, Dissertation, 2006. http://deposit.d-nb.de/cgi-bin/dokserv?idn=983002959\&dok_var= d1\&dok_ext=pdf\&filename=983002959.pdf 
Vekerdy \& Meijerink 1998

Vekerdy, Z. ; Meijerink, A. M. J.: Statistical and analytical study of the propagation of flood induced groundwater rise in an alluvial aquifer. In: Journal of Hydrology 205 (1998), S. 112-125

Vázquez-Suñé et al. 2007

Vázquez-Suñé, E. ; Capino, B. ; Abarca, E. ; Carrera, J.: Estimation of Recharge from Floods in Disconnected Stream-Aquifer-Systems. In: Ground Water 45 (2007), Nr. 5, S. 579-598. http://dx.doi.org/10. $1111 / j .1745-6584.2007 .00326 . x$

Walther \& Marre 2004

Walther, W. ; Marre, D.: Kopplung Fließgewässer - Grundwasser: Wirkung des Hochwassers auf den unterirdischen Raum. In: Köngeter, J. (Hrsg.): 34. IWASA, Internationales Wasserbau-Symposium Aachen 2004 : Hochwasserschutz-eindeichen oder ausweichen. Aachen : Shaker, 2004 (Mitteilungen / Lehrst. u. Inst. f. Wasserbau u. Wasserwirtschaft, Rheinisch-Westfälische Technische Hochschule Aachen 136). - ISBN 3-8322-3592-2, S. 173-202

Ward 1964

Ward, J. C.: Turbulent Flow in Porous Media. In: Journal Of The Hydraulics Division 90 (1964), Nr. HY5, S. $1-12$

Wasy GmbH 2005

Wasy GmbH: FEFLOW ${ }^{\circledR}$ White Papers Vol. 1. Berlin, 2005

Wasy GmbH 2006

Wasy GmbH: FEFLOW ${ }^{\circledR}$. 2006. http://www. feflow.de, Abruf: 15.12.2006

Wasy GmbH 2008

Wasy GmbH: FEFLOW ${ }^{\circledR}$ Online Help. Version 5.3, 2008

Weill \& Mouche 2006

Weill, S. ; Mouche, E.: Darcy multi-domain approach for integrated surface/subsurface hydrologic models. In: Binning, P. J. (Hrsg.) ; Engesgaard, P. K. (Hrsg.) ; Dahle, H. K. (Hrsg.) ; Pinder, G. F. (Hrsg.) ; Gray, W. G. (Hrsg.): Proceedings of the XVI International Conference on Computational Methods in Water Resources. Copenhagen, Denmark, June 2006, http: //proceedings.cmwr-xvi.org/contributionDisplay. py?contribId=77\&am\%p; session Id=10\&amp; conf Id=a0 51

Wieczorrek 2007

Wieczorrek, Y.: Grundwasserprojekt. E-Mail vom 23.10.2007 von yvonne.wieczorrek@steb-koeln.de an reuter@iww.rwth-aachen.de, 2007

Winter et al. 1998

Winter, T. C. ; Harvey, J. W. ; Franke, O. L. ; Alley, W. M.: Ground Water and Surface Water : A Single Resource. Denver, Colorado : U. S. Government Printing Office, 1998 (U. S. Geological Survey Circular 1139). - ISBN 0-607-89339-7 


\section{A Die Feflow-Programmierschnittstelle IFM}

(zu Abschnitt 2.4.1. Abschnitt 4.5 und Anhang C.3

Das Programm Feflow erlaubt es, über ein Modul an verschiedenen Stellen des Programmablaufs Daten zu modifizieren. An diesen Eingriffspunkten führt das Programm sogenannte CallbackFunktionen aus. In diese Funktionen kann der Anwender eigene Programmteile in den Programmiersprachen C oder C++ integrieren. Die Modifikation und die Abfrage der Daten des Strömungsproblems erfolgt mit IFM-Funktionen (siehe WASY 2005). Die Abkürzung IFM steht für Interface Manager. Zur Veranschaulichung sind einige IFM-Funktionen in Tabelle A.1 aufgeführt.

Tabelle A.1: Beispiele für IFM-Funktionen (siehe auch Wasy GmbH 2008)

\begin{tabular}{|c|c|c|}
\hline Name & Input (Auszug) & Beschreibung \\
\hline $\begin{array}{l}\text { IfmGetAbsolute } \\
\text { SimulationTime }\end{array}$ & - & $\begin{array}{l}\text { Gibt die aktuelle Simulationszeit } \\
\text { zurück }\end{array}$ \\
\hline $\begin{array}{l}\text { IfmGetResults } \\
\text { FlowHeadValue }\end{array}$ & Knotennummer & $\begin{array}{l}\text { Gibt die berechnete } \\
\text { Grundwasserdruckhöhe für die } \\
\text { aktuelle Simulationszeit und einen } \\
\text { Knoten zurück }\end{array}$ \\
\hline $\begin{array}{l}\text { IfmSetBCFlowTypeAnd } \\
\text { ValueAtCurrentTime }\end{array}$ & $\begin{array}{l}\text { Knotennummer, Randbe- } \\
\text { dingungswert, Randbedin- } \\
\text { gungstyp }\end{array}$ & $\begin{array}{l}\text { Setzt für den aktuellen Zeitschritt } \\
\text { für einen Knoten eine } \\
\text { Randbedingung }\end{array}$ \\
\hline
\end{tabular}

Abbildung A.1 liefert eine Übersicht über die verschiedenen Callback-Funktionen des Programms Feflow. Zentral angeordnet ist der Prozess der FlowSimulation, in dem das Gleichungssystem des Strömungsproblems gelöst wird. Vor und nach der Lösung des Gleichungssystems werden die Funktionen Preflowsimulation beziehungsweise PostFlowsimula tion ausgeführt. Die Lösung des Gleichungssystems wird zum Beispiel dann wiederholt, wenn Nebenbedingungen nicht erfüllt werden. Über die IFM-Funktion IFMSet SimulationControl Flag kann eine solche Wiederholung auch vom Anwender innerhalb eines Moduls ausgelöst werden (siehe z. B. Nowack 2007).

Vor und nach der Berechnung eines Zeitschrittes werden die Callback-Funktionen PreTime Step und PostTimestep ausgeführt, bis das Ende der Simulationszeit erreicht ist. Die Funktion OnBeginSimulation und OnEndSimulation beziehen sich auf den Beginn und das Ende einer Strömungsberechnung, während OnBeginDocument direkt beim Einlesen der *. fem- 
Datei, die ein Strömungsproblem beschreibt, ausgeführt wird. OnEndDocument wird ausgeführt, wenn das Strömungsproblem zugunsten einer anderen Datei oder bei Programmende gelöscht wird. Zusammen mit der Callback-Funktion onEnterSimulator sind die beiden zuletzt genannten Callback-Funktionen in Abbildung A.1 abgesetzt dargestellt, weil sie sich nicht auf die eigentliche Simulation beziehen. OnEnterS imulator wird ausgeführt, wenn der Anwender das Menü „Simulator“ anwählt oder wenn ein Berechnungslauf über den Batch-Modus gestartet wird. Nach der Simulation kann ein weiterer Rechenlauf über die Benutzeroberfläche gestartet werden. Der nächste Callback, in dem ein Eingriff in den Programmablauf möglich ist, wäre in diesem Fall wieder OnBeginsimulation.

Die Callback-Funktionen werden in eine standardisierte dynamische Programmbibliothek (DLL) eingebunden, die dann vom Hauptprogramm aufgerufen wird. Eine solche DLL wird IFM-Modul genannt. 


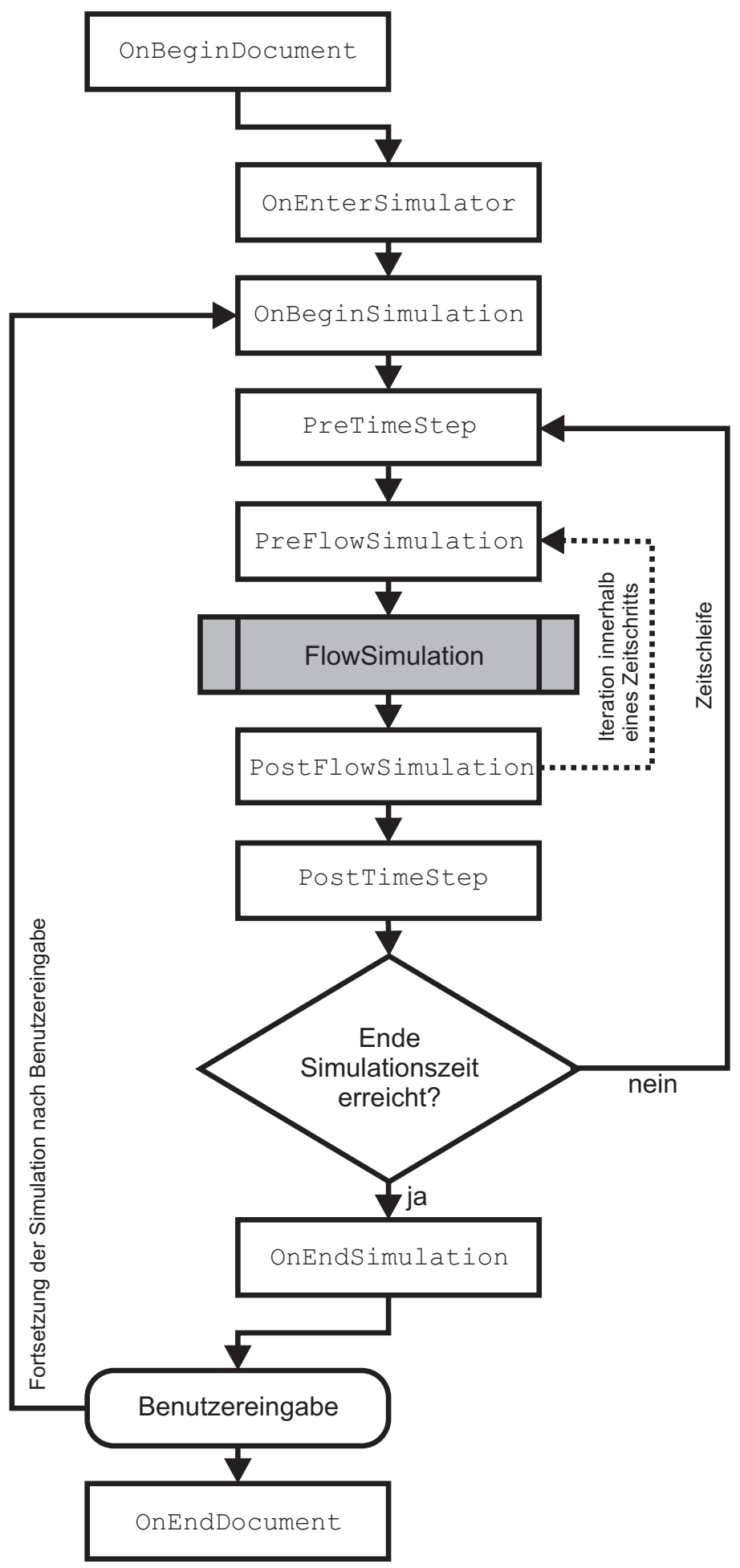

Abbildung A.1: Möglichkeiten des Programmeingriffs in die Feflow-Grundwasserströmungsberechnung über Callback-Funktionen 


\section{B Ausrüstung bestehender Programme mit einer OpenMI-Schnittstelle}

(zu Abschnitt 4.1, Anhang C.2 und Anhang C.3.1

Die Ausrüstung eines bestehenden Strömungsprogramms mit einer OpenMI-Schnittstelle wird Migration (migration, Gijsbers et al. 2005) genannt. Die OpenMI Association (2007) liefert Beispiele, die den Einstieg erleichtern. Die OpenMI-Dokumentation (Gijsbers et al. 2005) enthält eine Schritt-für-Schritt-Anweisung. Das migrierte Programm heißt OpenMI-kompatible Komponente und erfüllt folgende Anforderungen (Gijsbers et al. 2005):

1. das Modell muss zur Laufzeit durch eine externe Einheit steuerbar sein

2. die Struktur des Programmablaufs besteht aus einer Initialisierungsphase und einer Berechnungsphase

3. das Modell muss grundsätzliche Informationen über die modellierten Größen nach außen übergeben können

4. das Modell muss Werte für modellierte Größen für jeden Punkt in Zeit und Raum nach außen übergeben können

5. das Modell muss den Datenaustausch nach dem Frage-Antwort-Mechanismus unterstützen

6. das Modell muss wissen, welchen Zeitpunkt es in seiner Berechnung erreicht hat

7. Komponenten müssen interpolieren und extrapolieren können, wenn angefragte Punkte nicht auf dem Modell-Zeitgitter oder Raumgitter liegen

8. das Modell muss entscheiden, ob es angefragte Werte für einen bestimmten Zeitpunkt berechnet, interpoliert oder aus einem Zwischenspeicher entnimmt.

Während für die Anforderungen 3 bis 8 von der OpenMI-Umgebung entsprechende Funktionen bereit gestellt werden, gelten die Anforderungen 1 und 2 grundsätzlich für das Strömungsprogramm, das als OpenMI-Komponente umgerüstet werden soll. Der einfachste Weg, ein bestehendes Strömungsprogramm OpenMI-kompatibel zu machen, ist nach Gijsbers et al. (2005), den Quellcode einen sogenannten Wrapper (wrapper, dt. Übers. „Kapsel“, „Umschlag“ oder „Hül$1 \mathrm{e}^{\text {") }}$ einzubinden. Dieser Wrapper stellt alle nach dem OpenMI-Standard geforderten Funktionen bereit und kommuniziert mit dem Rechenkern des Strömungsprogramms.

Der Datenaustausch nach dem OpenMI-Standard erfolgt zur Laufzeit. Die OpenMI-Umgebung ist deshalb in der Programmiersprache C\# für die .NET-Plattform entwickelt. Wenn das Programm als herkömmlicher Quellcode (unmanaged code) zur Verfügung steht, bietet es sich 
an, das Programm als DLL (dynamic link library) zu erstellen. Die DLL wird nun über das Platform-Invoke-Verfahren (MSDN 2008) in den C\#-Wrapper eingebunden, der selbst als DLL vom OpenMI-Editor aufgerufen wird.

Als nächstes ist der Programmcode des zu migrierenden Programms so zu organisieren, dass er vier Grundfunktionen, die im Wrapper enthalten sind, bedienen kann (Gijsbers et al. 2005):

1. Initialize() Öffnen und Einlesen der Dateien mit Rand- und Anfangsbedingungen und Netzgeometrie

2. PerformtimeStep () Berechnung eines einzelnen Zeitschritts

3. Finish () Schließen aller Eingabe- und Ausgabedateien

4. Dispose () Freigabe von Speicher

Ist dies gelungen, so sind die Anforderung nach einer Ansteuerbarkeit von außen und die Aufteilung der Berechnung in eine Initialisierungsphase (Punkt 1 der obigen Liste) und eine Berechnungsphase (Punkt 2) erfüllt.

Nun wird die Funktion Initialize () um die Definition der Austauschobjekte erweitert, an denen Daten mit anderen Komponenten eines OpenMI-Systems ausgetauscht werden. Zur vollständigen OpenMI-Kompatibilität muss der Code des Strömungsprogramms so verändert werden, dass dieses weitere Funktionen des OpenMI-Wrappers bedient. Einige davon sind in folgender Auflistung wiedergegeben:

- GetCurrentTime (): Gibt die aktuelle Simulationszeit des Rechenkerns zurück.

- GetValues (QuantityID, ElementSetID): Gibt den Wert eines Ausgabeobjektes, definiert durch Quantity und ElementSet, zur aktuellen Simulationszeit zurück. Dies kann zum Beispiel der Wasserstand eines Modellknotens sein.

- SetValues(QuantityID, ElementSetID, values): Setzt für ein Eingabeobjekt (Quantity, ElementSet) zur aktuellen Simulationszeit den Wert value. Die Ausführung dieser Funktion entspricht meistens dem Setzen einer Randbedingung, zum Beispiel eine Zuflussmenge eines Modellknotens. 


\section{Aufbau eines OpenMI-Systems aus den Programmen IImoflood und Feflow}

(zu Abschnitt 4.1, Abschnitt 4.5 und Abschnitt 8.1)

\section{C.1 Einführung}

In diesem Kapitel wird erläutert, wie die beiden Programme Ilmoflood und Feflow mit OpenMI-Schnittstellen ausgerüstet wurden. Damit sind die Programme für eine externe Kopplung mit anderen OpenMl-Komponenten geeignet. Für Ilmoflood lässt sich die Migration in Richtung einer OpenMI-linkable component nach der Schritt-für-Schritt-Anleitung der OpenMI Association (Gijsbers et al. 2005) umsetzen. Deshalb wird zunächst die Migration von Ilmoflood erläutert. Die Migration von Feflow stellt eine besondere Herausforderung dar, auf die im Kapitel C.3 eingegangen wird. Der letzte Abschnitt enthält ein Anwendungsbeispiel, das zur Verifikation der korrekten Programmierung verwendet wurde. Scherf (2009) und Jansen (2009) haben das gekoppelte OpenMI-Systeme aus den Programmen Feflow und IImoflood bereits für ihre Untersuchungen verwendet und damit quasi einem Betatest ${ }^{1}$ unterzogen.

\section{C.2 Migration von IImoflood}

Die Migration des Programms Ilmoflood erforderte zunächst die Neuorganisation des Programmcodes und Einbindung in einen Wrapper (siehe Anhang B). Als Austauschobjekte wurden im Hinblick auf die Zielsetzung dieser Arbeit nur die Zellen der Überflutungsflächen berücksichtigt. Für die Eingabeobjekte war für die Kopplung die Implementierung einer Zuflussrandbedingung notwendig, für die Ausgabeobjekte konnte auf die interne Speicherung der Berechnungsergebnisse zugegriffen werden. Tabelle C.1 enthält eine Übersicht über die Austauschobjekte, die in das Programm Ilmoflood implementiert wurden.

Die Zuflussrandbedingung summiert einzelne Werte auf, es ist also möglich, eine Zelle mit mehreren Knoten eines anderen Modells zu verbinden. Für das Ausgabeobjekt „Wasserstand“ werden nur dann Werte übergeben, wenn die Zelle nicht trocken ist.

\footnotetext{
${ }^{1}$ erweiterter Softwaretest durch einen eingeschränkten Nutzerkreis, bei dem Programmfehler aufgedeckt werden sollen
} 
Tabelle C.1: Austauschobjekte für das Überflutungsflächenmodul von IImoflood

\begin{tabular}{cccccl}
\hline Name & Objektart & Größe & Einheit & Lokalisierung & Programmfunktion \\
\hline $\mathrm{Q}$ & Eingabe & Durchfluss & $\mathrm{m}^{3} / \mathrm{s}$ & Zellnummer & Zuflussrandbedingung \\
$\mathrm{h}$ & Ausgabe & Wasserstand & $\mathrm{m}$ & Zellnummer & berechneter Wasserstand im \\
& & & & & Fall einer benetzten Zelle \\
\hline
\end{tabular}

Die Ausgabe von Ilmoflood-Ergebnissen an eine angekoppelte Komponente und die Eingabe von Randbedingungen von einer angekoppelten Komponente in ein Ilmoflood-Modell erfolgt zu den Ausgabezeitpunkten, die vom Anwender durch die Vorgabe einer Ausgabezeitschrittweite, zum Beispiel eine Stunde, definiert werden. Zwischen diesen Ausgabezeitpunkten nimmt Ilmoflood eine interne Zeitdiskretisierung vor, die von den Strömungsverhältnissen im Modell abhängt. Eine Interpolation der zu den Ausgabezeitpunkten über die OpenMI-Schnittstelle empfangenen Randbedingungswerte auf die internen Zeitschritte erfolgt nicht, weil eine solche Interpolation unter Umständen numerisch Masse erzeugen oder vernichten würde.

\section{C.3 Erweiterung von Feflow mit einer OpenMI-Schnittstelle}

\section{C.3.1 Problemstellung und Lösungsansatz}

Die Ausrüstung des Programms Feflow mit einer OpenMI-Schnittstelle bereitet besondere Schwierigkeiten, weil es sich bei Feflow um ein kommerzielles ausführbares Programm handelt. Der Quellcode ist deshalb nicht verfügbar, so dass nur begrenzt Änderungen vorgenommen werden können.

Der Programmablauf von Feflow entspricht der zweiten Anforderung aus Abschnitt B. Wenn der Berechnungslauf, in der Feflow-Terminologie mit Simulation bezeichnet, beginnt, sind die Geometrie des Berechnungsnetzes, alle Anfangs- und alle Randbedingungen bereits bekannt. Den Anforderungen nach einer Übergabemöglichkeit der modellierten Größen selbst und der Werte für modellierte Größen (Anhang B, Anforderungen 3 und 4) lässt sich über die IFM-Schnittstelle nachkommen, denn Feflow stellt IFM-Funktionen zur Verfügung, mit denen während der Berechnung nahezu alle modellierten Größen abgefragt und manipuliert werden können (siehe Anhang A) . Die Erfüllung von Anforderungen 5, 7] und 8 aus Anhang B wird durch die Verwendung des Wrappers sichergestellt.

Der kritische Punkt ist damit die externe Steuerung der Berechnung von außen (Anforderung 1). Ohne den Quellcode führt der einzige Weg hin zu einer externen Steuerung über die IFMSchnittstelle.

Wird das Programm Feflow mit Hilfe der IFM-Schnittstelle erweitert, so entsteht eine Modul in Form einer IFM-DLL, die vom Programm Feflow geladen wird. Naheliegend ist also, diese IFMDLL mit entsprechenden Funktionen in einen OpenMI-Wrapper einzubinden, wie in Anhang 
B skizziert. Diese OpenMI-IFM-DLL würde dann sowohl die IFM-Schnittstelle und die OpenMISchnittstelle vereinigen, aber von zwei Programmen aufgerufen werden müssen, nämlich einmal von Feflow und einmal von der OpenMI-Umgebung, welche die Ansteuerung übernimmt. Da eine DLL beim wiederholtem Aufruf als neue Instanz in den Arbeitsspeicher des Computers geladen wird, besteht zunächst keine Kommunikationsmöglichkeit zwischen den beiden Programmen, denn beide Programme greifen auf voneinander völlig unabhängige Instanzen der gleichen DLL $\mathrm{zu}$.

Da für jede Instanz einer DLL grundsätzlich unterschiedliche Speicherbereiche reserviert werden, liegt es nahe, einen gemeinsamen Speicherbereich (shared memory) für alle Instanzen der OpenMI-IFM-DLL einzurichten. Für die externe Steuerung des Programms Feflow ist es aber notwendig, die IFM-Funktionen des Programms Feflow auszuführen. Dies erfordert den Zugriff auf den für Feflow reservierten Speicherbereich von außen. Ohne eine entsprechende Umgestaltung des Programms Feflow ist ein solcher Zugriff nicht möglich.

Die Ansteuerung des Programms Feflow wird daher dem Vorschlag von Gründler (2007) folgend über remote procedure calls (RPC, dt. Übers.: Prozedur-Fernaufruf) realisiert. Mit Hilfe des RPC-Protokolls kann ein Programm Funktionen eines anderen Programms aufrufen (Brockhaus-Enzyklopädie Online 2008). Der RPC arbeitet nach dem Client-Server-Modell, wobei ein Programm als Client eine Funktion des Servers ausführt. Der Server sendet den Rückgabewert der aufgerufenen Funktion an den Client. Die beiden Programme können auf dem gleichen Rechner oder auf unterschiedlichen Rechnern laufen. Das Programm Feflow wird also nicht wie in Abschnitt B beschrieben umorganisiert, sondern erweitert. Daher kann auch nicht von einer Migration im Sinne von Gijsbers et al. (2005) gesprochen werden.

\section{C.3.2 Umsetzung}

Das Funktionsprinzip der externen Steuerung des Programms Feflow ist in Abbildung C.1 dargestellt. Links im Bild dargestellt ist der FeflowController. Der FeflowController ist die OpenMI-kompatible Komponente, sie wird als DLL vom OpenMI-Editor (OpenMI . exe) geladen und enthält alle Funktionen des OpenMI-Standards. Eine Auswahl dieser Funktionen ist im Bild dargestellt:

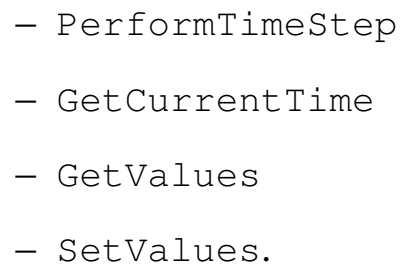

Das Strömungsprogramm Feflow (feflow. exe im rechten Teil des Bildes) lädt eine DLL namens FEopenMI. Es handelt sich hierbei um ein IFM-Modul (siehe dazu Anhang A) mit den Callback-Funktionen

- OnBeginSimulation 


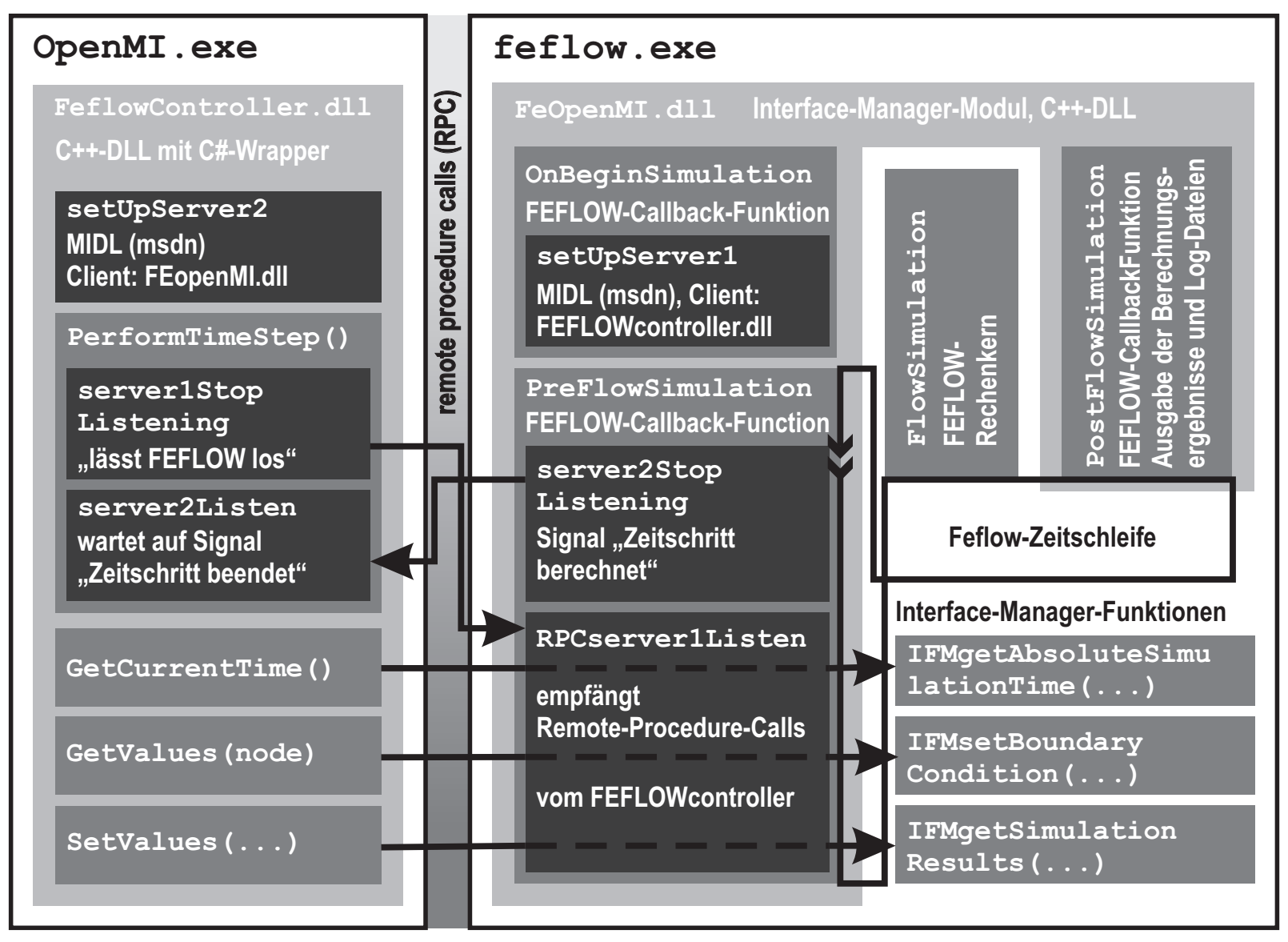

Abbildung C.1: Funktionsprinzip der externen Steuerung des Programms Feflow über ein IFM-Modul und RPC (nach Becker et al. 2008b)

- Preflowsimulation

- PostTimestep.

Darüber hinaus sind als Beispiel für IFM-Funktionen

- IfmGetAbsoluteSimulationTime

- IfmGetResultsFlowHeadValue

- IfmSetBcFlowTypeAndValueAtCurrentTime

dargestellt. Diese Funktionen sind in Feflow fest implementierte Funktionen, die in IFM-Modulen ausgeführt werden können, um Werte abzufragen oder zu modifizieren. I fmGetAbsoluteS imu lationTime gibt die momentane Simulationszeit zurück. IfmGetResultsFlowHeadValue gibt für einen Knoten die letzte berechnete Grundwasserdruckhöhe zurück. Die IFM-Funktion IfmSetBCF lowTypeAndValueAtCurrentTime ermöglicht, für einen Knoten einen Wert als Randbedingung eines bestimmten Typs für die aktuelle Simulationszeit und alle folgenden Zeitschritte zu setzen. 
Innerhalb des Blocks feflow.exe ist die Feflow-Zeitschleife angedeutet. Für die Berechnung eines Zeitschritts wird zunächst als Vorbereitung die Callback-Funktion OnBeginSimulation ausgeführt, dann erfolgt die Lösung des Strömungsgleichungssystems (F lowSimulation, Feflow-Rechenkern) und zum Abschluss wird die Callback-Funktion PostFlowsimulation ausgeführt.

Der FeflowController und das IFM-Modul FeOpenMI kommunizieren über Prozedur-Fernaufrufe (RPC). Die RPC-Steuerung wurde mit Microsoft-RPC (MSDN 2007) mit einer Interface-Definition entsprechend der Microsoft Interface Definition Language (MIDL) über das Named-Pipe-Netzwerkprotokoll umgesetzt. Da MIDL die Entwicklung mit herkömmlichen (unmanaged) Code in der Programmiersprache $\mathrm{C}^{++}$voraussetzt, das OpenMI-Interface aber in verwaltetem (managed) Code definiert ist, müssen die RPC-Funktionen wiederum in einer separaten DLL bereitgestellt werden. Diese DLL wird über das Platform-Invoke-Verfahren (MSDN 2008) in einen OpenMI-Wrapper eingebunden. In das IFM-Modul dagegen können die RPC-Funktionen direkt eingebunden werden, da IFM-Module in herkömmlichem $\mathrm{C}^{++}$-Code entwickelt werden.

Als Vorbereitung für eine gekoppelte Strömungsberechnung wird vom FeflowController ein Server eingerichtet (Server 2). Auch das Programm Feflow richtet einen Server ein (Server1), bevor die eigentliche Rechnung beginnt. Dieser Schritt ist in der Callback-Funktion OnBeg inSimula tion des IFM-Moduls FeOpenMI angesiedelt.

Zur Erläuterung der Kommunikation zwischen dem FeflowController und FeOpenMI während der Berechnung wird als Ausgangsposition angenommen, dass Feflow einen Zeitschritt $t-1$ vollständig berechnet und die Callback-Funktion Post TimeStep des Feflow-Moduls ausgeführt hat. Der vom FeflowController eingerichtete Server 2 befindet sich im Wartemodus und wartet auf Prozedur-Fernaufrufe von Feflow, das mit Hilfe des Moduls FeOpenMI die Rolle des Client übernimmt.

Als Vorbereitung für die Berechnung des neuen Zeitschrittes $t$ führt Feflow nun die CallbackFunktion PreFlowsimulation aus. Innerhalb dieser Callback-Funktion wird der Wartemodus des Servers 2 (FeflowController) durch Ausführung der Client-Funktion server2StopListen ing beendet. Damit ist die Vollendung der Berechnung für den Zeitschritt $t-1$ an den FeflowController gemeldet. Nächster Schritt innerhalb der Vorbereitung für die Berechnung des nächsten Zeitschritts $t$ ist die Ausführung der Funktion server1Listen. Damit wird Feflow in die Wartebereitschaft versetzt und fungiert als Server. Andere OpenMI-Komponenten können nun über die OpenMI-Schnittstelle Anfragen stellen, die der FeflowController als Client über RemoteProcedure-Calls an den Server 1 weiterleitet. Die Frage nach der momentanen Simulationszeit (GetCurrentTime) bewirkt beispielsweise über den Server 1 die Ausführung der Feflow-IFMFunktion IFMgetAbsoluteSimulationTime. Auf die gleiche Art werden Berechnungsergebnisse des Zeitschritts $t-1$ abgefragt (GetValues $\rightarrow$ IFMget SimulationResults) oder Randbedingungen gesetzt (SetValues $\rightarrow$ IFMsetBoundaryCondition).

Der Aufruf der OpenMI-Funktion PerformTimeStep beendet den Wartemodus des Servers 1 über den RPC server1StopListening und lässt Feflow seinen internen Programmablauf weiter ausführen, während der FeflowController die Rolle des Servers übernimmt. Hat Feflow 
seine Berechnung des Zeitschrittes $t$ ausgeführt, werden in der Callback-Funktion PostF lowSi mulation alle Randbedingungen, die über die OpenMl-Funktion SetValues gesetzt wurden, wieder gelöscht, da die mit der IFM-Funktion I fmSetBCF lowTypeAndVal ueAt Current Time gesetzten Randbedingungen als für den gesamten Simulationszeitraum interpretiert werden. Bei der nächsten Ausführung der Callback-Funktion PreFlowSimulation gibt das Feflow-IFMModul zunächst als Client über einen RPC die Meldung an Server 2 (FeflowController), dass die Berechnung des Zeitschritts $t$ abgeschlossen ist.

Anhand der Funktionsweise der externen Steuerung von Feflow mit dem Wechsel der ClientServer-Aufgaben wird deutlich, dass nur synchrone RPCs verwendet werden können. Beim synchronen RPC wartet der Client auf die Antwort, bevor weitere Programmschritte ausgeführt werden können. Der Programmablauf des Clients ist also streng sequentiell. Beim asynchronen RPC wartet der Client hingegen nicht zwangsläufig auf die Antwort des Servers und kann inzwischen andere Operationen ausführen. Auch OpenMI basiert auf einer single threaded architecture (Brinkmann et al. 2005). Operationen wie die Übergabe von Ergebnissen einer Modellkomponente an eine andere und die Ausführung von Simulationszeitschritten erfolgen konsekutiv. Dies kann anhand des Beispiels in Abschnitt C.4 nachvollzogen werden.

Die Vorbereitung der Server- und Client-Aufgaben, also das Festlegen der RPC-Protokolle und die Definition sogenannter binding handles ist in Abbildung C.1 nicht dargestellt, diese Schritte werden in einer Prepare-Funktion des FeflowControllers untergebracht und von der gleichnamigen Funktion des OpenMI-Wrappers aufgerufen. Entsprechend führt die Finish-Funktion den Rückbau der Server- und der Client-Funktionen im FeflowController und im IFM-Modul aus und sorgt jeweils für die Freigabe des benutzten Speichers.

Zur Verifikation wurden während der Entwicklung entsprechend dem Vorschlag der OpenMIDokumentation (Gijsbers et al. 2005) nUnit-Tests ${ }^{2}$ durchgeführt. Geeignete Ausgabefunktionen wurden in die entsprechenden Programmkomponenten eingebettet, um die korrekte Funktionsweise nachzuvollziehen. Darüber hinaus wurden an geeigneter Stelle Ausgabebefehle eingerichtet, die die Austauschwerte in Dateien schreiben.

\section{C.3.3 Austauschobjekte}

Die für Feflow-Modelle implementierten Austauschobjekte sind in Tabelle C.2 aufgelistet. Alle Austauschobjekte sind Zeitpunkten (time steps) zugeordnet und werden über die Knotennummer angesprochen. Das Setzen von Randbedingungen wird mit Hilfe der IFM-Funktion I fmSetBCC FlowTypeAndValueAtCurrentTime realisiert. Diese Funktion setzt eine eine bis zum Ende der Simulationszeit gültige und zeitlich konstante Randbedingung eines bestimmten Typs für einen Knoten. Bereits vorher auf dem Knoten gesetzte Randbedingungen werden überschrieben. Weil die Randbedingung ab dem Simulationszeitpunkt, für den sie gesetzt wird, bis zum Ende der Berechnung gilt, müssen nach der Lösung des Gleichungssystems im Callback PostF low Simulation alle Randbedingungen, die über die OpenMI-Schnittstelle gesetzt wurden, wieder

\footnotetext{
${ }^{2}$ Testprogramm für .NET-Anwendungen
} 
gelöscht werden. Zum Löschen von Randbedingungen wird ebenfalls die IFM-Funktion IfmSet BCCF lowTypeAndValueAtCurrent Time mit dem Typ-Parameter 0 verwendet.

Tabelle C.2: Austauschobjekte für Feflow. Alle Austauschobjekte beziehen sich auf einen Modellknoten, die Ansteuerung erfolgt über die Knotennummer.

\begin{tabular}{|c|c|c|c|c|}
\hline Name & Objektart & Größe & Einheit & Programmfunktion \\
\hline $\mathrm{h}$ & Eingabe & Wasserstand & $\mathrm{m}$ & Head-Randbedingung \\
\hline Leakage 1 & Eingabe & Wasserstand & $\mathrm{m}$ & $\begin{array}{l}\text { Transfer-Randbedingung (Kontaktfläche } \\
\text { abhängig vom Grundwasserstand) }\end{array}$ \\
\hline Leakage 2 & Eingabe & Wasserstand & $\mathrm{m}$ & $\begin{array}{l}\text { Transfer-Randbedingung (Kontaktfläche } \\
\text { nicht abhängig vom Grundwasserstand) }\end{array}$ \\
\hline Q & Eingabe & Durchfluss & $\mathrm{m}^{3} / \mathrm{d}$ & Well-Randbedingung \\
\hline $\mathrm{h}$ & Ausgabe & Wasserstand & $\mathrm{m}$ & Berechnungsergebnis \\
\hline Q & Ausgabe & Durchfluss & $\mathrm{m}^{3} / \mathrm{d}$ & Budget-Ergebnis \\
\hline Sickerfläche & Ausgabe & Durchfluss & $\mathrm{m}^{3} / \mathrm{d}$ & $\begin{array}{l}\text { Budget-Ergebnis, gleichzeitig Ansatz } \\
\text { einer Sickerflächen-Randbedingung }\end{array}$ \\
\hline
\end{tabular}

Zur Rückgabe des Grundwasserstands wird die Funktion IfmGetResultsFlowHeadValue verwendet. Der Durchfluss wird über den Rückgabewert der Funktion I fmBudget QueryF low AtNode ermittelt. Diese Funktion greift auf das Ergebnis einer nachgeordneten Berechnung zu und liefert die einer Randbedingung korrespondierenden $\mathrm{Zu}$ - oder Abflussmenge.

\section{C.4 Verifikationsbeispiel „Deichquerschnitt“}

Die Funktionsweise der OpenMI-Kopplung soll am Beispiel einer Berechnung der Sickerlinie für einen Deichquerschnitt bei instationärer Randbedingung gezeigt werden. Dieses Beispiel wurde verwendet, um korrekten Austausch der Daten zu überprüfen. Die korrekte Funktionsweise der verwendeten Programmkomponenten Feflow und IImoflood war nicht Gegenstand der Verifikationsrechnungen, das diese Programme bereits verifiziert sind.

Das Berechnungsnetz des Deichquerschnitts ist in Abbildung C.2 gezeigt. Es handelt sich um ein zweidimensional-vertikales Modellgebiet, für das mit Hilfe von Feflow die Richards-Gleichung gelöst wird. Auf der Landseite rechts sind auf der Böschung Sickerflächenrandbedingungen (Gleichungen 7.1 und 7.2) gesetzt. Für die zeitliche Diskretisierung werden konstante Zeitschritte von $1800 \mathrm{~s}$ festgelegt. Die Materialeigenschaften entsprechen den Werten für trockenen Sand aus Tabelle 6.1.

Den Wasserstand auf der Vorlandseite liefert ein einfaches Ilmoflood-Modell. Dieses besteht aus einer Kette quadratischer Rasterzellen, die der Steigung des Deichs an der Wasserseite folgen 


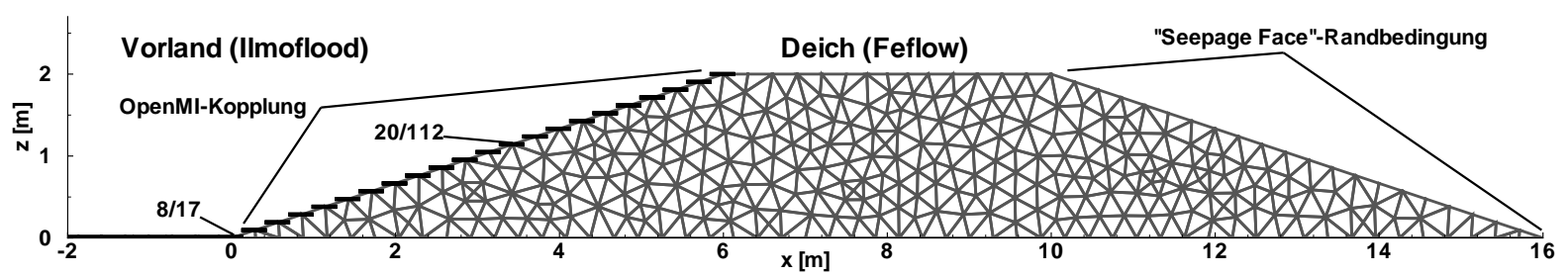

Abbildung C.2: Berechnungsnetz des Deichquerschnitts und des Vorlands, Randbedingungen an der Landseite des Deichs, OpenMI-Kopplungsbereiche und die Lage zweier Zellen-Knoten-Paare

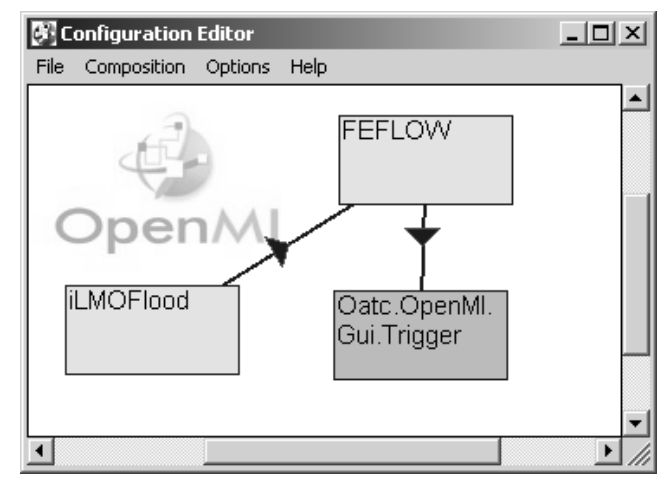

Abbildung C.3: Bildschirmfoto des OpenMI-Editors mit dem OpenMI-System des Deichquerschnitts

(Abbildung C.2). Das Modell hat links eine Zuflussrandbedingung, die den Wasserstand im Vorland so steuert, dass er ansteigt und wieder fällt. Die Zeitschrittweite beträgt 3600 s. Die Grundfläche des Modells ist um den Faktor 100 größer als in der Abbildung dargestellt. Dies wurde nötig, um numerische Probleme, die bei besonders kleinen Zellgrößen entstehen, zu umgehen. Für die Berechnung hat dies keine Auswirkungen, weil nur der Wasserstand übergeben wird. Von den Zellen des IImoflood-Modells zu den der Lage entsprechenden Knoten des Feflow-Modells werden Ein-Richtungs-Verbindungen hergestellt. Zwei solcher Zellen-Knoten-Paare sind in Abbildung C.2 gekennzeichnet. Wenn die Zelle des Ilmoflood-Modells nicht trocken ist, dann wird dem Feflow-Modell der Wasserstand als h-Randbedingung übergeben.

Das fertige OpenMI-System ist in Abbildung C.3 zu sehen. Zwischen den OpenMI-kompatiblen Komponenten ist die unidirektionale Verbindung angedeutet, die sich auf 22 Zellen-KnotenPaare bezieht. Das Trigger-Element ist notwendig, um die gekoppelte Berechnung nach dem Frage-und-Antwort-Mechanismus zu initialisieren, denn der Trigger erfragt vom Feflow-Modell ein Berechnungsergebnis, das das Feflow-Modell erst zum Ende der Simulationszeit liefern kann und muss deshalb mehrere Simulationszeitschritte durchlaufen. Für jeden Simulationszeitschritt benötigt es jedoch Berechnungsergebnisse des Ilmoflood-Modells. Eine Rückkopplung, bei der das Ilmoflood-Modell seinerseits wieder Werte vom Feflow-Modell benötigt, ist in diesem Beispiel nicht vorgesehen.

Der Frage-und-Antwort-Mechanismus ist in Abbildung C.4 grafisch dargestellt. Als Ausgangslage wird angenommen, dass das Ilmoflood-Modell für den Simulationszeitpunkt 7600 s die Be- 


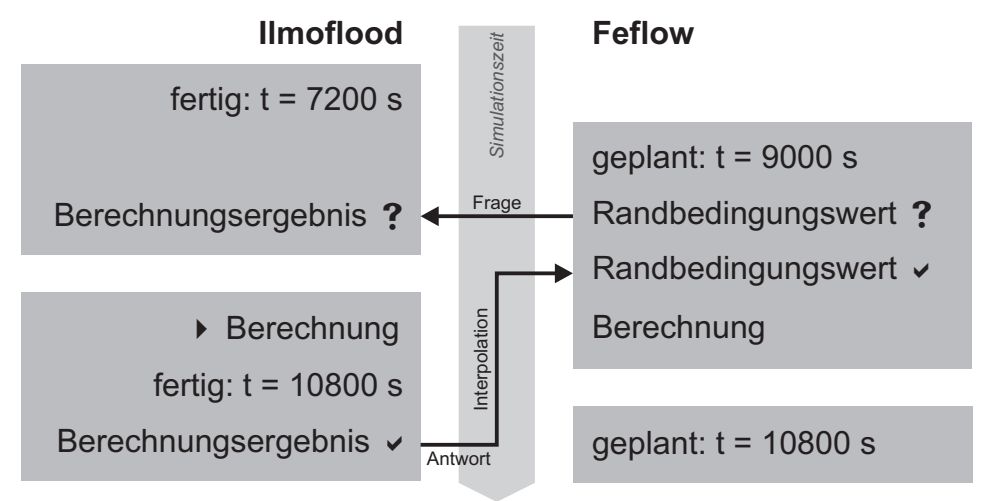

Abbildung C.4: Ablauf des Frage-Antwort-Mechanismus und der Interpolation für die unidirektionale Verbindung

rechnung abgeschlossen hat. Auf die Frage nach der momentanen Simulationszeit über die OpenMI-Funktion GetCurrentTime würde dieses Modell 7200 s zurückgeben. Das Feflow-Modell plant die Berechnung des nächsten Zeitschritts 9000 s und würde als Rückgabewert für den Funktionsaufruf von GetCurrent Time 9000 s zurückgeben, aber anders als llmoflood ist für diesen Zeitschritt noch kein Berechnungsergebnis verfügbar. Für die auszuführende Berechnung benötigt das Feflow-Modell für alle 22 Knoten mit OpenMl-Verbindung jeweils einen Randbedingungswert aus der IImoflood-Berechnung und stellt deshalb über den OpenMI-Schnittstelle eine entsprechende Frage. Für den Zeitpunkt 9000 s kann das Ilmoflood-Modell keinen Wert liefern. Die OpenMI-Umgebung initialisiert durch Aufruf der Funktion PerformTimeStep deshalb den nächsten Zeitschritt. Dieser liegt mit 10800 s im Beispiel zeitlich hinter dem, für den das Feflow-Modell einen Wert angefragt hat. Wäre das nicht der Fall, würde die Ausführung weiterer Berechnungsschritte erforderlich. Die Anfrage des Feflow-Modells wird nun von der OpenMIUmgebung mit einem aus den den Zeitpunkten $7200 \mathrm{~s}$ und $10800 \mathrm{~s}$ zugeordneten Wasserständen für $9000 \mathrm{~s}$ interpolierten Wert beantwortet. Berechnungsergebnisse des vorangegangenen Zeitschrittes sind dazu in einem buffer zwischengespeichert worden. Hat das Feflow-Modell nun den benötigten Randbedingungswert erhalten, kann es die Berechnung des Zeitschrittes $9000 \mathrm{~s}$ ausführen. Für die Berechnung des nächsten Zeitschritts bei 10800 s kann das IImoflood-Modell einen Wert liefern, so dass dafür keine weitere Berechnung notwendig ist.

Die Darstellung der Berechnungsergebnisse ist als Verlauf der Sickerlinie in Abbildung C.5 für verschiedene Simulationszeitpunkte gezeigt. Die Sickerlinie ist hier jeweils die Isolinie für eine Saugspannung mit dem Wert $\psi=0$. Der Anschlusspunkt der Sickerlinie an der Böschung folgt dem Wasserstand, solange dieser steigt. Bei ablaufendem Wasser folgt er dem Wasserstand im Vorland verzögert, weil sich das Wasser an der Oberfläche wesentlich schneller bewegt als im Deich. Das Wasser bewegt sich auch dann noch zur Landseite hin, wenn auf der Wasserseite der Wasserstand schon wieder stark zurückgegangen ist.

In diesem Beispiel hat das Feflow-Modell eine feinere zeitliche Auflösung als das IImofloodModell, so dass nur für jeden zweiten Feflow-Zeitschritt Berechnungsergebnisse des IImofloodModells vorhanden sind. Deshalb wird für die Zeitpunkte des Feflow-Modells, zu denen das IImo- 

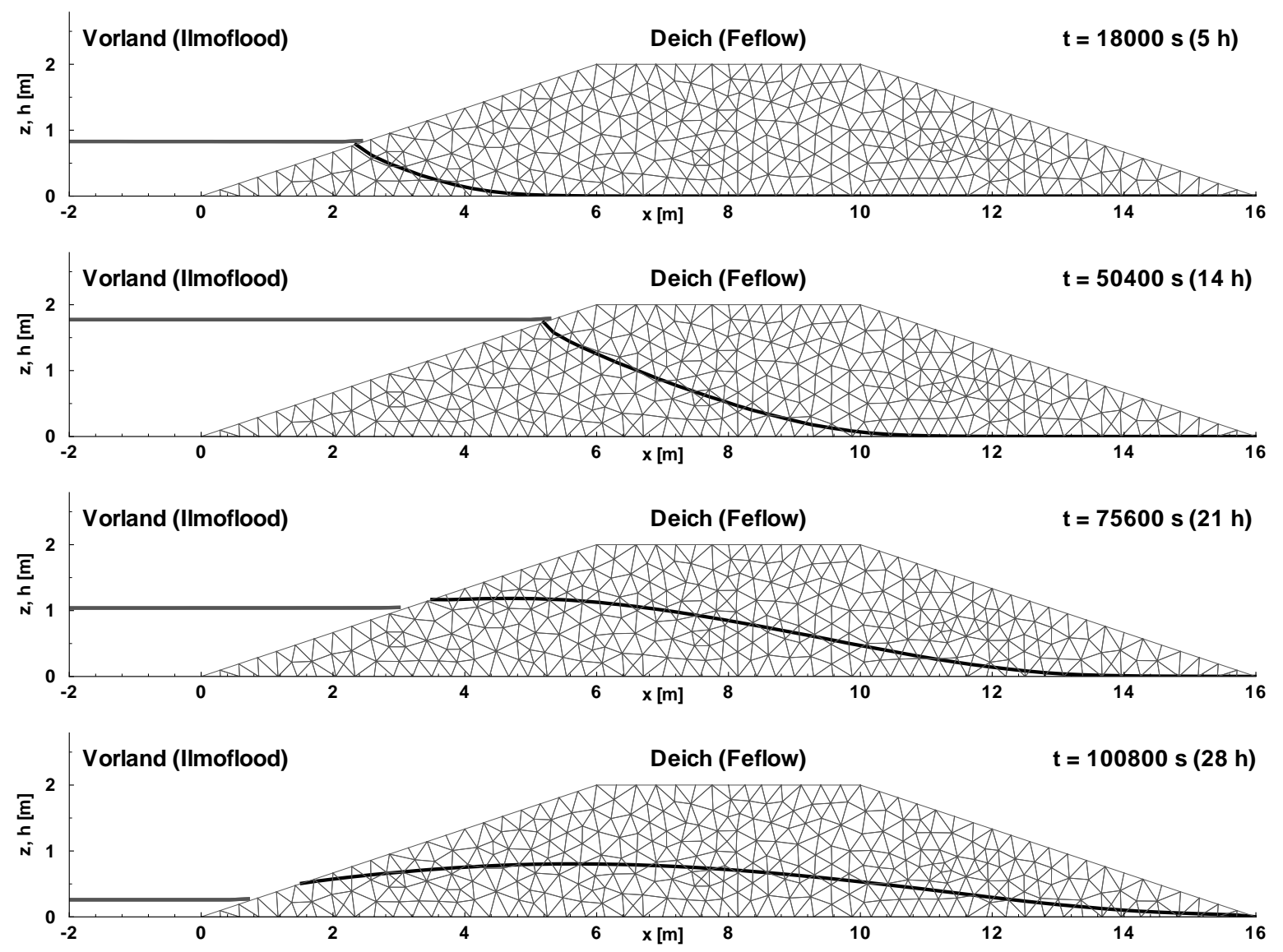

Abbildung C.5: Mit dem OpenMI-System aus einen Feflow-Modell und einem Ilmoflood-Modell berechnete Sickerlinie des Deichquerschnitts und berechneter Wassertand des Vorlandes für verschiedene Zeitpunkte $t$

flood-Modell keinen Wert liefert, eine zeitliche Interpolation durchgeführt. Diese kann anhand Abbildung C.6 nachvollzogen werden. Wenn nur eine Interpolationsgrenze verfügbar ist, weil das Ilmoflood-Modell für die betreffende trockene Zelle keinen Wasserstand ausgibt, wird der verfügbare Wert angesetzt.

Durch die geneigte Böschung des Deiches entsteht für jeden Knoten eine andere Randbedingungszeitreihe. Weil Knoten 17 am Fuß des Deiches liegt, wird hier schon bei einem niedrigem Wasserstand im Vorland ein Wert übergeben (Abbildung C.6b). Knoten 112 dagegen erhält erst dann einen Wert, wenn im Ilmoflood-Modell der Wasserstand bis zur Höhenlage dieses Knotens angestiegen ist und auf Zelle 20 Wasser steht (Abbildung C.6a). Nachdem Zelle 20 wieder trocken gefallen ist, endet die Übergabe des Wasserstands an Feflow-Knoten 112.

Für das gezeigte einfache Beispiel liegt der Nutzen der Modellkopplung darin, dass man sich die Erzeugung der Randbedingungszeitreihen für die einzelnen Knoten spart. Ein weiterer Vor- 


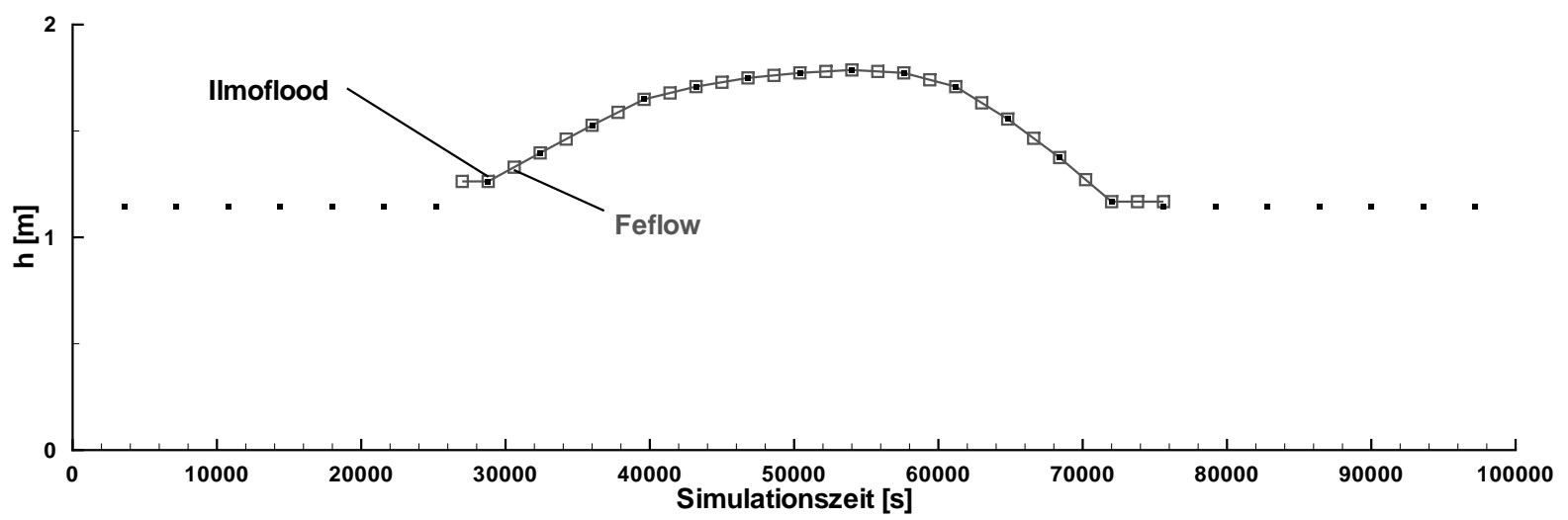

(a) Zelle 20-Knoten 112

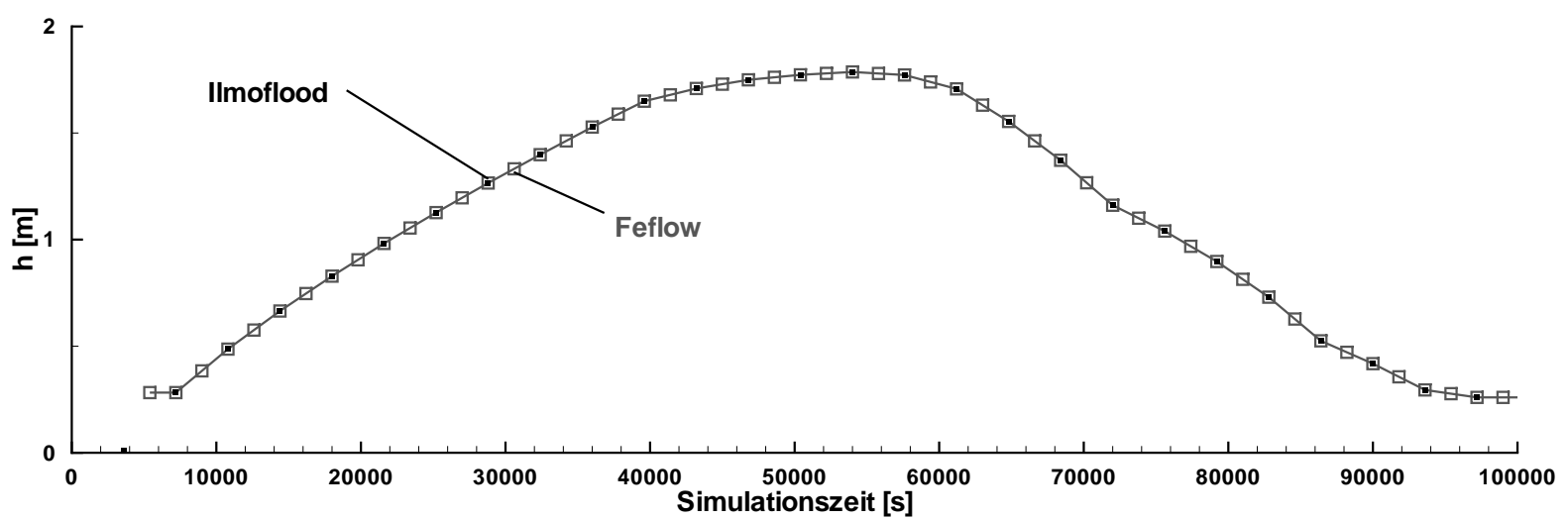

(b) Zelle 8-Knoten 17

Abbildung C.6: Ergebnis des Ilmoflood-Modells und angesetzter Randbedingungswert im Feflow-Modell für zwei verschiedene Zellen-Knoten-Verbindungen

teil liegt in der zeitlichen Interpolation, die von der OpenMI-Umgebung durchgeführt wird und deshalb nicht mehr im Rahmen der Vorbereitungsarbeiten vorgenommen werden muss. 


\section{Datengrundlage zum Rheinhochwasser 1983 im Neuwieder Becken}

(zu Abbildung 5.7 und Abschnitt 5.4.3

Tabelle D.1: Messwerte am Messprofil Urmitz vom April-Hochwasser 1983 nach Ubell (1987a) für Wasserstände des Rheins und der Grundwassermessstellen U01, U03 und U05 in m ü. NN als Tagesmittelwerte, berechnete Druckhöhendifferenzen $\Delta h$ in m und Austauschfluss $Q$ in $1 / \mathrm{s}$ pro km Länge des Flussabschnittes. Wasserstände wurden der Abbildung 4 (Ubell 1987a) entnommen und über die Tage gemittelt, Austauschflüsse stammen aus der bei Ubell (1987a) angegebenen Tabelle.

\begin{tabular}{crrrrrrrr}
\hline Tag & $Q$ & $h_{\text {Rhein }}$ & $h_{\mathrm{U} 01}$ & $\Delta h_{\mathrm{U} 01}$ & $h_{\mathrm{U} 03}$ & $\Delta h_{\mathrm{U} 03}$ & $h_{\mathrm{U} 05}$ & $\Delta h_{\mathrm{U} 05}$ \\
\hline 4 & 158,80 & 58,19 & 58,14 & 0,05 & 58,11 & 0,07 & 58,08 & 0,11 \\
5 & 287,83 & 58,37 & 58,26 & 0,10 & 58,18 & 0,18 & 58,08 & 0,29 \\
6 & 260,02 & 58,55 & 58,38 & 0,17 & 58,24 & 0,31 & 58,08 & 0,47 \\
7 & 498,76 & 58,90 & 58,58 & 0,32 & 58,34 & 0,56 & 58,10 & 0,80 \\
8 & 747,45 & 59,41 & 58,93 & 0,48 & 58,51 & 0,90 & 58,14 & 1,27 \\
9 & 1265,02 & 60,35 & 59,46 & 0,89 & 58,78 & 1,57 & 58,19 & 2,16 \\
10 & 1704,52 & 61,42 & 60,14 & 1,28 & 59,19 & 2,23 & 58,25 & 3,17 \\
11 & 2030,99 & 62,26 & 61,00 & 1,26 & 59,74 & 2,52 & 58,35 & 3,91 \\
12 & 2349,18 & 62,91 & 61,84 & 1,07 & 60,39 & 2,52 & 58,48 & 4,43 \\
13 & 2097,04 & 63,23 & 62,36 & 0,87 & 61,02 & 2,20 & 58,64 & 4,59 \\
14 & 1034,88 & 62,84 & 62,37 & 0,47 & 61,43 & 1,41 & 58,83 & 4,01 \\
15 & 164,50 & 61,82 & 61,81 & 0,01 & 61,42 & 0,40 & 59,03 & 2,79 \\
16 & $-27,16$ & 61,01 & 61,29 & $-0,27$ & 61,20 & $-0,18$ & 59,21 & 1,80 \\
17 & $-175,30$ & 60,51 & 60,92 & $-0,40$ & 60,95 & $-0,43$ & 59,35 & 1,16 \\
18 & $-306,49$ & 60,13 & 60,59 & $-0,45$ & 60,71 & $-0,58$ & 59,44 & 0,68 \\
19 & $-469,02$ & 59,82 & 60,25 & $-0,42$ & 60,47 & $-0,64$ & 59,49 & 0,33 \\
20 & $-407,59$ & 59,54 & 59,95 & $-0,40$ & 60,21 & $-0,66$ & 59,51 & 0,03 \\
21 & $-378,91$ & 59,30 & 59,71 & $-0,40$ & 59,97 & $-0,66$ & 59,52 & $-0,21$ \\
22 & $-405,62$ & 59,12 & 59,52 & $-0,39$ & 59,79 & $-0,66$ & 59,51 & $-0,38$ \\
23 & $-408,36$ & 58,97 & 59,34 & $-0,36$ & 59,63 & $-0,65$ & 59,50 & $-0,52$ \\
24 & $-356,46$ & 58,82 & 59,17 & $-0,34$ & 59,46 & $-0,64$ & 59,48 & $-0,65$ \\
25 & $-353,46$ & 58,65 & 59,00 & $-0,35$ & 59,32 & $-0,66$ & 59,45 & $-0,80$ \\
26 & $-296,75$ & 58,49 & 58,85 & $-0,35$ & 59,19 & $-0,69$ & 59,44 & $-0,94$ \\
\hline & & & & & & & &
\end{tabular}




\section{E Statistische Kennzahlen}

(zu Abschnitt5.4.3 und Abschnitt5.4.4.1)

\section{E.1 Empirischer Korrelationskoeffizient}

Der empirische Korrelationskoeffizient $R$ ist ein Maß für einen linearen Zusammenhang zwischen zwei Datenreihen. Er ist anwendbar auf linear abhängige Merkmale und kann deshalb auch für die Beurteilung der Kalibrierungsgüte verwendet werden. Er erlaubt eine Aussage über die Übereinstimmung der Kurvenverläufe, nicht jedoch eine quantitative Aussage über die Abweichung zwischen gemessenen und berechneten Grundwasserständen. Der Wertebereich für den Korrelationskoeffizienten ist

$$
-1 \leq R \leq 1
$$

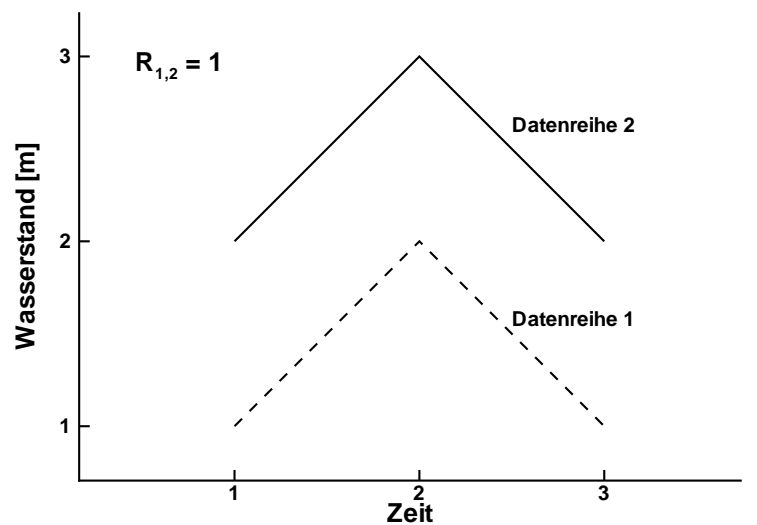

(a) exakte Korrelation mit $R_{1,2}=1$

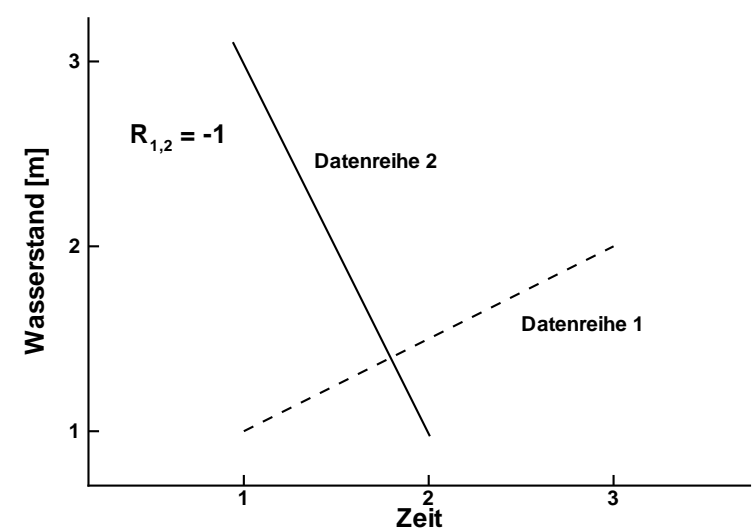

(b) keine Korrelation mit $R_{1,2}=-1$

Abbildung E.1: Beispiele für Korrelationen zweier Datenreihen (nach Jansen 2009)

Abbildung E.1 zeigt ein Beispiel für den Korrelationskoeffizienten. Die beiden Messreihen im linken Teil der Abbildung korrelieren exakt miteinander, $R$ nimmt hier den Wert Eins an. Im rechten Teil dagegen stehen die beiden Messreihen in keiner linearen Abhängigkeit zueinander, $R$ nimmt dann den Wert minus eins an. 
Der Korrelationskoeffizient wird wie folgt berechnet (siehe z. B. Bronstein et al. 1997):

$$
R_{h_{\mathrm{M}}, h_{\mathrm{R}}}=\frac{\frac{1}{n} \sum_{i=1}^{n}\left(h_{\mathrm{M} i}-\bar{h}_{\mathrm{M}}\right)\left(h_{\mathrm{R} i}-\bar{h}_{\mathrm{R}}\right)}{\sigma_{h_{\mathrm{M}}} \cdot \sigma_{h_{\mathrm{R}}}}
$$

Darin ist $h_{\mathrm{M}}$ der gemessene Wasserspiegel (Messwert) und $h_{\mathrm{R}}$ das Ergebnis einer Berechnung. $\sigma$ ist die Standardabweichung und $\bar{h}$ der Mittelwert der Reihen $h_{\mathrm{M}}$ und $h_{\mathrm{R}}$.

Für den Fall der einfachen Regression ist das Quadrat des Korrelationskoeffizienten $R^{2}$ das Bestimmtheitsmaß und gibt den Anteil der Variabilität (Varianz) einer von $X$ abhängigen Variablen $Y$ durch eine lineare Regression der Form

$$
Y_{i}=a+b \cdot X_{i}
$$

an.

\section{E.2 Nash-Sutcliffe-Effizienz}

Die Nash-Sutcliffe-Effizienz NSE (Nash \& Sutcliffe 1970) ist auf Zeitreihen - im vorliegenden Fall die Ganglinien der Grundwassermessstellen - anwendbar und lässt im Gegensatz zum Korrelationskoeffizienten eine quantitative Bewertung der Ergebnisse zu. Sie gibt wieder, wie gut die berechneten Werte die gemessenen Werte abbilden. Der Wertebereich der Nash-SutcliffeEffizienz beträgt

$$
-\infty<N S E<1 .
$$

Bei $N S E=1$ werden die Messwerte exakt durch die berechneten Werte abgebildet. Für negative Nash-Sutcliffe-Effizienzen werden die Messwerte besser durch ihren Mittelwert als durch die Simulationswerte abgebildet. Die Nash-Sutcliffe-Effizienz berechnet sich zu

$$
N S E=1-\frac{\sum_{t=1}^{t_{\mathrm{n}}}\left(h_{\mathrm{M}}^{t}-h_{\mathrm{R}}^{t}\right)^{2}}{\sum_{t=1}^{t_{\mathrm{n}}}\left(h_{\mathrm{M}}^{t}-\bar{h}_{\mathrm{M}}\right)^{2}}
$$

Darin ist $h_{\mathrm{M}}$ der gemessene Wasserspiegel und $h_{\mathrm{R}}$ der simulierte Wasserspiegel. $t$ bezeichnet die Stützstelle einer Zeitreihe mit $n$ Werten. 


\section{Danksagung}

Viele Menschen haben durch ihre Unterstützung zu dieser Arbeit beigetragen. Herr Professor Köngeter hat mich als wissenschaftlichen Mitarbeiter in sein Institut für Wasserbau und Wasserwirtschaft der RWTH (IWW) aufgenommen und mir so die Möglichkeit gegeben, diese Arbeit zu verwirklichen. Professor Schüttrumpf hat die Betreuung der Arbeit übernommen und engagiert weitergeführt. Professor Pirotton nahm mich als Gastwissenschaftler für drei Monate sehr freundlich auf in seine Arbeitsgruppe Hydrologie, Hydrodynamique Appliquée et Constructions Hydrauliques $(\mathrm{HACH})$ an der Université de Liège. Allen drei Professoren danke ich sehr für das Interesse an der Arbeit, die Betreuung, die Begutachtung und die vielen Anregungen.

Mein Dank gilt auch allen Studentinnen und Studenten, deren Arbeit und Ideen über Diplomarbeiten, eine Arbeit im Rahmen des RWTH Undergraduate Research Opportunities Programme, Seminararbeiten und ihre Tätigkeit als studentische Hilfskraft in diese Dissertation, aber auch in andere Projekte, einflossen.

Den Kollegen aus dem Kreis der wissenschaftlichen Mitarbeiterinnen und Mitarbeiter des IWW danke ich für alle Hilfe und die vielen fachlichen Diskussionen zur Forschungs- und Projektarbeit, für alles, was ich von ihnen gelernt habe und die schöne Arbeitsatmosphäre. Auch den Mitarbeitern des HACH danke ich für die freundliche Aufnahme und ebenfalls für alle Hilfe, Fachgespräche und die freundliche und fruchtbare Atmosphäre während meines Aufenthalts dort.

Unterstützung erhielt ich auch von den Mitarbeiterinnen und Mitarbeitern des IWW, die Aufgaben als Systemadministrator, in der Bibliothek, in den Werkstätten der Versuchshalle, im Zeichenbüro oder andere Organisationsaufgaben des IWW übernehmen. Ihnen allen danke ich für die Unterstützung und die vielen Erleichterungen während der Zeit am IWW.

Meiner Familie und meinen Freunden danke ich für die vielen schönen gemeinsamen Stunden und alle Hilfe und Unterstützung, die sie mir zukommen ließen. Meiner Freundin Anke Hauschild danke ich für die liebevolle Zuneigung, aber auch die fachliche Diskussion und Anregungen, die sie mir gegeben hat.

Besonderer Dank gilt den vielen Menschen, die in Form von Fachpublikationen, Vorträgen, Postern, Diskussionen, Telefongesprächen, E-Mail-Schriftverkehr oder im Internet ihr Wissen für diese Arbeit zur Verfügung gestellt haben.

Lüttich, im September 2009

Bernhard Becker 